\title{
Extended Calculations with Spectroscopic Accuracy: Energy Levels and Radiative Rates for O-like Ions between Ar XI and Cr XVII
}

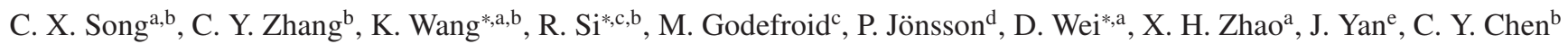 \\ ${ }^{a}$ Hebei Key Lab of Optic-electronic Information and Materials, The College of Physics Science and Technology, Hebei University, Baoding 071002, China; \\ ${ }^{b}$ Shanghai EBIT Lab, Key Laboratory of Nuclear Physics and Ion-beam Application, Institute of Modern Physics, Department of Nuclear Science and Technology, \\ Fudan University, Shanghai 200433, China; \\ ${ }^{c}$ Spectroscopy, Quantum Chemistry and Atmospheric Remote Sensing (SQUARES), CP160/09, Université libre de Bruxelles, Av. F.D. Roosevelt 50, 1050 Brussels, \\ Belgium; \\ ${ }^{d}$ Department of Materials Science and Applied Mathematics, Malmö University, SE-20506, Malmö, Sweden; \\ ${ }^{e}$ Institute of Applied Physics and Computational Mathematics, Beijing 100088, China;

\section{Abstract} \\ Using the multiconfiguration Dirac-Hartree-Fock and the relativistic configuration interaction methods, a consistent set of transition \\ energies and radiative transition data for the main states of the $2 s^{2} 2 p^{4}, 2 s 2 p^{5}, 2 p^{6}, 2 s^{2} 2 p^{3} 3 s, 2 s^{2} 2 p^{3} 3 p, 2 s^{2} 2 p^{3} 3 d, 2 s 2 p^{4} 3 s$, \\ $2 s 2 p^{4} 3 p$, and $2 s 2 p^{4} 3 d$ configurations in O-like Ions between $\operatorname{Ar} \mathrm{XI}(Z=18)$ and $\mathrm{Cr}$ XVII $(Z=24)$ is provided. Our data set is \\ compared with the NIST compiled values and previous calculations. The data are accurate enough for identification and deblending \\ 'of new emission lines from hot astrophysical and laboratory plasmas. The amount of data of high accuracy is significantly increased \\ for the $n=3$ states of several O-like ions, where experimental data are very scarce.
}

${ }^{*}$ Corresponding Author

Email addresses: wang_kai10@fudan.edu.cn (K. Wang),

rsi13@fudan.edu.cn (R. Si), dangwei@hbu.edu.cn (D. Wei) 


\section{Contents}

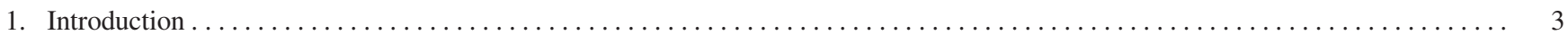

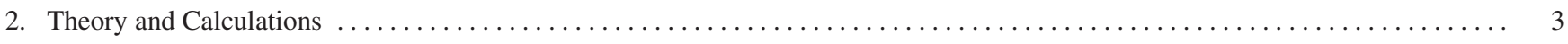

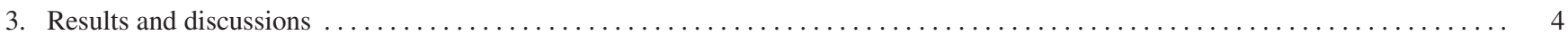

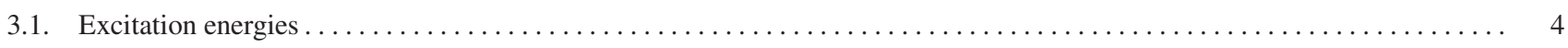

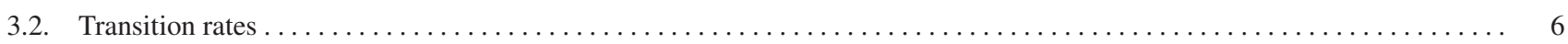

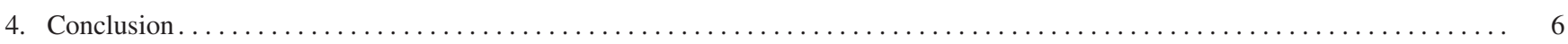

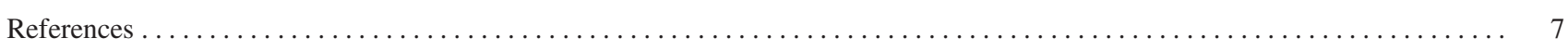

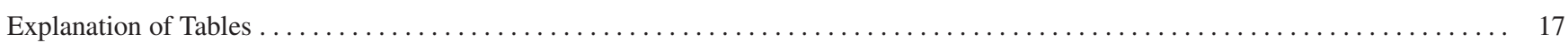

Tables

1. Excitation energies in $\mathrm{cm}^{-1}$ and radiative lifetimes in $\mathrm{s}$ for the lowest $156(179,184,196,200,200,200)$ states of the $n \leq 3$

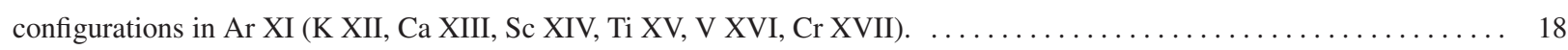

2. Transition wavelengths $\lambda$ (in $\AA$ ), transition rates $A$ (in $\mathrm{s}^{-1}$ ), weighted oscillator strengths $g f$, and line strengths $S$ (in a.u.) between the lowest $156(179,184,196,200,200,200)$ states of the $n \leq 3$ configurations in Ar XI (K XII, Ca XIII, Sc XIV, Ti XV, V XVI, Cr XVII) listed in Table 1. Transitions with the branching fraction (BF) $\geq 10^{-5}$ are presented. 


\section{Introduction}

Oxygen-like ions with nuclear charge numbers from eighteen to twenty four have long been observed in hot astrophysical and laboratory plasmas [1-5]. For example, spectra of Ar XI, Ca XIII, Ti XV, and Cr XVII have been observed in the solar corona and in flares [6-9]. Though strong lines from these ions of astrophysical abundant elements ( $\mathrm{Ar}, \mathrm{Ca}, \mathrm{K}, \mathrm{Ti}$, and $\mathrm{Cr}$ ) are well known, a large fraction of the lines from these ions are unclassified due to the lack of accurate atomic data [5, 10-13]. To remedy this situation, we have reported highly accurate excitation energies and radiative transition rates for L-shell and M-shell atomic ions of the above elements [14-23]. This work extends our effort to accurate atomic data of O-like from Ar XI to $\mathrm{Cr}$ XVII.

Using different methods, excitation energies and/or radiative decay rates of the $\left(1 s^{2}\right) 2 s^{2} 2 p^{4}, 2 s 2 p^{5}$, and $2 p^{6}$ configurations for O-like from $\mathrm{Ar}$ XI to $\mathrm{Cr}$ XVII have been provided [24-32]. Because of wide applications in modeling and diagnosing different kinds of plasmas, and in analyzing new observations of different astrophysical sources, energy structures and radiative transition parameters for higher-lying states of the $n \geq 3$ configurations are eagerly needed [4, 6, 33]. AUTOSTRUCTURE calculations for the lowest 86 levels of the $2 s^{2} 2 p^{4}$, $2 s 2 p^{5}, 2 p^{6}, 2 s^{2} 2 p^{3} 3 s, 2^{2} 2 p^{3} 3 p$, and $2 s^{2} 2 p^{3} 3 d$ configurations in Ar XI and Ca XIII were provided by [34, 35]. Bogdanovich and Karpuškienè [36] reported excitation energies and radiative transition data for the lowest 200 levels of the $n \leq 3$ configurations in Cr XVII using a configuration interaction method. In their calculations, the relativistic effects were taken into account within the traditional BreitPauli approximation.

Among the previous theoretical studies, the results for the $n=2$ levels provided by $\mathrm{Gu}$ [31] and Rynkun et al. [32] are, so far, the most accurate. By contrast, previous calculations [34-36] involving the $n \leq 3$ levels of Ar XI, Ca XIII, and Cr XVII are indeed not accurate enough due to restricted configuration interaction effects included in their works. Excitation energies of the calculations by Landi and Bhatia [34] for Ar XI, by Landi and Bhatia [35] for Ca XIII, and by Bogdanovich and
Karpuškienė [36] for Cr XVII differ from the corresponding experimental values in the Atomic Spectra Database (ASD) of the National Institute of Standards and Technology (NIST) [37] by up to $35000 \mathrm{~cm}^{-1}, 35000 \mathrm{~cm}^{-1}$ and $20000 \mathrm{~cm}^{-1}$, respectively. Therefore, systematic calculations of high accuracy involving the $n \geq 3$ states are greatly desired. Databases such as CHIANTI [38, 39] also demand complete and consistent data sets of high accuracy, with the view of offering the astrophysical community tools and data to carry out accurate plasma diagnostics.

Recently, using the multireference Møller-Plesset (MR-MP) perturbation method [40-42], Santana et al. [43] reported calculated excitation energies for L-shell ions of Argon. In their calculations, they adopted large configuration state function expansions and reported calculated excitation energies with high accuracy, including the $n \leq 3$ levels in Ar XI. Their data can be used to identify observed spectral lines.

The present work provides a consistent data sets of energy structures and radiative transition data with high accuracy for O-like ions in the range of nuclear charges $18 \leq Z \leq 24$. Using the multiconfiguration Dirac-Hartree-Fock (MCDHF) and the relativistic configuration interaction (RCI) methods implemented in the GRASP2K code [44], excitation energies and lifetimes for the main $n \leq 3$ in Ar XI (K XII, Ca XIII, Sc XIV, Ti XV, V XVI, Cr XVII), and multipole transition rates (electric dipole (E1), magnetic dipole (M1), and electric quadrupole (E2)) among these states are calculated. Compared with previous studies of O-like ions, our calculations result in a significant extension of accurate energy and transition data for higher-lying states of the $n=3$ configurations, which will greatly improve the assessment of blending for diagnostic lines of interest, and aid the analysis of new spectra from hot astrophysical and laboratory plasmas.

\section{Theory and Calculations}

The MCDHF method in the GRASP2K code $[44,45]$ is reviewed by Froese Fischer et al. [46]. This method is also described in our recent papers [47-60]. For this reason, in the 
sections below, only the computational strategies are described.

In our MCDHF calculations, the multireference (MR) sets for even and odd parities include

even configurations: $2 s^{2} 2 p^{4}, 2 p^{6}, 2 s^{2} 2 p^{3} 3 p, 2 s 2 p^{4} 3 s$, $2 s 2 p^{4} 3 d, 2 p^{5} 3 p, 2 s^{2} 2 p^{3} 4 p, 2 s^{2} 2 p^{3} 4 f$;

odd configurations: $2 s 2 p^{5}, 2 s^{2} 2 p^{3} 3 s, 2 s^{2} 2 p^{3} 3 d, 2 s 2 p^{4} 3 p$, $2 p^{5} 3 s, 2 p^{5} 3 d, 2 s^{2} 2 p^{3} 4 s, 2 s^{2} 2 p^{3} 4 d$.

We start the calculation without any excitation from the MR configurations, which is usually referred to as the Dirac-Fock (DF) calculation. Based on the active space (AS) approach [61] for the generation of the configuration state function (CSF) expansions, separate MCDF calculations are done for the even and odd parity states. Subsequently, the CSFs expansions are obtained through the single and double (SD) substitutions from subshells of the reference configurations up to a $n l$ orbital, with $n \leq 8$ and $l \leq 5$. To reduce the number of CSFs, the $1 s^{2}$ core is closed during the relativistic self-consistent field (RSCF) calculations, but is opened during the following RCI calculations, where the Breit interaction and the leading QED effects, i.e., vacuum polarization and self- energy, are included in the Hamiltonian. The number of CSFs in the final even and odd state expansions are approximately 1796000 and 3938000 , respectively, distributed over the different $J$ symmetries.

By using the $j j-L S J$ transformation approach [62, 63], the $j j$-coupled CSFs are transformed into $L S J$-coupled CSFs, from which the $L S J$ labels used by experimentalists are obtained.

\section{Results and discussions}

\subsection{Excitation energies}

Our MCDHF/RCI excitation energies $E_{\mathrm{MCDHF} / \mathrm{RCI}}\left(\mathrm{cm}^{-1}\right)$, together with MCDHF/RCI radiative lifetimes $\tau_{\mathrm{MCDHF} / \mathrm{RCI}}^{l}$ (in s) in the length form and $\tau_{\mathrm{MCDHF} / \mathrm{RCI}}^{v}$ (in s) in the velocity form, are reported in Table 1 for the lowest 156 (179, 184, 196, 200, 200, 200) states of the $2 s^{2} 2 p^{4}, 2 s 2 p^{5}, 2 p^{6}, 2 s^{2} 2 p^{3} 3 s, 2 s^{2} 2 p^{3} 3 p$, $2 s^{2} 2 p^{3} 3 d, 2 s 2 p^{4} 3 s, 2 s 2 p^{4} 3 p$, and $2 s 2 p^{4} 3 d$ configurations in Ar XI (K XII, Ca XIII, Sc XIV, Ti XV, V XVI, Cr XVII). All these states are below the first $2 s^{2} 2 p^{3} 4 s$ level. Observed values
$E_{\mathrm{NIST}}\left(\mathrm{cm}^{-1}\right)$ from the NIST ASD [37] are also listed in this table. As many levels are strongly mixed, their configuration label is not unique. Here, the parity, $J$ value and energy are used to match the levels in the NIST ASD. The levels in which our $L S J$-coupled labels are different from those in the NIST ASD are listed in Table A for reference.

Experimental and theoretical energy data are available for all $n=2$ levels along the isoelectronic sequence from Ar XI to $\mathrm{Cr}$ XVII. Computed excitation energies of the $n=2$ levels from the present MCDHF/RCI calculations, from the MCDHF/RCI calculations by Rynkun et al. [32] [hereafter referred to as MCDHF/RCI2], from the many-body perturbation theory (MBPT) calculations by Gu [31] [MBPT], from the SUPERSTRUCTURE calculations by Landi [30] [SS], are compared with experimental values from the NIST ASD [37] in Table B. Compared with the SS calculations, the present MCDHF/RCI calculations, as well as the MCDHF/RCI2 and MBPT calculations show better agreement with the NIST experimental values. The average absolute difference with the standard deviation [18] between computed excitation energies and the NIST values for all $n=2$ levels from Ar XI to Cr XIV are $4 \pm 197 \mathrm{~cm}^{-1}$ for MCDHF/RCI, $252 \pm 242 \mathrm{~cm}^{-1}$ for MCDHF/RCI2, and $-421 \pm 940 \mathrm{~cm}^{-1}$ for MBPT. The corresponding values for SS are $9154 \pm 10208 \mathrm{~cm}^{-1}$.

Among the seven O-like ions considered here, theoretical energy data of the lowest 200 levels up to the $n=3$ configurations for Cr XVII were provided by Bogdanovich and Karpuškienė [36] using a configuration interaction method. In our previous work, we also performed the calculations for the lowest 200 levels employing two state-of-the-art methods, MCDHF/RCI and MBPT. In what follows we will further access the accuracy of the present MCDHF/RCI excitation energies in Cr XVII, by comparing available results. In Table C, we present the MCDHF/RCI excitation energies of the lowest 200 levels for Cr XVII. Observed energies from the NIST ASD and computed values from different sources (MCDHF/RCI3: the MCDHF/RCI results from Wang et al. [18]; MBPT2: the MBPT results from Wang et al. [18]; CI: theoretical results 
from Bogdanovich and Karpuškienė [36]) are also included. As can be seen in Table $\mathrm{C}$, good agreement is obtained among the MCDHF/RCI, MCDHF/RCI3 and MBPT calculations for the lowest 200 levels. The average absolute difference with the standard deviation between computed excitation energies and the MCDHF/RCI values for all the $n \leq 3$ levels are $821 \pm 274 \mathrm{~cm}^{-1}$ for MCDHF/RCI3, and $202 \pm 497 \mathrm{~cm}^{-1}$ for MBPT2. This corresponds to an average relative difference of $0.014 \% \pm 0.009 \%$ for MCDHF/RCI3 and $0.002 \% \pm 0.018 \%$ for MBPT2, respectively, where the standard deviations are indicated after the values. The CI excitation energies from Bogdanovich and Karpuškienè [36] are generally higher than our MCDHF/RCI values by ten to twenty of thousand $\mathrm{cm}^{-1}$ for the $n=3$ levels. The average absolute difference with the standard deviation from the present MCDHF/RCI values are $15267 \pm 4016 \mathrm{~cm}^{-1}$ for the CI calculations.

Compiled values from the NIST ASD are also questionable for a few $n=3$ states of Cr XVII, for which the deviations from the present results are about or larger than 10000 $\mathrm{cm}^{-1}$. For example, the values $6074000 \mathrm{~cm}^{-1}$ for the level $2 s^{2} 2 p^{3}\left({ }_{3}^{2} D\right)^{2} D 3 d^{3} D_{3}^{\circ}$ with the key (\#68), $6131000 \mathrm{~cm}^{-1}$ for the level \#80/2 $s^{2} 2 p^{3}\left({ }_{1}^{2} P\right)^{2} P 3 d^{3} P_{2}^{\circ}$, and $6164800 \mathrm{~cm}^{-1}$ for the level \#87/2s $2 s^{2} p^{3}\left({ }_{1}^{2} P\right)^{2} P 3 d^{3} D_{3}^{\circ}$ do not have obvious counterparts in the present MCDHF/RCI calculations, as well as in the previous MCDHF/RCI3 and MBPT2 calculations.

The NIST compiled values differ from our MCDHF results by over $3000 \mathrm{~cm}^{-1}$ (about $0.1 \%$ ) for many states of seven Olike ions considered in our work, and all these values have been listed in Table D. As shown in our studies [14-18, 21, 22, $56,64]$, the plot of differences between calculated and measured excitation energies along the isoelectronic sequence is an useful tool to identify the possible of measured values with large uncertainty. As an example, we show the differences (in $\mathrm{cm}^{-1}$ ) between the present MCDHF/RCI excitation energies and the NIST values for three levels $\left(2 s^{2} 2 p^{3}\left({ }_{1}^{2} P\right){ }^{2} P 3 s^{1} P_{1}^{\circ}\right.$, $2 s^{2} 2 p^{3}\left({ }_{1}^{2} P\right){ }^{2} P 3 d^{3} D_{1}^{\circ}$, and $\left.2 s^{2} 2 p^{3}\left({ }_{1}^{2} P\right){ }^{2} P 3 d^{3} D_{3}^{\circ}\right)$ as a function of $Z$ with $Z=18-24$ in Figure 1 . The differences for the NIST values from the present MCDHF results are about -

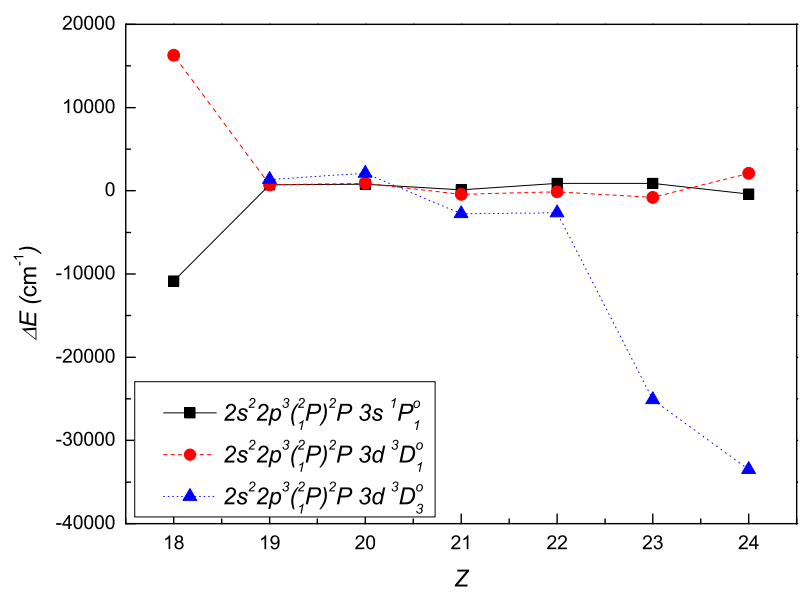

Fig. 1: Energy deviations $\Delta E=E_{\mathrm{NIST}}-E_{\mathrm{MCDHF} / \mathrm{RCI}}$ in $\mathrm{cm}^{-1}$ as a function of $Z$ for three levels $\left(2 s^{2} 2 p^{3}\left({ }_{1}^{2} P\right)^{2} P 3 s^{1} P_{1}^{\circ}, 2 s^{2} 2 p^{3}\left({ }_{1}^{2} P\right)^{2} P 3 d^{3} D_{1}^{\circ}\right.$, and $\left.2 s^{2} 2 p^{3}\left({ }_{1}^{2} P\right)^{2} P 3 d^{3} D_{3}^{\circ}\right)$.

$10000 \mathrm{~cm}^{-1}$ for the level $2 s^{2} 2 p^{3}\left({ }_{1}^{2} P\right)^{2} P 3 s^{1} P_{1}^{\circ}$ in Ar XI, about $15000 \mathrm{~cm}^{-1}$ for the level $2 s^{2} 2 p^{3}\left({ }_{1}^{2} P\right)^{2} P 3 d^{3} D_{1}^{\circ}$ in Ar XI, and $35000 \mathrm{~cm}^{-1}--25000 \mathrm{~cm}^{-1}$ for the level $2 s^{2} 2 p^{3}\left({ }_{1}^{2} P\right)^{2} P 3 d^{3} D_{3}^{\circ}$ in V XVI and Cr XVII. Whereas good agreement is obtained for the same levels in the other ions along the isoelectronic sequence. Since in the present MCDHF/RCI calculations the same computational strategies are used for each ion, the accuracy of our calculated excitation energies should be consistent and systematic for the same level along the sequence.

We also showed the deviations of the NIST excitation energies to the MCDHF/RCI3 results for two levels $2 s^{2} 2 p^{3}\left({ }_{3}^{4} S\right){ }^{4} S 3 d^{3} D_{3}^{\circ}$ and $2 s^{2} 2 p^{3}\left({ }_{3}^{2} D\right){ }^{2} D 3 d^{3} D_{3}^{\circ}$ of O-like ions as a function of the nuclear charge $Z$ with $Z=24-30$ in Figure 1 (a) of our previous work [18]. The deviations between the MBPT2 and MCDHF/RCI3 values for the same levels along the sequence were shown in Figure 1 (b) of our previous work. A few of the NIST compiled values, including excitation energies for the two levels $2 s^{2} 2 p^{3}\left({ }_{3}^{4} S\right){ }^{4} S 3 d^{3} D_{3}^{\circ}$ and $2 s^{2} 2 p^{3}\left({ }_{3}^{2} D\right)^{2} D 3 d^{3} D_{3}^{\circ}$ of Cr XVII listed in Table D, depart from the MCDHF/RCI3 data by over than $4000 \mathrm{~cm}^{-1}$, while good agreement is obtained between the computed MCDHF/RCI3 and MBPT2 data sets. Moreover the differences 
of two theoretical data sets vary smoothly along the isoelectronic sequence. Therefore, these values compiled by the NIST ASD, which have been listed in Table $\mathrm{D}$, seem to be wrong or at least are affected by large errors.

Recently, using the MR-MP method Santana et al. [43] reported calculated excitation energies for L-shell ions of Argon, including the $n \leq 3$ levels in Ar XI. Compared with the NIST experimental results (excluding seven cases for Ar XI in Table D), both the present MCDHF/RCI calculations and the MRMP calculations show good agreements. The average absolute difference with the standard deviation between computed excitation energies and the NIST values for the $n \leq 3$ levels in Ar XI are $-97 \pm 732 \mathrm{~cm}^{-1}$ for MCDHF/RCI, and $-221 \pm 886 \mathrm{~cm}^{-1}$ for MR-MP. High accuracy is achieved in both the MCDHF/RCI and MR-MP calculations.

\subsection{Transition rates}

Wavelengths $\lambda_{i j}$, transition rates $A_{j i}$, and branching fractions $\left(\mathrm{BF}_{j i}=A_{j i} / \sum_{k=1}^{j-1} A_{j k}\right)$ involving all levels considered in the present MCDHF/RCI calculations, as reported in Table 1, along with line strength $S_{j i}$ and weighted oscillator strengths $g f_{j i}$, are provided in Table 2. E1 and E2 transition data in both length $(l)$ and velocity $(v)$ forms are given. For E1 and E2 transitions, we provide (last column) the uncertainty estimations of line strengths $S$ adopting the NIST ASD [37] terminology (AA $\leq 1 \%, \mathrm{~A}^{+} \leq 2 \%, \mathrm{~A} \leq 3 \%, \mathrm{~B}^{+} \leq 7 \%, \mathrm{~B} \leq 10 \%, \mathrm{C}^{+} \leq 18 \%$, $\mathrm{C} \leq 25 \%, \mathrm{D}^{+} \leq 40 \%, \mathrm{D} \leq 50 \%$, and $\mathrm{E}>50 \%$ ) and using the method proposed by [65]. For each E1 transition, the deviation $\delta S$ of line strengths $S_{l}$ in the length form and $S_{v}$ in the velocity form is defined as $\delta S=\left|S_{v}-S_{l}\right| / \max \left(S_{v}, S_{l}\right)$. In various ranges of $S$, the averaged uncertainties $\delta S_{a v}$ of $\delta S$ for E1 transitions in Cr XVII are assessed to $0.5 \%$ for $S \geq 10^{0}$; $0.7 \%$ for $10^{0}>S \geq 10^{-1} ; 1.3 \%$ for $10^{-1}>S \geq 10^{-2} ; 3.3 \%$ for $10^{-2}>S \geq 10^{-3} ; 6.7 \%$ for $10^{-3}>S \geq 10^{-4}, 11 \%$ for $10^{-4}>S \geq 10^{-5}, 15 \%$ for $10^{-5}>S \geq 10^{-6}, 28 \%$ for $10^{-6}>S \geq 10^{-7}$. Then, the largest of $\delta S_{a v}$ and $\delta S_{i j}$ is considered to be the uncertainty of each particular transition.

In the spirit of the uncertainty estimation approach $[65,66]$, the estimated uncertainties of line strengths $S$ for E2 transitions in Cr XVII are estimated, as well as those for E1 and E2 transitions in Ar XI, K XII, Ca XIII, Sc XIV, Ti XV, and V XVI. The estimated uncertainties for all E1 and E2 transitions with $\mathrm{BF} \geq 10^{-5}$ in Ar XI, K XII, Ca XIII, Sc XIV, Ti XV, V XVI, and Cr XVII, are listed in Table 2.

Our MCDHF/RCI radiative lifetimes $\tau_{\mathrm{MCDHF} / \mathrm{RCI}}^{l}$ (in s) in the length form and $\tau_{\mathrm{MCDHF} / \mathrm{RCI}}^{v}$ (in s) in the velocity form, for the lowest $156(179,184,196,200,200,200)$ states of the $n \leq 3$ configurations in Ar XI (K XII, Ca XIII, Sc XIV, Ti XV, V XVI, Cr XVII), which are calculated by considering all possible E1, E2, M1, and M2 transitions, are provided in Table 1. Our MCDHF/RCI radiative lifetimes $\tau_{\mathrm{MCDHF} / \mathrm{RCI}}^{l}$ and $\tau_{\text {MCDHF/RCI }}^{v}$ show good agreement. For example, the average deviation between $\tau_{\mathrm{MCDHF} / \mathrm{RCI}}^{l}$ and $\tau_{\mathrm{MCDHF} / \mathrm{RCI}}^{v}$ for all 200 levels in Cr XVII is $1 \%$.

\section{Conclusion}

Employing a state-of-the-art method, MCDHF/RCI, the excitation energies and lifetimes of the lowest $156(179,184,196$, $200,200,200)$ states of the $n \leq 3$ configurations in Ar XI (K XII, Ca XIII, Sc XIV, Ti XV, V XVI, Cr XVII), have been calculated. Wavelengths, line strengths, transition rates, and oscillator strengths for the E1, M1, and E2 transitions with BF larger than $10^{-5}$ are also reported.

Our detailed discussion of excitation energies of these seven O-like ions have highlighted several discrepancies in the experimental energies in the NIST ASD. Those levels compiled by the NIST ASD have been listed in Table D. The above comparisons clearly show the importance of the present ab initio calculations to assess the correctness of level and line identifications. Further experimental work is encouraged to confirm our suggestions. We believe the present data could serve as benchmarks in future line identifications, and could make important contributions to modeling and diagnosing hot astrophyscial and laboratory plasmas. 


\section{Acknowledgments}

We acknowledge the support from the National Key Research and Development Program of China under Grant No. 2017YFA0403200, the Science Challenge Project of China Academy of Engineering Physics (CAEP) under Grant No. TZ2016005, the National Natural Science Foundation of China (Grant No. 11703004, No. 11674066, No. 11504421, and No. 11734013), the Natural Science Foundation of Hebei Province, China (A2019201300), and the Natural Science Foundation of Educational Department of Hebei Province, China (BJ2018058). This work is also supported by the Fonds de la Recherche Scientifique - (FNRS) and the Fonds Wetenschappelijk Onderzoek - Vlaanderen (FWO) under EOS Project $\mathrm{n}^{\mathrm{o}} \mathrm{O} 022818 \mathrm{~F}$, and by the Swedish research council under contracts 2015-04842 and 2016-04185. Kai Wang expresses his gratitude to the support from the visiting researcher program at the Fudan University.

\section{References}

[1] W. E. Behring, L. Cohen, U. Feldman, The Astrophysical Journal 175 (1972) 493.

[2] D. L. McKenzie, P. B. Landecker, The Astrophysical Journal 254 (1982) 309-317.

[3] U. Feldman, W. Curdt, G. A. Doschek, U. Schühle, K. Wilhelm, P. Lemaire, The Astrophysical Journal 503 (1998) 467-474.

[4] U. Feldman, E. Landi, W. Curdt, The Astrophysical Journal 607 (2004) 1039-1045.

[5] E. Träbert, P. Beiersdorfer, J. K. Lepson, M. L. Reinke, J. E. Rice, Astrophy. J. 865 (2018) 148.

[6] U. Feldman, W. Curdt, E. Landi, K. Wilhelm, The Astrophysical Journal 544 (2000) 508.

[7] W. Curdt, E. Landi, U. Feldman, Astronomy and Astrophysics 427 (2004) 1045-1054.

[8] G. Del Zanna, Astronomy and Astrophysics 537 (2012) A38.

[9] G. Del Zanna, E. E. DeLuca, The Astrophysical Journal 852 (2018) 52.

[10] P. Beiersdorfer, E. Träbert, J. K. Lepson, N. S. Brickhouse, L. Golub, The Astrophysical Journal 788 (2014) 25.

[11] E. Träbert, P. Beiersdorfer, N. S. Brickhouse, L. Golub, The Astrophysical Journal Supplement Series 215 (2014) 6.
[12] E. Träbert, P. Beiersdorfer, N. S. Brickhouse, L. Golub, The Astrophysical Journal Supplement Series 211 (2014) 14.

[13] P. Beiersdorfer, E. Träbert, The Astrophysical Journal 854 (2018) 114.

[14] K. Wang, D. F. Li, H. T. Liu, X. Y. Han, B. Duan, C. Y. Li, J. G. Li, X. L. Guo, C. Y. Chen, J. Yan, The Astrophysical Journal Supplement Series 215 (2014) 26.

[15] K. Wang, X. L. Guo, H. T. Liu, D. F. Li, F. Y. Long, X. Y. Han, B. Duan, J. G. Li, M. Huang, Y. S. Wang, R. Hutton, Y. M. Zou, J. L. Zeng, C. Y. Chen, J. Yan, The Astrophysical Journal Supplement Series 218 (2015) 16.

[16] K. Wang, R. Si, W. Dang, P. Jönsson, X. L. Guo, S. Li, Z. B. Chen, H. Zhang, F. Y. Long, H. T. Liu, D. F. Li, R. Hutton, C. Y. Chen, J. Yan, The Astrophysical Journal Supplement Series 223 (2016) 3.

[17] K. Wang, Z. B. Chen, R. Si, P. Jönsson, J. Ekman, X. L. Guo, S. Li, F. Y. Long, W. Dang, X. H. Zhao, R. Hutton, C. Y. Chen, J. Yan, X. Yang, The Astrophysical Journal Supplement Series 226 (2016) 14.

[18] K. Wang, P. Jönsson, J. Ekman, G. Gaigalas, M. R. Godefroid, R. Si, Z. B. Chen, S. Li, C. Y. Chen, J. Yan, The Astrophysical Journal Supplement Series 229 (2017) 37.

[19] K. Wang, P. Jönsson, G. Gaigalas, L. Radžiūtė, P. Rynkun, G. Del Zanna, C. Y. Chen, The Astrophysical Journal Supplement Series 235 (2018) 27.

[20] K. Wang, C. X. Song, P. Jönsson, G. Del Zanna, S. Schiffmann, M. Godefroid, G. Gaigalas, X. H. Zhao, R. Si, C. Y. Chen, J. Yan, The Astrophysical Journal Supplement Series 239 (2018) 30.

[21] K. Wang, P. Jönsson, G. D. Zanna, M. Godefroid, Z. B. Chen, C. Y. Chen, J. Yan, The Astrophysical Journal Supplement Series 246 (2020) 1.

[22] R. Si, S. Li, X. L. Guo, Z. B. Chen, T. Brage, P. Jönsson, K. Wang, J. Yan, C. Y. Chen, Y. M. Zou, The Astrophysical Journal Supplement Series 227 (2016) 16.

[23] R. Si, C. Y. Zhang, Z. Y. Cheng, K. Wang, P. Jönsson, K. Yao, M. F. Gu, C. Y. Chen, The Astrophysical Journal Supplement Series 239 (2018) 3.

[24] K. Cheng, Y. K. Kim, J. Desclaux, Atomic Data and Nuclear Data Tables 24 (1979) $111-189$.

[25] C. Froese Fischer, H. P. Saha, Physical Review A 28 (1983) 3169-3178.

[26] K. L. Baluja, C. J. Zeippen, Journal of Physics B Atomic Molecular Physics 21 (1988) 1455-1471.

[27] G. Gaigalas, J. Kaniauskas, R. Kisielius, G. Merkelis, M. J. Vilkas, Physica Scripta 49 (1994) 135-147.

[28] U. I. Safronova, A. S. Shlyaptseva, Physica Scripta 60 (1999) 36-45.

[29] H. L. Zhang, D. H. Sampson, Atomic Data and Nuclear Data Tables 82 (2002) 357-389.

[30] E. Landi, Astronomy and Astrophysics 434 (2005) 365-376. 
[31] M. F. Gu, Atomic Data and Nuclear Data Tables 89 (2005) 267 - 293.

[32] P. Rynkun, P. Jönsson, G. Gaigalas, C. Froese Fischer, Astronomy \& Astrophysics 557 (2013) A136.

[33] S. V. Shestov, S. A. Bozhenkov, I. A. Zhitnik, S. V. Kuzin, A. M. Urnov, I. L. Beigman, F. F. Goryaev, I. Y. Tolstikhina, Astronomy Letters 34 (2008) 33-51.

[34] E. Landi, A. Bhatia, Atomic Data and Nuclear Data Tables 92 (2006) 305 -374 .

[35] E. Landi, A. K. Bhatia, Astronomy and Astrophysics 444 (2005) 305317.

[36] P. Bogdanovich, R. Karpuškienè, Atomic Data and Nuclear Data Tables 94 (2008) 623-649.

[37] A. Kramida, Yu. Ralchenko, J. Reader, and NIST ASD Team, NIST Atomic Spectra Database (ver. 5.6.1), [Online]. Available: https://physics.nist.gov/asd [2019, May 27]. National Institute of Standards and Technology, Gaithersburg, MD., 2018.

[38] K. P. Dere, G. Del Zanna, P. R. Young, E. Landi, R. S. Sutherland, The Astrophysical Journal Supplement Series 241 (2019) 22.

[39] K. P. Dere, E. Landi, H. E. Mason, B. C. Monsignori Fossi, P. R. Young, Astronomy and Astrophysics Supplement Series 125 (1997) 149-173.

[40] J. A. Santana, N. A. Lopez-Dauphin, P. Beiersdorfer, The Astrophysical Journal Supplement Series 234 (2018) 13.

[41] J. A. Santana, E. L. Peña-Cotto, E. J. M. Butler, P. Beiersdorfer, G. V. Brown, The Astrophysical Journal Supplement Series 245 (2019) 9.

[42] J. A. Santana, Y. Ishikawa, P. Beiersdorfer, The Astrophysical Journal Supplement Series 247 (2020) 52.

[43] J. A. Santana, N. A. Lopez-Dauphin, E. J. M. Butler, P. Beiersdorfer, The Astrophysical Journal Supplement Series 238 (2018) 34.

[44] P. Jönsson, G. Gaigalas, J. Bieroń, C. Froese Fischer, I. P. Grant, Computer Physics Communications 184 (2013) 2197-2203.

[45] P. Jönsson, X. He, C. Froese Fischer, I. Grant, Computer Physics Communications 177 (2007) $597-622$.

[46] C. Froese Fischer, M. Godefroid, T. Brage, P. Jönsson, G. Gaigalas, J. Phys. B: At. Mol. Opt. Phys. 49 (2016) 182004.

[47] K. Wang, X. Yang, Z. B. Chen, R. Si, C. Y. Chen, J. Yan, X. H. Zhao, W. Dang, Atomic Data and Nuclear Data Tables 117-118 (2017) 1-173.

[48] K. Wang, Z. B. Chen, C. Y. Chen, J. Yan, W. Dang, X. H. Zhao, X. Yang, Atomic Data and Nuclear Data Tables 117-118 (2017) 174-319.

[49] K. Wang, C. Y. Zhang, R. Si, S. Li, Z. B. Chen, X. H. Zhao, C. Y. Chen, J. Yan, Atomic Data and Nuclear Data Tables 123 (2018) 114-167.

[50] K. Wang, W. Zheng, X. H. Zhao, Z. B. Chen, C. Y. Chen, J. Yan, Journal of Quantitative Spectroscopy and Radiative Transfer 236 (2019) 106586.
[51] K. Wang, Z. B. Chen, X. H. Zhao, C. Y. Chen, J. Yan, Journal of Quantitative Spectroscopy and Radiative Transfer 237 (2019) 106640.

[52] K. Wang, P. Jönsson, J. Ekman, T. Brage, C. Y. Chen, C. Froese Fischer, G. Gaigalas, M. Godefroid, Physical Review Letters 119 (2017) 189301.

[53] Y. Li, R. Si, J. Li, C. Zhang, K. Yao, K. Wang, M. Gu, C. Chen, Atomic Data and Nuclear Data Tables 133-134 (2020) 101339.

[54] J. Li, C. Zhang, R. Si, K. Wang, C. Chen, Atomic Data and Nuclear Data Tables 126 (2019) 158-294.

[55] Z. Zhao, K. Wang, S. Li, R. Si, C. Y. Chen, Z. B. Chen, J. Yan, Yu. Ralchenko, Atomic Data and Nuclear Data Tables 119 (2018) 314-353.

[56] C. X. Song, K. Wang, G. D. Zanna, P. Jönsson, R. Si, M. Godefroid, G. Gaigalas, L. Radžiūtė, P. Rynkun, X. H. Zhao, J. Yan, C. Y. Chen, The Astrophysical Journal Supplement Series 247 (2020) 70.

[57] Z.-B. Chen, Y.-S. Tian, C.-C. Sang, X.-L. Wang, K. Wang, X.-L. Guo, Atomic Data and Nuclear Data Tables 129-130 (2019) 101278.

[58] Z. B. Chen, X. L. Guo, K. Wang, Journal of Quantitative Spectroscopy and Radiative Transfer 206 (2018) 213-232.

[59] Z. B. Chen, K. Ma, H. J. Wang, K. Wang, X. B. Liu, J. L. Zeng, Atomic Data and Nuclear Data Tables 113 (2017) 258-292.

[60] Z. B. Chen, K. Wang, Atomic Data and Nuclear Data Tables 114 (2017) 61-261.

[61] L. Sturesson, P. Jönsson, C. Froese Fischer, Computer Physics Communications 177 (2007) 539-550.

[62] G. Gaigalas, C. Froese Fischer, P. Rynkun, P. Jönsson, Atoms 5 (2017) 6.

[63] G. Gaigalas, T. Zalandauskas, S. Fritzsche, Computer Physics Communications 157 (2004) 239-253.

[64] K. Wang, Z. B. Chen, C. Y. Zhang, R. Si, P. Jönsson, H. Hartman, M. F. Gu, C. Y. Chen, J. Yan, The Astrophysical Journal Supplement Series 234 (2018) 40.

[65] A. Kramida, The Astrophysical Journal Supplement Series 212 (2014) 11.

[66] A. Kramida, Fusion Science and Technology 63 (2013) 313-323.

[67] E. B. Saloman, Journal of Physical and Chemical Reference Data 39 (2010) 033101.

[68] B. C. Fawcett, R. W. Hayes, Monthly Notices of the Royal Astronomical Society 170 (1975) 185-197.

[69] J. Sugar, C. Corliss, Journal of Physical and Chemical Reference Data 14 (1985) $1-664$. 


\section{Tables}

Table A

The levels in Table 1 for which our $L S J$-coupled labels are different from those in the NIST ASD.

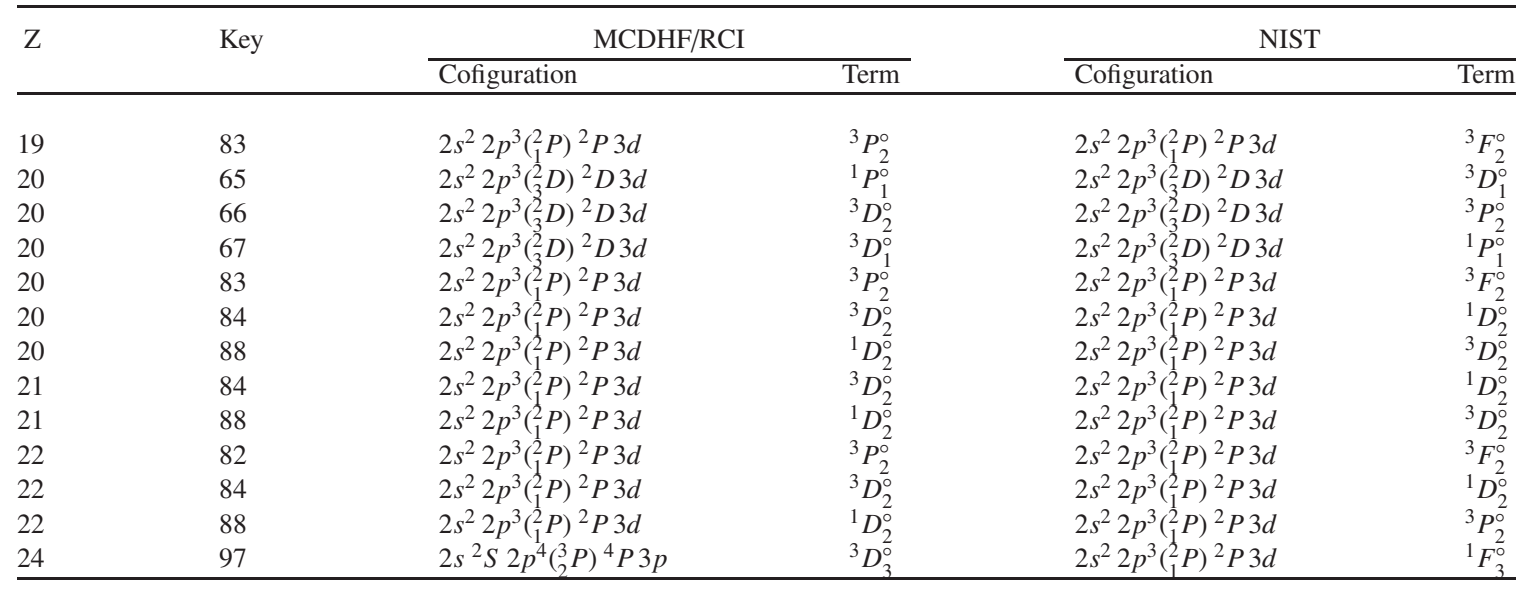


Table B

Comparison of the present MCDHF/RCI excitation energies (in $\mathrm{cm}^{-1}$ ) with the experimental and theoretical values for the lowest 10 levels of $\mathrm{O}-$ like ions with

$Z=18$ - 24. NIST: experimental excitation energies compiled in the NIST ASD [37]; MCDHF/RCI: the present MCDHF/RCI excitation energies; MCDHF/RCI2: the MCDHF/RCI excitation energies from Rynkun et al. [32]; MBPT: the MBPT results from Gu [31]; SS: the SUPERSTRUCTURE results from Landi [30]. Differences $\left(\Delta E_{x}=E_{x}-E_{\mathrm{NIST}}\right)$ in $\mathrm{cm}^{-1}$ from different theoretical results with the NIST values are also included.

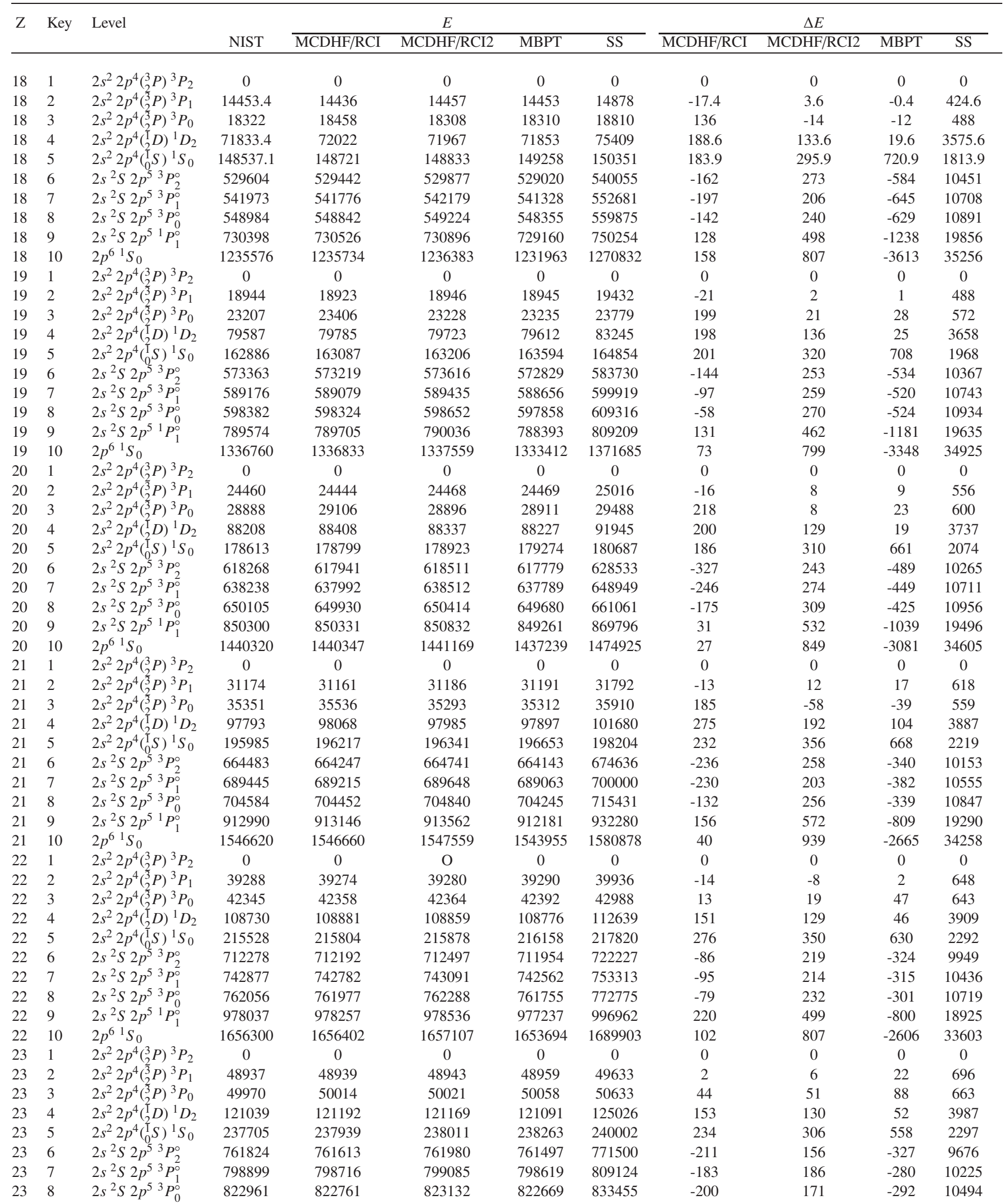


Table B (continued)

\begin{tabular}{|c|c|c|c|c|c|c|c|c|c|c|c|}
\hline \multirow[t]{2}{*}{$\mathrm{Z}$} & \multirow[t]{2}{*}{ Key } & \multirow[t]{2}{*}{ Level } & \multirow[b]{2}{*}{ NIST } & \multicolumn{4}{|c|}{$E$} & \multicolumn{4}{|c|}{$\Delta E$} \\
\hline & & & & $\overline{\mathrm{MCDHF} / \mathrm{RCI}}$ & MCDHF/RCI2 & MBPT & SS & MCDHF/RCI & MCDHF/RCI2 & MBPT & $\mathrm{SS}$ \\
\hline 23 & 9 & $2 s^{2} S 2 p^{5}{ }^{1} P_{1}^{\circ}$ & 1045590 & 1045742 & 1046086 & 1044867 & 1064162 & 152 & 496 & -723 & 18572 \\
\hline 23 & 10 & $2 p^{6}{ }^{1} S_{0}$ & 1769360 & 1769451 & 1770193 & 1766975 & 1802368 & 91 & 833 & -2385 & 33008 \\
\hline 24 & 1 & $2 s^{2} 2 p^{4}\left({ }_{2}^{3} P\right)^{3} P_{2}$ & 0 & 0 & 0 & 0 & 0 & 0 & 0 & 0 & 0 \\
\hline 24 & 2 & $2 s^{2} 2 p^{4}\left({ }_{2}^{3} P\right)^{3} P_{0}$ & 57680 & 58142 & 58150 & 58197 & 58728 & 462 & 470 & 517 & 1048 \\
\hline 24 & 3 & $2 s^{2} 2 p^{4}\left({ }_{2}^{3} P\right)^{3} P_{1}$ & 60376 & 60376 & 60378 & 60401 & 61081 & 0 & 2 & 25 & 705 \\
\hline 24 & 5 & $2 s^{2} 2 p^{4}\left({ }_{0}^{1} S\right){ }^{1} S_{0}$ & 262970 & 263198 & 263267 & 263497 & 265261 & 228 & 297 & 527 & 2291 \\
\hline 24 & 6 & $2 s^{2} S 2 p^{5}{ }^{3} P_{2}^{\circ}$ & 813180 & 812691 & 813417 & 812983 & 822657 & -489 & 237 & -197 & 9477 \\
\hline 24 & 7 & $2 s^{2} S 2 p^{5}{ }^{3} P_{1}^{\circ}$ & 857690 & 857169 & 857895 & 857480 & 867670 & -521 & 205 & -210 & 9980 \\
\hline 24 & 8 & $2 s^{2} S 2 p^{5}{ }^{3} P_{0}^{\circ}$ & 887540 & 887059 & 887785 & 887379 & 897851 & -481 & 245 & -161 & 10311 \\
\hline 24 & 9 & $2 s^{2} S 2 p^{5}{ }^{1} P_{1}^{\circ}$ & 1116080 & 1115887 & 1116590 & 1115448 & 1134246 & -193 & 510 & -632 & 18166 \\
\hline 24 & 10 & $2 p^{6}{ }^{1} S_{0}$ & 1886850 & 1886437 & 1887238 & 1884200 & 1918673 & -413 & 388 & -2650 & 31823 \\
\hline
\end{tabular}


Table C

Excitation energies (in $\mathrm{cm}^{-1}$ ) of O-like Cr XVII. MCDHF/RCI: the present MCDHF/RCI results; MCDHF/RCI3 - the MCDHF/RCI results from Wang et al. [18];

MBPT2: the MBPT results from Wang et al. [18]; CI: theoretical results from Bogdanovich and Karpuškienè [36]. NIST: experimental excitation energies compiled in the NIST ASD [37]. $\Delta E$ - differences (in $\mathrm{cm}^{-1}$ ) between different sources (see above) and the MCDHF/RCI values are also included.

\begin{tabular}{|c|c|c|c|c|c|c|c|c|c|c|}
\hline \multirow[b]{2}{*}{ Key } & \multirow[b]{2}{*}{ Level } & \multirow{2}{*}{$\frac{\mathrm{MCDHF} / \mathrm{RCI}}{E}$} & \multicolumn{2}{|c|}{ MCDHF/RCI3 } & \multicolumn{2}{|c|}{ MBPT2 } & \multicolumn{2}{|c|}{$\mathrm{CI}$} & \multicolumn{2}{|c|}{ NIST } \\
\hline & & & $E$ & $\Delta E$ & $E$ & $\Delta E$ & $E$ & $\Delta E$ & $E$ & $\Delta E$ \\
\hline 1 & $2 s^{2} 2 p^{4}\left({ }_{2}^{3} P\right)^{3} P_{2}$ & 0 & 0 & 0 & 0 & 0 & 0 & 0 & 0 & 0 \\
\hline 2 & $2 s^{2} 2 p^{4}\left({ }_{2}^{3} P\right)^{3} P_{0}$ & 58142 & 58159 & 17 & 58117 & -25 & 58315 & 173 & 57680 & -462 \\
\hline 3 & $2 s^{2} 2 p^{4}\left({ }_{2}^{3} P\right)^{3} P_{1}$ & 60376 & 60380 & 4 & 60456 & 80 & 60551 & 175 & 60376 & 0 \\
\hline 4 & $2 s^{2} 2 p^{4}\left({ }_{2}^{1} D\right){ }^{1} D_{2}$ & 135161 & 135132 & -29 & 134970 & -191 & 134872 & -289 & 134980 & -181 \\
\hline 5 & $2 s^{2} 2 p^{4}\left({ }_{0}^{1} S\right){ }^{1} S_{0}$ & 263198 & 263290 & 92 & 263008 & -190 & 262826 & -372 & 262970 & -228 \\
\hline 6 & $2 s^{2} S 2 p^{53} P_{2}^{\circ}$ & 812691 & 813320 & 629 & 812560 & -131 & 812005 & -686 & 813180 & 489 \\
\hline 7 & $2 s^{2} S 2 p^{5}{ }^{3} P_{1}^{\circ}$ & 857169 & 857798 & 629 & 857071 & -98 & 856388 & -781 & 857690 & 521 \\
\hline 8 & $2 s^{2} S 2 p^{5}{ }^{3} P_{0}^{\circ}$ & 887059 & 887702 & 643 & 886984 & -75 & 886318 & -741 & 887540 & 481 \\
\hline 9 & $2 s^{2} S 2 p^{5}{ }^{1} P_{1}^{\circ}$ & 1115887 & 1116521 & 634 & 1115563 & -324 & 1114559 & -1328 & 1116080 & 193 \\
\hline 10 & $2 p^{6}{ }^{1} S_{0}$ & 1886437 & 1887159 & 722 & 1885088 & -1349 & 1886990 & 553 & 1886850 & 413 \\
\hline 11 & $2 s^{2} 2 p^{3}\left({ }_{3}^{4} S\right){ }^{4} S 3 s^{5} S_{2}^{\circ}$ & 5408096 & 5408814 & 718 & 5409010 & 914 & 5427856 & 19760 & & \\
\hline 12 & $2 s^{2} 2 p^{3}\left({ }_{3}^{4} S\right)^{4} S 3 s^{3} S_{1}^{2}$ & 5455700 & 5456408 & 708 & 5456125 & 425 & 5475506 & 19806 & 5455000 & -700 \\
\hline 13 & $2 s^{2} 2 p^{3}\left({ }_{3}^{2} D\right)^{2} D 3 s^{3} D_{1}^{\circ}$ & 5549793 & 5550457 & 664 & 5550398 & 605 & 5569166 & 19373 & 5546800 & -2993 \\
\hline 14 & $2 s^{2} 2 p^{3}\left({ }_{3}^{2} D\right)^{2} D 3 s^{3} D_{2}^{\circ}$ & 5549925 & 5550582 & 657 & 5550579 & 654 & 5569395 & 19470 & 5549400 & -525 \\
\hline 15 & $2 s^{2} 2 p^{3}\left({ }_{3}^{2} D\right)^{2} D 3 s^{3} D_{3}^{\circ}$ & 5570024 & 5570713 & 689 & 5570624 & 600 & 5588657 & 18633 & 5568900 & -1124 \\
\hline 16 & $2 s^{2} 2 p^{3}\left({ }_{3}^{2} D\right)^{2} D 3 s^{1} D_{2}^{\circ}$ & 5588272 & 5588907 & 635 & 5588701 & 429 & 5606967 & 18695 & 5588700 & 428 \\
\hline 17 & $2 s^{2} 2 p^{3}\left({ }_{3}^{4} S\right){ }^{4} S 3 p^{5} P_{1}$ & 5618629 & 5619012 & 383 & 5619084 & 455 & 5637875 & 19246 & & \\
\hline 18 & $2 s^{2} 2 p^{3}\left({ }_{3}^{4} S\right){ }^{4} S 3 p^{5} P_{2}$ & 5621744 & 5622124 & 380 & 5622188 & 444 & 5640753 & 19009 & & \\
\hline 19 & $2 s^{2} 2 p^{3}\left({ }_{3}^{4} S\right){ }^{4} S 3 p^{5} P_{3}$ & 5634490 & 5634860 & 370 & 5634971 & 481 & 5652325 & 17835 & & \\
\hline 20 & $2 s^{2} 2 p^{3}\left({ }_{1}^{2} P\right)^{2} P 3 s^{3} P_{0}^{\circ}$ & 5646453 & 5647267 & 814 & 5647383 & 930 & 5664845 & 18392 & & \\
\hline 21 & $2 s^{2} 2 p^{3}\left({ }_{1}^{2} P\right)^{2} P 3 s^{3} P_{1}^{\circ}$ & 5652448 & 5653176 & 728 & 5653332 & 884 & 5670818 & 18370 & & \\
\hline 22 & $2 s^{2} 2 p^{3}\left({ }_{3}^{4} S\right){ }^{4} S 3 p^{3} P_{1}$ & 5666705 & 5667133 & 428 & 5666815 & 110 & 5684918 & 18213 & & \\
\hline 23 & $2 s^{2} 2 p^{3}\left({ }_{3}^{4} S\right){ }^{4} S 3 p^{3} P_{2}$ & 5678760 & 5679169 & 409 & 5678785 & 25 & 5696893 & 18133 & & \\
\hline 24 & $2 s^{2} 2 p^{3}\left({ }_{3}^{4} S\right){ }^{4} S 3 p^{3} P_{0}$ & 5683973 & 5684447 & 474 & 5683984 & 11 & 5701839 & 17866 & & \\
\hline 25 & $2 s^{2} 2 p^{3}\left({ }_{1}^{2} P\right)^{2} P 3 s^{3} P_{2}^{\circ}$ & 5686066 & 5686765 & 699 & 5686888 & 822 & 5703801 & 17735 & & \\
\hline 26 & $2 s^{2} 2 p^{3}\left({ }_{1}^{2} P\right)^{2} P 3 s^{1} P_{1}^{\circ}$ & 5701089 & 5701795 & 706 & 5701747 & 658 & 5718910 & 17821 & 5700700 & -389 \\
\hline 27 & $2 s^{2} 2 p^{3}\left({ }_{3}^{2} D\right)^{2} D 3 p^{3} D_{1}$ & 5736813 & 5737188 & 375 & 5737056 & 243 & 5755634 & 18821 & & \\
\hline 28 & $2 s^{2} 2 p^{3}\left({ }_{3}^{2} D\right)^{2} D 3 p^{3} F_{2}$ & 5755365 & 5755704 & 339 & 5755602 & 237 & 5774082 & 18717 & & \\
\hline 29 & $2 s^{2} 2 p^{3}\left({ }_{3}^{2} D\right)^{2} D 3 p^{3} D_{2}$ & 5770215 & 5770540 & 325 & 5770430 & 215 & 5788267 & 18052 & & \\
\hline 30 & $2 s^{2} 2 p^{3}\left({ }_{3}^{2} D\right)^{2} D 3 p^{3} F_{3}$ & 5770968 & 5771323 & 355 & 5771199 & 231 & 5788829 & 17861 & & \\
\hline 31 & $2 s^{2} 2 p^{3}\left({ }_{3}^{2} D\right)^{2} D 3 p^{1} P_{1}$ & 5777373 & 5777750 & 377 & 5777607 & 234 & 5794480 & 17107 & & \\
\hline 32 & $2 s^{2} 2 p^{3}\left({ }_{3}^{2} D\right)^{2} D 3 p^{3} D_{3}$ & 5782646 & 5782950 & 304 & 5782810 & 164 & 5800206 & 17560 & & \\
\hline 33 & $2 s^{2} 2 p^{3}\left({ }_{3}^{2} D\right)^{2} D 3 p^{1} F_{3}$ & 5794949 & 5795265 & 316 & 5795032 & 83 & 5812221 & 17272 & & \\
\hline 34 & $2 s^{2} 2 p^{3}\left({ }_{3}^{2} D\right)^{2} D 3 p^{3} F_{4}$ & 5798254 & 5798614 & 360 & 5798386 & 132 & 5815043 & 16789 & & \\
\hline 35 & $2 s^{2} 2 p^{3}\left({ }_{3}^{2} D\right)^{2} D 3 p^{3} P_{0}$ & 5827777 & 5828232 & 455 & 5827793 & 16 & 5845937 & 18160 & & \\
\hline 36 & $2 s^{2} 2 p^{3}\left({ }_{3}^{2} D\right)^{2} D 3 p^{3} P_{1}$ & 5835559 & 5836042 & 483 & 5835595 & 36 & 5853231 & 17672 & & \\
\hline 37 & $2 s^{2} 2 p^{3}\left({ }_{3}^{2} D\right)^{2} D 3 p^{3} P_{2}$ & 5850982 & 5851555 & 573 & 5850459 & -523 & 5867080 & 16098 & & \\
\hline 38 & $2 s^{2} 2 p^{3}\left({ }_{1}^{2} P\right)^{2} P 3 p^{3} D_{1}$ & 5860955 & 5861337 & 382 & 5861341 & 386 & 5878967 & 18012 & & \\
\hline 39 & $2 s^{2} 2 p^{3}\left({ }_{3}^{2} D\right)^{2} D 3 p^{1} D_{2}$ & 5874467 & 5874907 & 440 & 5874390 & -77 & 5892075 & 17608 & & \\
\hline 40 & $2 s^{2} 2 p^{3}\left({ }_{1}^{2} P\right)^{2} P 3 p^{3} S_{1}$ & 5881500 & 5881898 & 398 & 5881918 & 418 & 5897868 & 16368 & & \\
\hline 41 & $2 s^{2} 2 p^{3}\left({ }_{1}^{2} P\right)^{2} P 3 p^{3} D_{2}$ & 5881733 & 5882127 & 394 & 5882152 & 419 & 5898267 & 16534 & & \\
\hline 42 & $2 s^{2} 2 p^{3}\left({ }_{3}^{4} S\right){ }^{4} S 3 d^{5} D_{3}^{\circ}$ & 5891086 & 5892052 & 966 & 5891746 & 660 & 5909942 & 18856 & & \\
\hline 43 & $2 s^{2} 2 p^{3}\left({ }_{3}^{4} S\right){ }^{4} S 3 d^{5} D_{2}^{\circ}$ & 5891283 & 5892261 & 978 & 5891944 & 661 & 5910068 & 18785 & & \\
\hline 44 & $2 s^{2} 2 p^{3}\left({ }_{3}^{4} S\right){ }^{4} S 3 d^{5} D_{0}^{\circ}$ & 5891333 & 5892369 & 1036 & 5891989 & 656 & 5910036 & 18703 & & \\
\hline 45 & $2 s^{2} 2 p^{3}\left({ }_{3}^{4} S\right){ }^{4} S 3 d^{5} D_{1}^{\circ}$ & 5891477 & 5892471 & 994 & 5892140 & 663 & 5910205 & 18728 & & \\
\hline 46 & $2 s^{2} 2 p^{3}\left({ }_{3}^{4} S\right){ }^{4} S 3 d^{5} D_{4}^{\circ}$ & 5892882 & 5893850 & 968 & 5893552 & 670 & 5911757 & 18875 & & \\
\hline 47 & $2 s^{2} 2 p^{3}\left({ }_{1}^{2} P\right)^{2} P 3 p^{3} D_{3}$ & 5907865 & 5908240 & 375 & 5908258 & 393 & 5923803 & 15938 & & \\
\hline 48 & $2 s^{2} 2 p^{3}\left({ }_{1}^{2} P\right)^{2} P 3 p^{1} P_{1}$ & 5909389 & 5909854 & 465 & 5909520 & 131 & 5925431 & 16042 & & \\
\hline 49 & $2 s^{2} 2 p^{3}\left({ }_{1}^{2} P\right)^{2} P 3 p^{3} P_{0}$ & 5918962 & 5919582 & 620 & 5918696 & -266 & 5934780 & 15818 & & \\
\hline 50 & $2 s^{2} 2 p^{3}\left({ }_{1}^{2} P\right)^{2} P 3 p^{3} P_{1}$ & 5932333 & 5932788 & 455 & 5932380 & 47 & 5948527 & 16194 & & \\
\hline 51 & $2 s^{2} 2 p^{3}\left({ }_{1}^{2} P\right)^{2} P 3 p^{3} P_{2}$ & 5936634 & 5937028 & 394 & 5936973 & 339 & 5952459 & 15825 & & \\
\hline 52 & $2 s^{2} 2 p^{3}\left({ }_{3}^{4} S\right){ }^{4} S 3 d^{3} D_{2}^{\circ}$ & 5937322 & 5938289 & 967 & 5937552 & 230 & 5956841 & 19519 & & \\
\hline 53 & $2 s^{2} 2 p^{3}\left({ }_{3}^{4} S\right){ }^{4} S 3 d^{3} D_{0}^{\circ}$ & 5951536 & 5952531 & 995 & 5951656 & 120 & 5970690 & 19154 & & \\
\hline 54 & $2 s^{2} 2 p^{3}\left({ }_{3}^{4} S\right){ }^{4} S 3 d^{3} D_{3}^{\circ}$ & 5952597 & 5953561 & 964 & 5952739 & 142 & 5971539 & 18942 & 5948500 & -4097 \\
\hline 55 & $2 s^{2} 2 p^{3}\left({ }_{1}^{2} P\right)^{2} P 3 p^{1} D_{2}$ & 5962225 & 5962845 & 620 & 5961453 & -772 & 5977911 & 15686 & & \\
\hline 56 & $2 s^{2} 2 p^{3}\left({ }_{3}^{2} D\right)^{2} D 3 d^{3} F_{2}^{\circ}$ & 6015985 & 6016941 & 956 & 6016441 & 456 & 6034251 & 18266 & & \\
\hline 57 & $2 s^{2} 2 p^{3}\left({ }_{3}^{2} D\right)^{2} D 3 d^{3} F_{3}^{\circ}$ & 6025408 & 6026359 & 951 & 6025844 & 436 & 6043608 & 18200 & & \\
\hline 58 & $2 s^{2} 2 p^{3}\left({ }_{3}^{2} D\right)^{2} D 3 d^{1} S_{0}^{\circ}$ & 6028317 & 6029218 & 901 & 6028659 & 342 & 6046246 & 17929 & & \\
\hline 59 & $2 s^{2} 2 p^{3}\left({ }_{3}^{2} D\right)^{2} D 3 d^{3} G_{4}^{\circ}$ & 6033836 & 6034821 & 985 & 6034194 & 358 & 6052567 & 18731 & & \\
\hline
\end{tabular}


Table C (continued)

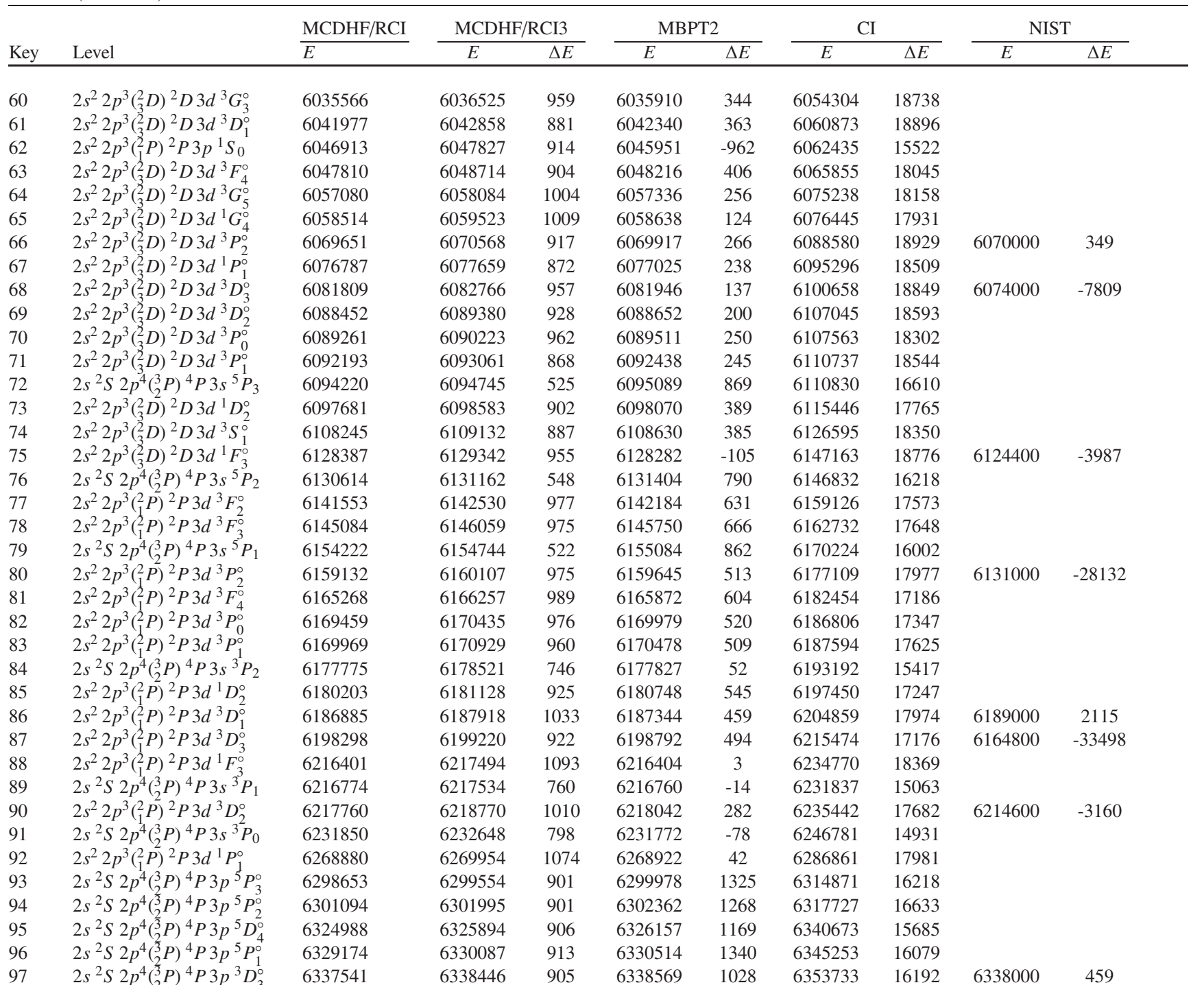

6358806

6359706

$\begin{array}{lllllll}6361557 & 6362057 & 500 & 6361665 & 108 & 6376563 & 15006\end{array}$

$900 \quad 6359907 \quad 1101$

16295

$100 \quad 2 s^{2} S 2 p^{4}\left({ }_{2}^{1} D\right)^{2} D 3 s^{3} D_{2} \quad 6363805$

$1012 s^{2} S 2 p^{4}\left({ }_{2}^{1} D\right)^{2} D 3 s^{3} D_{3} \quad 6368480$

6364312

6368987

6370337

6372331

6375261

6378565

6384467

6398367

6413884

6414968

6415899

6416471

6430265

6502673

6533867

6545175

6551868

6552799

6556585

6558800

6563360

6568007

$507 \quad 6363922$

117

6378921

15116

$6368612 \quad 132$

6383858

$6370591 \quad 1152 \quad 6385575$

$6372535 \quad 1142$

6387649

15378

$6375450 \quad 1087$

$6378357 \quad 759$

6389681

6393875

6399096

6412325

$6398368 \quad 995$

$6413808 \quad 858$

$6413891-407$

6428142

6428472

6429893

6430965

$6416150 \quad 680$

$6429906 \quad 658$

$6502284 \quad 243$

6444545

6516828

$6532942 \quad-205 \quad 6547676$

$6544525-105$

$6551983 \quad 853$

$6552950 \quad 854$

$6555852-174$

$6558949 \quad 850$

$6562901 \quad 402$

$6568152 \quad 846$

6559320

6566903

6567788

6570945

6573701

6577887

6582814

$6584720 \quad 15501$

$6338000 \quad 459$ 
Table C (continued)

\begin{tabular}{|c|c|c|c|c|c|c|c|c|c|c|}
\hline \multirow[b]{2}{*}{ Key } & \multirow[b]{2}{*}{ Level } & \multirow{2}{*}{$\frac{\mathrm{MCDHF} / \mathrm{RCI}}{E}$} & \multicolumn{2}{|c|}{ MCDHF/RCI3 } & \multicolumn{2}{|c|}{ MBPT2 } & \multicolumn{2}{|c|}{$\mathrm{CI}$} & \multicolumn{2}{|c|}{ NIST } \\
\hline & & & $E$ & $\Delta E$ & $E$ & $\Delta E$ & $E$ & $\Delta E$ & $E$ & $\Delta E$ \\
\hline 123 & $2 s^{2} S 2 p^{4}\left({ }_{2}^{1} D\right){ }^{2} D 3 p^{3} F_{3}^{\circ}$ & 6571449 & 6572319 & 870 & 6571862 & 413 & 6586719 & 15270 & & \\
\hline 124 & $2 s^{2} S 2 p^{4}\left({ }_{2}^{3} P\right){ }^{4} P 3 d^{5} F_{5}{ }^{3}$ & 6573707 & 6574648 & 941 & 6574403 & 696 & 6589649 & 15942 & & \\
\hline 125 & $2 s^{2} S 2 p^{4}\left({ }_{2}^{3} P\right)^{4} P 3 d^{5} D_{0}$ & 6578733 & 6579495 & 762 & 6579600 & 867 & 6594049 & 15316 & & \\
\hline 126 & $2 s^{2} S 2 p^{4}\left({ }_{2}^{1} D\right)^{2} D 3 p^{3} F_{4}^{\circ}$ & 6584882 & 6585767 & 885 & 6585315 & 433 & 6599346 & 14464 & & \\
\hline 127 & $2 s^{2} S 2 p^{4}\left({ }_{2}^{1} D\right)^{2} D 3 p^{1} F_{3}^{\circ}$ & 6592968 & 6593831 & 863 & 6593283 & 315 & 6607770 & 14802 & & \\
\hline 128 & $2 s^{2} S 2 p^{4}\left({ }_{2}^{3} P\right)^{4} P 3 d^{5} F_{4}{ }^{3}$ & 6597484 & 6598369 & 885 & 6598079 & 595 & 6613070 & 15586 & & \\
\hline 129 & $2 s^{2} S 2 p^{4}\left({ }_{2}^{1} D\right)^{2} D 3 p^{3} D_{2}^{\circ}$ & 6602451 & 6603372 & 921 & 6602797 & 346 & 6616862 & 14411 & & \\
\hline 130 & $2 s^{2} S 2 p^{4}\left({ }_{2}^{3} P\right){ }^{4} P 3 d^{5} P_{1}{ }^{2}$ & 6607339 & 6608062 & 723 & 6608096 & 757 & 6622854 & 15515 & & \\
\hline 131 & $2 s^{2} S 2 p^{4}\left({ }_{2}^{1} D\right){ }^{2} D 3 p^{3} D_{1}^{\circ}$ & 6608689 & 6609649 & 960 & 6608982 & 293 & 6623128 & 14439 & & \\
\hline 132 & $2 s^{2} S 2 p^{4}\left({ }_{2}^{3} P\right){ }^{4} P 3 d^{5} F_{3}$ & 6613816 & 6614689 & 873 & 6614455 & 639 & 6629096 & 15280 & & \\
\hline 133 & $2 s^{2} S 2 p^{4}\left({ }_{2}^{1} D\right)^{2} D 3 p^{3} D_{3}^{\circ}$ & 6613959 & 6614867 & 908 & 6614338 & 379 & 6628186 & 14227 & & \\
\hline 134 & $2 s^{2} S 2 p^{4}\left({ }_{2}^{3} P\right)^{2} P 3 s^{3} P_{1}$ & 6615268 & 6615833 & 565 & 6615211 & -57 & 6629066 & 13798 & & \\
\hline 135 & $2 s^{2} S 2 p^{4}\left({ }_{2}^{3} P\right)^{4} P 3 d^{5} P_{2}$ & 6619467 & 6620257 & 790 & 6620163 & 696 & 6634828 & 15361 & & \\
\hline 136 & $2 s^{2} S 2 p^{4}\left({ }_{2}^{1} D\right)^{2} D 3 p{ }^{1} D_{2}^{\circ}$ & 6620487 & 6621449 & 962 & 6620732 & 245 & 6634994 & 14507 & & \\
\hline 137 & $2 s^{2} S 2 p^{4}\left({ }_{2}^{3} P\right)^{2} P 3 s^{3} P_{0}{ }^{2}$ & 6624301 & 6624947 & 646 & 6624157 & -144 & 6637122 & 12821 & & \\
\hline 138 & $2 s^{2} S 2 p^{4}\left({ }_{2}^{1} D\right){ }^{2} D 3 p^{3} P_{1}^{\circ}$ & 6629157 & 6630330 & 1173 & 6629021 & -136 & 6643679 & 14522 & & \\
\hline 139 & $2 s^{2} S 2 p^{4}\left({ }_{2}^{3} P\right){ }^{4} P 3 d^{5} F_{2}$ & 6629369 & 6630202 & 833 & 6630079 & 710 & 6644480 & 15111 & & \\
\hline 140 & $2 s^{2} S 2 p^{4}\left({ }_{2}^{1} D\right)^{2} D 3 p^{3} P_{2}^{\circ}$ & 6630662 & 6631796 & 1134 & 6630527 & -135 & 6644204 & 13542 & & \\
\hline 141 & $2 s^{2} S 2 p^{4}\left({ }_{2}^{3} P\right){ }^{4} P 3 d^{5} F_{1}$ & 6631621 & 6632543 & 922 & 6632289 & 668 & 6646527 & 14906 & & \\
\hline 142 & $2 s^{2} S 2 p^{4}\left({ }_{2}^{1} D\right){ }^{2} D 3 p^{3} P_{0}^{\circ}$ & 6632694 & 6633944 & 1250 & 6632525 & -169 & 6647333 & 14639 & & \\
\hline 143 & $2 s^{2} S 2 p^{4}\left({ }_{2}^{3} P\right){ }^{4} P 3 d^{3} F_{4}$ & 6632913 & 6633748 & 835 & 6633276 & 363 & 6648928 & 16015 & & \\
\hline 144 & $2 s^{2} S 2 p^{4}\left({ }_{2}^{3} P\right){ }^{4} P 3 d^{5} P_{3}$ & 6640029 & 6640761 & 732 & 6640709 & 680 & 6655520 & 15491 & & \\
\hline 145 & $2 s^{2} S 2 p^{4}\left({ }_{2}^{3} P\right){ }^{4} P 3 d^{3} F_{3}$ & 6649620 & 6650419 & 799 & 6649969 & 349 & 6665720 & 16100 & & \\
\hline 146 & $2 s^{2} S 2 p^{4}\left({ }_{2}^{3} P\right){ }^{4} P 3 d^{3} P_{0}$ & 6656360 & 6657202 & 842 & 6656565 & 205 & 6672576 & 16216 & & \\
\hline 147 & $2 s^{2} S 2 p^{4}\left({ }_{2}^{3} P\right){ }^{4} P 3 d^{3} P_{1}$ & 6660032 & 6660826 & 794 & 6660255 & 223 & 6676393 & 16361 & & \\
\hline 148 & $2 s^{2} S 2 p^{4}\left({ }_{2}^{3} P\right){ }^{4} P 3 d^{3} D_{2}$ & 6660886 & 6661682 & 796 & 6661133 & 247 & 6677328 & 16442 & & \\
\hline 149 & $2 s^{2} S 2 p^{4}\left({ }_{2}^{3} P\right)^{4} P 3 d^{3} F_{2}$ & 6683722 & 6684546 & 824 & 6683925 & 203 & 6699701 & 15979 & & \\
\hline 150 & $2 s^{2} S 2 p^{4}\left({ }_{2}^{3} P\right){ }^{4} P 3 d^{3} D_{1}$ & 6694200 & 6694982 & 782 & 6694452 & 252 & 6710201 & 16001 & & \\
\hline 151 & $2 s^{2} S 2 p^{4}\left({ }_{2}^{3} P\right)^{4} P 3 d^{3} D_{3}$ & 6695430 & 6696233 & 803 & 6695640 & 210 & 6711516 & 16086 & & \\
\hline 152 & $2 s^{2} S 2 p^{4}\left({ }_{0}^{1} S\right){ }^{2} S 3 p^{3} P_{0}^{\circ}$ & 6706906 & 6708060 & 1154 & 6707286 & 380 & 6721933 & 15027 & & \\
\hline 153 & $2 s^{2} S 2 p^{4}\left({ }_{2}^{3} P\right)^{4} P 3 d^{3} P_{2}$ & 6711330 & 6712122 & 792 & 6711530 & 200 & 6727024 & 15695 & & \\
\hline 154 & $2 s^{2} S 2 p^{4}\left({ }_{0}^{1} S\right){ }^{2} S 3 p^{3} P_{1}^{\circ}$ & 6714532 & 6715570 & 1038 & 6714925 & 393 & 6729537 & 15005 & & \\
\hline 155 & $2 s^{2} S 2 p^{4}\left({ }_{0}^{1} S\right){ }^{2} S 3 p^{3} P_{2}^{\circ}$ & 6732906 & 6733923 & 1017 & 6733327 & 421 & 6746927 & 14021 & & \\
\hline 156 & $2 s^{2} S 2 p^{4}\left({ }_{0}^{1} S\right){ }^{2} S 3 p^{1} P_{1}^{\circ}$ & 6740804 & 6741873 & 1069 & 6741066 & 262 & 6754860 & 14056 & & \\
\hline 157 & $2 s^{2} S 2 p^{4}\left({ }_{2}^{3} P\right)^{2} P 3 p^{3} P_{2}^{\circ}$ & 6749432 & 6750472 & 1040 & 6749345 & -87 & 6764351 & 14919 & & \\
\hline 158 & $2 s^{2} S 2 p^{4}\left({ }_{2}^{3} P\right)^{2} P 3 p{ }^{1} D_{2}^{\circ}$ & 6765303 & 6766259 & 956 & 6765353 & 50 & 6779869 & 14566 & & \\
\hline 159 & $2 s^{2} S 2 p^{4}\left({ }_{2}^{3} P\right)^{2} P 3 p^{3} D_{3}^{\circ}$ & 6767173 & 6768069 & 896 & 6767333 & 160 & 6781190 & 14017 & & \\
\hline 160 & $2 s^{2} S 2 p^{4}\left({ }_{2}^{2} P\right)^{2} P 3 p^{3} P_{1}^{\circ}$ & 6775622 & 6776730 & 1108 & 6775435 & -187 & 6788829 & 13207 & & \\
\hline 161 & $2 s^{2} S 2 p^{4}\left({ }_{2}^{3} P\right)^{2} P 3 p^{1} S_{0}^{\circ}$ & 6789253 & 6790353 & 1100 & 6789142 & -111 & 6802524 & 13271 & & \\
\hline 162 & $2 s^{2} S 2 p^{4}\left({ }_{2}^{3} P\right)^{2} P 3 p^{3} D_{1}^{\circ}$ & 6806218 & 6807157 & 939 & 6806390 & 172 & 6820949 & 14731 & & \\
\hline 163 & $2 s^{2} S 2 p^{4}\left({ }_{2}^{1} D\right)^{2} D 3 d^{3} G_{3}$ & 6817033 & 6818088 & 1055 & 6816757 & -276 & 6831011 & 13978 & & \\
\hline 164 & $2 s^{2} S 2 p^{4}\left({ }_{2}^{1} D\right)^{2} D 3 d^{3} G_{4}$ & 6820961 & 6822027 & 1066 & 6820704 & -257 & 6835164 & 14203 & & \\
\hline 165 & $2 s^{2} S 2 p^{4}\left({ }_{2}^{1} D\right)^{2} D 3 d^{3} G_{5}$ & 6826258 & 6827345 & 1087 & 6826027 & -231 & 6840675 & 14417 & & \\
\hline 166 & $2 s^{2} S 2 p^{4}\left({ }_{2}^{3} P\right)^{2} P 3 p^{3} P_{0}^{\circ}$ & 6832794 & 6833821 & 1027 & 6832813 & 19 & 6845851 & 13057 & & \\
\hline 167 & $2 s^{2} S 2 p^{4}\left({ }_{2}^{3} P\right)^{2} P 3 p^{3} D_{2}^{\circ}$ & 6835294 & 6836240 & 946 & 6835506 & 212 & 6848335 & 13041 & & \\
\hline 168 & $2 s^{2} S 2 p^{4}\left({ }_{2}^{2} P\right)^{2} P 3 p^{3} S_{1}^{\circ}$ & 6840496 & 6841459 & 963 & 6840730 & 234 & 6853603 & 13107 & & \\
\hline 169 & $2 s^{2} S 2 p^{4}\left({ }_{2}^{1} D\right){ }^{2} D 3 d^{3} F_{2}$ & 6851799 & 6852769 & 970 & 6851588 & -211 & 6865855 & 14056 & & \\
\hline 170 & $2 s^{2} S 2 p^{4}\left({ }_{2}^{1} D\right){ }^{2} D 3 d^{3} S_{1}$ & 6855243 & 6855968 & 725 & 6855570 & 327 & 6870459 & 15216 & & \\
\hline 171 & $2 s^{2} S 2 p^{4}\left({ }_{2}^{1} D\right)^{2} D 3 d^{3} F_{3}$ & 6856396 & 6857349 & 953 & 6856206 & -190 & 6870590 & 14194 & & \\
\hline 172 & $2 s^{2} S 2 p^{4}\left({ }_{2}^{1} D\right)^{2} D 3 d^{3} F_{4}$ & 6858503 & 6859442 & 939 & 6858257 & -246 & 6872983 & 14480 & & \\
\hline 173 & $2 s^{2} S 2 p^{4}\left({ }_{2}^{1} D\right){ }^{2} D 3 d^{3} D_{1}$ & 6861443 & 6862285 & 842 & 6861236 & -207 & 6876202 & 14759 & & \\
\hline 174 & $2 s^{2} S 2 p^{4}\left({ }_{2}^{1} D\right){ }^{2} D 3 d^{3} P_{0}$ & 6864694 & 6865525 & 831 & 6864309 & -385 & 6879593 & 14899 & & \\
\hline 175 & $2 s^{2} S 2 p^{4}\left({ }_{2}^{1} D\right)^{2} D 3 d^{3} D_{2}$ & 6867498 & 6868376 & 878 & 6867217 & -281 & 6882205 & 14707 & & \\
\hline 176 & $2 s^{2} S 2 p^{4}\left({ }_{2}^{1} D\right)^{2} D 3 d^{1} G_{4}$ & 6867671 & 6868574 & 903 & 6867332 & -339 & 6882663 & 14992 & & \\
\hline 177 & $2 s^{2} S 2 p^{4}\left({ }_{2}^{1} D\right)^{2} D 3 d^{3} P_{2}$ & 6869690 & 6870541 & 851 & 6869348 & -342 & 6885016 & 15326 & & \\
\hline 178 & $2 s^{2} S 2 p^{4}\left({ }_{2}^{1} D\right)^{2} D 3 d^{3} D_{3}$ & 6869895 & 6870787 & 892 & 6869620 & -275 & 6884840 & 14945 & & \\
\hline 179 & $2 s^{2} S 2 p^{4}\left({ }_{2}^{1} D\right)^{2} D 3 d^{3} P_{1}$ & 6873924 & 6874776 & 852 & 6873591 & -333 & 6888804 & 14880 & & \\
\hline 180 & $2 s^{2} S 2 p^{4}\left({ }_{2}^{1} D\right){ }^{2} D 3 d^{1} P_{1}$ & 6889694 & 6890578 & 884 & 6889470 & -224 & 6904550 & 14856 & & \\
\hline 181 & $2 s^{2} S 2 p^{4}\left({ }_{2}^{1} D\right)^{2} D 3 d^{1} D_{2}$ & 6892388 & 6893284 & 896 & 6892081 & -307 & 6907213 & 14825 & & \\
\hline 182 & $2 s^{2} S 2 p^{4}\left({ }_{2}^{1} D\right){ }^{2} D 3 d^{1} F_{3}$ & 6899128 & 6899978 & 850 & 6898741 & -387 & 6914242 & 15114 & & \\
\hline 183 & $2 s^{2} S 2 p^{4}\left({ }_{2}^{3} P\right)^{2} P 3 p{ }^{1} P_{1}^{\circ}$ & 6902386 & 6903875 & 1489 & 6901003 & -1383 & 6914759 & 12373 & & \\
\hline 184 & $2 s^{2} S 2 p^{4}\left({ }_{2}^{1} D\right){ }^{2} D 3 d^{1} S_{0}$ & 6908434 & 6909252 & 818 & 6907425 & -1009 & 6923737 & 15303 & & \\
\hline 185 & $2 s^{2} S 2 p^{4}\left({ }_{0}^{1} S\right)^{2} S 3 d^{3} D_{1}$ & 6975119 & 6976283 & 1164 & 6974955 & -164 & 6988787 & 13668 & & \\
\hline
\end{tabular}


Table C (continued)

\begin{tabular}{|c|c|c|c|c|c|c|c|c|c|c|}
\hline \multirow[b]{2}{*}{ Key } & \multirow[b]{2}{*}{ Level } & \multirow{2}{*}{$\frac{\mathrm{MCDHF} / \mathrm{RCI}}{E}$} & \multicolumn{2}{|c|}{ MCDHF/RCI3 } & \multicolumn{2}{|c|}{ МBPT2 } & \multicolumn{2}{|c|}{$\mathrm{CI}$} & \multicolumn{2}{|c|}{ NIST } \\
\hline & & & $E$ & $\Delta E$ & $E$ & $\Delta E$ & $E$ & $\Delta E$ & $E$ & $\Delta E$ \\
\hline 186 & $2 s^{2} S 2 p^{4}\left({ }_{0}^{1} S\right){ }^{2} S 3 d^{3} D_{2}$ & 6980671 & 6981809 & 1138 & 6980535 & -136 & 6994407 & 13736 & & \\
\hline 187 & $2 s^{2} S 2 p^{4}\left({ }_{0}^{1} S\right){ }^{2} S 3 d^{3} D_{3}$ & 6984180 & 6985359 & 1179 & 6984015 & -165 & 6998173 & 13993 & & \\
\hline 188 & $2 s^{2} S 2 p^{4}\left({ }_{2}^{3} P\right)^{2} P 3 d^{3} D_{3}$ & 7000457 & 7001681 & 1224 & 6999884 & -573 & 7014377 & 13920 & & \\
\hline 189 & $2 s^{2} S 2 p^{4}\left({ }_{0}^{1} S\right){ }^{2} S 3 d^{1} D_{2}$ & 7005687 & 7006880 & 1193 & 7005144 & -543 & 7020021 & 14334 & & \\
\hline 190 & $2 s^{2} S 2 p^{4}\left({ }_{2}^{3} P\right)^{2} P 3 d^{3} D_{2}$ & 7015684 & 7016898 & 1214 & 7015203 & -481 & 7029544 & 13860 & & \\
\hline 191 & $2 s^{2} S 2 p^{4}\left({ }_{2}^{3} P\right)^{2} P 3 d^{1} F_{3}$ & 7024712 & 7025976 & 1264 & 7023946 & -766 & 7038382 & 13670 & & \\
\hline 192 & $2 s^{2} S 2 p^{4}\left({ }_{2}^{3} P\right)^{2} P 3 d^{3} F_{4}$ & 7024879 & 7026107 & 1228 & 7024244 & -635 & 7038967 & 14088 & & \\
\hline 193 & $2 s^{2} S 2 p^{4}\left({ }_{2}^{3} P\right)^{2} P 3 d^{3} D_{1}$ & 7027980 & 7029291 & 1311 & 7027393 & -587 & 7041390 & 13410 & & \\
\hline 194 & $2 s^{2} S 2 p^{4}\left({ }_{2}^{3} P\right)^{2} P 3 d^{3} P_{0}$ & 7044366 & 7045641 & 1275 & 7043719 & -647 & 7057600 & 13234 & & \\
\hline 195 & $2 s^{2} S 2 p^{4}\left({ }_{2}^{3} P\right)^{2} P 3 d^{3} P_{1}$ & 7056342 & 7057625 & 1283 & 7055739 & -603 & 7069206 & 12864 & & \\
\hline 196 & $2 s^{2} S 2 p^{4}\left({ }_{2}^{3} P\right)^{2} P 3 d^{3} F_{2}$ & 7059512 & 7060753 & 1241 & 7058739 & -773 & 7073071 & 13559 & & \\
\hline 197 & $2 s^{2} S 2 p^{4}\left({ }_{2}^{3} P\right)^{2} P 3 d^{3} F_{3}$ & 7087264 & 7088478 & 1214 & 7086718 & -546 & 7099986 & 12722 & & \\
\hline 198 & $2 s^{2} S 2 p^{4}\left({ }_{2}^{3} P\right)^{2} P 3 d^{3} P_{2}$ & 7099319 & 7100451 & 1132 & 7098876 & -443 & 7111562 & 12243 & & \\
\hline 199 & $2 s^{2} S 2 p^{4}\left({ }_{2}^{3} P\right)^{2} P 3 d^{1} P_{1}$ & 7122885 & 7124220 & 1335 & 7122264 & -621 & 7135852 & 12967 & & \\
\hline 200 & $2 s^{2} S 2 p^{4}\left({ }_{2}^{3} P\right)^{2} P 3 d^{1} D_{2}$ & 7132737 & 7134144 & 1407 & 7131445 & -1292 & 7146753 & 14016 & & \\
\hline
\end{tabular}


Table D

Excitation energies (in $\mathrm{cm}^{-1}$ ) for levels for which which the NIST values differ with the present MCDHF/RCI values by more than $3000 \mathrm{~cm}^{-1}$ (about $0.1 \%$ ).

$E_{\mathrm{MCDHF} / \mathrm{RCI}}$ : the present $\mathrm{MCDHF} / \mathrm{RCI}$ values; $\Delta E_{\mathrm{NIST}}$ : experimental excitation energies compiled in the NIST ASD [37]; $\Delta E=E_{\mathrm{MCDHF} / \mathrm{RCI}}-E_{\mathrm{MCDHF}}$; Reference: reference sources for the NIST values.

\begin{tabular}{|c|c|c|c|c|c|c|}
\hline $\mathrm{Z}$ & Key & Level & $E_{\mathrm{MCDHF} / \mathrm{RCI}}$ & $E_{\mathrm{NIST}}$ & $\Delta E$ & Reference \\
\hline 18 & 26 & $2 s^{2} 2 p^{3}\left({ }_{1}^{2} P\right)^{2} P 3 s^{1} P_{1}^{\circ}$ & 2660649 & 2649800 & -10849 & {$[67]$} \\
\hline 18 & 31 & $2 s^{2} 2 p^{3}\left(\frac{2}{3} D\right)^{2} D 3 p^{3} F_{2}$ & 2724663 & 2728790 & 4127 & {$[67]$} \\
\hline 18 & 61 & $2 s^{2} 2 p^{3}\left({ }_{3}^{2} D\right)^{2} D 3 d^{3} G_{3}^{\circ}$ & 2896390 & 2900300 & 3910 & [67] \\
\hline 18 & 72 & $2 s^{2} 2 p^{3}\left({ }_{3}^{2} D\right)^{2} D 3 d^{3} P_{1}^{\circ}$ & 2925253 & 2932610 & 7357 & [67] \\
\hline 18 & 79 & $2 s^{2} 2 p^{3}\left({ }_{1}^{2} P\right)^{2} P 3 d^{3} F_{3}^{\circ}$ & 2954139 & 2945040 & -9099 & [67] \\
\hline 18 & 81 & $2 s^{2} 2 p^{3}\left({ }_{1}^{2} P\right)^{2} P 3 d^{3} P_{1}^{\circ}$ & 2957524 & 2968000 & 10476 & [67] \\
\hline 18 & 85 & $2 s^{2} 2 p^{3}\left({ }_{1}^{2} P\right)^{2} P 3 d^{3} D_{1}^{\circ}$ & 2971222 & 2987530 & 16308 & {$[67]$} \\
\hline 19 & 67 & $2 s^{2} 2 p^{3}\left({ }_{3}^{2} D\right)^{2} D 3 d^{3} D_{1}^{\circ}$ & 3361421 & 3358100 & -3321 & [68] \\
\hline 19 & 84 & $2 s^{2} 2 p^{3}\left({ }_{1}^{2} P\right)^{2} P 3 d^{3} D_{2}^{\circ}$ & 3421283 & 3427000 & 5717 & [68] \\
\hline 19 & 87 & $2 s^{2} 2 p^{3}\left({ }_{1}^{2} P\right)^{2} P 3 d^{1} D_{2}^{\circ}$ & 3438950 & 3416600 & -22350 & {$[68]$} \\
\hline 20 & 72 & $2 s^{2} 2 p^{3}\left({ }_{3}^{2} D\right)^{2} D 3 d^{3} P_{1}^{\circ}$ & 3855862 & 3852000 & -3862 & [69] \\
\hline 20 & 82 & $2 s^{2} 2 p^{3}\left({ }_{1}^{2} P\right)^{2} P 3 d^{3} P_{1}^{\circ}$ & 3897467 & 3893000 & -4467 & [69] \\
\hline 20 & 88 & $2 s^{2} 2 p^{3}\left({ }_{1}^{2} P\right)^{2} P 3 d^{1} D_{2}^{\circ}$ & 3928517 & 3920000 & -8517 & [69] \\
\hline 21 & 22 & $2 s^{2} 2 p^{3}\left({ }_{1}^{2} P\right)^{2} P 3 s^{3} P_{2}^{\circ}$ & 4018824 & 4004600 & -14224 & [69] \\
\hline 21 & 82 & $2 s^{2} 2 p^{3}\left({ }_{1}^{2} P\right)^{2} P 3 d^{3} P_{2}^{\circ}$ & 4415589 & 4407000 & -8589 & [69] \\
\hline 21 & 88 & $2 s^{2} 2 p^{3}\left({ }_{1}^{2} P\right)^{2} P 3 d^{1} D_{2}^{\circ}$ & 4451019 & 4445000 & -6019 & [69] \\
\hline 22 & 23 & $2 s^{2} 2 p^{3}\left({ }_{1}^{2} P\right)^{2} P 3 s^{3} P_{2}^{\circ}$ & 4541430 & 4523000 & -18430 & [69] \\
\hline 22 & 52 & $2 s^{2} 2 p^{3}\left({ }_{3}^{4} S\right){ }^{4} S 3 d^{3} D_{2}^{\circ}$ & 4776970 & 4780000 & 3030 & [69] \\
\hline 22 & 83 & $2 s^{2} 2 p^{3}\left({ }_{1}^{2} P\right)^{2} P 3 d^{3} P_{1}^{\circ}$ & 4968183 & 4965000 & -3183 & [69] \\
\hline 22 & 92 & $2 s^{2} 2 p^{3}\left({ }_{1}^{2} P\right)^{2} P 3 d^{1} P_{1}^{\circ}$ & 5052035 & 5046000 & -6035 & [69] \\
\hline 23 & 24 & $2 s^{2} 2 p^{3}\left({ }_{1}^{2} P\right)^{2} P 3 s^{3} P_{2}^{\circ}$ & 5097146 & 5068000 & -29146 & [69] \\
\hline 23 & 66 & $2 s^{2} 2 p^{3}\left({ }_{3}^{2} D\right)^{2} D 3 d^{3} D_{2}^{\circ}$ & 5464936 & 5457000 & -7936 & [69] \\
\hline 23 & 69 & $2 s^{2} 2 p^{3}\left({ }_{3}^{2} D\right)^{2} D 3 d^{3} P_{2}^{\circ}$ & 5480564 & 5476000 & -4564 & [69] \\
\hline 23 & 80 & $2 s^{2} 2 p^{3}\left({ }_{1}^{2} P\right)^{2} P 3 d^{3} P_{2}^{\circ}$ & 5546098 & 5488000 & -58098 & [69] \\
\hline 23 & 87 & $2 s^{2} 2 p^{3}\left({ }_{1}^{2} P\right)^{2} P 3 d^{3} D_{3}^{\circ}$ & 5577101 & 5552000 & -25101 & [69] \\
\hline 24 & 54 & $2 s^{2} 2 p^{3}\left({ }_{3}^{4} S\right){ }^{4} S 3 d^{3} D_{3}^{\circ}$ & 5952597 & 5948500 & -4097 & [69] \\
\hline 24 & 68 & $2 s^{2} 2 p^{3}\left({ }_{3}^{2} D\right)^{2} D 3 d^{3} D_{3}^{\circ}$ & 6081809 & 6074000 & -7809 & [69] \\
\hline 24 & 75 & $2 s^{2} 2 p^{3}\left({ }_{3}^{2} D\right)^{2} D 3 d^{1} F_{3}^{\circ}$ & 6128387 & 6124400 & -3987 & [69] \\
\hline 24 & 80 & $2 s^{2} 2 p^{3}\left({ }_{1}^{2} P\right)^{2} P 3 d^{3} P_{2}^{\circ}$ & 6159132 & 6131000 & -28132 & [69] \\
\hline 24 & 87 & $2 s^{2} 2 p^{3}\left({ }_{1}^{2} P\right)^{2} P 3 d^{3} D_{3}^{\circ}$ & 6198298 & 6164800 & -33498 & [69] \\
\hline 24 & 90 & $2 s^{2} 2 p^{3}\left({ }_{1}^{2} P\right)^{2} P 3 d^{3} D_{2}^{\circ}$ & 6217760 & 6214600 & -3160 & [69] \\
\hline
\end{tabular}




\section{Explanation of Tables}

Table 1.Excitation energies in $\mathrm{cm}^{-1}$ and radiative lifetimes in $\mathrm{s}$ for the lowest $156(179,184,196,200,200,200)$ states of the $n \leq 3$ configurations in Ar XI (K XII, Ca XIII, Sc XIV, Ti XV, V XVI, Cr XVII).

$\begin{array}{ll}\text { Key } & \text { A number assigned to each level } \\ \text { Level } & \text { The } L S J \text {-coupled label for each level } \\ E_{\mathrm{NIST}} & \text { observed values compiled in the NIST ASD [37] } \\ E_{\mathrm{MCDHF} / \mathrm{RCI}} & \text { our MCDHF/RCI excitation energies } \\ \tau_{\mathrm{MCDHF} / \mathrm{RCI}}^{l} & \text { our MCDHF/RCI lifetimes in length form } \\ \tau_{\mathrm{MCDHF} / \mathrm{RCI}}^{v} & \text { our MCDHF/RCI lifetimes in velocity form } \\ L S \text {-composition } & \text { the } L S \text { eigenvector compositions }\end{array}$

Table 2. Transition wavelengths $\lambda$ (in $\AA$ ), transition rates $A$ (in $\mathrm{s}^{-1}$ ), weighted oscillator strengths $g f$, and line strengths $S$ (in a.u.) between the lowest $156(179,184,196,200,200,200)$ states of the $\boldsymbol{n} \leq \mathbf{3}$ configurations in Ar XI (K XII, Ca XIII, Sc XIV, Ti XV, V XVI, Cr XVII) listed in Table 1. Transitions with the branching fraction (BF) $\geq 10^{-5}$ are presented.

Z

$i$

$j$

$\lambda$

Type

$\mathrm{BF}$

$A^{l}$

$g f^{l}$

$S^{l}$

$A^{v}$

$g f^{v}$

$S^{v}$

Acc.
The nuclear charge number

The lower level of a transition

The upper level of a transition

Wavelength, in $\AA$.

Transition type (E1, M1, E2, M2).

The branching fraction from the upper level.

Transition rate (in $\mathrm{s}^{-1}$ ) in the length $(l)$ form

Weighted oscillator strength (dimensionless) in the length $(l)$ form

Line strength (in a.u.) in the length $(l)$ form

Transition rate (in $\mathrm{s}^{-1}$ ) in the velocity $(v)$ form

Weighted oscillator strength (dimensionless) in the velocity $(v)$ form

Line strength (in a.u.) in the velocity (v) form

the estimated accuracies of the $S$ values using the terminologies (AA $\leq 1 \%, \mathrm{~A}^{+} \leq 2 \%, \mathrm{~A} \leq 3 \%$, $\mathrm{B}^{+} \leq 7 \%, \mathrm{~B} \leq 10 \%, \mathrm{C}^{+} \leq 18 \%, \mathrm{C} \leq 25 \%, \mathrm{D}^{+} \leq 40 \%, \mathrm{D} \leq 50 \%$, and $\left.\mathrm{E}>50 \%\right)$ of the NIST ASD [37]. 


\begin{tabular}{|c|c|c|c|c|c|c|c|}
\hline $\mathrm{z}$ & Key & Level & $E_{\text {NIST }}$ & $E_{\mathrm{MCDHF} / \mathrm{RCI}}$ & $\tau_{\mathrm{MCDHF} / \mathrm{RCI}}^{l}$ & $\tau_{\mathrm{MCDHF} / \mathrm{RCI}}^{v}$ & $L S$-composition \\
\hline 18 & 1 & $2 s^{2} 2 p^{4}\left({ }_{2}^{3} P\right)^{3} P_{2}$ & 0 & 0 & $\ldots$ & $\ldots$ & 0.98 \\
\hline 18 & 2 & $2 s^{2} 2 p^{4}\left({ }_{2}^{2} P\right){ }^{3} P_{1}$ & 14453.4 & 14436 & $1.504 \mathrm{E}-02$ & $1.504 \mathrm{E}-02$ & 0.99 \\
\hline 18 & 3 & $2 s^{2} 2 p^{4}\left(\frac{2}{2} P\right){ }^{3} P_{0}$ & 18322 & 18458 & $2.908 \mathrm{E}-01$ & $2.908 \mathrm{E}-01$ & 0.97 \\
\hline 18 & 4 & $2 s^{2} 2 p^{4}\left({ }_{2}^{1} D\right)^{1} D_{2}$ & 71833.4 & 72022 & $3.646 \mathrm{E}-03$ & $3.646 \mathrm{E}-03$ & 0.98 \\
\hline 18 & 5 & $2 s^{2} 2 p^{4}\left({ }_{0}^{1} S\right){ }^{1} S_{0}$ & 148537.1 & 148721 & 3.953E-04 & 3.953E-04 & $0.95+0.032 p^{6}{ }^{1} S$ \\
\hline 18 & 6 & $2 s^{2} s 2 p^{53} P_{2}^{\circ}$ & 529604 & 529442 & $4.806 \mathrm{E}-11$ & $4.792 \mathrm{E}-11$ & 0.99 \\
\hline 18 & 7 & $2 s^{2} S 2 p^{53} P_{1}^{\circ}$ & 541973 & 541776 & $4.608 \mathrm{E}-11$ & $4.611 \mathrm{E}-11$ & 0.99 \\
\hline 18 & 8 & $2 s^{2} S 2 p^{5}{ }^{3} P_{0}^{\circ}$ & 548984 & 548842 & $4.547 \mathrm{E}-11$ & $4.555 \mathrm{E}-11$ & 0.99 \\
\hline 18 & 9 & $2 s^{2} S 2 p^{5}{ }^{1} P_{1}^{\circ}$ & 730398 & 730526 & $1.311 \mathrm{E}-11$ & $1.312 \mathrm{E}-11$ & 0.98 \\
\hline 18 & 10 & $2 p^{6}{ }^{1} s_{0}$ & 1235576 & 1235734 & $1.578 \mathrm{E}-11$ & $1.583 \mathrm{E}-11$ & $0.95+0.032 s^{2} 2 p^{4}\left({ }_{0}^{1} s\right)^{1} s$ \\
\hline 18 & 11 & $2 s^{2} 2 p^{3}\left({ }_{3}^{4} S\right)^{4} S 3 s^{5} S_{2}^{\circ}$ & 2483300 & 2483404 & $7.861 \mathrm{E}-10$ & 7.924E-10 & $0.95+0.032 s^{2} 2 p^{3}\left({ }_{3}^{4} s\right)^{4} S 4 s^{5} s^{\circ}$ \\
\hline 18 & 12 & $2 s^{2} 2 p^{3}\left({ }_{3}^{4} S\right)^{4} S 3 s^{3} s_{1}^{0}$ & & 2516400 & $1.764 \mathrm{E}-12$ & $1.766 \mathrm{E}-12$ & $0.94+0.032 s^{2} 2 p^{3}\left(\frac{4}{3} S\right)^{4} S 4 s^{3} S^{0}$ \\
\hline 18 & 13 & $2 s^{2} 2 p^{3}\left(\frac{2}{3} D\right)^{2} D 3 s^{3} D_{1}^{\circ}$ & 2587340 & 2586885 & $5.384 \mathrm{E}-12$ & $5.396 \mathrm{E}-12$ & $0.91+0.032 s^{2} 2 p^{3}\left(\frac{2}{3} D\right)^{2} D 4 s^{3} D^{\circ}+0.022 s^{2} 2 p^{3}\left({ }_{1}^{2} P\right)^{2} P 3 s^{1} P^{\circ}$ \\
\hline 18 & 14 & $2 s^{2} 2 p^{3}\left(_{2}^{3} D\right)^{2} D 3 s^{3} D_{2}^{\circ}$ & & 2587367 & $5.526 \mathrm{E}-12$ & $5.535 \mathrm{E}-12$ & $0.90+0.052 s^{2} 2 p^{3}\left(_{1}^{2} P\right)^{2} P 3 s^{3} P^{\circ}+0.032 s^{2} 2 p^{3}\left({ }_{2}^{2} D\right)^{2} D 4 s^{3} D^{\circ}$ \\
\hline 18 & 15 & $2 s^{2} 2 p^{3}\left(\frac{2}{3} D\right)^{2} D 3 s^{3} D_{3}^{\circ}$ & 2589610 & 2589486 & $5.541 \mathrm{E}-12$ & $5.548 \mathrm{E}-12$ & $0.96+0.032 s^{2} 2 p^{3}\left(\frac{2}{3} D\right)^{2} D 4 s^{3} D^{\circ}$ \\
\hline 18 & 16 & $2 s^{2} 2 p^{3}\left({ }_{3}^{2} D\right)^{2} D 3 s^{1} D_{2}^{\circ}$ & 2604370 & 2604383 & 2.238E-12 & 2.242E-12 & $0.90+0.042 s^{2} 2 p^{3}\left({ }_{1}^{2} P\right)^{2} P 3 s^{3} P^{\circ}+0.032 s^{2} 2 p^{3}\left({ }_{3}^{2} D\right)^{2} D 4 s^{1} D^{\circ}$ \\
\hline 18 & 17 & $2 s^{2} 2 p^{3}\left({ }_{3}^{4} S\right){ }^{4} S 3 p^{5} P_{1}^{2}$ & 2619080 & 2619269 & 6.919E-10 & 6.887E-10 & 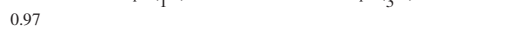 \\
\hline 18 & 18 & $2 s^{2} 2 p^{3}\left({ }_{3}^{4} S\right){ }^{4} S 3 p^{5} P_{2}$ & 2620200 & 2620396 & 6.987E-10 & 6.957E-10 & 0.96 \\
\hline 18 & 19 & $2 s^{2} 2 p^{3}\left({ }_{3}^{4} S\right)^{4} S 3 p^{5} P_{3}$ & 2622600 & 2622796 & $6.593 \mathrm{E}-10$ & $6.576 \mathrm{E}-10$ & 0.97 \\
\hline 18 & 20 & $2 s^{2} 2 p^{3}\left({ }_{1}^{2} P\right)^{2} P 3 s^{3} P_{0}^{\circ}$ & & 2641116 & $5.730 \mathrm{E}-12$ & 5.737E-12 & $0.94+0.032 s^{2} 2 p^{3}\left({ }_{1}^{2} P\right)^{2} P 4 s^{3} P^{\circ}$ \\
\hline 18 & 21 & $2 s^{2} 2 p^{3}\left({ }_{1}^{2} P\right)^{2} P 3 s^{3} P_{1}^{o}$ & & 2642472 & $5.517 \mathrm{E}-12$ & $5.523 \mathrm{E}-12$ & $0.90+0.032 s^{2} 2 p^{3}\left({ }_{1}^{2} P\right)^{2} P 4 s^{3} P^{\circ}$ \\
\hline 18 & 22 & $2 s^{2} 2 p^{3}\left({ }_{1}^{2} P\right)^{2} P 3 s^{3} P_{2}^{\circ}$ & 2644510 & 2646348 & 5.217E-12 & $5.221 \mathrm{E}-12$ & $0.85+0.052 s^{2} 2 p^{3}\left({ }_{3}^{2} D\right)^{2} D 3 s^{1} D^{\circ}+0.042 s^{2} 2 p^{3}\left({ }_{3}^{2} D\right)^{2} D 3 s^{3} D^{\circ}$ \\
\hline 18 & 23 & $2 s^{2} 2 p^{3}\left({ }_{3}^{4} S\right)^{4} S 3 p^{3} P_{1}^{2}$ & & 2650684 & $5.781 \mathrm{E}-10$ & $5.807 \mathrm{E}-10$ & $0.82+0.112 s^{2} 2 p^{3}\left(\frac{2}{3} D\right)^{2} D 3 p^{3} P+0.022 s^{2} 2 p^{3}\left({ }_{1}^{2} P\right)^{2} P 3 p^{3} P$ \\
\hline 18 & 24 & $2 s^{2} 2 p^{3}\left({ }_{3}^{4} S\right)^{4} S 3 p^{3} P_{2}$ & & 2651718 & 5.705E-10 & $5.729 \mathrm{E}-10$ & $0.83+0.112 s^{2} 2 p^{3}\left(\frac{2}{3} D\right)^{2} D 3 p^{3} P$ \\
\hline 18 & 25 & $2 s^{2} 2 p^{3}\left({ }_{3}^{4} S\right){ }^{4} S 3 p^{3} P_{0}$ & & 2652455 & $6.006 \mathrm{E}-10$ & $6.026 \mathrm{E}-10$ & $0.85+0.102 s^{2} 2 p^{3}\left({ }_{3}^{2} D\right)^{2} D 3 p^{3} P+0.032 s^{2} 2 p^{3}\left({ }_{1}^{2} P\right)^{2} P 3 p^{3} P$ \\
\hline 18 & 26 & $2 s^{2} 2 p^{3}\left({ }_{1}^{3} P\right)^{2} P 3 s^{1} P_{1}^{\circ}$ & 2649800 & 2660649 & $2.292 \mathrm{E}-12$ & $2.294 \mathrm{E}-12$ & $\left.0.89+0.032 s^{2} 2 p^{3}\left(\frac{2}{3} D\right)^{2} D 3 s^{3} D^{\circ}+0.032 s^{2} 2 p^{3}{ }_{1}^{2} P\right)^{2} P 4 s^{1} P^{\circ}$ \\
\hline 18 & 27 & $2 s^{2} 2 p^{3}\left({ }_{3}^{2} D\right){ }^{2} D 3 p^{1} P_{1}$ & & 2706788 & 4.643E-10 & $4.725 \mathrm{E}-10$ & $0.46+0.362 s^{2} 2 p^{3}\left({ }_{3}^{2} D\right)^{2} D 3 p^{3} D+0.102 s^{2} 2 p^{3}\left({ }_{1}^{2} P\right)^{2} P 3 p^{1} P$ \\
\hline 18 & 28 & $2 s^{2} 2 p^{3}\left(\frac{2}{3} D\right)^{2} D 3 p^{3} D_{2}$ & & 2714906 & $6.260 \mathrm{E}-10$ & $6.286 \mathrm{E}-10$ & $\left.0.83+0.082 s^{2} 2 p^{3}\left(\frac{2}{3} D\right)\right)^{2} D 3 p^{3} F+0.062 s^{2} 2 p^{3}\left({ }_{1}^{2} P\right)^{2} P 3 p^{3} D$ \\
\hline 18 & 29 & $2 s^{2} 2 p^{3}\left(\frac{3}{2} D\right)^{2} D 3 p^{3} D_{1}$ & & 2718097 & 4.075E-10 & $4.144 \mathrm{E}-10$ & $0.52+0.382 s^{2} 2 p^{3}\left({ }_{3}^{3} D\right)^{2} D 3 p^{1} P+0.052 s^{2} 2 p^{3}\left(_{1}^{2} P\right)^{2} P 3 p^{3} D$ \\
\hline 18 & 30 & $2 s^{2} 2 p^{3}\left(\frac{2}{3} D\right)^{2} D 3 p^{3} D_{3}$ & & 2718352 & $5.544 \mathrm{E}-10$ & $5.570 \mathrm{E}-10$ & $0.82+0.122 s^{2} 2 p^{3}\left(\frac{2}{3} D\right)^{2} D 3 p^{3} F+0.032 s^{2} 2 p^{3}\left({ }_{1}^{2} P\right)^{2} P 3 p^{3} D$ \\
\hline 18 & 31 & $2 s^{2} 2 p^{3}\left({ }_{3}^{2} D\right)^{2} D 3 p^{3} F_{2}$ & 2728790 & 2724663 & 4.974E-10 & 4.965E-10 & $0.86+0.092 s^{2} 2 p^{3}\left({ }_{3}^{2} D\right)^{2} D 3 p^{3} D+0.022 s^{2} 2 p^{3}\left({ }_{1}^{2} P\right)^{2} P 3 p^{1} D$ \\
\hline 18 & 32 & $2 s^{2} 2 p^{3}\left({ }_{3}^{2} D\right)^{2} D 3 p^{3} F_{3}$ & & 2727321 & $4.646 \mathrm{E}-10$ & $4.644 \mathrm{E}-10$ & $0.83+0.142 s^{2} 2 p^{3}\left({ }_{3}^{2} D\right)^{2} D 3 p^{3} D$ \\
\hline 18 & 33 & $2 s^{2} 2 p^{3}\left({ }_{3}^{2} D\right)^{2} D 3 p^{3} F_{4}$ & & 2729735 & $5.885 \mathrm{E}-10$ & $5.847 \mathrm{E}-10$ & $0.99+3+2+3$ \\
\hline 18 & 34 & $2 s^{2} 2 p^{3}\left(\frac{2}{3} D\right)^{2} D 3 p^{1} F_{3}$ & & 2732737 & $6.353 \mathrm{E}-10$ & $6.309 \mathrm{E}-10$ & $0.95+0.042 s^{2} 2 p^{3}\left({ }_{1}^{2} P\right)^{2} P 3 p^{3} D$ \\
\hline 18 & 35 & $2 s^{2} 2 p^{3}\left(\frac{2}{3} D\right)^{2} D 3 p^{3} P_{1}$ & & 2761742 & 5.675E-11 & $5.735 \mathrm{E}-11$ & $0.39+0.452 s^{2} 2 p^{3}\left({ }_{1}^{2} P\right)^{2} P 3 p^{3} S+0.072 s^{2} 2 p^{3}\left({ }_{3}^{4} S\right)^{4} S 3 p^{3} P$ \\
\hline 18 & 36 & $2 s^{2} 2 p^{3}\left({ }_{3}^{2} D\right)^{2} D 3 p^{3} P_{0}$ & & 2765720 & $5.800 \mathrm{E}-11$ & $5.850 \mathrm{E}-11$ & $0.78+0.132 s^{2} 2 p^{3}\left({ }_{1}^{2} P\right)^{2} P 3 p^{3} P+0.052 s^{2} 2 p^{3}\left({ }_{3}^{4} S\right)^{4} S 3 p^{3} P$ \\
\hline 18 & 37 & $2 s^{2} 2 p^{3}\left(\frac{2}{3} D\right)^{2} D 3 p^{3} P_{2}$ & 2769650 & 2769674 & $4.390 \mathrm{E}-11$ & 4.429E-11 & $0.82+0.112 s^{2} 2 p^{3}\left({ }_{3}^{4} S\right)^{4} S 3 p^{3} P$ \\
\hline 18 & 38 & $2 s^{2} 2 p^{3}\left({ }_{1}^{2} P\right)^{2} P 3 p^{3} s_{1}$ & & 2777858 & $5.770 \mathrm{E}-11$ & $5.829 \mathrm{E}-11$ & $0.47+0.372 s^{2} 2 p^{3}\left(\frac{2}{3} D\right)^{2} D 3 p^{3} P+0.032 s^{2} 2 p^{3}\left({ }_{1}^{2} P\right)^{2} P 3 p^{3} P$ \\
\hline 18 & 39 & $2 s^{2} 2 p^{3}\left({ }_{1}^{2} P\right)^{2} P 3 p^{3} D_{1}$ & & 2778874 & $1.448 \mathrm{E}-10$ & 1.454E-10 & $0.79+0.072 s^{2} 2 p^{3}\left({ }_{1}^{2} P\right)^{2} P 3 p^{1} P+0.052 s^{2} 2 p^{3}\left({ }_{3}^{2} D\right)^{2} D 3 p^{3} D$ \\
\hline 18 & 40 & $2 s^{2} 2 p^{3}\left({ }_{1}^{2} P\right)^{2} P 3 p^{3} D_{2}$ & & 2779604 & 1.434E-10 & $1.440 \mathrm{E}-10$ & $0.68+0.142 s^{2} 2 p^{3}\left({ }_{3}^{2} D\right)^{2} D 3 p^{1} D+0.052 s^{2} 2 p^{3}\left({ }_{1}^{2} P\right)^{2} P 3 p^{1} D$ \\
\hline 18 & 41 & $2 s^{2} 2 p^{3}\left({ }_{1}^{2} P\right)^{2} P 3 p^{3} D_{3}$ & & 2784347 & 1.693E-10 & 1.697E-10 & $0.88+0.042 s^{2} 2 p^{3}\left({ }_{3}^{3} D\right)^{2} D 3 p^{1} F+0.042 s^{2} 2 p^{3}\left(\frac{2}{3} D\right)^{2} D 3 p^{3} F$ \\
\hline 18 & 42 & $2 s^{2} 2 p^{3}\left(\frac{2}{3} D\right)^{2} D 3 p^{1} D_{2}$ & & 2786517 & $1.388 \mathrm{E}-10$ & $1.395 \mathrm{E}-10$ & $0.38+0.222 s^{2} 2 p^{3}\left({ }_{1}^{2} P\right)^{2} P 3 p^{1} D+0.202 s^{2} 2 p^{3}\left({ }_{1}^{2} P\right)^{2} P 3 p^{3} D$ \\
\hline 18 & 43 & $2 s^{2} 2 p^{3}\left({ }_{3}^{4} S\right)^{4} S 3 d^{5} D_{2}^{\circ}$ & & 2792116 & $1.730 \mathrm{E}-10$ & $1.724 \mathrm{E}-10$ & 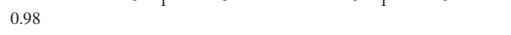 \\
\hline 18 & 44 & $2 s^{2} 2 p^{3}\left({ }_{3}^{4} S\right){ }^{4} S 3 d^{5} D_{1}^{\circ}$ & & 2792124 & 1.369E-10 & $1.363 \mathrm{E}-10$ & 0.98 \\
\hline 18 & 45 & $2 s^{2} 2 p^{3}\left({ }_{3}^{4} S\right){ }^{4} S 3 d^{5} D_{3}^{\circ}$ & & 2792132 & $3.202 \mathrm{E}-10$ & $3.201 \mathrm{E}-10$ & 0.98 \\
\hline 18 & 46 & $2 s^{2} 2 p^{3}\left({ }_{3}^{4} S\right){ }^{4} S 3 d^{5} D_{0}^{\circ}$ & & 2792139 & $1.340 \mathrm{E}-10$ & $1.332 \mathrm{E}-10$ & 0.98 \\
\hline 18 & 47 & $2 s^{2} 2 p^{3}\left({ }_{3}^{4} S\right){ }^{4} S 3 d^{5} D_{4}^{\circ}$ & & 2792309 & 3.734E-10 & $3.720 \mathrm{E}-10$ & 0.98 \\
\hline 18 & 48 & $2 s^{2} 2 p^{3}\left({ }_{1}^{2} P\right)^{2} P 3 p^{1} P_{1}^{4}$ & & 2792384 & $1.452 \mathrm{E}-10$ & 1.459E-10 & $0.68+0.092 s^{2} 2 p^{3}\left({ }_{3}^{2} D\right)^{2} D 3 p^{1} P+0.072 s^{2} 2 p^{3}\left({ }_{1}^{2} P\right)^{2} P 3 p^{3} D$ \\
\hline 18 & 49 & $2 s^{2} 2 p^{3}\left({ }_{1}^{2} P\right)^{2} P 3 p^{3} P_{2}$ & & 2803756 & $2.750 \mathrm{E}-10$ & 2.763E-10 & $\left.0.73+0.142 s^{2} 2 p^{3}\left({ }_{1}^{2} P\right)^{2} P 3 p{ }^{1} D+0.032 s^{2} 2 p^{3}\left(\frac{2}{3} D\right)\right)^{2} D 3 p^{1} D$ \\
\hline 18 & 50 & $2 s^{2} 2 p^{3}\left({ }_{1}^{2} P\right)^{2} P 3 p^{3} P_{1}$ & & 2804468 & $1.476 \mathrm{E}-10$ & 1.487E-10 & $0.78+0.052 s^{2} 2 p^{3}\left({ }_{3}^{4} S\right)^{4} S 3 p^{3} P+0.052 s^{2} 2 p^{3}\left(_{1}^{2} P\right)^{2} P 3 p^{1} P$ \\
\hline 18 & 51 & $2 s^{2} 2 p^{3}\left({ }_{1}^{2} P\right){ }^{2} P 3 p^{3} P_{0}$ & & 2804933 & $1.072 \mathrm{E}-10$ & $1.081 \mathrm{E}-10$ & $0.80+0.072 s^{2} 2 p^{3}\left({ }_{3}^{4} S\right)^{4} S 3 p^{3} P+0.072 s^{2} 2 p^{3}\left({ }_{3}^{2} D\right)^{2} D 3 p^{3} P$ \\
\hline 18 & 52 & $2 s^{2} 2 p^{3}\left({ }_{2}^{4} S\right)^{4} S 3 d^{3} D_{2}^{\circ}$ & 2826250 & 2825405 & $1.250 \mathrm{E}-12$ & $1.249 \mathrm{E}-12$ & $0.85+0.092 s^{2} 2 p^{3}\left(c_{3}^{2} D\right)^{2} D 3 d^{3} D^{\circ}$ \\
\hline 18 & 53 & $2 s^{2} 2 p^{3}\left(3_{3}^{4} S\right)^{4} S 3 d^{3} D_{1}^{2}$ & & 2826384 & 1.193E-12 & $1.192 \mathrm{E}-12$ & $0.87+0.082 s^{2} 2 p^{3}\left(c_{2}^{3} D\right)^{2} D 3 d^{3} D^{\circ}+0.032 s^{2} 2 p^{3}\left({ }_{1}^{2} P\right)^{2} P 3 d^{3} D^{\circ}$ \\
\hline 18 & 54 & $2 s^{2} 2 p^{3}\left({ }_{3}^{4} S\right){ }^{4} S 3 d^{3} D_{3}^{\circ}$ & 2826300 & 2827108 & $1.152 \mathrm{E}-12$ & $1.150 \mathrm{E}-12$ & $0.86+0.102 s^{2} 2 p^{3}\left({ }_{3}^{2} D\right)^{2} D 3 d^{3} D^{\circ}$ \\
\hline 18 & 55 & $2 s^{2} 2 p^{3}\left({ }_{1}^{2} P\right)^{2} P 3 p^{1} D_{2}^{3}$ & & 2828336 & $1.889 \mathrm{E}-10$ & 1.907E-10 & $0.50+0.422 s^{2} 2 p^{3}\left({ }_{3}^{2} D\right)^{2} D 3 p^{1} D+0.032 s^{2} 2 p^{3}\left({ }_{1}^{2} P\right)^{2} P 3 p^{3} P$ \\
\hline 18 & 56 & $2 s^{2} 2 p^{3}\left({ }_{1}^{2} P\right)^{2} P 3 p^{1} S_{0}$ & & 2879409 & 3.842E-11 & $3.870 \mathrm{E}-11$ & 0.93 \\
\hline 18 & 57 & $2 s^{2} 2 p^{3}\left({ }_{3}^{2} D\right)^{2} D 3 d^{3} F_{2}^{\circ}$ & & 2882794 & $3.148 \mathrm{E}-11$ & $3.139 \mathrm{E}-11$ & $0.85+0.102 s^{2} 2 p^{3}\left({ }_{1}^{2} P\right)^{2} P 3 d^{3} F^{\circ}$ \\
\hline 18 & 58 & $2 s^{2} 2 p^{3}\left({ }_{3}^{2} D\right)^{2} D 3 d^{3} F_{3}^{\circ}$ & & 2885698 & $2.678 \mathrm{E}-11$ & $2.677 \mathrm{E}-11$ & $0.85+0.092 s^{2} 2 p^{3}\left({ }_{1}^{2} P\right)^{2} P 3 d^{3} F^{\circ}+0.042 s^{2} 2 p^{3}\left({ }_{2}^{2} D\right)^{2} D 3 d^{3} G^{\circ}$ \\
\hline 18 & 59 & $2 s^{2} 2 p^{3}\left(\frac{2}{3} D\right)^{2} D 3 d^{1} S_{0}^{0}$ & & 2887376 & $8.448 \mathrm{E}-11$ & $8.488 \mathrm{E}-11$ & 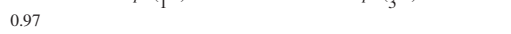 \\
\hline 18 & 60 & $2 s^{2} 2 p^{3}\left(\frac{2}{3} D\right)^{2} D 3 d^{3} F_{4}^{\circ}$ & & 2889110 & 3.959E-10 & $3.931 \mathrm{E}-10$ & $0.80+0.092 s^{2} 2 p^{3}\left({ }_{3}^{2} D\right)^{2} D 3 d^{3} G^{\circ}+0.062 s^{2} 2 p^{3}\left({ }_{1}^{2} P\right)^{2} P 3 d^{3} F^{\circ}$ \\
\hline 18 & 61 & $2 s^{2} 2 p^{3}\left({ }_{3}^{2} D\right)^{2} D 3 d^{3} G_{3}^{\circ}$ & 2900300 & 2896390 & 4.383E-11 & 4.379E-11 & $0.90+0.052 s^{2} 2 p^{3}\left({ }_{3}^{2} D\right)^{2} D 3 d^{3} F^{\circ}+0.022 s^{2} 2 p^{3}\left({ }_{1}^{2} P\right)^{2} P 3 d^{1} F^{\circ}$ \\
\hline 18 & 62 & $2 s^{2} 2 p^{3}\left(\frac{2}{3} D\right)^{2} D 3 d^{3} G_{4}^{0}$ & & 2897484 & $3.532 \mathrm{E}-10$ & $3.521 \mathrm{E}-10$ & $0.82+0.132 s^{2} 2 p^{3}\left({ }_{3}^{2} D\right)^{2} D 3 d^{3} F^{\circ}$ \\
\hline 18 & 63 & $2 s^{2} 2 p^{3}\left({ }_{3}^{3} D\right)^{2} D 3 d^{3} G_{5}^{\circ}$ & & 2898737 & $3.599 \mathrm{E}-10$ & $3.574 \mathrm{E}-10$ & 0.99 \\
\hline 18 & 64 & $2 s^{2} 2 p^{3}\left(\frac{2}{3} D\right)^{2} D 3 d^{1} G_{4}^{\mathrm{o}}$ & & 2901424 & $3.568 \mathrm{E}-10$ & 3.554E-10 & $0.92+0.042 s^{2} 2 p^{3}\left({ }_{3}^{2} D\right)^{2} D 3 d^{3} G^{\circ}+0.022 s^{2} 2 p^{3}\left({ }_{3}^{2} D\right)^{2} D 3 d^{3} F^{\circ}$ \\
\hline 18 & 65 & $2 s^{2} 2 p^{3}\left(\frac{2}{3} D\right)^{2} D 3 d^{1} P_{1}^{0}$ & & 2904680 & $7.871 \mathrm{E}-13$ & $7.868 \mathrm{E}-13$ & $0.47+0.372 s^{2} 2 p^{3}\left({ }_{3}^{2} D\right)^{2} D 3 d^{3} D^{\circ}+0.052 s^{2} 2 p^{3}\left(_{1}^{2} P\right)^{2} P 3 d^{1} P^{\circ}$ \\
\hline 18 & 66 & $2 s^{2} 2 p^{3}\left(\frac{2}{3} D\right)^{2} D 3 d^{3} D_{2}^{\circ}$ & 2911870 & 2911323 & $6.592 \mathrm{E}-13$ & $6.586 \mathrm{E}-13$ & $0.80+0.092 s^{2} 2 p^{3}\left({ }_{3}^{4} S\right)^{4} S 3 d^{3} D^{\circ}+0.032 s^{2} 2 p^{3}\left({ }_{3}^{2} D\right)^{2} D 3 d^{3} P^{\circ}$ \\
\hline 18 & 67 & $2 s^{2} 2 p^{3}\left({ }_{3}^{2} D\right)^{2} D 3 d^{3} D_{1}^{\circ}$ & & 2912584 & 7.483E-13 & 7.482E-13 & $0.50+0.412 s^{2} 2 p^{3}\left({ }_{3}^{2} D\right)^{2} D 3 d^{1} P^{\circ}+0.042 s^{2} 2 p^{3}\left({ }_{3}^{4} S\right)^{4} S 3 d^{3} D^{\circ}$ \\
\hline 18 & 68 & $2 s^{2} 2 p^{3}\left(c_{3}^{3} D\right)^{2} D 3 d^{3} D_{3}^{\circ}$ & 2913070 & 2912705 & $4.863 \mathrm{E}-13$ & $4.858 \mathrm{E}-13$ & $0.87+0.102 s^{2} 2 p^{3}\left({ }_{3}^{4} S\right)^{4} S 3 d^{3} D^{\circ}$ \\
\hline 18 & 69 & $2 s^{2} 2 p^{3}\left(\frac{2}{3} D\right)^{2} D 3 d^{3} P_{2}^{\circ}$ & 2922000 & 2920512 & $3.532 \mathrm{E}-13$ & $3.531 \mathrm{E}-13$ & $0.75+0.122 s^{2} 2 p^{3}\left({ }_{1}^{2} P\right)^{2} P 3 d^{3} P^{\circ}+0.052 s^{2} 2 p^{3}\left({ }_{3}^{2} D\right)^{2} D 3 d^{1} D^{\circ}$ \\
\hline 18 & 70 & $2 s^{2} 2 p^{3}\left({ }_{3}^{2} D\right)^{2} D 3 d^{1} D_{2}^{\circ}$ & & 2922234 & $1.118 \mathrm{E}-12$ & $1.118 \mathrm{E}-12$ & $0.53+0.312 s^{2} 2 p^{3}\left({ }_{1}^{2} P\right)^{2} P 3 d^{1} D^{\circ}+0.072 s^{2} 2 p^{3}\left({ }_{3}^{2} D\right)^{2} D 3 d^{3} P^{\circ}$ \\
\hline 18 & 71 & $2 s^{2} 2 p^{3}\left(\frac{2}{3} D\right)^{2} D 3 d^{3} P_{0}^{\circ}$ & & 2925090 & $5.640 \mathrm{E}-13$ & $5.638 \mathrm{E}-13$ & $0.96+0.022 s^{2} 2 p^{3}\left(2_{1}^{2} P\right)^{2} P 3 d^{3} P^{\circ}$ \\
\hline 18 & 72 & $2 s^{2} 2 p^{3}\left(\frac{2}{3} D\right)^{2} D 3 d^{3} P_{1}^{\circ}$ & 2932610 & 2925253 & $4.558 \mathrm{E}-13$ & 4.557E-13 & $0.95+0.022 s^{2} 2 p^{3}\left(\frac{1}{3} D\right)^{2} D 3 d^{1} P^{\circ}$ \\
\hline 18 & 73 & $2 s^{2} 2 p^{3}\left(\frac{2}{3} D\right)^{2} D 3 d^{3} S_{1}^{\circ}$ & 2933510 & 2932321 & $3.966 \mathrm{E}-13$ & $3.965 \mathrm{E}-13$ & $0.87+0.102 s^{2} 2 p^{3}\left({ }_{1}^{2} P\right)^{2} P 3 d^{3} P^{\circ}$ \\
\hline 18 & 74 & $2 s^{2} S 2 p^{4}\left({ }_{3}^{3} P\right)^{4} P 3 s^{5} P_{3}$ & & 2940888 & $1.035 \mathrm{E}-10$ & $1.032 \mathrm{E}-10$ & $0.98+10$ \\
\hline 18 & 75 & $2 s^{2} 2 p^{3}\left({ }_{3}^{2} D\right)^{2} D 3 d^{1} F_{3}^{\circ}$ & & 2944695 & $6.242 \mathrm{E}-13$ & $6.233 \mathrm{E}-13$ & $0.72+0.132 s^{2} 2 p^{3}\left({ }_{1}^{2} P\right)^{2} P 3 d^{3} F^{\circ}+0.052 s^{2} 2 p^{3}\left({ }_{1}^{2} P\right)^{2} P 3 d^{1} F^{\circ}$ \\
\hline 18 & 76 & $2 s^{2} S 2 p^{4}\left({ }_{2}^{3} P\right)^{4} P 3 s^{5} P_{2}$ & & 2951244 & 7.338E-11 & 7.354E-11 & 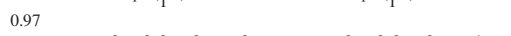 \\
\hline 18 & 77 & $2 s^{2} 2 p^{3}\left({ }_{1}^{2} P\right)^{2} P 3 d^{3} F_{4}^{\circ}$ & & 2953570 & $3.412 \mathrm{E}-10$ & 3.409E-10 & $0.87+0.032 s^{2} 2 p^{3}\left({ }_{3}^{2} D\right)^{2} D 3 d^{3} G^{\circ}+0.032 s^{2} 2 p^{3}\left({ }_{3}^{2} D\right)^{2} D 3 d^{1} G^{\circ}$ \\
\hline 18 & 78 & $2 s^{2} 2 p^{3}\left({ }_{1}^{2} P\right)^{2} P 3 d^{3} F_{2}^{0}$ & & 2953770 & $1.069 \mathrm{E}-11$ & $1.068 \mathrm{E}-11$ & $0.82+0.092 s^{2} 2 p^{3}\left({ }_{3}^{2} D\right)^{2} D 3 d^{3} F^{\circ}+0.022 s^{2} 2 p^{3}\left({ }_{1}^{2} P\right)^{2} P 3 d^{3} D^{\circ}$ \\
\hline 18 & 79 & $2 s^{2} 2 p^{3}\left({ }_{1}^{2} P\right)^{2} P 3 d^{3} F_{3}^{o}$ & 2945040 & 2954139 & 3.293E-12 & $3.289 \mathrm{E}-12$ & $0.69+0.132 s^{2} 2 p^{3}\left(_{1}^{2} P\right)^{2} P 3 d^{3} D^{\circ}+0.082 s^{2} 2 p^{3}\left({ }_{3}^{2} D\right)^{2} D 3 d^{1} F^{\circ}$ \\
\hline 18 & 80 & $2 s^{2} 2 p^{3}\left({ }_{1}^{2} P\right)^{2} P 3 d^{3} P_{0}^{\mathrm{o}}$ & & 2955060 & $8.298 \mathrm{E}-13$ & $8.297 \mathrm{E}-13$ & $0.92+0.022 s^{2} 2 p^{3}\left({ }_{3}^{2} D\right)^{2} D 3 d^{3} P^{\circ}$ \\
\hline 18 & 81 & $2 s^{2} 2 p^{3}\left({ }_{1}^{2} P\right)^{2} P 3 d^{3} P_{1}^{\circ}$ & 2968000 & 2957524 & 1.007E-12 & 1.007E-12 & $\left.0.82+0.112 s^{2} 2 p^{3}\left({ }_{3}^{2} D\right)\right)^{2} D 3 d^{3} s^{\circ}+0.022 s^{2} 2 p^{3}\left({ }_{3}^{2} D\right)^{2} D 3 d^{1} P^{\circ}$ \\
\hline 18 & 82 & $2 s^{2} S 2 p^{4}\left({ }_{3}^{3} P\right)^{4} P 3 s^{5} P_{1}$ & & 2957638 & 8.503E-11 & $8.541 \mathrm{E}-11$ & 0.98 \\
\hline
\end{tabular}




\begin{tabular}{|c|c|c|c|c|c|c|c|}
\hline $\mathrm{z}$ & Key & Level & $E_{\mathrm{NIST}}$ & $E_{\mathrm{MCDHF} / \mathrm{RCI}}$ & $\tau_{\mathrm{MCDHF} / \mathrm{RCI}}^{l}$ & $\tau_{\mathrm{MCDHF} / \mathrm{RCI}}^{v}$ & $L S$-composition \\
\hline 18 & 83 & $2 s^{2} 2 p^{3}\left({ }_{1}^{2} P\right)^{2} P 3 d^{3} P_{2}^{\circ}$ & 2958500 & 2959294 & $8.148 \mathrm{E}-12$ & $8.152 \mathrm{E}-12$ & $0.79+0.112 s^{2} 2 p^{3}\left({ }_{3}^{2} D\right)^{2} D 3 d^{3} P^{\circ}$ \\
\hline 18 & 84 & $2 s^{2} 2 p^{3}\left({ }_{1}^{2} P\right)^{2} P 3 d^{3} D_{2}^{2}$ & 2969540 & 2967490 & $5.364 \mathrm{E}-13$ & 5.359E-13 & $0.68+0.192 s^{2} 2 p^{3}\left({ }_{1}^{2} P\right)^{2} P 3 d^{1} D^{\circ}+0.052 s^{2} 2 p^{3}\left({ }_{3}^{2} D\right)^{2} D 3 d^{1} D^{\circ}$ \\
\hline 18 & 85 & $2 s^{2} 2 p^{3}\left({ }_{1}^{2} P\right)^{2} P 3 d^{3} D_{1}^{\circ}$ & 2987530 & 2971222 & 4.378E-13 & 4.372E-13 & $0.88+0.042 s^{2} 2 p^{3}\left({ }_{3}^{4} S\right)^{4} S 3 d^{3} D^{\circ}+0.022 s^{2} 2 p^{3}\left(\frac{2}{3} D\right)^{2} D 3 d^{3} D^{\circ}$ \\
\hline 18 & 86 & $2 s^{2} 2 p^{3}\left({ }_{1}^{2} P\right)^{2} P 3 d^{3} D_{3}^{\circ}$ & & 2971426 & 1.117E-12 & $1.116 \mathrm{E}-12$ & $0.74+0.082 s^{2} 2 p^{3}\left({ }_{1}^{2} P\right)^{2} P 3 d^{1} F^{\circ}+0.072 s^{2} 2 p^{3}\left({ }_{3}^{2} D\right)^{2} D 3 d^{1} F^{\circ}$ \\
\hline 18 & 87 & $2 s^{2} 2 p^{3}\left({ }_{1}^{2} P\right)^{2} P 3 d^{1} D_{2}^{0}$ & & 2981513 & $4.469 \mathrm{E}-13$ & $4.465 \mathrm{E}-13$ & $0.40+0.322 s^{2} 2 p^{3}\left(\frac{2}{3} D\right)^{2} D 3 d^{1} D^{\circ}+0.192 s^{2} 2 p^{3}\left(_{1}^{2} P\right)^{2} P 3 d^{3} D^{\circ}$ \\
\hline 18 & 88 & $2 s^{2} 2 p^{3}\left({ }_{1}^{2} P\right)^{2} P 3 d^{1} F_{3}^{\circ}$ & & 2984747 & 3.191E-13 & $3.187 \mathrm{E}-13$ & $0.80+0.112 s^{2} 2 p^{3}\left({ }_{3}^{2} D\right)^{2} D 3 d^{1} F^{\circ}+0.032 s^{2} 2 p^{3}\left({ }_{1}^{2} P\right)^{2} P 3 d^{3} D^{\circ}$ \\
\hline 18 & 89 & $2 s^{2} S 2 p^{4}\left({ }_{2}^{3} P\right){ }^{4} P 3 s^{3} P_{2}$ & & 2989973 & $2.781 \mathrm{E}-12$ & $2.785 \mathrm{E}-12$ & $0.95+3+1$ \\
\hline 18 & 90 & $2 s^{2} S 2 p^{4}\left({ }_{2}^{3} P\right)^{4} P 3 s^{3} P_{1}$ & & 3001336 & 2.703E-12 & $2.707 \mathrm{E}-12$ & 0.95 \\
\hline 18 & 91 & $\left.2 s^{2} S 2 p^{4}{ }_{2}^{3} P\right)^{4} P 3 s^{3} P_{0}$ & & 3006262 & 2.690E-12 & 2.693E-12 & 0.95 \\
\hline 18 & 92 & $2 s^{2} 2 p^{3}\left({ }_{1}^{2} P\right)^{2} P 3 d^{1} P_{1}^{\circ}$ & & 3015660 & $2.866 \mathrm{E}-13$ & $2.863 \mathrm{E}-13$ & $0.88+0.052 s^{2} 2 p^{3}\left(\frac{2}{3} D\right)^{2} D 3 d^{1} P^{\circ}$ \\
\hline 18 & 93 & $2 s^{2} S 2 p^{4}\left({ }_{2}^{3} P\right){ }^{4} P 3 p{ }^{5} P_{3}^{\circ}$ & & 3068065 & $1.011 \mathrm{E}-10$ & $1.005 \mathrm{E}-10$ & $0.92+0.082 s^{2} S 2 p^{4}\left({ }_{2}^{3} P\right)^{4} P 3 p^{5} D^{\circ}$ \\
\hline 18 & 94 & $2 s^{2} S 2 p^{4}\left({ }_{2}^{3} P\right)^{4} P 3 p^{5} P_{2}^{\circ}$ & & 3070456 & $7.860 \mathrm{E}-11$ & $7.829 \mathrm{E}-11$ & $\left.0.90+0.052 s^{2} S 2 p^{4}\left({ }_{2}^{3} P\right)^{4} P 3 p^{5} D^{\circ}+0.042 s^{2} S 2 p^{4}{ }_{2}^{3} P\right)^{4} P 3 p^{5} S^{\circ}$ \\
\hline 18 & 95 & $2 s^{2} S 2 p^{4}\left(\frac{3}{3} P\right)^{4} P 3 p^{5} P_{1}^{0}$ & & 3075944 & $7.837 \mathrm{E}-11$ & $7.823 \mathrm{E}-11$ & $0.96+0.032 s^{2} S 2 p^{4}\left(3_{2}^{3} P\right)^{4} P 3 p^{5} D^{\circ}$ \\
\hline 18 & 96 & $2 s^{2} S 2 p^{4}\left({ }_{2}^{3} P\right)^{4} P 3 p^{5} D_{4}^{\circ}$ & & 3083287 & $1.325 \mathrm{E}-10$ & $1.311 \mathrm{E}-10$ & 0.99 \\
\hline 18 & 97 & $2 s^{2} S 2 p^{4}\left({ }_{2}^{3} P\right){ }^{4} P 3 p^{5} D_{3}^{\circ}$ & & 3089202 & $1.932 \mathrm{E}-11$ & $1.929 \mathrm{E}-11$ & $\left.0.81+0.102 s^{2} S 2 p^{4}\left({ }_{2}^{3} P\right)^{4} P 3 p^{3} D^{\circ}+0.072 s^{2} S 2 p^{4} l_{2}^{3} P\right)^{4} P 3 p^{5} P^{\circ}$ \\
\hline 18 & 98 & $2 s^{2} S 2 p^{4}\left({ }_{2}^{3} P\right)^{4} P 3 p^{5} D_{2}^{\circ}$ & & 3093435 & 4.153E-11 & 4.147E-11 & $0.89+0.052 s^{2} S 2 p^{4}\left({ }_{2}^{3} P\right)^{4} P 3 p^{5} P^{\circ}+0.042 s^{2} S 2 p^{4}\left({ }_{2}^{3} P\right)^{4} P 3 p^{3} D^{\circ}$ \\
\hline 18 & 99 & $2 s^{2} S 2 p^{4}\left({ }_{2}^{3} P\right)^{4} P 3 p^{5} D_{1}^{\circ}$ & & 3095875 & 7.955E-11 & 7.943E-11 & $0.95+0.032 s^{2} S 2 p^{4}\left(2_{2}^{\frac{2}{3}} P\right)^{4} P 3 p^{5} P^{\circ}$ \\
\hline 18 & 100 & $2 s^{2} S 2 p^{4}\left({ }_{2}^{3} P\right){ }^{4} P 3 p^{5} D_{0}^{\circ}$ & & 3096816 & $1.203 \mathrm{E}-10$ & $1.200 \mathrm{E}-10$ & 0.99 \\
\hline 18 & 101 & $2 s^{2} S 2 p^{4}\left({ }_{2}^{3} P\right)^{4} P 3 p^{3} D_{3}^{\circ}$ & 3106170 & 3104626 & $2.340 \mathrm{E}-12$ & $2.336 \mathrm{E}-12$ & $0.88+0.102 s^{2} S 2 p^{4}\left({ }_{2}^{3} P\right)^{4} P 3 p^{5} D^{\circ}$ \\
\hline 18 & 102 & $2 s^{2} S 2 p^{4}\left({ }_{2}^{3} P\right)^{4} P 3 p^{3} D_{2}^{\circ}$ & & 3112508 & $2.210 \mathrm{E}-12$ & 2.207E-12 & $0.85+0.082 s^{2} S 2 p^{4}\left({ }_{2}^{3} P\right)^{4} P 3 p^{3} P^{\circ}+0.042 s^{2} S 2 p^{4}\left({ }_{2}^{3} P\right){ }^{4} P 3 p^{5} D^{\circ}$ \\
\hline 18 & 103 & $2 s^{2} S 2 p^{4}\left({ }_{2}^{3} P\right)^{4} P 3 p^{5} S_{2}^{2}$ & & 3114232 & 7.313E-11 & 7.335E-11 & $0.94+0.042 s^{2} S 2 p^{4}\left({ }_{2}^{3} P\right)^{4} P 3 p^{5} P^{\circ}$ \\
\hline 18 & 104 & $2 s^{2} S 2 p^{4}\left({ }_{2}^{3} P\right)^{4} P 3 p^{3} S_{1}^{2}$ & & 3115538 & $1.650 \mathrm{E}-12$ & 1.649E-12 & $0.66+0.182 s^{2} S 2 p^{4}\left({ }_{2}^{3} P\right)^{4} P 3 p^{3} P^{\circ}+0.112 s^{2} S 2 p^{4}\left({ }_{2}^{3} P\right){ }^{4} P 3 p^{3} D^{\circ}$ \\
\hline 18 & 105 & $2 s^{2} S 2 p^{4}\left({ }_{2}^{1} D\right)^{2} D 3 s^{3} D_{1}$ & & 3116032 & 4.090E-12 & $4.099 \mathrm{E}-12$ & $0.97-2+2$ \\
\hline 18 & 106 & $2 s^{2} S 2 p^{4}\left({ }_{2}^{1} D\right)^{2} D 3 s^{3} D_{2}$ & & 3116244 & 4.080E-12 & 4.087E-12 & 0.97 \\
\hline 18 & 107 & $2 s^{2} S 2 p^{4}\left(\frac{1}{2} D\right)^{2} D 3 s^{3} D_{3}$ & & 3116526 & $4.030 \mathrm{E}-12$ & $4.035 \mathrm{E}-12$ & 0.97 \\
\hline 18 & 108 & $2 s^{2} S 2 p^{4}\left({ }_{2}^{3} P\right)^{4} P 3 p^{3} D_{1}^{\circ}$ & & 3119641 & $2.058 \mathrm{E}-12$ & 2.056E-12 & $0.83+0.152 s^{2} S 2 p^{4}\left({ }_{2}^{3} P\right)^{4} P 3 p^{3} s^{\circ}$ \\
\hline 18 & 109 & $2 s^{2} S 2 p^{4}\left({ }_{2}^{3} P\right)^{4} P 3 p^{3} P_{2}^{\circ}$ & & 3127495 & $1.912 \mathrm{E}-12$ & $1.908 \mathrm{E}-12$ & $0.79+0.092 s^{2} S 2 p^{4}\left({ }_{2}^{3} P\right)^{4} P 3 p^{3} D^{\circ}+0.092 s^{2} S 2 p^{4}\left(\frac{1}{2} D\right)^{2} D 3 p^{3} P^{\circ}$ \\
\hline 18 & 110 & $2 s^{2} S 2 p^{4}\left(\frac{2}{3} P\right)^{4} P 3 p^{3} P_{0}^{\circ}$ & & 3130774 & 2.055E-12 & 2.051E-12 & $0.85+0.122 s^{2} S 2 p^{4}\left({ }_{2}^{1} D\right)^{2} D 3 p^{3} P^{\circ}$ \\
\hline 18 & 111 & $2 s^{2} S 2 p^{4}\left({ }_{2}^{3} P\right)^{4} P 3 p^{3} P_{1}^{\circ}$ & & 3132778 & 1.869E-12 & $1.867 \mathrm{E}-12$ & $0.68+0.172 s^{2} S 2 p^{4}\left({ }_{2}^{3} P\right)^{4} P 3 p^{3} S^{\circ}+0.092 s^{2} S 2 p^{4}\left({ }_{2}^{1} D\right)^{2} D 3 p^{3} P^{\circ}$ \\
\hline 18 & 112 & $2 s^{2} S 2 p^{4}\left({ }_{2}^{1} D\right)^{2} D 3 s^{1} D_{2}$ & & 3147557 & 7.418E-12 & 7.429E-12 & $0.98-2+2$ \\
\hline 18 & 113 & $2 s^{2} S 2 p^{4}\left({ }_{0}^{1} S\right)^{2} S 3 s^{3} S_{1}$ & & 3217541 & 3.657E-12 & 3.663E-12 & $0.92+0.042 s^{2} S 2 p^{4}\left({ }_{2}^{3} P\right)^{2} P 3 s^{3} P$ \\
\hline 18 & 114 & $2 s^{2} S 2 p^{4}\left({ }_{2}^{3} P\right){ }^{4} P 3 d^{5} D_{4}$ & & 3229873 & $1.139 \mathrm{E}-10$ & $1.151 \mathrm{E}-10$ & $\left.0.93+0.062 s^{2} S 2 p^{4}{ }_{2}^{3} P\right)^{4} P 3 d^{5} F$ \\
\hline 18 & 115 & $2 s^{2} S 2 p^{4}\left(\frac{3}{3} P\right){ }^{4} P 3 d^{5} D_{3}$ & & 3230841 & 1.108E-10 & $1.122 \mathrm{E}-10$ & $0.92+0.042 s^{2} S 2 p^{4}\left(3_{2}^{4} P\right)^{4} P 3 d^{5} F+0.032 s^{2} S 2 p^{4}\left(3_{2}^{3} P\right){ }^{4} P 3 d^{5} P$ \\
\hline 18 & 116 & $2 s^{2} S 2 p^{4}\left({ }_{2}^{3} P\right)^{4} P 3 d^{5} D_{2}$ & & 3232873 & $1.054 \mathrm{E}-10$ & $1.069 \mathrm{E}-10$ & $0.93+0.042 s^{2} S 2 p^{4}\left({ }_{2}^{3} P\right)^{4} P 3 d^{5} P+0.022 s^{2} S 2 p^{4}\left({ }_{2}^{3} P\right)^{4} P 3 d^{5} F$ \\
\hline 18 & 117 & $2 s^{2} S 2 p^{4}\left({ }_{2}^{3} P\right)^{4} P 3 d^{5} D_{1}$ & & 3235156 & $9.666 \mathrm{E}-11$ & $9.808 \mathrm{E}-11$ & $0.95+0.032 s^{2} S 2 p^{4}\left({ }_{2}^{3} P\right)^{4} P 3 d^{5} P$ \\
\hline 18 & 118 & $2 s^{2} S 2 p^{4}\left(3_{2}^{3} P\right)^{4} P 3 d^{5} D_{0}$ & & 3236784 & 8.961E-11 & $9.078 \mathrm{E}-11$ & 0.99 \\
\hline 18 & 119 & $2 s^{2} S 2 p^{4}\left({ }_{0}^{1} S\right)^{2} S 3 s^{1} S_{0}$ & & 3242987 & $5.023 \mathrm{E}-12$ & $5.033 \mathrm{E}-12$ & $0.73+0.252 s^{2} S 2 p^{4}\left({ }_{2}^{3} P\right)^{2} P 3 s^{3} P$ \\
\hline 18 & 120 & $2 s^{2} S 2 p^{4}\left(\frac{1}{2} D\right)^{2} D 3 p^{3} F_{2}^{\circ}$ & & 3245158 & $4.528 \mathrm{E}-11$ & 4.517E-11 & 0.98 \\
\hline 18 & 121 & $2 s^{2} S 2 p^{4}\left({ }_{2}^{3} P\right)^{4} P 3 d^{5} F_{5}^{2}$ & & 3245266 & 2.159E-10 & 2.054E-10 & 0.99 \\
\hline 18 & 122 & $2 s^{2} S 2 p^{4}\left({ }_{2}^{\top} D\right)^{2} D 3 p^{3} F_{3}^{\circ}$ & & 3246657 & 3.451E-11 & 3.443E-11 & 0.97 \\
\hline 18 & 123 & $2 s^{2} S 2 p^{4}\left(\frac{1}{2} D\right)^{2} D 3 p^{3} F_{4}^{\circ}$ & & 3249118 & $5.376 \mathrm{E}-11$ & $5.348 \mathrm{E}-11$ & 0.98 \\
\hline 18 & 124 & $2 s^{2} S 2 p^{4}\left({ }_{2}^{3} P\right)^{2} P 3 s^{3} P_{2}{ }^{4}$ & & 3249298 & $5.206 \mathrm{E}-12$ & $5.218 \mathrm{E}-12$ & 0.97 \\
\hline 18 & 125 & $2 s^{2} S 2 p^{4}\left({ }_{2}^{1} D\right)^{2} D 3 p^{1} P_{1}^{\circ}$ & & 3251172 & 1.339E-12 & $1.338 \mathrm{E}-12$ & $0.81+0.072 s^{2} S 2 p^{4}\left({ }_{2}^{3} P\right)^{2} P 3 p^{1} P^{\circ}+0.062 s^{2} S 2 p^{4}\left({ }_{2}^{1} D\right)^{2} D 3 p^{3} D^{\circ}$ \\
\hline 18 & 126 & $2 s^{2} S 2 p^{4}\left({ }_{2}^{3} P\right)^{4} P 3 d^{5} F_{4}$ & & 3251957 & 1.971E-10 & 1.895E-10 & $0.91+0.052 s^{2} S 2 p^{4}\left({ }_{2}^{3} P\right){ }^{4} P 3 d^{5} D+0.032 s^{2} S 2 p^{4}\left({ }_{2}^{2} P\right){ }^{4} P 3 d^{3} F$ \\
\hline 18 & 127 & $2 s^{2} S 2 p^{4}\left({ }_{2}^{3} P\right)^{2} P 3 s^{3} P_{1}$ & & 3255294 & 3.130E-12 & 3.138E-12 & $0.67+0.282 s^{2} S 2 p^{4}\left(\int_{2}^{3} P\right)^{2} P 3 s^{1} P$ \\
\hline 18 & 128 & $2 s^{2} S 2 p^{4}\left({ }_{2}^{3} P\right){ }^{4} P 3 d^{5} F_{3}$ & & 3256357 & $9.245 \mathrm{E}-11$ & $9.073 \mathrm{E}-11$ & $0.93+0.042 s^{2} S 2 p^{4}\left({ }_{2}^{3} P\right)^{4} P 3 d^{5} D$ \\
\hline 18 & 129 & $2 s^{2} S 2 p^{4}\left({ }_{2}^{3} P\right){ }^{4} P 3 d^{5} F_{2}$ & & 3259303 & 7.707E-11 & 7.592E-11 & $0.94+0.022 s^{2} S 2 p^{4}\left({ }_{2}^{3} P\right){ }^{4} P 3 d^{5} P$ \\
\hline 18 & 130 & $2 s^{2} S 2 p^{4}\left({ }_{2}^{\top} D\right)^{2} D 3 p p^{1} F_{3}^{\circ}$ & & 3260253 & $1.492 \mathrm{E}-12$ & $1.489 \mathrm{E}-12$ & $0.93+0.042 s^{2} S 2 p^{4}\left({ }_{2}^{\top} D\right)^{2} D 3 p^{3} D^{\circ}$ \\
\hline 18 & 131 & $2 s^{2} S 2 p^{4}\left({ }_{2}^{3} P\right)^{4} P 3 d^{5} P_{1}$ & & 3260810 & $5.245 \mathrm{E}-11$ & $5.322 \mathrm{E}-11$ & $0.56+0.422 s^{2} S 2 p^{4}\left({ }_{2}^{3} P\right)^{4} P 3 d^{5} F$ \\
\hline 18 & 132 & $2 s^{2} S 2 p^{4}\left(\frac{3}{2} P\right){ }^{4} P 3 d^{5} F_{1}$ & & 3261528 & 7.395E-11 & 7.482E-11 & $0.56+0.402 s^{2} S 2 p^{4}\left(\int_{2}^{3} P\right)^{4} P 3 d^{5} P+0.032 s^{2} S 2 p^{4}\left({ }_{2}^{3} P\right){ }^{4} P 3 d^{5} D$ \\
\hline 18 & 133 & $2 s^{2} S 2 p^{4}\left({ }_{2}^{3} P\right)^{4} P 3 d^{5} P_{2}$ & & 3265359 & 4.902E-11 & $5.070 \mathrm{E}-11$ & $0.93+0.042 s^{2} S 2 p^{4}\left(\int_{2}^{2} P\right)^{4} P 3 d^{5} D$ \\
\hline 18 & 134 & $2 s^{2} S 2 p^{4}\left(\frac{1}{2} D\right)^{2} D 3 p^{3} D_{2}^{\circ}$ & & 3266305 & 3.273E-11 & 3.273E-11 & 0.96 \\
\hline 18 & 135 & $2 s^{2} S 2 p^{4}\left({ }_{2}^{1} D\right)^{2} D 3 p^{3} D_{1}^{\circ}$ & & 3266629 & $1.326 \mathrm{E}-11$ & $1.325 \mathrm{E}-11$ & $0.92+0.052 s^{2} S 2 p^{4}\left({ }_{2}^{1} D\right)^{2} D 3 p^{1} P^{\circ}$ \\
\hline 18 & 136 & $2 s^{2} S 2 p^{4}\left({ }_{2}^{1} D\right)^{2} D 3 p^{3} D_{3}^{\circ}$ & & 3267794 & 2.024E-11 & 2.021E-11 & $0.93+0.042 s^{2} S 2 p^{4}\left({ }_{2}^{1} D\right){ }^{2} D 3 p^{1} F^{\circ}$ \\
\hline 18 & 137 & $2 s^{2} S 2 p^{4}\left({ }_{2}^{3} P\right){ }^{2} P 3 s^{3} P_{0}{ }^{3}$ & & 3269140 & $5.430 \mathrm{E}-12$ & $5.440 \mathrm{E}-12$ & $0.72+0.242 s^{2} S 2 p^{4}\left({ }_{0}^{\mathrm{T}} S\right)^{2} S 3 s^{1} S$ \\
\hline 18 & 138 & $2 s^{2} S 2 p^{4}\left(3_{2}^{3} P\right)^{4} P 3 d^{5} P_{3}$ & & 3270363 & $3.766 \mathrm{E}-11$ & $3.870 \mathrm{E}-11$ & $\left.0.95+0.032 s^{2} S 2 p^{4} \int_{2}^{3} P\right)^{4} P 3 d^{5} D$ \\
\hline 18 & 139 & $2 s^{2} S 2 p^{4}\left({ }_{2}^{3} P\right)^{2} P 3 s^{1} P_{1}$ & & 3271066 & $1.926 \mathrm{E}-12$ & $1.931 \mathrm{E}-12$ & $\left.0.67+0.262 s^{2} S 2 p^{4}{ }_{2}^{3} P\right)^{2} P 3 s^{3} P+0.042 s^{2} S 2 p^{4}\left({ }_{0}^{1} S\right)^{2} S 3 s^{3} S$ \\
\hline 18 & 140 & $2 s^{2} S 2 p^{4}\left({ }_{2}^{3} P\right)^{4} P 3 d^{3} F_{4}$ & & 3273575 & $1.782 \mathrm{E}-10$ & $1.727 \mathrm{E}-10$ & $\left.0.96+0.022 s^{2} S 2 p^{4}{ }_{2}^{2} P\right)^{4} P 3 d^{5} F$ \\
\hline 18 & 141 & $2 s^{2} S 2 p^{4}\left({ }_{2}^{1} D\right){ }^{2} D 3 p^{3} P_{2}^{\circ}$ & & 3276881 & $2.459 \mathrm{E}-12$ & $2.453 \mathrm{E}-12$ & $0.46+0.452 s^{2} S 2 p^{4}\left({ }_{2}^{1} D\right)^{2} D 3 p^{1} D^{\circ}+0.032 s^{2} S 2 p^{4}\left({ }_{2}^{3} P\right)^{4} P 3 p^{3} P^{\circ}$ \\
\hline 18 & 142 & $2 s^{2} S 2 p^{4}\left({ }_{2}^{1} D\right)^{2} D 3 p^{3} P_{1}^{\circ}$ & & 3280032 & 6.631E-12 & $6.602 \mathrm{E}-12$ & $0.82+0.102 s^{2} S 2 p^{4}\left({ }_{2}^{3} P\right)^{4} P 3 p^{3} P^{\circ}+0.052 s^{2} S 2 p^{4}\left(3_{2}^{2} P\right)^{2} P 3 p^{3} P^{\circ}$ \\
\hline 18 & 143 & $2 s^{2} S 2 p^{4}\left({ }_{2}^{\top} D\right)^{2} D 3 p^{1} D_{2}^{\circ}$ & & 3280057 & 2.125E-12 & $2.121 \mathrm{E}-12$ & $\left.0.52+0.392 s^{2} S 2 p^{4}\left({ }_{2}^{1} D\right)^{2} D 3 p^{3} P^{\circ}+0.052 s^{2} S 2 p^{4}{ }_{2}^{3} P\right)^{4} P 3 p^{3} P^{\circ}$ \\
\hline 18 & 144 & $2 s^{2} S 2 p^{4}\left({ }_{2}^{3} P\right){ }^{4} P 3 d^{3} F_{3}{ }^{2}$ & & 3280120 & $9.100 \mathrm{E}-12$ & $9.079 \mathrm{E}-12$ & $0.91+0.062 s^{2} S 2 p^{4}\left(2_{2}^{3} P\right)^{4} P 3 d^{3} D$ \\
\hline 18 & 145 & $2 s^{2} S 2 p^{4}\left({ }_{2}^{1} D\right)^{2} D 3 p^{3} P_{0}^{\circ}$ & & 3281244 & $6.214 \mathrm{E}-12$ & $6.185 \mathrm{E}-12$ & $0.81+0.112 s^{2} S 2 p^{4}\left({ }_{2}^{3} P\right)^{4} P 3 p^{3} P^{\circ}+0.072 s^{2} S 2 p^{4}\left(_{2}^{3} P\right)^{2} P 3 p^{3} P^{\circ}$ \\
\hline 18 & 146 & $2 s^{2} S 2 p^{4}\left({ }_{2}^{3} P\right){ }^{4} P 3 d^{3} F_{2}$ & & 3285825 & $6.656 \mathrm{E}-12$ & $6.646 \mathrm{E}-12$ & $0.89+0.072 s^{2} S 2 p^{4}\left({ }_{2}^{3} P\right)^{4} P 3 d^{3} D$ \\
\hline 18 & 147 & $2 s^{2} S 2 p^{4}\left({ }_{2}^{3} P\right)^{4} P 3 d^{3} P_{0}$ & & 3293195 & 1.444E-12 & $1.445 \mathrm{E}-12$ & $0.91+0.062 s^{2} S 2 p^{4}\left({ }_{2}^{1} D\right)^{2} D 3 d^{3} P$ \\
\hline 18 & 148 & $2 s^{2} S 2 p^{4}\left({ }_{2}^{2} P\right){ }^{4} P 3 d^{3} P_{1}$ & & 3294290 & 1.055E-12 & $1.056 \mathrm{E}-12$ & $0.72+0.202 s^{2} S 2 p^{4}\left({ }_{2}^{3} P\right){ }^{4} P 3 d^{3} D+0.042 s^{2} S 2 p^{4}\left({ }_{2}^{1} D\right)^{2} D 3 d^{3} P$ \\
\hline 18 & 149 & $2 s^{2} S 2 p^{4}\left({ }_{2}^{3} P\right)^{4} P 3 d^{3} D_{2}$ & & 3297752 & $8.148 \mathrm{E}-13$ & $8.147 \mathrm{E}-13$ & $\left.0.45+0.422 s^{2} S 2 p^{4}{ }_{2}^{3} P\right)^{4} P 3 d^{3} P+0.082 s^{2} S 2 p^{4}\left({ }_{2}^{3} P\right)^{4} P 3 d^{3} F$ \\
\hline 18 & 150 & $2 s^{2} S 2 p^{4}\left({ }_{2}^{3} P\right)^{4} P 3 d^{3} D_{3}$ & & 3302488 & $5.560 \mathrm{E}-13$ & $5.557 \mathrm{E}-13$ & $\left.0.89+0.062 s^{2} S 2 p^{4}{ }_{2}^{3} P\right)^{4} P 3 d^{3} F+0.032 s^{2} S 2 p^{4}\left({ }_{2}^{1} D\right)^{2} D 3 d^{3} D$ \\
\hline 18 & 151 & $2 s^{2} S 2 p^{4}\left({ }_{2}^{3} P\right){ }^{4} P 3 d^{3} D_{1}$ & & 3305043 & $6.843 \mathrm{E}-13$ & $6.843 \mathrm{E}-13$ & $\left.0.73+0.192 s^{2} S 2 p^{4} \int_{2}^{3} P\right)^{4} P 3 d^{3} P+0.042 s^{2} S 2 p^{4}\left({ }_{2}^{4} D\right)^{2} D 3 d^{3} D$ \\
\hline 18 & 152 & $2 s^{2} S 2 p^{4}\left({ }_{2}^{3} P\right)^{4} P 3 d^{3} P_{2}$ & & 3309076 & $8.412 \mathrm{E}-13$ & $8.412 \mathrm{E}-13$ & $0.50+0.412 s^{2} S 2 p^{4}\left({ }_{2}^{3} P\right)^{4} P 3 d^{3} D+0.032 s^{2} S 2 p^{4}\left({ }_{2}^{1} D\right)^{2} D 3 d^{3} P$ \\
\hline 18 & 153 & $2 s^{2} S 2 p^{4}\left({ }_{0}^{1} S\right)^{2} S 3 p^{3} P_{0}^{\circ}$ & & 3351803 & $7.875 \mathrm{E}-12$ & $7.890 \mathrm{E}-12$ & $0.77+0.182 s^{2} S 2 p^{4}\left({ }_{2}^{3} P\right)^{2} P 3 p^{3} P^{\circ}$ \\
\hline 18 & 154 & $2 s^{2} S 2 p^{4}\left({ }_{0}^{1} S\right)^{2} S 3 p^{3} P_{1}^{\circ}$ & & 3354599 & 7.748E-12 & $7.761 \mathrm{E}-12$ & $0.78+0.142 s^{2} S 2 p^{4}\left({ }_{2}^{3} P\right)^{2} P 3 p^{3} P^{\circ}+0.032 s^{2} S 2 p^{4}\left({ }_{2}^{3} P\right)^{2} P 3 p^{3} D^{\circ}$ \\
\hline 18 & 155 & $2 s^{2} S 2 p^{4}\left({ }_{0}^{1} S\right)^{2} S 3 p^{3} P_{2}^{0}$ & & 3360084 & 1.012E-11 & 1.014E-11 & $\left.0.84+0.062 s^{2} S 2 p^{4}\left({ }_{2}^{3} P\right)^{2} P 3 p^{3} D^{\circ}+0.052 s^{2} S 2 p^{4}{ }_{2}^{3} P\right)^{2} P 3 p^{3} P^{\circ}$ \\
\hline 18 & 156 & $2 s^{2} S 2 p^{4}\left({ }_{0}^{1} S\right)^{2} S 3 p p^{1} P_{1}^{2}$ & & 3367538 & $1.533 \mathrm{E}-12$ & $1.531 \mathrm{E}-12$ & $\left.0.81+0.062 s^{2} S 2 p^{4}\left(_{2}^{3} P\right)^{2} P 3 p^{3} D^{\circ}+0.052 s^{2} S 2 p^{4}{ }_{2}^{3} P\right)^{2} P 3 p^{3} P^{\circ}$ \\
\hline 19 & 1 & $2 s^{2} 2 p^{4}\left({ }_{2}^{3} P\right)^{3} P_{2}$ & 0 & 0 & $1.000 \mathrm{E}+40$ & $1.000 \mathrm{E}+40$ & $0.97+0.022 s^{2} 2 p^{4}\left({ }_{2}^{1} D\right){ }^{1} D$ \\
\hline 19 & 2 & $2 s^{2} 2 p^{4}\left({ }_{2}^{3} P\right){ }^{3} P_{1}$ & 18944 & 18923 & $6.718 \mathrm{E}-03$ & $6.718 \mathrm{E}-03$ & $0.99+2$ \\
\hline 19 & 3 & $2 s^{2} 2 p^{4}\left(\frac{2}{2} P\right)^{3} P_{0}$ & 23207 & 23406 & 2.119E-01 & 2.119E-01 & $0.96+0.032 s^{2} 2 p^{4}\left({ }_{0}^{1} S\right){ }^{1} S$ \\
\hline 19 & 4 & $2 s^{2} 2 p^{4}\left({ }_{2}^{1} D\right){ }^{1} D_{2}$ & 79587 & 79785 & $1.998 \mathrm{E}-03$ & $1.998 \mathrm{E}-03$ & $0.97+0.022 s^{2} 2 p^{4}\left(3_{2}^{3} P\right)^{3} P$ \\
\hline 19 & 5 & $2 s^{2} 2 p^{4}\left({ }_{0}^{\top} s\right){ }^{1} S_{0}$ & 162886 & 163087 & $2.173 \mathrm{E}-04$ & $2.173 \mathrm{E}-04$ & $\left.0.94+0.032 s^{2} 2 p^{4}{ }^{4}{ }_{2}^{3} P\right)^{3} P+0.022 p^{6}{ }^{1} S$ \\
\hline 19 & 6 & $2 s^{2} S 2 p^{5}{ }^{3} P_{2}^{\circ}$ & 573363 & 573219 & $4.308 \mathrm{E}-11$ & $4.295 \mathrm{E}-11$ & 0.99 \\
\hline 19 & 7 & $2 s^{2} S 2 p^{5} P_{1}^{\mathrm{o}}$ & 589176 & 589079 & 4.091E-11 & 4.094E-11 & 0.99 \\
\hline 19 & 8 & $2 s^{2} S 2 p^{5}{ }^{3} P_{0}^{\mathrm{o}}$ & 598382 & 598324 & 4.034E-11 & $4.042 \mathrm{E}-11$ & 0.99 \\
\hline 19 & 9 & $2 s^{2} S 2 p^{5}{ }^{1} P_{1}^{\circ}$ & 789574 & 789705 & $1.184 \mathrm{E}-11$ & $1.185 \mathrm{E}-11$ & 0.98 \\
\hline 19 & 10 & $2 p^{6}{ }^{1} s_{0}$ & 1336760 & 1336833 & 1.400E-11 & 1.403E-11 & $0.96+0.022 s^{2} 2 p^{4}\left({ }_{0}^{1} S\right){ }^{1} S$ \\
\hline
\end{tabular}




\begin{tabular}{|c|c|c|c|c|c|c|c|}
\hline $\mathrm{z}$ & Key & Level & $E_{\text {NIST }}$ & $E_{\mathrm{MCDHF} / \mathrm{RCI}}$ & $\tau_{\mathrm{MCDHF} / \mathrm{RCI}}^{l}$ & $\tau_{\mathrm{MCDHF} / \mathrm{RCI}}^{v}$ & $L S$-composition \\
\hline 19 & 11 & $2 s^{2} 2 p^{3}\left({ }_{3}^{4} S\right)^{4} S 3 s^{5} S_{2}^{0}$ & & 2893775 & 4.049E-10 & $4.076 \mathrm{E}-10$ & 0.98 \\
\hline 19 & 12 & $2 s^{2} 2 p^{3}\left({ }_{3}^{4} S\right)^{4} S 3 s^{3} S_{1}^{2}$ & 2930400 & 2929502 & $1.326 \mathrm{E}-12$ & $1.327 \mathrm{E}-12$ & 0.97 \\
\hline 19 & 13 & $2 s^{2} 2 p^{3}\left({ }_{3}^{2} D\right)^{2} D 3 s^{3} D_{1}^{\circ}$ & 3004100 & 3003977 & $3.986 \mathrm{E}-12$ & 3.993E-12 & $0.92+0.032 s^{2} 2 p^{3}\left({ }_{1}^{2} P\right)^{2} P 3 s^{1} P^{\circ}+0.032 s^{2} 2 p^{3}\left({ }_{1}^{2} P\right)^{2} P 3 s^{3} P^{\circ}$ \\
\hline 19 & 14 & $2 s^{2} 2 p^{3}\left({ }_{3}^{2} D\right)^{2} D 3 s^{3} D_{2}^{\circ}$ & 3005200 & 3004650 & $4.107 \mathrm{E}-12$ & $4.113 \mathrm{E}-12$ & $0.90+0.072 s^{2} 2 p^{3}\left({ }_{1}^{2} P\right)^{2} P 3 s^{3} P^{\circ}$ \\
\hline 19 & 15 & $2 s^{2} 2 p^{3}\left(\frac{2}{3} D\right)^{2} D 3 s^{3} D_{3}^{\circ}$ & 3008700 & 3008066 & 4.154E-12 & $4.158 \mathrm{E}-12$ & 0.99 \\
\hline 19 & 16 & $2 s^{2} 2 p^{3}\left(\frac{2}{3} D\right)^{2} D 3 s^{1} D_{2}^{\circ}$ & 3024600 & 3023664 & $1.704 \mathrm{E}-12$ & $1.706 \mathrm{E}-12$ & $0.91+0.042 s^{2} 2 p^{3}\left({ }_{1}^{2} P\right)^{2} P 3 s^{3} P^{\circ}+0.032 s^{2} 2 p^{3}\left({ }_{3}^{2} D\right)^{2} D 3 s^{3} D^{\circ}$ \\
\hline 19 & 17 & $2 s^{2} 2 p^{3}\left({ }_{3}^{4} S\right)^{4} S 3 p^{5} P_{1}$ & & 3041848 & $6.059 \mathrm{E}-10$ & $6.036 \mathrm{E}-10$ & $0.97+13+3$ \\
\hline 19 & 18 & $2 s^{2} 2 p^{3}\left({ }_{3}^{4} S\right)^{4} S 3 p^{5} P_{2}$ & & 3043279 & $6.214 \mathrm{E}-10$ & 6.193E-10 & 0.95 \\
\hline 19 & 19 & $2 s^{2} 2 p^{3}\left({ }_{3}^{4} S\right)^{4} S 3 p^{5} P_{3}$ & & 3046556 & $5.780 \mathrm{E}-10$ & $5.773 \mathrm{E}-10$ & 0.97 \\
\hline 19 & 20 & $2 s^{2} 2 p^{3}\left({ }_{1}^{2} P\right)^{2} P 3 s^{3} P_{0}^{\circ}$ & & 3063632 & $4.327 \mathrm{E}-12$ & $4.331 \mathrm{E}-12$ & 0.97 \\
\hline 19 & 21 & $2 s^{2} 2 p^{3}\left({ }_{1}^{2} P\right)^{2} P 3 s^{3} P_{1}^{\circ}$ & & 3065600 & $4.066 \mathrm{E}-12$ & 4.069E-12 & $0.92+0.032 s^{2} 2 p^{3}\left({ }_{1}^{2} P\right)^{2} P 3 s^{1} P^{\circ}+0.022 s^{2} 2 p^{3}\left({ }_{3}^{2} D\right)^{2} D 3 s^{3} D^{\circ}$ \\
\hline 19 & 22 & $2 s^{2} 2 p^{3}\left({ }_{1}^{2} P\right)^{2} P 3 s^{3} P_{2}^{\circ}$ & & 3071592 & $3.844 \mathrm{E}-12$ & $3.846 \mathrm{E}-12$ & $0.85+0.072 s^{2} 2 p^{3}\left({ }_{3}^{2} D\right)^{2} D 3 s^{1} D^{\circ}+0.052 s^{2} 2 p^{3}\left({ }_{3}^{2} D\right)^{2} D 3 s^{3} D^{\circ}$ \\
\hline 19 & 23 & $2 s^{2} 2 p^{3}\left({ }_{3}^{4} S\right)^{4} S 3 p^{3} P_{1}^{2}$ & & 3076174 & $4.836 \mathrm{E}-10$ & 4.862E-10 & $0.81+0.112 s^{2} 2 p^{3}\left({ }_{3}^{2} D\right)^{2} D 3 p^{3} P+0.022 s^{2} 2 p^{3}\left({ }_{1}^{2} P\right)^{2} P 3 p^{3} P$ \\
\hline 19 & 24 & $2 s^{2} 2 p^{3}\left({ }_{3}^{4} S\right)^{4} S 3 p^{3} P_{2}$ & & 3077863 & 4.787E-10 & $4.812 \mathrm{E}-10$ & $0.82+0.122 s^{2} 2 p^{3}\left(\frac{2}{3} D\right)^{2} D 3 p^{3} P+0.022 s^{2} 2 p^{3}\left({ }_{3}^{4} S\right)^{4} S 3 p^{5} P$ \\
\hline 19 & 25 & $2 s^{2} 2 p^{3}\left({ }_{3}^{4} S\right)^{4} S 3 p^{3} P_{0}$ & & 3078924 & $5.124 \mathrm{E}-10$ & $5.146 \mathrm{E}-10$ & $0.85+0.102 s^{2} 2 p^{3}\left({ }_{3}^{2} D\right)^{2} D 3 p^{3} P+0.032 s^{2} 2 p^{3}\left({ }_{1}^{2} P\right)^{2} P 3 p^{3} P$ \\
\hline 19 & 26 & $2 s^{2} 2 p^{3}\left({ }_{1}^{2} P\right)^{2} P 3 s^{1} P_{1}^{\circ}$ & 3087000 & 3086273 & $1.741 \mathrm{E}-12$ & $1.743 \mathrm{E}-12$ & $0.90+0.052 s^{2} 2 p^{3}\left({ }_{3}^{2} D\right)^{2} D 3 s^{3} D^{\circ}$ \\
\hline 19 & 27 & $2 s^{2} 2 p^{3}\left(\frac{1}{2} D\right)^{2} D 3 p{ }^{1} P_{1}$ & & 3134579 & $3.848 \mathrm{E}-10$ & $3.906 \mathrm{E}-10$ & $0.42+0.392 s^{2} 2 p^{3}\left(\frac{2}{3} D\right)^{2} D 3 p^{3} D+0.102 s^{2} 2 p^{3}\left(_{1}^{2} P\right)^{2} P 3 p^{1} P$ \\
\hline 19 & 28 & $2 s^{2} 2 p^{3}\left(\frac{2}{3} D\right)^{2} D 3 p^{3} D_{2}$ & & 3144256 & $5.570 \mathrm{E}-10$ & $5.590 \mathrm{E}-10$ & $\left.0.78+0.112 s^{2} 2 p^{3}\left({ }_{3}^{2} D\right)\right)^{2} D 3 p^{3} F+0.072 s^{2} 2 p^{3}\left({ }_{1}^{2} P\right)^{2} P 3 p^{3} D$ \\
\hline 19 & 29 & $2 s^{2} 2 p^{3}\left(\frac{2}{2} D\right)^{2} D 3 p^{3} D_{3}$ & & 3148918 & 4.893E-10 & 4.913E-10 & $0.77+0.182 s^{2} 2 p^{3}\left({ }_{2}^{2} D\right)^{2} D 3 p^{3} F+0.042 s^{2} 2 p^{3}\left({ }_{1}^{2} P\right)^{2} P 3 p^{3} D$ \\
\hline 19 & 30 & $2 s^{2} 2 p^{3}\left({ }_{3}^{2} D\right)^{2} D 3 p^{3} D_{1}$ & & 3149078 & $3.155 \mathrm{E}-10$ & 3.205E-10 & $0.48+0.422 s^{2} 2 p^{3}\left({ }_{3}^{2} D\right)^{2} D 3 p^{1} P+0.052 s^{2} 2 p^{3}\left({ }_{1}^{2} P\right)^{2} P 3 p^{3} D$ \\
\hline 19 & 31 & $2 s^{2} 2 p^{3}\left({ }_{3}^{2} D\right)^{2} D 3 p^{3} F_{2}$ & & 3154678 & $3.920 \mathrm{E}-10$ & $3.921 \mathrm{E}-10$ & $0.81+0.132 s^{2} 2 p^{3}\left({ }_{3}^{2} D\right)^{2} D 3 p^{3} D+0.032 s^{2} 2 p^{3}\left({ }_{1}^{2} P\right)^{2} P 3 p^{1} D$ \\
\hline 19 & 32 & $2 s^{2} 2 p^{3}\left(\frac{2}{3} D\right)^{2} D 3 p^{3} F_{3}$ & & 3158524 & $3.484 \mathrm{E}-10$ & $3.491 \mathrm{E}-10$ & $0.76+0.202 s^{2} 2 p^{3}\left(\frac{2}{2} D\right)^{2} D 3 p^{3} D+0.022 s^{2} 2 p^{3}\left({ }_{1}^{2} P\right)^{2} P 3 p^{3} D$ \\
\hline 19 & 33 & $2 s^{2} 2 p^{3}\left(\frac{2}{3} D\right)^{2} D 3 p^{3} F_{4}$ & & 3161873 & $5.091 \mathrm{E}-10$ & $5.068 \mathrm{E}-10$ & 0.99 \\
\hline 19 & 34 & $2 s^{2} 2 p^{3}\left(\frac{2}{3} D\right)^{2} D 3 p^{1} F_{3}$ & & 3164133 & $5.350 \mathrm{E}-10$ & $5.322 \mathrm{E}-10$ & $0.94+0.042 s^{2} 2 p^{3}\left({ }_{1}^{2} P\right)^{2} P 3 p^{3} D$ \\
\hline 19 & 35 & $2 s^{2} 2 p^{3}\left(\frac{2}{3} D\right)^{2} D 3 p^{3} P_{1}$ & & 3195103 & $4.479 \mathrm{E}-11$ & $4.519 \mathrm{E}-11$ & $0.38+0.442 s^{2} 2 p^{3}\left({ }_{1}^{2} P\right)^{2} P 3 p^{3} S+0.072 s^{2} 2 p^{3}\left({ }_{3}^{4} S\right)^{4} S 3 p^{3} P$ \\
\hline 19 & 36 & $2 s^{2} 2 p^{3}\left({ }_{3}^{2} D\right)^{2} D 3 p^{3} P_{0}$ & & 3199229 & 4.663E-11 & 4.697E-11 & $0.77+0.152 s^{2} 2 p^{3}\left({ }_{1}^{2} P\right)^{2} P 3 p^{3} P+0.052 s^{2} 2 p^{3}\left({ }_{3}^{4} S\right)^{4} S 3 p^{3} P$ \\
\hline 19 & 37 & $2 s^{2} 2 p^{3}\left({ }_{3}^{2} D\right)^{2} D 3 p^{3} P_{2}$ & & 3205070 & 3.327E-11 & $3.352 \mathrm{E}-11$ & $0.81+0.112 s^{2} 2 p^{3}\left({ }_{3}^{4} S\right)^{4} S 3 p^{3} P+0.022 s^{2} 2 p^{3}\left({ }_{1}^{2} P\right)^{2} P 3 p^{1} D$ \\
\hline 19 & 38 & $2 s^{2} 2 p^{3}\left({ }_{1}^{2} P\right)^{2} P 3 p^{3} D_{1}$ & & 3213876 & 1.137E-10 & $1.141 \mathrm{E}-10$ & $0.74+0.122 s^{2} 2 p^{3}\left({ }_{1}^{2} P\right)^{2} P 3 p^{1} P+0.052 s^{2} 2 p^{3}\left({ }_{3}^{2} D\right)^{2} D 3 p^{3} D$ \\
\hline 19 & 39 & $2 s^{2} 2 p^{3}\left({ }_{1}^{2} P\right)^{2} P 3 p^{3} S_{1}$ & & 3215533 & 4.671E-11 & $4.710 \mathrm{E}-11$ & $0.45+0.372 s^{2} 2 p^{3}\left({ }_{3}^{2} D\right)^{2} D 3 p^{3} P+0.062 s^{2} 2 p^{3}\left({ }_{1}^{2} P\right)^{2} P 3 p^{3} D$ \\
\hline 19 & 40 & $2 s^{2} 2 p^{3}\left({ }_{1}^{2} P\right)^{2} P 3 p^{3} D_{2}$ & & 3215790 & $1.116 \mathrm{E}-10$ & $1.120 \mathrm{E}-10$ & $0.65+0.162 s^{2} 2 p^{3}\left({ }_{3}^{2} D\right)^{2} D 3 p^{1} D+0.052 s^{2} 2 p^{3}\left({ }_{3}^{2} D\right)^{2} D 3 p^{3} F$ \\
\hline 19 & 41 & $2 s^{2} 2 p^{3}\left({ }_{1}^{2} P\right)^{2} P 3 p^{3} D_{3}$ & & 3222581 & 1.371E-10 & $1.374 \mathrm{E}-10$ & $0.86+0.052 s^{2} 2 p^{3}\left({ }_{3}^{2} D\right)^{2} D 3 p^{1} F+0.052 s^{2} 2 p^{3}\left(\frac{3}{3} D\right)^{2} D 3 p^{3} F$ \\
\hline 19 & 42 & $2 s^{2} 2 p^{3}\left(\frac{2}{2} D\right)^{2} D 3 p^{1} D_{2}$ & & 3223093 & $1.147 \mathrm{E}-10$ & $1.152 \mathrm{E}-10$ & $0.35+0.222 s^{2} 2 p^{3}\left({ }_{1}^{2} P\right)^{2} P 3 p^{3} D+0.202 s^{2} 2 p^{3}\left(_{1}^{3} P\right)^{2} P 3 p^{1} D$ \\
\hline 19 & 43 & $2 s^{2} 2 p^{3}\left({ }_{1}^{2} P\right)^{2} P 3 p^{1} P_{1}$ & & 3230460 & $1.008 \mathrm{E}-10$ & $1.013 \mathrm{E}-10$ & $0.63+0.082 s^{2} 2 p^{3}\left({ }_{1}^{2} P\right)^{2} P 3 p^{3} P+0.082 s^{2} 2 p^{3}\left({ }_{1}^{2} P\right)^{2} P 3 p^{3} D$ \\
\hline 19 & 44 & $2 s^{2} 2 p^{3}\left({ }_{2}^{4} S\right)^{4} S 3 d^{5} D_{2}^{\circ}$ & & 3230603 & $1.098 \mathrm{E}-10$ & $1.095 \mathrm{E}-10$ & 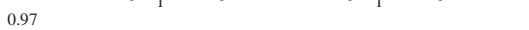 \\
\hline 19 & 45 & $2 s^{2} 2 p^{3}\left({ }_{3}^{4} S\right)^{4} S 3 d^{5} D_{1}^{\circ}$ & & 3230605 & $8.447 \mathrm{E}-11$ & $8.406 \mathrm{E}-11$ & 0.97 \\
\hline 19 & 46 & $2 s^{2} 2 p^{3}\left({ }_{3}^{4} S\right)^{4} S 3 d^{5} D_{0}^{\circ}$ & & 3230611 & $8.577 \mathrm{E}-11$ & $8.526 \mathrm{E}-11$ & 0.97 \\
\hline 19 & 47 & $2 s^{2} 2 p^{3}\left({ }_{3}^{4} S\right)^{4} S 3 d^{5} D_{3}^{0}$ & & 3230626 & 2.650E-10 & 2.654E-10 & 0.97 \\
\hline 19 & 48 & $2 s^{2} 2 p^{3}\left({ }_{3}^{4} S\right)^{4} S 3 d^{5} D_{4}^{\circ}$ & & 3230898 & 3.435E-10 & 3.424E-10 & 0.97 \\
\hline 19 & 49 & $2 s^{2} 2 p^{3}\left(_{1}^{3} P\right)^{2} P 3 p^{3} P_{2}^{4}$ & & 3243657 & $2.219 \mathrm{E}-10$ & $2.229 \mathrm{E}-10$ & $0.67+0.182 s^{2} 2 p^{3}\left({ }_{1}^{2} P\right)^{2} P 3 p^{1} D+0.042 s^{2} 2 p^{3}\left({ }_{2}^{2} D\right)^{2} D 3 p^{1} D$ \\
\hline 19 & 50 & $2 s^{2} 2 p^{3}\left({ }_{1}^{2} P\right)^{2} P 3 p^{3} P_{1}$ & & 3244177 & $1.098 \mathrm{E}-10$ & $1.106 \mathrm{E}-10$ & $0.74+0.072 s^{2} 2 p^{3}\left({ }_{1}^{2} P\right)^{2} P 3 p^{1} P+0.062 s^{2} 2 p^{3}\left({ }_{3}^{4} S\right)^{4} S 3 p^{3} P$ \\
\hline 19 & 51 & $2 s^{2} 2 p^{3}\left({ }_{1}^{2} P\right)^{2} P 3 p^{3} P_{0}$ & & 3244342 & 7.621E-11 & 7.681E-11 & $\left.0.77+0.082 s^{2} 2 p^{3}\left({ }_{3}^{2} D\right)\right)^{2} D 3 p^{3} P+0.082 s^{2} 2 p^{3}\left({ }_{3}^{4} S\right)^{4} S 3 p^{3} P$ \\
\hline 19 & 52 & $2 s^{2} 2 p^{3}\left({ }_{3}^{4} S\right)^{4} S 3 d^{3} D_{2}^{\circ}$ & 3265700 & 3267084 & $9.789 \mathrm{E}-13$ & $9.779 \mathrm{E}-13$ & $0.82+0.102 s^{2} 2 p^{3}\left({ }_{3}^{2} D\right)^{2} D 3 d^{3} D^{\circ}+0.022 s^{2} 2 p^{3}\left({ }_{1}^{2} P\right)^{2} P 3 d^{3} D^{\circ}$ \\
\hline 19 & 53 & $2 s^{2} 2 p^{3}\left({ }_{3}^{4} S\right){ }^{4} S 3 d^{3} D_{1}^{\circ}$ & & 3268814 & $9.070 \mathrm{E}-13$ & $9.063 \mathrm{E}-13$ & $0.86+0.082 s^{2} 2 p^{3}\left({ }_{3}^{2} D\right)^{2} D 3 d^{3} D^{\circ}+0.032 s^{2} 2 p^{3}\left({ }_{1}^{2} P\right)^{2} P 3 d^{3} D^{\circ}$ \\
\hline 19 & 54 & $2 s^{2} 2 p^{3}\left({ }_{3}^{4} S\right)^{4} S 3 d^{3} D_{3}^{\circ}$ & 3271100 & 3269653 & $8.790 \mathrm{E}-13$ & $8.776 \mathrm{E}-13$ & $0.84+0.112 s^{2} 2 p^{3}\left({ }_{3}^{2} D\right)^{2} D 3 d^{3} D^{\circ}$ \\
\hline 19 & 55 & $2 s^{2} 2 p^{3}\left({ }_{1}^{2} P\right)^{2} P 3 p^{1} D_{2}^{3}$ & & 3270077 & $1.597 \mathrm{E}-10$ & $1.612 \mathrm{E}-10$ & $0.48+0.432 s^{2} 2 p^{3}\left({ }_{3}^{2} D\right)^{2} D 3 p^{1} D+0.042 s^{2} 2 p^{3}\left({ }_{1}^{2} P\right)^{2} P 3 p^{3} P$ \\
\hline 19 & 56 & $2 s^{2} 2 p^{3}\left({ }_{1}^{2} P\right)^{2} P 3 p^{1} S_{0}$ & & 3325896 & 2.917E-11 & $2.935 \mathrm{E}-11$ & $0.93+0.022 s^{2} 2 p^{3}\left(\frac{2}{3} D\right)^{2} D 3 p^{3} P$ \\
\hline 19 & 57 & $2 s^{2} 2 p^{3}\left(\frac{2}{3} D\right)^{2} D 3 d^{3} F_{2}^{\circ}$ & & 3326754 & $1.698 \mathrm{E}-11$ & $1.694 \mathrm{E}-11$ & $0.82+0.112 s^{2} 2 p^{3}\left(_{1}^{2} P\right)^{2} P 3 d^{3} F^{\circ}+0.022 s^{2} 2 p^{3}\left({ }_{1}^{2} P\right)^{2} P 3 d^{1} D^{\circ}$ \\
\hline 19 & 58 & $2 s^{2} 2 p^{3}\left({ }_{3}^{2} D\right)^{2} D 3 d^{3} F_{3}^{\circ}$ & & 3330575 & 1.451E-11 & 1.451E-11 & $0.82+0.092 s^{2} 2 p^{3}\left({ }_{1}^{2} P\right)^{2} P 3 d^{3} F^{\circ}+0.052 s^{2} 2 p^{3}\left({ }_{3}^{2} D\right)^{2} D 3 d^{3} G^{\circ}$ \\
\hline 19 & 59 & $2 s^{2} 2 p^{3}\left(\frac{2}{2} D\right)^{2} D 3 d^{1} S_{0}^{3}$ & & 3332643 & $6.246 \mathrm{E}-11$ & $6.276 \mathrm{E}-11$ & $0.95+0.032 s^{2} 2 p^{3}\left({ }_{1}^{2} P\right)^{2} P 3 d^{3} P^{\circ}$ \\
\hline 19 & 60 & $2 s^{2} 2 p^{3}\left(\frac{2}{3} D\right)^{2} D 3 d^{3} F_{4}^{\circ}$ & & 3335027 & 3.629E-10 & 3.605E-10 & $0.74+0.132 s^{2} 2 p^{3}\left(\frac{2}{3} D\right)^{2} D 3 d^{3} G^{\circ}+0.072 s^{2} 2 p^{3}\left({ }_{1}^{2} P\right)^{2} P 3 d^{3} F^{\circ}$ \\
\hline 19 & 61 & $2 s^{2} 2 p^{3}\left(\frac{2}{3} D\right)^{2} D 3 d^{3} G_{3}^{\circ}$ & & 3342132 & $2.315 \mathrm{E}-11$ & 2.313E-11 & $0.87+0.072 s^{2} 2 p^{3}\left({ }_{3}^{2} D\right)^{2} D 3 d^{3} F^{\circ}+0.032 s^{2} 2 p^{3}\left({ }_{1}^{2} P\right)^{2} P 3 d^{1} F^{\circ}$ \\
\hline 19 & 62 & $2 s^{2} 2 p^{3}\left({ }_{3}^{2} D\right)^{2} D 3 d^{3} G_{4}^{3}$ & & 3343807 & $3.236 \mathrm{E}-10$ & $3.228 \mathrm{E}-10$ & $0.73+0.202 s^{2} 2 p^{3}\left({ }_{3}^{2} D\right)^{2} D 3 d^{3} F^{\circ}+0.032 s^{2} 2 p^{3}\left({ }_{3}^{2} D\right)^{2} D 3 d^{1} G^{\circ}$ \\
\hline 19 & 63 & $\left.2 s^{2} 2 p^{3}\left({ }_{3}^{2} D\right)\right)^{2} D 3 d^{3} G_{5}^{\mathrm{o}}$ & & 3345853 & $3.299 \mathrm{E}-10$ & $3.279 \mathrm{E}-10$ & $0.99+35+35$ \\
\hline 19 & 64 & $2 s^{2} 2 p^{3}\left(\frac{2}{3} D\right)^{2} D 3 d^{1} G_{4}^{\circ}$ & & 3348529 & $3.224 \mathrm{E}-10$ & $3.212 \mathrm{E}-10$ & $0.87+0.082 s^{2} 2 p^{3}\left({ }_{3}^{2} D\right)^{2} D 3 d^{3} G^{\circ}$ \\
\hline 19 & 65 & $2 s^{2} 2 p^{3}\left({ }_{3}^{2} D\right)^{2} D 3 d^{1} P_{1}^{\circ}$ & & 3350575 & $5.957 \mathrm{E}-13$ & $5.953 \mathrm{E}-13$ & $0.44+0.372 s^{2} 2 p^{3}\left({ }_{3}^{2} D\right)^{2} D 3 d^{3} D^{\circ}+0.052 s^{2} 2 p^{3}\left(_{1}^{2} P\right)^{2} P 3 d^{1} P^{\circ}$ \\
\hline 19 & 66 & $2 s^{2} 2 p^{3}\left(\frac{2}{3} D\right)^{2} D 3 d^{3} D_{2}^{\circ}$ & 3361100 & 3359747 & 4.764E-13 & $4.759 \mathrm{E}-13$ & $0.74+0.092 s^{2} 2 p^{3}\left({ }_{3}^{4} S\right)^{4} S 3 d^{3} D^{\circ}+0.052 s^{2} 2 p^{3}\left({ }_{3}^{2} D\right)^{2} D 3 d^{3} P^{\circ}$ \\
\hline 19 & 67 & $2 s^{2} 2 p^{3}\left({ }_{2}^{3} D\right)^{2} D 3 d^{3} D_{1}^{\circ}$ & 3358100 & 3361421 & $5.629 \mathrm{E}-13$ & $5.628 \mathrm{E}-13$ & $0.47+0.422 s^{2} 2 p^{3}\left({ }_{3}^{2} D\right)^{2} D 3 d^{1} P^{\circ}+0.042 s^{2} 2 p^{3}\left({ }_{2}^{4} S\right)^{4} S 3 d^{3} D^{\circ}$ \\
\hline 19 & 68 & $2 s^{2} 2 p^{3}\left(c_{3}^{2} D\right)^{2} D 3 d^{3} D_{3}^{\circ}$ & 3363000 & 3361970 & $3.402 \mathrm{E}-13$ & $3.399 \mathrm{E}-13$ & $0.85+0.102 s^{2} 2 p^{3}\left(3_{3}^{4} S\right)^{4} S 3 d^{3} D^{\circ}$ \\
\hline 19 & 69 & $2 s^{2} 2 p^{3}\left({ }_{3}^{3} D\right)^{2} D 3 d^{3} P_{2}^{0}$ & 3370700 & 3369335 & $2.636 \mathrm{E}-13$ & 2.634E-13 & $0.66+0.132 s^{2} 2 p^{3}\left({ }_{1}^{2} P\right)^{2} P 3 d^{3} P^{\circ}+0.082 s^{2} 2 p^{3}\left({ }_{3}^{2} D\right)^{2} D 3 d^{1} D^{\circ}$ \\
\hline 19 & 70 & $2 s^{2} 2 p^{3}\left(\frac{2}{3} D\right)^{2} D 3 d^{1} D_{2}^{2}$ & 3374400 & 3371913 & $8.060 \mathrm{E}-13$ & $8.058 \mathrm{E}-13$ & $0.48+0.302 s^{2} 2 p^{3}\left({ }_{1}^{2} P\right)^{2} P 3 d^{1} D^{\circ}+0.112 s^{2} 2 p^{3}\left({ }_{3}^{2} D\right)^{2} D 3 d^{3} P^{\circ}$ \\
\hline 19 & 71 & $2 s^{2} 2 p^{3}\left(\frac{2}{3} D\right)^{2} D 3 d^{3} P_{0}^{\circ}$ & & 3374369 & 4.607E-13 & 4.604E-13 & $0.92+0.042 s^{2} 2 p^{3}\left({ }_{1}^{2} P\right)^{2} P 3 d^{3} P^{\circ}$ \\
\hline 19 & 72 & $2 s^{2} 2 p^{3}\left(\frac{2}{3} D\right)^{2} D 3 d^{3} P_{1}^{\circ}$ & & 3375125 & $3.600 \mathrm{E}-13$ & $3.598 \mathrm{E}-13$ & 0.94 \\
\hline 19 & 73 & $2 s^{2} 2 p^{3}\left({ }_{3}^{3} D\right)^{2} D 3 d^{3} S_{1}^{\circ}$ & 3383100 & 3382355 & $2.876 \mathrm{E}-13$ & $2.875 \mathrm{E}-13$ & $0.83+0.132 s^{2} 2 p^{3}\left({ }_{1}^{2} P\right)^{2} P 3 d^{3} P^{\circ}$ \\
\hline 19 & 74 & $2 s^{2} S 2 p^{4}\left({ }_{2}^{3} P\right){ }^{4} P 3 s^{5} P_{3}$ & & 3387034 & 9.027E-11 & $9.003 \mathrm{E}-11$ & $0.98+1$ \\
\hline 19 & 75 & $2 s^{2} 2 p^{3}\left({ }_{3}^{2} D\right)^{2} D 3 d^{1} F_{3}^{\circ}$ & 3396300 & 3396381 & $4.656 \mathrm{E}-13$ & $4.650 \mathrm{E}-13$ & $0.67+0.152 s^{2} 2 p^{3}\left({ }_{1}^{2} P\right)^{2} P 3 d^{3} F^{\circ}+0.052 s^{2} 2 p^{3}\left({ }_{1}^{2} P\right)^{2} P 3 d^{3} D^{\circ}$ \\
\hline 19 & 76 & $2 s^{2} S 2 p^{4}\left({ }_{2}^{3} P\right){ }^{4} P 3 s^{5} P_{2}$ & & 3400320 & $5.331 \mathrm{E}-11$ & $5.343 \mathrm{E}-11$ & 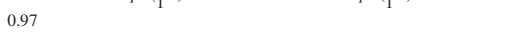 \\
\hline 19 & 77 & $2 s^{2} 2 p^{3}\left({ }_{1}^{2} P\right)^{2} P 3 d^{3} F_{2}^{\circ}$ & 3405200 & 3405592 & 6.033E-12 & $6.026 \mathrm{E}-12$ & $0.79+0.102 s^{2} 2 p^{3}\left({ }_{3}^{2} D\right)^{2} D 3 d^{3} F^{\circ}+0.032 s^{2} 2 p^{3}\left({ }_{1}^{2} P\right)^{2} P 3 d^{3} D^{\circ}$ \\
\hline 19 & 78 & $2 s^{2} 2 p^{3}\left({ }_{1}^{2} P\right)^{2} P 3 d^{3} F_{3}^{\circ}$ & & 3406243 & $2.186 \mathrm{E}-12$ & 2.183E-12 & $0.65+0.172 s^{2} 2 p^{3}\left({ }_{1}^{2} P\right)^{2} P 3 d^{3} D^{\circ}+0.082 s^{2} 2 p^{3}\left({ }_{3}^{2} D\right)^{2} D 3 d^{1} F^{\circ}$ \\
\hline 19 & 79 & $2 s^{2} 2 p^{3}\left({ }_{1}^{2} P\right)^{2} P 3 d^{3} F_{4}^{0}$ & & 3406608 & $3.139 \mathrm{E}-10$ & $3.138 \mathrm{E}-10$ & $0.85+0.052 s^{2} 2 p^{3}\left(\frac{2}{2} D\right)^{2} D 3 d^{3} G^{\circ}+0.042 s^{2} 2 p^{3}\left(\frac{2}{2} D\right)^{2} D 3 d^{1} G^{\circ}$ \\
\hline 19 & 80 & $2 s^{2} 2 p^{3}\left({ }_{1}^{2} P\right)^{2} P 3 d^{3} P_{0}^{\circ}$ & & 3408324 & $5.272 \mathrm{E}-13$ & $5.270 \mathrm{E}-13$ & $0.88+0.052 s^{2} 2 p^{3}\left({ }_{3}^{2} D\right)^{2} D 3 d^{3} P^{\circ}+0.022 s^{2} 2 p^{3}\left({ }_{3}^{2} D\right)^{2} D 3 d^{1} S^{\circ}$ \\
\hline 19 & 81 & $2 s^{2} S 2 p^{4}\left({ }_{2}^{3} P\right)^{4} P 3 s^{5} P_{1}$ & & 3408502 & $6.924 \mathrm{E}-11$ & $6.957 \mathrm{E}-11$ & 0.98 \\
\hline 19 & 82 & $2 s^{2} 2 p^{3}\left({ }_{1}^{2} P\right)^{2} P 3 d^{3} P_{1}^{\circ}$ & 3410000 & 3411391 & $6.376 \mathrm{E}-13$ & $6.376 \mathrm{E}-13$ & $0.77+0.142 s^{2} 2 p^{3}\left({ }_{3}^{2} D\right)^{2} D 3 d^{3} s^{\circ}+0.032 s^{2} 2 p^{3}\left({ }_{3}^{2} D\right)^{2} D 3 d^{1} P^{\circ}$ \\
\hline 19 & 83 & $2 s^{2} 2 p^{3}\left({ }_{1}^{2} P\right)^{2} P 3 d^{3} P_{2}^{\circ}$ & & 3412800 & $4.421 \mathrm{E}-12$ & 4.420E-12 & $0.74+0.112 s^{2} 2 p^{3}\left({ }_{3}^{2} D\right)^{2} D 3 d^{3} P^{\circ}+0.042 s^{2} 2 p^{3}\left({ }_{1}^{2} P\right)^{2} P 3 d^{3} D^{\circ}$ \\
\hline 19 & 84 & $2 s^{2} 2 p^{3}\left({ }_{1}^{2} P\right)^{2} P 3 d^{3} D_{2}^{\circ}$ & 3427000 & 3421283 & 3.873E-13 & 3.869E-13 & $0.62+0.212 s^{2} 2 p^{3}\left({ }_{1}^{2} P\right)^{2} P 3 d^{1} D^{\circ}+0.052 s^{2} 2 p^{3}\left({ }_{3}^{2} D\right)^{2} D 3 d^{1} D^{\circ}$ \\
\hline 19 & 85 & $2 s^{2} 2 p^{3}\left({ }_{1}^{2} P\right)^{2} P 3 d^{3} D_{1}^{\circ}$ & 3427000 & 3426279 & 3.009E-13 & 3.005E-13 & $0.85+0.042 s^{2} 2 p^{3}\left({ }_{3}^{4} S\right)^{4} S 3 d^{3} D^{\circ}+0.032 s^{2} 2 p^{3}\left({ }_{3}^{2} D\right)^{2} D 3 d^{3} D^{\circ}$ \\
\hline 19 & 86 & $2 s^{2} 2 p^{3}\left({ }_{1}^{2} P\right)^{2} P 3 d^{3} D_{3}^{\circ}$ & 3428400 & 3427050 & $9.609 \mathrm{E}-13$ & $9.600 \mathrm{E}-13$ & $0.67+0.102 s^{2} 2 p^{3}\left(_{1}^{2} P\right)^{2} P 3 d^{1} F^{\circ}+0.082 s^{2} 2 p^{3}\left(_{2}^{3} D\right)^{2} D 3 d^{1} F^{\circ}$ \\
\hline 19 & 87 & $2 s^{2} 2 p^{3}\left({ }_{1}^{2} P\right)^{2} P 3 d^{1} D_{2}^{\circ}$ & 3416600 & 3438950 & 3.203E-13 & $3.200 \mathrm{E}-13$ & $0.36+0.312 s^{2} 2 p^{3}\left({ }_{3}^{2} D\right)^{2} D 3 d^{1} D^{\circ}+0.212 s^{2} 2 p^{3}\left({ }_{1}^{2} P\right)^{2} P 3 d^{3} D^{\circ}$ \\
\hline 19 & 88 & $2 s^{2} S 2 p^{4}\left({ }_{2}^{3} P\right){ }^{4} P 3 s^{3} P_{2}$ & & 3441147 & $2.108 \mathrm{E}-12$ & $2.110 \mathrm{E}-12$ & 0.94 \\
\hline 19 & 89 & $2 s^{2} 2 p^{3}\left({ }_{1}^{2} P\right)^{2} P 3 d^{1} F_{3}^{\circ}$ & 3442700 & 3441814 & $2.218 \mathrm{E}-13$ & $2.216 \mathrm{E}-13$ & $0.75+0.132 s^{2} 2 p^{3}\left({ }_{3}^{2} D\right)^{2} D 3 d d^{1} F^{\circ}+0.042 s^{2} 2 p^{3}\left({ }_{1}^{2} P\right)^{2} P 3 d^{3} D^{\circ}$ \\
\hline 19 & 90 & $2 s^{2} S 2 p^{4}\left({ }_{2}^{3} P\right){ }^{4} P 3 s^{3} P_{1}$ & & 3455707 & 2.031E-12 & 2.033E-12 & 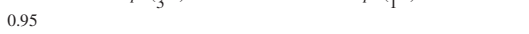 \\
\hline 19 & 91 & $2 s^{2} S 2 p^{4}\left({ }_{2}^{3} P\right){ }^{4} P 3 s^{3} P_{0}$ & & 3461905 & $2.022 \mathrm{E}-12$ & $2.023 \mathrm{E}-12$ & 0.95 \\
\hline 19 & 92 & $2 s^{2} 2 p^{3}\left({ }_{1}^{2} P\right)^{2} P 3 d^{1} P_{1}^{\circ}$ & 3474100 & 3476145 & $2.071 \mathrm{E}-13$ & 2.069E-13 & $0.85+0.052 s^{2} 2 p^{3}\left({ }_{3}^{2} D\right)^{2} D 3 d^{1} P^{\circ}$ \\
\hline 19 & 93 & $2 s^{2} S 2 p^{4}\left({ }_{2}^{3} P\right)^{4} P 3 p^{5} P_{3}^{\circ}$ & & 3526562 & $9.051 \mathrm{E}-11$ & $8.996 \mathrm{E}-11$ & $0.89+0.102 s^{2} S 2 p^{4}\left({ }_{2}^{3} P\right)^{4} P 3 p^{5} D^{\circ}$ \\
\hline 19 & 94 & $\left.2 s^{2} S 2 p^{4}{ }_{2}^{3} P\right)^{4} P 3 p^{5} P_{2}^{\circ}$ & & 3529265 & $6.096 \mathrm{E}-11$ & $6.073 \mathrm{E}-11$ & $0.87+0.062 s^{2} S 2 p^{4}\left({ }_{2}^{3} P\right)^{4} P 3 p^{5} D^{\circ}+0.052 s^{2} S 2 p^{4}\left({ }_{2}^{3} P\right)^{4} P 3 p^{5} S^{\circ}$ \\
\hline
\end{tabular}




\begin{tabular}{|c|c|c|c|c|c|c|c|}
\hline $\mathrm{z}$ & Key & Level & $E_{\text {NIST }}$ & $E_{\mathrm{MCDHF} / \mathrm{RCI}}$ & $\tau_{\mathrm{MCDHF} / \mathrm{RCI}}^{l}$ & $\tau_{\mathrm{MCDHF} / \mathrm{RCI}}^{v}$ & $L S$-composition \\
\hline 19 & 95 & $2 s^{2} S 2 p^{4}\left({ }_{2}^{3} P\right){ }^{4} P 3 p^{5} P_{1}^{\circ}$ & & 3536791 & $6.051 \mathrm{E}-11$ & $6.040 \mathrm{E}-11$ & $0.95+0.042 s^{2} S 2 p^{4}\left({ }_{2}^{3} P\right){ }^{4} P 3 p^{5} D^{\circ}$ \\
\hline 19 & 96 & $2 s^{2} S 2 p^{4}\left({ }_{2}^{3} P\right){ }^{4} P 3 p^{5} D_{4}^{\circ}$ & & 3543272 & 1.195E-10 & $1.183 \mathrm{E}-10$ & 0.98 \\
\hline 19 & 97 & $\left.2 s^{2} S 2 p^{4}{ }_{2}^{3} P\right)^{4} P 3 p^{5} D_{3}^{\circ}$ & & 3550591 & $9.837 \mathrm{E}-12$ & $9.821 \mathrm{E}-12$ & $0.74+0.162 s^{2} S 2 p^{4}\left({ }_{2}^{3} P\right)^{4} P 3 p^{3} D^{\circ}+0.092 s^{2} S 2 p^{4}\left({ }_{2}^{3} P\right)^{4} P 3 p^{5} P^{\circ}$ \\
\hline 19 & 98 & $\left.2 s^{2} S 2 p^{4}{ }_{2}^{2} P\right)^{4} P 3 p^{5} D_{2}^{3}$ & & 3556199 & $2.572 \mathrm{E}-11$ & $2.569 \mathrm{E}-11$ & $0.87+0.062 s^{2} S 2 p^{4}\left({ }_{2}^{3} P\right){ }^{4} P 3 p^{3} D^{\circ}+0.052 s^{2} S 2 p^{4}\left({ }_{2}^{3} P\right)^{4} P 3 p^{5} P^{\circ}$ \\
\hline 19 & 99 & $2 s^{2} S 2 p^{4}\left({ }_{2}^{3} P\right)^{4} P 3 p^{5} D_{1}^{\circ}$ & & 3559371 & $5.924 \mathrm{E}-11$ & $5.920 \mathrm{E}-11$ & $0.94+0.042 s^{2} S 2 p^{4}\left(\int_{2}^{3} P\right)^{4} P 3 p^{5} P^{\circ}$ \\
\hline 19 & 100 & $2 s^{2} S 2 p^{4}\left({ }_{2}^{2} P\right){ }^{4} P 3 p^{5} D_{0}^{\circ}$ & & 3560488 & $1.057 \mathrm{E}-10$ & $1.056 \mathrm{E}-10$ & 0.99 \\
\hline 19 & 101 & $2 s^{2} S 2 p^{4}\left({ }_{2}^{2} P\right){ }^{4} P 3 p^{3} D_{3}^{\circ}$ & & 3567274 & $1.810 \mathrm{E}-12$ & $1.806 \mathrm{E}-12$ & $0.82+0.152 s^{2} S 2 p^{4}\left({ }_{2}^{3} P\right){ }^{4} P 3 p^{5} D^{\circ}$ \\
\hline 19 & 102 & $2 s^{2} S 2 p^{4}\left({ }_{2}^{1} D\right)^{2} D 3 s^{3} D_{1}^{3}$ & & 3576237 & $3.122 \mathrm{E}-12$ & $3.127 \mathrm{E}-12$ & $0.97 \quad-2$ \\
\hline 19 & 103 & $2 s^{2} S 2 p^{4}\left({ }_{2}^{1} D\right)^{2} D 3 s^{3} D_{2}$ & & 3576602 & $3.116 \mathrm{E}-12$ & $3.120 \mathrm{E}-12$ & 0.97 \\
\hline 19 & 104 & $2 s^{2} S 2 p^{4} \overbrace{3}^{3} P)^{4} P 3 p^{3} D_{2}^{\circ}$ & & 3576628 & $1.622 \mathrm{E}-12$ & $1.619 \mathrm{E}-12$ & $0.80+0.122 s^{2} S 2 p^{4}\left({ }_{2}^{3} P\right)^{4} P 3 p^{3} P^{\circ}+0.052 s^{2} S 2 p^{4}\left({ }_{2}^{3} P\right)^{4} P 3 p^{5} D^{\circ}$ \\
\hline 19 & 105 & $2 s^{2} S 2 p^{4}\left(\frac{1}{2} D\right)^{2} D 3 s^{3} D_{3}^{2}$ & & 3577114 & 3.066E-12 & 3.068E-12 & 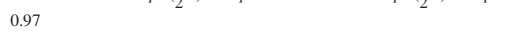 \\
\hline 19 & 106 & $2 s^{2} S 2 p^{4}\left(\frac{2}{3} P\right){ }^{4} P 3 p^{3} S_{1}^{0}$ & & 3578755 & $1.196 \mathrm{E}-12$ & $1.194 \mathrm{E}-12$ & $0.68+0.202 s^{2} S 2 p^{4}\left({ }_{2}^{3} P\right)^{4} P 3 p^{3} P^{\circ}+0.072 s^{2} S 2 p^{4}\left({ }_{2}^{3} P\right)^{4} P 3 p^{3} D^{\circ}$ \\
\hline 19 & 107 & $2 s^{2} S 2 p^{4}\left({ }_{2}^{2} P\right){ }^{4} P 3 p^{5} S_{2}^{\circ}$ & & 3579185 & $6.766 \mathrm{E}-11$ & $6.783 \mathrm{E}-11$ & $0.92+0.062 s^{2} S 2 p^{4}\left({ }_{2}^{3} P\right){ }^{4} P 3 p^{5} P^{\circ}$ \\
\hline 19 & 108 & $2 s^{2} S 2 p^{4}\left({ }_{2}^{2} P\right){ }^{4} P 3 p^{3} D_{1}^{\circ}$ & & 3585857 & $1.539 \mathrm{E}-12$ & $1.537 \mathrm{E}-12$ & $0.86+0.112 s^{2} S 2 p^{4}\left({ }_{2}^{3} P\right){ }^{4} P 3 p^{3} S^{\circ}$ \\
\hline 19 & 109 & $2 s^{2} S 2 p^{4}\left({ }_{2}^{3} P\right)^{4} P 3 p^{3} P_{2}^{\circ}$ & & 3592858 & $1.397 \mathrm{E}-12$ & $1.394 \mathrm{E}-12$ & $0.75+0.132 s^{2} S 2 p^{4}\left({ }_{2}^{3} P\right)^{4} P 3 p^{3} D^{\circ}+0.092 s^{2} S 2 p^{4}\left({ }_{2}^{1} D\right)^{2} D 3 p^{3} P^{\circ}$ \\
\hline 19 & 110 & $2 s^{2} S 2 p^{4}\left({ }_{2}^{3} P\right){ }^{4} P 3 p^{3} P_{0}^{\circ}$ & & 3596431 & $1.516 \mathrm{E}-12$ & $1.513 \mathrm{E}-12$ & $0.84+0.132 s^{2} S 2 p^{4}\left({ }_{2}^{1} D\right)^{2} D 3 p^{3} P^{\circ}$ \\
\hline 19 & 111 & $2 s^{2} S 2 p^{4}\left({ }_{2}^{3} P\right)^{4} P 3 p^{3} P_{1}^{\circ}$ & & 3599576 & $1.355 \mathrm{E}-12$ & $1.353 \mathrm{E}-12$ & $0.64+0.202 s^{2} S 2 p^{4}\left({ }_{2}^{3} P\right)^{4} P 3 p^{3} S^{\circ}+0.092 s^{2} S 2 p^{4}\left({ }_{2}^{1} D\right)^{2} D 3 p^{3} P^{\circ}$ \\
\hline 19 & 112 & $2 s^{2} S 2 p^{4}\left({ }_{2}^{1} D\right)^{2} D 3 s^{1} D_{2}$ & & 3610898 & $5.620 \mathrm{E}-12$ & $5.625 \mathrm{E}-12$ & $0.98+2$ \\
\hline 19 & 113 & $2 s^{2} S 2 p^{4}\left({ }_{0}^{1} S\right)^{2} S 3 s^{3} S_{1}$ & & 3684430 & 2.859E-12 & $2.862 \mathrm{E}-12$ & $0.90+0.052 s^{2} S 2 p^{4}\left({ }_{2}^{3} P\right)^{2} P 3 s^{3} P$ \\
\hline 19 & 114 & $2 s^{2} S 2 p^{4}\left({ }_{2}^{3} P\right){ }^{4} P 3 d^{5} D_{4}$ & & 3702677 & $1.040 \mathrm{E}-10$ & $1.048 \mathrm{E}-10$ & $0.92+0.072 s^{2} S 2 p^{4}\left({ }_{2}^{3} P\right){ }^{4} P 3 d^{5} F$ \\
\hline 19 & 115 & $2 s^{2} S 2 p^{4}\left({ }_{2}^{3} P\right){ }^{4} P 3 d^{5} D_{3}$ & & 3703749 & $1.006 \mathrm{E}-10$ & $1.017 \mathrm{E}-10$ & $0.91+0.052 s^{2} S 2 p^{4}\left({ }_{2}^{3} P\right){ }^{4} P 3 d^{5} F+0.032 s^{2} S 2 p^{4}\left({ }_{2}^{3} P\right)^{4} P 3 d^{5} P$ \\
\hline 19 & 116 & $2 s^{2} S 2 p^{4}\left({ }_{2}^{3} P\right){ }^{4} P 3 d^{5} D_{2}$ & & 3706320 & 9.434E-11 & $9.553 \mathrm{E}-11$ & $\left.0.91+0.052 s^{2} S 2 p^{4}{ }_{2}^{3} P\right)^{4} P 3 d^{5} P+0.032 s^{2} S 2 p^{4}\left({ }_{2}^{2} P\right)^{4} P 3 d^{5} F$ \\
\hline 19 & 117 & $2 s^{2} S 2 p^{4}\left({ }_{3}^{3} P\right)^{4} P 3 d^{5} D_{1}$ & & 3709376 & $8.261 \mathrm{E}-11$ & $8.367 \mathrm{E}-11$ & $0.94+0.042 s^{2} S 2 p^{4}\left({ }_{3}^{3} P\right)^{4} P 3 d^{5} P$ \\
\hline 19 & 118 & $2 s^{2} S 2 p^{4}\left({ }_{0}^{2} S\right)^{2} S 3 s^{1} s_{0}$ & & 3711153 & $3.907 \mathrm{E}-12$ & $3.911 \mathrm{E}-12$ & $0.69+0.282 s^{2} S 2 p^{4}\left({ }_{2}^{2} P\right)^{2} P 3 s^{3} P$ \\
\hline 19 & 119 & $2 s^{2} S 2 p^{4}\left({ }_{2}^{3} P\right){ }^{4} P 3 d^{5} D_{0}$ & & 3711694 & 7.479E-11 & 7.564E-11 & $0.98-23$ \\
\hline 19 & 120 & $2 s^{2} S 2 p^{4}\left({ }_{1}^{1} D\right)^{2} D 3 p^{3} F_{2}^{\circ}$ & & 3717215 & $3.688 \mathrm{E}-11$ & $3.682 \mathrm{E}-11$ & 0.97 \\
\hline 19 & 121 & $2 s^{2} S 2 p^{4}\left({ }_{2}^{3} P\right)^{2} P 3 s^{3} P_{2}{ }^{2}$ & & 3717579 & $4.191 \mathrm{E}-12$ & $4.197 \mathrm{E}-12$ & 0.97 \\
\hline 19 & 122 & $2 s^{2} S 2 p^{4}\left({ }_{2}^{1} D\right)^{2} D 3 p^{3} F_{3}^{\circ}$ & & 3719291 & $2.574 \mathrm{E}-11$ & $2.569 \mathrm{E}-11$ & 0.96 \\
\hline 19 & 123 & $2 s^{2} S 2 p^{4}\left({ }_{2}^{2} P\right){ }^{4} P 3 d^{5} F_{5}^{3}$ & & 3719538 & $1.830 \mathrm{E}-10$ & $1.745 \mathrm{E}-10$ & 0.99 \\
\hline 19 & 124 & $2 s^{2} S 2 p^{4}\left({ }_{2}^{1} D\right)^{2} D 3 p^{3} F_{4}^{\circ}$ & & 3722702 & 4.843E-11 & $4.819 \mathrm{E}-11$ & 0.98 \\
\hline 19 & 125 & $2 s^{2} S 2 p^{4}\left({ }_{2}^{1} D\right)^{2} D 3 p^{1} P_{1}^{\circ}$ & & 3723576 & $1.012 \mathrm{E}-12$ & $1.011 \mathrm{E}-12$ & $\left.0.78+0.072 s^{2} S 2 p^{4}\left({ }_{2}^{1} D\right)^{2} D 3 p^{3} D^{\circ}+0.072 s^{2} S 2 p^{4}{ }_{2}^{3} P\right)^{2} P 3 p^{1} P^{\circ}$ \\
\hline 19 & 126 & $2 s^{2} S 2 p^{4}\left({ }_{2}^{3} P\right)^{2} P 3 s^{3} P_{1}$ & & 3724633 & $2.255 \mathrm{E}-12$ & $2.260 \mathrm{E}-12$ & $0.61+0.342 s^{2} S 2 p^{4}\left(3_{2}^{3} P\right)^{2} P 3 s^{1} P$ \\
\hline 19 & 127 & $2 s^{2} S 2 p^{4}\left({ }_{2}^{3} P\right){ }^{4} P 3 d^{5} F_{4}$ & & 3728229 & $1.642 \mathrm{E}-10$ & $1.584 \mathrm{E}-10$ & $\left.0.88+0.072 s^{2} S 2 p^{4}{ }_{2}^{3} P\right)^{4} P 3 d^{5} D+0.042 s^{2} S 2 p^{4}\left({ }_{2}^{3} P\right)^{4} P 3 d^{3} F$ \\
\hline 19 & 128 & $2 s^{2} S 2 p^{4}\left({ }_{2}^{3} P\right){ }^{4} P 3 d^{5} F_{3}$ & & 3733847 & 5.465E-11 & $5.396 \mathrm{E}-11$ & $0.91+0.042 s^{2} S 2 p^{4}\left({ }_{2}^{3} P\right)^{4} P 3 d^{5} D+0.022 s^{2} S 2 p^{4}\left({ }_{2}^{3} P\right){ }^{4} P 3 d^{3} F$ \\
\hline 19 & 129 & $2 s^{2} S 2 p^{4}\left({ }_{2}^{1} D\right)^{2} D 3 p^{1} F_{3}^{\circ}$ & & 3734243 & $1.126 \mathrm{E}-12$ & $1.123 \mathrm{E}-12$ & $0.91+0.052 s^{2} S 2 p^{4}\left({ }_{2}^{1} D\right)^{2} D 3 p^{3} D^{\circ}$ \\
\hline 19 & 130 & $2 s^{2} S 2 p^{4}\left({ }_{2}^{3} P\right){ }^{4} P 3 d^{5} P_{1}{ }^{3}$ & & 3737396 & 3.186E-11 & $3.248 \mathrm{E}-11$ & $\left.0.92+0.042 s^{2} S 2 p^{4}{ }_{2}^{2} P\right)^{4} P 3 d^{5} F+0.032 s^{2} S 2 p^{4}\left({ }_{2}^{3} P\right)^{4} P 3 d^{5} D$ \\
\hline 19 & 131 & $2 s^{2} S 2 p^{4}\left({ }_{2}^{2} P\right){ }^{4} P 3 d^{5} F_{2}$ & & 3737521 & $4.380 \mathrm{E}-11$ & $4.341 \mathrm{E}-11$ & $0.92+0.052 s^{2} S 2 p^{4}\left({ }_{2}^{3} P\right)^{4} P 3 d^{5} P$ \\
\hline 19 & 132 & $2 s^{2} S 2 p^{4}\left({ }_{2}^{3} P\right){ }^{4} P 3 d^{5} F_{1}$ & & 3740137 & 5.803E-11 & $5.736 \mathrm{E}-11$ & $0.93+0.032 s^{2} S 2 p^{4}\left(\int_{2}^{2} P\right)^{4} P 3 d^{5} P+0.022 s^{2} S 2 p^{4}\left({ }_{2}^{3} P\right)^{4} P 3 d^{5} D$ \\
\hline 19 & 133 & $2 s^{2} S 2 p^{4}\left({ }_{2}^{1} D\right)^{2} D 3 p^{3} D_{2}^{\circ}$ & & 3741054 & 2.614E-11 & 2.613E-11 & $0.96-2+2+2$ \\
\hline 19 & 134 & $2 s^{2} S 2 p^{4}\left({ }_{1}^{1} D\right)^{2} D 3 p^{3} D^{2}$ & & 3741686 & 8.373E-12 & $8.361 \mathrm{E}-12$ & $0.90+0.072 s^{2} S 2 p^{4}\left({ }_{1}^{1} D\right)^{2} D 3 p^{1} P^{\circ}$ \\
\hline 19 & 135 & $2 s^{2} S 2 p^{4}\left(3_{3}^{3} P\right)^{4} P 3 d^{5} P_{2}$ & & 3743164 & 3.622E-11 & 3.713E-11 & $0.89+0.062 s^{2} S 2 p^{4}\left({ }_{2}^{3} P\right)^{4} P 3 d^{5} D+0.032 s^{2} S 2 p^{4}\left({ }_{2}^{3} P\right)^{4} P 3 d^{5} F$ \\
\hline 19 & 136 & $2 s^{2} S 2 p^{4}\left({ }_{2}^{1} D\right)^{2} D 3 p^{3} D_{3}^{\circ}$ & & 3743250 & $1.401 \mathrm{E}-11$ & $1.398 \mathrm{E}-11$ & $0.92+0.052 s^{2} S 2 p^{4}\left({ }_{2}^{1} D\right)^{2} D 3 p^{1} F^{\circ}$ \\
\hline 19 & 137 & $2 s^{2} S 2 p^{4}\left({ }_{2}^{3} P\right)^{2} P 3 s^{3} P_{0}{ }^{3}$ & & 3743787 & $4.358 \mathrm{E}-12$ & 4.363E-12 & $0.69+0.282 s^{2} S 2 p^{4}\left({ }_{0}^{1} S\right)^{2} S 3 s^{1} S$ \\
\hline 19 & 138 & $2 s^{2} S 2 p^{4}\left({ }_{2}^{3} P\right)^{2} P 3 s^{1} P_{1}$ & & 3744342 & $1.560 \mathrm{E}-12$ & $1.563 \mathrm{E}-12$ & $0.61+0.312 s^{2} S 2 p^{4}\left({ }_{2}^{3} P\right)^{2} P 3 s^{3} P+0.052 s^{2} S 2 p^{4}\left({ }_{0}^{1} S\right)^{2} S 3 s^{3} S$ \\
\hline 19 & 139 & $2 s^{2} S 2 p^{4}\left({ }_{3}^{3} P\right)^{4} P 3 d^{5} P_{3}$ & & 3749463 & $2.511 \mathrm{E}-11$ & $2.558 \mathrm{E}-11$ & $0.93+0.042 s^{2} S 2 p^{4}\left(3_{2}^{3} P\right)^{4} P 3 d^{5} D$ \\
\hline 19 & 140 & $2 s^{2} S 2 p^{4}\left({ }_{2}^{3} P\right){ }^{4} P 3 d^{3} F_{4}$ & & 3751888 & $1.464 \mathrm{E}-10$ & 1.423E-10 & $0.94+0.032 s^{2} S 2 p^{4}\left({ }_{2}^{3} P\right)^{4} P 3 d^{5} F$ \\
\hline 19 & 141 & $2 s^{2} S 2 p^{4}\left({ }_{2}^{1} D\right)^{2} D 3 p^{1} D_{2}^{\circ}$ & & 3752428 & $1.675 \mathrm{E}-12$ & $1.671 \mathrm{E}-12$ & $0.51+0.422 s^{2} S 2 p^{4}\left({ }_{2}^{1} D\right)^{2} D 3 p^{3} P^{\circ}+0.032 s^{2} S 2 p^{4}\left({ }_{2}^{3} P\right)^{2} P 3 p^{3} P^{\circ}$ \\
\hline 19 & 142 & $2 s^{2} S 2 p^{4}\left({ }_{2}^{1} D\right)^{2} D 3 p^{3} P_{1}^{\circ}$ & & 3756533 & $4.486 \mathrm{E}-12$ & $4.468 \mathrm{E}-12$ & $0.80+0.102 s^{2} S 2 p^{4}\left({ }_{2}^{3} P\right)^{4} P 3 p^{3} P^{\circ}+0.062 s^{2} S 2 p^{4}\left(_{2}^{3} P\right)^{2} P 3 p^{3} P^{\circ}$ \\
\hline 19 & 143 & $2 s^{2} S 2 p^{4}\left({ }_{2}^{1} D\right){ }^{2} D 3 p^{3} P_{2}^{\circ}$ & & 3756556 & $1.680 \mathrm{E}-12$ & $1.676 \mathrm{E}-12$ & $0.43+0.472 s^{2} S 2 p^{4}\left({ }_{2}^{1} D\right)^{2} D 3 p^{1} D^{\circ}+0.062 s^{2} S 2 p^{4}\left({ }_{2}^{3} P\right)^{4} P 3 p^{3} P^{\circ}$ \\
\hline 19 & 144 & $2 s^{2} S 2 p^{4}\left({ }_{2}^{1} D\right)^{2} D 3 p^{3} P_{0}^{\circ}$ & & 3758047 & 4.183E-12 & 4.165E-12 & $0.79+0.122 s^{2} S 2 p^{4}\left({ }_{2}^{3} P\right){ }^{4} P 3 p^{3} P^{\circ}+0.082 s^{2} S 2 p^{4}\left(3_{2}^{2} P\right)^{2} P 3 p^{3} P^{\circ}$ \\
\hline 19 & 145 & $2 s^{2} S 2 p^{4}\left({ }_{2}^{3} P\right){ }^{4} P 3 d^{3} F_{3}$ & & 3759993 & 5.291E-12 & $5.280 \mathrm{E}-12$ & $0.88+0.072 s^{2} S 2 p^{4}\left({ }_{2}^{3} P\right){ }^{4} P 3 d^{3} D+0.022 s^{2} S 2 p^{4}\left({ }_{2}^{3} P\right){ }^{4} P 3 d^{5} F$ \\
\hline 19 & 146 & $2 s^{2} S 2 p^{4}\left({ }_{2}^{2} P\right){ }^{4} P 3 d^{3} F_{2}$ & & 3767115 & 3.194E-12 & $3.190 \mathrm{E}-12$ & $\left.0.84+0.112 s^{2} S 2 p^{4}{ }_{2}^{3} P\right)^{4} P 3 d^{3} D+0.022 s^{2} S 2 p^{4}\left({ }_{2}^{2} P\right)^{4} P 3 d^{3} P$ \\
\hline 19 & 147 & $2 s^{2} S 2 p^{4}\left({ }_{2}^{3} P\right){ }^{4} P 3 d^{3} P_{0}$ & & 3772937 & $1.115 \mathrm{E}-12$ & $1.115 \mathrm{E}-12$ & $0.90+0.072 s^{2} S 2 p^{4}\left({ }_{2}^{4} D\right)^{2} D 3 d^{3} P$ \\
\hline 19 & 148 & $2 s^{2} S 2 p^{4}\left({ }_{2}^{2} P\right){ }^{4} P 3 d^{3} P_{1}$ & & 3774379 & $7.998 \mathrm{E}-13$ & 7.997E-13 & $0.72+0.202 s^{2} S 2 p^{4}\left({ }_{2}^{2} P\right)^{4} P 3 d^{3} D+0.052 s^{2} S 2 p^{4}\left({ }_{2}^{1} D\right)^{2} D 3 d^{3} P$ \\
\hline 19 & 149 & $2 s^{2} S 2 p^{4}\left({ }_{2}^{3} P\right){ }^{4} P 3 d^{3} D_{2}$ & & 3779275 & 6.488E-13 & 6.485E-13 & $0.41+0.402 s^{2} S 2 p^{4}\left({ }_{2}^{2} P\right)^{4} P 3 d^{3} P+0.132 s^{2} S 2 p^{4}\left({ }_{2}^{3} P\right)^{4} P 3 d^{3} F$ \\
\hline 19 & 150 & $2 s^{2} S 2 p^{4}\left({ }_{2}^{3} P\right){ }^{4} P 3 d^{3} D_{3}$ & & 3785331 & $4.169 \mathrm{E}-13$ & $4.165 \mathrm{E}-13$ & $0.87+0.082 s^{2} S 2 p^{4}\left({ }_{2}^{3} P\right)^{4} P 3 d^{3} F+0.032 s^{2} S 2 p^{4}\left({ }_{2}^{T} D\right)^{2} D 3 d^{3} D$ \\
\hline 19 & 151 & $2 s^{2} S 2 p^{4}\left({ }_{2}^{3} P\right){ }^{4} P 3 d^{3} D_{1}$ & & 3787975 & $5.210 \mathrm{E}-13$ & $5.208 \mathrm{E}-13$ & $0.72+0.192 s^{2} S 2 p^{4}\left({ }_{2}^{3} P\right)^{4} P 3 d^{3} P+0.042 s^{2} S 2 p^{4}\left({ }_{2}^{1} D\right)^{2} D 3 d^{3} D$ \\
\hline 19 & 152 & $2 s^{2} S 2 p^{4}\left({ }_{2}^{3} P\right){ }^{4} P 3 d^{3} P_{2}$ & & 3793325 & $6.383 \mathrm{E}-13$ & $6.380 \mathrm{E}-13$ & $0.50+0.412 s^{2} S 2 p^{4}\left({ }_{2}^{3} P\right)^{4} P 3 d^{3} D+0.042 s^{2} S 2 p^{4}\left({ }_{2}^{1} D\right)^{2} D 3 d^{3} P$ \\
\hline 19 & 153 & $\left.2 s^{2} S 2 p^{4}(1)\right)^{2} S 3 p^{3} P_{0}^{\circ}$ & & 3830586 & $6.308 \mathrm{E}-12$ & $6.315 \mathrm{E}-12$ & $0.76+0.192 s^{2} S 2 p^{4}\left({ }_{2}^{3} P\right)^{2} P 3 p^{3} P^{\circ}+0.022 s^{2} S 2 p^{4}\left(\frac{1}{2} D\right)^{2} D 3 p^{3} P^{\circ}$ \\
\hline 19 & 154 & $\left.2 s^{2} S 2 p^{4}(1)_{0}^{1} S\right)^{2} p^{3} P_{1}^{\circ}$ & & 3834105 & $5.893 \mathrm{E}-12$ & $5.897 \mathrm{E}-12$ & $0.76+0.142 s^{2} S 2 p^{4}\left(\int_{2}^{3} P\right)^{2} P 3 p^{3} P^{\circ}+0.052 s^{2} S 2 p^{4}\left({ }_{2}^{3} P\right)^{2} P 3 p^{3} D^{\circ}$ \\
\hline 19 & 155 & $2 s^{2} S 2 p^{4}\left({ }_{0}^{1} S\right)^{2} S 3 p^{3} P_{2}^{\circ}$ & & 3841090 & $8.031 \mathrm{E}-12$ & $8.038 \mathrm{E}-12$ & $0.83+0.092 s^{2} S 2 p^{4}\left({ }_{2}^{3} P\right)^{2} P 3 p^{3} D^{\circ}+0.042 s^{2} S 2 p^{4}\left({ }_{2}^{3} P\right)^{2} P 3 p^{3} P^{\circ}$ \\
\hline 19 & 156 & $2 s^{2} S 2 p^{4}\left({ }_{0}^{1} S\right)^{2} S 3 p^{1} P_{1}^{\circ}$ & & 3848435 & 1.177E-12 & $1.175 \mathrm{E}-12$ & $0.78+0.072 s^{2} S 2 p^{4}\left({ }_{2}^{3} P\right)^{2} P 3 p^{3} D^{\circ}+0.072 s^{2} S 2 p^{4}\left({ }_{2}^{3} P\right)^{2} P 3 p^{3} P^{\circ}$ \\
\hline 19 & 157 & $2 s^{2} S 2 p^{4}\left({ }_{2}^{3} P\right)^{2} P 3 p^{3} P_{2}^{\circ}$ & & 3858636 & $4.457 \mathrm{E}-12$ & 4.457E-12 & $0.76+0.082 s^{2} S 2 p^{4}\left({ }_{0}^{1} S\right)^{2} S 3 p^{3} P^{\circ}+0.072 s^{2} S 2 p^{4}\left({ }_{2}^{3} P\right)^{2} P 3 p^{3} D^{\circ}$ \\
\hline 19 & 158 & $\left.2 s^{2} S 2 p^{4}{ }_{2}^{2} P\right)^{2} P 3 p^{3} D_{3}^{\circ}$ & & 3867617 & $2.328 \mathrm{E}-12$ & $2.325 \mathrm{E}-12$ & 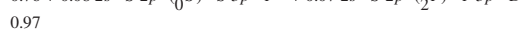 \\
\hline 19 & 159 & $2 s^{2} S 2 p^{4}\left({ }_{2}^{3} P\right)^{2} P 3 p^{3} D_{2}^{\circ}$ & & 3868134 & $3.416 \mathrm{E}-12$ & $3.414 \mathrm{E}-12$ & $0.48+0.402 s^{2} S 2 p^{4}\left({ }_{2}^{3} P\right)^{2} P 3 p^{1} D^{\circ}+0.082 s^{2} S 2 p^{4}\left({ }_{2}^{3} P\right)^{2} P 3 p^{3} P^{\circ}$ \\
\hline 19 & 160 & $2 s^{2} S 2 p^{4}\left({ }_{2}^{3} P\right)^{2} P 3 p^{3} P_{1}^{\circ}$ & & 3869498 & 4.918E-12 & $4.916 \mathrm{E}-12$ & $\left.0.56+0.182 s^{2} S 2 p^{4}\left({ }_{0}^{1} S\right)^{2} S 3 p^{3} P^{\circ}+0.092 s^{2} S 2 p^{4}{ }_{2}^{3} P\right)^{2} P 3 p^{3} S^{\circ}$ \\
\hline 19 & 161 & $2 s^{2} S 2 p^{4}\left({ }_{2}^{3} P\right)^{2} P 3 p^{3} P_{0}^{\circ}$ & & 3875753 & $6.710 \mathrm{E}-12$ & $6.716 \mathrm{E}-12$ & $0.59+0.172 s^{2} S 2 p^{4}\left({ }_{2}^{3} P\right)^{2} P 3 p^{1} S^{\circ}+0.152 s^{2} S 2 p^{4}\left({ }_{0}^{1} S\right)^{2} S 3 p^{3} P^{\circ}$ \\
\hline 19 & 162 & $\left.2 s^{2} S 2 p^{4}{ }_{3}^{3} P\right)^{2} P 3 p^{3} D_{1}^{\circ}$ & & 3882017 & $2.052 \mathrm{E}-12$ & $2.049 \mathrm{E}-12$ & $0.77+0.092 s^{2} S 2 p^{4}\left({ }_{0}^{1} S\right)^{2} S 3 p^{1} P^{\circ}+0.052 s^{2} S 2 p^{4}\left(\int_{2}^{2} P\right)^{2} P 3 p^{3} P^{\circ}$ \\
\hline 19 & 163 & $\left.2 s^{2} S 2 p^{4}{ }_{2}^{2} P\right)^{2} P 3 p^{1} D_{2}^{1}$ & & 3891052 & 4.777E-12 & $4.777 \mathrm{E}-12$ & $0.54+0.342 s^{2} S 2 p^{4}\left(\int_{2}^{3} P\right)^{2} P 3 p^{3} D^{\circ}+0.072 s^{2} S 2 p^{4}\left({ }_{0}^{1} S\right)^{2} S 3 p^{3} P^{\circ}$ \\
\hline 19 & 164 & $2 s^{2} S 2 p^{4}\left(\frac{1}{2} D\right)^{2} D 3 d^{3} G_{3}^{2}$ & & 3891497 & $6.731 \mathrm{E}-11$ & $6.649 \mathrm{E}-11$ & 0.98 \\
\hline 19 & 165 & $2 s^{2} S 2 p^{4}\left(\frac{1}{2} D\right)^{2} D 3 d^{3} G_{4}$ & & 3892203 & $6.858 \mathrm{E}-11$ & $6.767 \mathrm{E}-11$ & 0.98 \\
\hline 19 & 166 & $2 s^{2} S 2 p^{4}\left({ }_{2}^{1} D\right)^{2} D 3 d^{3} G_{5}$ & & 3893092 & 7.018E-11 & $6.925 \mathrm{E}-11$ & 0.98 \\
\hline 19 & 167 & $2 s^{2} S 2 p^{4}\left({ }_{2}^{3} P\right)^{2} P 3 p p^{1} S_{0}^{0}$ & & 3896348 & 1.203E-11 & $1.204 \mathrm{E}-11$ & $0.81+0.102 s^{2} S 2 p^{4}\left({ }_{2}^{3} P\right)^{2} P 3 p^{3} P^{\circ}+0.072 s^{2} S 2 p^{4}\left({ }_{0}^{1} S\right)^{2} S 3 p^{3} P^{\circ}$ \\
\hline 19 & 168 & $2 s^{2} S 2 p^{4}\left({ }_{2}^{3} P\right)^{2} P 3 p^{3} S_{1}^{0}$ & & 3901030 & $3.640 \mathrm{E}-12$ & $3.619 \mathrm{E}-12$ & $0.80+0.072 s^{2} S 2 p^{4}\left({ }_{2}^{3} P\right)^{2} P 3 p^{3} P^{\circ}+0.042 s^{2} S 2 p^{4}\left({ }_{0}^{1} S\right)^{2} S 3 p^{1} P^{\circ}$ \\
\hline 19 & 169 & $2 s^{2} S 2 p^{4}\left({ }_{2}^{1} D\right)^{2} D 3 d^{3} S_{1}$ & & 3914962 & $2.492 \mathrm{E}-13$ & 2.493E-13 & $0.96-2+2$ \\
\hline 19 & 170 & $2 s^{2} S 2 p^{4}\left(\frac{1}{2} D\right)^{2} D 3 d^{3} F_{2}$ & & 3916541 & $3.534 \mathrm{E}-11$ & $3.582 \mathrm{E}-11$ & 0.96 \\
\hline 19 & 171 & $2 s^{2} S 2 p^{4}\left({ }_{2}^{1} D\right)^{2} D 3 d^{3} F_{3}$ & & 3917258 & $2.412 \mathrm{E}-11$ & 2.431E-11 & 0.97 \\
\hline 19 & 172 & $2 s^{2} S 2 p^{4}\left(\frac{1}{2} D\right)^{2} D 3 d^{3} F_{4}$ & & 3917712 & $5.741 \mathrm{E}-11$ & $5.815 \mathrm{E}-11$ & $0.85+0.132 s^{2} S 2 p^{4}\left({ }_{2}^{1} D\right)^{2} D 3 d^{1} G$ \\
\hline 19 & 173 & $2 s^{2} S 2 p^{4}\left(\frac{1}{2} D\right)^{2} D 3 d^{1} G_{4}$ & & 3920583 & $5.419 \mathrm{E}-11$ & $5.421 \mathrm{E}-11$ & $0.86+0.122 s^{2} S 2 p^{4}\left({ }_{2}^{1} D\right)^{2} D 3 d^{3} F$ \\
\hline 19 & 174 & $2 s^{2} S 2 p^{4}\left({ }_{2}^{1} D\right)^{2} D 3 d^{3} P_{2}$ & & 3922123 & $2.746 \mathrm{E}-13$ & $2.747 \mathrm{E}-13$ & $0.86+0.062 s^{2} S 2 p^{4}\left({ }_{2}^{2} D\right)^{2} D 3 d^{3} D+0.052 s^{2} S 2 p^{4}\left({ }_{2}^{3} P\right)^{4} P 3 d^{3} P$ \\
\hline 19 & 175 & $2 s^{2} S 2 p^{4}\left({ }_{2}^{1} D\right)^{2} D 3 d^{3} D_{1}$ & & 3922473 & $4.006 \mathrm{E}-13$ & 4.005E-13 & $\left.0.52+0.302 s^{2} S 2 p^{4}\left({ }_{2}^{1} D\right)\right)^{2} D 3 d^{3} P+0.082 s^{2} S 2 p^{4}\left({ }_{2}^{2} D\right)^{2} D 3 d^{1} P$ \\
\hline 19 & 176 & $2 s^{2} S 2 p^{4}\left({ }_{2}^{1} D\right)^{2} D 3 d^{3} D_{3}$ & & 3922751 & $4.481 \mathrm{E}-13$ & $4.478 \mathrm{E}-13$ & $0.92+0.032 s^{2} S 2 p^{4}\left(3_{2}^{3} P\right)^{4} P 3 d^{3} D$ \\
\hline 19 & 177 & $2 s^{2} S 2 p^{4}\left({ }_{2}^{1} D\right)^{2} D 3 d^{3} P_{0}$ & & 3923092 & $2.761 \mathrm{E}-13$ & $2.762 \mathrm{E}-13$ & $0.88+0.072 s^{2} S 2 p^{4}\left({ }_{2}^{3} P\right)^{4} P 3 d^{3} P+0.032 s^{2} S 2 p^{4}\left({ }_{2}^{1} D\right)^{2} D 3 d^{1} S$ \\
\hline 19 & 178 & $2 s^{2} S 2 p^{4}\left({ }_{1}^{1} D\right)^{2} D 3 d^{3} D_{2}$ & & 3923357 & $4.289 \mathrm{E}-13$ & $4.286 \mathrm{E}-13$ & $0.85+0.062 s^{2} S 2 p^{4}\left(c_{2}^{1} D\right)^{2} D 3 d^{3} P+0.042 s^{2} S 2 p^{4}\left(\frac{3}{2} P\right)^{4} P 3 d^{3} D$ \\
\hline
\end{tabular}




\begin{tabular}{|c|c|c|c|c|c|c|c|}
\hline $\mathrm{z}$ & Key & Level & $E_{\mathrm{NIST}}$ & $E_{\mathrm{MCDHF} / \mathrm{RCI}}$ & $\tau_{\mathrm{MCDHF} / \mathrm{RCI}}^{l}$ & $\tau_{\mathrm{MCDHF} / \mathrm{RCI}}^{v}$ & $L S$-composition \\
\hline 19 & 179 & $2 s^{2} S 2 p^{4}\left({ }_{2}^{1} D\right)^{2} D 3 d^{3} P_{1}$ & & 3924071 & $3.060 \mathrm{E}-13$ & $3.060 \mathrm{E}-13$ & $0.59+0.282 s^{2} S 2 p^{4}\left({ }_{2}^{1} D\right)^{2} D 3 d^{3} D+0.052 s^{2} S 2 p^{4}\left({ }_{2}^{3} P\right)^{4} P 3 d^{3} P$ \\
\hline 20 & 1 & $2 s^{2} 2 p^{4}\left({ }_{2}^{3} P\right)^{3} P_{2}$ & 0 & 0 & $\ldots$ & $\ldots$ & $0.96+0.032 s^{2} 2 p^{4}\left({ }_{2}^{1} D\right){ }^{1} D$ \\
\hline 20 & 2 & $2 s^{2} 2 p^{4}\left({ }_{2}^{3} P\right)^{3} P_{1}$ & 24460 & 24444 & $3.141 \mathrm{E}-03$ & $3.141 \mathrm{E}-03$ & 0.99 \\
\hline 20 & 3 & $2 s^{2} 2 p^{4}\left(\frac{3}{2} P\right)^{3} P_{0}$ & 28888 & 29106 & $1.906 \mathrm{E}-01$ & $1.906 \mathrm{E}-01$ & $0.95+0.042 s^{2} 2 p^{4}\left({ }_{0}^{1} s\right){ }^{1} S$ \\
\hline 20 & 4 & $2 s^{2} 2 p^{4}\left({ }_{2}^{1} D\right){ }^{1} D_{2}$ & 88208 & 88408 & $1.129 \mathrm{E}-03$ & $1.129 \mathrm{E}-03$ & $0.96+0.032 s^{2} 2 p^{4}\left({ }_{2}^{3} P\right)^{3} P$ \\
\hline 20 & 5 & $2 s^{2} 2 p^{4}\left({ }_{0}^{1} s\right){ }^{1} s_{0}$ & 178613 & 178799 & $1.235 \mathrm{E}-04$ & $1.236 \mathrm{E}-04$ & $0.93+0.042 s^{2} 2 p^{4}\left({ }_{2}^{3} P\right)^{3} P+0.022 p^{6}{ }^{1} S$ \\
\hline 20 & 6 & $2 s^{2} S 2 p^{5}{ }^{3} P_{2}^{\circ}$ & 618268 & 617941 & $3.889 \mathrm{E}-11$ & 3.873E-11 & 0.99 \\
\hline 20 & 7 & $2 s^{2} S 2 p^{5}{ }^{3} P_{1}^{2}$ & 638238 & 637992 & 3.652E-11 & 3.652E-11 & 0.99 \\
\hline 20 & 8 & $2 s^{2} S 2 p^{5}{ }^{3} P_{0}^{\circ}$ & 650105 & 649930 & $3.599 \mathrm{E}-11$ & 3.605E-11 & 0.99 \\
\hline 20 & 9 & $2 s^{2} S 2 p^{5}{ }^{1} P_{1}^{\circ}$ & 850300 & 850331 & $1.076 \mathrm{E}-11$ & $1.076 \mathrm{E}-11$ & 0.98 \\
\hline 20 & 10 & $2 p^{6}{ }^{1} S_{0}$ & \multirow[t]{2}{*}{1440320} & 1440347 & $1.250 \mathrm{E}-11$ & $1.252 \mathrm{E}-11$ & $0.96+0.022 s^{2} 2 p^{4}\left({ }_{0}^{1} s\right)^{1} S$ \\
\hline 20 & 11 & $2 s^{2} 2 p^{3}\left({ }_{3}^{4} s\right)^{4} s 3 s^{5} s_{2}^{\circ}$ & & 3334846 & $2.164 \mathrm{E}-10$ & $2.177 \mathrm{E}-10$ & 0.97 \\
\hline 20 & 12 & $2 s^{2} 2 p^{3}\left({ }_{3}^{4} S\right)^{4} S 3 s^{3} S_{1}^{0}$ & 3374600 & 3373246 & $1.018 \mathrm{E}-12$ & $1.019 \mathrm{E}-12$ & 0.97 \\
\hline 20 & 13 & $2 s^{2} 2 p^{3}\left(\frac{2}{3} D\right)^{2} D 3 s^{3} D_{1}^{\circ}$ & 3452700 & 3451512 & $3.000 \mathrm{E}-12$ & 3.004E-12 & $\left.0.90+0.042 s^{2} 2 p^{3}{ }_{1}^{2} P\right)^{2} P 3 s^{1} P^{\circ}+0.042 s^{2} 2 p^{3}\left({ }_{1}^{2} P\right)^{2} P 3 s^{3} P^{\circ}$ \\
\hline 20 & 14 & $2 s^{2} 2 p^{3}\left(\frac{2}{3} D\right)^{2} D 3 s^{3} D_{2}^{\circ}$ & 3453200 & 3452356 & $3.101 \mathrm{E}-12$ & $3.104 \mathrm{E}-12$ & $0.87+0.092 s^{2} 2 p^{3}\left({ }_{1}^{2} P\right)^{2} P 3 s^{3} P^{\circ}+0.032 s^{2} 2 p^{3}\left(\frac{2}{3} D\right)^{2} D 3 s^{1} D^{\circ}$ \\
\hline 20 & 15 & $2 s^{2} 2 p^{3}\left({ }_{3}^{2} D\right)^{2} D 3 s^{3} D_{3}^{\circ}$ & 3458300 & 3457620 & $3.177 \mathrm{E}-12$ & $3.179 \mathrm{E}-12$ & $0.99+13+3$ \\
\hline 20 & 16 & $2 s^{2} 2 p^{3}\left({ }_{3}^{3} D\right)^{2} D 3 s^{1} D_{2}^{\circ}$ & \multirow[t]{10}{*}{3474600} & 3473806 & $1.327 \mathrm{E}-12$ & $1.328 \mathrm{E}-12$ & $0.88+0.062 s^{2} 2 p^{3}\left({ }_{3}^{2} D\right)^{2} D 3 s^{3} D^{\circ}+0.052 s^{2} 2 p^{3}\left({ }_{1}^{2} P\right)^{2} P 3 s^{3} P^{\circ}$ \\
\hline 20 & 17 & $2 s^{2} 2 p^{3}\left({ }_{3}^{4} S\right)^{4} S 3 p^{5} P_{1}^{2}$ & & 3495370 & $5.273 \mathrm{E}-10$ & $5.260 \mathrm{E}-10$ & 0.96 \\
\hline 20 & 18 & $2 s^{2} 2 p^{3}\left({ }_{3}^{4} S\right)^{4} S 3 p^{5} P_{2}$ & & 3497134 & $5.531 \mathrm{E}-10$ & $5.519 \mathrm{E}-10$ & $0.94+0.022 s^{2} 2 p^{3}\left({ }_{3}^{4} S\right)^{4} S 3 p^{3} P$ \\
\hline 20 & 19 & $2 s^{2} 2 p^{3}\left({ }_{3}^{4} S\right)^{4} S 3 p^{5} P_{3}$ & & 3501540 & $5.064 \mathrm{E}-10$ & $5.063 \mathrm{E}-10$ & 0.97 \\
\hline 20 & 20 & $2 s^{2} 2 p^{3}\left({ }_{1}^{2} P\right)^{2} P 3 s^{3} P_{0}^{\circ}$ & & 3517201 & $3.332 \mathrm{E}-12$ & $3.334 \mathrm{E}-12$ & 0.97 \\
\hline 20 & 21 & $2 s^{2} 2 p^{3}\left({ }_{1}^{2} P\right)^{2} P 3 s^{3} P_{1}^{\circ}$ & & 3519893 & 3.028E-12 & 3.029E-12 & $0.90+0.052 s^{2} 2 p^{3}\left({ }_{1}^{2} P\right)^{2} P 3 s^{1} P^{\circ}+0.022 s^{2} 2 p^{3}\left({ }_{3}^{2} D\right)^{2} D 3 s^{3} D^{\circ}$ \\
\hline 20 & 22 & $2 s^{2} 2 p^{3}\left({ }_{1}^{2} P\right)^{2} P 3 s^{3} P_{2}^{\circ}$ & & 3528842 & $2.888 \mathrm{E}-12$ & $2.889 \mathrm{E}-12$ & $0.82+0.082 s^{2} 2 p^{3}\left(\frac{2}{3} D\right)^{2} D 3 s^{1} D^{\circ}+0.062 s^{2} 2 p^{3}\left(\frac{2}{3} D\right)^{2} D 3 s^{3} D^{\circ}$ \\
\hline 20 & 23 & $2 s^{2} 2 p^{3}\left({ }_{3}^{4} S\right)^{4} S 3 p^{3} P_{1}^{2}$ & & 3532586 & 4.019E-10 & $4.042 \mathrm{E}-10$ & $\left.\left.0.79+0.112 s^{2} 2 p^{3}\left({ }_{3}^{3} D\right)\right)^{2} D 3 p^{3} P+0.032 s^{2} 2 p^{3}{ }^{2}{ }_{1}^{3} P\right)^{2} P 3 p^{3} P$ \\
\hline 20 & 24 & $2 s^{2} 2 p^{3}\left({ }_{3}^{4} S\right)^{4} S 3 p^{3} P_{2}$ & & 3535250 & 4.010E-10 & 4.033E-10 & $0.81+0.122 s^{2} 2 p^{3}\left({ }_{3}^{2} D\right)^{2} D 3 p^{3} P+0.032 s^{2} 2 p^{3}\left({ }_{3}^{4} S\right)^{4} S 3 p^{5} P$ \\
\hline 20 & 25 & $2 s^{2} 2 p^{3}\left({ }_{3}^{4} S\right)^{4} S 3 p^{3} P_{0}$ & & 3536742 & $4.361 \mathrm{E}-10$ & $4.380 \mathrm{E}-10$ & $0.85+0.102 s^{2} 2 p^{3}\left(\frac{2}{2} D\right)^{2} D 3 p^{3} P+0.042 s^{2} 2 p^{3}\left({ }_{1}^{2} P\right)^{2} P 3 p^{3} P$ \\
\hline 20 & 26 & $2 s^{2} 2 p^{3}\left({ }_{1}^{2} P\right)^{2} P 3 s^{1} P_{1}^{\circ}$ & \multirow[t]{26}{*}{3544500} & 3543725 & $1.355 \mathrm{E}-12$ & $1.356 \mathrm{E}-12$ & $0.87+0.062 s^{2} 2 p^{3}\left(\frac{3}{3} D\right)^{2} D 3 s^{3} D^{\circ}+0.032 s^{2} 2 p^{3}\left({ }_{1}^{2} P\right)^{2} P 3 s^{3} P^{\circ}$ \\
\hline 20 & 27 & $2 s^{2} 2 p^{3}\left({ }_{3}^{2} D\right)^{2} D 3 p{ }^{1} P_{1}$ & & 3593132 & 3.163E-10 & 3.205E-10 & $0.38+0.412 s^{2} 2 p^{3}\left({ }_{3}^{2} D\right)^{2} D 3 p^{3} D+0.092 s^{2} 2 p^{3}\left({ }_{1}^{2} P\right)^{2} P 3 p^{1} P$ \\
\hline 20 & 28 & $2 s^{2} 2 p^{3}\left({ }_{3}^{2} D\right)^{2} D 3 p^{3} D_{2}$ & & 3604523 & $5.026 \mathrm{E}-10$ & $5.043 \mathrm{E}-10$ & $0.73+0.152 s^{2} 2 p^{3}\left({ }_{3}^{2} D\right)^{2} D 3 p^{3} F+0.082 s^{2} 2 p^{3}\left({ }_{1}^{2} P\right)^{2} P 3 p^{3} D$ \\
\hline 20 & 29 & $2 s^{2} 2 p^{3}\left(\frac{3}{3} D\right)^{2} D 3 p^{3} D_{3}$ & & 3610698 & 4.442E-10 & 4.459E-10 & $\left.0.69+0.242 s^{2} 2 p^{3}\left(\frac{2}{3} D\right)\right)^{2} D 3 p^{3} F+0.042 s^{2} 2 p^{3}\left({ }_{1}^{2} P\right)^{2} P 3 p^{3} D$ \\
\hline 20 & 30 & $2 s^{2} 2 p^{3}\left(\frac{2}{3} D\right)^{2} D 3 p^{3} D_{1}$ & & 3611480 & 2.444E-10 & $2.480 \mathrm{E}-10$ & $0.45+0.452 s^{2} 2 p^{3}\left({ }_{3}^{2} D\right){ }^{2} D 3 p^{1} P+0.052 s^{2} 2 p^{3}\left({ }_{1}^{2} P\right)^{2} P 3 p^{3} D$ \\
\hline 20 & 31 & $2 s^{2} 2 p^{3}\left({ }_{3}^{3} D\right)^{2} D 3 p^{3} F_{2}$ & & 3615570 & 3.049E-10 & $3.055 \mathrm{E}-10$ & $0.75+0.182 s^{2} 2 p^{3}\left({ }_{3}^{2} D\right)^{2} D 3 p^{3} D+0.042 s^{2} 2 p^{3}\left({ }_{1}^{2} P\right)^{2} P 3 p^{1} D$ \\
\hline 20 & 32 & $2 s^{2} 2 p^{3}\left(\frac{2}{3} D\right)^{2} D 3 p^{3} F_{3}$ & & 3620896 & 2.523E-10 & 2.532E-10 & $0.66+0.282 s^{2} 2 p^{3}\left({ }_{3}^{2} D\right)^{2} D 3 p^{3} D+0.032 s^{2} 2 p^{3}\left({ }_{1}^{2} P\right)^{2} P 3 p^{3} D$ \\
\hline 20 & 33 & $2 s^{2} 2 p^{3}\left({ }_{3}^{2} D\right)^{2} D 3 p^{3} F_{4}$ & & 3625506 & $4.404 \mathrm{E}-10$ & $4.390 \mathrm{E}-10$ & 0.99 \\
\hline 20 & 34 & $2 s^{2} 2 p^{3}\left(\frac{2}{3} D\right)^{2} D 3 p^{1} F_{3}$ & & 3626728 & 4.679E-10 & $4.658 \mathrm{E}-10$ & $0.92+0.042 s^{2} 2 p^{3}\left({ }_{1}^{2} P\right)^{2} P 3 p^{3} D+0.022 s^{2} 2 p^{3}\left({ }_{3}^{2} D\right)^{2} D 3 p^{3} F$ \\
\hline 20 & 35 & $2 s^{2} 2 p^{3}\left(\frac{3}{3} D\right)^{2} D 3 p^{3} P_{1}$ & & 3659719 & 3.603E-11 & $3.630 \mathrm{E}-11$ & $0.38+0.412 s^{2} 2 p^{3}\left({ }_{1}^{2} P\right)^{2} P 3 p^{3} S+0.082 s^{2} 2 p^{3}\left({ }_{3}^{4} S\right)^{4} S 3 p^{3} P$ \\
\hline 20 & 36 & $\left.2 s^{2} 2 p^{3}\left({ }_{3}^{3} D\right)\right)^{2} D 3 p^{3} P_{0}$ & & 3663380 & $3.811 \mathrm{E}-11$ & 3.834E-11 & $0.75+0.172 s^{2} 2 p^{3}\left({ }_{1}^{2} P\right){ }^{2} P 3 p^{3} P+0.042 s^{2} 2 p^{3}\left({ }_{3}^{4} S\right)^{4} S 3 p^{3} P$ \\
\hline 20 & 37 & $2 s^{2} 2 p^{3}\left(\frac{3}{3} D\right){ }^{2} D 3 p^{3} P_{2}$ & & 3671601 & $2.566 \mathrm{E}-11$ & $2.582 \mathrm{E}-11$ & $0.80+0.122 s^{2} 2 p^{3}\left({ }_{3}^{4} S\right)^{4} S 3 p^{3} P+0.032 s^{2} 2 p^{3}\left({ }_{1}^{2} P\right)^{2} P 3 p^{1} D$ \\
\hline 20 & 38 & $2 s^{2} 2 p^{3}\left({ }_{1}^{3} P\right)^{2} P 3 p^{3} D_{1}$ & & 3680279 & $9.648 \mathrm{E}-11$ & $9.676 \mathrm{E}-11$ & $0.74+0.132 s^{2} 2 p^{3}\left({ }_{1}^{2} P\right)^{2} P 3 p^{1} P+0.052 s^{2} 2 p^{3}\left({ }_{3}^{2} D\right){ }^{2} D 3 p^{3} D$ \\
\hline 20 & 39 & $2 s^{2} 2 p^{3}\left({ }_{1}^{2} P\right)^{2} P 3 p^{3} D_{2}$ & & 3683660 & $8.856 \mathrm{E}-11$ & $8.885 \mathrm{E}-11$ & $0.60+0.192 s^{2} 2 p^{3}\left(\frac{1}{3} D\right)^{2} D 3 p^{1} D+0.072 s^{2} 2 p^{3}\left({ }_{3}^{2} D\right)^{2} D 3 p^{3} F$ \\
\hline 20 & 40 & $2 s^{2} 2 p^{3}\left({ }_{1}^{2} P\right)^{2} P 3 p^{3} s_{1}$ & & 3684805 & $3.835 \mathrm{E}-11$ & 3.863E-11 & $0.48+0.342 s^{2} 2 p^{3}\left({ }_{3}^{2} D\right)^{2} D 3 p^{3} P+0.082 s^{2} 2 p^{3}\left(_{1}^{2} P\right)^{2} P 3 p^{3} P$ \\
\hline 20 & 41 & $2 s^{2} 2 p^{3}\left(\frac{2}{3} D\right)^{2} D 3 p^{1} D_{2}$ & & 3690981 & $9.744 \mathrm{E}-11$ & $9.780 \mathrm{E}-11$ & $0.32+0.252 s^{2} 2 p^{3}\left({ }_{1}^{2} P\right)^{2} P 3 p^{3} D+0.232 s^{2} 2 p^{3}\left({ }_{1}^{2} P\right)^{2} P 3 p^{3} P$ \\
\hline 20 & 42 & $2 s^{2} 2 p^{3}\left({ }_{1}^{2} P\right)^{2} P 3 p^{3} D_{3}$ & & 3693254 & $1.130 \mathrm{E}-10$ & $1.131 \mathrm{E}-10$ & $0.84+0.062 s^{2} 2 p^{3}\left(\frac{1}{3} D\right)^{2} D 3 p^{1} F+0.062 s^{2} 2 p^{3}\left({ }_{3}^{2} D\right)^{2} D 3 p^{3} F$ \\
\hline 20 & 43 & $2 s^{2} 2 p^{3}\left({ }_{3}^{4} S\right)^{4} S 3 d^{5} D_{0}^{\circ}$ & & 3700051 & $5.450 \mathrm{E}-11$ & $5.417 \mathrm{E}-11$ & 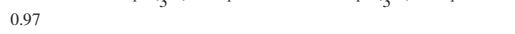 \\
\hline 20 & 44 & $2 s^{2} 2 p^{3}\left({ }_{3}^{4} S\right)^{4} S 3 d^{5} D_{1}^{\circ}$ & & 3700061 & 5.075E-11 & 5.051E-11 & 0.97 \\
\hline 20 & 45 & $2 s^{2} 2 p^{3}\left({ }_{3}^{4} S\right){ }^{4} S 3 d^{5} D_{2}^{\circ}$ & & 3700061 & 6.653E-11 & 6.632E-11 & 0.97 \\
\hline 20 & 46 & $2 s^{2} 2 p^{3}\left({ }_{3}^{4} S\right)^{4} S 3 d^{5} D_{3}^{\circ}$ & & 3700086 & $2.096 \mathrm{E}-10$ & 2.101E-10 & 0.96 \\
\hline 20 & 47 & $2 s^{2} 2 p^{3}\left({ }_{3}^{4} S\right){ }^{4} S 3 d^{5} D_{4}^{\circ}$ & & 3700497 & $3.190 \mathrm{E}-10$ & 3.178E-10 & 0.97 \\
\hline 20 & 48 & $2 s^{2} 2 p^{3}\left({ }_{1}^{2} P\right)^{2} P 3 p p^{1} P_{1}^{4}$ & & 3700572 & $6.891 \mathrm{E}-11$ & $6.928 \mathrm{E}-11$ & $0.57+0.112 s^{2} 2 p^{3}\left({ }_{3}^{2} D\right)^{2} D 3 p^{3} P+0.102 s^{2} 2 p^{3}\left({ }_{1}^{2} P\right)^{2} P 3 p^{3} P$ \\
\hline 20 & 49 & $2 s^{2} 2 p^{3}\left({ }_{1}^{2} P\right)^{2} P 3 p^{3} P_{0}$ & & 3715488 & $5.570 \mathrm{E}-11$ & $5.608 \mathrm{E}-11$ & $0.74+0.102 s^{2} 2 p^{3}\left(\frac{2}{3} D\right)^{2} D 3 p^{3} P+0.082 s^{2} 2 p^{3}\left(\frac{4}{3} S\right)^{4} S 3 p^{3} P$ \\
\hline 20 & 50 & $2 s^{2} 2 p^{3}\left({ }_{1}^{2} P\right)^{2} P 3 p^{3} P_{2}$ & & 3715974 & $1.769 \mathrm{E}-10$ & $1.777 \mathrm{E}-10$ & $0.61+0.212 s^{2} 2 p^{3}\left({ }_{1}^{2} P\right)^{2} P 3 p^{1} D+0.042 s^{2} 2 p^{3}\left({ }_{3}^{3} D\right)^{2} D 3 p^{1} D$ \\
\hline 20 & 51 & $2 s^{2} 2 p^{3}\left({ }_{1}^{2} P\right)^{2} P 3 p^{3} P_{1}$ & & 3716067 & $8.377 \mathrm{E}-11$ & 8.429E-11 & $0.69+0.092 s^{2} 2 p^{3}\left({ }_{1}^{2} P\right){ }^{2} P 3 p^{1} P+0.072 s^{2} 2 p^{3}\left({ }_{3}^{4} S\right)^{4} S 3 p^{3} P$ \\
\hline 20 & 52 & $2 s^{2} 2 p^{3}\left({ }_{3}^{4} S\right)^{4} S 3 d^{3} D_{2}^{\circ}$ & 3739000 & 3739430 & $7.871 \mathrm{E}-13$ & $7.862 \mathrm{E}-13$ & $\left.0.80+0.112 s^{2} 2 p^{3}\left(\frac{1}{2} D\right)^{2} D 3 d^{3} D^{\circ}+0.022 s^{2} 2 p^{3}{ }_{1}^{2} P\right)^{2} P 3 d^{1} D^{\circ}$ \\
\hline 20 & 53 & $2 s^{2} 2 p^{3}\left({ }_{3}^{4} S\right)^{4} S 3 d^{3} D_{1}^{\circ}$ & & 3742300 & 7.007E-13 & $7.000 \mathrm{E}-13$ & $0.85+0.092 s^{2} 2 p^{3}\left({ }_{3}^{2} D\right)^{2} D 3 d^{3} D^{\circ}+0.032 s^{2} 2 p^{3}\left({ }_{1}^{2} P\right)^{2} P 3 d^{3} D^{\circ}$ \\
\hline 20 & 54 & $2 s^{2} 2 p^{3}\left({ }_{3}^{4} S\right)^{4} S 3 d^{3} D_{3}^{\circ}$ & 3743000 & 3743244 & $6.824 \mathrm{E}-13$ & $6.812 \mathrm{E}-13$ & $0.83+0.112 s^{2} 2 p^{3}\left({ }_{3}^{2} D\right)^{2} D 3 d^{3} D^{\circ}$ \\
\hline 20 & 55 & $2 s^{2} 2 p^{3}\left({ }_{1}^{2} P\right)^{2} P 3 p^{1} D_{2}$ & & 3743648 & $1.345 \mathrm{E}-10$ & $1.357 \mathrm{E}-10$ & $0.46+0.432 s^{2} 2 p^{3}\left({ }_{3}^{2} D\right)^{2} D 3 p p^{1} D+0.052 s^{2} 2 p^{3}\left({ }_{1}^{2} P\right)^{2} P 3 p^{3} P$ \\
\hline 20 & 56 & $2 s^{2} 2 p^{3}\left(\frac{1}{3} D\right)^{2} D 3 d^{3} F_{2}^{\circ}$ & & 3801577 & $9.460 \mathrm{E}-12$ & $9.434 \mathrm{E}-12$ & $0.80+0.112 s^{2} 2 p^{3}\left({ }_{1}^{2} P\right)^{2} P 3 d^{3} F^{\circ}+0.022 s^{2} 2 p^{3}\left({ }_{1}^{2} P\right)^{2} P 3 d^{1} D^{\circ}$ \\
\hline 20 & 57 & $2 s^{2} 2 p^{3}\left({ }_{1}^{2} P\right)^{2} P 3 p p^{1} s_{0}$ & & 3804402 & $2.246 \mathrm{E}-11$ & $2.258 \mathrm{E}-11$ & $0.92+0.032 s^{2} 2 p^{3}\left({ }_{3}^{2} D\right)^{2} D 3 p^{3} P$ \\
\hline 20 & 58 & $2 s^{2} 2 p^{3}\left(\frac{1}{3} D\right)^{2} D 3 d^{3} F_{3}^{\circ}$ & & 3806445 & 8.094E-12 & $8.089 \mathrm{E}-12$ & $0.80+0.102 s^{2} 2 p^{3}\left({ }_{1}^{2} P\right)^{2} P 3 d^{3} F^{\circ}+0.072 s^{2} 2 p^{3}\left({ }_{3}^{2} D\right)^{2} D 3 d^{3} G^{\circ}$ \\
\hline 20 & 59 & $2 s^{2} 2 p^{3}\left({ }_{3}^{2} D\right)^{2} D 3 d^{1} s_{0}^{\circ}$ & & 3808898 & $4.996 \mathrm{E}-11$ & $5.021 \mathrm{E}-11$ & $0.95+0.042 s^{2} 2 p^{3}\left({ }_{1}^{2} P\right)^{2} P 3 d^{3} P^{\circ}$ \\
\hline 20 & 60 & $2 s^{2} 2 p^{3}\left(\frac{2}{2} D\right)^{2} D 3 d^{3} F_{4}^{\circ}$ & & 3812061 & $3.351 \mathrm{E}-10$ & $3.328 \mathrm{E}-10$ & $0.65+0.192 s^{2} 2 p^{3}\left({ }_{3}^{2} D\right)^{2} D 3 d^{3} G^{\circ}+0.082 s^{2} 2 p^{3}\left({ }_{1}^{2} P\right)^{2} P 3 d^{3} F^{\circ}$ \\
\hline 20 & 61 & $2 s^{2} 2 p^{3}\left(\frac{3}{3} D\right)^{2} D 3 d^{3} G_{3}^{\circ}$ & & 3818597 & $1.243 \mathrm{E}-11$ & $1.242 \mathrm{E}-11$ & $\left.0.84+0.092 s^{2} 2 p^{3}\left(\frac{2}{3} D\right)\right)^{2} D 3 d^{3} F^{\circ}+0.042 s^{2} 2 p^{3}\left({ }_{1}^{2} P\right)^{2} P 3 d^{1} F^{\circ}$ \\
\hline 20 & 62 & $2 s^{2} 2 p^{3}\left({ }_{3}^{2} D\right)^{2} D 3 d^{3} G_{4}^{\circ}$ & & 3821091 & $2.996 \mathrm{E}-10$ & $2.990 \mathrm{E}-10$ & $0.60+0.302 s^{2} 2 p^{3}\left(\frac{2}{2} D\right)^{2} D 3 d^{3} F^{\circ}+0.052 s^{2} 2 p^{3}\left(\frac{1}{2} D\right)^{2} D 3 d^{1} G^{\circ}$ \\
\hline 20 & 63 & $2 s^{2} 2 p^{3}\left({ }_{3}^{3} D\right)^{2} D 3 d^{3} G_{5}^{\mathrm{o}}$ & & 3824256 & 3.055E-10 & 3.037E-10 & $0.99+3$ \\
\hline 20 & 64 & $2 s^{2} 2 p^{3}\left(\frac{2}{3} D\right)^{2} D 3 d^{1} G_{4}^{\mathrm{o}}$ & & 3826822 & $2.940 \mathrm{E}-10$ & $2.928 \mathrm{E}-10$ & $0.83+0.142 s^{2} 2 p^{3}\left({ }_{3}^{2} D\right)^{2} D 3 d^{3} G^{\circ}$ \\
\hline 20 & 65 & $2 s^{2} 2 p^{3}\left(\frac{2}{3} D\right)^{2} D 3 d^{1} P_{1}^{\circ}$ & 3828000 & 3826970 & 4.643E-13 & $4.639 \mathrm{E}-13$ & $0.41+0.382 s^{2} 2 p^{3}\left({ }_{3}^{2} D\right)^{2} D 3 d^{3} D^{\circ}+0.052 s^{2} 2 p^{3}\left({ }_{1}^{2} P\right)^{2} P 3 d^{3} D^{\circ}$ \\
\hline 20 & 66 & $2 s^{2} 2 p^{3}\left({ }_{3}^{2} D\right)^{2} D 3 d^{3} D_{2}^{\circ}$ & 3838000 & 3839057 & 3.485E-13 & 3.482E-13 & $0.68+0.102 s^{2} 2 p^{3}\left({ }_{3}^{4} S\right)^{4} S 3 d^{3} D^{\circ}+0.092 s^{2} 2 p^{3}\left({ }_{3}^{2} D\right)^{2} D 3 d^{3} P^{\circ}$ \\
\hline 20 & 67 & $2 s^{2} 2 p^{3}\left(\frac{2}{3} D\right)^{2} D 3 d^{3} D_{1}^{\circ}$ & & 3841415 & $4.351 \mathrm{E}-13$ & $4.349 \mathrm{E}-13$ & $0.45+0.452 s^{2} 2 p^{3}\left({ }_{3}^{2} D\right)^{2} D 3 d^{1} P^{\circ}+0.042 s^{2} 2 p^{3}\left({ }_{3}^{4} S\right)^{4} S 3 d^{3} D^{\circ}$ \\
\hline 20 & 68 & $2 s^{2} 2 p^{3}\left(\frac{3}{3} D\right)^{2} D 3 d^{3} D_{3}^{\circ}$ & 3841000 & 3842444 & $2.448 \mathrm{E}-13$ & $2.445 \mathrm{E}-13$ & $0.84+0.112 s^{2} 2 p^{3}\left({ }_{3}^{4} S\right)^{4} S 3 d^{3} D^{\circ}$ \\
\hline 20 & 69 & $2 s^{2} 2 p^{3}\left(\frac{3}{3} D\right)^{2} D 3 d^{3} P_{2}^{\circ}$ & & 3849310 & $2.028 \mathrm{E}-13$ & $2.026 \mathrm{E}-13$ & $\left.0.57+0.132 s^{2} 2 p^{3}\left({ }_{1}^{2} P\right)\right)^{2} P 3 d^{3} P^{\circ}+0.112 s^{2} 2 p^{3}\left({ }_{3}^{2} D\right)^{2} D 3 d^{1} D^{\circ}$ \\
\hline 20 & 70 & $2 s^{2} 2 p^{3}\left({ }_{3}^{2} D\right)^{2} D 3 d^{1} D_{2}^{\circ}$ & & 3852997 & $5.964 \mathrm{E}-13$ & $5.961 \mathrm{E}-13$ & $\left.0.45+0.292 s^{2} 2 p^{3}{ }_{1}^{\frac{1}{2}} P\right)^{2} P 3 d^{1} D^{\circ}+0.152 s^{2} 2 p^{3}\left(\frac{2}{2} D\right)^{2} D 3 d^{3} P^{\circ}$ \\
\hline 20 & 71 & $2 s^{2} 2 p^{3}\left(\frac{2}{3} D\right)^{2} D 3 d^{3} P_{0}^{\circ}$ & & 3854435 & $3.870 \mathrm{E}-13$ & 3.867E-13 & $\left.0.90+0.072 s^{2} 2 p^{3}{ }_{1}^{2} P\right)^{2} P 3 d^{3} P^{\circ}$ \\
\hline 20 & 72 & $2 s^{2} 2 p^{3}\left(\frac{2}{3} D\right)^{2} D 3 d^{3} P_{1}^{\circ}$ & 3852000 & 3855862 & $2.980 \mathrm{E}-13$ & $2.978 \mathrm{E}-13$ & $0.91+0.022 s^{2} 2 p^{3}\left({ }_{1}^{2} P\right)^{2} P 3 d^{3} P^{\circ}$ \\
\hline 20 & 73 & $2 s^{2} 2 p^{3}\left(\frac{2}{3} D\right)^{2} D 3 d^{3} S_{1}^{\circ}$ & 3864000 & 3863490 & 2.093E-13 & 2.091E-13 & $0.79+0.132 s^{2} 2 p^{3}\left({ }_{1}^{2} P\right)^{2} P 3 d^{3} P^{\circ}+0.032 s^{2} 2 p^{3}\left({ }_{3}^{2} D\right)^{2} D 3 d^{3} P^{\circ}$ \\
\hline 20 & 74 & $2 s^{2} S 2 p^{4}\left({ }_{2}^{3} P\right){ }^{4} P 3 s^{5} P_{3}$ & & 3864735 & $7.762 \mathrm{E}-11$ & $7.744 \mathrm{E}-11$ & 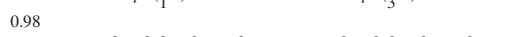 \\
\hline 20 & 75 & $2 s^{2} 2 p^{3}\left({ }_{3}^{2} D\right)^{2} D 3 d^{1} F_{3}^{\circ}$ & & 3879217 & 3.433E-13 & $3.428 \mathrm{E}-13$ & $0.66+0.152 s^{2} 2 p^{3}\left({ }_{1}^{2} P\right)^{2} P 3 d^{3} F^{\circ}+0.062 s^{2} 2 p^{3}\left({ }_{1}^{2} P\right)^{2} P 3 d^{3} D^{\circ}$ \\
\hline 20 & 76 & $2 s^{2} S 2 p^{4}\left({ }_{2}^{3} P\right)^{4} P 3 s^{5} P_{2}$ & & 3881495 & 3.614E-11 & 3.623E-11 & $0.96+0.022 s^{2} S 2 p^{4}\left({ }_{2}^{3} P\right)^{4} P 3 s^{3} P$ \\
\hline 20 & 77 & $2 s^{2} 2 p^{3}\left({ }_{1}^{2} P\right)^{2} P 3 d^{3} F_{2}^{\circ}$ & 3890000 & 3888785 & 3.563E-12 & $3.558 \mathrm{E}-12$ & $0.77+0.102 s^{2} 2 p^{3}\left({ }_{3}^{2} D\right)^{2} D 3 d^{3} F^{\circ}+0.042 s^{2} 2 p^{3}\left({ }_{1}^{2} P\right)^{2} P 3 d^{3} D^{\circ}$ \\
\hline 20 & 78 & $2 s^{2} 2 p^{3}\left({ }_{1}^{2} P\right){ }^{2} P 3 d^{3} F_{3}^{\circ}$ & & 3889718 & $1.699 \mathrm{E}-12$ & $1.696 \mathrm{E}-12$ & $0.63+0.202 s^{2} 2 p^{3}\left({ }_{1}^{2} P\right)^{2} P 3 d^{3} D^{\circ}+0.072 s^{2} 2 p^{3}\left({ }_{3}^{\frac{1}{2}} D\right)^{2} D 3 d^{1} F^{\circ}$ \\
\hline 20 & 79 & $2 s^{2} S 2 p^{4}\left({ }_{2}^{3} P\right)^{4} P 3 s^{5} P_{1}$ & & 3891816 & 5.483E-11 & $5.511 \mathrm{E}-11$ & $0.98+1-50$ \\
\hline 20 & 80 & $2 s^{2} 2 p^{3}\left({ }_{1}^{2} P\right)^{2} P 3 d^{3} F_{4}^{\circ}$ & & 3891817 & 2.924E-10 & $2.921 \mathrm{E}-10$ & $0.83+0.062 s^{2} 2 p^{3}\left({ }_{3}^{2} D\right)^{2} D 3 d^{3} G^{\circ}+0.052 s^{2} 2 p^{3}\left({ }_{3}^{2} D\right)^{2} D 3 d^{1} G^{\circ}$ \\
\hline 20 & 81 & $2 s^{2} 2 p^{3}\left({ }_{1}^{2} P\right)^{2} P 3 d^{3} P_{0}^{\circ}$ & & 3893903 & $3.522 \mathrm{E}-13$ & $3.519 \mathrm{E}-13$ & $0.85+0.082 s^{2} 2 p^{3}\left({ }_{3}^{2} D\right)^{2} D 3 d^{3} P^{\circ}+0.032 s^{2} 2 p^{3}\left({ }_{3}^{2} D\right)^{2} D 3 d^{1} s^{\circ}$ \\
\hline 20 & 82 & $2 s^{2} 2 p^{3}\left({ }_{1}^{2} P\right)^{2} P 3 d^{3} P_{1}^{\circ}$ & 3893000 & 3897467 & $4.252 \mathrm{E}-13$ & $4.251 \mathrm{E}-13$ & $0.73+0.162 s^{2} 2 p^{3}\left(\frac{2}{3} D\right)^{2} D 3 d^{3} s^{\circ}+0.042 s^{2} 2 p^{3}\left(\frac{2}{3} D\right)^{2} D 3 d^{1} P^{\circ}$ \\
\hline 20 & 83 & $2 s^{2} 2 p^{3}\left({ }_{1}^{2} P\right)^{2} P 3 d^{3} P_{2}^{\circ}$ & & 3898064 & $1.844 \mathrm{E}-12$ & $1.842 \mathrm{E}-12$ & $0.69+0.102 s^{2} 2 p^{3}\left({ }_{3}^{2} D\right)^{2} D 3 d^{3} P^{\circ}+0.082 s^{2} 2 p^{3}\left({ }_{1}^{2} P\right)^{2} P 3 d^{3} D^{\circ}$ \\
\hline
\end{tabular}




\begin{tabular}{|c|c|c|c|c|c|c|c|}
\hline $\mathrm{z}$ & Key & Level & $E_{\mathrm{NIST}}$ & $E_{\mathrm{MCDHF} / \mathrm{RCI}}$ & $\tau_{\mathrm{MCDHF} / \mathrm{RCI}}^{l}$ & $\tau_{\mathrm{MCDHF} / \mathrm{RCI}}^{v}$ & $L S$-composition \\
\hline 20 & 84 & $2 s^{2} 2 p^{3}\left({ }_{1}^{2} P\right)^{2} P 3 d^{3} D_{2}^{\circ}$ & 3905000 & 3906800 & $3.014 \mathrm{E}-13$ & $3.010 \mathrm{E}-13$ & $0.54+0.242 s^{2} 2 p^{3}\left({ }_{1}^{2} P\right)^{2} P 3 d^{1} D^{\circ}+0.062 s^{2} 2 p^{3}\left({ }_{3}^{2} D\right)^{2} D 3 d^{3} P^{\circ}$ \\
\hline 20 & 85 & $2 s^{2} 2 p^{3}\left({ }_{1}^{2} P\right)^{2} P 3 d^{3} D_{1}^{\circ}$ & 3914000 & 3913114 & $2.143 \mathrm{E}-13$ & $2.140 \mathrm{E}-13$ & $0.82+0.052 s^{2} 2 p^{3}\left({ }_{3}^{4} S\right)^{4} S 3 d^{3} D^{\circ}+0.042 s^{2} 2 p^{3}\left({ }_{3}^{2} D\right)^{2} D 3 d^{3} D^{\circ}$ \\
\hline 20 & 86 & $2 s^{2} 2 p^{3}\left({ }_{1}^{2} P\right)^{2} P 3 d^{3} D_{3}^{\circ}$ & 3917000 & 3914887 & 8.803E-13 & $8.793 \mathrm{E}-13$ & $0.61+0.142 s^{2} 2 p^{3}\left({ }_{1}^{2} P\right)^{2} P 3 d^{1} F^{\circ}+0.092 s^{2} 2 p^{3}\left({ }_{3}^{2} D\right)^{2} D 3 d^{1} F^{\circ}$ \\
\hline 20 & 87 & $2 s^{2} S 2 p^{4}\left({ }_{2}^{3} P\right){ }^{4} P 3 s^{3} P_{2}$ & & 3924035 & $1.636 \mathrm{E}-12$ & $1.637 \mathrm{E}-12$ & $0.93+0.022 s^{2} S 2 p^{4}\left({ }_{2}^{3} P\right)^{4} P 3 s^{5} P$ \\
\hline 20 & 88 & $2 s^{2} 2 p^{3}\left({ }_{1}^{2} P\right)^{2} P 3 d^{1} D_{2}^{\circ}$ & 3920000 & 3928517 & $2.372 \mathrm{E}-13$ & $2.369 \mathrm{E}-13$ & $0.33+0.312 s^{2} 2 p^{3}\left({ }_{3}^{2} D\right)^{2} D 3 d^{1} D^{\circ}+0.232 s^{2} 2 p^{3}\left({ }_{1}^{2} P\right)^{2} P 3 d^{3} D^{\circ}$ \\
\hline 20 & 89 & $2 s^{2} 2 p^{3}\left({ }_{1}^{2} P\right)^{2} P 3 d^{1} F_{3}^{\circ}$ & 3929000 & 3930864 & $1.600 \mathrm{E}-13$ & $1.598 \mathrm{E}-13$ & $0.71+0.152 s^{2} 2 p^{3}\left({ }_{3}^{2} D\right)^{2} D 3 d^{1} F^{\circ}+0.052 s^{2} 2 p^{3}\left({ }_{1}^{2} P\right)^{2} P 3 d^{3} D^{\circ}$ \\
\hline 20 & 90 & $2 s^{2} S 2 p^{4}\left({ }_{2}^{3} P\right){ }^{4} P 3 s^{3} P_{1}$ & & 3942367 & $1.556 \mathrm{E}-12$ & $1.556 \mathrm{E}-12$ & 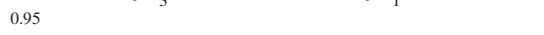 \\
\hline 20 & 91 & $2 s^{2} S 2 p^{4}\left({ }_{2}^{3} P\right){ }^{4} P 3 s^{3} P_{0}$ & & 3950025 & $1.550 \mathrm{E}-12$ & $1.551 \mathrm{E}-12$ & 0.95 \\
\hline 20 & 92 & $2 s^{2} 2 p^{3}\left({ }_{1}^{2} P\right)^{2} P 3 d^{1} P_{1}^{\circ}$ & 3969000 & 3968610 & $1.536 \mathrm{E}-13$ & $1.534 \mathrm{E}-13$ & $0.84+0.052 s^{2} 2 p^{3}\left({ }_{3}^{2} D\right)^{2} D 3 d^{1} P^{\circ}$ \\
\hline 20 & 93 & $2 s^{2} S 2 p^{4}\left({ }_{2}^{3} P\right)^{4} P 3 p^{5} P_{3}^{\circ}$ & & 4016660 & 8.031E-11 & 7.970E-11 & $0.87+0.122 s^{2} S 2 p^{4}\left({ }_{2}^{3} P\right)^{4} P 3 p^{5} D^{\circ}$ \\
\hline 20 & 94 & $2 s^{2} S 2 p^{4}\left({ }_{2}^{3} P\right)^{4} P 3 p^{5} P_{2}^{\circ}$ & & 4019601 & $4.531 \mathrm{E}-11$ & $4.511 \mathrm{E}-11$ & $0.85+0.072 s^{2} S 2 p^{4}\left({ }_{2}^{3} P\right)^{4} P 3 p^{5} D^{\circ}+0.062 s^{2} S 2 p^{4}\left({ }_{2}^{3} P\right)^{4} P 3 p^{5} S^{\circ}$ \\
\hline 20 & 95 & $2 s^{2} S 2 p^{4}\left({ }_{2}^{2} P\right)^{4} P 3 p^{5} P_{1}^{\circ}$ & & 4029736 & 4.394E-11 & 4.382E-11 & $0.93+0.042 s^{2} S 2 p^{4}\left({ }_{2}^{3} P\right)^{4} P 3 p^{5} D^{\circ}$ \\
\hline 20 & 96 & $2 s^{2} S 2 p^{4}\left({ }_{2}^{3} P\right)^{4} P 3 p^{5} D_{4}^{\circ}$ & & 4034948 & $1.084 \mathrm{E}-10$ & $1.073 \mathrm{E}-10$ & 0.98 \\
\hline 20 & 97 & $2 s^{2} S 2 p^{4}\left({ }_{2}^{2} P\right)^{4} P 3 p^{5} D_{3}^{\circ}$ & & 4043695 & 5.173E-12 & $5.163 \mathrm{E}-12$ & $0.65+0.232 s^{2} S 2 p^{4}\left({ }_{2}^{3} P\right)^{4} P 3 p^{3} D^{\circ}+0.102 s^{2} S 2 p^{4}\left({ }_{2}^{3} P\right)^{4} P 3 p^{5} P^{\circ}$ \\
\hline 20 & 98 & $2 s^{2} S 2 p^{4}\left({ }_{2}^{3} P\right)^{4} P 3 p^{5} D_{2}^{\circ}$ & & 4051119 & $1.583 \mathrm{E}-11$ & $1.580 \mathrm{E}-11$ & $0.83+0.072 s^{2} S 2 p^{4}\left({ }_{2}^{3} P\right)^{4} P 3 p^{3} D^{\circ}+0.062 s^{2} S 2 p^{4}\left({ }_{2}^{3} P\right)^{4} P 3 p^{5} P^{\circ}$ \\
\hline 20 & 99 & $2 s^{2} S 2 p^{4}\left(\frac{3}{3} P\right)^{4} P 3 p^{5} D_{1}^{\circ}$ & & 4055227 & $4.317 \mathrm{E}-11$ & 4.314E-11 & $0.92+0.042 s^{2} S 2 p^{4}\left(\frac{3}{3} P\right)^{4} P 3 p^{5} P^{\circ}$ \\
\hline 20 & 100 & $2 s^{2} S 2 p^{4}\left({ }_{2}^{3} P\right)^{4} P 3 p^{5} D_{0}^{\circ}$ & & 4056521 & $9.233 \mathrm{E}-11$ & $9.229 \mathrm{E}-11$ & 0.98 \\
\hline 20 & 101 & $2 s^{2} S 2 p^{4}\left({ }_{2}^{2} P\right){ }^{2} P 3 p^{3} D_{3}^{\circ}$ & & 4062130 & $1.466 \mathrm{E}-12$ & $1.463 \mathrm{E}-12$ & $0.75+0.212 s^{2} S 2 p^{4}\left({ }_{2}^{3} P\right){ }^{4} P 3 p^{5} D^{\circ}+0.022 s^{2} S 2 p^{4}\left({ }_{2}^{3} P\right)^{4} P 3 p^{5} P^{\circ}$ \\
\hline 20 & 102 & $2 s^{2} S 2 p^{4}\left({ }_{2}^{1} D\right)^{2} D 3 s^{3} D_{1}$ & & 4068422 & 2.423E-12 & 2.427E-12 & 0.97 \\
\hline 20 & 103 & $2 s^{2} S 2 p^{4}\left({ }_{2}^{1} D\right)^{2} D 3 s^{3} D_{2}$ & & 4069006 & $2.421 \mathrm{E}-12$ & $2.424 \mathrm{E}-12$ & 0.97 \\
\hline 20 & 104 & $2 s^{2} S 2 p^{4}\left({ }_{2}^{1} D\right)^{2} D 3 s^{3} D_{3}$ & & 4069871 & $2.372 \mathrm{E}-12$ & 2.373E-12 & 0.97 \\
\hline 20 & 105 & $2 s^{2} S 2 p^{4}\left({ }_{2}^{3} P\right)^{4} P 3 p^{3} D_{2}^{\circ}$ & & 4072732 & $1.213 \mathrm{E}-12$ & $1.210 \mathrm{E}-12$ & $0.73+0.172 s^{2} S 2 p^{4}\left({ }_{2}^{3} P\right)^{4} P 3 p^{3} P^{\circ}+0.072 s^{2} S 2 p^{4}\left({ }_{2}^{3} P\right)^{4} P 3 p^{5} D^{\circ}$ \\
\hline 20 & 106 & $2 s^{2} S 2 p^{4}\left(3_{2}^{3} P\right)^{4} P 3 p^{3} s_{1}^{\circ}$ & & 4073636 & 8.935E-13 & $8.921 \mathrm{E}-13$ & $0.67+0.222 s^{2} S 2 p^{4}\left({ }_{2}^{3} P\right)^{4} P 3 p^{3} P^{\circ}+0.052 s^{2} S 2 p^{4}\left(_{2}^{3} P\right)^{4} P 3 p^{3} D^{\circ}$ \\
\hline 20 & 107 & $2 s^{2} S 2 p^{4}\left({ }_{2}^{2} P\right)^{4} P 3 p^{5} S_{2}^{1}$ & & 4076425 & $6.299 \mathrm{E}-11$ & $6.313 \mathrm{E}-11$ & $0.90+0.072 s^{2} S 2 p^{4}\left({ }_{2}^{3} P\right)^{4} P 3 p^{5} P^{\circ}$ \\
\hline 20 & 108 & $2 s^{2} S 2 p^{4}\left({ }_{2}^{3} P\right)^{4} P 3 p^{3} D_{1}^{\circ}$ & & 4084621 & $1.161 \mathrm{E}-12$ & $1.159 \mathrm{E}-12$ & $0.86+0.102 s^{2} S 2 p^{4}\left({ }_{2}^{3} P\right)^{4} P 3 p^{3} S^{\circ}$ \\
\hline 20 & 109 & $\left.2 s^{2} S 2 p^{4}{ }_{2}^{3} P\right)^{4} P 3 p^{3} P_{2}^{\circ}$ & & 4090624 & 1.049E-12 & $1.047 \mathrm{E}-12$ & $0.69+0.182 s^{2} S 2 p^{4}\left({ }_{2}^{3} P\right)^{4} P 3 p^{3} D^{\circ}+0.082 s^{2} S 2 p^{4}\left({ }_{2}^{1} D\right)^{2} D 3 p^{3} P^{\circ}$ \\
\hline 20 & 110 & $2 s^{2} S 2 p^{4}\left({ }_{2}^{2} P\right){ }^{4} P 3 p^{3} P_{0}^{\circ}$ & & 4094251 & 1.147E-12 & $1.145 \mathrm{E}-12$ & $0.83+0.142 s^{2} S 2 p^{4}\left({ }_{2}^{1} D\right)^{2} D 3 p^{3} P^{\circ}$ \\
\hline 20 & 111 & $\left.2 s^{2} S 2 p^{4}{ }_{2}^{3} P\right)^{4} P 3 p^{3} P_{1}^{\circ}$ & & 4098874 & 1.007E-12 & $1.005 \mathrm{E}-12$ & $0.61+0.222 s^{2} S 2 p^{4}\left({ }_{2}^{3} P\right)^{4} P 3 p^{3} S^{\circ}+0.082 s^{2} S 2 p^{4}\left({ }_{2}^{1} D\right)^{2} D 3 p^{3} P^{\circ}$ \\
\hline 20 & 112 & $2 s^{2} S 2 p^{4}\left({ }_{2}^{1} D\right)^{2} D 3 s^{1} D_{2}$ & & 4106327 & $4.320 \mathrm{E}-12$ & $4.323 \mathrm{E}-12$ & $0.97+2+2+2$ \\
\hline 20 & 113 & $2 s^{2} S 2 p^{4}\left(1_{0}^{1} S\right)^{2} S 3 s^{3} S_{1}$ & & 4183168 & $2.269 \mathrm{E}-12$ & $2.271 \mathrm{E}-12$ & $0.88+0.062 s^{2} S 2 p^{4}\left({ }_{2}^{3} P\right)^{2} P 3 s^{3} P$ \\
\hline 20 & 114 & $2 s^{2} S 2 p^{4}\left({ }_{2}^{3} P\right)^{4} P 3 d^{5} D_{4}$ & & 4207489 & 9.525E-11 & $9.600 \mathrm{E}-11$ & $0.90+0.092 s^{2} S 2 p^{4}\left({ }_{2}^{2} P\right)^{4} P 3 d^{5} F$ \\
\hline 20 & 115 & $2 s^{2} S 2 p^{4}\left({ }_{2}^{2} P\right)^{4} P 3 d^{5} D_{3}$ & & 4208634 & $9.155 \mathrm{E}-11$ & $9.255 \mathrm{E}-11$ & $0.89+0.062 s^{2} S 2 p^{4}\left({ }_{2}^{3} P\right)^{4} P 3 d^{5} F+0.042 s^{2} S 2 p^{4}\left({ }_{2}^{3} P\right)^{4} P 3 d^{5} P$ \\
\hline 20 & 116 & $2 s^{2} S 2 p^{4}\left(1_{0}^{1} S\right)^{2} S 3 s^{1} s_{0}$ & & 4211026 & $3.074 \mathrm{E}-12$ & $3.077 \mathrm{E}-12$ & $0.67+0.312 s^{2} S 2 p^{4}\left({ }_{2}^{3} P\right)^{2} P 3 s^{3} P$ \\
\hline 20 & 117 & $2 s^{2} S 2 p^{4}\left({ }_{2}^{3} P\right)^{4} P 3 d^{5} D_{2}$ & & 4211814 & 8.433E-11 & 8.537E-11 & $0.89+0.062 s^{2} S 2 p^{4}\left({ }_{2}^{3} P\right)^{4} P 3 d^{5} P+0.032 s^{2} S 2 p^{4}\left({ }_{2}^{3} P\right)^{4} P 3 d^{5} F$ \\
\hline 20 & 118 & $2 s^{2} S 2 p^{4}\left({ }_{2}^{3} P\right){ }^{4} P 3 d^{5} D_{1}$ & & 4215814 & $6.893 \mathrm{E}-11$ & $6.977 \mathrm{E}-11$ & $\left.0.92+0.052 s^{2} S 2 p^{4}{ }_{2}^{2} P\right)^{4} P 3 d^{5} P$ \\
\hline 20 & 119 & $2 s^{2} S 2 p^{4}\left({ }_{2}^{3} P\right)^{2} P 3 s^{3} P_{2}$ & & 4217807 & $3.402 \mathrm{E}-12$ & $3.406 \mathrm{E}-12$ & 0.96 \\
\hline 20 & 120 & $2 s^{2} S 2 p^{4}\left({ }_{2}^{3} P\right){ }^{4} P 3 d^{5} D_{0}$ & & 4219075 & $5.899 \mathrm{E}-11$ & $5.966 \mathrm{E}-11$ & 0.98 \\
\hline 20 & 121 & $2 s^{2} S 2 p^{4}\left(\frac{1}{2} D\right)^{2} D 3 p^{3} F_{2}^{\circ}$ & & 4221149 & $2.902 \mathrm{E}-11$ & 2.897E-11 & 0.97 \\
\hline 20 & 122 & $2 s^{2} S 2 p^{4}\left({ }_{2}^{1} D\right)^{2} D 3 p^{3} F_{3}^{\circ}$ & & 4223987 & $1.885 \mathrm{E}-11$ & $1.882 \mathrm{E}-11$ & 0.95 \\
\hline 20 & 123 & $2 s^{2} S 2 p^{4}\left(3_{2}^{3} P\right)^{4} P 3 d^{5} F_{5}{ }^{3}$ & & 4225683 & $1.589 \mathrm{E}-10$ & $1.521 \mathrm{E}-10$ & 0.99 \\
\hline 20 & 124 & $2 s^{2} S 2 p^{4}\left({ }_{2}^{3} P\right)^{2} P 3 s^{3} P_{1}$ & & 4225880 & $1.660 \mathrm{E}-12$ & $1.662 \mathrm{E}-12$ & $0.56+0.392 s^{2} S 2 p^{4}\left({ }_{2}^{3} P\right)^{2} P 3 s^{1} P$ \\
\hline 20 & 125 & $2 s^{2} S 2 p^{4}\left({ }_{2}^{1} D\right)^{2} D 3 p p^{1} P_{1}^{\circ}$ & & 4227823 & $7.872 \mathrm{E}-13$ & $7.859 \mathrm{E}-13$ & $\left.0.75+0.102 s^{2} S 2 p^{4}\left({ }_{2}^{1} D\right)^{2} D 3 p^{3} D^{\circ}+0.072 s^{2} S 2 p^{4}{ }_{2}^{3} P\right)^{2} P 3 p^{1} P^{\circ}$ \\
\hline 20 & 126 & $2 s^{2} S 2 p^{4}\left({ }_{2}^{1} D\right)^{2} D 3 p^{3} F_{4}^{\circ}$ & & 4228622 & $4.419 \mathrm{E}-11$ & 4.395E-11 & 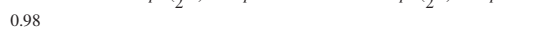 \\
\hline 20 & 127 & $2 s^{2} S 2 p^{4}\left({ }_{2}^{3} P\right)^{4} P 3 d^{5} F_{4}^{4}$ & & 4236752 & $1.398 \mathrm{E}-10$ & $1.356 \mathrm{E}-10$ & $0.85+0.082 s^{2} S 2 p^{4}\left({ }_{2}^{3} P\right)^{4} P 3 d^{5} D+0.062 s^{2} S 2 p^{4}\left({ }_{2}^{3} P\right){ }^{4} P 3 d^{3} F$ \\
\hline 20 & 128 & $2 s^{2} S 2 p^{4}\left({ }_{2}^{1} D\right)^{2} D 3 p^{1} F_{3}^{\circ}$ & & 4240307 & 8.677E-13 & 8.657E-13 & $0.90+0.062 s^{2} S 2 p^{4}\left({ }_{2}^{1} D\right)^{2} D 3 p^{3} D^{\circ}+0.022 s^{2} S 2 p^{4}\left({ }_{2}^{1} D\right)^{2} D 3 p^{3} F^{\circ}$ \\
\hline 20 & 129 & $2 s^{2} S 2 p^{4}\left(3_{2}^{3} P\right)^{4} P 3 d^{5} F_{3}$ & & 4243820 & $3.068 \mathrm{E}-11$ & $3.046 \mathrm{E}-11$ & $0.89+0.052 s^{2} S 2 p^{4}\left(_{3}^{3} P\right)^{4} P 3 d^{5} D+0.032 s^{2} S 2 p^{4}\left({ }_{2}^{3} P\right)^{4} P 3 d^{3} F$ \\
\hline 20 & 130 & $2 s^{2} S 2 p^{4}\left({ }_{2}^{3} P\right){ }^{4} P 3 d^{5} P_{1}$ & & 4245887 & $2.048 \mathrm{E}-11$ & 2.074E-11 & $0.92+0.042 s^{2} S 2 p^{4}\left(\int_{2}^{3} P\right)^{4} P 3 d^{5} D+0.022 s^{2} S 2 p^{4}\left({ }_{2}^{3} P\right){ }^{4} P 3 d^{5} F$ \\
\hline 20 & 131 & $2 s^{2} S 2 p^{4}\left({ }_{2}^{1} D\right)^{2} D 3 p^{3} D_{2}^{\circ}$ & & 4247783 & $1.974 \mathrm{E}-11$ & 1.971E-11 & $0.95+25$ \\
\hline 20 & 132 & $2 s^{2} S 2 p^{4}\left({ }_{2}^{3} P\right)^{4} P 3 d^{5} F_{2}{ }^{2}$ & & 4248240 & 2.313E-11 & $2.304 \mathrm{E}-11$ & $0.86+0.112 s^{2} S 2 p^{4}\left({ }_{2}^{3} P\right){ }^{4} P 3 d^{5} P$ \\
\hline 20 & 133 & $2 s^{2} S 2 p^{4}\left({ }_{2}^{1} D\right)^{2} D 3 p^{3} D_{1}^{\circ}$ & & 4248910 & $5.244 \mathrm{E}-12$ & $5.234 \mathrm{E}-12$ & $0.88+0.102 s^{2} S 2 p^{4}\left({ }_{2}^{1} D\right)^{2} D 3 p p^{1} P^{\circ}$ \\
\hline 20 & 134 & $2 s^{2} S 2 p^{4}\left({ }_{2}^{3} P\right)^{2} P 3 s^{1} P_{1}$ & & 4250620 & $1.283 \mathrm{E}-12$ & $1.285 \mathrm{E}-12$ & $\left.0.55+0.352 s^{2} S 2 p^{4}{ }_{2}^{3} P\right)^{2} P 3 s^{3} P+0.072 s^{2} S 2 p^{4}\left({ }_{0}^{1} S\right)^{2} S 3 s^{3} S$ \\
\hline 20 & 135 & $2 s^{2} S 2 p^{4}\left({ }_{2}^{1} D\right)^{2} D 3 p^{3} D_{3}^{\circ}$ & & 4250955 & 9.403E-12 & $9.378 \mathrm{E}-12$ & $0.91+0.062 s^{2} S 2 p^{4}\left(\frac{1}{2} D\right)^{2} D 3 p^{1} F^{\circ}$ \\
\hline 20 & 136 & $2 s^{2} S 2 p^{4}\left({ }_{3}^{3} P\right)^{2} P 3 s^{3} P_{0}{ }^{3}$ & & 4251655 & $3.546 \mathrm{E}-12$ & $3.549 \mathrm{E}-12$ & $0.67+0.302 s^{2} S 2 p^{4}\left({ }_{0}^{1} S\right)^{2} S 3 s^{1} S$ \\
\hline 20 & 137 & $2 s^{2} S 2 p^{4}\left({ }_{2}^{3} P\right)^{4} P 3 d^{5} F_{1}$ & & 4251675 & 3.730E-11 & 3.698E-11 & $0.95+0.022 s^{2} S 2 p^{4}\left({ }_{2}^{3} P\right)^{4} P 3 d^{5} D$ \\
\hline 20 & 138 & $2 s^{2} S 2 p^{4}\left({ }_{2}^{3} P\right){ }^{4} P 3 d d^{5} P_{2}$ & & 4253487 & $2.728 \mathrm{E}-11$ & $2.778 \mathrm{E}-11$ & $0.82+0.082 s^{2} S 2 p^{4}\left(_{2}^{3} P\right)^{4} P 3 d^{5} D+0.082 s^{2} S 2 p^{4}\left({ }_{2}^{3} P\right){ }^{4} P 3 d^{5} F$ \\
\hline 20 & 139 & $2 s^{2} S 2 p^{4}\left({ }_{2}^{1} D\right)^{2} D 3 p^{1} D_{2}^{\circ}$ & & 4260053 & $1.211 \mathrm{E}-12$ & $1.207 \mathrm{E}-12$ & $0.54+0.392 s^{2} S 2 p^{4}\left({ }_{2}^{1} D\right)^{2} D 3 p^{3} P^{\circ}+0.032 s^{2} S 2 p^{4}\left({ }_{2}^{3} P\right)^{2} P 3 p^{3} P^{\circ}$ \\
\hline 20 & 140 & $2 s^{2} S 2 p^{4}\left({ }_{2}^{2} P\right)^{4} P 3 d^{5} P_{3}{ }^{2}$ & & 4261153 & $1.576 \mathrm{E}-11$ & $1.594 \mathrm{E}-11$ & $\left.0.91+0.052 s^{2} S 2 p^{4}{ }_{2}^{3} P\right)^{4} P 3 d^{5} D$ \\
\hline 20 & 141 & $2 s^{2} S 2 p^{4}\left({ }_{2}^{3} P\right){ }^{4} P 3 d^{3} F_{4}$ & & 4262329 & $1.226 \mathrm{E}-10$ & $1.196 \mathrm{E}-10$ & $0.92+0.052 s^{2} S 2 p^{4}\left({ }_{2}^{3} P\right)^{4} P 3 d^{5} F$ \\
\hline 20 & 142 & $2 s^{2} S 2 p^{4}\left({ }_{2}^{1} D\right)^{2} D 3 p^{3} P_{1}^{\circ}$ & & 4265161 & 3.092E-12 & 3.079E-12 & $0.78+0.112 s^{2} S 2 p^{4}\left({ }_{2}^{3} P\right)^{4} P 3 p^{3} P^{\circ}+0.072 s^{2} S 2 p^{4}\left({ }_{2}^{3} P\right)^{2} P 3 p^{3} P^{\circ}$ \\
\hline 20 & 143 & $2 s^{2} S 2 p^{4}\left({ }_{2}^{1} D\right)^{2} D 3 p^{3} P_{2}^{\circ}$ & & 4265291 & $1.314 \mathrm{E}-12$ & $1.310 \mathrm{E}-12$ & $\left.0.46+0.442 s^{2} S 2 p^{4}\left({ }_{2}^{1} D\right)^{2} D 3 p^{1} D^{\circ}+0.072 s^{2} S 2 p^{4}{ }_{2}^{2} P\right)^{4} P 3 p^{3} P^{\circ}$ \\
\hline 20 & 144 & $2 s^{2} S 2 p^{4}\left({ }_{2}^{1} D\right)^{2} D 3 p^{3} P_{0}^{\circ}$ & & 4267024 & $2.879 \mathrm{E}-12$ & 2.867E-12 & $\left.0.77+0.122 s^{2} S 2 p^{4}{ }_{2}^{3} P\right)^{4} P 3 p^{3} P^{\circ}+0.092 s^{2} S 2 p^{4}\left({ }_{2}^{3} P\right)^{2} P 3 p^{3} P^{\circ}$ \\
\hline 20 & 145 & $2 s^{2} S 2 p^{4}\left({ }_{2}^{2} P\right)^{4} P 3 d^{3} F_{3}$ & & 4272123 & $3.244 \mathrm{E}-12$ & $3.238 \mathrm{E}-12$ & $0.85+0.092 s^{2} S 2 p^{4}\left(_{2}^{3} P\right)^{4} P 3 d^{3} D+0.032 s^{2} S 2 p^{4}\left(3_{2}^{2} P\right)^{4} P 3 d^{5} F$ \\
\hline 20 & 146 & $2 s^{2} S 2 p^{4}\left({ }_{2}^{3} P\right){ }^{4} P 3 d^{3} F_{2}$ & & 4280691 & $1.599 \mathrm{E}-12$ & $1.597 \mathrm{E}-12$ & $0.75+0.172 s^{2} S 2 p^{4}\left({ }_{2}^{3} P\right){ }^{4} P 3 d^{3} D+0.042 s^{2} S 2 p^{4}\left({ }_{2}^{3} P\right)^{4} P 3 d^{3} P$ \\
\hline 20 & 147 & $2 s^{2} S 2 p^{4}\left({ }_{2}^{3} P\right){ }^{4} P 3 d^{3} P_{0}$ & & 4284567 & $8.770 \mathrm{E}-13$ & $8.769 \mathrm{E}-13$ & $0.89+0.082 s^{2} S 2 p^{4}\left({ }_{2}^{1} D\right)^{2} D 3 d^{3} P$ \\
\hline 20 & 148 & $2 s^{2} S 2 p^{4}\left(\frac{3}{2} P\right)^{4} P 3 d^{3} P_{1}$ & & 4286409 & $6.190 \mathrm{E}-13$ & $6.189 \mathrm{E}-13$ & $0.71+0.192 s^{2} S 2 p^{4}\left({ }_{2}^{3} P\right){ }^{4} P 3 d^{3} D+0.052 s^{2} S 2 p^{4}\left({ }_{2}^{1} D\right)^{2} D 3 d^{3} P$ \\
\hline 20 & 149 & $2 s^{2} S 2 p^{4}\left({ }_{2}^{3} P\right){ }^{4} P 3 d^{3} D_{2}$ & & 4293300 & $5.510 \mathrm{E}-13$ & $5.507 \mathrm{E}-13$ & $0.35+0.382 s^{2} S 2 p^{4}\left({ }_{2}^{3} P\right)^{4} P 3 d^{3} P+0.202 s^{2} S 2 p^{4}\left({ }_{2}^{3} P\right)^{4} P 3 d^{3} F$ \\
\hline 20 & 150 & $2 s^{2} S 2 p^{4}\left({ }_{2}^{3} P\right){ }^{4} P 3 d^{3} D_{3}$ & & 4300772 & $3.206 \mathrm{E}-13$ & 3.203E-13 & $\left.0.84+0.102 s^{2} S 2 p^{4}{ }_{2}^{3} P\right)^{4} P 3 d^{3} F+0.032 s^{2} S 2 p^{4}\left({ }_{2}^{1} D\right)^{2} D 3 d^{3} D$ \\
\hline 20 & 151 & $2 s^{2} S 2 p^{4}\left(\frac{3}{3} P\right)^{4} P 3 d^{3} D_{1}$ & & 4303307 & $4.071 \mathrm{E}-13$ & 4.069E-13 & $0.71+0.192 s^{2} S 2 p^{4}\left(\int_{2}^{3} P\right)^{4} P 3 d^{3} P+0.052 s^{2} S 2 p^{4}\left(1_{2}^{2} D\right)^{2} D 3 d^{3} D$ \\
\hline 20 & 152 & $2 s^{2} S 2 p^{4}\left({ }_{2}^{2} P\right){ }^{4} P 3 d^{3} P_{2}$ & & 4310257 & $4.958 \mathrm{E}-13$ & 4.955E-13 & $0.50+0.402 s^{2} S 2 p^{4}\left({ }_{2}^{3} P\right)^{4} P 3 d^{3} D+0.042 s^{2} S 2 p^{4}\left({ }_{2}^{1} D\right)^{2} D 3 d^{3} P$ \\
\hline 20 & 153 & $2 s^{2} S 2 p^{4}\left({ }_{0}^{1} S\right)^{2} S 3 p^{3} P_{0}^{\circ}$ & & 4340968 & 5.037E-12 & 5.039E-12 & $0.74+0.202 s^{2} S 2 p^{4}\left({ }_{2}^{3} P\right)^{2} P 3 p^{3} P^{\circ}+0.032 s^{2} S 2 p^{4}\left({ }_{2}^{1} D\right)^{2} D 3 p^{3} P^{\circ}$ \\
\hline 20 & 154 & $2 s^{2} S 2 p^{4}\left({ }_{0}^{O} S\right)^{2} S 3 p^{3} P_{1}^{\circ}$ & & 4345246 & $4.378 \mathrm{E}-12$ & $4.377 \mathrm{E}-12$ & $\left.0.73+0.152 s^{2} S 2 p^{4}{ }_{2}^{3} P\right)^{2} P 3 p^{3} P^{\circ}+0.062 s^{2} S 2 p^{4}\left({ }_{2}^{3} P\right)^{2} P 3 p^{3} D^{\circ}$ \\
\hline 20 & 155 & $2 s^{2} S 2 p^{4}\left({ }_{0}^{1} S\right)^{2} S 3 p^{3} P_{2}^{\circ}$ & & 4353941 & $6.121 \mathrm{E}-12$ & $6.119 \mathrm{E}-12$ & $\left.\left.0.80+0.112 s^{2} S 2 p^{4}{ }_{2}^{3} P\right)^{2} P 3 p^{3} D^{\circ}+0.042 s^{2} S 2 p^{4}{ }_{2}^{3} P\right)^{2} P 3 p^{3} P^{\circ}$ \\
\hline 20 & 156 & $2 s^{2} S 2 p^{4}\left({ }_{0}^{1} S\right)^{2} S 3 p^{1} P_{1}^{\circ}$ & & 4361217 & $9.250 \mathrm{E}-13$ & $9.230 \mathrm{E}-13$ & $\left.\left.0.75+0.082 s^{2} S 2 p^{4}{ }_{2}^{3} P\right)^{2} P 3 p^{3} D^{\circ}+0.082 s^{2} S 2 p^{4}{ }_{2}^{3} P\right)^{2} P 3 p^{3} P^{\circ}$ \\
\hline 20 & 157 & $2 s^{2} S 2 p^{4}\left({ }_{2}^{3} P\right)^{2} P 3 p^{3} P_{2}^{\circ}$ & & 4371445 & $3.710 \mathrm{E}-12$ & 3.707E-12 & $\left.0.73+0.082 s^{2} S 2 p^{4}\left({ }_{0}^{1} S\right)^{2} S 3 p^{3} P^{\circ}+0.082 s^{2} S 2 p^{4}{ }_{2}^{3} P\right)^{2} P 3 p^{3} D^{\circ}$ \\
\hline 20 & 158 & $2 s^{2} S 2 p^{4}\left(3_{3}^{2} P\right)^{2} P 3 p^{3} D_{3}^{\circ}$ & & 4381557 & $1.867 \mathrm{E}-12$ & $1.863 \mathrm{E}-12$ & 0.97 \\
\hline 20 & 159 & $2 s^{2} S 2 p^{4}\left({ }_{2}^{3} P\right)^{2} P 3 p^{3} D_{2}^{\circ}$ & & 4381781 & 2.929E-12 & $2.925 \mathrm{E}-12$ & $\left.0.43+0.432 s^{2} S 2 p^{4}\left({ }_{2}^{3} P\right)^{2} P 3 p^{1} D^{\circ}+0.112 s^{2} S 2 p^{4}{ }_{2}^{3} P\right)^{2} P 3 p^{3} P^{\circ}$ \\
\hline 20 & 160 & $2 s^{2} S 2 p^{4}\left({ }_{2}^{3} P\right)^{2} P 3 p^{3} P_{1}^{\mathrm{o}}$ & & 4384818 & 4.095E-12 & $4.091 \mathrm{E}-12$ & $\left.0.52+0.182 s^{2} S 2 p^{4}\left({ }_{0}^{1} S\right)^{2} S 3 p^{3} P^{\circ}+0.122 s^{2} S 2 p^{4}{ }_{2}^{3} P\right)^{2} P 3 p^{3} S^{\circ}$ \\
\hline 20 & 161 & $2 s^{2} S 2 p^{4}\left({ }_{2}^{2} P\right)^{2} P 3 p^{3} P_{0}^{\mathrm{O}}$ & & 4392449 & $6.214 \mathrm{E}-12$ & $6.216 \mathrm{E}-12$ & $0.53+0.242 s^{2} S 2 p^{4}\left(_{2}^{3} P\right)^{2} P 3 p^{1} S^{\circ}+0.132 s^{2} S 2 p^{4}\left({ }_{0}^{1} S\right)^{2} S 3 p^{3} P^{\circ}$ \\
\hline 20 & 162 & $2 s^{2} S 2 p^{4}\left({ }_{2}^{3} P\right)^{2} P 3 p^{3} D_{1}^{\circ}$ & & 4399980 & $1.611 \mathrm{E}-12$ & $1.607 \mathrm{E}-12$ & $\left.0.74+0.102 s^{2} S 2 p^{4}\left({ }_{0}^{1} S\right)^{2} S 3 p^{1} P^{\circ}+0.072 s^{2} S 2 p^{4}{ }_{2}^{3} P\right)^{2} P 3 p^{3} S^{\circ}$ \\
\hline 20 & 163 & $2 s^{2} S 2 p^{4}\left({ }_{2}^{1} D\right)^{2} D 3 d^{3} G_{3}$ & & 4410797 & $5.763 \mathrm{E}-11$ & $5.700 \mathrm{E}-11$ & 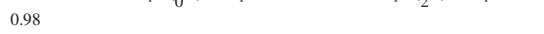 \\
\hline 20 & 164 & $2 s^{2} S 2 p^{4}\left({ }_{2}^{3} P\right)^{2} P 3 p{ }^{1} D_{2}^{\circ}$ & & 4411156 & 3.914E-12 & $3.911 \mathrm{E}-12$ & $0.50+0.352 s^{2} S 2 p^{4}\left({ }_{2}^{3} P\right)^{2} P 3 p^{3} D^{\circ}+0.092 s^{2} S 2 p^{4}\left({ }_{0}^{1} S\right)^{2} S 3 p^{3} P^{\circ}$ \\
\hline 20 & 165 & $2 s^{2} S 2 p^{4}\left({ }_{2}^{1} D\right)^{2} D 3 d^{3} G_{4}$ & & 4411850 & 5.917E-11 & $5.845 \mathrm{E}-11$ & $0.98+2-1<+0$ \\
\hline 20 & 166 & $2 s^{2} S 2 p^{4}\left({ }_{2}^{1} D\right)^{2} D 3 d^{3} G_{5}$ & & 4413170 & 6.107E-11 & $6.033 \mathrm{E}-11$ & 0.98 \\
\hline 20 & 167 & $2 s^{2} S 2 p^{4}\left({ }_{2}^{2} P\right)^{2} P 3 p^{1} S_{0}^{0}$ & & 4414972 & $1.042 \mathrm{E}-11$ & $1.042 \mathrm{E}-11$ & $\left.0.73+0.152 s^{2} S 2 p^{4}{ }_{2}^{3} P\right)^{2} P 3 p^{3} P^{\circ}+0.102 s^{2} S 2 p^{4}\left({ }_{0}^{1} S\right)^{2} S 3 p^{3} P^{\circ}$ \\
\hline
\end{tabular}




\begin{tabular}{|c|c|c|c|c|c|c|c|}
\hline $\mathrm{z}$ & Key & Level & $E_{\text {NIST }}$ & $E_{\mathrm{MCDHF} / \mathrm{RCI}}$ & $\tau_{\mathrm{MCDHF} / \mathrm{RCI}}^{l}$ & $\tau_{\mathrm{MCDHF} / \mathrm{RCI}}^{v}$ & $L S$-composition \\
\hline 20 & 168 & $2 s^{2} S 2 p^{4}\left({ }_{2}^{3} P\right)^{2} P 3 p^{3} S_{1}^{\circ}$ & & 4420820 & $2.299 \mathrm{E}-12$ & $2.289 \mathrm{E}-12$ & $0.77+0.092 s^{2} S 2 p^{4}\left({ }_{2}^{3} P\right)^{2} P 3 p^{3} P^{\circ}+0.062 s^{2} S 2 p^{4}\left({ }_{0}^{1} S\right)^{2} S 3 p^{1} P^{\circ}$ \\
\hline 20 & 169 & $2 s^{2} S 2 p^{4}\left({ }_{2}^{1} D\right)^{2} D 3 d^{3} S_{1}$ & & 4436781 & $1.870 \mathrm{E}-13$ & $1.870 \mathrm{E}-13$ & $0.96-2$ \\
\hline 20 & 170 & $2 s^{2} S 2 p^{4}\left({ }_{2}^{1} D\right)^{2} D 3 d^{3} F_{2}$ & & 4438153 & 2.709E-11 & $2.738 \mathrm{E}-11$ & 0.96 \\
\hline 20 & 171 & $2 s^{2} S 2 p^{4}\left({ }_{2}^{1} D\right)^{2} D 3 d^{3} F_{3}$ & & 4439247 & $1.584 \mathrm{E}-11$ & $1.592 \mathrm{E}-11$ & 0.96 \\
\hline 20 & 172 & $2 s^{2} S 2 p^{4}\left({ }_{2}^{1} D\right)^{2} D 3 d^{3} F_{4}$ & & 4439878 & $5.022 \mathrm{E}-11$ & 5.071E-11 & $0.82+0.162 s^{2} S 2 p^{4}\left({ }_{2}^{1} D\right)^{2} D 3 d^{1} G$ \\
\hline 20 & 173 & $2 s^{2} S 2 p^{4}\left({ }_{2}^{1} D\right)^{2} D 3 d^{1} G_{4}$ & & 4443516 & $4.673 \mathrm{E}-11$ & 4.679E-11 & $0.82+0.162 s^{2} S 2 p^{4}\left({ }_{2}^{1} D\right)^{2} D 3 d^{3} F$ \\
\hline 20 & 174 & $2 s^{2} S 2 p^{4}\left(c_{1}^{1} D\right)^{2} D 3 d^{3} D_{1}$ & & 4445023 & $3.310 \mathrm{E}-13$ & $3.308 \mathrm{E}-13$ & $0.57+0.212 s^{2} S 2 p^{4}\left({ }_{1}^{1} D\right)^{2} D 3 d^{3} P+0.122 s^{2} S 2 p^{4}\left({ }_{1}^{1} D\right)^{2} D 3 d^{1} P$ \\
\hline 20 & 175 & $2 s^{2} S 2 p^{4}\left({ }_{2}^{1} D\right)^{2} D 3 d^{3} P_{2}$ & & 4445363 & $2.078 \mathrm{E}-13$ & 2.078E-13 & $0.82+0.092 s^{2} S 2 p^{4}\left({ }_{2}^{1} D\right)^{2} D 3 d^{3} D+0.052 s^{2} S 2 p^{4}\left(3_{2}^{2} P\right)^{4} P 3 d^{3} P$ \\
\hline 20 & 176 & $2 s^{2} S 2 p^{4}\left({ }_{2}^{1} D\right)^{2} D 3 d^{3} D_{3}$ & & 4445928 & $3.298 \mathrm{E}-13$ & $3.296 \mathrm{E}-13$ & $\left.0.91+0.032 s^{2} S 2 p^{4}{ }_{2}^{3} P\right)^{4} P 3 d^{3} D$ \\
\hline 20 & 177 & $2 s^{2} S 2 p^{4}\left({ }_{2}^{1} D\right)^{2} D 3 d^{3} P_{0}$ & & 4446104 & 2.074E-13 & $2.074 \mathrm{E}-13$ & $0.85+0.072 s^{2} S 2 p^{4}\left({ }_{2}^{2} P\right){ }^{4} P 3 d^{3} P+0.042 s^{2} S 2 p^{4}\left({ }_{2}^{1} D\right)^{2} D 3 d^{1} S$ \\
\hline 20 & 178 & $2 s^{2} S 2 p^{4}\left({ }_{2}^{1} D\right)^{2} D 3 d^{3} D_{2}$ & & 4446480 & $3.068 \mathrm{E}-13$ & $3.066 \mathrm{E}-13$ & $0.80+0.102 s^{2} S 2 p^{4}\left({ }_{2}^{1} D\right)^{2} D 3 d^{3} P+0.052 s^{2} S 2 p^{4}\left({ }_{2}^{3} P\right)^{4} P 3 d^{3} D$ \\
\hline 20 & 179 & $2 s^{2} S 2 p^{4}\left({ }_{2}^{1} D\right)^{2} D 3 d^{3} P_{1}$ & & 4447704 & 2.149E-13 & $2.149 \mathrm{E}-13$ & $0.67+0.192 s^{2} S 2 p^{4}\left({ }_{2}^{4} D\right)^{2} D 3 d^{3} D+0.062 s^{2} S 2 p^{4}\left(3_{2}^{3} P\right)^{4} P 3 d^{3} P$ \\
\hline 20 & 180 & $2 s^{2} S 2 p^{4}\left({ }_{2}^{1} D\right)^{2} D 3 d^{1} P_{1}$ & & 4457644 & $5.313 \mathrm{E}-13$ & $5.312 \mathrm{E}-13$ & $\left.0.77+0.122 s^{2} S 2 p^{4}\left(\frac{1}{2} D\right){ }^{2} D 3 d^{3} D+0.052 s^{2} S 2 p^{4}{ }_{2}^{4} P\right)^{2} P 3 d^{1} P$ \\
\hline 20 & 181 & $2 s^{2} S 2 p^{4}\left({ }_{2}^{1} D\right)^{2} D 3 d^{1} D_{2}$ & & 4461878 & $1.550 \mathrm{E}-12$ & $1.548 \mathrm{E}-12$ & $\left.0.86+0.052 s^{2} S 2 p^{4}{ }_{2}^{3} P\right)^{2} P 3 d^{1} D$ \\
\hline 20 & 182 & $2 s^{2} S 2 p^{4}\left({ }_{1}^{1} D\right)^{2} D 3 d^{1} F_{3}$ & & 4468633 & 7.918E-12 & 7.953E-12 & 0.97 \\
\hline 20 & 183 & $2 s^{2} S 2 p^{4}\left({ }_{2}^{1} D\right)^{2} D 3 d^{1} S_{0}$ & & 4472918 & 2.223E-13 & $2.223 \mathrm{E}-13$ & $0.93+0.032 s^{2} S 2 p^{4}\left({ }_{2}^{1} D\right)^{2} D 3 d^{3} P$ \\
\hline 20 & 184 & $2 s^{2} S 2 p^{4}\left(3_{2}^{3} P\right)^{2} P 3 p{ }^{1} P_{1}^{\circ}$ & & 4475006 & 3.614E-12 & $3.602 \mathrm{E}-12$ & $0.85+0.062 s^{2} S 2 p^{4}\left({ }_{2}^{1} D\right)^{2} D 3 p^{1} P^{\circ}+0.032 s^{2} S 2 p^{4}\left({ }_{0}^{1} S\right)^{2} S 3 p^{1} P^{\circ}$ \\
\hline 21 & 1 & $2 s^{2} 2 p^{4}\left({ }_{2}^{3} P\right)^{3} P_{2}$ & 0 & 0 & $\ldots$ & $\ldots$ & $\left.0.96+0.042 s^{2} 2 p^{4}\left({ }_{2}^{1} D\right)\right)^{1} D$ \\
\hline 21 & 2 & $2 s^{2} 2 p^{4}\left({ }_{2}^{3} P\right)^{3} P_{1}$ & 31174 & 31161 & $1.530 \mathrm{E}-03$ & $1.530 \mathrm{E}-03$ & 0.99 \\
\hline 21 & 3 & $2 s^{2} 2 p^{4}\left({ }_{3}^{3} P\right)^{3} P_{0}$ & 35351 & 35536 & $2.339 \mathrm{E}-01$ & $2.340 \mathrm{E}-01$ & $0.94+0.062 s^{2} 2 p^{4}\left({ }_{0}^{1} s\right)^{1} S$ \\
\hline 21 & 4 & $2 s^{2} 2 p^{4}\left({ }_{2}^{1} D\right){ }^{1} D_{2}$ & 97793 & 98068 & $6.547 \mathrm{E}-04$ & $6.547 \mathrm{E}-04$ & $0.96+0.042 s^{2} 2 p^{4}\left({ }_{2}^{3} P\right)^{3} P$ \\
\hline 21 & 5 & $2 s^{2} 2 p^{4}\left({ }_{0}^{1} S\right){ }^{1} S_{0}$ & 195985 & 196217 & $7.241 \mathrm{E}-05$ & $7.241 \mathrm{E}-05$ & $\left.0.92+0.062 s^{2} 2 p^{4}{ }_{2}^{3} P\right)^{3} P+0.022 p^{6}{ }^{1} S$ \\
\hline 21 & 6 & $2 s^{2} S 2 p^{5} P_{2}^{\circ}$ & 664483 & 664247 & 3.523E-11 & $3.509 \mathrm{E}-11$ & 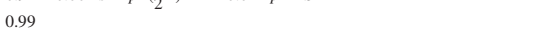 \\
\hline 21 & 7 & $2 s^{2} S 2 p^{5}{ }^{3} P_{1}^{2}$ & 689445 & 689215 & $3.265 \mathrm{E}-11$ & $3.266 \mathrm{E}-11$ & 0.99 \\
\hline 21 & 8 & $2 s^{2} S 2 p^{53} P_{0}^{\circ}$ & 704584 & 704452 & $3.220 \mathrm{E}-11$ & $3.226 \mathrm{E}-11$ & 0.99 \\
\hline 21 & 9 & $2 s^{2} S 2 p^{5}{ }^{1} P_{1}^{0}$ & 912990 & 913146 & $9.812 \mathrm{E}-12$ & $9.810 \mathrm{E}-12$ & 0.98 \\
\hline 21 & 10 & $2 p^{6}{ }^{1} s_{0}$ & 1546620 & 1546660 & 1.123E-11 & $1.124 \mathrm{E}-11$ & $0.96+0.022 s^{2} 2 p^{4}\left({ }_{0}^{1} S\right)^{1} S$ \\
\hline 21 & 11 & $2 s^{2} 2 p^{3}\left({ }_{3}^{4} S\right)^{4} S 3 s^{5} S_{2}^{\circ}$ & & 3807070 & $1.194 \mathrm{E}-10$ & $1.200 \mathrm{E}-10$ & $0.97+0.022 s^{2} 2 p^{3}\left({ }_{1}^{2} P\right)^{2} P 3 s^{3} P^{\circ}$ \\
\hline 21 & 12 & $2 s^{2} 2 p^{3}\left({ }_{3}^{3} s\right){ }^{4} s 3 s^{3} s_{1}^{2}$ & 3848500 & 3848044 & 7.969E-13 & 7.975E-13 & 0.96 \\
\hline 21 & 13 & $2 s^{2} 2 p^{3}\left(\frac{2}{3} D\right)^{2} D 3 s^{3} D_{1}^{\circ}$ & 3929400 & 3929975 & 2.287E-12 & $2.290 \mathrm{E}-12$ & $0.88+0.052 s^{2} 2 p^{3}\left({ }_{1}^{2} P\right)^{2} P 3 s^{1} P^{\circ}+0.042 s^{2} 2 p^{3}\left({ }_{1}^{2} P\right)^{2} P 3 s^{3} P^{\circ}$ \\
\hline 21 & 14 & $2 s^{2} 2 p^{3}\left({ }_{3}^{2} D\right)^{2} D 3 s^{3} D_{2}^{\circ}$ & 3930800 & 3930916 & $2.372 \mathrm{E}-12$ & $2.374 \mathrm{E}-12$ & $0.83+0.112 s^{2} 2 p^{3}\left({ }_{1}^{2} P\right)^{2} P 3 s^{3} P^{\circ}+0.042 s^{2} 2 p^{3}\left({ }_{3}^{2} D\right)^{2} D 3 s^{1} D^{\circ}$ \\
\hline 21 & 15 & $2 s^{2} 2 p^{3}\left(\frac{2}{3} D\right)^{2} D 3 s^{3} D_{3}^{\circ}$ & 3938200 & 3938702 & $2.471 \mathrm{E}-12$ & $2.472 \mathrm{E}-12$ & 0.99 \\
\hline 21 & 16 & $2 s^{2} 2 p^{3}\left({ }_{3}^{2} D\right)^{2} D 3 s^{1} D_{2}^{\circ}$ & 3955700 & 3955404 & $1.054 \mathrm{E}-12$ & $1.055 \mathrm{E}-12$ & $0.86+0.092 s^{2} 2 p^{3}\left({ }_{3}^{2} D\right)^{2} D 3 s^{3} D^{\circ}+0.052 s^{2} 2 p^{3}\left({ }_{1}^{2} P\right)^{2} P 3 s^{3} P^{\circ}$ \\
\hline 21 & 17 & $2 s^{2} 2 p^{3}\left(\frac{4}{3} S\right)^{4} S 3 p^{5} P_{1}^{2}$ & & 3979846 & 4.577E-10 & $4.558 \mathrm{E}-10$ & 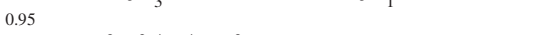 \\
\hline 21 & 18 & $2 s^{2} 2 p^{3}\left({ }_{2}^{4} S\right)^{4} S 3 p^{5} P_{2}$ & & 3981962 & $4.950 \mathrm{E}-10$ & 4.929E-10 & $0.92+0.032 s^{2} 2 p^{3}\left({ }_{3}^{4} S\right)^{4} S 3 p^{3} P$ \\
\hline 21 & 19 & $2 s^{2} 2 p^{3}\left({ }_{3}^{4} S\right)^{4} S 3 p^{5} P_{3}$ & & 3987805 & $4.455 \mathrm{E}-10$ & $4.446 \mathrm{E}-10$ & $0.96+0.022 s^{2} 2 p^{3}\left({ }_{1}^{2} P\right)^{2} P 3 p^{3} D$ \\
\hline 21 & 20 & $2 s^{2} 2 p^{3}\left({ }_{1}^{2} P\right)^{2} P 3 s^{3} P_{0}^{\circ}$ & & 4002364 & 2.609E-12 & $2.611 \mathrm{E}-12$ & 0.97 \\
\hline 21 & 21 & $2 s^{2} 2 p^{3}\left({ }_{1}^{2} P\right)^{2} P 3 s^{3} P_{1}^{\circ}$ & & 4005859 & $2.275 \mathrm{E}-12$ & $2.276 \mathrm{E}-12$ & $0.87+0.082 s^{2} 2 p^{3}\left({ }_{1}^{2} P\right)^{2} P 3 s^{1} P^{\circ}+0.022 s^{2} 2 p^{3}\left({ }_{3}^{2} D\right)^{2} D 3 s^{3} D^{\circ}$ \\
\hline 21 & 22 & $2 s^{2} 2 p^{3}\left({ }_{1}^{2} P\right)^{2} P 3 s^{3} P_{2}^{o}$ & 4004600 & 4018824 & $2.210 \mathrm{E}-12$ & $2.209 \mathrm{E}-12$ & $0.79+0.092 s^{2} 2 p^{3}\left({ }_{3}^{2} D\right)^{2} D 3 s^{1} D^{\circ}+0.072 s^{2} 2 p^{3}\left({ }_{3}^{2} D\right)^{2} D 3 s^{3} D^{\circ}$ \\
\hline 21 & 23 & $2 s^{2} 2 p^{3}\left({ }_{3}^{4} S\right){ }^{4} S 3 p^{3} P_{1}$ & & 4019911 & $3.330 \mathrm{E}-10$ & $3.344 \mathrm{E}-10$ & $0.77+0.112 s^{2} 2 p^{3}\left({ }_{3}^{2} D\right)^{2} D 3 p^{3} P+0.032 s^{2} 2 p^{3}\left({ }_{1}^{2} P\right)^{2} P 3 p^{1} P$ \\
\hline 21 & 24 & $2 s^{2} 2 p^{3}\left({ }_{3}^{4} S\right){ }^{4} S 3 p^{3} P_{2}$ & & 4023977 & 3.370E-10 & 3.384E-10 & $0.79+0.122 s^{2} 2 p^{3}\left({ }_{3}^{2} D\right)^{2} D 3 p^{3} P+0.052 s^{2} 2 p^{3}\left({ }_{3}^{4} S\right)^{4} S 3 p^{5} P$ \\
\hline 21 & 25 & $2 s^{2} 2 p^{3}\left({ }_{3}^{4} s\right){ }^{4} S 3 p^{3} P_{0}$ & & 4026039 & 3.709E-10 & $3.718 \mathrm{E}-10$ & $0.85+0.092 s^{2} 2 p^{3}\left({ }_{3}^{2} D\right)^{2} D 3 p^{3} P+0.042 s^{2} 2 p^{3}\left({ }_{1}^{2} P\right)^{2} P 3 p^{3} P$ \\
\hline 21 & 26 & $2 s^{2} 2 p^{3}\left({ }_{1}^{2} P\right){ }^{2} P 3 s^{1} P_{1}^{\circ}$ & 4033900 & 4033778 & $1.078 \mathrm{E}-12$ & 1.079E-12 & $0.83+0.082 s^{2} 2 p^{3}\left({ }_{3}^{2} D\right)^{2} D 3 s^{3} D^{\circ}+0.052 s^{2} 2 p^{3}\left({ }_{1}^{2} P\right)^{2} P 3 s^{3} P^{\circ}$ \\
\hline 21 & 27 & $2 s^{2} 2 p^{3}\left({ }_{3}^{2} D\right)^{2} D 3 p^{3} D_{1}$ & & 4082522 & $2.576 \mathrm{E}-10$ & 2.603E-10 & $0.43+0.352 s^{2} 2 p^{3}\left({ }_{3}^{2} D\right)^{2} D 3 p^{1} P+0.092 s^{2} 2 p^{3}\left({ }_{1}^{2} P\right)^{2} P 3 p^{1} P$ \\
\hline 21 & 28 & $2 s^{2} 2 p^{3}\left({ }_{3}^{2} D\right)^{2} D 3 p^{3} D_{2}$ & & 4095729 & $4.589 \mathrm{E}-10$ & $4.589 \mathrm{E}-10$ & $0.66+0.212 s^{2} 2 p^{3}\left({ }_{3}^{2} D\right)^{2} D 3 p^{3} F+0.082 s^{2} 2 p^{3}\left({ }_{1}^{2} P\right)^{2} P 3 p^{3} D$ \\
\hline 21 & 29 & $2 s^{2} 2 p^{3}\left(\frac{2}{3} D\right)^{2} D 3 p^{3} D_{3}$ & & 4103749 & 4.169E-10 & $4.171 \mathrm{E}-10$ & $0.61+0.332 s^{2} 2 p^{3}\left({ }_{3}^{2} D\right)^{2} D 3 p^{3} F+0.052 s^{2} 2 p^{3}\left({ }_{1}^{2} P\right)^{2} P 3 p^{3} D$ \\
\hline 21 & 30 & $2 s^{2} 2 p^{3}\left(c_{3}^{2} D\right)^{2} D 3 p^{1} P_{1}$ & & 4105402 & $1.903 \mathrm{E}-10$ & $1.927 \mathrm{E}-10$ & $0.46+0.422 s^{2} 2 p^{3}\left({ }_{3}^{2} D\right)^{2} D 3 p^{3} D+0.062 s^{2} 2 p^{3}\left({ }_{1}^{2} P\right)^{2} P 3 p^{3} D$ \\
\hline 21 & 31 & $2 s^{2} 2 p^{3}\left({ }_{3}^{2} D\right)^{2} D 3 p^{3} F_{2}$ & & 4107428 & 2.375E-10 & 2.379E-10 & $0.67+0.242 s^{2} 2 p^{3}\left({ }_{3}^{2} D\right)^{2} D 3 p^{3} D+0.052 s^{2} 2 p^{3}\left({ }_{1}^{2} P\right)^{2} P 3 p^{1} D$ \\
\hline 21 & 32 & $2 s^{2} 2 p^{3}\left({ }_{3}^{2} D\right)^{2} D 3 p^{3} F_{3}$ & & 4114449 & $1.817 \mathrm{E}-10$ & $1.822 \mathrm{E}-10$ & $0.52+0.362 s^{2} 2 p^{3}\left({ }_{3}^{2} D\right)^{2} D 3 p^{3} D+0.062 s^{2} 2 p^{3}\left({ }_{3}^{2} D\right)^{2} D 3 p^{1} F$ \\
\hline 21 & 33 & $2 s^{2} 2 p^{3}\left({ }_{3}^{3} D\right)^{2} D 3 p^{3} F_{4}$ & & 4120793 & $3.830 \mathrm{E}-10$ & $3.813 \mathrm{E}-10$ & 0.99 \\
\hline 21 & 34 & $2 s^{2} 2 p^{3}\left({ }_{3}^{2} D\right)^{2} D 3 p^{1} F_{3}$ & & 4120795 & 4.275E-10 & $4.249 \mathrm{E}-10$ & $0.86+0.072 s^{2} 2 p^{3}\left(\frac{2}{3} D\right)^{2} D 3 p^{3} F+0.042 s^{2} 2 p^{3}\left({ }_{1}^{2} P\right)^{2} P 3 p^{3} D$ \\
\hline 21 & 35 & $2 s^{2} 2 p^{3}\left(\frac{2}{3} D\right)^{2} D 3 p^{3} P_{1}$ & & 4155821 & 2.954E-11 & 2.972E-11 & $0.38+0.372 s^{2} 2 p^{3}\left({ }_{1}^{2} P\right)^{2} P 3 p^{3} S+0.092 s^{2} 2 p^{3}\left({ }_{3}^{4} S\right)^{4} S 3 p^{3} P$ \\
\hline 21 & 36 & $2 s^{2} 2 p^{3}\left(\frac{2}{3} D\right)^{2} D 3 p^{3} P_{0}$ & & 4158247 & $3.159 \mathrm{E}-11$ & $3.174 \mathrm{E}-11$ & $0.74+0.192 s^{2} 2 p^{3}\left({ }_{1}^{2} P\right)^{2} P 3 p^{3} P+0.032 s^{2} 2 p^{3}\left({ }_{3}^{4} S\right)^{4} S 3 p^{3} P$ \\
\hline 21 & 37 & $2 s^{2} 2 p^{3}\left({ }_{3}^{2} D\right)^{2} D 3 p^{3} P_{2}$ & & 4169388 & $2.016 \mathrm{E}-11$ & $2.025 \mathrm{E}-11$ & $0.79+0.122 s^{2} 2 p^{3}\left({ }_{3}^{4} S\right)^{4} S 3 p^{3} P+0.032 s^{2} 2 p^{3}\left({ }_{1}^{2} P\right)^{2} P 3 p^{1} D$ \\
\hline 21 & 38 & $2 s^{2} 2 p^{3}\left({ }_{1}^{2} P\right)^{2} P 3 p^{3} D_{1}$ & & 4178039 & $7.895 \mathrm{E}-11$ & $7.908 \mathrm{E}-11$ & $0.73+0.152 s^{2} 2 p^{3}\left({ }_{1}^{2} P\right)^{2} P 3 p^{1} P+0.052 s^{2} 2 p^{3}\left({ }_{3}^{2} D\right)^{2} D 3 p^{3} D$ \\
\hline 21 & 39 & $2 s^{2} 2 p^{3}\left({ }_{1}^{2} P\right)^{2} P 3 p^{3} D_{2}$ & & 4183371 & 7.197E-11 & 7.213E-11 & $0.52+0.242 s^{2} 2 p^{3}\left({ }_{3}^{2} D\right)^{2} D 3 p^{1} D+0.082 s^{2} 2 p^{3}\left({ }_{3}^{2} D\right)^{2} D 3 p^{3} F$ \\
\hline 21 & 40 & $2 s^{2} 2 p^{3}\left({ }_{1}^{2} P\right)^{2} P 3 p^{3} s_{1}$ & & 4185957 & $3.347 \mathrm{E}-11$ & $3.366 \mathrm{E}-11$ & $0.49+0.282 s^{2} 2 p^{3}\left({ }_{3}^{2} D\right)^{2} D 3 p^{3} P+0.112 s^{2} 2 p^{3}\left({ }_{1}^{2} P\right)^{2} P 3 p^{3} P$ \\
\hline 21 & 41 & $2 s^{2} 2 p^{3}\left(\frac{2}{3} D\right)^{2} D 3 p^{1} D_{2}$ & & 4190432 & 8.437E-11 & 8.454E-11 & $0.27+0.312 s^{2} 2 p^{3}\left({ }_{1}^{2} P\right)^{2} P 3 p^{3} D+0.242 s^{2} 2 p^{3}\left({ }_{1}^{2} P\right)^{2} P 3 p^{3} P$ \\
\hline 21 & 42 & $2 s^{2} 2 p^{3}\left({ }_{1}^{2} P\right)^{2} P 3 p^{3} D_{3}$ & & 4196735 & $9.465 \mathrm{E}-11$ & 9.467E-11 & $0.81+0.072 s^{2} 2 p^{3}\left(\frac{2}{3} D\right)^{2} D 3 p^{1} F+0.072 s^{2} 2 p^{3}\left({ }_{3}^{2} D\right)^{2} D 3 p^{3} F$ \\
\hline 21 & 43 & $2 s^{2} 2 p^{3}\left({ }_{3}^{4} S\right)^{4} S 3 d^{5} D_{0}^{\circ}$ & & 4200994 & $3.494 \mathrm{E}-11$ & $3.474 \mathrm{E}-11$ & 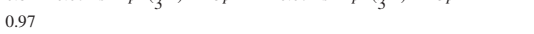 \\
\hline 21 & 44 & $2 s^{2} 2 p^{3}\left({ }_{3}^{4} S\right)^{4} S 3 d^{5} D_{2}^{\circ}$ & & 4201020 & $3.934 \mathrm{E}-11$ & $3.923 \mathrm{E}-11$ & 0.96 \\
\hline 21 & 45 & $2 s^{2} 2 p^{3}\left({ }_{3}^{4} S\right){ }^{4} S 3 d^{5} D_{1}^{2}$ & & 4201025 & $3.022 \mathrm{E}-11$ & $3.009 \mathrm{E}-11$ & 0.97 \\
\hline 21 & 46 & $2 s^{2} 2 p^{3}\left({ }_{3}^{4} S\right)^{4} S 3 d^{5} D_{3}^{\circ}$ & & 4201033 & $1.556 \mathrm{E}-10$ & $1.565 \mathrm{E}-10$ & 0.96 \\
\hline 21 & 47 & $2 s^{2} 2 p^{3}\left({ }_{3}^{4} S\right)^{4} S 3 d^{5} D_{4}^{\circ}$ & & 4201640 & $2.966 \mathrm{E}-10$ & $2.962 \mathrm{E}-10$ & $0.97+0.022 s^{2} 2 p^{3}\left({ }_{1}^{2} P\right)^{2} P 3 d^{3} F^{\circ}$ \\
\hline 21 & 48 & $\left.2 s^{2} 2 p^{3}{ }_{1}^{2} P\right)^{2} P 3 p^{1} P_{1}^{4}$ & & 4203093 & 4.703E-11 & $4.724 \mathrm{E}-11$ & $0.51+0.152 s^{2} 2 p^{3}\left({ }_{3}^{2} D\right)^{2} D 3 p^{3} P+0.112 s^{2} 2 p^{3}\left(_{1}^{2} P\right)^{2} P 3 p^{3} P$ \\
\hline 21 & 49 & $2 s^{2} 2 p^{3}\left({ }_{1}^{2} P\right)^{2} P 3 p^{3} P_{0}$ & & 4218477 & 4.193E-11 & $4.215 \mathrm{E}-11$ & $0.72+0.102 s^{2} 2 p^{3}\left(\frac{2}{3} D\right)^{2} D 3 p^{3} P+0.092 s^{2} 2 p^{3}\left({ }_{3}^{4} S\right)^{4} S 3 p^{3} P$ \\
\hline 21 & 50 & $2 s^{2} 2 p^{3}\left({ }_{1}^{2} P\right)^{2} P 3 p^{3} P_{1}$ & & 4220476 & $6.587 \mathrm{E}-11$ & $6.616 \mathrm{E}-11$ & $0.64+0.112 s^{2} 2 p^{3}\left({ }_{1}^{2} P\right)^{2} P 3 p^{1} P+0.072 s^{2} 2 p^{3}\left({ }_{3}^{4} S\right){ }^{4} S 3 p^{3} P$ \\
\hline 21 & 51 & $2 s^{2} 2 p^{3}\left({ }_{1}^{2} P\right)^{2} P 3 p^{3} P_{2}$ & & 4221065 & $1.410 \mathrm{E}-10$ & $1.414 \mathrm{E}-10$ & $0.56+0.242 s^{2} 2 p^{3}\left({ }_{1}^{2} P\right)^{2} P 3 p^{1} D+0.052 s^{2} 2 p^{3}\left({ }_{3}^{2} D\right)^{2} D 3 p^{1} D$ \\
\hline 21 & 52 & $2 s^{2} 2 p^{3}\left({ }_{3}^{4} S\right)^{4} S 3 d^{3} D_{2}^{\circ}$ & 4245000 & 4242884 & $6.479 \mathrm{E}-13$ & $6.472 \mathrm{E}-13$ & $0.78+0.122 s^{2} 2 p^{3}\left({ }_{3}^{2} D\right)^{2} D 3 d^{3} D^{\circ}+0.032 s^{2} 2 p^{3}\left({ }_{1}^{2} P\right)^{2} P 3 d^{1} D^{\circ}$ \\
\hline 21 & 53 & $2 s^{2} 2 p^{3}\left({ }_{3}^{4} S\right)^{4} S 3 d^{3} D_{1}^{\circ}$ & & 4247416 & $5.475 \mathrm{E}-13$ & $5.470 \mathrm{E}-13$ & $0.85+0.092 s^{2} 2 p^{3}\left({ }_{3}^{2} D\right)^{2} D 3 d^{3} D^{\circ}+0.042 s^{2} 2 p^{3}\left({ }_{1}^{2} P\right)^{2} P 3 d^{3} D^{\circ}$ \\
\hline 21 & 54 & $2 s^{2} 2 p^{3}\left({ }_{3}^{4} S\right){ }^{4} S 3 d^{3} D_{3}^{\circ}$ & 4249000 & 4248446 & $5.363 \mathrm{E}-13$ & 5.353E-13 & $0.82+0.122 s^{2} 2 p^{3}\left({ }_{3}^{2} D\right)^{2} D 3 d^{3} D^{\circ}$ \\
\hline 21 & 55 & $2 s^{2} 2 p^{3}\left({ }_{1}^{2} P\right)^{2} P 3 p^{1} D_{2}^{3}$ & & 4249346 & $1.131 \mathrm{E}-10$ & $1.138 \mathrm{E}-10$ & $0.44+0.432 s^{2} 2 p^{3}\left({ }_{3}^{2} D\right)^{2} D 3 p^{1} D+0.062 s^{2} 2 p^{3}\left({ }_{1}^{2} P\right)^{2} P 3 p^{3} P$ \\
\hline 21 & 56 & $2 s^{2} 2 p^{3}\left(\frac{2}{3} D\right)^{2} D 3 d^{3} F_{2}^{\circ}$ & & 4307892 & $5.464 \mathrm{E}-12$ & $5.450 \mathrm{E}-12$ & $0.79+0.112 s^{2} 2 p^{3}\left({ }_{1}^{3} P\right)^{2} P 3 d^{3} F^{\circ}+0.032 s^{2} 2 p^{3}\left({ }_{3}^{4} S\right)^{4} S 3 d^{3} D^{\circ}$ \\
\hline 21 & 57 & $2 s^{2} 2 p^{3}\left(\frac{2}{3} D\right)^{2} D 3 d^{3} F_{3}^{\circ}$ & & 4313900 & 4.653E-12 & $4.650 \mathrm{E}-12$ & $0.77+0.102 s^{2} 2 p^{3}\left({ }_{1}^{2} P\right)^{2} P 3 d^{3} F^{\circ}+0.082 s^{2} 2 p^{3}\left({ }_{3}^{2} D\right)^{2} D 3 d^{3} G^{\circ}$ \\
\hline 21 & 58 & $2 s^{2} 2 p^{3}\left({ }_{1}^{2} P\right)^{2} P 3 p^{1} s_{0}$ & & 4315333 & $1.753 \mathrm{E}-11$ & $1.760 \mathrm{E}-11$ & $0.90+0.042 s^{2} 2 p^{3}\left(\frac{2}{3} D\right)^{2} D 3 p^{3} P$ \\
\hline 21 & 59 & $2 s^{2} 2 p^{3}\left(\frac{2}{3} D\right)^{2} D 3 d^{1} S_{0}^{\circ}$ & & 4316711 & 4.413E-11 & $4.440 \mathrm{E}-11$ & $0.93+0.052 s^{2} 2 p^{3}\left({ }_{1}^{2} P\right)^{2} P 3 d^{3} P^{\circ}$ \\
\hline 21 & 60 & $2 s^{2} 2 p^{3}\left(c_{2}^{2} D\right)^{2} D 3 d^{3} F_{4}^{\circ}$ & & 4320693 & 3.087E-10 & 3.074E-10 & $0.53+0.272 s^{2} 2 p^{3}\left({ }_{3}^{2} D\right)^{2} D 3 d^{3} G^{\circ}+0.102 s^{2} 2 p^{3}\left({ }_{1}^{2} P\right)^{2} P 3 d^{3} F^{\circ}$ \\
\hline 21 & 61 & $2 s^{2} 2 p^{3}\left({ }_{3}^{2} D\right)^{2} D 3 d^{3} G_{3}^{\circ}$ & & 4326283 & 6.899E-12 & 6.893E-12 & $0.80+0.102 s^{2} 2 p^{3}\left({ }_{3}^{2} D\right)^{2} D 3 d^{3} F^{\circ}+0.052 s^{2} 2 p^{3}\left({ }_{1}^{2} P\right)^{2} P 3 d^{1} F^{\circ}$ \\
\hline 21 & 62 & $2 s^{2} 2 p^{3}\left({ }_{2}^{3} D\right)^{2} D 3 d^{3} G_{4}^{\circ}$ & & 4329982 & 2.783E-10 & 2.784E-10 & $0.45+0.442 s^{2} 2 p^{3}\left({ }_{2}^{2} D\right)^{2} D 3 d^{3} F^{\circ}+0.062 s^{2} 2 p^{3}\left({ }_{2}^{2} D\right)^{2} D 3 d^{1} G^{\circ}$ \\
\hline 21 & 63 & $2 s^{2} 2 p^{3}\left({ }_{3}^{2} D\right)^{2} D 3 d^{3} D_{1}^{\circ}$ & 4333000 & 4334403 & $3.706 \mathrm{E}-13$ & $3.701 \mathrm{E}-13$ & $0.40+0.382 s^{2} 2 p^{3}\left({ }_{3}^{2} D\right)^{2} D 3 d^{1} P^{\circ}+0.062 s^{2} 2 p^{3}\left({ }_{1}^{2} P\right)^{2} P 3 d^{3} D^{\circ}$ \\
\hline 21 & 64 & $2 s^{2} 2 p^{3}\left(\frac{2}{3} D\right)^{2} D 3 d^{3} G_{5}^{\mathrm{O}}$ & & 4334575 & 2.833E-10 & $2.824 \mathrm{E}-10$ & 1.00 \\
\hline 21 & 65 & $2 s^{2} 2 p^{3}\left({ }_{3}^{2} D\right)^{2} D 3 d^{1} G_{4}^{0}$ & & 4336956 & 2.685E-10 & 2.679E-10 & $0.78+0.202 s^{2} 2 p^{3}\left({ }_{3}^{2} D\right)^{2} D 3 d^{3} G^{\circ}$ \\
\hline 21 & 66 & $2 s^{2} 2 p^{3}\left({ }_{3}^{2} D\right)^{2} D 3 d^{3} D_{2}^{\circ}$ & 4350000 & 4349770 & $2.569 \mathrm{E}-13$ & $2.566 \mathrm{E}-13$ & $0.60+0.142 s^{2} 2 p^{3}\left({ }_{3}^{2} D\right)^{2} D 3 d^{3} P^{\circ}+0.102 s^{2} 2 p^{3}\left({ }_{3}^{4} S\right)^{4} S 3 d^{3} D^{\circ}$ \\
\hline 21 & 67 & $2 s^{2} 2 p^{3}\left({ }_{3}^{2} D\right)^{2} D 3 d^{1} P_{1}^{\circ}$ & & 4353139 & 3.454E-13 & $3.452 \mathrm{E}-13$ & $0.47+0.422 s^{2} 2 p^{3}\left({ }_{3}^{2} D\right)^{2} D 3 d^{3} D^{\circ}+0.032 s^{2} 2 p^{3}\left({ }_{3}^{4} S\right)^{4} S 3 d^{3} D^{\circ}$ \\
\hline
\end{tabular}




\begin{tabular}{|c|c|c|c|c|c|c|c|}
\hline $\mathrm{z}$ & Key & Level & $E_{\mathrm{NIST}}$ & $E_{\mathrm{MCDHF} / \mathrm{RCI}}$ & $\tau_{\mathrm{MCDHF} / \mathrm{RCI}}^{l}$ & $\tau_{\mathrm{MCDHF} / \mathrm{RCI}}^{v}$ & $L S$-composition \\
\hline 21 & 68 & $2 s^{2} 2 p^{3}\left({ }_{3}^{2} D\right)^{2} D 3 d^{3} D_{3}^{\circ}$ & \multirow{3}{*}{$\begin{array}{l}4354000 \\
4362000\end{array}$} & 4354720 & $1.804 \mathrm{E}-13$ & $1.802 \mathrm{E}-13$ & $0.83+0.122 s^{2} 2 p^{3}\left({ }_{3}^{4} S\right)^{4} S 3 d^{3} D^{\circ}$ \\
\hline 21 & 69 & $2 s^{2} 2 p^{3}\left(\frac{3}{3} D\right)^{2} D 3 d^{3} P_{2}^{\circ}$ & & 4361171 & $1.602 \mathrm{E}-13$ & $1.600 \mathrm{E}-13$ & $0.49+0.162 s^{2} 2 p^{3}\left(\frac{2}{3} D\right){ }^{2} D 3 d^{3} D^{\circ}+0.122 s^{2} 2 p^{3}\left({ }_{3}^{2} D\right)^{2} D 3 d^{1} D^{\circ}$ \\
\hline 21 & 70 & $2 s^{2} 2 p^{3}\left({ }_{3}^{2} D\right)^{2} D 3 d^{3} P_{0}^{\circ}$ & & 4365923 & $3.292 \mathrm{E}-13$ & $3.288 \mathrm{E}-13$ & $0.87+0.092 s^{2} 2 p^{3}\left(_{1}^{2} P\right)^{2} P 3 d^{3} P^{\circ}$ \\
\hline 21 & 71 & $2 s^{2} 2 p^{3}\left(\frac{2}{3} D\right)^{2} D 3 d^{1} D_{2}^{\circ}$ & \multirow{3}{*}{$\begin{array}{l}4367000 \\
4368000\end{array}$} & 4366138 & $4.531 \mathrm{E}-13$ & $4.528 \mathrm{E}-13$ & $0.42+0.282 s^{2} 2 p^{3}\left({ }_{1}^{2} P\right)^{2} P 3 d^{1} D^{\circ}+0.182 s^{2} 2 p^{3}\left({ }_{3}^{2} D\right)^{2} D 3 d^{3} P^{\circ}$ \\
\hline 21 & 72 & $2 s^{2} 2 p^{3}\left(\frac{2}{3} D\right)^{2} D 3 d^{3} P_{1}^{\circ}$ & & 4367909 & $2.508 \mathrm{E}-13$ & $2.506 \mathrm{E}-13$ & $0.86+0.052 s^{2} 2 p^{3}\left({ }_{1}^{2} P\right)^{2} P 3 d^{3} P^{\circ}+0.052 s^{2} 2 p^{3}\left({ }_{3}^{2} D\right)^{2} D 3 d^{3} S^{\circ}$ \\
\hline 21 & 73 & $2 s^{2} S 2 p^{4}\left({ }_{2}^{3} P\right)^{4} P 3 s^{5} P_{3}$ & & 4374152 & $6.560 \mathrm{E}-11$ & $6.543 \mathrm{E}-11$ & 0.98 \\
\hline 21 & 74 & $2 s^{2} 2 p^{3}\left(\frac{2}{3} D\right)^{2} D 3 d^{3} S_{1}^{\circ}$ & \multirow{8}{*}{$\begin{array}{l}4378000 \\
4395000\end{array}$} & 4376594 & $1.549 \mathrm{E}-13$ & $1.547 \mathrm{E}-13$ & $0.74+0.122 s^{2} 2 p^{3}\left({ }_{1}^{2} P\right)^{2} P 3 d^{3} P^{\circ}+0.082 s^{2} 2 p^{3}\left({ }_{3}^{2} D\right)^{2} D 3 d^{3} P^{\circ}$ \\
\hline 21 & 75 & $2 s^{2} 2 p^{3}\left(\frac{2}{3} D\right)^{2} D 3 d^{1} F_{3}^{\circ}$ & & 4393867 & $2.521 \mathrm{E}-13$ & $2.518 \mathrm{E}-13$ & $0.67+0.132 s^{2} 2 p^{3}\left(_{1}^{2} P\right)^{2} P 3 d^{3} F^{\circ}+0.082 s^{2} 2 p^{3}\left(_{1}^{2} P\right)^{2} P 3 d^{3} D^{\circ}$ \\
\hline 21 & 76 & $2 s^{2} S 2 p^{4}\left({ }_{2}^{3} P\right)^{4} P 3 s^{5} P_{2}$ & & 4394966 & 2.303E-11 & $2.308 \mathrm{E}-11$ & $0.95+0.032 s^{2} S 2 p^{4}\left({ }_{2}^{3} P\right)^{4} P 3 s^{3} P$ \\
\hline 21 & 77 & $2 s^{2} 2 p^{3}\left({ }_{1}^{2} P\right)^{2} P 3 d^{3} F_{2}^{\circ}$ & & 4403990 & $2.196 \mathrm{E}-12$ & 2.193E-12 & $0.75+0.102 s^{2} 2 p^{3}\left({ }_{3}^{2} D\right)^{2} D 3 d^{3} F^{\circ}+0.052 s^{2} 2 p^{3}\left({ }_{1}^{2} P\right)^{2} P 3 d^{3} D^{\circ}$ \\
\hline 21 & 78 & $2 s^{2} 2 p^{3}\left({ }_{1}^{2} P\right){ }^{2} P 3 d^{3} F_{3}^{\circ}$ & & 4405250 & $1.514 \mathrm{E}-12$ & $1.512 \mathrm{E}-12$ & $0.63+0.222 s^{2} 2 p^{3}\left({ }_{1}^{2} P\right)^{2} P 3 d^{3} D^{\circ}+0.052 s^{2} 2 p^{3}\left({ }_{3}^{2} D\right)^{2} D 3 d^{1} F^{\circ}$ \\
\hline 21 & 79 & $2 s^{2} S 2 p^{4}\left({ }_{2}^{3} P\right){ }^{4} P 3 s^{5} P_{1}$ & & 4407829 & $4.226 \mathrm{E}-11$ & $4.246 \mathrm{E}-11$ & $0.97+13+3$ \\
\hline 21 & 80 & $2 s^{2} 2 p^{3}\left({ }_{1}^{2} P\right)^{2} P 3 d^{3} F_{4}^{\circ}$ & & 4410058 & $2.722 \mathrm{E}-10$ & $2.725 \mathrm{E}-10$ & $0.81+0.072 s^{2} 2 p^{3}\left({ }_{3}^{2} D\right)^{2} D 3 d^{3} G^{\circ}+0.072 s^{2} 2 p^{3}\left({ }_{3}^{2} D\right)^{2} D 3 d^{1} G^{\circ}$ \\
\hline 21 & 81 & $2 s^{2} 2 p^{3}\left({ }_{1}^{2} P\right)^{2} P 3 d^{3} P_{0}^{\circ}$ & & 4412649 & $2.469 \mathrm{E}-13$ & $2.466 \mathrm{E}-13$ & $\left.0.81+0.112 s^{2} 2 p^{3}\left({ }_{3}^{2} D\right)\right)^{2} D 3 d^{3} P^{\circ}+0.042 s^{2} 2 p^{3}\left({ }_{3}^{2} D\right)^{2} D 3 d^{1} S^{\circ}$ \\
\hline 21 & 82 & $2 s^{2} 2 p^{3}\left({ }_{1}^{2} P\right)^{2} P 3 d^{3} P_{2}^{\circ}$ & 4407000 & 4415589 & $7.332 \mathrm{E}-13$ & $7.324 \mathrm{E}-13$ & $0.61+0.142 s^{2} 2 p^{3}\left({ }_{1}^{2} P\right)^{2} P 3 d^{3} D^{\circ}+0.072 s^{2} 2 p^{3}\left({ }_{3}^{2} D\right)^{2} D 3 d^{3} P^{\circ}$ \\
\hline 21 & 83 & $2 s^{2} 2 p^{3}\left({ }_{1}^{2} P\right)^{2} P 3 d^{3} P_{1}^{\circ}$ & 4419000 & 4416475 & $2.961 \mathrm{E}-13$ & 2.959E-13 & $0.70+0.182 s^{2} 2 p^{3}\left(\frac{1}{3} D\right)^{2} D 3 d^{3} s^{\circ}+0.052 s^{2} 2 p^{3}\left(\frac{2}{3} D\right)^{2} D 3 d^{1} P^{\circ}$ \\
\hline 21 & 84 & $2 s^{2} 2 p^{3}\left({ }_{1}^{2} P\right)^{2} P 3 d^{3} D_{2}^{\circ}$ & 4426000 & 4425016 & $2.622 \mathrm{E}-13$ & 2.619E-13 & $0.44+0.262 s^{2} 2 p^{3}\left({ }_{1}^{2} P\right)^{2} P 3 d^{1} D^{\circ}+0.092 s^{2} 2 p^{3}\left({ }_{3}^{3} D\right)^{2} D 3 d^{3} P^{\circ}$ \\
\hline 21 & 85 & $2 s^{2} 2 p^{3}\left({ }_{1}^{2} P\right)^{2} P 3 d^{3} D_{1}^{\circ}$ & 4432000 & 4432423 & $1.578 \mathrm{E}-13$ & $1.575 \mathrm{E}-13$ & $0.79+0.052 s^{2} 2 p^{3}\left({ }_{3}^{4} S\right)^{4} S 3 d^{3} D^{\circ}+0.052 s^{2} 2 p^{3}\left({ }_{3}^{2} D\right)^{2} D 3 d^{3} D^{\circ}$ \\
\hline 21 & 86 & $2 s^{2} 2 p^{3}\left({ }_{1}^{2} P\right)^{2} P 3 d^{3} D_{3}^{\circ}$ & 4433000 & 4435753 & $8.520 \mathrm{E}-13$ & $8.510 \mathrm{E}-13$ & $\left.0.56+0.172 s^{2} 2 p^{3}\left({ }_{1}^{2} P\right)^{2} P 3 d^{1} F^{\circ}+0.102 s^{2} 2 p^{3}{ }_{3}^{2} D\right)^{2} D 3 d^{1} F^{\circ}$ \\
\hline 21 & 87 & $2 s^{2} S 2 p^{4}\left({ }_{3}^{3} P\right)^{4} P 3 s^{3}{ }^{3} P_{2}$ & & 4438878 & $1.297 \mathrm{E}-12$ & $1.297 \mathrm{E}-12$ & $0.92+0.032 s^{2} S 2 p^{4}\left({ }_{2}^{3} P\right)^{4} P 3 s^{5} P$ \\
\hline 21 & 88 & $2 s^{2} 2 p^{3}\left({ }_{1}^{2} P\right)^{2} P 3 d{ }^{1} D_{2}^{\circ}$ & 4445000 & 4451019 & $1.804 \mathrm{E}-13$ & $1.801 \mathrm{E}-13$ & $0.31+0.312 s^{2} 2 p^{3}\left({ }_{3}^{2} D\right)^{2} D 3 d^{1} D^{\circ}+0.252 s^{2} 2 p^{3}\left({ }_{1}^{2} P\right)^{2} P 3 d^{3} D^{\circ}$ \\
\hline 21 & 89 & $2 s^{2} 2 p^{3}\left({ }_{1}^{2} P\right)^{2} P 3 d^{1} F_{3}^{\circ}$ & 4454000 & 4452673 & $1.192 \mathrm{E}-13$ & $1.191 \mathrm{E}-13$ & $0.67+0.172 s^{2} 2 p^{3}\left({ }_{3}^{3} D\right)^{2} D 3 d^{1} F^{\circ}+0.062 s^{2} 2 p^{3}\left({ }_{1}^{2} P\right)^{2} P 3 d^{3} D^{\circ}$ \\
\hline 21 & 90 & $2 s^{2} S 2 p^{4}\left({ }_{2}^{3} P\right){ }^{4} P 3 s^{3} P_{1}$ & & 4461581 & $1.211 \mathrm{E}-12$ & $1.212 \mathrm{E}-12$ & 0.94 \\
\hline 21 & 91 & $2 s^{2} S 2 p^{4}\left({ }_{2}^{3} P\right){ }^{4} P 3 s^{3} P_{0}$ & & 4470884 & $1.210 \mathrm{E}-12$ & $1.210 \mathrm{E}-12$ & 0.94 \\
\hline 21 & 92 & $2 s^{2} 2 p^{3}\left({ }_{1}^{2} P\right)^{2} P 3 d^{1} P_{1}^{\circ}$ & 4493000 & 4493897 & $1.164 \mathrm{E}-13$ & $1.162 \mathrm{E}-13$ & $\left.0.83+0.052 s^{2} 2 p^{3}\left({ }_{3}^{2} D\right)^{2} D 3 d^{1} P^{\circ}+0.022 s^{2} 2 p^{3}{ }_{1}^{2} P\right)^{2} P 3 d^{3} D^{\circ}$ \\
\hline 21 & 93 & $2 s^{2} S 2 p^{4}\left({ }_{2}^{3} P\right)^{4} P 3 p{ }^{5} P_{3}^{\circ}$ & & 4538967 & $6.904 \mathrm{E}-11$ & $6.854 \mathrm{E}-11$ & $0.84+0.142 s^{2} S 2 p^{4}\left({ }_{2}^{3} P\right)^{4} P 3 p^{5} D^{\circ}$ \\
\hline 21 & 94 & $2 s^{2} S 2 p^{4}\left({ }_{2}^{3} P\right){ }^{4} P 3 p^{5} P_{2}^{\circ}$ & & 4542036 & $3.238 \mathrm{E}-11$ & $3.225 \mathrm{E}-11$ & $\left.0.82+0.082 s^{2} S 2 p^{4}\left({ }_{2}^{3} P\right)^{4} P 3 p^{5} D^{\circ}+0.072 s^{2} S 2 p^{4}{ }_{2}^{3} P\right)^{4} P 3 p^{5} S^{\circ}$ \\
\hline 21 & 95 & $2 s^{2} S 2 p^{4}\left({ }_{2}^{3} P\right)^{4} P 3 p^{5} P_{1}^{\circ}$ & & 4555448 & 2.974E-11 & 2.967E-11 & $0.92+0.052 s^{2} S 2 p^{4}\left({ }_{2}^{3} P\right)^{4} P 3 p^{5} D^{\circ}$ \\
\hline 21 & 96 & $2 s^{2} S 2 p^{4}\left({ }_{2}^{2} P\right){ }^{4} P 3 p^{5} D_{4}^{\circ}$ & & 4558961 & $9.846 \mathrm{E}-11$ & $9.749 \mathrm{E}-11$ & $0.98+2+2+25$ \\
\hline 21 & 97 & $2 s^{2} S 2 p^{4}\left({ }_{2}^{3} P\right)^{4} P 3 p^{5} D_{3}^{\circ}$ & & 4569038 & 2.895E-12 & $2.889 \mathrm{E}-12$ & $0.55+0.322 s^{2} S 2 p^{4}\left({ }_{2}^{3} P\right)^{4} P 3 p^{3} D^{\circ}+0.112 s^{2} S 2 p^{4}\left({ }_{2}^{3} P\right){ }^{4} P 3 p^{5} P^{\circ}$ \\
\hline 21 & 98 & $2 s^{2} S 2 p^{4}\left({ }_{2}^{2} P\right){ }^{4} P 3 p^{5} D_{2}^{\circ}$ & & 4578850 & 9.817E-12 & $9.799 \mathrm{E}-12$ & $\left.0.80+0.102 s^{2} S 2 p^{4}\left({ }_{2}^{3} P\right)^{4} P 3 p^{3} D^{\circ}+0.062 s^{2} S 2 p^{4}{ }_{2}^{\frac{2}{3}} P\right)^{4} P 3 p^{5} P^{\circ}$ \\
\hline 21 & 99 & $2 s^{2} S 2 p^{4}\left({ }_{2}^{3} P\right){ }^{4} P 3 p^{5} D_{1}^{\circ}$ & & 4584148 & 3.083E-11 & 3.081E-11 & $0.91+0.052 s^{2} S 2 p^{4}\left({ }_{2}^{3} P\right)^{4} P 3 p^{5} P^{\circ}+0.022 s^{2} S 2 p^{4}\left({ }_{2}^{3} P\right){ }^{4} P 3 p^{3} D^{\circ}$ \\
\hline 21 & 100 & $2 s^{2} S 2 p^{4}\left({ }_{2}^{3} P\right)^{4} P 3 p p^{5} D_{0}^{\circ}$ & & 4585617 & 7.892E-11 & 7.897E-11 & $0.98-2+2$ \\
\hline 21 & 101 & $2 s^{2} S 2 p^{4}\left({ }_{2}^{3} P\right)^{4} P 3 p^{3} D_{3}^{\circ}$ & & 4590061 & $1.240 \mathrm{E}-12$ & $1.237 \mathrm{E}-12$ & $0.66+0.292 s^{2} S 2 p^{4}\left({ }_{2}^{3} P\right)^{4} P 3 p^{5} D^{\circ}+0.042 s^{2} S 2 p^{4}\left({ }_{2}^{3} P\right)^{4} P 3 p^{5} P^{\circ}$ \\
\hline 21 & 102 & $2 s^{2} S 2 p^{4}\left({ }_{2}^{\top} D\right)^{2} D 3 s^{3} D_{1}$ & & 4592820 & $1.911 \mathrm{E}-12$ & $1.913 \mathrm{E}-12$ & 0.96 \\
\hline 21 & 103 & $2 s^{2} S 2 p^{4}\left({ }_{2}^{4} D\right)^{2} D 3 s^{3} D_{2}$ & & 4593700 & $1.913 \mathrm{E}-12$ & $1.915 \mathrm{E}-12$ & 0.96 \\
\hline 21 & 104 & $2 s^{2} S 2 p^{4}\left({ }_{2}^{1} D\right)^{2} D 3 s^{3} D_{3}$ & & 4595087 & 1.864E-12 & $1.864 \mathrm{E}-12$ & 0.97 \\
\hline 21 & 105 & $2 s^{2} S 2 p^{4}\left({ }_{2}^{3} P\right){ }^{4} P 3 p p^{3} S_{1}^{\circ}$ & & 4600888 & $6.830 \mathrm{E}-13$ & $6.817 \mathrm{E}-13$ & $\left.0.65+0.232 s^{2} S 2 p^{4}{ }_{2}^{3} P\right)^{4} P 3 p^{3} P^{\circ}+0.042 s^{2} S 2 p^{4}\left({ }_{2}^{1} D\right)^{2} D 3 p^{3} P^{\circ}$ \\
\hline 21 & 106 & $2 s^{2} S 2 p^{4}\left({ }_{2}^{3} P\right)^{4} P 3 p^{3} D_{2}^{\circ}$ & & 4601459 & $9.218 \mathrm{E}-13$ & $9.195 \mathrm{E}-13$ & $0.64+0.222 s^{2} S 2 p^{4}\left({ }_{2}^{3} P\right)^{4} P 3 p^{3} P^{\circ}+0.092 s^{2} S 2 p^{4}\left({ }_{2}^{3} P\right){ }^{4} P 3 p^{5} D^{\circ}$ \\
\hline 21 & 107 & $2 s^{2} S 2 p^{4}\left({ }_{2}^{3} P\right)^{4} P 3 p^{5} S_{2}^{0}$ & & 4606742 & $5.587 \mathrm{E}-11$ & $5.602 \mathrm{E}-11$ & $0.88+0.092 s^{2} S 2 p^{4}\left({ }_{2}^{3} P\right)^{4} P 3 p^{5} P^{\circ}$ \\
\hline 21 & 108 & $2 s^{2} S 2 p^{4}\left(\frac{3}{2} P\right){ }^{4} P 3 p^{3} D_{1}^{\circ}$ & & 4616647 & $8.870 \mathrm{E}-13$ & $8.849 \mathrm{E}-13$ & $0.85+0.102 s^{2} S 2 p^{4}\left({ }_{2}^{3} P\right)^{4} P 3 p^{3} S^{\circ}$ \\
\hline 21 & 109 & $2 s^{2} S 2 p^{4}\left({ }_{2}^{3} P\right)^{4} P 3 p^{3} P_{2}^{\circ}$ & & 4621615 & $8.082 \mathrm{E}-13$ & $8.062 \mathrm{E}-13$ & $0.63+0.242 s^{2} S 2 p^{4}\left({ }_{2}^{3} P\right)^{4} P 3 p^{3} D^{\circ}+0.082 s^{2} S 2 p^{4}\left(\frac{1}{2} D\right)^{2} D 3 p^{3} P^{\circ}$ \\
\hline 21 & 110 & $2 s^{2} S 2 p^{4}\left({ }_{2}^{3} P\right)^{4} P 3 p^{3} P_{0}^{\circ}$ & & 4624939 & $8.881 \mathrm{E}-13$ & $8.859 \mathrm{E}-13$ & $0.82+0.152 s^{2} S 2 p^{4}\left({ }_{2}^{1} D\right)^{2} D 3 p^{3} P^{\circ}$ \\
\hline 21 & 111 & $2 s^{2} S 2 p^{4}\left({ }_{2}^{3} P\right){ }^{4} P 3 p^{3} P_{1}^{\circ}$ & & 4631418 & 7.653E-13 & 7.637E-13 & $0.57+0.232 s^{2} S 2 p^{4}\left({ }_{2}^{3} P\right)^{4} P 3 p^{3} s^{\circ}+0.082 s^{2} S 2 p^{4}\left({ }_{2}^{1} D\right)^{2} D 3 p^{3} P^{\circ}$ \\
\hline 21 & 112 & $2 s^{2} S 2 p^{4}\left({ }_{2}^{1} D\right)^{2} D 3 s^{1} D_{2}$ & & 4634113 & $3.362 \mathrm{E}-12$ & 3.363E-12 & $0.97-2+2$ \\
\hline 21 & 113 & $2 s^{2} S 2 p^{4}\left({ }_{0}^{1} S\right)^{2} S 3 s^{3} S_{1}$ & & 4714008 & $1.827 \mathrm{E}-12$ & $1.828 \mathrm{E}-12$ & $0.86+0.082 s^{2} S 2 p^{4}\left({ }_{2}^{3} P\right)^{2} P 3 s^{3} P+0.022 s^{2} S 2 p^{4}\left({ }_{2}^{3} P\right)^{2} P 3 s^{1} P$ \\
\hline 21 & 114 & $2 s^{2} S 2 p^{4}\left({ }_{0}^{1} S\right)^{2} S 3 s^{1} S_{0}$ & & 4742871 & $2.440 \mathrm{E}-12$ & 2.441E-12 & $0.65+0.332 s^{2} S 2 p^{4}\left(\frac{3}{3} P\right)^{2} P 3 s^{3} P$ \\
\hline 21 & 115 & $2 s^{2} S 2 p^{4}\left({ }_{2}^{3} P\right){ }^{4} P 3 d^{5} D_{4}$ & & 4744527 & $8.802 \mathrm{E}-11$ & $8.812 \mathrm{E}-11$ & $0.88+0.112 s^{2} S 2 p^{4}\left(3_{2}^{3} P\right)^{4} P 3 d^{5} F$ \\
\hline 21 & 116 & $2 s^{2} S 2 p^{4}\left({ }_{2}^{3} P\right){ }^{4} P 3 d^{5} D_{3}$ & & 4745708 & $8.391 \mathrm{E}-11$ & 8.425E-11 & $\left.0.87+0.072 s^{2} S 2 p^{4}{ }_{2}^{3} P\right)^{4} P 3 d^{5} F+0.052 s^{2} S 2 p^{4}\left({ }_{2}^{3} P\right)^{4} P 3 d^{5} P$ \\
\hline 21 & 117 & $2 s^{2} S 2 p^{4}\left({ }_{2}^{3} P\right){ }^{4} P 3 d^{5} D_{2}$ & & 4749551 & $6.765 \mathrm{E}-11$ & $6.792 \mathrm{E}-11$ & $\left.0.86+0.082 s^{2} S 2 p^{4}{ }_{2}^{4} P\right)^{4} P 3 d^{5} P+0.042 s^{2} S 2 p^{4}\left({ }_{2}^{4} P\right)^{4} P 3 d^{5} F$ \\
\hline 21 & 118 & $2 s^{2} S 2 p^{4}\left({ }_{2}^{3} P\right)^{2} P 3 s^{3} P_{2}$ & & 4750306 & 2.795E-12 & $2.797 \mathrm{E}-12$ & $0.96-2+2+2+2$ \\
\hline 21 & 119 & $2 s^{2} S 2 p^{4}\left({ }_{2}^{3} P\right){ }^{4} P 3 d^{5} D_{1}$ & & 4754667 & $5.504 \mathrm{E}-11$ & $5.537 \mathrm{E}-11$ & $0.89+0.072 s^{2} S 2 p^{4}\left({ }_{2}^{3} P\right)^{4} P 3 d^{5} P$ \\
\hline 21 & 120 & $2 s^{2} S 2 p^{4}\left({ }_{2}^{\mathrm{T}} D\right)^{2} D 3 p^{3} F_{2}^{\circ}$ & & 4757625 & 2.157E-11 & 2.153E-11 & 0.96 \\
\hline 21 & 121 & $2 s^{2} S 2 p^{4}\left({ }_{2}^{3} P\right){ }^{4} P 3 d^{5} D_{0}^{2}$ & & 4759214 & 4.423E-11 & $4.450 \mathrm{E}-11$ & 0.98 \\
\hline 21 & 122 & $2 s^{2} S 2 p^{4}\left({ }_{2}^{3} P\right)^{2} P 3 s^{3} P_{1}$ & & 4759339 & $1.250 \mathrm{E}-12$ & $1.252 \mathrm{E}-12$ & $0.51+0.442 s^{2} S 2 p^{4}\left({ }_{2}^{3} P\right)^{2} P 3 s^{1} P$ \\
\hline 21 & 123 & $2 s^{2} S 2 p^{4}\left(\frac{1}{2} D\right)^{2} D 3 p^{3} F_{3}^{\circ}$ & & 4761463 & $1.359 \mathrm{E}-11$ & $1.356 \mathrm{E}-11$ & $0.94+0.022 s^{2} S 2 p^{4}\left({ }_{2}^{1} D\right)^{2} D 3 p^{1} F^{\circ}$ \\
\hline 21 & 124 & $\left.2 s^{2} S 2 p^{4}{ }_{2}^{3} P\right)^{4} P 3 d^{5} F_{5}$ & & 4763942 & $1.408 \mathrm{E}-10$ & $1.351 \mathrm{E}-10$ & 0.99 \\
\hline 21 & 125 & $2 s^{2} S 2 p^{4}\left({ }_{2}^{1} D\right)^{2} D 3 p^{1} P_{1}^{\circ}$ & & 4764544 & $6.287 \mathrm{E}-13$ & $6.275 \mathrm{E}-13$ & $0.71+0.122 s^{2} S 2 p^{4}\left({ }_{2}^{1} D\right)^{2} D 3 p^{3} D^{\circ}+0.072 s^{2} S 2 p^{4}\left({ }_{2}^{3} P\right)^{2} P 3 p^{1} P^{\circ}$ \\
\hline 21 & 126 & $2 s^{2} S 2 p^{4}\left(\frac{1}{2} D\right)^{2} D 3 p^{3} F_{4}^{\circ}$ & & 4767652 & $3.974 \mathrm{E}-11$ & 3.953E-11 & 0.98 \\
\hline 21 & 127 & $2 s^{2} S 2 p^{4}\left({ }_{2}^{3} P\right)^{4} P 3 d^{5} F_{4}{ }^{4}$ & & 4777768 & $1.210 \mathrm{E}-10$ & $1.176 \mathrm{E}-10$ & $\left.0.81+0.092 s^{2} S 2 p^{4}{ }_{2}^{3} P\right)^{4} P 3 d^{5} D+0.092 s^{2} S 2 p^{4}\left({ }_{2}^{3} P\right){ }^{4} P 3 d^{3} F$ \\
\hline 21 & 128 & $2 s^{2} S 2 p^{4}\left({ }_{2}^{1} D\right)^{2} D 3 p^{1} F_{3}^{\circ}$ & & 4779151 & $6.815 \mathrm{E}-13$ & $6.798 \mathrm{E}-13$ & $0.87+0.072 s^{2} S 2 p^{4}\left({ }_{2}^{1} D\right){ }^{2} D 3 p^{3} D^{\circ}+0.032 s^{2} S 2 p^{4}\left({ }_{2}^{1} D\right)^{2} D 3 p^{3} F^{\circ}$ \\
\hline 21 & 129 & $2 s^{2} S 2 p^{4}\left({ }_{2}^{3} P\right)^{4} P 3 d^{5} F_{3}^{3}$ & & 4786562 & 1.659E-11 & $1.650 \mathrm{E}-11$ & $0.87+0.052 s^{2} S 2 p^{4}\left({ }_{2}^{3} P\right){ }^{4} P 3 d^{5} D+0.042 s^{2} S 2 p^{4}\left({ }_{2}^{3} P\right){ }^{4} P 3 d^{3} F$ \\
\hline 21 & 130 & $2 s^{2} S 2 p^{4}\left({ }_{2}^{3} P\right){ }^{4} P 3 d^{5} P_{1}$ & & 4786794 & $1.268 \mathrm{E}-11$ & $1.273 \mathrm{E}-11$ & $0.90+0.062 s^{2} S 2 p^{4}\left({ }_{2}^{3} P\right)^{4} P 3 d^{5} D$ \\
\hline 21 & 131 & $2 s^{2} S 2 p^{4}\left({ }_{2}^{\dagger} D\right)^{2} D 3 p^{3} D_{2}^{\circ}$ & & 4787224 & $1.409 \mathrm{E}-11$ & $1.406 \mathrm{E}-11$ & 0.93 \\
\hline 21 & 132 & $2 s^{2} S 2 p^{4}\left({ }_{2}^{1} D\right)^{2} D 3 p^{3} D_{1}^{\circ}$ & & 4789103 & $3.346 \mathrm{E}-12$ & $3.339 \mathrm{E}-12$ & $0.85+0.122 s^{2} S 2 p^{4}\left({ }_{2}^{1} D\right)^{2} D 3 p^{1} P^{\circ}$ \\
\hline 21 & 133 & $2 s^{2} S 2 p^{4}\left({ }_{2}^{3} P\right)^{2} P 3 s^{1} P_{1}$ & & 4790390 & 1.065E-12 & $1.065 \mathrm{E}-12$ & $0.50+0.382 s^{2} S 2 p^{4}\left({ }_{2}^{3} P\right)^{2} P 3 s^{3} P+0.092 s^{2} S 2 p^{4}\left({ }_{0}^{1} S\right)^{2} S 3 s^{3} S$ \\
\hline 21 & 134 & $2 s^{2} S 2 p^{4}\left({ }_{2}^{3} P\right){ }^{4} P 3 d^{5} F_{2}$ & & 4791628 & $1.161 \mathrm{E}-11$ & $1.159 \mathrm{E}-11$ & $0.75+0.222 s^{2} S 2 p^{4}\left({ }_{2}^{3} P\right)^{4} P 3 d^{5} P$ \\
\hline 21 & 135 & $2 s^{2} S 2 p^{4}\left({ }_{2}^{1} D\right)^{2} D 3 p^{3} D_{3}^{\circ}$ & & 4791719 & $6.264 \mathrm{E}-12$ & $6.247 \mathrm{E}-12$ & $0.89+0.082 s^{2} S 2 p^{4}\left({ }_{2}^{1} D\right)^{2} D 3 p^{1} F^{\circ}$ \\
\hline 21 & 136 & $2 s^{2} S 2 p^{4}\left({ }_{2}^{3} P\right)^{2} P 3 s^{3} P_{0}{ }^{3}$ & & 4793193 & 2.915E-12 & $2.916 \mathrm{E}-12$ & $0.65+0.322 s^{2} S 2 p^{4}\left({ }_{0}^{1} S\right)^{2} S 3 s^{1} S$ \\
\hline 21 & 137 & $2 s^{2} S 2 p^{4}\left({ }_{2}^{3} P\right)^{4} P 3 d^{5} F_{1}$ & & 4796303 & 2.495E-11 & $2.476 \mathrm{E}-11$ & $0.95+0.022 s^{2} S 2 p^{4}\left(\frac{3}{2} P\right)^{4} P 3 d^{5} D$ \\
\hline 21 & 138 & $2 s^{2} S 2 p^{4}\left({ }_{2}^{3} P\right){ }^{4} P 3 d^{5} P_{2}$ & & 4796840 & $2.250 \mathrm{E}-11$ & $2.268 \mathrm{E}-11$ & $\left.0.68+0.192 s^{2} S 2 p^{4}\left({ }_{2}^{3} P\right)\right)^{4} P 3 d^{5} F+0.112 s^{2} S 2 p^{4}\left({ }_{2}^{3} P\right)^{4} P 3 d^{5} D$ \\
\hline 21 & 139 & $2 s^{2} S 2 p^{4}\left({ }_{2}^{1} D\right)^{2} D 3 p^{1} D_{2}^{\circ}$ & & 4800518 & $9.145 \mathrm{E}-13$ & $9.119 \mathrm{E}-13$ & $0.55+0.372 s^{2} S 2 p^{4}\left({ }_{2}^{1} D\right)^{2} D 3 p^{3} P^{\circ}+0.032 s^{2} S 2 p^{4}\left({ }_{2}^{3} P\right)^{2} P 3 p^{3} P^{\circ}$ \\
\hline 21 & 140 & $2 s^{2} S 2 p^{4}\left({ }_{2}^{3} P\right){ }^{4} P 3 d^{3} F_{4}{ }^{2}$ & & 4805245 & 1.040E-10 & $1.017 \mathrm{E}-10$ & $\left.\left.0.89+0.072 s^{2} S 2 p^{4} \int_{2}^{2} P\right)^{4} P 3 d^{5} F+0.022 s^{2} S 2 p^{4}{ }_{2}^{3} P\right)^{4} P 3 d^{5} D$ \\
\hline 21 & 141 & $2 s^{2} S 2 p^{4}\left({ }_{2}^{2} P\right){ }^{4} P 3 d^{5} P_{3}$ & & 4805789 & $9.424 \mathrm{E}-12$ & $9.464 \mathrm{E}-12$ & $\left.0.89+0.062 s^{2} S 2 p^{4} \int_{2}^{2} P\right)^{4} P 3 d^{5} D$ \\
\hline 21 & 142 & $2 s^{2} S 2 p^{4}\left({ }_{2}^{1} D\right)^{2} D 3 p^{3} P_{1}^{\circ}$ & & 4806620 & $2.164 \mathrm{E}-12$ & $2.155 \mathrm{E}-12$ & $\left.0.76+0.112 s^{2} S 2 p^{4}{ }_{2}^{3} P\right)^{4} P 3 p^{3} P^{\circ}+0.072 s^{2} S 2 p^{4}\left({ }_{2}^{3} P\right)^{2} P 3 p^{3} P^{\circ}$ \\
\hline 21 & 143 & $2 s^{2} S 2 p^{4}\left({ }_{2}^{1} D\right)^{2} D 3 p^{3} P_{2}^{\circ}$ & & 4806975 & $1.024 \mathrm{E}-12$ & $1.021 \mathrm{E}-12$ & $\left.0.47+0.422 s^{2} S 2 p^{4}\left({ }_{2}^{1} D\right)^{2} D 3 p^{1} D^{\circ}+0.072 s^{2} S 2 p^{4}{ }_{2}^{3} P\right)^{4} P 3 p^{3} P^{\circ}$ \\
\hline 21 & 144 & $2 s^{2} S 2 p^{4}\left({ }_{2}^{1} D\right)^{2} D 3 p^{3} P_{0}^{\circ}$ & & 4808875 & 2.024E-12 & $2.016 \mathrm{E}-12$ & $\left.0.75+0.132 s^{2} S 2 p^{4}{ }_{2}^{3} P\right)^{4} P 3 p^{3} P^{\circ}+0.102 s^{2} S 2 p^{4}\left({ }_{2}^{2} P\right)^{2} P 3 p^{3} P^{\circ}$ \\
\hline 21 & 145 & $2 s^{2} S 2 p^{4}\left({ }_{2}^{3} P\right){ }^{4} P 3 d^{3} F_{3}$ & & 4816804 & $2.107 \mathrm{E}-12$ & $2.104 \mathrm{E}-12$ & $\left.0.81+0.112 s^{2} S 2 p^{4}\left({ }_{2}^{4} P\right){ }^{4} P 3 d^{3} D+0.042 s^{2} S 2 p^{4} \int_{2}^{2} P\right){ }^{4} P 3 d^{5} F$ \\
\hline 21 & 146 & $2 s^{2} S 2 p^{4}\left({ }_{2}^{3} P\right){ }^{4} P 3 d^{3} F_{2}$ & & 4826685 & $8.614 \mathrm{E}-13$ & $8.604 \mathrm{E}-13$ & $0.64+0.232 s^{2} S 2 p^{4}\left({ }_{2}^{3} P\right)^{4} P 3 d^{3} D+0.072 s^{2} S 2 p^{4}\left({ }_{2}^{3} P\right)^{4} P 3 d^{3} P$ \\
\hline 21 & 147 & $2 s^{2} S 2 p^{4}\left({ }_{2}^{3} P\right){ }^{4} P 3 d^{3} P_{0}$ & & 4828408 & 7.012E-13 & $7.008 \mathrm{E}-13$ & $0.88+0.082 s^{2} S 2 p^{4}\left({ }_{2}^{4} D\right)^{2} D 3 d^{3} P$ \\
\hline 21 & 148 & $2 s^{2} S 2 p^{4}\left({ }_{2}^{3} P\right){ }^{4} P 3 d^{3} P_{1}$ & & 4830688 & $4.877 \mathrm{E}-13$ & $4.874 \mathrm{E}-13$ & $0.71+0.192 s^{2} S 2 p^{4}\left({ }_{2}^{3} P\right)^{4} P 3 d^{3} D+0.062 s^{2} S 2 p^{4}\left({ }_{2}^{1} D\right)^{2} D 3 d^{3} P$ \\
\hline 21 & 149 & $2 s^{2} S 2 p^{4}\left({ }_{2}^{3} P\right){ }^{4} P 3 d^{3} D_{2}$ & & 4840326 & $5.027 \mathrm{E}-13$ & $5.023 \mathrm{E}-13$ & $0.28+0.352 s^{2} S 2 p^{4}\left({ }_{2}^{3} P\right)^{4} P 3 d^{3} P+0.302 s^{2} S 2 p^{4}\left({ }_{2}^{3} P\right)^{4} P 3 d^{3} F$ \\
\hline 21 & 150 & $2 s^{2} S 2 p^{4}\left({ }_{2}^{3} P\right){ }^{4} P 3 d^{3} D_{3}$ & & 4849166 & $2.520 \mathrm{E}-13$ & $2.517 \mathrm{E}-13$ & $0.81+0.122 s^{2} S 2 p^{4}\left(c_{2}^{3} P\right)^{4} P 3 d^{3} F+0.032 s^{2} S 2 p^{4}\left({ }_{2}^{1} D\right)^{2} D 3 d^{3} D$ \\
\hline 21 & 151 & $2 s^{2} S 2 p^{4}\left({ }_{2}^{3} P\right){ }^{4} P 3 d^{3} D_{1}$ & & 4851329 & $3.258 \mathrm{E}-13$ & $3.255 \mathrm{E}-13$ & $0.70+0.182 s^{2} S 2 p^{4}\left({ }_{2}^{3} P\right)^{4} P 3 d^{3} P+0.062 s^{2} S 2 p^{4}\left({ }_{2}^{1} D\right)^{2} D 3 d^{3} D$ \\
\hline
\end{tabular}




\begin{tabular}{|c|c|c|c|c|c|c|c|}
\hline $\mathrm{z}$ & Key & Level & $E_{\mathrm{NIST}}$ & $E_{\mathrm{MCDHF} / \mathrm{RCI}}$ & $\tau_{\mathrm{MCDHF} / \mathrm{RCI}}^{l}$ & $\tau_{\mathrm{MCDHF} / \mathrm{RCI}}^{v}$ & $L S$-composition \\
\hline 21 & 152 & $2 s^{2} S 2 p^{4}\left({ }_{2}^{3} P\right)^{4} P 3 d^{3} P_{2}$ & & 4860225 & 3.935E-13 & $3.932 \mathrm{E}-13$ & $0.49+0.392 s^{2} S 2 p^{4}\left({ }_{2}^{3} P\right)^{4} P 3 d^{3} D+0.042 s^{2} S 2 p^{4}\left({ }_{2}^{1} D\right)^{2} D 3 d^{3} P$ \\
\hline 21 & 153 & $2 s^{2} S 2 p^{4}\left({ }_{0}^{1} S\right)^{2} S 3 p^{3} P_{0}^{\circ}$ & & 4883685 & 4.030E-12 & 4.029E-12 & $0.72+0.202 s^{2} S 2 p^{4}\left({ }_{2}^{3} P\right)^{2} P 3 p^{3} P^{\circ}+0.032 s^{2} S 2 p^{4}\left({ }_{2}^{1} D\right)^{2} D 3 p^{3} P^{\circ}$ \\
\hline 21 & 154 & $2 s^{2} S 2 p^{4}\left({ }_{0}^{1} S\right)^{2} S 3 p^{3} P_{1}^{\circ}$ & & 4888762 & $3.206 \mathrm{E}-12$ & 3.204E-12 & $\left.0.70+0.142 s^{2} S 2 p^{4}\left({ }_{2}^{3} P\right)^{2} P 3 p^{3} P^{\circ}+0.082 s^{2} S 2 p^{4}{ }_{2}^{3} P\right)^{2} P 3 p^{3} D^{\circ}$ \\
\hline 21 & 155 & $2 s^{2} S 2 p^{4}\left({ }_{0}^{9} S\right)^{2} S 3 p^{3} P_{2}^{\circ}$ & & 4899414 & $4.566 \mathrm{E}-12$ & $4.561 \mathrm{E}-12$ & $0.77+0.142 s^{2} S 2 p^{4}\left({ }_{2}^{3} P\right)^{2} P 3 p^{3} D^{\circ}+0.032 s^{2} S 2 p^{4}\left({ }_{2}^{3} P\right)^{2} P 3 p^{3} P^{\circ}$ \\
\hline 21 & 156 & $2 s^{2} S 2 p^{4}\left({ }_{0}^{1} S\right)^{2} S 3 p^{1} P_{1}^{\circ}$ & & 4906675 & 7.415E-13 & 7.397E-13 & $0.71+0.092 s^{2} S 2 p^{4}\left({ }_{2}^{3} P\right)^{2} P 3 p^{3} D^{\circ}+0.092 s^{2} S 2 p^{4}\left({ }_{2}^{3} P\right)^{2} P 3 p^{3} P^{\circ}$ \\
\hline 21 & 157 & $2 s^{2} S 2 p^{4}\left({ }_{2}^{3} P\right)^{2} P 3 p^{3} P_{2}^{\circ}$ & & 4916836 & 3.072E-12 & $3.068 \mathrm{E}-12$ & $0.69+0.102 s^{2} S 2 p^{4}\left({ }_{2}^{3} P\right)^{2} P 3 p^{3} D^{\circ}+0.092 s^{2} S 2 p^{4}\left({ }_{0}^{1} S\right)^{2} S 3 p^{3} P^{\circ}$ \\
\hline 21 & 158 & $\left.2 s^{2} S 2 p^{4}{ }_{2}^{2} P\right)^{2} P 3 p^{3} D_{2}^{\circ}$ & & 4928096 & $2.511 \mathrm{E}-12$ & $2.507 \mathrm{E}-12$ & $0.36+0.452 s^{2} S 2 p^{4}\left({ }_{2}^{3} P\right)^{2} P 3 p^{1} D^{\circ}+0.142 s^{2} S 2 p^{4}\left({ }_{2}^{3} P\right)^{2} P 3 p^{3} P^{\circ}$ \\
\hline 21 & 159 & $2 s^{2} S 2 p^{4}\left({ }_{2}^{3} P\right)^{2} P 3 p^{3} D_{3}^{\circ}$ & & 4928286 & $1.493 \mathrm{E}-12$ & $1.490 \mathrm{E}-12$ & 0.97 \\
\hline 21 & 160 & $2 s^{2} S 2 p^{4}\left({ }_{2}^{3} P\right)^{2} P 3 p^{3} P_{1}^{\circ}$ & & 4932988 & $3.381 \mathrm{E}-12$ & $3.376 \mathrm{E}-12$ & $0.48+0.192 s^{2} S 2 p^{4}\left({ }_{0}^{1} S\right)^{2} S 3 p^{3} P^{\circ}+0.152 s^{2} S 2 p^{4}\left({ }_{2}^{3} P\right)^{2} P 3 p^{3} S^{\circ}$ \\
\hline 21 & 161 & $\left.2 s^{2} S 2 p^{4}{ }_{3}^{3} P\right)^{2} P 3 p^{3} P_{0}^{\circ}$ & & 4942037 & $5.803 \mathrm{E}-12$ & $5.802 \mathrm{E}-12$ & $0.46+0.332 s^{2} S 2 p^{4}\left({ }_{2}^{3} P\right)^{2} P 3 p^{1} S^{\circ}+0.112 s^{2} S 2 p^{4}\left({ }_{0}^{1} S\right)^{2} S 3 p^{3} P^{\circ}$ \\
\hline 21 & 162 & $2 s^{2} S 2 p^{4}\left({ }_{2}^{2} P\right)^{2} P 3 p^{3} D_{1}^{\circ}$ & & 4951260 & $1.290 \mathrm{E}-12$ & $1.287 \mathrm{E}-12$ & $0.70+0.102 s^{2} S 2 p^{4}\left({ }_{2}^{3} P\right)^{2} P 3 p^{3} S^{\circ}+0.092 s^{2} S 2 p^{4}\left({ }_{0}^{1} S\right)^{2} S 3 p^{1} P^{\circ}$ \\
\hline 21 & 163 & $2 s^{2} S 2 p^{4}\left({ }_{2}^{1} D\right)^{2} D 3 d^{3} G_{3}$ & & 4962682 & $5.009 \mathrm{E}-11$ & $4.941 \mathrm{E}-11$ & 0.98 \\
\hline 21 & 164 & $2 s^{2} S 2 p^{4}\left(\frac{1}{2} D\right)^{2} D 3 d^{3} G_{4}$ & & 4964211 & $5.196 \mathrm{E}-11$ & $5.120 \mathrm{E}-11$ & 0.98 \\
\hline 21 & 165 & $2 s^{2} S 2 p^{4}\left({ }_{2}^{3} P\right)^{2} P 3 p^{1} D_{2}^{\circ}$ & & 4965258 & $3.190 \mathrm{E}-12$ & $3.185 \mathrm{E}-12$ & $0.47+0.362 s^{2} S 2 p^{4}\left({ }_{2}^{3} P\right)^{2} P 3 p^{3} D^{\circ}+0.112 s^{2} S 2 p^{4}\left({ }_{0}^{1} S\right)^{2} S 3 p^{3} P^{\circ}$ \\
\hline 21 & 166 & $2 s^{2} S 2 p^{4}\left(\frac{1}{2} D\right)^{2} D 3 d^{3} G_{5}^{2}$ & & 4966132 & $5.416 \mathrm{E}-11$ & $5.338 \mathrm{E}-11$ & 0.98 \\
\hline 21 & 167 & $2 s^{2} S 2 p^{4}\left({ }_{2}^{2} P\right)^{2} P 3 p p^{1} S_{0}^{0}$ & & 4967632 & $8.959 \mathrm{E}-12$ & $8.960 \mathrm{E}-12$ & $0.64+0.192 s^{2} S 2 p^{4}\left({ }_{2}^{3} P\right)^{2} P 3 p^{3} P^{\circ}+0.142 s^{2} S 2 p^{4}\left({ }_{0}^{1} S\right)^{2} S 3 p^{3} P^{\circ}$ \\
\hline 21 & 168 & $\left.2 s^{2} S 2 p^{4}{ }_{2}^{3} P\right)^{2} P 3 p^{3} S_{1}^{0}$ & & 4973943 & $1.618 \mathrm{E}-12$ & $1.612 \mathrm{E}-12$ & $0.69+0.122 s^{2} S 2 p^{4}\left({ }_{2}^{3} P\right)^{2} P 3 p^{3} P^{\circ}+0.092 s^{2} S 2 p^{4}\left({ }_{0}^{1} S\right)^{2} S 3 p^{1} P^{\circ}$ \\
\hline 21 & 169 & $2 s^{2} S 2 p^{4}\left({ }_{2}^{1} D\right)^{2} D 3 d^{3} s_{1}$ & & 4991390 & 1.433E-13 & $1.433 \mathrm{E}-13$ & 0.96 \\
\hline 21 & 170 & $2 s^{2} S 2 p^{4}\left({ }_{2}^{2} D\right)^{2} D 3 d^{3} F_{2}$ & & 4992191 & $2.066 \mathrm{E}-11$ & $2.072 \mathrm{E}-11$ & 0.95 \\
\hline 21 & 171 & $2 s^{2} S 2 p^{4}\left(\frac{1}{2} D\right)^{2} D 3 d^{3} F_{3}$ & & 4993821 & $1.006 \mathrm{E}-11$ & $1.006 \mathrm{E}-11$ & 0.96 \\
\hline 21 & 172 & $2 s^{2} S 2 p^{4}\left({ }_{2}^{1} D\right)^{2} D 3 d^{3} F_{4}$ & & 4994695 & 4.434E-11 & 4.433E-11 & $0.78+0.202 s^{2} S 2 p^{4}\left(\frac{1}{2} D\right)^{2} D 3 d^{1} G$ \\
\hline 21 & 173 & $2 s^{2} S 2 p^{4}\left(\frac{1}{2} D\right)^{2} D 3 d^{1} G_{4}$ & & 4999301 & 4.091E-11 & $4.080 \mathrm{E}-11$ & $0.78+0.202 s^{2} S 2 p^{4}\left({ }_{2}^{1} D\right)^{2} D 3 d^{3} F$ \\
\hline 21 & 174 & $2 s^{2} S 2 p^{4}\left({ }_{2}^{1} D\right)^{2} D 3 d^{3} D_{1}$ & & 4999907 & $2.751 \mathrm{E}-13$ & $2.749 \mathrm{E}-13$ & $0.56+0.172 s^{2} S 2 p^{4}\left({ }_{2}^{1} D\right)^{2} D 3 d^{1} P+0.162 s^{2} S 2 p^{4}\left({ }_{2}^{1} D\right)^{2} D 3 d^{3} P$ \\
\hline 21 & 175 & $2 s^{2} S 2 p^{4}\left(\frac{1}{2} D\right)^{2} D 3 d^{3} P_{2}$ & & 5001362 & $1.663 \mathrm{E}-13$ & $1.663 \mathrm{E}-13$ & $0.72+0.182 s^{2} S 2 p^{4}\left({ }_{2}^{1} D\right)^{2} D 3 d^{3} D+0.052 s^{2} S 2 p^{4}\left({ }_{2}^{3} P\right)^{4} P 3 d^{3} P$ \\
\hline 21 & 176 & $2 s^{2} S 2 p^{4}\left(\frac{1}{1} D\right)^{2} D 3 d^{3} P_{0}$ & & 5001579 & $1.604 \mathrm{E}-13$ & 1.603E-13 & $0.82+0.072 s^{2} S 2 p^{4}\left({ }_{2}^{2} P\right)^{4} P 3 d^{3} P+0.062 s^{2} S 2 p^{4}\left({ }_{2}^{1} D\right)^{2} D 3 d^{1} S$ \\
\hline 21 & 177 & $2 s^{2} S 2 p^{4}\left({ }_{2}^{1} D\right)^{2} D 3 d^{3} D_{3}$ & & 5001858 & $2.496 \mathrm{E}-13$ & 2.494E-13 & $0.91+0.042 s^{2} S 2 p^{4}\left({ }_{2}^{3} P\right)^{4} P 3 d^{3} D$ \\
\hline 21 & 178 & $2 s^{2} S 2 p^{4}\left({ }_{2}^{1} D\right)^{2} D 3 d^{3} D_{2}$ & & 5002219 & 2.173E-13 & $2.171 \mathrm{E}-13$ & $0.71+0.182 s^{2} S 2 p^{4}\left({ }_{2}^{1} D\right)^{2} D 3 d^{3} P+0.052 s^{2} S 2 p^{4}\left({ }_{2}^{3} P\right)^{4} P 3 d^{3} D$ \\
\hline 21 & 179 & $2 s^{2} S 2 p^{4}\left(\frac{1}{2} D\right)^{2} D 3 d^{3} P_{1}$ & & 5004179 & $1.586 \mathrm{E}-13$ & $1.586 \mathrm{E}-13$ & $0.71+0.152 s^{2} S 2 p^{4}\left({ }_{2}^{1} D\right)^{2} D 3 d^{3} D+0.072 s^{2} S 2 p^{4}\left(\int_{2}^{3} P\right)^{4} P 3 d^{3} P$ \\
\hline 21 & 180 & $2 s^{2} S 2 p^{4}\left({ }_{2}^{1} D\right)^{2} D 3 d^{1} P_{1}$ & & 5015078 & 3.785E-13 & $3.782 \mathrm{E}-13$ & $\left.\left.0.73+0.162 s^{2} S 2 p^{4}\left({ }_{2}^{1} D\right)\right)^{2} D 3 d^{3} D+0.052 s^{2} S 2 p^{4}{ }_{2}^{3} P\right)^{2} P 3 d^{1} P$ \\
\hline 21 & 181 & $2 s^{2} S 2 p^{4}\left(\frac{1}{2} D\right){ }^{2} D 3 d^{1} D_{2}$ & & 5019167 & $1.056 \mathrm{E}-12$ & $1.054 \mathrm{E}-12$ & $0.84+0.052 s^{2} S 2 p^{4}\left({ }_{2}^{2} P\right)^{2} P 3 d^{1} D+0.032 s^{2} S 2 p^{4}\left({ }_{2}^{2} D\right)^{2} D 3 d^{3} F$ \\
\hline 21 & 182 & $2 s^{2} S 2 p^{4}\left({ }_{2}^{1} D\right)^{2} D 3 d^{1} F_{3}$ & & 5026368 & 4.695E-12 & $4.700 \mathrm{E}-12$ & 0.96 \\
\hline 21 & 183 & $2 s^{2} S 2 p^{4}\left({ }_{2}^{1} D\right)^{2} D 3 d^{1} s_{0}$ & & 5031212 & $1.674 \mathrm{E}-13$ & $1.673 \mathrm{E}-13$ & $0.90+0.052 s^{2} S 2 p^{4}\left({ }_{2}^{1} D\right)^{2} D 3 d^{3} P+0.022 s^{2} S 2 p^{4}\left({ }_{2}^{3} P\right){ }^{4} P 3 d^{3} P$ \\
\hline 21 & 184 & $2 s^{2} S 2 p^{4}\left({ }_{2}^{3} P\right)^{2} P 3 p^{1} P_{1}^{\circ}$ & & 5031354 & 2.835E-12 & 2.825E-12 & $0.84+0.072 s^{2} S 2 p^{4}\left({ }_{2}^{1} D\right)^{2} D 3 p^{1} P^{\circ}+0.032 s^{2} S 2 p^{4}\left({ }_{0}^{1} S\right)^{2} S 3 p^{1} P^{\circ}$ \\
\hline 21 & 185 & $2 s^{2} S 2 p^{4}\left({ }_{0}^{1} S\right)^{2} S 3 d^{3} D_{1}$ & & 5098972 & 6.817E-12 & 6.837E-12 & $0.67+0.272 s^{2} S 2 p^{4}\left({ }_{2}^{3} P\right)^{2} P 3 d^{3} D+0.032 s^{2} S 2 p^{4}\left({ }_{2}^{1} D\right)^{2} D 3 d^{3} D$ \\
\hline 21 & 186 & $2 s^{2} S 2 p^{4}\left({ }_{0}^{1} S\right)^{2} S 3 d^{3} D_{2}$ & & 5102500 & $3.245 \mathrm{E}-12$ & $3.248 \mathrm{E}-12$ & $0.73+0.152 s^{2} S 2 p^{4}\left(3_{2}^{3} P\right)^{2} P 3 d^{3} D+0.062 s^{2} S 2 p^{4}\left(\int_{2}^{3} P\right)^{2} P 3 d^{3} F$ \\
\hline 21 & 187 & $2 s^{2} S 2 p^{4}\left({ }_{0}^{1} S\right)^{2} S 3 d^{3} D_{3}$ & & 5105057 & $6.902 \mathrm{E}-13$ & $6.896 \mathrm{E}-13$ & $0.85+0.062 s^{2} S 2 p^{4}\left({ }_{2}^{3} P\right)^{2} P 3 d^{3} F+0.032 s^{2} S 2 p^{4}\left(_{2}^{3} P\right)^{2} P 3 d^{1} F$ \\
\hline 21 & 188 & $2 s^{2} S 2 p^{4}\left(3^{3} P\right)^{2} P 3 d^{3} D_{3}$ & & 5117487 & $7.519 \mathrm{E}-13$ & $7.515 \mathrm{E}-13$ & $0.87+0.052 s^{2} S 2 p^{4}\left(_{3}^{3} P\right)^{2} P 3 d^{3} F$ \\
\hline 21 & 189 & $2 s^{2} S 2 p^{4}\left({ }_{2}^{3} P\right)^{2} P 3 d^{3} D_{2}$ & & 5121252 & $4.374 \mathrm{E}-13$ & $4.369 \mathrm{E}-13$ & $0.52+0.252 s^{2} S 2 p^{4}\left(c_{0}^{1} S\right)^{2} S 3 d^{1} D+0.082 s^{2} S 2 p^{4}\left(1_{0}^{1} S\right)^{2} S 3 d^{3} D$ \\
\hline 21 & 190 & $2 s^{2} S 2 p^{4}\left({ }_{0}^{1} S\right)^{2} S 3 d^{1} D_{2}$ & & 5130818 & $1.056 \mathrm{E}-12$ & $1.056 \mathrm{E}-12$ & $\left.0.40+0.212 s^{2} S 2 p^{4}\left({ }_{2}^{3} P\right)^{2} P 3 d^{3} P+0.172 s^{2} S 2 p^{4}{ }_{2}^{3} P\right)^{2} P 3 d^{3} D$ \\
\hline 21 & 191 & $2 s^{2} S 2 p^{4}\left({ }_{2}^{3} P\right)^{2} P 3 d^{3} D_{1}$ & & 5134948 & $2.798 \mathrm{E}-13$ & $2.795 \mathrm{E}-13$ & $0.56+0.202 s^{2} S 2 p^{4}\left({ }_{0}^{1} S\right)^{2} S 3 d^{3} D+0.152 s^{2} S 2 p^{4}\left({ }_{2}^{3} P\right)^{2} P 3 d^{3} P$ \\
\hline 21 & 192 & $2 s^{2} S 2 p^{4}\left({ }_{2}^{3} P\right)^{2} P 3 d^{3} F_{4}$ & & 5139100 & $1.160 \mathrm{E}-11$ & $1.180 \mathrm{E}-11$ & 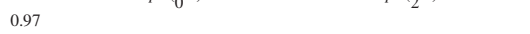 \\
\hline 21 & 193 & $2 s^{2} S 2 p^{4}\left({ }_{2}^{2} P\right)^{2} P 3 d^{1} F_{3}$ & & 5139900 & $1.191 \mathrm{E}-11$ & $1.207 \mathrm{E}-11$ & $0.51+0.452 s^{2} S 2 p^{4}\left({ }_{2}^{3} P\right)^{2} P 3 d^{3} F$ \\
\hline 21 & 194 & $2 s^{2} S 2 p^{4}\left({ }_{2}^{3} P\right)^{2} P 3 d^{3} P_{0}$ & & 5153564 & $6.538 \mathrm{E}-13$ & $6.533 \mathrm{E}-13$ & $0.94+0.022 s^{2} S 2 p^{4}\left({ }_{2}^{1} D\right)^{2} D 3 d^{3} P$ \\
\hline 21 & 195 & $2 s^{2} S 2 p^{4}\left({ }_{2}^{3} P\right)^{2} P 3 d^{3} P_{1}$ & & 5159398 & 5.083E-13 & $5.079 \mathrm{E}-13$ & $\left.0.75+0.082 s^{2} S 2 p^{4}{ }_{2}^{3} P\right)^{2} P 3 d^{1} P+0.072 s^{2} S 2 p^{4}\left({ }_{2}^{3} P\right)^{2} P 3 d^{3} D$ \\
\hline 21 & 196 & $2 s^{2} S 2 p^{4}\left({ }_{2}^{3} P\right)^{2} P 3 d^{3} F_{2}$ & & 5160538 & $6.959 \mathrm{E}-13$ & $6.955 \mathrm{E}-13$ & $\left.0.60+0.182 s^{2} S 2 p^{4}{ }_{2}^{3} P\right)^{2} P 3 d^{3} P+0.122 s^{2} S 2 p^{4}\left({ }_{2}^{3} P\right)^{2} P 3 d^{1} D$ \\
\hline 22 & 1 & $2 s^{2} 2 p^{4}\left({ }_{2}^{3} P\right){ }^{2} P_{2}$ & 0 & 0 & $\ldots$ & $\ldots$ & $0.95+0.052 s^{2} 2 p^{4}\left(2_{2}^{1} D\right){ }^{1} D$ \\
\hline 22 & 2 & $2 s^{2} 2 p^{4}\left({ }_{2}^{2} P\right)^{3} P_{1}$ & 39288 & 39274 & 7.722E-04 & $7.722 \mathrm{E}-04$ & $0.99+2>1-290$ \\
\hline 22 & 3 & $2 s^{2} 2 p^{4}\left({ }_{2}^{2} P\right){ }^{3} P_{0}$ & 42345 & 42358 & $6.616 \mathrm{E}-01$ & 6.631E-01 & $0.92+0.082 s^{2} 2 p^{4}\left({ }_{0}^{1} s\right){ }^{1} S$ \\
\hline 22 & 4 & $2 s^{2} 2 p^{4}\left({ }_{2}^{1} D\right){ }^{1} D_{2}$ & 108730 & 108881 & 3.902E-04 & $3.902 \mathrm{E}-04$ & $\left.0.95+0.052 s^{2} 2 p^{4}{ }_{2}^{3} P\right)^{3} P$ \\
\hline 22 & 5 & $2 s^{2} 2 p^{4}\left({ }_{0}^{1} s\right){ }^{1} s_{0}$ & 215528 & 215804 & 4.388E-05 & 4.388E-05 & $0.90+0.082 s^{2} 2 p^{4}\left({ }_{2}^{3} P\right){ }^{3} P+0.022 p^{6}{ }^{1} S$ \\
\hline 22 & 6 & $2 s^{2} S 2 p^{5}{ }^{3} P_{2}^{\circ}$ & 712278 & 712192 & $3.202 \mathrm{E}-11$ & $3.189 \mathrm{E}-11$ & 0.99 \\
\hline 22 & 7 & $2 s^{2} S 2 p^{5}{ }^{3} P_{1}^{2}$ & 742877 & 742782 & 2.923E-11 & 2.925E-11 & 0.98 \\
\hline 22 & 8 & $2 s^{2} S 2 p^{5} P_{P_{0}^{1}}^{1}$ & 762056 & 761977 & $2.888 \mathrm{E}-11$ & $2.895 \mathrm{E}-11$ & 0.99 \\
\hline 22 & 9 & $2 s^{2} S 2 p^{5}{ }^{1} P_{1}^{\circ}$ & 978037 & 978257 & $8.976 \mathrm{E}-12$ & $8.973 \mathrm{E}-12$ & 0.98 \\
\hline 22 & 10 & $2 p^{6}{ }^{1} s_{0} \quad{ }_{1}$ & 1656300 & 1656402 & $1.012 \mathrm{E}-11$ & $1.013 \mathrm{E}-11$ & $0.96+0.022 s^{2} 2 p^{4}\left({ }_{0}^{1} s\right)^{1} S$ \\
\hline 22 & 11 & $2 s^{2} 2 p^{3}\left({ }_{3}^{4} s\right)^{4} s 3 s^{5} s_{2}^{0}$ & & 4310000 & $6.754 \mathrm{E}-11$ & $6.782 \mathrm{E}-11$ & $0.96+0.032 s^{2} 2 p^{3}\left({ }_{1}^{2} P\right)^{2} P 3 s^{3} P^{\circ}$ \\
\hline 22 & 12 & $2 s^{2} 2 p^{3}\left(\frac{3}{3} S\right)^{4} S 3 s^{3} s_{1}^{\circ}$ & 4354100 & 4353399 & $6.348 \mathrm{E}-13$ & $6.352 \mathrm{E}-13$ & $0.94+0.022 s^{2} 2 p^{3}\left({ }_{1}^{2} P\right)^{2} P 3 s^{1} P^{\circ}$ \\
\hline 22 & 13 & $2 s^{2} 2 p^{3}\left({ }_{3}^{2} D\right)^{2} D 3 s^{3} D_{1}^{\circ}$ & 4440200 & 4439052 & $1.761 \mathrm{E}-12$ & $1.764 \mathrm{E}-12$ & $\left.0.86+0.062 s^{2} 2 p^{3}{ }_{1}^{2} P\right)^{2} P 3 s^{1} P^{\circ}+0.052 s^{2} 2 p^{3}\left({ }_{1}^{2} P\right)^{2} P 3 s^{3} P^{\circ}$ \\
\hline 22 & 14 & $2 s^{2} 2 p^{3}\left(\frac{3}{3} D\right)^{2} D 3 s^{3} D_{2}^{\circ}$ & 4440400 & 4439949 & $1.841 \mathrm{E}-12$ & $1.842 \mathrm{E}-12$ & $0.78+0.132 s^{2} 2 p^{3}\left({ }_{1}^{2} P\right)^{2} P 3 s^{3} P^{\circ}+0.062 s^{2} 2 p^{3}\left({ }_{3}^{2} D\right)^{2} D 3 s^{1} D^{\circ}$ \\
\hline 22 & 15 & $2 s^{2} 2 p^{3}\left({ }_{2}^{2} D\right)^{2} D 3 s^{3} D_{3}^{\circ}$ & 4451600 & 4450976 & $1.952 \mathrm{E}-12$ & $1.952 \mathrm{E}-12$ & 0.99 \\
\hline 22 & 16 & $2 s^{2} 2 p^{3}\left({ }_{3}^{2} D\right)^{2} D 3 s^{1} D_{2}^{\circ}$ & 4469100 & 4468181 & $8.504 \mathrm{E}-13$ & $8.510 \mathrm{E}-13$ & $0.83+0.122 s^{2} 2 p^{3}\left({ }_{3}^{2} D\right)^{2} D 3 s^{3} D^{\circ}+0.042 s^{2} 2 p^{3}\left({ }_{1}^{2} P\right)^{2} P 3 s^{3} P^{\circ}$ \\
\hline 22 & 17 & $2 s^{2} 2 p^{3}\left({ }_{3}^{4} S\right)^{4} S 3 p^{5} P_{1}^{2}$ & & 4495126 & 3.925E-10 & $3.906 \mathrm{E}-10$ & 0.94 \\
\hline 22 & 18 & $2 s^{2} 2 p^{3}\left({ }_{3}^{4} S\right)^{4} S 3 p^{5} P_{2}$ & & 4497588 & 4.407E-10 & $4.384 \mathrm{E}-10$ & $0.90+0.042 s^{2} 2 p^{3}\left({ }_{3}^{4} S\right)^{4} S 3 p^{3} P+0.022 s^{2} 2 p^{3}\left({ }_{1}^{2} P\right)^{2} P 3 p^{3} P$ \\
\hline 22 & 19 & $2 s^{2} 2 p^{3}\left({ }_{3}^{4} S\right){ }^{4} S 3 p^{5} P_{3}$ & & 4505254 & $3.901 \mathrm{E}-10$ & $3.889 \mathrm{E}-10$ & $0.95+0.032 s^{2} 2 p^{3}\left({ }_{1}^{2} P\right)^{2} P 3 p^{3} D$ \\
\hline 22 & 20 & $2 s^{2} 2 p^{3}\left({ }_{1}^{2} P\right)^{2} P 3 s^{3} P_{0}^{\circ}$ & & 4518848 & $2.074 \mathrm{E}-12$ & $2.075 \mathrm{E}-12$ & 0.97 \\
\hline 22 & 21 & $2 s^{2} 2 p^{3}\left({ }_{1}^{2} P\right)^{2} P 3 s^{3} P_{1}^{\circ}$ & & 4523182 & $1.729 \mathrm{E}-12$ & $1.729 \mathrm{E}-12$ & $0.84+0.112 s^{2} 2 p^{3}\left({ }_{1}^{2} P\right)^{2} P 3 s^{1} P^{\circ}+0.022 s^{2} 2 p^{3}\left({ }_{3}^{2} D\right)^{2} D 3 s^{3} D^{\circ}$ \\
\hline 22 & 22 & $2 s^{2} 2 p^{3}\left({ }_{3}^{4} S\right)^{4} S 3 p^{3} P_{1}$ & & 4537919 & $2.736 \mathrm{E}-10$ & $2.745 \mathrm{E}-10$ & $\left.0.75+0.112 s^{2} 2 p^{3}\left({ }_{3}^{2} D\right)^{2} D 3 p^{3} P+0.042 s^{2} 2 p^{3}{ }_{1}^{2} P\right)^{2} P 3 p^{1} P$ \\
\hline 22 & 23 & $2 s^{2} 2 p^{3}\left({ }_{1}^{2} P\right)^{2} P 3 s^{3} P_{2}^{\circ}$ & 4523000 & 4541430 & $1.721 \mathrm{E}-12$ & $1.720 \mathrm{E}-12$ & $0.77+0.102 s^{2} 2 p^{3}\left({ }_{3}^{2} D\right)^{2} D 3 s^{1} D^{\circ}+0.092 s^{2} 2 p^{3}\left({ }_{3}^{2} D\right)^{2} D 3 s^{3} D^{\circ}$ \\
\hline 22 & 24 & $2 s^{2} 2 p^{3}\left({ }_{3}^{4} S\right)^{4} S 3 p^{3} P_{2}$ & & 4543986 & 2.827E-10 & $2.835 \mathrm{E}-10$ & $0.77+0.122 s^{2} 2 p^{3}\left({ }_{3}^{2} D\right)^{2} D 3 p^{3} P+0.062 s^{2} 2 p^{3}\left({ }_{3}^{4} S\right)^{4} S 3 p^{5} P$ \\
\hline 22 & 25 & $2 s^{2} 2 p^{3}\left({ }_{3}^{4} S\right){ }^{4} S 3 p^{3} P_{0}$ & & 4546874 & 3.124E-10 & $3.127 \mathrm{E}-10$ & $0.85+0.092 s^{2} 2 p^{3}\left({ }_{3}^{2} D\right)^{2} D 3 p^{3} P+0.052 s^{2} 2 p^{3}\left({ }_{1}^{2} P\right)^{2} P 3 p^{3} P$ \\
\hline 22 & 26 & $2 s^{2} 2 p^{3}\left({ }_{1}^{2} P\right)^{2} P 3 s^{1} P_{1}^{\circ}$ & 4557300 & 4556402 & $8.738 \mathrm{E}-13$ & $8.741 \mathrm{E}-13$ & $0.78+0.102 s^{2} 2 p^{3}\left({ }_{3}^{2} D\right)^{2} D 3 s^{3} D^{\circ}+0.062 s^{2} 2 p^{3}\left({ }_{1}^{2} P\right)^{2} P 3 s^{3} P^{\circ}$ \\
\hline 22 & 27 & $2 s^{2} 2 p^{3}\left({ }_{3}^{2} D\right)^{2} D 3 p^{3} D_{1}$ & & 4602751 & 2.067E-10 & $2.084 \mathrm{E}-10$ & $0.43+0.332 s^{2} 2 p^{3}\left({ }_{3}^{2} D\right)^{2} D 3 p^{1} P+0.082 s^{2} 2 p^{3}\left({ }_{1}^{2} P\right)^{2} P 3 p^{1} P$ \\
\hline 22 & 28 & $2 s^{2} 2 p^{3}\left(\frac{3}{2} D\right)^{2} D 3 p^{3} D_{2}$ & & 4617778 & $4.141 \mathrm{E}-10$ & $4.131 \mathrm{E}-10$ & $0.58+0.272 s^{2} 2 p^{3}\left({ }_{3}^{2} D\right)^{2} D 3 p^{3} F+0.092 s^{2} 2 p^{3}\left({ }_{1}^{2} P\right)^{2} P 3 p^{3} D$ \\
\hline 22 & 29 & $2 s^{2} 2 p^{3}\left(\frac{2}{3} D\right)^{2} D 3 p^{3} D_{3}$ & & 4627980 & $3.961 \mathrm{E}-10$ & $3.952 \mathrm{E}-10$ & $0.50+0.422 s^{2} 2 p^{3}\left({ }_{3}^{2} D\right)^{2} D 3 p^{3} F+0.062 s^{2} 2 p^{3}\left({ }_{1}^{2} P\right)^{2} P 3 p^{3} D$ \\
\hline 22 & 30 & $2 s^{2} 2 p^{3}\left(\frac{2}{3} D\right)^{2} D 3 p^{3} F_{2}$ & & 4630251 & $1.868 \mathrm{E}-10$ & $1.872 \mathrm{E}-10$ & $0.58+0.312 s^{2} 2 p^{3}\left(\frac{2}{3} D\right)^{2} D 3 p^{3} D+0.062 s^{2} 2 p^{3}\left({ }_{1}^{2} P\right)^{2} P 3 p^{1} D$ \\
\hline 22 & 31 & $2 s^{2} 2 p^{3}\left(\frac{2}{3} D\right)^{2} D 3 p{ }^{1} P_{1}$ & & 4630804 & $1.493 \mathrm{E}-10$ & $1.509 \mathrm{E}-10$ & $0.47+0.402 s^{2} 2 p^{3}\left({ }_{3}^{2} D\right)^{2} D 3 p^{3} D+0.062 s^{2} 2 p^{3}\left({ }_{1}^{2} P\right)^{2} P 3 p^{3} D$ \\
\hline 22 & 32 & $2 s^{2} 2 p^{3}\left({ }_{3}^{2} D\right)^{2} D 3 p^{3} F_{3}$ & & 4639055 & $1.350 \mathrm{E}-10$ & $1.353 \mathrm{E}-10$ & $0.36+0.422 s^{2} 2 p^{3}\left({ }_{3}^{2} D\right)^{2} D 3 p^{3} D+0.142 s^{2} 2 p^{3}\left({ }_{3}^{2} D\right)^{2} D 3 p^{1} F$ \\
\hline 22 & 33 & $2 s^{2} 2 p^{3}\left({ }_{3}^{2} D\right)^{2} D 3 p^{1} F_{3}$ & & 4646526 & $3.860 \mathrm{E}-10$ & $3.835 \mathrm{E}-10$ & $0.77+0.132 s^{2} 2 p^{3}\left({ }_{3}^{2} D\right)^{2} D 3 p^{3} F+0.062 s^{2} 2 p^{3}\left({ }_{3}^{2} D\right)^{2} D 3 p^{3} D$ \\
\hline 22 & 34 & $2 s^{2} 2 p^{3}\left({ }_{3}^{2} D\right)^{2} D 3 p^{3} F_{4}$ & & 4647749 & $3.326 \mathrm{E}-10$ & $3.310 \mathrm{E}-10$ & 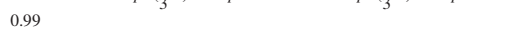 \\
\hline 22 & 35 & $2 s^{2} 2 p^{3}\left({ }_{2}^{2} D\right)^{2} D 3 p^{3} P_{1}$ & & 4683603 & $2.461 \mathrm{E}-11$ & $2.473 \mathrm{E}-11$ & $0.39+0.332 s^{2} 2 p^{3}\left({ }_{1}^{2} P\right)^{2} P 3 p^{3} S+0.112 s^{2} 2 p^{3}\left({ }_{3}^{4} S\right)^{4} S 3 p^{3} P$ \\
\hline 22 & 36 & $2 s^{2} 2 p^{3}\left(\frac{2}{3} D\right)^{2} D 3 p^{3} P_{0}$ & & 4683740 & $2.644 \mathrm{E}-11$ & $2.653 \mathrm{E}-11$ & $0.73+0.202 s^{2} 2 p^{3}\left({ }_{1}^{2} P\right)^{2} P 3 p^{3} P+0.032 s^{2} 2 p^{3}\left({ }_{1}^{2} P\right)^{2} P 3 p^{1} S$ \\
\hline 22 & 37 & $2 s^{2} 2 p^{3}\left({ }_{3}^{2} D\right)^{2} D 3 p^{3} P_{2}$ & & 4698379 & 1.613E-11 & $1.619 \mathrm{E}-11$ & $0.77+0.122 s^{2} 2 p^{3}\left({ }_{3}^{4} S\right)^{4} S 3 p^{3} P+0.032 s^{2} 2 p^{3}\left({ }_{1}^{2} P\right)^{2} P 3 p^{3} P$ \\
\hline 22 & 38 & $2 s^{2} 2 p^{3}\left(_{1}^{3} P\right)^{2} P 3 p^{3} D_{1}$ & & 4707201 & $6.488 \mathrm{E}-11$ & 6.494E-11 & $\left.0.71+0.162 s^{2} 2 p^{3}\left(_{1}^{2} P\right)^{2} P 3 p^{1} P+0.052 s^{2} 2 p^{3}{ }_{2}^{2} D\right)^{2} D 3 p^{3} D$ \\
\hline 22 & 39 & $2 s^{2} 2 p^{3}\left({ }_{1}^{2} P\right)^{2} P 3 p^{3} D_{2}$ & & 4714927 & 5.943E-11 & $5.951 \mathrm{E}-11$ & $0.42+0.302 s^{2} 2 p^{3}\left({ }_{3}^{2} D\right)^{2} D 3 p^{1} D+0.102 s^{2} 2 p^{3}\left({ }_{3}^{2} D\right)^{2} D 3 p^{3} F$ \\
\hline
\end{tabular}




\begin{tabular}{|c|c|c|c|c|c|c|c|}
\hline $\mathrm{z}$ & Key & Level & $E_{\mathrm{NIST}}$ & $E_{\mathrm{MCDHF} / \mathrm{RCI}}$ & $\tau_{\mathrm{MCDHF} / \mathrm{RCI}}^{l}$ & $\tau_{\mathrm{MCDHF} / \mathrm{RCI}}^{v}$ & $L S$-composition \\
\hline 22 & 40 & $2 s^{2} 2 p^{3}\left({ }_{1}^{2} P\right)^{2} P 3 p^{3} S_{1}$ & & 4718928 & $3.028 \mathrm{E}-11$ & 3.042E-11 & $0.48+0.222 s^{2} 2 p^{3}\left({ }_{3}^{2} D\right)^{2} D 3 p^{3} P+0.142 s^{2} 2 p^{3}\left({ }_{1}^{2} P\right)^{2} P 3 p^{3} P$ \\
\hline 22 & 41 & $2 s^{2} 2 p^{3}\left({ }_{1}^{2} P\right)^{2} P 3 p^{1} D_{2}$ & & 4721666 & $7.325 \mathrm{E}-11$ & 7.331E-11 & $0.13+0.402 s^{2} 2 p^{3}\left({ }_{1}^{3} P\right)^{2} P 3 p^{3} D+0.232 s^{2} 2 p^{3}\left({ }_{1}^{2} P\right)^{2} P 3 p^{3} P$ \\
\hline 22 & 42 & $2 s^{2} 2 p^{3}\left({ }_{3}^{4} S\right)^{4} S 3 d^{5} D_{0}^{\circ}$ & & 4733128 & $2.322 \mathrm{E}-11$ & $2.310 \mathrm{E}-11$ & $0.96+0.032 s^{2} 2 p^{3}\left({ }_{1}^{2} P\right)^{2} P 3 d^{3} P^{\circ}$ \\
\hline 22 & 43 & $2 s^{2} 2 p^{3}\left({ }_{3}^{\circ} S\right){ }^{4} S 3 d^{5} D_{3}^{\circ}$ & & 4733135 & $1.079 \mathrm{E}-10$ & $1.086 \mathrm{E}-10$ & 0.94 \\
\hline 22 & 44 & $2 s^{2} 2 p^{3}\left({ }_{3}^{4} S\right)^{4} S 3 d^{5} D_{2}^{\circ}$ & & 4733160 & 2.305E-11 & $2.298 \mathrm{E}-11$ & 0.95 \\
\hline 22 & 45 & $2 s^{2} 2 p^{3}\left({ }_{3}^{4} S\right)^{4} S 3 d^{5} D_{1}^{\circ}$ & & 4733188 & $1.810 \mathrm{E}-11$ & $1.802 \mathrm{E}-11$ & 0.96 \\
\hline 22 & 46 & $2 s^{2} 2 p^{3}\left({ }_{1}^{2} P\right)^{2} P 3 p^{3} D_{3}$ & & 4733290 & 8.023E-11 & $8.019 \mathrm{E}-11$ & $0.79+0.092 s^{2} 2 p^{3}\left({ }_{3}^{2} D\right)^{2} D 3 p^{3} F+0.082 s^{2} 2 p^{3}\left({ }_{3}^{2} D\right)^{2} D 3 p^{1} F$ \\
\hline 22 & 47 & $2 s^{2} 2 p^{3}\left({ }_{3}^{4} S\right){ }^{4} S 3 d^{5} D_{4}^{\circ}$ & & 4734025 & $2.767 \mathrm{E}-10$ & $2.766 \mathrm{E}-10$ & $0.96+0.032 s^{2} 2 p^{3}\left({ }_{1}^{2} P\right)^{2} P 3 d^{3} F^{\circ}$ \\
\hline 22 & 48 & $2 s^{2} 2 p^{3}\left({ }_{1}^{2} P\right)^{2} P 3 p^{1} P_{1}^{4}$ & & 4738341 & $3.270 \mathrm{E}-11$ & $3.283 \mathrm{E}-11$ & $0.44+0.212 s^{2} 2 p^{3}\left(\frac{2}{3} D\right)^{2} D 3 p^{3} P+0.112 s^{2} 2 p^{3}\left({ }_{1}^{2} P\right)^{2} P 3 p^{3} P$ \\
\hline 22 & 49 & $2 s^{2} 2 p^{3}\left({ }_{1}^{2} P\right)^{2} P 3 p^{3} P_{0}$ & & 4753210 & $3.250 \mathrm{E}-11$ & 3.262E-11 & $0.70+0.112 s^{2} 2 p^{3}\left(\frac{2}{3} D\right)^{2} D 3 p^{3} P+0.092 s^{2} 2 p^{3}\left({ }_{3}^{4} S\right)^{4} S 3 p^{3} P$ \\
\hline 22 & 50 & $2 s^{2} 2 p^{3}\left({ }_{1}^{2} P\right){ }^{2} P 3 p^{3} P_{1}$ & & 4757629 & $5.336 \mathrm{E}-11$ & $5.353 \mathrm{E}-11$ & $0.58+0.132 s^{2} 2 p^{3}\left({ }_{1}^{2} P\right)^{2} P 3 p^{1} P+0.082 s^{2} 2 p^{3}\left({ }_{3}^{4} S\right)^{4} S 3 p^{3} P$ \\
\hline 22 & 51 & $2 s^{2} 2 p^{3}\left({ }_{1}^{2} P\right)^{2} P 3 p^{3} P_{2}$ & & 4759182 & $1.129 \mathrm{E}-10$ & $1.131 \mathrm{E}-10$ & $0.52+0.262 s^{2} 2 p^{3}\left({ }_{1}^{2} P\right)^{2} P 3 p^{1} D+0.052 s^{2} 2 p^{3}\left({ }_{3}^{2} D\right)^{2} D 3 p^{3} D$ \\
\hline 22 & 52 & $2 s^{2} 2 p^{3}\left({ }_{3}^{4} S\right)^{4} S 3 d^{3} D_{2}^{\circ}$ & 4780000 & 4776970 & $5.455 \mathrm{E}-13$ & $5.449 \mathrm{E}-13$ & $\left.\left.0.74+0.122 s^{2} 2 p^{3}\left({ }_{3}^{2} D\right)\right)^{2} D 3 d^{3} D^{\circ}+0.042 s^{2} 2 p^{3}{ }_{1}^{2} P\right)^{2} P 3 d^{1} D^{\circ}$ \\
\hline 22 & 53 & $2 s^{2} 2 p^{3}\left({ }_{3}^{4} S\right)^{4} S 3 d^{3} D_{1}^{\circ}$ & & 4783929 & $4.309 \mathrm{E}-13$ & 4.305E-13 & $0.85+0.082 s^{2} 2 p^{3}\left({ }_{3}^{2} D\right)^{2} D 3 d^{3} D^{\circ}+0.042 s^{2} 2 p^{3}\left({ }_{1}^{2} P\right)^{2} P 3 d^{3} D^{\circ}$ \\
\hline 22 & 54 & $2 s^{2} 2 p^{3}\left({ }_{3}^{4} S\right)^{4} S 3 d^{3} D_{3}^{\circ}$ & 4785000 & 4784989 & $4.245 \mathrm{E}-13$ & $4.237 \mathrm{E}-13$ & $\left.0.80+0.122 s^{2} 2 p^{3}\left(\frac{2}{3} D\right)\right)^{2} D 3 d^{3} D^{\circ}$ \\
\hline 22 & 55 & $2 s^{2} 2 p^{3}\left({ }_{3}^{2} D\right)^{2} D 3 p^{1} D_{2}^{3}$ & & 4787330 & $9.472 \mathrm{E}-11$ & $9.521 \mathrm{E}-11$ & $0.43+0.422 s^{2} 2 p^{3}\left({ }_{1}^{2} P\right)^{2} P 3 p^{1} D+0.082 s^{2} 2 p^{3}\left({ }_{1}^{2} P\right)^{2} P 3 p^{3} P$ \\
\hline 22 & 56 & $\left.2 s^{2} 2 p^{3}\left({ }_{3}^{2} D\right)\right)^{2} D 3 d^{3} F_{2}^{\circ}$ & & 4845576 & 3.307E-12 & $3.299 \mathrm{E}-12$ & $0.76+0.112 s^{2} 2 p^{3}\left(2_{1}^{2} P\right)^{2} P 3 d^{3} F^{\circ}+0.042 s^{2} 2 p^{3}\left({ }_{3}^{4} S\right)^{4} S 3 d^{3} D^{\circ}$ \\
\hline 22 & 57 & $2 s^{2} 2 p^{3}\left({ }_{3}^{2} D\right)^{2} D 3 d^{3} F_{3}^{\circ}$ & & 4852729 & $2.769 \mathrm{E}-12$ & $2.767 \mathrm{E}-12$ & $0.75+0.102 s^{2} 2 p^{3}\left({ }_{1}^{2} P\right)^{2} P 3 d^{3} F^{\circ}+0.092 s^{2} 2 p^{3}\left({ }_{3}^{2} D\right)^{2} D 3 d^{3} G^{\circ}$ \\
\hline 22 & 58 & $2 s^{2} 2 p^{3}\left(\frac{2}{3} D\right)^{2} D 3 d^{1} s_{0}^{0}$ & & 4855768 & 4.485E-11 & $4.520 \mathrm{E}-11$ & $0.91+0.062 s^{2} 2 p^{3}\left(2_{1}^{2} P\right)^{2} P 3 d^{3} P^{\circ}$ \\
\hline 22 & 59 & $2 s^{2} 2 p^{3}\left({ }_{1}^{3} P\right)^{2} P 3 p^{1} S_{0}$ & & 4859010 & $1.384 \mathrm{E}-11$ & $1.388 \mathrm{E}-11$ & $\left.0.88+0.052 s^{2} 2 p^{3}\left(\frac{2}{3} D\right)\right)^{2} D 3 p^{3} P+0.022 s^{2} 2 p^{3}\left({ }_{3}^{4} S\right)^{4} S 3 p^{3} P$ \\
\hline 22 & 60 & $2 s^{2} 2 p^{3}\left({ }_{3}^{2} D\right)^{2} D 3 d^{3} G_{4}^{\circ}$ & & 4860534 & $2.840 \mathrm{E}-10$ & $2.831 \mathrm{E}-10$ & $\left.0.37+0.372 s^{2} 2 p^{3}\left({ }_{3}^{2} D\right)^{2} D 3 d^{3} F^{\circ}+0.132 s^{2} 2 p^{3}{ }^{3}{ }_{1}^{2} P\right)^{2} P 3 d^{3} F^{\circ}$ \\
\hline 22 & 61 & $2 s^{2} 2 p^{3}\left({ }_{3}^{2} D\right)^{2} D 3 d^{3} G_{3}^{\circ}$ & & 4864902 & $4.036 \mathrm{E}-12$ & $4.032 \mathrm{E}-12$ & $\left.0.76+0.122 s^{2} 2 p^{3}\left({ }_{3}^{2} D\right)\right)^{2} D 3 d^{3} F^{\circ}+0.062 s^{2} 2 p^{3}\left({ }_{1}^{2} P\right)^{2} P 3 d^{1} F^{\circ}$ \\
\hline 22 & 62 & $2 s^{2} 2 p^{3}\left(\frac{2}{3} D\right)^{2} D 3 d^{3} F_{4}^{\circ}$ & & 4870423 & 2.603E-10 & 2.607E-10 & $0.61+0.302 s^{2} 2 p^{3}\left({ }_{3}^{2} D\right){ }^{2} D 3 d^{3} G^{\circ}+0.062 s^{2} 2 p^{3}\left(\frac{2}{3} D\right)^{2} D 3 d^{1} G^{\circ}$ \\
\hline 22 & 63 & $2 s^{2} 2 p^{3}\left(\frac{2}{3} D\right)^{2} D 3 d^{3} D_{1}^{\circ}$ & 4873000 & 4872594 & $3.017 \mathrm{E}-13$ & $3.013 \mathrm{E}-13$ & $\left.0.41+0.352 s^{2} 2 p^{3}\left({ }_{3}^{2} D\right)^{2} D 3 d^{1} P^{\circ}+0.072 s^{2} 2 p^{3}{ }_{1}^{2} P\right)^{2} P 3 d^{3} D^{\circ}$ \\
\hline 22 & 64 & $2 s^{2} 2 p^{3}\left(\frac{2}{3} D\right)^{2} D 3 d^{3} G_{5}^{\circ}$ & & 4876626 & $2.639 \mathrm{E}-10$ & $2.632 \mathrm{E}-10$ & 1.00 \\
\hline 22 & 65 & $2 s^{2} 2 p^{3}\left(\frac{2}{3} D\right)^{2} D 3 d^{1} G_{4}^{\circ}$ & & 4878758 & 2.463E-10 & $2.460 \mathrm{E}-10$ & $0.73+0.252 s^{2} 2 p^{3}\left({ }_{3}^{2} D\right)^{2} D 3 d^{3} G^{\circ}$ \\
\hline 22 & 66 & $2 s^{2} 2 p^{3}\left({ }_{3}^{3} D\right)^{2} D 3 d^{3} D_{2}^{\circ}$ & 4891000 & 4891656 & $1.915 \mathrm{E}-13$ & $1.913 \mathrm{E}-13$ & $0.51+0.182 s^{2} 2 p^{3}\left({ }_{3}^{2} D\right){ }^{2} D 3 d^{3} P^{\circ}+0.102 s^{2} 2 p^{3}\left({ }_{3}^{4} S\right)^{4} S 3 d^{3} D^{\circ}$ \\
\hline 22 & 67 & $2 s^{2} 2 p^{3}\left({ }_{3}^{2} D\right)^{2} D 3 d^{1} P_{1}^{\circ}$ & & 4896218 & $2.821 \mathrm{E}-13$ & $2.819 \mathrm{E}-13$ & $0.48+0.392 s^{2} 2 p^{3}\left({ }_{3}^{2} D\right)^{2} D 3 d^{3} D^{\circ}+0.042 s^{2} 2 p^{3}\left({ }_{1}^{2} P\right)^{2} P 3 d^{3} P^{\circ}$ \\
\hline 22 & 68 & $2 s^{2} 2 p^{3}\left(\frac{2}{3} D\right)^{2} D 3 d^{3} D_{3}^{\circ}$ & 4898000 & 4898535 & $1.361 \mathrm{E}-13$ & $1.359 \mathrm{E}-13$ & $0.81+0.132 s^{2} 2 p^{3}\left({ }_{3}^{4} S\right)^{4} S 3 d^{3} D^{\circ}$ \\
\hline 22 & 69 & $2 s^{2} 2 p^{3}\left(\frac{2}{2} D\right)^{2} D 3 d^{3} P_{2}^{0}$ & 4905000 & 4904783 & $1.292 \mathrm{E}-13$ & $1.290 \mathrm{E}-13$ & $0.42+0.232 s^{2} 2 p^{3}\left(\frac{2}{3} D\right){ }^{2} D 3 d^{3} D^{\circ}+0.132 s^{2} 2 p^{3}\left(\frac{2}{3} D\right)^{2} D 3 d^{1} D^{\circ}$ \\
\hline 22 & 70 & $2 s^{2} 2 p^{3}\left(\frac{3}{3} D\right)^{2} D 3 d^{3} P_{0}^{\circ}$ & & 4908661 & $2.798 \mathrm{E}-13$ & 2.794E-13 & $0.84+0.112 s^{2} 2 p^{3}\left({ }_{1}^{2} P\right)^{2} P 3 d^{3} P^{\circ}+0.032 s^{2} 2 p^{3}\left({ }_{3}^{2} D\right)^{2} D 3 d^{1} S^{\circ}$ \\
\hline 22 & 71 & $2 s^{2} 2 p^{3}\left(\frac{2}{3} D\right)^{2} D 3 d^{3} P_{1}^{\circ}$ & 4908000 & 4910999 & $2.058 \mathrm{E}-13$ & 2.056E-13 & $0.78+0.082 s^{2} 2 p^{3}\left(\frac{1}{3} D\right)^{2} D 3 d^{3} s^{\circ}+0.072 s^{2} 2 p^{3}\left({ }_{1}^{2} P\right)^{2} P 3 d^{3} P^{\circ}$ \\
\hline 22 & 72 & $2 s^{2} 2 p^{3}\left(\frac{2}{2} D\right)^{2} D 3 d^{1} D_{2}^{0}$ & 4911000 & 4911124 & $3.522 \mathrm{E}-13$ & $3.520 \mathrm{E}-13$ & $0.40+0.272 s^{2} 2 p^{3}\left({ }_{1}^{2} P\right)^{2} P 3 d^{1} D^{\circ}+0.202 s^{2} 2 p^{3}\left({ }_{3}^{\frac{2}{2}} D\right)^{2} D 3 d^{3} P^{\circ}$ \\
\hline 22 & 73 & $2 s^{2} S 2 p^{4}\left({ }_{2}^{3} P\right){ }^{4} P 3 s^{5} P_{3}$ & & 4915429 & $5.423 \mathrm{E}-11$ & $5.410 \mathrm{E}-11$ & $0.98+12+3=0$ \\
\hline 22 & 74 & $2 s^{2} 2 p^{3}\left({ }_{3}^{2} D\right)^{2} D 3 d^{3} s_{1}^{\circ}$ & & 4921579 & $1.185 \mathrm{E}-13$ & $1.183 \mathrm{E}-13$ & $\left.0.69+0.152 s^{2} 2 p^{3}\left({ }_{3}^{2} D\right)^{2} D 3 d^{3} P^{\circ}+0.092 s^{2} 2 p^{3}{ }_{1}^{2} P\right)^{2} P 3 d^{3} P^{\circ}$ \\
\hline 22 & 75 & $2 s^{2} 2 p^{3}\left({ }_{3}^{2} D\right)^{2} D 3 d^{1} F_{3}^{\circ}$ & 4940000 & 4940131 & $1.874 \mathrm{E}-13$ & $1.871 \mathrm{E}-13$ & $\left.0.67+0.102 s^{2} 2 p^{3}\left({ }_{1}^{2} P\right)^{2} P 3 d^{3} D^{\circ}+0.102 s^{2} 2 p^{3}{ }_{1}^{2} P\right)^{2} P 3 d^{3} F^{\circ}$ \\
\hline 22 & 76 & $2 s^{2} S 2 p^{4}\left({ }_{2}^{3} P\right){ }^{4} P 3 s^{5} P_{2}$ & & 4940903 & $1.397 \mathrm{E}-11$ & $1.399 \mathrm{E}-11$ & $0.93+0.052 s^{2} S 2 p^{4}\left({ }_{2}^{3} P\right)^{4} P 3 s^{3} P$ \\
\hline 22 & 77 & $2 s^{2} 2 p^{3}\left({ }_{1}^{2} P\right)^{2} P 3 d^{3} F_{2}^{\circ}$ & 4950000 & 4951036 & $1.409 \mathrm{E}-12$ & 1.407E-12 & $0.73+0.102 s^{2} 2 p^{3}\left({ }_{3}^{2} D\right)^{2} D 3 d^{3} F^{\circ}+0.062 s^{2} 2 p^{3}\left({ }_{1}^{2} P\right)^{2} P 3 d^{3} D^{\circ}$ \\
\hline 22 & 78 & $2 s^{2} 2 p^{3}\left({ }_{1}^{2} P\right)^{2} P 3 d^{3} F_{3}^{\circ}$ & & 4952778 & 1.473E-12 & 1.471E-12 & $0.64+0.222 s^{2} 2 p^{3}\left({ }_{1}^{2} P\right)^{2} P 3 d^{3} D^{\circ}+0.042 s^{2} 2 p^{3}\left({ }_{3}^{2} D\right)^{2} D 3 d^{3} G^{\circ}$ \\
\hline 22 & 79 & $2 s^{2} S 2 p^{4}\left({ }_{2}^{3} P\right)^{4} P 3 s^{5} P_{1}$ & & 4956628 & $3.212 \mathrm{E}-11$ & $3.226 \mathrm{E}-11$ & 0.97 \\
\hline 22 & 80 & $2 s^{2} 2 p^{3}\left({ }_{1}^{2} P\right)^{2} P 3 d^{3} F_{4}^{\circ}$ & & 4961406 & $2.549 \mathrm{E}-10$ & 2.554E-10 & $0.79+0.082 s^{2} 2 p^{3}\left({ }_{3}^{2} D\right){ }^{2} D 3 d^{3} G^{\circ}+0.082 s^{2} 2 p^{3}\left({ }_{3}^{2} D\right)^{2} D 3 d^{1} G^{\circ}$ \\
\hline 22 & 81 & $2 s^{2} 2 p^{3}\left({ }_{1}^{2} P\right)^{2} P 3 d^{3} P_{0}^{\circ}$ & & 4964549 & $1.809 \mathrm{E}-13$ & $1.807 \mathrm{E}-13$ & $0.78+0.142 s^{2} 2 p^{3}\left({ }_{3}^{2} D\right)^{2} D 3 d^{3} P^{\circ}+0.042 s^{2} 2 p^{3}\left({ }_{3}^{2} D\right)^{2} D 3 d^{1} S^{\circ}$ \\
\hline 22 & 82 & $2 s^{2} 2 p^{3}\left({ }_{1}^{2} P\right)^{2} P 3 d^{3} P_{2}^{0}$ & 4962000 & 4964889 & $3.387 \mathrm{E}-13$ & 3.383E-13 & $0.51+0.222 s^{2} 2 p^{3}\left({ }_{1}^{2} P\right)^{2} P 3 d^{3} D^{\circ}+0.092 s^{2} 2 p^{3}\left({ }_{3}^{2} D\right)^{2} D 3 d^{1} D^{\circ}$ \\
\hline 22 & 83 & $2 s^{2} 2 p^{3}\left({ }_{1}^{2} P\right)^{2} P 3 d^{3} P_{1}^{\mathrm{o}}$ & 4965000 & 4968183 & $2.125 \mathrm{E}-13$ & $2.124 \mathrm{E}-13$ & $0.65+0.182 s^{2} 2 p^{3}\left(\frac{2}{3} D\right)^{2} D 3 d^{3} s^{\circ}+0.062 s^{2} 2 p^{3}\left(\frac{2}{3} D\right)^{2} D 3 d^{1} P^{\circ}$ \\
\hline 22 & 84 & $2 s^{2} 2 p^{3}\left({ }_{1}^{2} P\right)^{2} P 3 d^{3} D_{2}^{\circ}$ & & 4976250 & $2.605 \mathrm{E}-13$ & $2.602 \mathrm{E}-13$ & $0.32+0.272 s^{2} 2 p^{3}\left({ }_{1}^{2} P\right)^{2} P 3 d^{1} D^{\circ}+0.162 s^{2} 2 p^{3}\left({ }_{1}^{2} P\right)^{2} P 3 d^{3} P^{\circ}$ \\
\hline 22 & 85 & $2 s^{2} 2 p^{3}\left({ }_{1}^{2} P\right)^{2} P 3 d^{3} D_{1}^{\circ}$ & 4984000 & 4984124 & $1.204 \mathrm{E}-13$ & $1.202 \mathrm{E}-13$ & $0.74+0.062 s^{2} 2 p^{3}\left({ }_{1}^{2} P\right)^{2} P 3 d^{1} P^{\circ}+0.062 s^{2} 2 p^{3}\left({ }_{3}^{2} D\right)^{2} D 3 d^{3} D^{\circ}$ \\
\hline 22 & 86 & $2 s^{2} S 2 p^{4}\left({ }_{2}^{3} P\right)^{4} P 3 s^{3} P_{2}$ & & 4985913 & $1.051 \mathrm{E}-12$ & $1.051 \mathrm{E}-12$ & $0.90+0.052 s^{2} S 2 p^{4}\left({ }_{2}^{3} P\right)^{4} P 3 s^{5} P$ \\
\hline 22 & 87 & $2 s^{2} 2 p^{3}\left({ }_{1}^{2} P\right)^{2} P 3 d^{3} D_{3}^{\circ}$ & 4987000 & 4989645 & $8.564 \mathrm{E}-13$ & $8.554 \mathrm{E}-13$ & $0.52+0.202 s^{2} 2 p^{3}\left({ }_{1}^{2} P\right)^{2} P 3 d^{1} F^{\circ}+0.102 s^{2} 2 p^{3}\left({ }_{3}^{2} D\right)^{2} D 3 d^{1} F^{\circ}$ \\
\hline 22 & 88 & $2 s^{2} 2 p^{3}\left({ }_{1}^{2} P\right)^{2} P 3 d^{1} D_{2}^{0}$ & 5006000 & 5006405 & $1.404 \mathrm{E}-13$ & $1.402 \mathrm{E}-13$ & $0.28+0.292 s^{2} 2 p^{3}\left({ }_{3}^{2} D\right){ }^{2} D 3 d^{1} D^{\circ}+0.262 s^{2} 2 p^{3}\left({ }_{1}^{2} P\right)^{2} P 3 d^{3} D^{\circ}$ \\
\hline 22 & 89 & $2 s^{2} 2 p^{3}\left({ }_{1}^{2} P\right)^{2} P 3 d^{1} F_{3}^{\circ}$ & 5006000 & 5007229 & $9.157 \mathrm{E}-14$ & $9.143 \mathrm{E}-14$ & $0.63+0.182 s^{2} 2 p^{3}\left({ }_{3}^{2} D\right)^{2} D 3 d^{1} F^{\circ}+0.072 s^{2} 2 p^{3}\left({ }_{1}^{2} P\right)^{2} P 3 d^{3} D^{\circ}$ \\
\hline 22 & 90 & $2 s^{2} S 2 p^{4}\left({ }_{2}^{3} P\right){ }^{4} P 3 s^{3} P_{1}$ & & 5013538 & $9.566 \mathrm{E}-13$ & $9.565 \mathrm{E}-13$ & 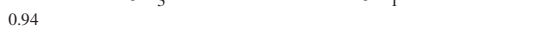 \\
\hline 22 & 91 & $2 s^{2} S 2 p^{4}\left({ }_{2}^{3} P\right){ }^{4} P 3 s^{3} P_{0}$ & & 5024511 & $9.596 \mathrm{E}-13$ & $9.594 \mathrm{E}-13$ & 0.94 \\
\hline 22 & 92 & $2 s^{2} 2 p^{3}\left({ }_{1}^{2} P\right)^{2} P 3 d^{1} P_{1}^{\circ}$ & 5046000 & 5052035 & $8.984 \mathrm{E}-14$ & $8.972 \mathrm{E}-14$ & $0.81+0.052 s^{2} 2 p^{3}\left({ }_{3}^{2} D\right){ }^{2} D 3 d^{1} P^{\circ}+0.032 s^{2} 2 p^{3}\left({ }_{1}^{2} P\right)^{2} P 3 d^{3} D^{\circ}$ \\
\hline 22 & 93 & $2 s^{2} S 2 p^{4}\left({ }_{2}^{3} P\right)^{4} P 3 p p^{5} P_{3}^{\circ}$ & & 5093362 & $5.614 \mathrm{E}-11$ & $5.578 \mathrm{E}-11$ & $0.82+0.172 s^{2} S 2 p^{4}\left({ }_{2}^{3} P\right)^{4} P 3 p^{5} D^{\circ}$ \\
\hline 22 & 94 & $2 s^{2} S 2 p^{4}\left(\frac{3}{3} P\right)^{4} P 3 p^{5} P_{2}^{\circ}$ & & 5096397 & 2.262E-11 & 2.253E-11 & $0.79+0.092 s^{2} S 2 p^{4}\left({ }_{2}^{3} P\right)^{4} P 3 p^{5} D^{\circ}+0.082 s^{2} S 2 p^{4}\left({ }_{2}^{3} P\right)^{4} P 3 p^{5} S^{\circ}$ \\
\hline 22 & 95 & $2 s^{2} S 2 p^{4}\left({ }_{2}^{3} P\right)^{4} P 3 p^{5} P_{1}^{\circ}$ & & 5113868 & 1.893E-11 & $1.888 \mathrm{E}-11$ & $0.90+0.062 s^{2} S 2 p^{4}\left({ }_{2}^{3} P\right)^{4} P 3 p^{5} D^{\circ}$ \\
\hline 22 & 96 & $2 s^{2} S 2 p^{4}\left({ }_{2}^{3} P\right){ }^{4} P 3 p^{5} D_{4}^{\circ}$ & & 5115235 & $8.969 \mathrm{E}-11$ & 8.883E-11 & $0.98+2+25$ \\
\hline 22 & 97 & $\left.2 s^{2} S 2 p^{4}{ }_{2}^{2} P\right)^{3} P 3 p^{5} D_{3}^{\circ}$ & & 5126439 & $1.749 \mathrm{E}-12$ & $1.745 \mathrm{E}-12$ & $0.45+0.412 s^{2} S 2 p^{4}\left({ }_{2}^{3} P\right)^{4} P 3 p^{3} D^{\circ}+0.122 s^{2} S 2 p^{4}\left({ }_{2}^{3} P\right)^{4} P 3 p^{5} P^{\circ}$ \\
\hline 22 & 98 & $2 s^{2} S 2 p^{4}\left({ }_{2}^{3} P\right)^{4} P 3 p^{5} D_{2}^{\circ}$ & & 5139291 & $6.233 \mathrm{E}-12$ & $6.220 \mathrm{E}-12$ & $\left.0.76+0.122 s^{2} S 2 p^{4}\left({ }_{2}^{3} P\right)^{4} P 3 p^{3} D^{\circ}+0.062 s^{2} S 2 p^{4}{ }_{2}^{3} P\right)^{4} P 3 p^{5} P^{\circ}$ \\
\hline 22 & 99 & $2 s^{2} S 2 p^{4}\left({ }_{2}^{3} P\right){ }^{4} P 3 p p^{5} D_{1}^{\circ}$ & & 5145975 & 2.171E-11 & 2.169E-11 & $0.89+0.062 s^{2} S 2 p^{4}\left({ }_{2}^{3} P\right)^{4} P 3 p^{5} P^{\circ}+0.022 s^{2} S 2 p^{4}\left({ }_{2}^{3} P\right){ }^{4} P 3 p^{3} D^{\circ}$ \\
\hline 22 & 100 & $\left.2 s^{2} S 2 p^{4}{ }_{2}^{\frac{2}{3}} P\right)^{4} P 3 p^{5} D_{0}^{\circ}$ & & 5147578 & $6.446 \mathrm{E}-11$ & $6.454 \mathrm{E}-11$ & $0.98-2+2+2$ \\
\hline 22 & 101 & $2 s^{2} S 2 p^{4}\left({ }_{2}^{1} D\right)^{2} D 3 s^{3} D_{1}$ & & 5149611 & $1.527 \mathrm{E}-12$ & $1.528 \mathrm{E}-12$ & 0.96 \\
\hline 22 & 102 & $2 s^{2} S 2 p^{4}\left({ }_{2}^{1} D\right)^{2} D 3 s^{3} D_{2}$ & & 5150865 & $1.535 \mathrm{E}-12$ & $1.535 \mathrm{E}-12$ & 0.96 \\
\hline 22 & 103 & $2 s^{2} S 2 p^{4}\left({ }_{2}^{3} P\right)^{4} P 3 p^{3} D_{3}^{\circ}$ & & 5151229 & $1.087 \mathrm{E}-12$ & $1.084 \mathrm{E}-12$ & $0.56+0.372 s^{2} S 2 p^{4}\left({ }_{2}^{3} P\right)^{4} P 3 p^{5} D^{\circ}+0.052 s^{2} S 2 p^{4}\left({ }_{2}^{3} P\right)^{4} P 3 p^{5} P^{\circ}$ \\
\hline 22 & 104 & $2 s^{2} S 2 p^{4}\left({ }_{2}^{1} D\right)^{2} D 3 s^{3} D_{3}^{3}$ & & 5152994 & $1.485 \mathrm{E}-12$ & 1.484E-12 & 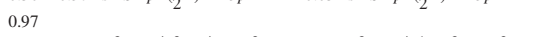 \\
\hline 22 & 105 & $2 s^{2} S 2 p^{4}\left({ }_{2}^{3} P\right)^{4} P 3 p^{3} S_{1}^{0}$ & & 5160492 & $5.324 \mathrm{E}-13$ & $5.312 \mathrm{E}-13$ & $0.64+0.242 s^{2} S 2 p^{4}\left({ }_{2}^{3} P\right)^{4} P 3 p^{3} P^{\circ}+0.042 s^{2} S 2 p^{4}\left({ }_{2}^{1} D\right)^{2} D 3 p^{3} P^{\circ}$ \\
\hline 22 & 106 & $2 s^{2} S 2 p^{4}\left({ }_{2}^{3} P\right)^{4} P 3 p^{3} D_{2}^{\circ}$ & & 5162720 & $7.121 \mathrm{E}-13$ & $7.102 \mathrm{E}-13$ & $\left.0.56+0.282 s^{2} S 2 p^{4}{ }_{2}^{3} P\right)^{4} P 3 p^{3} P^{\circ}+0.112 s^{2} S 2 p^{4}\left({ }_{2}^{3} P\right)^{4} P 3 p^{5} D^{\circ}$ \\
\hline 22 & 107 & $2 s^{2} S 2 p^{4}\left({ }_{2}^{3} P\right)^{4} P 3 p^{5} S_{2}^{0}$ & & 5170132 & 4.304E-11 & 4.313E-11 & $0.86+0.112 s^{2} S 2 p^{4}\left({ }_{2}^{3} P\right)^{4} P 3 p^{5} P^{\circ}$ \\
\hline 22 & 108 & $2 s^{2} S 2 p^{4}\left({ }_{2}^{3} P\right)^{4} P 3 p^{3} D_{1}^{\circ}$ & & 5181856 & $6.881 \mathrm{E}-13$ & $6.862 \mathrm{E}-13$ & $0.83+0.102 s^{2} S 2 p^{4}\left({ }_{2}^{3} P\right)^{4} P 3 p^{3} s^{\circ}+0.032 s^{2} S 2 p^{4}\left({ }_{2}^{3} P\right)^{4} P 3 p^{3} P^{\circ}$ \\
\hline 22 & 109 & $2 s^{2} S 2 p^{4}\left(\frac{3}{2} P\right)^{4} P 3 p^{3} P_{2}^{0}$ & & 5185879 & $6.370 \mathrm{E}-13$ & $6.353 \mathrm{E}-13$ & $0.57+0.292 s^{2} S 2 p^{4}\left(3_{2}^{3} P\right)^{4} P 3 p^{3} D^{\circ}+0.072 s^{2} S 2 p^{4}\left({ }_{2}^{1} D\right)^{2} D 3 p^{3} P^{\circ}$ \\
\hline 22 & 110 & $\left.2 s^{2} S 2 p^{4}{ }_{2}^{3} P\right)^{4} P 3 p^{3} P_{0}^{\circ}$ & & 5188482 & 7.024E-13 & 7.005E-13 & $0.81+0.162 s^{2} S 2 p^{4}\left({ }_{2}^{1} D\right)^{2} D 3 p^{3} P^{\circ}$ \\
\hline 22 & 111 & $2 s^{2} S 2 p^{4}\left({ }_{2}^{1} D\right)^{2} D 3 s^{1} D_{2}$ & & 5194465 & 2.643E-12 & 2.643E-12 & 0.96 \\
\hline 22 & 112 & $\left.2 s^{2} S 2 p^{4}{ }_{2}^{3} P\right)^{4} P 3 p^{3} P_{1}^{\circ}$ & & 5197086 & $5.927 \mathrm{E}-13$ & $5.913 \mathrm{E}-13$ & $\left.0.54+0.242 s^{2} S 2 p^{4}\left({ }_{2}^{3} P\right)^{4} P 3 p^{3} S^{\circ}+0.092 s^{2} S 2 p^{4}{ }_{2}^{3} P\right)^{4} P 3 p^{3} D^{\circ}$ \\
\hline 22 & 113 & $2 s^{2} S 2 p^{4}\left({ }_{0}^{1} S\right)^{2} S 3 s^{3} S_{1}^{1}$ & & 5277315 & $1.490 \mathrm{E}-12$ & $1.490 \mathrm{E}-12$ & $0.84+0.102 s^{2} S 2 p^{4}\left(_{2}^{3} P\right)^{2} P 3 s^{3} P+0.032 s^{2} S 2 p^{4}\left({ }_{2}^{3} P\right)^{2} P 3 s^{1} P$ \\
\hline 22 & 114 & $2 s^{2} S 2 p^{4}\left({ }_{0}^{1} S\right)^{2} S 3 s^{1} s_{0}$ & & 5307035 & 1.949E-12 & $1.949 \mathrm{E}-12$ & $0.63+0.342 s^{2} S 2 p^{4}\left({ }_{2}^{2} P\right)^{2} P 3 s^{3} P$ \\
\hline 22 & 115 & $2 s^{2} S 2 p^{4}\left({ }_{2}^{3} P\right){ }^{4} P 3 d^{5} D_{4}$ & & 5314000 & $8.146 \mathrm{E}-11$ & $8.130 \mathrm{E}-11$ & $0.86+0.132 s^{2} S 2 p^{4}\left({ }_{2}^{2} P\right)^{4} P 3 d^{5} F$ \\
\hline 22 & 116 & $2 s^{2} S 2 p^{4}\left({ }_{2}^{3} P\right)^{4} P 3 d^{5} D_{3}$ & & 5315152 & 7.683E-11 & 7.692E-11 & $0.85+0.082 s^{2} S 2 p^{4}\left({ }_{2}^{2} P\right)^{4} P 3 d^{5} F+0.052 s^{2} S 2 p^{4}\left({ }_{2}^{3} P\right){ }^{4} P 3 d^{5} P$ \\
\hline 22 & 117 & $2 s^{2} S 2 p^{4}\left({ }_{2}^{3} P\right)^{2} P 3 s^{3} P_{2}$ & & 5315356 & $2.292 \mathrm{E}-12$ & $2.292 \mathrm{E}-12$ & 0.95 \\
\hline 22 & 118 & $2 s^{2} S 2 p^{4}\left({ }_{2}^{3} P\right)^{4} P 3 d^{5} D_{2}$ & & 5319709 & $6.707 \mathrm{E}-11$ & $6.720 \mathrm{E}-11$ & $\left.0.84+0.102 s^{2} S 2 p^{4}\left({ }_{2}^{3} P\right)^{4} P 3 d^{5} P+0.042 s^{2} S 2 p^{4}{ }_{2}^{3} P\right){ }^{4} P 3 d^{5} F$ \\
\hline 22 & 119 & $2 s^{2} S 2 p^{4}\left({ }_{2}^{3} P\right)^{2} P 3 s^{1} P_{1}$ & & 5325270 & $9.663 \mathrm{E}-13$ & $9.668 \mathrm{E}-13$ & $0.47+0.472 s^{2} S 2 p^{4}\left(\int_{2}^{2} P\right)^{2} P 3 s^{3} P$ \\
\hline 22 & 120 & $2 s^{2} S 2 p^{4}\left({ }_{2}^{3} P\right){ }^{4} P 3 d^{5} D_{1}$ & & 5326110 & $3.736 \mathrm{E}-11$ & 3.744E-11 & $0.85+0.102 s^{2} S 2 p^{4}\left({ }_{2}^{2} P\right)^{4} P 3 d^{5} P$ \\
\hline 22 & 121 & $2 s^{2} S 2 p^{4}\left({ }_{2}^{1} D\right)^{2} D 3 p^{3} F_{2}^{\circ}$ & & 5326557 & $1.545 \mathrm{E}-11$ & $1.543 \mathrm{E}-11$ & 0.96 \\
\hline 22 & 122 & $2 s^{2} S 2 p^{4}\left({ }_{2}^{1} D\right)^{2} D 3 p^{3} F_{3}^{\circ}$ & & 5331684 & $9.831 \mathrm{E}-12$ & $9.814 \mathrm{E}-12$ & $0.94+0.022 s^{2} S 2 p^{4}\left({ }_{2}^{1} D\right)^{2} D 3 p^{1} F^{\circ}$ \\
\hline 22 & 123 & $2 s^{2} S 2 p^{4}\left({ }_{2}^{3} P\right)^{4} P 3 d^{5} D_{0}$ & & 5332386 & $3.136 \mathrm{E}-11$ & $3.151 \mathrm{E}-11$ & $0.97+0.022 s^{2} S 2 p^{4}\left({ }_{2}^{3} P\right)^{4} P 3 d^{3} P$ \\
\hline
\end{tabular}




\begin{tabular}{|c|c|c|c|c|c|c|c|}
\hline $\mathrm{z}$ & Key & Level & $E_{\mathrm{NIST}}$ & $E_{\mathrm{MCDHF} / \mathrm{RCI}}$ & $\tau_{\mathrm{MCDHF} / \mathrm{RCI}}^{l}$ & $\tau_{\mathrm{MCDHF} / \mathrm{RCI}}^{v}$ & $L S$-composition \\
\hline 22 & 124 & $2 s^{2} S 2 p^{4}\left({ }_{2}^{1} D\right)^{2} D 3 p^{1} P_{1}^{\circ}$ & & 5333605 & $5.140 \mathrm{E}-13$ & $5.129 \mathrm{E}-13$ & $0.67+0.152 s^{2} S 2 p^{4}\left({ }_{2}^{1} D\right)^{2} D 3 p^{3} D^{\circ}+0.072 s^{2} S 2 p^{4}\left({ }_{2}^{3} P\right)^{2} P 3 p^{1} P^{\circ}$ \\
\hline 22 & 125 & $2 s^{2} S 2 p^{4}\left({ }_{2}^{2} P\right)^{4} P 3 d^{5} F_{5}$ & & 5334541 & $1.261 \mathrm{E}-10$ & $1.211 \mathrm{E}-10$ & 0.98 \\
\hline 22 & 126 & $2 s^{2} S 2 p^{4}\left({ }_{2}^{1} D\right)^{2} D 3 p^{3} F_{4}^{\circ}$ & & 5339808 & $3.609 \mathrm{E}-11$ & $3.591 \mathrm{E}-11$ & 0.98 \\
\hline 22 & 127 & $2 s^{2} S 2 p^{4}\left({ }_{2}^{2} D\right)^{2} D 3 p^{1} F_{3}^{\circ}$ & & 5350723 & $5.448 \mathrm{E}-13$ & 5.433E-13 & $0.85+0.092 s^{2} S 2 p^{4}\left({ }_{2}^{1} D\right)^{2} D 3 p^{3} D^{\circ}+0.032 s^{2} S 2 p^{4}\left({ }_{2}^{1} D\right)^{2} D 3 p^{3} F^{\circ}$ \\
\hline 22 & 128 & $2 s^{2} S 2 p^{4}\left({ }_{2}^{3} P\right)^{4} P 3 d^{5} F_{4}{ }^{3}$ & & 5351479 & 1.053E-10 & $1.025 \mathrm{E}-10$ & $0.76+0.132 s^{2} S 2 p^{4}\left({ }_{2}^{3} P\right)^{4} P 3 d^{3} F+0.102 s^{2} S 2 p^{4}{ }_{2}^{3} P{ }_{2}^{2}{ }^{4} P 3 d^{5} D$ \\
\hline 22 & 129 & $2 s^{2} S 2 p^{4}\left({ }_{2}^{1} D\right)^{2} D 3 p^{3} D_{2}^{\circ}$ & & 5359328 & $9.685 \mathrm{E}-12$ & $9.662 \mathrm{E}-12$ & $0.92+0.022 s^{2} S 2 p^{4}\left({ }_{2}^{3} P\right)^{2} P 3 p^{3} P^{\circ}+0.022 s^{2} S 2 p^{4}\left({ }_{2}^{1} D\right)^{2} D 3 p^{3} P^{\circ}$ \\
\hline 22 & 130 & $2 s^{2} S 2 p^{4}\left(\frac{3}{3} P\right)^{4} P 3 d^{5} P_{1}$ & & 5360455 & $7.716 \mathrm{E}-12$ & $7.724 \mathrm{E}-12$ & $0.87+0.092 s^{2} S 2 p^{4}\left(3_{2}^{3} P\right)^{4} P 3 d^{5} D$ \\
\hline 22 & 131 & $2 s^{2} S 2 p^{4}\left({ }_{2}^{1} D\right)^{2} D 3 p^{3} D_{1}^{\circ}$ & & 5362276 & $2.222 \mathrm{E}-12$ & $2.216 \mathrm{E}-12$ & $0.82+0.142 s^{2} S 2 p^{4}\left({ }_{2}^{1} D\right)^{2} D 3 p^{1} P^{\circ}$ \\
\hline 22 & 132 & $2 s^{2} S 2 p^{4}\left(_{2}^{3} P\right)^{4} P 3 d^{5} F_{3}$ & & 5362311 & $8.766 \mathrm{E}-12$ & $8.737 \mathrm{E}-12$ & $0.84+0.062 s^{2} S 2 p^{4}\left({ }_{2}^{3} P\right)^{4} P 3 d^{3} F+0.052 s^{2} S 2 p^{4}\left({ }_{2}^{3} P\right)^{4} P 3 d^{5} D$ \\
\hline 22 & 133 & $2 s^{2} S 2 p^{4}\left({ }_{2}^{3} P\right)^{2} P 3 s^{3} P_{1}$ & & 5364004 & $8.886 \mathrm{E}-13$ & $8.886 \mathrm{E}-13$ & $0.40+0.462 s^{2} S 2 p^{4}\left({ }_{2}^{3} P\right)^{2} P 3 s^{1} P+0.112 s^{2} S 2 p^{4}\left({ }_{0}^{1} S\right)^{2} S 3 s^{3} S$ \\
\hline 22 & 134 & $2 s^{2} S 2 p^{4}\left({ }_{2}^{1} D\right)^{2} D 3 p^{3} D_{3}^{\circ}$ & & 5365571 & 4.223E-12 & $4.210 \mathrm{E}-12$ & $0.87+0.102 s^{2} S 2 p^{4}\left({ }_{2}^{1} D\right)^{2} D 3 p^{1} F^{\circ}$ \\
\hline 22 & 135 & $\left.2 s^{2} S 2 p^{4}{ }_{2}^{\frac{2}{3}} P\right)^{4} P 3 d^{5} F_{2}{ }^{3}$ & & 5367789 & 5.943E-12 & $5.936 \mathrm{E}-12$ & $0.60+0.362 s^{2} S 2 p^{4}\left({ }_{2}^{2} P\right)^{4} P 3 d^{5} P$ \\
\hline 22 & 136 & $2 s^{2} S 2 p^{4}\left({ }_{2}^{3} P\right)^{2} P 3 s^{3} P_{0}$ & & 5368744 & $2.410 \mathrm{E}-12$ & $2.410 \mathrm{E}-12$ & $0.63+0.342 s^{2} S 2 p^{4}\left({ }_{0}^{1} S\right)^{2} S 3 s^{1} S$ \\
\hline 22 & 137 & $2 s^{2} S 2 p^{4}\left({ }_{2}^{3} P\right)^{4} P 3 d^{5} P_{2}$ & & 5373616 & $2.019 \mathrm{E}-11$ & $2.028 \mathrm{E}-11$ & $0.52+0.322 s^{2} S 2 p^{4}\left(\int_{2}^{3} P\right)^{4} P 3 d^{5} F+0.132 s^{2} S 2 p^{4}\left({ }_{2}^{3} P\right)^{4} P 3 d^{5} D$ \\
\hline 22 & 138 & $2 s^{2} S 2 p^{4}\left({ }_{2}^{1} D\right)^{2} D 3 p^{1} D_{2}^{\circ}$ & & 5373861 & 7.119E-13 & 7.097E-13 & $0.55+0.362 s^{2} S 2 p^{4}\left({ }_{2}^{1} D\right)^{2} D 3 p^{3} P^{\circ}+0.032 s^{2} S 2 p^{4}\left({ }_{2}^{3} P\right)^{2} P 3 p^{3} P^{\circ}$ \\
\hline 22 & 139 & $2 s^{2} S 2 p^{4}\left(\frac{3}{2} P\right)^{4} P 3 d^{5} F_{1}$ & & 5374182 & $1.703 \mathrm{E}-11$ & 1.693E-11 & $0.95+0.032 s^{2} S 2 p^{4}\left(3_{2}^{3} P\right)^{4} P 3 d^{5} D$ \\
\hline 22 & 140 & $2 s^{2} S 2 p^{4}\left({ }_{2}^{1} D\right)^{2} D 3 p^{3} P_{1}^{\circ}$ & & 5380898 & $1.530 \mathrm{E}-12$ & $1.525 \mathrm{E}-12$ & $\left.0.74+0.112 s^{2} S 2 p^{4}\left({ }_{2}^{3} P\right)^{4} P 3 p^{3} P^{\circ}+0.082 s^{2} S 2 p^{4}{ }_{2}^{3} P\right)^{2} P 3 p^{3} P^{\circ}$ \\
\hline 22 & 141 & $2 s^{2} S 2 p^{4}\left({ }_{2}^{3} P\right)^{4} P 3 d^{3} F_{4}$ & & 5381021 & $8.862 \mathrm{E}-11$ & $8.685 \mathrm{E}-11$ & $0.85+0.102 s^{2} S 2 p^{4}\left({ }_{2}^{3} P\right)^{4} P 3 d^{5} F+0.032 s^{2} S 2 p^{4}\left({ }_{2}^{3} P\right)^{4} P 3 d^{5} D$ \\
\hline 22 & 142 & $2 s^{2} S 2 p^{4}\left({ }_{2}^{1} D\right)^{2} D 3 p^{3} P_{2}^{\circ}$ & & 5381579 & $8.036 \mathrm{E}-13$ & $8.013 \mathrm{E}-13$ & $0.47+0.412 s^{2} S 2 p^{4}\left({ }_{2}^{1} D\right)^{2} D 3 p^{1} D^{\circ}+0.082 s^{2} S 2 p^{4}\left({ }_{2}^{3} P\right)^{4} P 3 p^{3} P^{\circ}$ \\
\hline 22 & 143 & $2 s^{2} S 2 p^{4}\left({ }_{2}^{3} P\right)^{4} P 3 d^{5} P_{3}^{2}$ & & 5383569 & 5.373E-12 & $5.380 \mathrm{E}-12$ & $0.85+0.072 s^{2} S 2 p^{4}\left({ }_{2}^{3} P\right)^{4} P 3 d^{5} D+0.032 s^{2} S 2 p^{4}\left({ }_{2}^{3} P\right)^{2} P 3 d^{3} D$ \\
\hline 22 & 144 & $2 s^{2} S 2 p^{4}\left({ }_{2}^{1} D\right)^{2} D 3 p^{3} P_{0}^{\circ}$ & & 5383615 & 1.448E-12 & 1.443E-12 & $\left.\left.0.73+0.142 s^{2} S 2 p^{4}{ }_{2}^{3} P\right)^{4} P 3 p^{3} P^{\circ}+0.112 s^{2} S 2 p^{4}{ }_{2}^{3} P\right)^{2} P 3 p^{3} P^{\circ}$ \\
\hline 22 & 145 & $2 s^{2} S 2 p^{4}\left({ }_{3}^{3} P\right)^{4} P 3 d^{3} F_{3}$ & & 5394347 & $1.471 \mathrm{E}-12$ & $1.468 \mathrm{E}-12$ & $0.76+0.122 s^{2} S 2 p^{4}\left(3_{2}^{3} P\right)^{4} P 3 d^{3} D+0.052 s^{2} S 2 p^{4}\left(3_{2}^{2} P\right)^{4} P 3 d^{5} F$ \\
\hline 22 & 146 & $2 s^{2} S 2 p^{4}\left({ }_{2}^{3} P\right)^{4} P 3 d^{3} P_{0}$ & & 5404762 & $5.687 \mathrm{E}-13$ & $5.682 \mathrm{E}-13$ & $0.87+0.092 s^{2} S 2 p^{4}\left({ }_{2}^{4} D\right)^{2} D 3 d^{3} P+0.032 s^{2} S 2 p^{4}\left(3_{2}^{3} P\right)^{4} P 3 d^{5} D$ \\
\hline 22 & 147 & $2 s^{2} S 2 p^{4}\left({ }_{2}^{3} P\right){ }^{4} P 3 d^{3} F_{2}$ & & 5405199 & $5.162 \mathrm{E}-13$ & $5.156 \mathrm{E}-13$ & $\left.0.53+0.302 s^{2} S 2 p^{4}\left({ }_{2}^{2} P\right)^{4} P 3 d^{3} D+0.112 s^{2} S 2 p^{4}{ }_{2}^{3} P\right)^{4} P 3 d^{3} P$ \\
\hline 22 & 148 & $2 s^{2} S 2 p^{4}\left({ }_{2}^{3} P\right){ }^{4} P 3 d^{3} P_{1}$ & & 5407506 & 3.905E-13 & $3.902 \mathrm{E}-13$ & $0.70+0.192 s^{2} S 2 p^{4}\left({ }_{2}^{2} P\right)^{4} P 3 d^{3} D+0.062 s^{2} S 2 p^{4}\left({ }_{2}^{1} D\right)^{2} D 3 d^{3} P$ \\
\hline 22 & 149 & $2 s^{2} S 2 p^{4}\left({ }_{2}^{3} P\right){ }^{4} P 3 d^{3} D_{2}$ & & 5420762 & $4.860 \mathrm{E}-13$ & $4.856 \mathrm{E}-13$ & $\left.0.21+0.412 s^{2} S 2 p^{4}\left({ }_{2}^{3} P\right)^{4} P 3 d^{3} F+0.312 s^{2} S 2 p^{4}{ }_{2}^{3} P\right)^{4} P 3 d^{3} P$ \\
\hline 22 & 150 & $2 s^{2} S 2 p^{4}\left({ }_{2}^{3} P\right){ }^{4} P 3 d^{3} D_{3}$ & & 5430816 & $2.016 \mathrm{E}-13$ & 2.013E-13 & $0.78+0.152 s^{2} S 2 p^{4}\left({ }_{2}^{2} P\right)^{4} P 3 d^{3} F+0.032 s^{2} S 2 p^{4}\left({ }_{2}^{1} D\right)^{2} D 3 d^{3} D$ \\
\hline 22 & 151 & $2 s^{2} S 2 p^{4}\left({ }_{2}^{3} P\right){ }^{4} P 3 d^{3} D_{1}$ & & 5432297 & $2.659 \mathrm{E}-13$ & $2.656 \mathrm{E}-13$ & $0.69+0.182 s^{2} S 2 p^{4}\left({ }_{2}^{3} P\right)^{4} P 3 d^{3} P+0.072 s^{2} S 2 p^{4}\left({ }_{2}^{1} D\right)^{2} D 3 d^{3} D$ \\
\hline 22 & 152 & $2 s^{2} S 2 p^{4}\left({ }_{3}^{3} P\right)^{4} P 3 d^{3} P_{2}$ & & 5443409 & $3.179 \mathrm{E}-13$ & $3.175 \mathrm{E}-13$ & $0.49+0.382 s^{2} S 2 p^{4}\left(3_{2}^{3} P\right)^{4} P 3 d^{3} D+0.052 s^{2} S 2 p^{4}\left({ }_{2}^{1} D\right)^{2} D 3 d^{3} P$ \\
\hline 22 & 153 & $2 s^{2} S 2 p^{4}\left({ }_{0}^{1} S\right)^{2} S 3 p^{3} P_{0}^{\circ}$ & & 5458851 & $3.241 \mathrm{E}-12$ & $3.239 \mathrm{E}-12$ & $0.71+0.212 s^{2} S 2 p^{4}\left({ }_{2}^{3} P\right)^{2} P 3 p^{3} P^{\circ}+0.032 s^{2} S 2 p^{4}\left({ }_{2}^{1} D\right)^{2} D 3 p^{3} P^{\circ}$ \\
\hline 22 & 154 & $2 s^{2} S 2 p^{4}\left({ }_{0}^{1} S\right)^{2} S 3 p^{3} P_{1}^{\circ}$ & & 5464752 & 2.335E-12 & 2.332E-12 & $\left.0.67+0.142 s^{2} S 2 p^{4}{ }_{2}^{3} P\right)^{2} P 3 p^{3} P^{\circ}+0.112 s^{2} S 2 p^{4}\left({ }_{2}^{3} P\right)^{2} P 3 p^{3} D^{\circ}$ \\
\hline 22 & 155 & $2 s^{2} S 2 p^{4}\left({ }_{0}^{1} S\right)^{2} S 3 p^{3} P_{2}^{0}$ & & 5477649 & 3.357E-12 & $3.352 \mathrm{E}-12$ & $\left.\left.0.74+0.182 s^{2} S 2 p^{4}{ }_{2}^{3} P\right)^{2} P 3 p^{3} D^{\circ}+0.032 s^{2} S 2 p^{4}{ }_{2}^{3} P\right)^{2} P 3 p^{3} P^{\circ}$ \\
\hline 22 & 156 & $2 s^{2} S 2 p^{4}\left({ }_{0}^{1} S\right)^{2} S 3 p^{1} P_{1}^{\circ}$ & & 5484980 & $6.066 \mathrm{E}-13$ & $6.050 \mathrm{E}-13$ & $\left.0.68+0.102 s^{2} S 2 p^{4}\left({ }_{2}^{3} P\right)^{2} P 3 p^{3} P^{\circ}+0.102 s^{2} S 2 p^{4}{ }_{2}^{3} P\right)^{2} P 3 p^{3} D^{\circ}$ \\
\hline 22 & 157 & $2 s^{2} S 2 p^{4}\left({ }_{2}^{3} P\right)^{2} P 3 p^{3} P_{2}^{\circ}$ & & 5494829 & $2.555 \mathrm{E}-12$ & $2.551 \mathrm{E}-12$ & $0.64+0.122 s^{2} S 2 p^{4}\left({ }_{2}^{3} P\right)^{2} P 3 p^{3} D^{\circ}+0.092 s^{2} S 2 p^{4}\left({ }_{0}^{1} S\right)^{2} S 3 p^{3} P^{\circ}$ \\
\hline 22 & 158 & $2 s^{2} S 2 p^{4}\left({ }_{2}^{3} P\right)^{2} P 3 p p^{1} D_{2}^{\circ}$ & & 5507219 & 2.161E-12 & 2.157E-12 & $\left.0.47+0.302 s^{2} S 2 p^{4}{ }_{2}^{3} P\right)^{2} P 3 p^{3} D^{\circ}+0.182 s^{2} S 2 p^{4}\left({ }_{2}^{3} P\right)^{2} P 3 p^{3} P^{\circ}$ \\
\hline 22 & 159 & $\left.2 s^{2} S 2 p^{4}{ }_{2}^{2} P\right)^{2} P 3 p^{3} D_{3}^{\circ}$ & & 5507918 & $1.200 \mathrm{E}-12$ & 1.197E-12 & $0.96+2+2+2$ \\
\hline 22 & 160 & $2 s^{2} S 2 p^{4}\left({ }_{2}^{2} P\right)^{2} P 3 p^{3} P_{1}^{0}$ & & 5514021 & 2.765E-12 & $2.759 \mathrm{E}-12$ & $0.45+0.192 s^{2} S 2 p^{4}\left({ }_{2}^{3} P\right)^{2} P 3 p^{3} S^{\circ}+0.182 s^{2} S 2 p^{4}\left({ }_{0}^{1} S\right)^{2} S 3 p^{3} P^{\circ}$ \\
\hline 22 & 161 & $\left.2 s^{2} S 2 p^{4}{ }_{2}^{3} P\right)^{2} P 3 p^{3} P_{0}^{\circ}$ & & 5524492 & $5.460 \mathrm{E}-12$ & $5.458 \mathrm{E}-12$ & $\left.0.40+0.402 s^{2} S 2 p^{4} \int_{2}^{3} P\right)^{2} P 3 p^{1} S^{\circ}+0.082 s^{2} S 2 p^{4}\left(_{0}^{1} S\right)^{2} S 3 p^{3} P^{\circ}$ \\
\hline 22 & 162 & $\left.2 s^{2} S 2 p^{4}{ }_{2}^{3} P\right)^{2} P 3 p^{3} D_{1}^{\circ}$ & & 5535843 & 1.061E-12 & $1.059 \mathrm{E}-12$ & $\left.0.66+0.142 s^{2} S 2 p^{4}{ }_{2}^{3} P\right)^{2} P 3 p^{3} S^{\circ}+0.092 s^{2} S 2 p^{4}\left({ }_{0}^{1} S\right)^{2} S 3 p^{1} P^{\circ}$ \\
\hline 22 & 163 & $2 s^{2} S 2 p^{4}\left({ }_{2}^{1} D\right)^{2} D 3 d^{3} G_{3}$ & & 5547430 & $4.352 \mathrm{E}-11$ & 4.293E-11 & 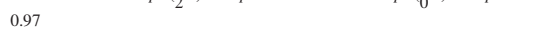 \\
\hline 22 & 164 & $2 s^{2} S 2 p^{4}\left({ }_{2}^{2} D\right)^{2} D 3 d^{3} G_{4}$ & & 5549579 & $4.595 \mathrm{E}-11$ & $4.526 \mathrm{E}-11$ & 0.98 \\
\hline 22 & 165 & $2 s^{2} S 2 p^{4}\left({ }_{2}^{1} D\right)^{2} D 3 d^{3} G_{5}$ & & 5552311 & $4.845 \mathrm{E}-11$ & $4.772 \mathrm{E}-11$ & 0.98 \\
\hline 22 & 166 & $2 s^{2} S 2 p^{4}\left({ }_{2}^{3} P\right)^{2} P 3 p^{3} D_{2}^{\circ}$ & & 5553583 & 2.624E-12 & 2.619E-12 & $0.37+0.432 s^{2} S 2 p^{4}\left({ }_{2}^{3} P\right)^{2} P 3 p^{1} D^{\circ}+0.132 s^{2} S 2 p^{4}\left({ }_{0}^{1} S\right)^{2} S 3 p^{3} P^{\circ}$ \\
\hline 22 & 167 & $2 s^{2} S 2 p^{4}\left(3_{2}^{2} P\right)^{2} P 3 p^{1} S_{0}^{0}$ & & 5554467 & $7.746 \mathrm{E}-12$ & $7.747 \mathrm{E}-12$ & $0.55+0.242 s^{2} S 2 p^{4}\left(\int_{2}^{3} P\right)^{2} P 3 p^{3} P^{\circ}+0.172 s^{2} S 2 p^{4}\left({ }_{0}^{1} S\right)^{2} S 3 p^{3} P^{\circ}$ \\
\hline 22 & 168 & $2 s^{2} S 2 p^{4}\left({ }_{2}^{3} P\right)^{2} P 3 p^{3} S_{1}^{0}$ & & 5561179 & $1.151 \mathrm{E}-12$ & $1.146 \mathrm{E}-12$ & $0.61+0.152 s^{2} S 2 p^{4}\left({ }_{2}^{2} P\right)^{2} P 3 p^{3} P^{\circ}+0.122 s^{2} S 2 p^{4}\left({ }_{0}^{1} S\right)^{2} S 3 p^{1} P^{\circ}$ \\
\hline 22 & 169 & $2 s^{2} S 2 p^{4}\left({ }_{2}^{1} D\right)^{2} D 3 d^{3} F_{2}$ & & 5578924 & $1.584 \mathrm{E}-11$ & $1.585 \mathrm{E}-11$ & $0.94+0.032 s^{2} S 2 p^{4}\left({ }_{2}^{1} D\right)^{2} D 3 d^{1} D$ \\
\hline 22 & 170 & $2 s^{2} S 2 p^{4}\left({ }_{2}^{1} D\right)^{2} D 3 d^{3} s_{1}$ & & 5579073 & $1.119 \mathrm{E}-13$ & $1.118 \mathrm{E}-13$ & 0.96 \\
\hline 22 & 171 & $2 s^{2} S 2 p^{4}\left({ }_{2}^{1} D\right)^{2} D 3 d^{3} F_{3}$ & & 5581283 & $6.317 \mathrm{E}-12$ & $6.311 \mathrm{E}-12$ & $0.95+0.032 s^{2} S 2 p^{4}\left({ }_{2}^{1} D\right)^{2} D 3 d^{3} D$ \\
\hline 22 & 172 & $2 s^{2} S 2 p^{4}\left({ }_{2}^{1} D\right)^{2} D 3 d^{3} F_{4}$ & & 5582466 & 3.910E-11 & 3.895E-11 & $0.73+0.242 s^{2} S 2 p^{4}\left({ }_{2}^{\mathrm{T}} D\right)^{2} D 3 d^{1} G$ \\
\hline 22 & 173 & $2 s^{2} S 2 p^{4}\left({ }_{2}^{1} D\right)^{2} D 3 d^{3} D_{1}$ & & 5587371 & $2.308 \mathrm{E}-13$ & $2.305 \mathrm{E}-13$ & $0.54+0.202 s^{2} S 2 p^{4}\left({ }_{2}^{1} D\right)^{2} D 3 d^{1} P+0.142 s^{2} S 2 p^{4}\left({ }_{2}^{1} D\right)^{2} D 3 d^{3} P$ \\
\hline 22 & 174 & $2 s^{2} S 2 p^{4}\left({ }_{2}^{1} D\right)^{2} D 3 d^{1} G_{4}$ & & 5588265 & 3.603E-11 & $3.590 \mathrm{E}-11$ & $0.73+0.242 s^{2} S 2 p^{4}\left({ }_{2}^{1} D\right)^{2} D 3 d^{3} F$ \\
\hline 22 & 175 & $2 s^{2} S 2 p^{4}\left({ }_{2}^{1} D\right)^{2} D 3 d^{3} P_{0}$ & & 5589677 & $1.272 \mathrm{E}-13$ & $1.271 \mathrm{E}-13$ & $\left.0.79+0.092 s^{2} S 2 p^{4}\left({ }_{2}^{1} D\right)^{2} D 3 d^{1} S+0.072 s^{2} S 2 p^{4}{ }_{2}^{3} P\right)^{4} P 3 d^{3} P$ \\
\hline 22 & 176 & $2 s^{2} S 2 p^{4}\left({ }_{2}^{1} D\right)^{2} D 3 d^{3} D_{2}$ & & 5590336 & $1.602 \mathrm{E}-13$ & $1.600 \mathrm{E}-13$ & $0.49+0.392 s^{2} S 2 p^{4}\left({ }_{2}^{1} D\right)^{2} D 3 d^{3} P+0.032 s^{2} S 2 p^{4}\left({ }_{2}^{1} D\right)^{2} D 3 d^{1} D$ \\
\hline 22 & 177 & $2 s^{2} S 2 p^{4}\left({ }_{2}^{2} D\right)^{2} D 3 d^{3} D_{3}$ & & 5590867 & $1.934 \mathrm{E}-13$ & $1.932 \mathrm{E}-13$ & $0.90+0.042 s^{2} S 2 p^{4}\left({ }_{2}^{2} P\right)^{4} P 3 d^{3} D+0.022 s^{2} S 2 p^{4}\left({ }_{2}^{1} D\right)^{2} D 3 d^{3} F$ \\
\hline 22 & 178 & $2 s^{2} S 2 p^{4}\left({ }_{2}^{1} D\right)^{2} D 3 d^{3} P_{2}$ & & 5590882 & $1.353 \mathrm{E}-13$ & $1.352 \mathrm{E}-13$ & $0.51+0.382 s^{2} S 2 p^{4}\left({ }_{2}^{1} D\right)^{2} D 3 d^{3} D+0.042 s^{2} S 2 p^{4}\left({ }_{2}^{3} P\right)^{4} P 3 d^{3} D$ \\
\hline 22 & 179 & $2 s^{2} S 2 p^{4}\left({ }_{2}^{1} D\right)^{2} D 3 d^{3} P_{1}$ & & 5593779 & $1.209 \mathrm{E}-13$ & $1.208 \mathrm{E}-13$ & $0.72+0.122 s^{2} S 2 p^{4}\left({ }_{2}^{1} D\right)^{2} D 3 d^{3} D+0.082 s^{2} S 2 p^{4}\left({ }_{2}^{2} P\right)^{4} P 3 d^{3} P$ \\
\hline 22 & 180 & $2 s^{2} S 2 p^{4}\left({ }_{2}^{1} D\right)^{2} D 3 d^{1} P_{1}$ & & 5605901 & 2.753E-13 & $2.751 \mathrm{E}-13$ & $0.68+0.192 s^{2} S 2 p^{4}\left({ }_{2}^{4} D\right)^{2} D 3 d^{3} D+0.052 s^{2} S 2 p^{4}\left(_{2}^{3} P\right)^{2} P 3 d^{1} P$ \\
\hline 22 & 181 & $2 s^{2} S 2 p^{4}\left({ }_{2}^{1} D\right)^{2} D 3 d^{1} D_{2}$ & & 5609675 & $7.207 \mathrm{E}-13$ & 7.193E-13 & $0.82+0.052 s^{2} S 2 p^{4}\left({ }_{2}^{2} P\right)^{2} P 3 d^{1} D+0.042 s^{2} S 2 p^{4}\left({ }_{2}^{2} D\right)^{2} D 3 d^{3} F$ \\
\hline 22 & 182 & $2 s^{2} S 2 p^{4}\left(I_{1}^{2} D\right)^{2} D 3 d^{1} F_{3}$ & & 5617081 & $2.843 \mathrm{E}-12$ & $2.843 \mathrm{E}-12$ & 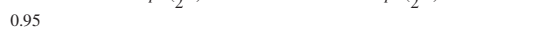 \\
\hline 22 & 183 & $2 s^{2} S 2 p^{4}\left({ }_{2}^{3} P\right)^{2} P 3 p p^{1} P_{1}^{\circ}$ & & 5621027 & $2.253 \mathrm{E}-12$ & $2.245 \mathrm{E}-12$ & $0.83+0.062 s^{2} S 2 p^{4}\left({ }_{2}^{1} D\right)^{2} D 3 p^{1} P^{\circ}+0.022 s^{2} S 2 p^{4}\left(1_{0}^{1} S\right)^{2} S 3 p^{1} P^{\circ}$ \\
\hline 22 & 184 & $2 s^{2} S 2 p^{4}\left({ }_{2}^{1} D\right)^{2} D 3 d^{1} S_{0}^{1}$ & & 5622882 & $1.280 \mathrm{E}-13$ & $1.279 \mathrm{E}-13$ & $0.88+0.072 s^{2} S 2 p^{4}\left({ }_{2}^{1} D\right)^{2} D 3 d^{3} P+0.032 s^{2} S 2 p^{4}\left({ }_{2}^{3} P\right)^{4} P 3 d^{3} P$ \\
\hline 22 & 185 & $2 s^{2} S 2 p^{4}\left({ }_{0}^{1} S\right)^{2} S 3 d^{3} D_{1}$ & & 5691092 & 6.205E-12 & $6.212 \mathrm{E}-12$ & $0.65+0.282 s^{2} S 2 p^{4}\left({ }_{2}^{2} P\right)^{2} P 3 d^{3} D+0.042 s^{2} S 2 p^{4}\left({ }_{2}^{1} D\right)^{2} D 3 d^{3} D$ \\
\hline 22 & 186 & $2 s^{2} S 2 p^{4}\left({ }_{0}^{1} S\right)^{2} S 3 d^{3} D_{2}$ & & 5695271 & 2.874E-12 & $2.875 \mathrm{E}-12$ & $\left.0.70+0.162 s^{2} S 2 p^{4}\left({ }_{2}^{2} P\right)^{2} P 3 d^{3} D+0.072 s^{2} S 2 p^{4}{ }_{2}^{3} P\right)^{2} P 3 d^{3} F$ \\
\hline 22 & 187 & $2 s^{2} S 2 p^{4}\left({ }_{0}^{1} S\right)^{2} S 3 d^{3} D_{3}$ & & 5698232 & $5.214 \mathrm{E}-13$ & $5.208 \mathrm{E}-13$ & $\left.\left.0.82+0.072 s^{2} S 2 p^{4}{ }_{2}^{3} P\right)^{2} P 3 d^{3} F+0.042 s^{2} S 2 p^{4}{ }_{2}^{3} P\right)^{2} P 3 d^{1} F$ \\
\hline 22 & 188 & $2 s^{2} S 2 p^{4}\left({ }_{2}^{3} P\right)^{2} P 3 d^{3} D_{3}$ & & 5711499 & $6.224 \mathrm{E}-13$ & $6.218 \mathrm{E}-13$ & $\left.0.85+0.072 s^{2} S 2 p^{4}{ }_{2}^{3} P\right)^{2} P 3 d^{3} F$ \\
\hline 22 & 189 & $\left.2 s^{2} S 2 p^{4}{ }_{2}^{2} P\right)^{2} P 3 d^{3} D_{2}$ & & 5716003 & 3.424E-13 & $3.420 \mathrm{E}-13$ & $0.48+0.282 s^{2} S 2 p^{4}\left({ }_{0}^{1} S\right)^{2} S 3 d^{1} D+0.072 s^{2} S 2 p^{4}\left({ }_{0}^{1} S\right)^{2} S 3 d^{3} D$ \\
\hline 22 & 190 & $2 s^{2} S 2 p^{4}\left({ }_{0}^{1} S\right)^{2} S 3 d^{1} D_{2}$ & & 5725774 & 7.038E-13 & $7.029 \mathrm{E}-13$ & $0.34+0.252 s^{2} S 2 p^{4}\left(\int_{2}^{3} P\right)^{2} P 3 d^{3} P+0.182 s^{2} S 2 p^{4}\left({ }_{2}^{3} P\right)^{2} P 3 d^{3} D$ \\
\hline 22 & 191 & $2 s^{2} S 2 p^{4}\left({ }_{2}^{3} P\right)^{2} P 3 d^{3} D_{1}$ & & 5732355 & $2.130 \mathrm{E}-13$ & $2.127 \mathrm{E}-13$ & $\left.0.51+0.212 s^{2} S 2 p^{4}{ }_{2}^{3} P\right)^{2} P 3 d^{3} P+0.192 s^{2} S 2 p^{4}\left({ }_{0}^{1} S\right)^{2} S 3 d^{3} D$ \\
\hline 22 & 192 & $2 s^{2} S 2 p^{4}\left({ }_{2}^{3} P\right){ }^{2} P 3 d^{3} F_{4}$ & & 5734180 & $1.052 \mathrm{E}-11$ & $1.066 \mathrm{E}-11$ & 0.97 \\
\hline 22 & 193 & $2 s^{2} S 2 p^{4}\left({ }_{2}^{3} P\right)^{2} P 3 d^{1} F_{3}$ & & 5734799 & $1.075 \mathrm{E}-11$ & 1.084E-11 & $0.53+0.432 s^{2} S 2 p^{4}\left({ }_{2}^{3} P\right)^{2} P 3 d^{3} F$ \\
\hline 22 & 194 & $\left.2 s^{2} S 2 p^{4}{ }_{3}^{3} P\right)^{2} P 3 d^{3} P_{0}$ & & 5750292 & $4.656 \mathrm{E}-13$ & 4.651E-13 & $0.93+0.032 s^{2} S 2 p^{4}\left({ }_{1}^{2} D\right)^{2} D 3 d^{3} P$ \\
\hline 22 & 195 & $2 s^{2} S 2 p^{4}\left({ }_{2}^{3} P\right)^{2} P 3 d^{3} P_{1}$ & & 5757827 & $3.350 \mathrm{E}-13$ & $3.346 \mathrm{E}-13$ & $0.69+0.102 s^{2} S 2 p^{4}\left({ }_{2}^{3} P\right)^{2} P 3 d^{1} P+0.092 s^{2} S 2 p^{4}\left({ }_{2}^{3} P\right)^{2} P 3 d^{3} D$ \\
\hline 22 & 196 & $2 s^{2} S 2 p^{4}\left({ }_{2}^{2} P\right)^{2} P 3 d^{3} F_{2}$ & & 5759755 & 4.406E-13 & 4.401E-13 & $\left.\left.0.55+0.192 s^{2} S 2 p^{4}{ }_{2}^{2} P\right)^{2} P 3 d^{3} P+0.152 s^{2} S 2 p^{4}{ }_{2}^{2} P\right)^{2} P 3 d^{1} D$ \\
\hline 22 & 197 & $2 s^{2} S 2 p^{4}\left({ }_{2}^{3} P\right)^{2} P 3 d^{3} F_{3}$ & & 5774988 & $1.123 \mathrm{E}-11$ & $1.132 \mathrm{E}-11$ & $0.41+0.392 s^{2} S 2 p^{4}\left({ }_{2}^{3} P\right)^{2} P 3 d^{1} F+0.132 s^{2} S 2 p^{4}\left({ }_{0}^{1} S\right)^{2} S 3 d^{3} D$ \\
\hline 22 & 198 & $2 s^{2} S 2 p^{4}\left({ }_{2}^{3} P\right)^{2} P 3 d^{3} P_{2}$ & & 5784126 & $1.353 \mathrm{E}-12$ & $1.351 \mathrm{E}-12$ & $0.49+0.222 s^{2} S 2 p^{4}\left(r_{0}^{1} S\right)^{2} S 3 d^{1} D+0.122 s^{2} S 2 p^{4}\left({ }_{2}^{3} P\right)^{2} P 3 d^{3} F$ \\
\hline 22 & 199 & $2 s^{2} S 2 p^{4}\left({ }_{3}^{3} P\right)^{2} P 3 d^{1} P_{1}$ & & 5809581 & $1.227 \mathrm{E}-13$ & $1.225 \mathrm{E}-13$ & $0.77+0.062 s^{2} S 2 p^{4}\left({ }_{2}^{1} D\right)^{2} D 3 d^{1} P+0.052 s^{2} S 2 p^{4}\left(C_{0}^{1} S\right)^{2} S 3 d^{3} D$ \\
\hline 22 & 200 & $2 s^{2} S 2 p^{4}\left({ }_{2}^{3} P\right)^{2} P 3 d^{1} D_{2}$ & & 5817188 & 7.971E-14 & 7.961E-14 & $0.69+0.102 s^{2} S 2 p^{4}\left(l_{0}^{1} S\right)^{2} S 3 d^{1} D+0.082 s^{2} S 2 p^{4}\left({ }_{2}^{3} P\right)^{2} P 3 d^{3} F$ \\
\hline 23 & 1 & $2 s^{2} 2 p^{4}\left({ }_{2}^{3} P\right)^{3} P_{2}$ & 0 & 0 & $\ldots$ & $\ldots$ & $0.94+0.062 s^{2} 2 p^{4}\left({ }_{2}^{1} D\right){ }^{1} D$ \\
\hline 23 & 2 & $\left.2 s^{2} 2 p^{4}{ }_{3}^{3} P\right)^{3} P_{1}$ & 48937 & 48939 & $4.039 \mathrm{E}-04$ & $4.039 \mathrm{E}-04$ & 1.00 \\
\hline 23 & 3 & $2 s^{2} 2 p^{4}\left({ }_{2}^{2} P\right)^{3} P_{0}$ & 49970 & 50014 & $6.073 \mathrm{E}+00$ & $6.314 \mathrm{E}+00$ & $0.89+0.102 s^{2} 2 p^{4}\left({ }_{0}^{1} S\right){ }^{1} S$ \\
\hline 23 & 4 & $2 s^{2} 2 p^{4}\left({ }_{2}^{1} D\right){ }^{1} D_{2}$ & 121039 & 121192 & $2.356 \mathrm{E}-04$ & $2.356 \mathrm{E}-04$ & $0.94+0.062 s^{2} 2 p^{4}\left({ }_{2}^{3} P\right)^{3} P$ \\
\hline 23 & 5 & $2 s^{2} 2 p^{4}\left({ }_{0}^{1} s\right){ }^{1} s_{0}$ & 237705 & 237939 & $2.706 \mathrm{E}-05$ & $2.706 \mathrm{E}-05$ & $0.87+0.102 s^{2} 2 p^{4}\left({ }_{2}^{3} P\right)^{3} P+0.022 p^{6}{ }^{1} S$ \\
\hline 23 & 6 & $2 s^{2} S 2 p^{5}{ }^{3} P_{2}^{\circ}$ & 761824 & 761613 & $2.922 \mathrm{E}-11$ & $2.909 \mathrm{E}-11$ & 0.99 \\
\hline 23 & 7 & $2 s^{2} S 2 p^{53} P_{1}^{0}$ & 798899 & 798716 & $2.620 \mathrm{E}-11$ & $2.622 \mathrm{E}-11$ & 0.98 \\
\hline
\end{tabular}




\begin{tabular}{|c|c|c|c|c|c|c|c|}
\hline $\mathrm{z}$ & Key & Level & $E_{\text {NIST }}$ & $E_{\mathrm{MCDHF} / \mathrm{RCI}}$ & $\tau_{\mathrm{MCDHF} / \mathrm{RCI}}^{l}$ & $\tau_{\mathrm{MCDHF} / \mathrm{RCI}}^{v}$ & $L S$-composition \\
\hline 23 & 8 & $2 s^{2} S 2 p^{5}{ }^{3} P_{0}^{\circ}$ & 822961 & 822761 & $2.598 \mathrm{E}-11$ & $2.603 \mathrm{E}-11$ & 0.99 \\
\hline 23 & 9 & $2 s^{2} S 2 p^{5}{ }^{1} P^{0}$ & 1045590 & 1045742 & $8.242 \mathrm{E}-12$ & $8.236 \mathrm{E}-12$ & 0.98 \\
\hline 23 & 10 & $2 p^{6}{ }^{1} S_{0} \quad 1$ & \multirow[t]{2}{*}{1769360} & 1769451 & $9.152 \mathrm{E}-12$ & $9.159 \mathrm{E}-12$ & $0.97+0.022 s^{2} 2 p^{4}\left({ }_{0}^{1} S\right)^{1} S$ \\
\hline 23 & 11 & $2 s^{2} 2 p^{3}\left({ }_{3}^{4} S\right){ }^{4} S 3 s^{5} S_{2}^{\circ}$ & & 4843789 & $3.926 \mathrm{E}-11$ & $3.940 \mathrm{E}-11$ & $0.95+0.042 s^{2} 2 p^{3}\left({ }_{1}^{2} P\right)^{2} P 3 s^{3} P^{\circ}$ \\
\hline 23 & 12 & $2 s^{2} 2 p^{3}\left({ }_{2}^{4} S\right)^{4} S 3 s^{3} S_{1}^{2}$ & 4891000 & 4889424 & $5.141 \mathrm{E}-13$ & $5.143 \mathrm{E}-13$ & $0.92+0.032 s^{2} 2 p^{3}\left({ }_{1}^{2} P\right)^{2} P 3 s^{1} P^{\circ}+0.022 s^{2} 2 p^{3}\left({ }_{1}^{2} P\right)^{2} P 3 s^{3} P^{\circ}$ \\
\hline 23 & 13 & $2 s^{2} 2 p^{3}\left({ }_{3}^{2} D\right)^{2} D 3 s^{3} D_{1}^{\circ}$ & 4981000 & 4979007 & $1.367 \mathrm{E}-12$ & $1.368 \mathrm{E}-12$ & $0.83+0.072 s^{2} 2 p^{3}\left({ }_{1}^{2} P\right)^{2} P 3 s^{1} P^{\circ}+0.052 s^{2} 2 p^{3}\left({ }_{1}^{2} P\right)^{2} P 3 s^{3} P^{\circ}$ \\
\hline 23 & 14 & $2 s^{2} 2 p^{3}\left(\frac{2}{3} D\right)^{2} D 3 s^{3} D_{2}^{\circ}$ & 4980000 & 4979660 & $1.450 \mathrm{E}-12$ & $1.450 \mathrm{E}-12$ & $0.74+0.152 s^{2} 2 p^{3}\left({ }_{1}^{2} P\right)^{2} P 3 s^{3} P^{\circ}+0.082 s^{2} 2 p^{3}\left({ }_{3}^{2} D\right)^{2} D 3 s^{1} D^{\circ}$ \\
\hline 23 & 15 & $2 s^{2} 2 p^{3}\left({ }_{3}^{3} D\right)^{2} D 3 s^{3} D_{3}^{\circ}$ & 4996000 & 4994799 & $1.562 \mathrm{E}-12$ & $1.562 \mathrm{E}-12$ & 0.99 \\
\hline 23 & 16 & $2 s^{2} 2 p^{3}\left({ }_{2}^{2} D\right)^{2} D 3 s^{1} D_{2}^{\circ}$ & \multirow[t]{8}{*}{5012000} & 5012507 & $6.959 \mathrm{E}-13$ & $6.963 \mathrm{E}-13$ & $0.80+0.152 s^{2} 2 p^{3}\left({ }_{3}^{2} D\right)^{2} D 3 s^{3} D^{\circ}+0.042 s^{2} 2 p^{3}\left({ }_{1}^{2} P\right)^{2} P 3 s^{3} P^{\circ}$ \\
\hline 23 & 17 & $\left.2 s^{2} 2 p^{3}{ }^{3} S\right)^{4} S 3 p^{5} P_{1}$ & & 5041453 & $3.311 \mathrm{E}-10$ & $3.298 \mathrm{E}-10$ & $0.92 \quad-3$ का \\
\hline 23 & 18 & $2 s^{2} 2 p^{3}\left({ }_{3}^{4} S\right)^{4} S 3 p^{5} P_{2}$ & & 5044254 & $3.880 \mathrm{E}-10$ & $3.861 \mathrm{E}-10$ & $0.87+0.062 s^{2} 2 p^{3}\left({ }_{3}^{4} S\right)^{4} S 3 p^{3} P+0.032 s^{2} 2 p^{3}\left({ }_{1}^{2} P\right)^{2} P 3 p^{3} P$ \\
\hline 23 & 19 & $2 s^{2} 2 p^{3}\left({ }_{3}^{4} S\right){ }^{4} S 3 p^{5} P_{3}$ & & 5054191 & $3.385 \mathrm{E}-10$ & $3.376 \mathrm{E}-10$ & $0.94+0.042 s^{2} 2 p^{3}\left({ }_{1}^{2} P\right)^{2} P 3 p^{3} D$ \\
\hline 23 & 20 & $2 s^{2} 2 p^{3}\left({ }_{1}^{2} P\right)^{2} P 3 s^{3} P_{0}^{\circ}$ & & 5066908 & $1.671 \mathrm{E}-12$ & $1.672 \mathrm{E}-12$ & 0.97 \\
\hline 23 & 21 & $2 s^{2} 2 p^{3}\left({ }_{1}^{2} P\right)^{2} P 3 s^{3} P_{1}^{\circ}$ & & 5072086 & $1.330 \mathrm{E}-12$ & $1.330 \mathrm{E}-12$ & $0.82+0.142 s^{2} 2 p^{3}\left({ }_{1}^{2} P\right)^{2} P 3 s^{1} P^{\circ}$ \\
\hline 23 & 22 & $2 s^{2} 2 p^{3}\left({ }_{3}^{4} S\right)^{4} S 3 p^{3} P_{1}$ & & 5086927 & 2.229E-10 & $2.236 \mathrm{E}-10$ & $0.71+0.112 s^{2} 2 p^{3}\left({ }_{3}^{2} D\right)^{2} D 3 p^{3} P+0.052 s^{2} 2 p^{3}\left({ }_{1}^{2} P\right)^{2} P 3 p^{1} P$ \\
\hline 23 & 23 & $2 s^{2} 2 p^{3}\left({ }_{2}^{4} S\right)^{4} S 3 p^{3} P_{2}$ & & 5095611 & 2.363E-10 & 2.369E-10 & $\left.0.74+0.112 s^{2} 2 p^{3}\left({ }_{2}^{2} D\right)^{2} D 3 p^{3} P+0.082 s^{2} 2 p^{3}{ }^{4} S\right)^{4} S{ }^{4} 3 p^{5} P$ \\
\hline 23 & 24 & $2 s^{2} 2 p^{3}\left({ }_{1}^{3} P\right)^{2} P 3 s^{3} P_{2}^{\circ}$ & \multirow[t]{2}{*}{5068000} & 5097146 & $1.360 \mathrm{E}-12$ & $1.360 \mathrm{E}-12$ & $0.74+0.112 s^{2} 2 p^{3}\left(\frac{2}{2} D\right)^{2} D 3 s^{1} D^{\circ}+0.102 s^{2} 2 p^{3}\left(\frac{2}{2} D\right)^{2} D 3 s^{3} D^{\circ}$ \\
\hline 23 & 25 & $\left.2 s^{2} 2 p^{3}{ }^{4} S\right)^{4} S 3 p^{3} P_{0}^{2}$ & & 5099499 & $2.588 \mathrm{E}-10$ & $2.590 \mathrm{E}-10$ & $\left.0.85+0.082 s^{2} 2 p^{3}\left(2_{2}^{2} D\right)^{2} D 3 p^{3} P+0.052 s^{2} 2 p^{3} 2_{2}^{3} P\right)^{2} P 3 p^{3} P$ \\
\hline 23 & 26 & $2 s^{2} 2 p^{3}\left({ }_{1}^{2} P\right)^{2} P 3 s^{1} P_{1}^{\circ}$ & 5113000 & 5112119 & 7.187E-13 & $7.188 \mathrm{E}-13$ & $\left.0.74+0.132 s^{2} 2 p^{3}\left({ }_{3}^{2} D\right)^{2} D 3 s^{3} D^{\circ}+0.082 s^{2} 2 p^{3}{ }_{1}^{2} P\right)^{2} P 3 s^{3} P^{\circ}$ \\
\hline 23 & 27 & $2 s^{2} 2 p^{3}\left({ }_{3}^{2} D\right)^{2} D 3 p^{3} D_{1}$ & & 5154158 & $1.639 \mathrm{E}-10$ & $1.650 \mathrm{E}-10$ & $0.42+0.312 s^{2} 2 p^{3}\left({ }_{3}^{2} D\right)^{2} D 3 p^{1} P+0.072 s^{2} 2 p^{3}\left({ }_{1}^{2} P\right)^{2} P 3 p^{1} P$ \\
\hline 23 & 28 & $2 s^{2} 2 p^{3}\left({ }_{3}^{2} D\right)^{2} D 3 p^{3} D_{2}$ & & 5170991 & $3.625 \mathrm{E}-10$ & $3.612 \mathrm{E}-10$ & $0.49+0.332 s^{2} 2 p^{3}\left({ }_{3}^{2} D\right)^{2} D 3 p^{3} F+0.102 s^{2} 2 p^{3}\left({ }_{1}^{2} P\right)^{2} P 3 p^{3} D$ \\
\hline 23 & 29 & $2 s^{2} 2 p^{3}\left(\frac{2}{3} D\right)^{2} D 3 p^{3} F_{3}$ & & 5183736 & 3.699E-10 & 3.684E-10 & $0.51+0.392 s^{2} 2 p^{3}\left({ }_{3}^{2} D\right)^{2} D 3 p^{3} D+0.082 s^{2} 2 p^{3}\left({ }_{1}^{2} P\right)^{2} P 3 p^{3} D$ \\
\hline 23 & 30 & $2 s^{2} 2 p^{3}\left({ }_{2}^{3} D\right)^{2} D 3 p^{3} F_{2}$ & & 5184483 & $1.493 \mathrm{E}-10$ & $1.497 \mathrm{E}-10$ & $0.49+0.392 s^{2} 2 p^{3}\left({ }_{2}^{2} D\right)^{2} D 3 p^{3} D+0.072 s^{2} 2 p^{3}\left(_{1}^{2} P\right)^{2} P 3 p^{1} D$ \\
\hline 23 & 31 & $2 s^{2} 2 p^{3}\left(\frac{2}{3} D\right)^{2} D 3 p{ }^{1} P_{1}$ & & 5188118 & $1.180 \mathrm{E}-10$ & $1.191 \mathrm{E}-10$ & $0.48+0.382 s^{2} 2 p^{3}\left({ }_{3}^{2} D\right)^{2} D 3 p^{3} D+0.062 s^{2} 2 p^{3}\left({ }_{1}^{2} P\right)^{2} P 3 p^{3} D$ \\
\hline 23 & 32 & $2 s^{2} 2 p^{3}\left(2^{2} D\right)^{2} D 3 p^{3} D_{3}$ & & 5195083 & $1.054 \mathrm{E}-10$ & $1.057 \mathrm{E}-10$ & $0.47+0.232 s^{2} 2 p^{3}\left({ }_{2}^{2} D\right)^{2} D 3 p^{1} F+0.212 s^{2} 2 p^{3}\left({ }_{2}^{2} D\right)^{2} D 3 p^{3} F$ \\
\hline 23 & 33 & $2 s^{2} 2 p^{3}\left({ }_{3}^{2} D\right)^{2} D 3 p^{1} F_{3}$ & & 5204539 & $3.254 \mathrm{E}-10$ & $3.240 \mathrm{E}-10$ & $0.67+0.182 s^{2} 2 p^{3}\left({ }_{2}^{2} D\right)^{2} D 3 p^{3} F+0.122 s^{2} 2 p^{3}\left({ }_{3}^{2} D\right)^{2} D 3 p^{3} D$ \\
\hline 23 & 34 & $2 s^{2} 2 p^{3}\left({ }_{3}^{2} D\right)^{2} D 3 p^{3} F_{4}$ & & 5206881 & $2.880 \mathrm{E}-10$ & $2.868 \mathrm{E}-10$ & 0.99 \\
\hline 23 & 35 & $2 s^{2} 2 p^{3}\left(\frac{2}{3} D\right)^{2} D 3 p^{3} P_{0}$ & & 5240259 & $2.233 \mathrm{E}-11$ & $2.240 \mathrm{E}-11$ & $\left.0.72+0.212 s^{2} 2 p^{3}\left({ }_{1}^{2} P\right)^{2} P 3 p^{3} P+0.042 s^{2} 2 p^{3}{ }_{1}^{2} P\right)^{2} P 3 p^{1} S$ \\
\hline 23 & 36 & $2 s^{2} 2 p^{3}\left(\frac{2}{3} D\right)^{2} D 3 p^{3} P_{1}$ & & 5243436 & $2.079 \mathrm{E}-11$ & 2.087E-11 & $0.39+0.282 s^{2} 2 p^{3}\left({ }_{1}^{2} P\right)^{2} P 3 p^{3} S+0.122 s^{2} 2 p^{3}\left({ }_{3}^{4} S\right)^{4} S 3 p^{3} P$ \\
\hline 23 & 37 & $2 s^{2} 2 p^{3}\left({ }_{2}^{3} D\right)^{2} D 3 p^{3} P_{2}$ & & 5258917 & $1.317 \mathrm{E}-11$ & $1.322 \mathrm{E}-11$ & $0.75+0.132 s^{2} 2 p^{3}\left({ }_{3}^{4} S\right)^{4} S 3 p^{3} P+0.052 s^{2} 2 p^{3}\left(_{1}^{2} P\right)^{2} P 3 p^{3} P$ \\
\hline 23 & 38 & $2 s^{2} 2 p^{3}\left({ }_{1}^{2} P\right)^{2} P 3 p^{3} D_{1}$ & & 5268147 & $5.364 \mathrm{E}-11$ & 5.367E-11 & $0.69+0.172 s^{2} 2 p^{3}\left({ }_{1}^{2} P\right)^{2} P 3 p^{1} P+0.052 s^{2} 2 p^{3}\left({ }_{3}^{2} D\right)^{2} D 3 p^{1} P$ \\
\hline 23 & 39 & $2 s^{2} 2 p^{3}\left({ }_{1}^{2} P\right)^{2} P 3 p{ }^{1} D_{2}$ & & 5278634 & $4.921 \mathrm{E}-11$ & $4.928 \mathrm{E}-11$ & $0.07+0.382 s^{2} 2 p^{3}\left({ }_{3}^{2} D\right)^{2} D 3 p{ }^{1} D+0.282 s^{2} 2 p^{3}\left({ }_{1}^{2} P\right)^{2} P 3 p^{3} D$ \\
\hline 23 & 40 & $2 s^{2} 2 p^{3}\left({ }_{1}^{2} P\right)^{2} P 3 p^{3} S_{1}$ & & 5284099 & 2.797E-11 & $2.808 \mathrm{E}-11$ & $0.47+0.182 s^{2} 2 p^{3}\left({ }_{1}^{2} P\right)^{2} P 3 p^{3} P+0.162 s^{2} 2 p^{3}\left({ }_{3}^{2} D\right)^{2} D 3 p^{3} P$ \\
\hline 23 & 41 & $2 s^{2} 2 p^{3}\left({ }_{1}^{2} P\right)^{2} P 3 p^{3} D_{2}$ & & 5285320 & $6.318 \mathrm{E}-11$ & $6.317 \mathrm{E}-11$ & $0.53+0.192 s^{2} 2 p^{3}\left({ }_{1}^{2} P\right)^{2} P 3 p^{3} P+0.122 s^{2} 2 p^{3}\left({ }_{3}^{2} D\right)^{2} D 3 p^{1} D$ \\
\hline 23 & 42 & $2 s^{2} 2 p^{3}\left({ }_{3}^{1} S\right)^{4} S 3 d^{5} D_{3}^{\circ}$ & & 5296588 & $7.158 \mathrm{E}-11$ & $7.211 \mathrm{E}-11$ & $0.93+0.022 s^{2} 2 p^{3}\left({ }_{1}^{2} P\right)^{2} P 3 d^{3} D^{\circ}$ \\
\hline 23 & 43 & $2 s^{2} 2 p^{3}\left({ }_{3}^{4} S\right)^{4} S 3 d^{5} D_{0}^{\circ}$ & & 5296666 & $1.566 \mathrm{E}-11$ & $1.558 \mathrm{E}-11$ & $0.95+0.042 s^{2} 2 p^{3}\left({ }_{1}^{2} P\right)^{2} P 3 d^{3} P^{\circ}$ \\
\hline 23 & 44 & $2 s^{2} 2 p^{3}\left({ }_{3}^{4} S\right)^{4} S 3 d^{5} D_{2}^{\circ}$ & & 5296680 & $1.375 \mathrm{E}-11$ & $1.371 \mathrm{E}-11$ & $0.94+0.022 s^{2} 2 p^{3}\left({ }_{1}^{2} P\right)^{2} P 3 d^{3} D^{\circ}$ \\
\hline 23 & 45 & $2 s^{2} 2 p^{3}\left({ }_{2}^{4} S\right)^{4} S 3 d^{5} D_{1}^{\circ}$ & & 5296762 & $1.094 \mathrm{E}-11$ & $1.089 \mathrm{E}-11$ & $0.95+0.032 s^{2} 2 p^{3}\left({ }_{1}^{2} P\right)^{2} P 3 d^{3} P^{\circ}$ \\
\hline 23 & 46 & $2 s^{2} 2 p^{3}\left({ }_{3}^{4} S\right)^{4} S 3 d^{5} D_{4}^{\circ}$ & & 5297863 & 2.593E-10 & $2.591 \mathrm{E}-10$ & $0.95+0.042 s^{2} 2 p^{3}\left({ }_{1}^{2} P\right)^{2} P 3 d^{3} F^{\circ}$ \\
\hline 23 & 47 & $\left.2 s^{2} 2 p^{3} c^{2} P\right)^{2} P 3 p^{3} D_{3}$ & & 5303558 & $6.866 \mathrm{E}-11$ & $6.860 \mathrm{E}-11$ & $\left.0.76+0.102 s^{2} 2 p^{3}\left(c^{2} D\right)^{2} D 3 p^{3} F+0.102 s^{2} 2 p^{3} c^{2} D\right)^{2} D 3 p^{1} F$ \\
\hline 23 & 48 & $2 s^{2} 2 p^{3}\left({ }_{1}^{2} P\right)^{2} P 3 p^{1} P_{1}$ & & 5306983 & 2.347E-11 & $2.355 \mathrm{E}-11$ & $0.38+0.262 s^{2} 2 p^{3}\left(\frac{2}{3} D\right)^{2} D 3 p^{3} P+0.122 s^{2} 2 p^{3}\left({ }_{1}^{2} P\right)^{2} P 3 p^{3} P$ \\
\hline 23 & 49 & $2 s^{2} 2 p^{3}\left(C_{1}^{2} P\right)^{2} P 3 p^{3} P_{0}$ & & 5320069 & $2.573 \mathrm{E}-11$ & $2.580 \mathrm{E}-11$ & $0.68+0.112 s^{2} 2 p^{3}\left({ }_{3}^{2} D\right)^{2} D 3 p^{3} P+0.092 s^{2} 2 p^{3}\left({ }_{3}^{4} S\right)^{4} S 3 p^{3} P$ \\
\hline 23 & 50 & $2 s^{2} 2 p^{3}\left({ }_{1}^{2} P\right)^{2} P 3 p^{3} P_{1}$ & & 5328145 & $4.450 \mathrm{E}-11$ & $4.462 \mathrm{E}-11$ & $0.53+0.162 s^{2} 2 p^{3}\left({ }_{1}^{2} P\right)^{2} P 3 p p^{1} P+0.082 s^{2} 2 p^{3}\left({ }_{3}^{4} S\right)^{4} S 3 p^{3} P$ \\
\hline 23 & 51 & $2 s^{2} 2 p^{3}\left({ }_{1}^{2} P\right)^{2} P 3 p^{3} P_{2}$ & & 5330933 & $9.108 \mathrm{E}-11$ & $9.122 \mathrm{E}-11$ & $0.47+0.282 s^{2} 2 p^{3}\left({ }_{1}^{2} P\right)^{2} P 3 p^{1} D+0.062 s^{2} 2 p^{3}\left({ }_{3}^{2} D\right)^{2} D 3 p^{3} D$ \\
\hline 23 & 52 & $2 s^{2} 2 p^{3}\left({ }^{4} S\right)^{4} S 3 d^{3} D_{2}^{\circ}$ & 5343000 & 5341910 & $4.670 \mathrm{E}-13$ & $4.665 \mathrm{E}-13$ & $0.70+0.122 s^{2} 2 p^{3}\left(\frac{2}{2} D\right)^{2} D 3 d^{3} D^{\circ}+0.062 s^{2} 2 p^{3}\left({ }_{1}^{2} P\right)^{2} P 3 d^{1} D^{\circ}$ \\
\hline 23 & 53 & $2 s^{2} 2 p^{3}\left({ }_{3}^{4} S\right){ }^{4} S 3 d^{3} D_{1}^{\circ}$ & & 5352028 & 3.413E-13 & $3.410 \mathrm{E}-13$ & $0.84+0.082 s^{2} 2 p^{3}\left({ }_{3}^{2} D\right)^{2} D 3 d^{3} D^{\circ}+0.042 s^{2} 2 p^{3}\left({ }_{1}^{2} P\right)^{2} P 3 d^{3} D^{\circ}$ \\
\hline 23 & 54 & $2 s^{2} 2 p^{3}\left({ }_{3}^{4} S\right)^{4} S 3 d^{3} D_{3}^{\circ}$ & 5351000 & 5353110 & $3.376 \mathrm{E}-13$ & $3.369 \mathrm{E}-13$ & $0.78+0.122 s^{2} 2 p^{3}\left({ }_{3}^{3} D\right)^{2} D 3 d^{3} D^{\circ}+0.032 s^{2} 2 p^{3}\left({ }_{3}^{4} S\right)^{4} S 3 d^{5} D^{\circ}$ \\
\hline 23 & 55 & $2 s^{2} 2 p^{3}\left(2^{2} D\right)^{2} D 3 p^{1} D_{2}$ & & 5358203 & $7.895 \mathrm{E}-11$ & $7.930 \mathrm{E}-11$ & $0.42+0.402 s^{2} 2 p^{3}\left(c_{1}^{2} P\right)^{2} P 3 p^{1} D+0.092 s^{2} 2 p^{3}\left(c_{1}^{2} P\right)^{2} P 3 p^{3} P$ \\
\hline 23 & 56 & $2 s^{2} 2 p^{3}\left(\frac{2}{3} D\right)^{2} D 3 d^{3} F_{2}^{\circ}$ & & 5414943 & $2.072 \mathrm{E}-12$ & $2.066 \mathrm{E}-12$ & $0.74+0.112 s^{2} 2 p^{3}\left({ }_{1}^{2} P\right)^{2} P 3 d^{3} F^{\circ}+0.062 s^{2} 2 p^{3}\left({ }_{3}^{4} S\right)^{4} S 3 d^{3} D^{\circ}$ \\
\hline 23 & 57 & $2 s^{2} 2 p^{3}\left({ }_{2}^{2} D\right)^{2} D 3 d^{3} F_{3}^{\circ}$ & & 5423260 & $1.685 \mathrm{E}-12$ & $1.684 \mathrm{E}-12$ & $0.72+0.112 s^{2} 2 p^{3}\left({ }_{3}^{2} D\right)^{2} D 3 d^{3} G^{\circ}+0.102 s^{2} 2 p^{3}\left({ }_{1}^{2} P\right)^{2} P 3 d^{3} F^{\circ}$ \\
\hline 23 & 58 & $2 s^{2} 2 p^{3}\left(\frac{2}{3} D\right)^{2} D 3 d^{1} S_{0}^{\circ}$ & & 5426355 & $5.453 \mathrm{E}-11$ & $5.507 \mathrm{E}-11$ & $0.89+0.082 s^{2} 2 p^{3}\left({ }_{1}^{2} P\right)^{2} P 3 d^{3} P^{\circ}$ \\
\hline 23 & 59 & $2 s^{2} 2 p^{3}\left({ }_{3}^{2} D\right)^{2} D 3 d^{3} G_{4}^{\circ}$ & & 5431663 & 2.614E-10 & $2.607 \mathrm{E}-10$ & $0.45+0.222 s^{2} 2 p^{3}\left({ }_{3}^{2} D\right)^{2} D 3 d^{3} F^{\circ}+0.162 s^{2} 2 p^{3}\left({ }_{3}^{2} D\right)^{2} D 3 d^{1} G^{\circ}$ \\
\hline 23 & 60 & $2 s^{2} 2 p^{3}\left({ }_{2}^{3} D\right)^{2} D 3 d^{3} G_{3}^{\circ}$ & & 5434709 & 2.503E-12 & $2.500 \mathrm{E}-12$ & $0.71+0.142 s^{2} 2 p^{3}\left(_{2}^{2} D\right)^{2} D 3 d^{3} F^{\circ}+0.072 s^{2} 2 p^{3}\left(_{1}^{2} P\right)^{2} P 3 d^{1} F^{\circ}$ \\
\hline 23 & 61 & $2 s^{2} 2 p^{3}\left({ }_{1}^{2} P\right)^{2} P 3 p^{1} S_{0}^{3}$ & & 5436059 & 1.103E-11 & $1.106 \mathrm{E}-11$ & $0.85+0.062 s^{2} 2 p^{3}\left({ }_{3}^{2} D\right)^{2} D 3 p^{3} P+0.032 s^{2} 2 p^{3}\left({ }_{3}^{4} S\right)^{4} S 3 p^{3} P$ \\
\hline 23 & 62 & $2 s^{2} 2 p^{3}(2 D){ }^{2} D 3 d^{3} D^{\circ}$ & & 5441813 & $2.500 \mathrm{E}-13$ & $2.496 \mathrm{E}-13$ & $\left.0.42+0.322 s^{2} 2 p^{3}(2 D)\right)^{2} D 3 d^{1} P^{\circ}+0.082 s^{2} 2 p^{3}\left({ }^{3} P\right)^{2} P 3 d^{3} D^{\circ}$ \\
\hline 23 & 63 & $2 s^{2} 2 p^{3}\left(\frac{2}{3} D\right)^{2} D 3 d^{3} F_{4}^{\circ}$ & & 5443005 & $2.452 \mathrm{E}-10$ & $2.455 \mathrm{E}-10$ & $0.77+0.152 s^{2} 2 p^{3}\left(\frac{2}{2} D\right)^{2} D 3 d^{3} G^{\circ}+0.052 s^{2} 2 p^{3}\left({ }_{2}^{2} D\right)^{2} D 3 d^{1} G^{\circ}$ \\
\hline 23 & 64 & $2 s^{2} 2 p^{3}\left({ }_{3}^{2} D\right)^{2} D 3 d^{3} G_{5}^{\circ}$ & & 5450823 & $2.469 \mathrm{E}-10$ & $2.464 \mathrm{E}-10$ & 1.00 \\
\hline 23 & 65 & $2 s^{2} 2 p^{3}\left(\frac{2}{3} D\right)^{2} D 3 d^{1} G_{4}^{\circ}$ & & 5452639 & $2.272 \mathrm{E}-10$ & $2.267 \mathrm{E}-10$ & $0.70+0.292 s^{2} 2 p^{3}\left({ }_{3}^{2} D\right)^{2} D 3 d^{3} G^{\circ}$ \\
\hline 23 & 66 & $2 s^{2} 2 p^{3}\left({ }_{3}^{2} D\right)^{2} D 3 d^{3} D_{2}^{\circ}$ & 5457000 & 5464936 & $1.454 \mathrm{E}-13$ & $1.452 \mathrm{E}-13$ & $0.42+0.222 s^{2} 2 p^{3}\left({ }_{3}^{2} D\right)^{2} D 3 d^{3} P^{\circ}+0.122 s^{2} 2 p^{3}\left({ }_{1}^{2} P\right)^{2} P 3 d^{3} P^{\circ}$ \\
\hline 23 & 67 & $2 s^{2} 2 p^{3}\left(\frac{2}{2} D\right)^{2} D 3 d^{1} P_{1}^{\circ}$ & & 5470893 & 2.375E-13 & 2.373E-13 & $0.48+0.362 s^{2} 2 p^{3}(2 D)^{2} D 3 d^{3} D^{\circ}+0.062 s^{2} 2 p^{3}\left({ }_{1}^{2} P\right)^{2} P 3 d^{3} P^{\circ}$ \\
\hline 23 & 68 & $2 s^{2} 2 p^{3}\left({ }_{3}^{2} D\right)^{2} D 3 d^{3} D_{3}^{\circ}$ & 5475000 & 5474264 & $1.048 \mathrm{E}-13$ & $1.046 \mathrm{E}-13$ & $0.79+0.132 s^{2} 2 p^{3}\left({ }_{3}^{4} S\right)^{4} S 3 d^{3} D^{\circ}+0.022 s^{2} 2 p^{3}\left({ }_{3}^{2} D\right)^{2} D 3 d^{3} G^{\circ}$ \\
\hline 23 & 69 & $2 s^{2} 2 p^{3}\left(\frac{2}{3} D\right)^{2} D 3 d^{3} P_{2}^{\circ}$ & 5476000 & 5480564 & $1.057 \mathrm{E}-13$ & $1.056 \mathrm{E}-13$ & $0.36+0.302 s^{2} 2 p^{3}\left(\frac{2}{3} D\right)^{2} D 3 d^{3} D^{\circ}+0.122 s^{2} 2 p^{3}\left({ }_{3}^{2} D\right)^{2} D 3 d^{1} D^{\circ}$ \\
\hline 23 & 70 & $2 s^{2} 2 p^{3}\left(\frac{2}{3} D\right)^{2} D 3 d^{3} P_{0}^{\circ}$ & & 5483089 & $2.374 \mathrm{E}-13$ & $2.371 \mathrm{E}-13$ & $0.81+0.122 s^{2} 2 p^{3}\left({ }_{1}^{2} P\right)^{2} P 3 d^{3} P^{\circ}+0.052 s^{2} 2 p^{3}\left({ }_{2}^{2} D\right)^{2} D 3 d^{1} S^{\circ}$ \\
\hline 23 & 71 & $2 s^{2} 2 p^{3}\left(2_{2}^{2} D\right)^{2} D 3 d^{3} P_{0}^{0}$ & & 5485665 & $1.636 \mathrm{E}-13$ & $1.635 \mathrm{E}-13$ & $0.70+0.112 s^{2} 2 p^{3}\left({ }_{3}^{2} D\right)^{2} D 3 d^{3} s^{\circ}+0.082 s^{2} 2 p^{3}\left({ }_{1}^{2} P\right)^{2} P 3 d^{3} P^{\circ}$ \\
\hline 23 & 72 & $2 s^{2} 2 p^{3}\left({ }_{3}^{2} D\right)^{2} D 3 d^{1} D_{2}^{\circ}$ & 5489000 & 5488330 & $2.789 \mathrm{E}-13$ & $2.787 \mathrm{E}-13$ & $0.39+0.252 s^{2} 2 p^{3}\left({ }_{1}^{2} P\right)^{2} P 3 d^{1} D^{\circ}+0.222 s^{2} 2 p^{3}\left({ }_{3}^{2} D\right)^{2} D 3 d^{3} P^{\circ}$ \\
\hline 23 & 73 & $2 s^{2} S 2 p^{4}\left({ }_{2}^{3} P\right){ }^{4} P 3 s^{5} P_{3}^{2}$ & & 5488732 & $4.375 \mathrm{E}-11$ & $4.366 \mathrm{E}-11$ & 0.97 \\
\hline 23 & 74 & $2 s^{2} 2 p^{3}\left(_{2}^{2} D\right)^{2} D 3 d^{3} S^{0}$ & & 5498832 & $9.338 \mathrm{E}-14$ & $9.326 \mathrm{E}-14$ & $\left.0.64+0.212 s^{2} 2 p^{3}{ }^{2}{ }_{2}^{2} D\right)^{2} D 3 d^{3} P^{\circ}+0.072 s^{2} 2 p^{3}\left(_{2}^{2} P\right)^{2} P 3 d^{3} P^{\circ}$ \\
\hline 23 & 75 & $2 s^{2} 2 p^{3}\left({ }_{3}^{2} D\right)^{2} D 3 d^{1} F_{3}^{\circ}$ & 5518000 & 5518323 & $1.431 \mathrm{E}-13$ & $1.429 \mathrm{E}-13$ & $0.68+0.122 s^{2} 2 p^{3}\left({ }_{1}^{2} P\right)^{2} P 3 d^{3} D^{\circ}+0.072 s^{2} 2 p^{3}\left({ }_{1}^{2} P\right)^{2} P 3 d^{3} F^{\circ}$ \\
\hline 23 & 76 & $2 s^{2} S 2 p^{4}\left({ }_{2}^{3} P\right){ }^{4} P 3 s^{5} P_{2}$ & & 5519419 & $8.255 \mathrm{E}-12$ & $8.265 \mathrm{E}-12$ & $0.91+0.072 s^{2} S 2 p^{4}\left({ }_{2}^{3} P\right){ }^{4} P 3 s^{3} P$ \\
\hline 23 & 77 & $2 s^{2} 2 p^{3}(2 P)^{2} P 3 d^{3} F_{0}^{\circ}$ & & 5530240 & $9.367 \mathrm{E}-13$ & $9.351 \mathrm{E}-13$ & $0.70+0.102 s^{2} 2 p^{3}(2 D D)^{2} D 3 d^{3} F^{\circ}+0.072 s^{2} 2 p^{3}\left(_{2}^{2} P\right)^{2} P 3 d^{3} D^{\circ}$ \\
\hline 23 & 78 & $2 s^{2} 2 p^{3}\left({ }_{1}^{2} P\right)^{2} P 3 d^{3} F_{3}^{\circ}$ & & 5532713 & $1.387 \mathrm{E}-12$ & $1.385 \mathrm{E}-12$ & $0.65+0.212 s^{2} 2 p^{3}\left(_{1}^{2} P\right)^{2} P 3 d^{3} D^{\circ}+0.052 s^{2} 2 p^{3}\left({ }_{3}^{2} D\right)^{2} D 3 d^{3} G^{\circ}$ \\
\hline 23 & 79 & $2 s^{2} S 2 p^{4}\left({ }_{2}^{3} P\right){ }^{4} P 3 s^{5} P_{1}$ & & 5538725 & 2.373E-11 & 2.383E-11 & 0.96 \\
\hline 23 & 80 & $2 s^{2} 2 p^{3}\left({ }_{1}^{2} P\right)^{2} P 3 d^{3} P_{2}^{\circ}$ & 5488000 & 5546098 & $1.913 \mathrm{E}-13$ & $1.911 \mathrm{E}-13$ & $0.42+0.292 s^{2} 2 p^{3}\left({ }_{1}^{2} P\right)^{2} P 3 d^{3} D^{\circ}+0.122 s^{2} 2 p^{3}\left({ }_{3}^{2} D\right)^{2} D 3 d^{1} D^{\circ}$ \\
\hline 23 & 81 & $2 s^{2} 2 p^{3}\left({ }_{1}^{2} P\right)^{2} P 3 d^{3} F_{4}^{\circ}$ & & 5546410 & $2.399 \mathrm{E}-10$ & $2.402 \mathrm{E}-10$ & $0.76+0.102 s^{2} 2 p^{3}\left(\frac{2}{3} D\right)^{2} D 3 d^{3} G^{\circ}+0.092 s^{2} 2 p^{3}\left({ }_{3}^{2} D\right)^{2} D 3 d^{1} G^{\circ}$ \\
\hline 23 & 82 & $2 s^{2} 2 p^{3}\left(c_{P}^{2} P\right)^{2} P 3 d^{3} P_{0}^{\circ}$ & & 5550108 & $1.370 \mathrm{E}-13$ & $1.368 \mathrm{E}-13$ & $0.74+0.162 s^{2} 2 p^{3}\left(_{2}^{2} D\right)^{2} D 3 d^{3} P^{\circ}+0.052 s^{2} 2 p^{3}\left({ }_{2}^{2} D\right)^{2} D 3 d^{1} S^{\circ}$ \\
\hline 23 & 83 & $2 s^{2} 2 p^{3}\left({ }_{1}^{2} P\right)^{2} P 3 d^{3} P_{1}^{\circ}$ & & 5552828 & $1.534 \mathrm{E}-13$ & $1.533 \mathrm{E}-13$ & $0.60+0.162 s^{2} 2 p^{3}\left({ }_{2}^{2} D\right)^{2} D 3 d^{3} S^{\circ}+0.102 s^{2} 2 p^{3}\left(_{1}^{2} P\right)^{2} P 3 d^{3} D^{\circ}$ \\
\hline 23 & 84 & $2 s^{2} 2 p^{3}\left({ }_{1}^{2} P\right)^{2} P 3 d^{1} D_{2}^{\circ}$ & & 5561228 & $2.851 \mathrm{E}-13$ & $2.847 \mathrm{E}-13$ & $0.26+0.232 s^{2} 2 p^{3}\left({ }_{1}^{2} P\right)^{2} P 3 d^{3} P^{\circ}+0.222 s^{2} 2 p^{3}\left({ }_{1}^{2} P\right)^{2} P 3 d^{3} D^{\circ}$ \\
\hline 23 & 85 & $2 s^{2} S 2 p^{4}\left({ }_{2}^{3} P\right){ }^{4} P 3 s^{3} P_{2}$ & & 5565430 & $8.714 \mathrm{E}-13$ & $8.713 \mathrm{E}-13$ & $0.87+0.072 s^{2} S 2 p^{4}\left({ }_{2}^{3} P\right){ }^{4} P 3 s^{5} P$ \\
\hline 23 & 86 & $2 s^{2} 2 p^{3}\left({ }_{1}^{2} P\right)^{2} P 3 d^{3} D_{1}^{\circ}$ & 5568000 & 5568800 & $9.547 \mathrm{E}-14$ & $9.530 \mathrm{E}-14$ & $0.67+0.082 s^{2} 2 p^{3}\left({ }_{1}^{2} P\right)^{2} P 3 d^{1} P^{\circ}+0.072 s^{2} 2 p^{3}\left({ }_{1}^{2} P\right)^{2} P 3 d^{3} P^{\circ}$ \\
\hline 23 & 87 & $2 s^{2} 2 p^{3}\left({ }_{1}^{2} P\right)^{2} P 3 d^{3} D_{3}^{\circ}$ & 5552000 & 5577101 & $9.004 \mathrm{E}-13$ & 8.993E-13 & $0.48+0.232 s^{2} 2 p^{3}\left({ }_{1}^{2} P\right)^{2} P 3 d^{1} F^{\circ}+0.102 s^{2} 2 p^{3}\left({ }_{3}^{2} D\right)^{2} D 3 d^{1} F^{\circ}$ \\
\hline 23 & 88 & $2 s^{2} 2 p^{3}\left({ }_{1}^{2} P\right)^{2} P 3 d^{1} F_{3}^{o}$ & 5596000 & 5595079 & $7.216 \mathrm{E}-14$ & $7.204 \mathrm{E}-14$ & $0.58+0.192 s^{2} 2 p^{3}\left(\frac{2}{3} D\right)^{2} D 3 d^{1} F^{\circ}+0.082 s^{2} 2 p^{3}\left(_{1}^{2} P\right)^{2} P 3 d^{3} D^{\circ}$ \\
\hline 23 & 89 & $2 s^{2} 2 p^{3}\left({ }_{1}^{2} P\right)^{2} P 3 d^{3} D_{2}^{\circ}$ & & 5595271 & $1.114 \mathrm{E}-13$ & $1.113 \mathrm{E}-13$ & $0.27+0.282 s^{2} 2 p^{3}\left({ }_{3}^{2} D\right)^{2} D 3 d^{1} D^{\circ}+0.262 s^{2} 2 p^{3}\left({ }_{1}^{2} P\right)^{2} P 3 d^{1} D^{\circ}$ \\
\hline 23 & 90 & $2 s^{2} S 2 p^{4}\left({ }_{3}^{3} P\right)^{4} P 3 s^{3}{ }^{2} P_{1}$ & & 5598537 & 7.654E-13 & $7.652 \mathrm{E}-13$ & $0.93+3$ \\
\hline 23 & 91 & $2 s^{2} S 2 p^{4}\left({ }_{2}^{2} P\right){ }^{4} P 3 s^{3} P_{0}$ & & 5611485 & $7.719 \mathrm{E}-13$ & $7.716 \mathrm{E}-13$ & $0.93+0.022 s^{2} S 2 p^{4}\left({ }_{0}^{1} S\right)^{2} S 3 s^{1} S$ \\
\hline
\end{tabular}




\begin{tabular}{|c|c|c|c|c|c|c|c|}
\hline $\mathrm{z}$ & Key & Level & $E_{\text {NIST }}$ & $E_{\mathrm{MCDHF} / \mathrm{RCI}}$ & $\tau_{\mathrm{MCDHF} / \mathrm{RCI}}^{l}$ & $\tau_{\mathrm{MCDHF} / \mathrm{RCI}}^{v}$ & $L S$-composition \\
\hline 23 & 92 & $2 s^{2} 2 p^{3}\left({ }_{1}^{2} P\right)^{2} P 3 d^{1} P_{1}^{\circ}$ & & 5643608 & $7.048 \mathrm{E}-14$ & $7.038 \mathrm{E}-14$ & $0.78+0.052 s^{2} 2 p^{3}\left({ }_{3}^{2} D\right)^{2} D 3 d^{1} P^{\circ}+0.042 s^{2} 2 p^{3}\left({ }_{1}^{2} P\right)^{2} P 3 d^{3} D^{\circ}$ \\
\hline 23 & 93 & $2 s^{2} S 2 p^{4}\left({ }_{2}^{3} P\right)^{4} P 3 p p^{5} P_{3}^{\circ}$ & & 5679925 & $4.230 \mathrm{E}-11$ & $4.204 \mathrm{E}-11$ & $0.79+0.192 s^{2} S 2 p^{4}\left({ }_{2}^{3} P\right)^{4} P 3 p^{5} D^{\circ}$ \\
\hline 23 & 94 & $2 s^{2} S 2 p^{4}\left({ }_{2}^{3} P\right)^{4} P 3 p p^{5} P_{2}^{\circ}$ & & 5682764 & $1.566 \mathrm{E}-11$ & $1.560 \mathrm{E}-11$ & $\left.\left.0.76+0.102 s^{2} S 2 p^{4}{ }_{2}^{3} P\right)^{4} P 3 p^{5} S^{\circ}+0.092 s^{2} S 2 p^{4}{ }_{2}^{3} P\right)^{4} P 3 p^{5} D^{\circ}$ \\
\hline 23 & 95 & $2 s^{2} S 2 p^{4}\left({ }_{2}^{2} P\right){ }^{4} P 3 p^{5} D_{4}^{\circ}$ & & 5703893 & $8.199 \mathrm{E}-11$ & $8.115 \mathrm{E}-11$ & 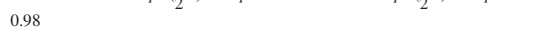 \\
\hline 23 & 96 & $2 s^{2} S 2 p^{4}\left({ }_{2}^{3} P\right)^{4} P 3 p^{5} P_{1}^{\circ}$ & & 5705098 & $1.151 \mathrm{E}-11$ & $1.147 \mathrm{E}-11$ & $\left.0.87+0.072 s^{2} S 2 p^{4}\left({ }_{2}^{3} P\right)^{4} P 3 p^{5} D^{\circ}+0.032 s^{2} S 2 p^{4}{ }_{2}^{3} P\right)^{4} P 3 p^{3} P^{\circ}$ \\
\hline 23 & 97 & $2 s^{2} S 2 p^{4}\left({ }_{2}^{2} P\right){ }^{4} P 3 p^{3} D_{3}^{\circ}$ & & 5715928 & 1.143E-12 & $1.140 \mathrm{E}-12$ & $\left.0.49+0.362 s^{2} S 2 p^{4}\left({ }_{2}^{3} P\right)^{4} P 3 p^{5} D^{\circ}+0.132 s^{2} S 2 p^{4}{ }_{2}^{3} P\right)^{4} P 3 p^{5} P^{\circ}$ \\
\hline 23 & 98 & $2 s^{2} S 2 p^{4}\left({ }_{2}^{2} P\right){ }^{4} P 3 p^{5} D_{2}^{\circ}$ & & 5732607 & $4.016 \mathrm{E}-12$ & $4.006 \mathrm{E}-12$ & $\left.\left.0.71+0.152 s^{2} S 2 p^{4}{ }_{2}^{3} P\right)^{4} P 3 p^{3} D^{\circ}+0.062 s^{2} S 2 p^{4} \int_{2}^{3} P\right)^{4} P 3 p^{5} P^{\circ}$ \\
\hline 23 & 99 & $2 s^{2} S 2 p^{4}\left({ }_{2}^{4} D\right)^{2} D 3 s^{3} D_{1}^{2}$ & & 5739116 & $1.235 \mathrm{E}-12$ & $1.236 \mathrm{E}-12$ & 0.95 \\
\hline 23 & 100 & $2 s^{2} S 2 p^{4}\left({ }_{2}^{1} D\right)^{2} D 3 s^{3} D_{2}$ & & 5740834 & $1.248 \mathrm{E}-12$ & $1.248 \mathrm{E}-12$ & 0.95 \\
\hline 23 & 101 & $2 s^{2} S 2 p^{4}\left({ }_{2}^{3} P\right)^{4} P 3 p^{5} D_{1}^{\circ}$ & & 5741103 & 1.413E-11 & 1.412E-11 & $0.88+0.062 s^{2} S 2 p^{4}\left({ }_{2}^{3} P\right)^{4} P 3 p^{5} P^{\circ}+0.032 s^{2} S 2 p^{4}\left({ }_{2}^{3} P\right){ }^{4} P 3 p^{3} D^{\circ}$ \\
\hline 23 & 102 & $2 s^{2} S 2 p^{4}\left({ }_{2}^{3} P\right){ }^{4} P 3 p^{5} D_{0}^{\circ}$ & & 5742863 & 5.013E-11 & $5.019 \mathrm{E}-11$ & 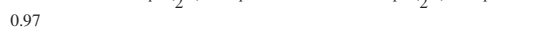 \\
\hline 23 & 103 & $2 s^{2} s 2 p^{4}\left({ }_{2}^{1} D\right)^{2} D 3 s^{3} D_{3}$ & & 5744027 & 1.197E-12 & 1.197E-12 & 0.97 \\
\hline 23 & 104 & $2 s^{2} S 2 p^{4}\left({ }_{2}^{2} P\right){ }^{4} P 3 p^{5} D_{3}^{\circ}$ & & 5745923 & $9.731 \mathrm{E}-13$ & $9.702 \mathrm{E}-13$ & $0.43+0.482 s^{2} S 2 p^{4}\left({ }_{2}^{3} P\right)^{4} P 3 p^{3} D^{\circ}+0.072 s^{2} S 2 p^{4}\left({ }_{2}^{3} P\right)^{4} P 3 p^{5} P^{\circ}$ \\
\hline 23 & 105 & $2 s^{2} S 2 p^{4}\left({ }_{2}^{2} P\right){ }^{4} P 3 p^{3} S_{1}^{0}$ & & 5752660 & $4.232 \mathrm{E}-13$ & $4.222 \mathrm{E}-13$ & $0.62+0.242 s^{2} S 2 p^{4}\left(_{2}^{3} P\right)^{4} P 3 p^{3} P^{\circ}+0.042 s^{2} S 2 p^{4}\left({ }_{2}^{3} P\right){ }^{4} P 3 p^{5} P^{\circ}$ \\
\hline 23 & 106 & $2 s^{2} S 2 p^{4}\left({ }_{2}^{3} P\right){ }^{4} P 3 p^{3} D_{2}^{\circ}$ & & 5756692 & $5.592 \mathrm{E}-13$ & $5.576 \mathrm{E}-13$ & $0.47+0.342 s^{2} S 2 p^{4}\left({ }_{2}^{3} P\right)^{4} P 3 p^{3} P^{\circ}+0.132 s^{2} S 2 p^{4}\left({ }_{2}^{3} P\right){ }^{4} P 3 p^{5} D^{\circ}$ \\
\hline 23 & 107 & $2 s^{2} S 2 p^{4}\left({ }_{2}^{3} P\right)^{4} P 3 p^{5} S_{2}^{0}$ & & 5766966 & 2.831E-11 & $2.832 \mathrm{E}-11$ & $0.83+0.132 s^{2} S 2 p^{4}\left(\frac{3}{2} P\right)^{4} P 3 p^{5} P^{\circ}$ \\
\hline 23 & 108 & $2 s^{2} S 2 p^{4}\left({ }_{2}^{3} P\right){ }^{4} P 3 p^{3} D_{1}^{\circ}$ & & 5780601 & 5.414E-13 & $5.398 \mathrm{E}-13$ & $\left.0.81+0.112 s^{2} S 2 p^{4}{ }_{2}^{3} P\right)^{4} P 3 p^{3} s^{\circ}+0.042 s^{2} S 2 p^{4}\left({ }_{2}^{3} P\right)^{4} P 3 p^{3} P^{\circ}$ \\
\hline 23 & 109 & $2 s^{2} S 2 p^{4}\left({ }_{2}^{3} P\right){ }^{4} P 3 p^{3} P_{2}^{\circ}$ & & 5783782 & $5.131 \mathrm{E}-13$ & $5.116 \mathrm{E}-13$ & $0.50+0.342 s^{2} S 2 p^{4}\left({ }_{2}^{3} P\right)^{4} P 3 p^{3} D^{\circ}+0.062 s^{2} S 2 p^{4}\left({ }_{2}^{1} D\right)^{2} D 3 p^{3} P^{\circ}$ \\
\hline 23 & 110 & $2 s^{2} S 2 p^{4}\left({ }_{2}^{2} P\right)^{4} P 3 p^{3} P_{0}^{\circ}$ & & 5785059 & $5.669 \mathrm{E}-13$ & 5.653E-13 & $0.80+0.172 s^{2} S 2 p^{4}\left({ }_{2}^{2} D\right)^{2} D 3 p^{3} P^{\circ}$ \\
\hline 23 & 111 & $2 s^{2} S 2 p^{4}\left({ }_{2}^{1} D\right)^{2} D 3 s^{1} D_{2}$ & & 5787769 & 2.093E-12 & $2.094 \mathrm{E}-12$ & 0.95 \\
\hline 23 & 112 & $\left.2 s^{2} S 2 p^{4}{ }_{2}^{3} P\right)^{4} P 3 p^{3} P_{1}^{\circ}$ & & 5796356 & 4.673E-13 & $4.660 \mathrm{E}-13$ & $\left.0.51+0.242 s^{2} S 2 p^{4}\left({ }_{2}^{3} P\right)^{4} P 3 p^{3} s^{\circ}+0.112 s^{2} S 2 p^{4}{ }_{2}^{3} P\right)^{4} P 3 p^{3} D^{\circ}$ \\
\hline 23 & 113 & $2 s^{2} S 2 p^{4}\left({ }_{0}^{1} S\right)^{2} S 3 s^{3} S_{1}$ & & 5873184 & $1.230 \mathrm{E}-12$ & $1.230 \mathrm{E}-12$ & $0.81+0.112 s^{2} S 2 p^{4}\left({ }_{2}^{3} P\right)^{2} P 3 s^{3} P+0.032 s^{2} S 2 p^{4}\left({ }_{2}^{2} P\right)^{2} P 3 s^{1} P$ \\
\hline 23 & 114 & $2 s^{2} S 2 p^{4}\left(Y_{0} S\right)^{2} S 3 s^{1} s_{0}$ & & 5903662 & $1.565 \mathrm{E}-12$ & $1.565 \mathrm{E}-12$ & $0.61+0.362 s^{2} S 2 p^{4}\left({ }_{3}^{3} P\right)^{2} P 3 s^{3} P$ \\
\hline 23 & 115 & $2 s^{2} S 2 p^{4}\left({ }_{2}^{3} P\right)^{2} P 3 s^{3} P_{2}$ & & 5913347 & $1.897 \mathrm{E}-12$ & $1.897 \mathrm{E}-12$ & 0.95 \\
\hline 23 & 116 & $2 s^{2} S 2 p^{4}\left({ }_{3}^{3} P\right)^{4} P 3 d^{5} D_{4}$ & & 5916127 & $7.551 \mathrm{E}-11$ & 7.531E-11 & $0.84+0.152 s^{2} S 2 p^{4}\left(3_{2}^{3} P\right)^{4} P 3 d^{5} F$ \\
\hline 23 & 117 & $2 s^{2} S 2 p^{4}\left({ }_{2}^{2} P\right){ }^{4} P 3 d^{5} D_{3}$ & & 5917215 & 7.025E-11 & 7.033E-11 & $0.83+0.092 s^{2} S 2 p^{4}\left({ }_{2}^{3} P\right)^{4} P 3 d^{5} F+0.062 s^{2} S 2 p^{4}\left({ }_{2}^{3} P\right)^{4} P 3 d^{5} P$ \\
\hline 23 & 118 & $2 s^{2} S 2 p^{4}\left({ }_{2}^{3} P\right){ }^{4} P 3 d^{5} D_{2}$ & & 5922497 & $5.929 \mathrm{E}-11$ & $5.932 \mathrm{E}-11$ & $0.81+0.122 s^{2} S 2 p^{4}\left({ }_{2}^{3} P\right){ }^{4} P 3 d^{5} P+0.042 s^{2} S 2 p^{4}\left({ }_{2}^{3} P\right)^{4} P 3 d^{5} F$ \\
\hline 23 & 119 & $2 s^{2} S 2 p^{4}\left({ }_{2}^{3} P\right)^{2} P 3 s^{1} P_{1}$ & & 5924060 & $7.552 \mathrm{E}-13$ & 7.554E-13 & $0.50+0.442 s^{2} S 2 p^{4}\left({ }_{2}^{3} P\right)^{2} P 3 s^{3} P$ \\
\hline 23 & 120 & $2 s^{2} S 2 p^{4}\left(\frac{1}{2} D\right)^{2} D 3 p^{3} F_{2}^{\circ}$ & & 5928165 & $1.069 \mathrm{E}-11$ & $1.067 \mathrm{E}-11$ & $0.95+23$ \\
\hline 23 & 121 & $2 s^{2} S 2 p^{4}\left({ }_{3}^{3} P\right){ }^{4} P 3 d^{5} D_{1}$ & & 5930271 & 3.483E-11 & $3.488 \mathrm{E}-11$ & $0.81+0.142 s^{2} S 2 p^{4}\left({ }_{2}^{3} P\right)^{4} P 3 d^{5} P$ \\
\hline 23 & 122 & $2 s^{2} S 2 p^{4}\left({ }_{2}^{\mathrm{T}} D\right)^{2} D 3 p^{3} F_{3}^{\circ}$ & & 5934969 & 7.223E-12 & $7.209 \mathrm{E}-12$ & $0.93+0.032 s^{2} S 2 p^{4}\left({ }_{2}^{1} D\right)^{2} D 3 p^{1} F^{\circ}$ \\
\hline 23 & 123 & $2 s^{2} S 2 p^{4}\left({ }_{2}^{1} D\right)^{2} D 3 p^{1} P_{1}^{\circ}$ & & 5935160 & $4.292 \mathrm{E}-13$ & $4.282 \mathrm{E}-13$ & $\left.0.63+0.182 s^{2} S 2 p^{4}\left({ }_{2}^{1} D\right)^{2} D 3 p^{3} D^{\circ}+0.072 s^{2} S 2 p^{4}{ }_{2}^{3} P\right)^{2} P 3 p^{1} P^{\circ}$ \\
\hline 23 & 124 & $2 s^{2} S 2 p^{4}\left({ }_{2}^{2} P\right){ }^{4} P 3 d^{5} F_{5}$ & & 5937720 & $1.137 \mathrm{E}-10$ & $1.094 \mathrm{E}-10$ & 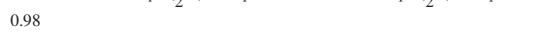 \\
\hline 23 & 125 & $2 s^{2} S 2 p^{4}\left({ }_{2}^{3} P\right){ }^{4} P 3 d^{5} D_{0}$ & & 5938811 & $2.116 \mathrm{E}-11$ & 2.125E-11 & $0.95+0.032 s^{2} S 2 p^{4}\left({ }_{2}^{3} P\right)^{4} P 3 d^{3} P$ \\
\hline 23 & 126 & $2 s^{2} S 2 p^{4}\left({ }_{2}^{1} D\right)^{2} D 3 p^{3} F_{4}^{\circ}$ & & 5945487 & $3.286 \mathrm{E}-11$ & $3.268 \mathrm{E}-11$ & 0.97 \\
\hline 23 & 127 & $2 s^{2} S 2 p^{4}\left({ }_{2}^{1} D\right)^{2} D 3 p^{1} F_{3}^{\circ}$ & & 5955298 & 4.423E-13 & $4.410 \mathrm{E}-13$ & $0.82+0.102 s^{2} S 2 p^{4}\left({ }_{2}^{1} D\right)^{2} D 3 p^{3} D^{\circ}+0.042 s^{2} S 2 p^{4}\left({ }_{2}^{1} D\right)^{2} D 3 p^{3} F^{\circ}$ \\
\hline 23 & 128 & $2 s^{2} S 2 p^{4}\left({ }_{2}^{2} P\right){ }^{4} P 3 d^{5} F_{4}^{3}$ & & 5958013 & $9.166 \mathrm{E}-11$ & $8.950 \mathrm{E}-11$ & $0.70+0.182 s^{2} S 2 p^{4}\left({ }_{2}^{3} P\right){ }^{4} P 3 d^{3} F+0.112 s^{2} S 2 p^{4}\left({ }_{2}^{3} P\right)^{4} P 3 d^{5} D$ \\
\hline 23 & 129 & $2 s^{2} S 2 p^{4}\left(\frac{1}{2} D\right)^{2} D 3 p^{3} D_{2}^{\circ}$ & & 5964372 & $6.497 \mathrm{E}-12$ & $6.478 \mathrm{E}-12$ & $0.90+0.032 s^{2} S 2 p^{4}\left(\frac{3}{2} P\right)^{2} P 3 p^{3} P^{\circ}+0.032 s^{2} S 2 p^{4}\left({ }_{2}^{1} D\right)^{2} D 3 p^{3} P^{\circ}$ \\
\hline 23 & 130 & $2 s^{2} S 2 p^{4}\left({ }_{2}^{2} P\right){ }^{4} P 3 d^{5} P_{1}$ & & 5967195 & $4.651 \mathrm{E}-12$ & $4.650 \mathrm{E}-12$ & $0.84+0.122 s^{2} S 2 p^{4}\left({ }_{2}^{3} P\right)^{4} P 3 d^{5} D$ \\
\hline 23 & 131 & $2 s^{2} S 2 p^{4}\left({ }_{1}^{1} D\right)^{2} D 3 p^{3} D_{1}^{\circ}$ & & 5968771 & $1.549 \mathrm{E}-12$ & $1.545 \mathrm{E}-12$ & $0.79+0.172 s^{2} S 2 p^{4}\left({ }_{1}^{1} D\right)^{2} D 3 p^{1} P^{\circ}$ \\
\hline 23 & 132 & $2 s^{2} S 2 p^{4}\left({ }_{2}^{3} P\right){ }^{4} P 3 d^{5} F_{3}$ & & 5971337 & 4.615E-12 & $4.604 \mathrm{E}-12$ & $0.80+0.082 s^{2} S 2 p^{4}\left(\int_{2}^{3} P\right)^{4} P 3 d^{3} F+0.062 s^{2} S 2 p^{4}\left({ }_{2}^{3} P\right)^{4} P 3 d^{5} D$ \\
\hline 23 & 133 & $2 s^{2} S 2 p^{4}\left({ }_{2}^{2} P\right)^{2} P 3 s^{3} P_{1}$ & & 5972156 & $7.448 \mathrm{E}-13$ & $7.445 \mathrm{E}-13$ & $0.42+0.422 s^{2} S 2 p^{4}\left({ }_{2}^{3} P\right)^{2} P 3 s^{1} P+0.132 s^{2} S 2 p^{4}\left({ }_{0}^{1} S\right)^{2} S 3 s^{3} S$ \\
\hline 23 & 134 & $2 s^{2} S 2 p^{4}\left({ }_{2}^{1} D\right)^{2} D 3 p^{3} D_{3}^{\circ}$ & & 5972917 & $2.902 \mathrm{E}-12$ & $2.892 \mathrm{E}-12$ & $0.85+0.122 s^{2} S 2 p^{4}\left({ }_{2}^{1} D\right)^{2} D 3 p^{1} F^{\circ}$ \\
\hline 23 & 135 & $\left.2 s^{2} S 2 p^{4}{ }_{2}^{3} P\right)^{4} P 3 d^{5} P_{2}{ }^{3}$ & & 5976971 & $3.199 \mathrm{E}-12$ & 3.197E-12 & $0.48+0.462 s^{2} S 2 p^{4}\left({ }_{2}^{3} P\right)^{4} P 3 d^{5} F$ \\
\hline 23 & 136 & $2 s^{2} S 2 p^{4}\left({ }_{3}^{3} P\right)^{2} P 3 s^{3} P_{0}$ & & 5978969 & 2.004E-12 & 2.003E-12 & $0.62+0.352 s^{2} S 2 p^{4}\left(C^{1} S\right)^{2} S 3 s^{1} S$ \\
\hline 23 & 137 & $2 s^{2} S 2 p^{4}\left({ }_{2}^{1} D\right)^{2} D 3 p^{1} D_{2}^{\circ}$ & & 5980444 & $5.699 \mathrm{E}-13$ & $5.681 \mathrm{E}-13$ & $0.55+0.352 s^{2} S 2 p^{4}\left({ }_{2} D\right)^{2} D 3 p^{3} P^{\circ}+0.042 s^{2} S 2 p^{4}\left({ }_{2}^{1} D\right)^{2} D 3 p^{3} D^{\circ}$ \\
\hline 23 & 138 & $2 s^{2} S 2 p^{4}\left({ }_{2}^{3} P\right){ }^{4} P 3 d^{5} F_{2}{ }^{2}$ & & 5984402 & $1.826 \mathrm{E}-11$ & $1.831 \mathrm{E}-11$ & $0.46+0.372 s^{2} S 2 p^{4}\left({ }_{2}^{3} P\right)^{4} P 3 d^{5} P+0.142 s^{2} S 2 p^{4}\left({ }_{2}^{3} P\right)^{4} P 3 d^{5} D$ \\
\hline 23 & 139 & $2 s^{2} S 2 p^{4}\left({ }_{2}^{3} P\right){ }^{4} P 3 d^{5} F_{1}$ & & 5985897 & $1.166 \mathrm{E}-11$ & $1.160 \mathrm{E}-11$ & $0.95+0.032 s^{2} S 2 p^{4}\left({ }_{2}^{2} P\right)^{4} P 3 d^{5} D$ \\
\hline 23 & 140 & $2 s^{2} S 2 p^{4}\left({ }_{2}^{1} D\right)^{2} D 3 p^{3} P_{1}^{\circ}$ & & 5988349 & $1.086 \mathrm{E}-12$ & $1.082 \mathrm{E}-12$ & $\left.0.70+0.112 s^{2} S 2 p^{4}{ }_{2}^{3} P\right)^{4} P 3 p^{3} P^{\circ}+0.082 s^{2} S 2 p^{4}\left({ }_{2}^{3} P\right)^{2} P 3 p^{3} P^{\circ}$ \\
\hline 23 & 141 & $2 s^{2} S 2 p^{4}\left({ }_{2}^{1} D\right)^{2} D 3 p^{3} P_{2}^{\circ}$ & & 5989436 & $6.359 \mathrm{E}-13$ & $6.340 \mathrm{E}-13$ & $\left.0.48+0.402 s^{2} S 2 p^{4}\left({ }_{2}^{1} D\right)^{2} D 3 p^{1} D^{\circ}+0.082 s^{2} S 2 p^{4}{ }_{2}^{3} P\right)^{4} P 3 p^{3} P^{\circ}$ \\
\hline 23 & 142 & $2 s^{2} S 2 p^{4}\left({ }_{2}^{3} P\right){ }^{4} P 3 d^{3} F_{4}^{2}$ & & 5990082 & $7.580 \mathrm{E}-11$ & 7.447E-11 & $\left.\left.0.80+0.142 s^{2} S 2 p^{4}{ }_{2}^{3} P\right)^{4} P 3 d^{5} F+0.052 s^{2} S 2 p^{4}{ }_{2}^{3} P\right)^{2} P 3 d^{5} D$ \\
\hline 23 & 143 & $2 s^{2} S 2 p^{4}\left({ }_{2}^{1} D\right)^{2} D 3 p^{3} P_{0}^{\circ}$ & & 5991503 & $1.052 \mathrm{E}-12$ & $1.048 \mathrm{E}-12$ & $\left.\left.0.70+0.152 s^{2} S 2 p^{4}{ }_{2}^{3} P\right)^{4} P 3 p^{3} P^{\circ}+0.132 s^{2} S 2 p^{4}{ }_{2}^{3} P\right)^{2} P 3 p^{3} P^{\circ}$ \\
\hline 23 & 144 & $2 s^{2} S 2 p^{4}\left({ }_{3}^{3} P\right)^{4} P 3 d^{5} P_{3}$ & & 5994943 & $2.959 \mathrm{E}-12$ & $2.959 \mathrm{E}-12$ & $0.79+0.082 s^{2} S 2 p^{4}\left(3_{2}^{2} P\right)^{4} P 3 d^{5} D+0.042 s^{2} S 2 p^{4}\left(3_{2}^{2} P\right)^{4} P 3 d^{3} D$ \\
\hline 23 & 145 & $2 s^{2} S 2 p^{4}\left({ }_{2}^{2} P\right){ }^{4} P 3 d^{3} F_{3}$ & & 6005131 & $1.112 \mathrm{E}-12$ & $1.109 \mathrm{E}-12$ & $\left.0.70+0.132 s^{2} S 2 p^{4}\left({ }_{2}^{2} P\right)^{4} P 3 d^{3} D+0.082 s^{2} S 2 p^{4}{ }_{2}^{3} P\right)^{4} P 3 d^{5} P$ \\
\hline 23 & 146 & $2 s^{2} S 2 p^{4}\left({ }_{2}^{2} P\right){ }^{4} P 3 d^{3} P_{0}$ & & 6013955 & 4.667E-13 & 4.663E-13 & $0.85+0.092 s^{2} S 2 p^{4}\left({ }_{2}^{1} D\right)^{2} D 3 d^{3} P+0.042 s^{2} S 2 p^{4}\left({ }_{2}^{2} P\right)^{4} P 3 d^{5} D$ \\
\hline 23 & 147 & $2 s^{2} S 2 p^{4}\left({ }_{2}^{3} P\right){ }^{4} P 3 d^{3} D_{2}$ & & 6016502 & $3.401 \mathrm{E}-13$ & 3.397E-13 & $0.35+0.412 s^{2} S 2 p^{4}\left({ }_{2}^{2} P\right)^{4} P 3 d^{3} F+0.142 s^{2} S 2 p^{4}\left({ }_{2}^{3} P\right){ }^{4} P 3 d^{3} P$ \\
\hline 23 & 148 & $2 s^{2} S 2 p^{4}\left({ }_{2}^{3} P\right){ }^{4} P 3 d^{3} P_{1}$ & & 6017171 & $3.172 \mathrm{E}-13$ & $3.170 \mathrm{E}-13$ & $0.69+0.182 s^{2} S 2 p^{4}\left({ }_{2}^{3} P\right)^{4} P 3 d^{3} D+0.062 s^{2} S 2 p^{4}\left({ }_{2}^{1} D\right)^{2} D 3 d^{3} P$ \\
\hline 23 & 149 & $2 s^{2} S 2 p^{4}\left({ }_{2}^{3} P\right){ }^{4} P 3 d^{3} F_{2}$ & & 6035134 & 4.897E-13 & $4.892 \mathrm{E}-13$ & $0.52+0.272 s^{2} S 2 p^{4}\left({ }_{2}^{3} P\right)^{4} P 3 d^{3} P+0.152 s^{2} S 2 p^{4}\left({ }_{2}^{3} P\right){ }^{4} P 3 d^{3} D$ \\
\hline 23 & 150 & $2 s^{2} S 2 p^{4}\left({ }_{2}^{3} P\right){ }^{4} P 3 d^{3} D_{3}$ & & 6046135 & $1.636 \mathrm{E}-13$ & $1.634 \mathrm{E}-13$ & $0.75+0.182 s^{2} S 2 p^{4}\left({ }_{2}^{2} P\right)^{4} P 3 d^{3} F+0.032 s^{2} S 2 p^{4}\left({ }_{2}^{1} D\right)^{2} D 3 d^{3} D$ \\
\hline 23 & 151 & $\left.2 s^{2} S 2 p^{4}{ }_{3}^{3} P\right)^{4} P 3 d^{3} D_{1}$ & & 6046501 & $2.211 \mathrm{E}-13$ & $2.209 \mathrm{E}-13$ & $0.68+0.182 s^{2} S 2 p^{4}\left(\int_{2}^{2} P\right)^{4} P 3 d^{3} P+0.072 s^{2} S 2 p^{4}\left({ }_{2}^{1} D\right)^{2} D 3 d^{3} D$ \\
\hline 23 & 152 & $2 s^{2} S 2 p^{4}\left({ }_{2}^{3} P\right){ }^{4} P 3 d^{3} P_{2}$ & & 6060378 & $2.607 \mathrm{E}-13$ & $2.604 \mathrm{E}-13$ & $0.49+0.372 s^{2} S 2 p^{4}\left(3_{2}^{3} P\right)^{4} P 3 d^{3} D+0.052 s^{2} S 2 p^{4}\left(\frac{1}{2} D\right)^{2} D 3 d^{3} P$ \\
\hline 23 & 153 & $2 s^{2} S 2 p^{4}\left({ }_{0}^{\mathrm{T}} S\right)^{2} S 3 p^{3} P_{0}^{\circ}$ & & 6066508 & $2.630 \mathrm{E}-12$ & 2.627E-12 & $\left.0.69+0.212 s^{2} S 2 p^{4}{ }_{2}^{3} P\right)^{2} P 3 p^{3} P^{\circ}+0.042 s^{2} S 2 p^{4}\left({ }_{2}^{1} D\right)^{2} D 3 p^{3} P^{\circ}$ \\
\hline 23 & 154 & $2 s^{2} S 2 p^{4}\left({ }_{0}^{1} S\right)^{2} S 3 p^{3} P_{1}^{\circ}$ & & 6073259 & $1.706 \mathrm{E}-12$ & 1.703E-12 & $\left.\left.0.63+0.142 s^{2} S 2 p^{4}{ }_{2}^{3} P\right)^{2} P 3 p^{3} P^{\circ}+0.132 s^{2} S 2 p^{4}{ }_{2}^{3} P\right)^{2} P 3 p^{3} D^{\circ}$ \\
\hline 23 & 155 & $2 s^{2} S 2 p^{4}\left({ }_{0}^{1} S\right)^{2} S 3 p^{3} P_{2}^{\circ}$ & & 6088716 & $2.467 \mathrm{E}-12$ & $2.461 \mathrm{E}-12$ & $\left.0.71+0.212 s^{2} S 2 p^{4}\left({ }_{2}^{3} P\right)^{2} P 3 p^{3} D^{\circ}+0.022 s^{2} S 2 p^{4}{ }_{2}^{3} P\right)^{2} P 3 p^{3} P^{\circ}$ \\
\hline 23 & 156 & $2 s^{2} S 2 p^{4}\left({ }_{0}^{1} S\right)^{2} S 3 p^{1} P_{1}^{\circ}$ & & 6096246 & $5.054 \mathrm{E}-13$ & $5.039 \mathrm{E}-13$ & $\left.\left.0.64+0.112 s^{2} S 2 p^{4}{ }_{2}^{3} P\right)^{2} P 3 p^{3} P^{\circ}+0.102 s^{2} S 2 p^{4}{ }_{2}^{3} P\right)^{2} P 3 p^{3} D^{\circ}$ \\
\hline 23 & 157 & $2 s^{2} S 2 p^{4}\left({ }_{2}^{3} P\right)^{2} P 3 p^{3} P_{2}^{\circ}$ & & 6105637 & $2.129 \mathrm{E}-12$ & $2.125 \mathrm{E}-12$ & $0.60+0.142 s^{2} S 2 p^{4}\left({ }_{2}^{2} P\right)^{2} P 3 p^{3} D^{\circ}+0.102 s^{2} S 2 p^{4}\left(1_{0}^{2} S\right)^{2} S 3 p^{3} P^{\circ}$ \\
\hline 23 & 158 & $\left.2 s^{2} S 2 p^{4}{ }_{2}^{2} P\right)^{2} P 3 p^{1} D_{2}^{\circ}$ & & 6119529 & 1.867E-12 & $1.863 \mathrm{E}-12$ & $\left.\left.0.47+0.252 s^{2} S 2 p^{4}{ }_{2}^{2} P\right)^{2} P 3 p^{3} D^{\circ}+0.222 s^{2} S 2 p^{4}{ }_{2}{ }_{2} P\right)^{2} P 3 p^{3} P^{\circ}$ \\
\hline 23 & 159 & $\left.2 s^{2} S 2 p^{4}{ }_{3}^{3} P\right)^{2} P 3 p^{3} D_{3}^{\circ}$ & & 6120797 & $9.712 \mathrm{E}-13$ & $9.684 \mathrm{E}-13$ & $0.96-2$ \\
\hline 23 & 160 & $2 s^{2} S 2 p^{4}\left({ }_{2}^{2} P\right)^{2} P 3 p^{3} P_{1}^{3}$ & & 6128173 & $2.237 \mathrm{E}-12$ & $2.232 \mathrm{E}-12$ & $0.42+0.222 s^{2} S 2 p^{4}\left({ }_{2}^{3} P\right)^{2} P 3 p^{3} S^{\circ}+0.182 s^{2} S 2 p^{4}\left({ }_{0}^{1} S\right)^{2} S 3 p^{3} P^{\circ}$ \\
\hline 23 & 161 & $\left.2 s^{2} S 2 p^{4}{ }_{2}^{2} P\right)^{2} P 3 p^{3} P_{0}^{\circ}$ & & 6140151 & $5.162 \mathrm{E}-12$ & 5.157E-12 & $0.35+0.472 s^{2} S 2 p^{4}\left({ }_{2}^{2} P\right)^{2} P 3 p^{1} S^{\circ}+0.082 s^{2} S 2 p^{4}\left({ }_{2}^{1} D\right)^{2} D 3 p^{3} P^{\circ}$ \\
\hline 23 & 162 & $2 s^{2} S 2 p^{4}\left({ }_{2}^{3} P\right)^{2} P 3 p^{3} D_{1}^{\circ}$ & & 6154119 & $8.982 \mathrm{E}-13$ & $8.955 \mathrm{E}-13$ & $0.61+0.172 s^{2} S 2 p^{4}\left({ }_{2}^{3} P\right)^{2} P 3 p^{3} S^{\circ}+0.072 s^{2} S 2 p^{4}\left({ }_{0}^{1} S\right)^{2} S 3 p^{1} P^{\circ}$ \\
\hline 23 & 163 & $2 s^{2} S 2 p^{4}\left({ }_{2}^{1} D\right)^{2} D 3 d^{3} G_{3}$ & & 6165448 & $3.736 \mathrm{E}-11$ & $3.690 \mathrm{E}-11$ & 0.97 \\
\hline 23 & 164 & $2 s^{2} S 2 p^{4}\left(\frac{1}{2} D\right)^{2} D 3 d^{3} G_{4}$ & & 6168395 & 4.077E-11 & $4.020 \mathrm{E}-11$ & 0.97 \\
\hline 23 & 165 & $2 s^{2} S 2 p^{4}\left({ }_{2}^{1} D\right)^{2} D 3 d^{3} G_{5}$ & & 6172228 & $4.361 \mathrm{E}-11$ & $4.298 \mathrm{E}-11$ & 0.98 \\
\hline 23 & 166 & $2 s^{2} S 2 p^{4}\left({ }_{2}^{3} P\right)^{2} P 3 p p^{1} S_{0}^{0}$ & & 6176103 & $6.780 \mathrm{E}-12$ & $6.779 \mathrm{E}-12$ & $0.47+0.282 s^{2} S 2 p^{4}\left({ }_{2}^{3} P\right)^{2} P 3 p^{3} P^{\circ}+0.202 s^{2} S 2 p^{4}\left({ }_{0}^{1} S\right)^{2} S 3 p^{3} P^{\circ}$ \\
\hline 23 & 167 & $2 s^{2} S 2 p^{4}\left({ }_{2}^{3} P\right)^{2} P 3 p^{3} D_{2}^{\circ}$ & & 6176817 & $2.184 \mathrm{E}-12$ & $2.179 \mathrm{E}-12$ & $0.37+0.402 s^{2} S 2 p^{4}\left(3_{2}^{3} P\right)^{2} P 3 p^{1} D^{\circ}+0.162 s^{2} S 2 p^{4}\left(\frac{1}{0} S\right)^{2} S 3 p^{3} P^{\circ}$ \\
\hline 23 & 168 & $\left.2 s^{2} S 2 p^{4}{ }_{2}^{2} P\right)^{2} P 3 p^{3} S_{1}^{\circ}$ & & 6183234 & $8.229 \mathrm{E}-13$ & $8.199 \mathrm{E}-13$ & $0.52+0.172 s^{2} S 2 p^{4}\left({ }_{2}^{3} P\right)^{2} P 3 p^{3} P^{\circ}+0.162 s^{2} S 2 p^{4}\left({ }_{0}^{1} S\right)^{2} S 3 p^{1} P^{\circ}$ \\
\hline 23 & 169 & $2 s^{2} S 2 p^{4}\left({ }_{2}^{1} D\right)^{2} D 3 d^{3} F_{2}$ & & 6198711 & $1.225 \mathrm{E}-11$ & $1.225 \mathrm{E}-11$ & $0.92+0.032 s^{2} S 2 p^{4}\left({ }_{2}^{1} D\right)^{2} D 3 d^{1} D$ \\
\hline 23 & 170 & $2 s^{2} S 2 p^{4}\left({ }_{2}^{1} D\right)^{2} D 3 d^{3} s_{1}$ & & 6200268 & $8.889 \mathrm{E}-14$ & $8.882 \mathrm{E}-14$ & 0.95 \\
\hline 23 & 171 & $2 s^{2} S 2 p^{4}\left({ }_{2}^{1} D\right)^{2} D 3 d^{3} F_{3}$ & & 6202048 & 3.933E-12 & $3.929 \mathrm{E}-12$ & $0.93+0.032 s^{2} S 2 p^{4}\left({ }_{2}^{1} D\right)^{2} D 3 d^{3} D$ \\
\hline 23 & 172 & $2 s^{2} S 2 p^{4}\left({ }_{2}^{2} D\right)^{2} D 3 d^{3} F_{4}$ & & 6203631 & 3.441E-11 & $3.426 \mathrm{E}-11$ & $0.68+0.292 s^{2} S 2 p^{4}\left({ }_{2}^{2} D\right)^{2} D 3 d^{1} G$ \\
\hline 23 & 173 & $2 s^{2} S 2 p^{4}\left({ }_{2}^{1} D\right)^{2} D 3 d^{3} D_{1}$ & & 6207772 & $1.958 \mathrm{E}-13$ & $1.955 \mathrm{E}-13$ & $0.50+0.232 s^{2} S 2 p^{4}\left({ }_{2}^{1} D\right)^{2} D 3 d^{1} P+0.122 s^{2} S 2 p^{4}\left({ }_{2}^{1} D\right)^{2} D 3 d^{3} P$ \\
\hline 23 & 174 & $2 s^{2} S 2 p^{4}\left({ }_{2}^{1} D\right)^{2} D 3 d^{3} P_{0}$ & & 6210660 & 1.033E-13 & 1.032E-13 & $0.75+0.112 s^{2} S 2 p^{4}\left({ }_{2}^{2} D\right)^{2} D 3 d^{1} S+0.072 s^{2} S 2 p^{4}\left({ }_{2}^{3} P\right)^{4} P 3 d^{3} P$ \\
\hline 23 & 175 & $2 s^{2} S 2 p^{4}\left({ }_{2}^{1} D\right)^{2} D 3 d^{1} G_{4}$ & & 6210928 & $3.190 \mathrm{E}-11$ & $3.180 \mathrm{E}-11$ & $0.68+0.292 s^{2} S 2 p^{4}\left({ }_{2}^{1} D\right)^{2} D 3 d^{3} F$ \\
\hline
\end{tabular}




\begin{tabular}{|c|c|c|c|c|c|c|c|}
\hline $\mathrm{z}$ & Key & Level & $E_{\mathrm{NIST}}$ & $E_{\mathrm{MCDHF} / \mathrm{RCI}}$ & $\tau_{\mathrm{MCDHF} / \mathrm{RCI}}^{l}$ & $\tau_{\mathrm{MCDHF} / \mathrm{RCI}}^{v}$ & $L S$-composition \\
\hline 23 & 176 & $2 s^{2} S 2 p^{4}\left({ }_{2}^{1} D\right)^{2} D 3 d^{3} D_{2}$ & & 6212319 & $1.682 \mathrm{E}-13$ & $1.680 \mathrm{E}-13$ & $0.85+0.052 s^{2} S 2 p^{4}\left({ }_{2}^{3} P\right)^{4} P 3 d^{3} D+0.042 s^{2} S 2 p^{4}\left({ }_{2}^{1} D\right){ }^{2} D 3 d^{1} D$ \\
\hline 23 & 177 & $2 s^{2} S 2 p^{4}\left({ }_{2}^{1} D\right)^{2} D 3 d^{3} P_{2}$ & & 6213248 & $8.921 \mathrm{E}-14$ & 8.913E-14 & $0.89+0.062 s^{2} S 2 p^{4}\left(\frac{3}{2} P\right)^{4} P 3 d^{3} P$ \\
\hline 23 & 178 & $2 s^{2} S 2 p^{4}\left({ }_{2}^{1} D\right)^{2} D 3 d^{3} D_{3}$ & & 6213427 & $1.531 \mathrm{E}-13$ & $1.530 \mathrm{E}-13$ & $0.89+0.042 s^{2} S 2 p^{4}\left({ }_{2}^{2} P\right)^{4} P 3 d^{3} D+0.032 s^{2} S 2 p^{4}\left(\frac{1}{2} D\right)^{2} D 3 d^{3} F$ \\
\hline 23 & 179 & $2 s^{2} S 2 p^{4}\left({ }_{2}^{1} D\right)^{2} D 3 d^{3} P_{1}$ & & 6216920 & $9.430 \mathrm{E}-14$ & $9.422 \mathrm{E}-14$ & $\left.\left.0.73+0.102 s^{2} S 2 p^{4}\left({ }_{2}^{4} D\right)\right)^{2} D 3 d^{3} D+0.092 s^{2} S 2 p^{4}{ }_{2}^{3} P\right)^{4} P 3 d^{3} P$ \\
\hline 23 & 180 & $2 s^{2} S 2 p^{4}\left({ }_{2}^{1} D\right)^{2} D 3 d^{1} P_{1}$ & & 6230650 & $2.047 \mathrm{E}-13$ & $2.044 \mathrm{E}-13$ & $\left.0.64+0.222 s^{2} S 2 p^{4}\left({ }_{2}^{4} D\right)^{2} D 3 d^{3} D+0.052 s^{2} S 2 p^{4}{ }_{2}^{3} P\right)^{2} P 3 d^{1} P$ \\
\hline 23 & 181 & $2 s^{2} S 2 p^{4}\left({ }_{2}^{1} D\right)^{2} D 3 d^{1} D_{2}$ & & 6233945 & $4.946 \mathrm{E}-13$ & $4.936 \mathrm{E}-13$ & $0.79+0.052 s^{2} S 2 p^{4}\left({ }_{2}^{3} P\right)^{2} P 3 d^{1} D+0.052 s^{2} S 2 p^{4}\left({ }_{2}^{2} D\right)^{2} D 3 d^{3} F$ \\
\hline 23 & 182 & $2 s^{2} S 2 p^{4}\left({ }_{2}^{1} D\right)^{2} D 3 d^{1} F_{3}$ & & 6241225 & $1.762 \mathrm{E}-12$ & $1.761 \mathrm{E}-12$ & 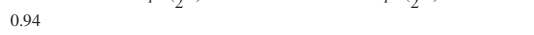 \\
\hline 23 & 183 & $\left.2 s^{2} S 2 p^{4}{ }_{2}^{3} P\right)^{2} P 3 p p^{1} P_{1}^{\circ}$ & & 6244583 & $1.821 \mathrm{E}-12$ & 1.815E-12 & $0.81+0.062 s^{2} S 2 p^{4}\left({ }_{2}^{1} D\right)^{2} D 3 p^{1} P^{\circ}+0.032 s^{2} S 2 p^{4}\left({ }_{0}^{1} S\right)^{2} S 3 p^{3} P^{\circ}$ \\
\hline 23 & 184 & $2 s^{2} S 2 p^{4}\left({ }_{2}^{1} D\right)^{2} D 3 d^{1} S_{0}$ & & 6248486 & $9.918 \mathrm{E}-14$ & $9.910 \mathrm{E}-14$ & $0.84+0.102 s^{2} S 2 p^{4}\left({ }_{2}^{1} D\right)^{2} D 3 d^{3} P+0.042 s^{2} S 2 p^{4}\left({ }_{2}^{3} P\right)^{4} P 3 d^{3} P$ \\
\hline 23 & 185 & $2 s^{2} S 2 p^{4}\left({ }_{0}^{1} S\right)^{2} S 3 d^{3} D_{1}$ & & 6316309 & $5.353 \mathrm{E}-12$ & $5.355 \mathrm{E}-12$ & $0.62+0.292 s^{2} S 2 p^{4}\left(\hat{2}_{2}^{3} P\right)^{2} P 3 d^{3} D+0.042 s^{2} S 2 p^{4}\left({ }_{2}^{1} D\right)^{2} D 3 d^{3} D$ \\
\hline 23 & 186 & $2 s^{2} S 2 p^{4}\left({ }_{0}^{1} S\right)^{2} S 3 d^{3} D_{2}$ & & 6321160 & 2.464E-12 & $2.465 \mathrm{E}-12$ & $0.68+0.172 s^{2} S 2 p^{4}\left({ }_{2}^{2} P\right)^{2} P 3 d^{3} D+0.082 s^{2} S 2 p^{4}\left(_{2}^{3} P\right)^{2} P 3 d^{3} F$ \\
\hline 23 & 187 & $2 s^{2} S 2 p^{4}\left({ }_{0}^{1} S\right)^{2} S 3 d^{3} D_{3}$ & & 6324439 & $4.026 \mathrm{E}-13$ & $4.021 \mathrm{E}-13$ & $\left.0.80+0.082 s^{2} S 2 p^{4}\left({ }_{2}^{3} P\right)^{2} P 3 d^{3} F+0.042 s^{2} S 2 p^{4}{ }_{2}^{3} P\right)^{2} P 3 d^{1} F$ \\
\hline 23 & 188 & $2 s^{2} S 2 p^{4}\left({ }_{2}^{3} P\right)^{2} P 3 d^{3} D_{3}$ & & 6338994 & $5.325 \mathrm{E}-13$ & $5.319 \mathrm{E}-13$ & $0.82+0.082 s^{2} S 2 p^{4}\left({ }_{2}^{3} P\right)^{2} P 3 d^{3} F+0.032 s^{2} S 2 p^{4}\left({ }_{2}^{1} D\right){ }^{2} D 3 d^{1} F$ \\
\hline 23 & 189 & $2 s^{2} S 2 p^{4}\left({ }_{2}^{3} P\right)^{2} P 3 d^{3} D_{2}$ & & 6344023 & $2.832 \mathrm{E}-13$ & $2.828 \mathrm{E}-13$ & $0.43+0.322 s^{2} S 2 p^{4}\left({ }_{0}^{1} S\right)^{2} S 3 d^{1} D+0.062 s^{2} S 2 p^{4}\left({ }_{2}^{2} P\right)^{2} P 3 d^{3} F$ \\
\hline 23 & 190 & $2 s^{2} S 2 p^{4}\left({ }_{0}^{1} S\right)^{2} S 3 d^{1} D_{2}$ & & 6353866 & 4.615E-13 & $4.609 \mathrm{E}-13$ & $0.28+0.282 s^{2} S 2 p^{4}\left({ }_{2}^{3} P\right)^{2} P 3 d^{3} P+0.212 s^{2} S 2 p^{4}\left({ }_{2}^{3} P\right)^{2} P 3 d^{3} D$ \\
\hline 23 & 191 & $2 s^{2} S 2 p^{4}\left({ }_{2}^{3} P\right)^{2} P 3 d^{3} F_{4}$ & & 6362615 & $9.572 \mathrm{E}-12$ & $9.685 \mathrm{E}-12$ & $0.96-2+2$ \\
\hline 23 & 192 & $2 s^{2} S 2 p^{4}\left({ }_{2}^{3} P\right)^{2} P 3 d^{1} F_{3}$ & & 6362915 & $9.716 \mathrm{E}-12$ & $9.793 \mathrm{E}-12$ & $0.54+0.422 s^{2} S 2 p^{4}\left({ }_{2}^{3} P\right)^{2} P 3 d^{3} F$ \\
\hline 23 & 193 & $2 s^{2} S 2 p^{4}\left({ }_{2}^{3} P\right){ }^{2} P 3 d^{3} D_{1}$ & & 6363244 & $1.669 \mathrm{E}-13$ & $1.667 \mathrm{E}-13$ & $\left.0.45+0.272 s^{2} S 2 p^{4}{ }_{2}^{4} P\right)^{2} P 3 d^{3} P+0.182 s^{2} S 2 p^{4}\left({ }_{0}^{1} S\right)^{2} S 3 d^{3} D$ \\
\hline 23 & 194 & $2 s^{2} S 2 p^{4}\left({ }_{3}^{3} P\right)^{2} P 3 d^{3} P_{0}$ & & 6380400 & $3.369 \mathrm{E}-13$ & $3.366 \mathrm{E}-13$ & $0.93+0.032 s^{2} S 2 p^{4}\left({ }_{2}^{1} D\right)^{2} D 3 d^{3} P$ \\
\hline 23 & 195 & $2 s^{2} S 2 p^{4}\left({ }_{2}^{3} P\right)^{2} P 3 d^{3} P_{1}$ & & 6389991 & 2.235E-13 & 2.233E-13 & $\left.0.61+0.142 s^{2} S 2 p^{4}{ }_{2}^{3} P\right)^{2} P 3 d^{1} P+0.122 s^{2} S 2 p^{4}\left({ }_{2}^{3} P\right)^{2} P 3 d^{3} D$ \\
\hline 23 & 196 & $2 s^{2} S 2 p^{4}\left({ }_{2}^{3} P\right)^{2} P 3 d^{3} F_{2}$ & & 6392619 & $2.865 \mathrm{E}-13$ & $2.861 \mathrm{E}-13$ & $\left.0.51+0.192 s^{2} S 2 p^{4}\left({ }_{2}^{3} P\right){ }^{2} P 3 d^{3} P+0.172 s^{2} S 2 p^{4}{ }_{2}^{3} P\right)^{2} P 3 d^{1} D$ \\
\hline 23 & 197 & $2 s^{2} S 2 p^{4}\left({ }_{2}^{3} P\right)^{2} P 3 d^{3} F_{3}$ & & 6413358 & $1.015 \mathrm{E}-11$ & $1.023 \mathrm{E}-11$ & $0.40+0.372 s^{2} S 2 p^{4}\left({ }_{2}^{3} P\right)^{2} P 3 d^{1} F+0.152 s^{2} S 2 p^{4}\left({ }_{0}^{1} S\right)^{2} S 3 d^{3} D$ \\
\hline 23 & 198 & $2 s^{2} S 2 p^{4}\left({ }_{2}^{3} P\right)^{2} P 3 d^{3} P_{2}$ & & 6423897 & $1.141 \mathrm{E}-12$ & $1.140 \mathrm{E}-12$ & $\left.0.45+0.242 s^{2} S 2 p^{4}\left({ }_{0}^{1} S\right)^{2} S 3 d^{1} D+0.122 s^{2} S 2 p^{4}{ }_{2}^{3} P\right)^{2} P 3 d^{3} F$ \\
\hline 23 & 199 & $2 s^{2} S 2 p^{4}\left({ }_{2}^{3} P\right)^{2} P 3 d^{1} P_{1}$ & & 6448694 & $9.816 \mathrm{E}-14$ & $9.805 \mathrm{E}-14$ & $0.73+0.072 s^{2} S 2 p^{4}\left({ }_{0}^{4} S\right)^{2} S 3 d^{3} D+0.062 s^{2} S 2 p^{4}\left({ }_{2}^{4} D\right)^{2} D 3 d^{1} P$ \\
\hline 23 & 200 & $2 s^{2} S 2 p^{4}\left({ }_{2}^{3} P\right)^{2} P 3 d^{1} D_{2}$ & & 6457412 & $6.463 \mathrm{E}-14$ & $6.454 \mathrm{E}-14$ & $0.66+0.102 s^{2} S 2 p^{4}\left(1_{0}^{1} S\right)^{2} S 3 d^{1} D+0.102 s^{2} S 2 p^{4}\left({ }_{2}^{3} P\right)^{2} P 3 d^{3} F$ \\
\hline 24 & 1 & $2 s^{2} 2 p^{4}\left({ }_{2}^{3} P\right)^{3} P_{2}$ & 0 & 0 & $\ldots$ & $\ldots$ & $0.93+0.072 s^{2} 2 p^{4}\left(1_{2}^{1} D\right)^{1} D$ \\
\hline 24 & 2 & $2 s^{2} 2 p^{4}\left({ }_{2}^{3} P\right)^{3} P_{0}$ & 57680 & 58142 & $5.358 \mathrm{E}+00$ & $5.681 \mathrm{E}+00$ & $0.87+0.132 s^{2} 2 p^{4}\left({ }_{0}^{1} s\right){ }^{1} s$ \\
\hline 24 & 3 & $2 s^{2} 2 p^{4}\left({ }_{2}^{3} P\right){ }^{3} P_{1}$ & 60376 & 60376 & $2.179 \mathrm{E}-04$ & $2.179 \mathrm{E}-04$ & 1.00 \\
\hline 24 & 4 & $2 s^{2} 2 p^{4}\left({ }_{2}^{1} D\right)^{1} D_{2}$ & 134980 & 135161 & $1.446 \mathrm{E}-04$ & $1.446 \mathrm{E}-04$ & $0.93+0.072 s^{2} 2 p^{4}\left({ }_{2}^{3} P\right){ }^{3} P$ \\
\hline 24 & 5 & $2 s^{2} 2 p^{4}\left({ }_{0}^{1} s\right){ }^{1} s_{0}$ & 262970 & 263198 & $1.705 \mathrm{E}-05$ & $1.705 \mathrm{E}-05$ & $0.85+0.132 s^{2} 2 p^{4}\left({ }_{2}^{3} P\right)^{3} P$ \\
\hline 24 & 6 & $2 s^{2} S 2 p^{5}{ }^{3} P_{2}^{\circ}$ & 813180 & 812691 & 2.675E-11 & 2.660E-11 & 0.99 \\
\hline 24 & 7 & $2 s^{2} S 2 p^{53} P_{1}^{\circ}$ & 857690 & 857169 & $2.351 \mathrm{E}-11$ & $2.350 \mathrm{E}-11$ & 0.98 \\
\hline 24 & 8 & $2 s^{2} S 2 p^{5}{ }^{3} P_{0}^{\circ}$ & 887540 & 887059 & 2.341E-11 & 2.344E-11 & 0.99 \\
\hline 24 & 9 & $2 s^{2} S 2 p^{5}{ }^{1} P_{1}^{0}$ & 1116080 & 1115887 & 7.593E-12 & $7.580 \mathrm{E}-12$ & 0.97 \\
\hline 24 & 10 & $2 p^{6}{ }^{1} S_{0}$ & 1886850 & 1886437 & $8.288 \mathrm{E}-12$ & $8.298 \mathrm{E}-12$ & $0.97+0.022 s^{2} 2 p^{4}\left({ }_{0}^{1} s\right)^{1} S$ \\
\hline 24 & 11 & $2 s^{2} 2 p^{3}\left({ }_{3}^{4} S\right)^{4} S 3 s^{5} S_{2}^{\circ}$ & & 5408096 & $2.336 \mathrm{E}-11$ & 2.343E-11 & $0.93+0.052 s^{2} 2 p^{3}\left({ }_{1}^{2} P\right)^{2} P 3 s^{3} P^{\circ}$ \\
\hline 24 & 12 & $2 s^{2} 2 p^{3}\left({ }_{3}^{4} S\right)^{4} S 3 s^{3} s_{1}^{2}$ & 5455000 & 5455700 & $4.231 \mathrm{E}-13$ & $4.231 \mathrm{E}-13$ & $0.90+0.042 s^{2} 2 p^{3}\left({ }_{1}^{2} P\right)^{2} P 3 s^{1} P^{\circ}+0.032 s^{2} 2 p^{3}\left({ }_{1}^{2} P\right)^{2} P 3 s^{3} P^{\circ}$ \\
\hline 24 & 13 & $2 s^{2} 2 p^{3}\left(\frac{3}{3} D\right)^{2} D 3 s^{3} D_{1}^{\circ}$ & 5546800 & 5549793 & $1.066 \mathrm{E}-12$ & 1.067E-12 & $0.81+0.072 s^{2} 2 p^{3}\left({ }_{1}^{2} P\right)^{2} P 3 s^{1} P^{\circ}+0.062 s^{2} 2 p^{3}\left({ }_{1}^{2} P\right)^{2} P 3 s^{3} P^{\circ}$ \\
\hline 24 & 14 & $2 s^{2} 2 p^{3}\left({ }_{3}^{3} D\right)^{2} D 3 s^{3} D_{2}^{\circ}$ & 5549400 & 5549925 & $1.160 \mathrm{E}-12$ & $1.160 \mathrm{E}-12$ & $0.70+0.172 s^{2} 2 p^{3}\left({ }_{1}^{2} P\right)^{2} P 3 s^{3} P^{\circ}+0.092 s^{2} 2 p^{3}\left({ }_{3}^{2} D\right)^{2} D 3 s^{1} D^{\circ}$ \\
\hline 24 & 15 & $2 s^{2} 2 p^{3}\left({ }_{3}^{2} D\right)^{2} D 3 s^{3} D_{3}^{\circ}$ & 5568900 & 5570024 & $1.265 \mathrm{E}-12$ & $1.265 \mathrm{E}-12$ & 0.99 \\
\hline 24 & 16 & $2 s^{2} 2 p^{3}\left({ }_{3}^{3} D\right)^{2} D 3 s^{1} D_{2}^{\circ}$ & 5588700 & 5588272 & $5.758 \mathrm{E}-13$ & $5.759 \mathrm{E}-13$ & $0.78+0.182 s^{2} 2 p^{3}\left({ }_{3}^{2} D\right)^{2} D 3 s^{3} D^{\circ}+0.032 s^{2} 2 p^{3}\left({ }_{1}^{2} P\right)^{2} P 3 s^{3} P^{\circ}$ \\
\hline 24 & 17 & $2 s^{2} 2 p^{3}\left({ }_{3}^{4} S\right)^{4} S 3 p^{5} P_{1}^{2}$ & & 5618629 & 2.743E-10 & $2.739 \mathrm{E}-10$ & $0.90+0.022 s^{2} 2 p^{3}\left({ }_{1}^{2} P\right)^{2} P 3 p^{3} P+0.022 s^{2} 2 p^{3}\left({ }_{3}^{4} S\right)^{4} S 3 p^{3} P$ \\
\hline 24 & 18 & $2 s^{2} 2 p^{3}\left({ }_{3}^{4} S\right)^{4} S 3 p^{5} P_{2}$ & & 5621744 & $3.355 \mathrm{E}-10$ & $3.349 \mathrm{E}-10$ & $0.84+0.072 s^{2} 2 p^{3}\left({ }_{3}^{4} S\right)^{4} S 3 p^{3} P+0.042 s^{2} 2 p^{3}\left({ }_{1}^{2} P\right)^{2} P 3 p^{3} P$ \\
\hline 24 & 19 & $2 s^{2} 2 p^{3}\left({ }_{3}^{4} S\right){ }^{4} S 3 p^{5} P_{3}$ & & 5634490 & $2.899 \mathrm{E}-10$ & $2.898 \mathrm{E}-10$ & $0.93+0.052 s^{2} 2 p^{3}\left({ }_{1}^{2} P\right)^{2} P 3 p^{3} D$ \\
\hline 24 & 20 & $2 s^{2} 2 p^{3}\left({ }_{1}^{3} P\right)^{2} P 3 s^{3} P_{0}^{\circ}$ & & 5646453 & 1.363E-12 & $1.363 \mathrm{E}-12$ & 0.97 \\
\hline 24 & 21 & $2 s^{2} 2 p^{3}\left({ }_{1}^{2} P\right)^{2} P 3 s^{3} P_{1}^{\circ}$ & & 5652448 & $1.039 \mathrm{E}-12$ & 1.039E-12 & $0.79+0.172 s^{2} 2 p^{3}\left({ }_{1}^{2} P\right)^{2} P 3 s^{1} P^{\circ}$ \\
\hline 24 & 22 & $2 s^{2} 2 p^{3}\left({ }_{3}^{4} S\right)^{4} S 3 p^{3} P_{1}$ & & 5666705 & $1.801 \mathrm{E}-10$ & $1.808 \mathrm{E}-10$ & $0.68+0.112 s^{2} 2 p^{3}\left(\frac{2}{3} D\right){ }^{2} D 3 p^{3} P+0.072 s^{2} 2 p^{3}\left({ }_{1}^{2} P\right)^{2} P 3 p^{1} P$ \\
\hline 24 & 23 & $2 s^{2} 2 p^{3}\left({ }_{3}^{4} S\right)^{4} S 3 p^{3} P_{2}$ & & 5678760 & $1.967 \mathrm{E}-10$ & $1.974 \mathrm{E}-10$ & $0.71+0.112 s^{2} 2 p^{3}\left({ }_{3}^{2} D\right)^{2} D 3 p^{3} P+0.112 s^{2} 2 p^{3}\left({ }_{3}^{4} S\right)^{4} S 3 p^{5} P$ \\
\hline 24 & 24 & $2 s^{2} 2 p^{3}\left({ }_{3}^{4} S\right)^{4} S 3 p^{3} P_{0}$ & & 5683973 & 2.098E-10 & $2.101 \mathrm{E}-10$ & $0.85+0.072 s^{2} 2 p^{3}\left({ }_{3}^{2} D\right)^{2} D 3 p^{3} P+0.062 s^{2} 2 p^{3}\left(_{1}^{2} P\right)^{2} P 3 p^{3} P$ \\
\hline 24 & 25 & $2 s^{2} 2 p^{3}\left({ }_{1}^{3} P\right)^{2} P 3 s^{3} P_{2}^{\circ}$ & & 5686066 & $1.091 \mathrm{E}-12$ & $1.090 \mathrm{E}-12$ & $0.72+0.122 s^{2} 2 p^{3}\left(\frac{2}{3} D\right)^{2} D 3 s^{1} D^{\circ}+0.112 s^{2} 2 p^{3}\left(\frac{2}{3} D\right)^{2} D 3 s^{3} D^{\circ}$ \\
\hline 24 & 26 & $2 s^{2} 2 p^{3}\left({ }_{1}^{2} P\right){ }^{2} P 3 s^{1} P_{1}^{\circ}$ & 5700700 & 5701089 & $5.978 \mathrm{E}-13$ & $5.977 \mathrm{E}-13$ & $0.69+0.152 s^{2} 2 p^{3}\left(\frac{2}{3} D\right)^{2} D 3 s^{3} D^{\circ}+0.102 s^{2} 2 p^{3}\left({ }_{1}^{2} P\right)^{2} P 3 s^{3} P^{\circ}$ \\
\hline 24 & 27 & $2 s^{2} 2 p^{3}\left(\frac{1}{2} D\right)^{2} D 3 p^{3} D_{1}$ & & 5736813 & $1.290 \mathrm{E}-10$ & $1.299 \mathrm{E}-10$ & $0.41+0.292 s^{2} 2 p^{3}\left({ }_{3}^{2} D\right)^{2} D 3 p^{1} P+0.072 s^{2} 2 p^{3}\left({ }_{1}^{2} P\right)^{2} P 3 p^{1} P$ \\
\hline 24 & 28 & $2 s^{2} 2 p^{3}\left({ }_{3}^{3} D\right)^{2} D 3 p^{3} F_{2}$ & & 5755365 & 3.039E-10 & 3.032E-10 & $0.39+0.402 s^{2} 2 p^{3}\left({ }_{3}^{2} D\right)^{2} D 3 p^{3} D+0.102 s^{2} 2 p^{3}\left({ }_{1}^{2} P\right)^{2} P 3 p^{3} D$ \\
\hline 24 & 29 & $2 s^{2} 2 p^{3}\left({ }_{3}^{3} D\right)^{2} D 3 p^{3} D_{2}$ & & 5770215 & $1.218 \mathrm{E}-10$ & $1.223 \mathrm{E}-10$ & $0.46+0.402 s^{2} 2 p^{3}\left(\frac{2}{3} D\right)^{2} D 3 p^{3} F+0.072 s^{2} 2 p^{3}\left({ }_{1}^{2} P\right)^{2} P 3 p^{1} D$ \\
\hline 24 & 30 & $2 s^{2} 2 p^{3}\left(\frac{2}{3} D\right)^{2} D 3 p^{3} F_{3}$ & & 5770968 & $3.220 \mathrm{E}-10$ & $3.211 \mathrm{E}-10$ & $0.59+0.282 s^{2} 2 p^{3}\left({ }_{3}^{2} D\right)^{2} D 3 p^{3} D+0.092 s^{2} 2 p^{3}\left({ }_{1}^{2} P\right)^{2} P 3 p^{3} D$ \\
\hline 24 & 31 & $2 s^{2} 2 p^{3}\left(\frac{2}{3} D\right)^{2} D 3 p^{1} P_{1}$ & & 5777373 & $9.461 \mathrm{E}-11$ & $9.548 \mathrm{E}-11$ & $0.47+0.372 s^{2} 2 p^{3}\left(\frac{3}{3} D\right)^{2} D 3 p^{3} D+0.072 s^{2} 2 p^{3}\left({ }_{1}^{2} P\right)^{2} P 3 p^{3} D$ \\
\hline 24 & 32 & $2 s^{2} 2 p^{3}\left(\frac{2}{3} D\right)^{2} D 3 p^{3} D_{3}$ & & 5782646 & 8.674E-11 & $8.699 \mathrm{E}-11$ & $0.50+0.302 s^{2} 2 p^{3}\left(\frac{2}{3} D\right)^{2} D 3 p^{1} F+0.102 s^{2} 2 p^{3}\left(\frac{1}{3} D\right)^{2} D 3 p^{3} F$ \\
\hline 24 & 33 & $2 s^{2} 2 p^{3}\left(\frac{2}{3} D\right)^{2} D 3 p^{1} F_{3}$ & & 5794949 & $2.584 \mathrm{E}-10$ & 2.583E-10 & $0.58+0.202 s^{2} 2 p^{3}\left({ }_{3}^{2} D\right)^{2} D 3 p^{3} D+0.202 s^{2} 2 p^{3}\left({ }_{3}^{2} D\right)^{2} D 3 p^{3} F$ \\
\hline 24 & 34 & $2 s^{2} 2 p^{3}\left(\frac{3}{3} D\right)^{2} D 3 p^{3} F_{4}$ & & 5798254 & $2.480 \mathrm{E}-10$ & $2.477 \mathrm{E}-10$ & 0.99 \\
\hline 24 & 35 & $\left.2 s^{2} 2 p^{3}\left(\frac{3}{3} D\right)\right)^{2} D 3 p^{3} P_{0}$ & & 5827777 & $1.901 \mathrm{E}-11$ & $1.907 \mathrm{E}-11$ & $0.72+0.222 s^{2} 2 p^{3}\left({ }_{1}^{2} P\right){ }^{2} P 3 p^{3} P+0.042 s^{2} 2 p^{3}\left({ }_{1}^{2} P\right)^{2} P 3 p^{1} S$ \\
\hline 24 & 36 & $2 s^{2} 2 p^{3}\left({ }_{3}^{3} D\right)^{2} D 3 p^{3} P_{1}$ & & 5835559 & $1.776 \mathrm{E}-11$ & 1.783E-11 & $0.39+0.232 s^{2} 2 p^{3}\left({ }_{1}^{2} P\right)^{2} P 3 p^{3} S+0.132 s^{2} 2 p^{3}\left({ }_{3}^{4} S\right)^{4} S 3 p^{3} P$ \\
\hline 24 & 37 & $2 s^{2} 2 p^{3}\left({ }_{3}^{3} D\right)^{2} D 3 p^{3} P_{2}$ & & 5850982 & $1.099 \mathrm{E}-11$ & $1.102 \mathrm{E}-11$ & $0.73+0.132 s^{2} 2 p^{3}\left({ }_{3}^{4} S\right)^{4} S 3 p^{3} P+0.062 s^{2} 2 p^{3}\left({ }_{1}^{2} P\right)^{2} P 3 p^{3} P$ \\
\hline 24 & 38 & $2 s^{2} 2 p^{3}\left({ }_{1}^{2} P\right)^{2} P 3 p^{3} D_{1}$ & & 5860955 & 4.457E-11 & $4.460 \mathrm{E}-11$ & $0.67+0.182 s^{2} 2 p^{3}\left({ }_{1}^{2} P\right)^{2} P 3 p^{1} P+0.062 s^{2} 2 p^{3}\left({ }_{3}^{2} D\right)^{2} D 3 p^{1} P$ \\
\hline 24 & 39 & $2 s^{2} 2 p^{3}\left(\frac{2}{3} D\right)^{2} D 3 p^{1} D_{2}$ & & 5874467 & 4.028E-11 & 4.035E-11 & $\left.0.45+0.152 s^{2} 2 p^{3}\left({ }_{1}^{2} P\right)^{2} P 3 p^{3} D+0.122 s^{2} 2 p^{3}{ }_{1}^{2} P\right)^{2} P 3 p^{3} P$ \\
\hline 24 & 40 & $2 s^{2} 2 p^{3}\left({ }_{1}^{2} P\right){ }^{2} P 3 p^{3} S_{1}$ & & 5881500 & $2.586 \mathrm{E}-11$ & $2.595 \mathrm{E}-11$ & $0.45+0.212 s^{2} 2 p^{3}\left({ }_{1}^{2} P\right)^{2} P 3 p^{3} P+0.112 s^{2} 2 p^{3}\left({ }_{3}^{2} D\right)^{2} D 3 p^{3} P$ \\
\hline 24 & 41 & $2 s^{2} 2 p^{3}\left({ }_{1}^{2} P\right)^{2} P 3 p^{3} D_{2}$ & & 5881733 & $5.398 \mathrm{E}-11$ & $5.398 \mathrm{E}-11$ & $\left.0.65+0.152 s^{2} 2 p^{3}\left({ }_{1}^{2} P\right)^{2} P 3 p^{3} P+0.082 s^{2} 2 p^{3}{ }_{1}^{2} P\right)^{2} P 3 p^{1} D$ \\
\hline 24 & 42 & $2 s^{2} 2 p^{3}\left({ }_{3}^{4} S\right)^{4} S 3 d^{5} D_{3}^{\circ}$ & & 5891086 & 4.593E-11 & 4.624E-11 & $\left.0.91+0.032 s^{2} 2 p^{3}\left({ }_{1}^{2} P\right)^{2} P 3 d^{3} D^{\circ}+0.022 s^{2} 2 p^{3}{ }_{1}^{2} P\right)^{2} P 3 d^{3} F^{\circ}$ \\
\hline 24 & 43 & $2 s^{2} 2 p^{3}\left({ }_{3}^{4} S\right)^{4} S 3 d^{5} D_{2}^{\circ}$ & & 5891283 & $8.384 \mathrm{E}-12$ & $8.355 \mathrm{E}-12$ & $0.92+0.032 s^{2} 2 p^{3}\left({ }_{1}^{2} P\right)^{2} P 3 d^{3} D^{\circ}$ \\
\hline 24 & 44 & $2 s^{2} 2 p^{3}\left({ }_{3}^{4} S\right)^{4} S 3 d^{5} D_{0}^{\circ}$ & & 5891333 & $1.092 \mathrm{E}-11$ & $1.086 \mathrm{E}-11$ & $\left.0.94+0.052 s^{2} 2 p^{3}{ }_{1}^{2} P\right)^{2} P 3 d^{3} P^{\circ}$ \\
\hline 24 & 45 & $2 s^{2} 2 p^{3}\left({ }_{3}^{4} S\right)^{4} S 3 d^{5} D_{1}^{\circ}$ & & 5891477 & $6.714 \mathrm{E}-12$ & $6.684 \mathrm{E}-12$ & $0.94+0.032 s^{2} 2 p^{3}\left(\left(_{1}^{2} P\right)^{2} P 3 d^{3} P^{\circ}\right.$ \\
\hline 24 & 46 & $2 s^{2} 2 p^{3}\left({ }_{3}^{4} S\right)^{4} S 3 d^{5} D_{4}^{\circ}$ & & 5892882 & 2.443E-10 & $2.436 \mathrm{E}-10$ & $0.93+0.052 s^{2} 2 p^{3}\left({ }_{1}^{2} P\right)^{2} P 3 d^{3} F^{\circ}$ \\
\hline 24 & 47 & $2 s^{2} 2 p^{3}\left({ }_{1}^{3} P\right)^{2} P 3 p^{3} D_{3}^{4}$ & & 5907865 & 5.913E-11 & $5.910 \mathrm{E}-11$ & $0.74+0.112 s^{2} 2 p^{3}\left(\frac{2}{3} D\right)^{2} D 3 p^{3} F+0.102 s^{2} 2 p^{3}\left({ }_{3}^{2} D\right)^{2} D 3 p^{1} F$ \\
\hline 24 & 48 & $2 s^{2} 2 p^{3}\left({ }_{1}^{2} P\right){ }^{2} P 3 p^{1} P_{1}$ & & 5909389 & 1.753E-11 & $1.760 \mathrm{E}-11$ & $0.32+0.302 s^{2} 2 p^{3}\left({ }_{3}^{2} D\right)^{2} D 3 p^{3} P+0.122 s^{2} 2 p^{3}\left({ }_{1}^{2} P\right)^{2} P 3 p^{3} S$ \\
\hline 24 & 49 & $2 s^{2} 2 p^{3}\left({ }_{1}^{2} P\right)^{2} P 3 p^{3} P_{0}$ & & 5918962 & $2.080 \mathrm{E}-11$ & $2.086 \mathrm{E}-11$ & $0.66+0.102 s^{2} 2 p^{3}\left({ }_{1}^{2} P\right)^{2} P 3 p^{1} S+0.102 s^{2} 2 p^{3}\left({ }_{3}^{2} D\right)^{2} D 3 p^{3} P$ \\
\hline 24 & 50 & $2 s^{2} 2 p^{3}\left({ }_{1}^{2} P\right)^{2} P 3 p^{3} P_{1}$ & & 5932333 & 3.815E-11 & $3.825 \mathrm{E}-11$ & $0.48+0.182 s^{2} 2 p^{3}\left({ }_{1}^{2} P\right)^{2} P 3 p^{1} P+0.092 s^{2} 2 p^{3}\left({ }_{3}^{4} S\right)^{4} S 3 p^{3} P$ \\
\hline 24 & 51 & $2 s^{2} 2 p^{3}\left({ }_{1}^{2} P\right)^{2} P 3 p^{3} P_{2}$ & & 5936634 & 7.431E-11 & 7.445E-11 & $0.44+0.302 s^{2} 2 p^{3}\left({ }_{1}^{2} P\right)^{2} P 3 p^{1} D+0.072 s^{2} 2 p^{3}\left(\frac{2}{3} D\right)^{2} D 3 p^{3} D$ \\
\hline 24 & 52 & $2 s^{2} 2 p^{3}\left({ }_{3}^{4} S\right)^{4} S 3 d^{3} D_{2}^{\circ}$ & & 5937322 & 4.053E-13 & $4.048 \mathrm{E}-13$ & $\left.0.66+0.132 s^{2} 2 p^{3}\left({ }_{3}^{2} D\right)^{2} D 3 d^{3} D^{\circ}+0.072 s^{2} 2 p^{3}{ }_{1}^{2} P\right)^{2} P 3 d^{1} D^{\circ}$ \\
\hline 24 & 53 & $2 s^{2} 2 p^{3}\left({ }_{3}^{4} S\right)^{4} S 3 d^{3} D_{1}^{\circ}$ & & 5951536 & $2.711 \mathrm{E}-13$ & $2.708 \mathrm{E}-13$ & $\left.0.83+0.072 s^{2} 2 p^{3}\left({ }_{3}^{2} D\right)^{2} D 3 d^{3} D^{\circ}+0.052 s^{2} 2 p^{3}{ }_{1}^{2} P\right)^{2} P 3 d^{3} D^{\circ}$ \\
\hline 24 & 54 & $2 s^{2} 2 p^{3}\left({ }_{3}^{4} S\right)^{4} S 3 d^{3} D_{3}^{\circ}$ & 5948500 & 5952597 & $2.684 \mathrm{E}-13$ & $2.678 \mathrm{E}-13$ & $0.76+0.122 s^{2} 2 p^{3}\left({ }_{3}^{2} D\right)^{2} D 3 d^{3} D^{\circ}+0.042 s^{2} 2 p^{3}\left({ }_{3}^{4} S\right)^{4} S 3 d^{5} D^{\circ}$ \\
\hline 24 & 55 & $2 s^{2} 2 p^{3}\left({ }_{1}^{2} P\right)^{2} P 3 p^{1} D_{2}$ & & 5962225 & $6.563 \mathrm{E}-11$ & $6.596 \mathrm{E}-11$ & $0.37+0.412 s^{2} 2 p^{3}\left(\frac{2}{3} D\right)^{2} D 3 p^{1} D+0.112 s^{2} 2 p^{3}\left({ }_{1}^{2} P\right)^{2} P 3 p^{3} P$ \\
\hline 24 & 56 & $2 s^{2} 2 p^{3}\left({ }_{3}^{2} D\right)^{2} D 3 d^{3} F_{2}^{\circ}$ & & 6015985 & $1.352 \mathrm{E}-12$ & $1.348 \mathrm{E}-12$ & $0.71+0.112 s^{2} 2 p^{3}\left({ }_{1}^{2} P\right){ }^{2} P 3 d^{3} F^{\circ}+0.072 s^{2} 2 p^{3}\left({ }_{3}^{4} S\right)^{4} S 3 d^{3} D^{\circ}$ \\
\hline 24 & 57 & $2 s^{2} 2 p^{3}\left(\frac{2}{3} D\right)^{2} D 3 d^{3} F_{3}^{\circ}$ & & 6025408 & $1.047 \mathrm{E}-12$ & $1.046 \mathrm{E}-12$ & $0.69+0.112 s^{2} 2 p^{3}\left({ }_{3}^{2} D\right)^{2} D 3 d^{3} G^{\circ}+0.102 s^{2} 2 p^{3}\left({ }_{1}^{2} P\right)^{2} P 3 d^{3} F^{\circ}$ \\
\hline 24 & 58 & $2 s^{2} 2 p^{3}\left(\frac{2}{3} D\right)^{2} D 3 d^{1} S_{0}^{0}$ & & 6028317 & $8.681 \mathrm{E}-11$ & $8.792 \mathrm{E}-11$ & $0.86+0.092 s^{2} 2 p^{3}\left({ }_{1}^{2} P\right)^{2} P 3 d^{3} P^{\circ}+0.022 s^{2} 2 p^{3}\left({ }_{3}^{2} D\right)^{2} D 3 d^{3} P^{\circ}$ \\
\hline 24 & 59 & $2 s^{2} 2 p^{3}\left(\frac{2}{3} D\right)^{2} D 3 d^{3} G_{4}^{\circ}$ & & 6033836 & 2.419E-10 & $2.409 \mathrm{E}-10$ & $0.50+0.192 s^{2} 2 p^{3}\left({ }_{3}^{2} D\right)^{2} D 3 d^{1} G^{\circ}+0.172 s^{2} 2 p^{3}\left({ }_{1}^{2} P\right)^{2} P 3 d^{3} F^{\circ}$ \\
\hline
\end{tabular}




\begin{tabular}{|c|c|c|c|c|c|c|c|}
\hline $\mathrm{z}$ & Key & Level & $E_{\text {NIST }}$ & $E_{\mathrm{MCDHF} / \mathrm{RCI}}$ & $\tau_{\mathrm{MCDHF} / \mathrm{RCI}}^{l}$ & $\tau_{\mathrm{MCDHF} / \mathrm{RCI}}^{v}$ & $L S$-composition \\
\hline 24 & 60 & $2 s^{2} 2 p^{3}\left({ }_{3}^{2} D\right)^{2} D 3 d^{3} G_{3}^{\circ}$ & \multirow{8}{*}{6070000} & 6035566 & $1.682 \mathrm{E}-12$ & $1.680 \mathrm{E}-12$ & $0.67+0.142 s^{2} 2 p^{3}\left({ }_{3}^{2} D\right){ }^{2} D 3 d^{3} F^{\circ}+0.082 s^{2} 2 p^{3}\left({ }_{1}^{2} P\right)^{2} P 3 d^{1} F^{\circ}$ \\
\hline 24 & 61 & $2 s^{2} 2 p^{3}\left(\frac{2}{3} D\right)^{2} D 3 d^{3} D_{1}^{\circ}$ & & 6041977 & $2.106 \mathrm{E}-13$ & $2.101 \mathrm{E}-13$ & $0.44+0.302 s^{2} 2 p^{3}\left({ }_{3}^{2} D\right)^{2} D 3 d^{1} P^{\circ}+0.092 s^{2} 2 p^{3}\left({ }_{1}^{2} P\right)^{2} P 3 d^{3} D^{\circ}$ \\
\hline 24 & 62 & $2 s^{2} 2 p^{3}\left({ }_{1}^{2} P\right)^{2} P 3 p^{1} S_{0}$ & & 6046913 & $8.869 \mathrm{E}-12$ & $8.889 \mathrm{E}-12$ & $0.82+0.082 s^{2} 2 p^{3}\left({ }_{3}^{2} D\right)^{2} D 3 p^{3} P+0.042 s^{2} 2 p^{3}\left({ }_{3}^{4} S\right)^{4} S 3 p^{3} P$ \\
\hline 24 & 63 & $2 s^{2} 2 p^{3}\left({ }_{2}^{2} D\right)^{2} D 3 d^{3} F_{4}^{\circ}$ & & 6047810 & $2.321 \mathrm{E}-10$ & $2.318 \mathrm{E}-10$ & $0.88+0.062 s^{2} 2 p^{3}\left({ }_{2}^{2} D\right)^{2} D 3 d^{3} G^{\circ}+0.042 s^{2} 2 p^{3}\left({ }_{3}^{2} D\right)^{2} D 3 d^{1} G^{\circ}$ \\
\hline 24 & 64 & $2 s^{2} 2 p^{3}\left(\frac{3}{2} D\right)^{2} D 3 d^{3} G_{5}^{\circ}$ & & 6057080 & 2.325E-10 & $2.316 \mathrm{E}-10$ & 1.00 \\
\hline 24 & 65 & $2 s^{2} 2 p^{3}\left(\frac{2}{3} D\right)^{2} D 3 d^{1} G_{4}^{\mathrm{o}}$ & & 6058514 & $2.109 \mathrm{E}-10$ & $2.101 \mathrm{E}-10$ & $0.67+0.322 s^{2} 2 p^{3}\left({ }_{3}^{2} D\right)^{2} D 3 d^{3} G^{\circ}$ \\
\hline 24 & 66 & $2 s^{2} 2 p^{3}\left({ }_{3}^{2} D\right)^{2} D 3 d^{3} P_{2}^{\circ}$ & & 6069651 & $1.128 \mathrm{E}-13$ & $1.127 \mathrm{E}-13$ & $0.26+0.352 s^{2} 2 p^{3}\left({ }_{3}^{2} D\right)^{2} D 3 d^{3} D^{\circ}+0.142 s^{2} 2 p^{3}\left({ }_{1}^{2} P\right)^{2} P 3 d^{3} P^{\circ}$ \\
\hline 24 & 67 & $2 s^{2} 2 p^{3}\left({ }_{3}^{2} D\right)^{2} D 3 d^{1} P_{1}^{\circ}$ & & 6076787 & $2.040 \mathrm{E}-13$ & $2.038 \mathrm{E}-13$ & $0.46+0.322 s^{2} 2 p^{3}\left({ }_{3}^{2} D\right)^{2} D 3 d^{3} D^{\circ}+0.092 s^{2} 2 p^{3}\left({ }_{1}^{2} P\right)^{2} P 3 d^{3} P^{\circ}$ \\
\hline 24 & 68 & $2 s^{2} 2 p^{3}\left({ }_{3}^{2} D\right)^{2} D 3 d^{3} D_{3}^{\circ}$ & \multirow[t]{7}{*}{6074000} & 6081809 & $8.220 \mathrm{E}-14$ & $8.206 \mathrm{E}-14$ & $0.77+0.132 s^{2} 2 p^{3}\left({ }_{3}^{2} S\right)^{4} S 3 d^{3} D^{\circ}+0.032 s^{2} 2 p^{3}\left({ }_{3}^{2} D\right)^{2} D 3 d^{3} G^{\circ}$ \\
\hline 24 & 69 & $2 s^{2} 2 p^{3}\left(\frac{2}{3} D\right)^{2} D 3 d^{3} D_{2}^{\circ}$ & & 6088452 & $8.734 \mathrm{E}-14$ & $8.718 \mathrm{E}-14$ & $0.35+0.312 s^{2} 2 p^{3}\left(\frac{2}{3} D\right)^{2} D 3 d^{3} P^{\circ}+0.122 s^{2} 2 p^{3}\left({ }_{3}^{2} D\right)^{2} D 3 d^{1} D^{\circ}$ \\
\hline 24 & 70 & $2 s^{2} 2 p^{3}\left({ }_{2}^{3} D\right)^{2} D 3 d^{3} P_{0}^{\circ}$ & & 6089261 & $2.002 \mathrm{E}-13$ & $1.999 \mathrm{E}-13$ & $0.78+0.122 s^{2} 2 p^{3}\left({ }_{1}^{2} P\right)^{2} P 3 d^{3} P^{\circ}+0.072 s^{2} 2 p^{3}\left({ }_{2}^{2} D\right)^{2} D 3 d^{1} S^{\circ}$ \\
\hline 24 & 71 & $2 s^{2} 2 p^{3}\left({ }_{3}^{2} D\right)^{2} D 3 d^{3} P_{1}^{\circ}$ & & 6092193 & $1.280 \mathrm{E}-13$ & $1.278 \mathrm{E}-13$ & $0.63+0.112 s^{2} 2 p^{3}\left({ }_{3}^{2} D\right)^{2} D 3 d^{3} s^{\circ}+0.072 s^{2} 2 p^{3}\left({ }_{1}^{2} P\right)^{2} P 3 d^{3} P^{\circ}$ \\
\hline 24 & 72 & $2 s^{2} S 2 p^{4}\left({ }_{2}^{3} P\right){ }^{4} P 3 s^{5} P_{3}$ & & 6094220 & $3.450 \mathrm{E}-11$ & $3.446 \mathrm{E}-11$ & 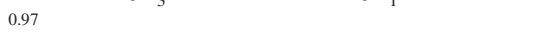 \\
\hline 24 & 73 & $2 s^{2} 2 p^{3}\left({ }_{3}^{2} D\right)^{2} D 3 d^{1} D_{2}^{\circ}$ & & 6097681 & 2.243E-13 & $2.241 \mathrm{E}-13$ & $0.37+0.242 s^{2} 2 p^{3}\left({ }_{1}^{2} P\right)^{2} P 3 d^{1} D^{\circ}+0.232 s^{2} 2 p^{3}\left({ }_{3}^{2} D\right)^{2} D 3 d^{3} P^{\circ}$ \\
\hline 24 & 74 & $2 s^{2} 2 p^{3}\left({ }_{2}^{3} D\right)^{2} D 3 d^{3} s_{1}^{2}$ & & 6108245 & $7.526 \mathrm{E}-14$ & $7.514 \mathrm{E}-14$ & $0.61+0.252 s^{2} 2 p^{3}\left({ }_{3}^{2} D\right)^{2} D 3 d^{3} P^{\circ}+0.062 s^{2} 2 p^{3}\left({ }_{3}^{2} D\right)^{2} D 3 d^{1} P^{\circ}$ \\
\hline 24 & 75 & $2 s^{2} 2 p^{3}\left({ }_{3}^{2} D\right)^{2} D 3 d^{1} F_{3}^{\circ}$ & \multirow[t]{5}{*}{6124400} & 6128387 & $1.131 \mathrm{E}-13$ & $1.129 \mathrm{E}-13$ & $0.68+0.132 s^{2} 2 p^{3}\left({ }_{1}^{2} P\right)^{2} P 3 d^{3} D^{\circ}+0.042 s^{2} 2 p^{3}\left({ }_{3}^{2} D\right)^{2} D 3 d^{3} G^{\circ}$ \\
\hline 24 & 76 & $2 s^{2} S 2 p^{4}\left({ }_{2}^{3} P\right){ }^{4} P 3 s^{5} P_{2}$ & & 6130614 & $4.839 \mathrm{E}-12$ & 4.844E-12 & $0.87+0.102 s^{2} S 2 p^{4}\left({ }_{2}^{3} P\right)^{4} P 3 s^{3} P$ \\
\hline 24 & 77 & $2 s^{2} 2 p^{3}\left({ }_{1}^{2} P\right)^{2} P 3 d^{3} F_{2}^{\circ}$ & & 6141553 & $6.430 \mathrm{E}-13$ & $6.418 \mathrm{E}-13$ & $0.68+0.102 s^{2} 2 p^{3}\left({ }_{3}^{2} D\right)^{2} D 3 d^{3} F^{\circ}+0.072 s^{2} 2 p^{3}\left({ }_{1}^{2} P\right)^{2} P 3 d^{3} D^{\circ}$ \\
\hline 24 & 78 & $2 s^{2} 2 p^{3}\left({ }_{1}^{2} P\right)^{2} P 3 d^{3} F_{3}^{\circ}$ & & 6145084 & $1.095 \mathrm{E}-12$ & $1.094 \mathrm{E}-12$ & $0.66+0.202 s^{2} 2 p^{3}\left({ }_{1}^{2} P\right)^{2} P 3 d^{3} D^{\circ}+0.052 s^{2} 2 p^{3}\left({ }_{3}^{2} D\right)^{2} D 3 d^{3} G^{\circ}$ \\
\hline 24 & 79 & $2 s^{2} S 2 p^{4}\left({ }_{2}^{3} P\right){ }^{4} P 3 s^{5} P_{1}$ & & 6154222 & $1.732 \mathrm{E}-11$ & $1.739 \mathrm{E}-11$ & 0.96 \\
\hline 24 & 80 & $2 s^{2} 2 p^{3}\left({ }_{1}^{2} P\right)^{2} P 3 d^{3} P_{2}^{\circ}$ & 6131000 & 6159132 & $1.263 \mathrm{E}-13$ & $1.261 \mathrm{E}-13$ & $0.35+0.322 s^{2} 2 p^{3}\left({ }_{1}^{2} P\right)^{2} P 3 d^{3} D^{\circ}+0.142 s^{2} 2 p^{3}\left({ }_{3}^{2} D\right)^{2} D 3 d^{1} D^{\circ}$ \\
\hline 24 & 81 & $2 s^{2} 2 p^{3}\left({ }_{1}^{2} P\right)^{2} P 3 d^{3} F_{4}^{\circ}$ & & 6165268 & $2.271 \mathrm{E}-10$ & $2.269 \mathrm{E}-10$ & $0.74+0.112 s^{2} 2 p^{3}\left(\frac{1}{2} D\right)^{2} D 3 d^{3} G^{\circ}+0.102 s^{2} 2 p^{3}\left({ }_{3}^{2} D\right)^{2} D 3 d^{1} G^{\circ}$ \\
\hline 24 & 82 & $2 s^{2} 2 p^{3}\left({ }_{1}^{2} P\right)^{2} P 3 d^{3} P_{0}^{\circ}$ & & 6169459 & $1.068 \mathrm{E}-13$ & $1.066 \mathrm{E}-13$ & $0.71+0.182 s^{2} 2 p^{3}\left({ }_{3}^{2} D\right){ }^{2} D 3 d^{3} P^{\circ}+0.052 s^{2} 2 p^{3}\left({ }_{3}^{2} D\right)^{2} D 3 d^{1} S^{\circ}$ \\
\hline 24 & 83 & $2 s^{2} 2 p^{3}\left({ }_{1}^{2} P\right)^{2} P 3 d^{3} P_{1}^{\mathrm{o}}$ & & 6169969 & $1.098 \mathrm{E}-13$ & $1.096 \mathrm{E}-13$ & $0.51+0.192 s^{2} 2 p^{3}\left(_{1}^{2} P\right)^{2} P 3 d^{3} D^{\circ}+0.132 s^{2} 2 p^{3}\left(\frac{2}{2} D\right)^{2} D 3 d^{3} S^{\circ}$ \\
\hline 24 & 84 & $2 s^{2} S 2 p^{4}\left({ }_{2}^{3} P\right){ }^{4} P 3 s^{3} P_{2}$ & & 6177775 & 7.421E-13 & 7.419E-13 & $0.84+0.112 s^{2} S 2 p^{4}\left({ }_{2}^{3} P\right)^{4} P 3 s^{5} P$ \\
\hline 24 & 85 & $2 s^{2} 2 p^{3}\left({ }_{1}^{2} P\right)^{2} P 3 d^{1} D_{2}^{\circ}$ & & 6180203 & $3.200 \mathrm{E}-13$ & $3.195 \mathrm{E}-13$ & $0.25+0.282 s^{2} 2 p^{3}\left({ }_{1}^{2} P\right)^{2} P 3 d^{3} P^{\circ}+0.172 s^{2} 2 p^{3}\left({ }_{3}^{2} D\right)^{2} D 3 d^{3} P^{\circ}$ \\
\hline 24 & 86 & $2 s^{2} 2 p^{3}\left({ }_{1}^{2} P\right)^{2} P 3 d^{3} D_{1}^{\circ}$ & 6189000 & 6186885 & $7.950 \mathrm{E}-14$ & 7.934E-14 & $\left.0.56+0.142 s^{2} 2 p^{3}\left({ }_{1}^{2} P\right)^{2} P 3 d^{3} P^{\circ}+0.082 s^{2} 2 p^{3}{ }_{1}^{2} P\right)^{2} P 3 d^{1} P^{\circ}$ \\
\hline 24 & 87 & $2 s^{2} 2 p^{3}\left({ }_{1}^{2} P\right)^{2} P 3 d^{3} D_{3}^{\circ}$ & 6164800 & 6198298 & $9.695 \mathrm{E}-13$ & $9.682 \mathrm{E}-13$ & $0.44+0.252 s^{2} 2 p^{3}\left({ }_{1}^{2} P\right)^{2} P 3 d^{1} F^{\circ}+0.102 s^{2} 2 p^{3}\left({ }_{3}^{2} D\right)^{2} D 3 d^{1} F^{\circ}$ \\
\hline 24 & 88 & $2 s^{2} 2 p^{3}\left({ }_{1}^{2} P\right)^{2} P 3 d{ }^{1} F_{3}^{\circ}$ & & 6216401 & $5.824 \mathrm{E}-14$ & $5.813 \mathrm{E}-14$ & $\left.0.53+0.202 s^{2} 2 p^{3}\left(\frac{2}{3} D\right)^{2} D 3 d^{1} F^{\circ}+0.102 s^{2} 2 p^{3}{ }_{1}^{3} P\right)^{2} P 3 d^{3} D^{\circ}$ \\
\hline 24 & 89 & $2 s^{2} S 2 p^{4}\left({ }_{2}^{3} P\right){ }^{4} P 3 s^{3^{3}} P_{1}$ & & 6216774 & $6.192 \mathrm{E}-13$ & $6.190 \mathrm{E}-13$ & $0.92+32^{3}+1$ \\
\hline 24 & 90 & $2 s^{2} 2 p^{3}\left({ }_{1}^{2} P\right)^{2} P 3 d^{3} D_{2}^{\circ}$ & 6214600 & 6217760 & 8.993E-14 & 8.977E-14 & $0.28+0.272 s^{2} 2 p^{3}\left({ }_{3}^{2} D\right){ }^{2} D 3 d^{1} D^{\circ}+0.242 s^{2} 2 p^{3}\left({ }_{1}^{2} P\right)^{2} P 3 d^{1} D^{\circ}$ \\
\hline 24 & 91 & $2 s^{2} S 2 p^{4}\left({ }_{2}^{3} P\right){ }^{4} P 3 s^{3} P_{0}$ & & 6231850 & $6.293 \mathrm{E}-13$ & $6.290 \mathrm{E}-13$ & $0.93+0.032 s^{2} S 2 p^{\frac{3}{4}}\left(\frac{1}{0} S\right)^{2} S 3 s^{1} S$ \\
\hline 24 & 92 & $2 s^{2} 2 p^{3}\left({ }_{1}^{2} P\right)^{2} P 3 d^{1} P_{1}^{\circ}$ & & 6268880 & $5.608 \mathrm{E}-14$ & $5.598 \mathrm{E}-14$ & $0.75+0.052 s^{2} 2 p^{3}\left({ }_{1}^{2} P\right)^{2} P 3 d^{3} D^{\circ}+0.042 s^{2} 2 p^{3}\left({ }_{3}^{2} D\right)^{2} D 3 d^{1} P^{\circ}$ \\
\hline 24 & 93 & $2 s^{2} S 2 p^{4}\left({ }_{2}^{3} P\right){ }^{4} P 3 p p^{5} P_{3}^{\circ}$ & & 6298653 & 2.934E-11 & $2.916 \mathrm{E}-11$ & $0.75+0.222 s^{2} S 2 p^{4}\left({ }_{2}^{3} P\right)^{4} P 3 p^{5} D^{\circ}$ \\
\hline 24 & 94 & $2 s^{2} S 2 p^{4}\left({ }_{2}^{3} P\right){ }^{4} P 3 p^{5} P_{2}^{\circ}$ & & 6301094 & $1.090 \mathrm{E}-11$ & $1.085 \mathrm{E}-11$ & $0.73+0.112 s^{2} S 2 p^{4}\left(_{2}^{3} P\right){ }^{4} P 3 p^{5} S^{\circ}+0.102 s^{2} S 2 p^{4}\left({ }_{2}^{3} P\right)^{4} P 3 p^{5} D^{\circ}$ \\
\hline 24 & 95 & $2 s^{2} S 2 p^{4}\left({ }_{2}^{2} P\right){ }^{4} P 3 p^{5} D_{4}^{\circ}$ & & 6324988 & 7.519E-11 & 7.429E-11 & $0.97-2+2+2+2$ \\
\hline 24 & 96 & $\left.2 s^{2} S 2 p^{4}{ }_{2}^{3} P\right)^{4} P 3 p^{5} P_{1}^{\circ}$ & & 6329174 & 6.803E-12 & $6.781 \mathrm{E}-12$ & $0.84+0.082 s^{2} S 2 p^{4}\left({ }_{2}^{3} P\right)^{4} P 3 p^{5} D^{\circ}+0.042 s^{2} S 2 p^{4}\left({ }_{2}^{3} P\right)^{4} P 3 p^{3} P^{\circ}$ \\
\hline 24 & 97 & $2 s^{2} S 2 p^{4}\left({ }_{2}^{3} P\right){ }^{4} P 3 p^{3} D_{3}^{\circ}$ & 6338000 & 6337541 & $7.980 \mathrm{E}-13$ & $7.956 \mathrm{E}-13$ & $0.55+0.282 s^{2} S 2 p^{4}\left({ }_{2}^{3} P\right){ }^{4} P 3 p^{5} D^{\circ}+0.142 s^{2} S 2 p^{4}\left({ }_{2}^{3} P\right)^{4} P 3 p^{5} P^{\circ}$ \\
\hline 24 & 98 & $\left.2 s^{2} S 2 p^{4}{ }_{2}^{2} P\right)^{4} P 3 p^{5} D_{2}^{\circ}$ & & 6358806 & $2.642 \mathrm{E}-12$ & $2.635 \mathrm{E}-12$ & $0.66+0.192 s^{2} S 2 p^{4}\left({ }_{2}^{3} P\right)^{4} P 3 p^{3} D^{\circ}+0.072 s^{2} S 2 p^{4}\left(_{2}^{\frac{3}{3}} P\right)^{4} P 3 p^{5} S^{\circ}$ \\
\hline 24 & 99 & $2 s^{2} S 2 p^{4}\left({ }_{2}^{4} D\right)^{2} D 3 s^{3} D_{1}^{2}$ & & 6361557 & $1.010 \mathrm{E}-12$ & $1.011 \mathrm{E}-12$ & 0.94 \\
\hline 24 & 100 & $2 s^{2} S 2 p^{4}\left(\frac{1}{2} D\right)^{2} D 3 s^{3} D_{2}$ & & 6363805 & $1.028 \mathrm{E}-12$ & $1.029 \mathrm{E}-12$ & 0.94 \\
\hline 24 & 101 & $2 s^{2} S 2 p^{4}\left(\frac{1}{1} D\right)^{2} D 3 s^{3} D_{3}$ & & 6368480 & $9.759 \mathrm{E}-13$ & $9.753 \mathrm{E}-13$ & 0.96 \\
\hline 24 & 102 & $2 s^{2} S 2 p^{4}\left({ }_{2}^{2} P\right){ }^{4} P 3 p p^{5} D_{1}^{\circ}$ & & 6369439 & $7.216 \mathrm{E}-12$ & $7.202 \mathrm{E}-12$ & $0.86+0.062 s^{2} S 2 p^{4}\left({ }_{2}^{3} P\right){ }^{4} P 3 p^{5} P^{\circ}+0.032 s^{2} S 2 p^{4}\left({ }_{2}^{3} P\right){ }^{4} P 3 p^{3} D^{\circ}$ \\
\hline 24 & 103 & $2 s^{2} S 2 p^{4}\left({ }_{2}^{3} P\right)^{4} P 3 p^{5} D_{0}^{\circ}$ & & 6371393 & 3.635E-11 & $3.635 \mathrm{E}-11$ & $0.96+0.022 s^{2} S 2 p^{4}\left({ }_{0}^{1} S\right)^{2} S 3 p^{3} p^{\circ}$ \\
\hline 24 & 104 & $2 s^{2} S 2 p^{4}\left({ }_{2}^{2} P\right){ }^{4} P 3 p^{5} D_{3}^{\circ}$ & & 6374363 & $8.767 \mathrm{E}-13$ & $8.738 \mathrm{E}-13$ & $0.48+0.412 s^{2} S 2 p^{4}\left({ }_{2}^{3} P\right)^{4} P 3 p^{3} D^{\circ}+0.092 s^{2} S 2 p^{4}\left({ }_{2}^{3} P\right)^{4} P 3 p^{5} P^{\circ}$ \\
\hline 24 & 105 & $2 s^{2} S 2 p^{4}\left({ }_{2}^{2} P\right){ }^{4} P 3 p^{3} S_{1}^{0}$ & & 6377598 & $3.461 \mathrm{E}-13$ & $3.451 \mathrm{E}-13$ & $0.60+0.232 s^{2} S 2 p^{4}\left({ }_{2}^{3} P\right)^{4} P 3 p^{3} P^{\circ}+0.072 s^{2} S 2 p^{4}\left(_{2}^{3} P\right)^{4} P 3 p^{5} P^{\circ}$ \\
\hline 24 & 106 & $2 s^{2} S 2 p^{4}\left({ }_{2}^{3} P\right)^{4} P 3 p^{3} D_{2}^{\circ}$ & & 6383465 & $4.466 \mathrm{E}-13$ & $4.452 \mathrm{E}-13$ & $0.38+0.382 s^{2} S 2 p^{4}\left({ }_{2}^{3} P\right){ }^{4} P 3 p^{3} P^{\circ}+0.162 s^{2} S 2 p^{4}\left({ }_{2}^{3} P\right){ }^{4} P 3 p^{5} D^{\circ}$ \\
\hline 24 & 107 & $2 s^{2} S 2 p^{4}\left({ }_{2}^{2} P\right){ }^{4} P 3 p^{5} S_{2}^{\circ}$ & & 6397373 & 1.713E-11 & $1.711 \mathrm{E}-11$ & $0.79+0.152 s^{2} S 2 p^{4}\left({ }_{2}^{\frac{2}{3}} P\right)^{4} P 3 p^{5} P^{\circ}$ \\
\hline 24 & 108 & $\left.2 s^{2} S 2 p^{4}{ }_{2}^{2} P\right)^{4} P 3 p^{3} D_{1}^{\circ}$ & & 6412950 & $4.321 \mathrm{E}-13$ & 4.308E-13 & $0.78+0.112 s^{2} S 2 p^{4}\left({ }_{2}^{3} P\right)^{4} P 3 p^{3} S^{\circ}+0.052 s^{2} S 2 p^{4}\left({ }_{2}^{3} P\right)^{4} P 3 p^{3} P^{\circ}$ \\
\hline 24 & 109 & $2 s^{2} s 2 p^{4}\left({ }_{2}^{1} D\right)^{2} D 3 s^{1} D_{2}$ & & 6414298 & $1.669 \mathrm{E}-12$ & $1.669 \mathrm{E}-12$ & $\left.0.94+0.022 s^{2} S 2 p^{4}{ }_{2}^{3} P\right)^{2} P 3 s^{3} P$ \\
\hline 24 & 110 & $2 s^{2} S 2 p^{4}\left({ }_{2}^{2} P\right){ }^{4} P 3 p^{3} P_{0}^{\circ}$ & & 6414786 & $4.666 \mathrm{E}-13$ & $4.651 \mathrm{E}-13$ & $0.78+0.182 s^{2} S 2 p^{4}\left({ }_{2}^{1} D\right)^{2} D 3 p^{3} P^{\circ}$ \\
\hline 24 & 111 & $2 s^{2} S 2 p^{4}\left({ }_{2}^{3} P\right)^{4} P 3 p^{3} P_{2}^{0}$ & & 6415470 & 4.213E-13 & $4.200 \mathrm{E}-13$ & $0.45+0.382 s^{2} S 2 p^{4}\left({ }_{2}^{2} P\right){ }^{4} P 3 p^{3} D^{\circ}+0.062 s^{2} S 2 p^{4}\left({ }_{2}^{1} D\right)^{2} D 3 p^{3} P^{\circ}$ \\
\hline 24 & 112 & $2 s^{2} S 2 p^{4}\left({ }_{2}^{2} P\right)^{4} P 3 p^{3} P_{1}^{\circ}$ & & 6429248 & $3.740 \mathrm{E}-13$ & $3.729 \mathrm{E}-13$ & $0.48+0.232 s^{2} S 2 p^{4}\left(_{2}^{3} P\right)^{4} P 3 p^{3} S^{\circ}+0.132 s^{2} S 2 p^{4}\left({ }_{2}^{3} P\right)^{4} P 3 p^{3} D^{\circ}$ \\
\hline 24 & 113 & $2 s^{2} S 2 p^{4}\left({ }_{0}^{2} S\right)^{2} S 3 s^{3} S_{1}$ & & 6502041 & $1.026 \mathrm{E}-12$ & $1.026 \mathrm{E}-12$ & $0.78+0.132 s^{2} S 2 p^{4}\left({ }_{2}^{3} P\right)^{2} P 3 s^{3} P+0.042 s^{2} S 2 p^{4}\left({ }_{2}^{3} P\right)^{2} P 3 s^{1} P$ \\
\hline 24 & 114 & $2 s^{2} S 2 p^{4}\left({ }_{0} S\right)^{2} S 3 s^{1} s_{0}$ & & 6533147 & $1.262 \mathrm{E}-12$ & $1.262 \mathrm{E}-12$ & $0.60+0.372 s^{2} S 2 p^{4}\left({ }_{2}^{3} P\right)^{2} P 3 s^{3} P$ \\
\hline 24 & 115 & $2 s^{2} S 2 p^{4}\left({ }_{2}^{3} P\right)^{2} P 3 s^{3} P_{2}$ & & 6544630 & 1.579E-12 & $1.578 \mathrm{E}-12$ & $0.94+0.022 s^{2} S 2 p^{4}\left({ }_{2}^{1} D\right)^{2} D 3 s^{1} D$ \\
\hline 24 & 116 & $2 s^{2} S 2 p^{4}\left({ }_{2}^{2} P\right){ }^{4} P 3 d^{5} D_{4}$ & & 6551130 & $7.000 \mathrm{E}-11$ & $6.986 \mathrm{E}-11$ & $0.82+0.172 s^{2} S 2 p^{4}\left({ }_{2}^{2} P\right)^{4} P 3 d^{5} F$ \\
\hline 24 & 117 & $2 s^{2} S 2 p^{4}\left({ }_{2}^{3} P\right){ }^{4} P 3 d^{5} D_{3}$ & & 6552096 & $6.400 \mathrm{E}-11$ & $6.417 \mathrm{E}-11$ & $0.81+0.092 s^{2} S 2 p^{4}\left({ }_{2}^{2} P\right)^{4} P 3 d^{5} F+0.072 s^{2} S 2 p^{4}\left({ }_{2}^{3} P\right)^{4} P 3 d^{5} P$ \\
\hline 24 & 118 & $2 s^{2} S 2 p^{4}\left({ }_{2}^{2} P\right)^{2} P 3 s^{1} P_{1}$ & & 6556026 & $6.040 \mathrm{E}-13$ & $6.042 \mathrm{E}-13$ & $0.53+0.412 s^{2} S 2 p^{4}\left({ }_{2}^{2} P\right)^{2} P 3 s^{3} P+0.032 s^{2} S 2 p^{4}\left({ }_{2}^{1} D\right)^{2} D 3 s^{3} D$ \\
\hline 24 & 119 & $2 s^{2} S 2 p^{4}\left({ }_{2}^{3} P\right){ }^{4} P 3 d^{5} D_{2}$ & & 6558099 & $5.130 \mathrm{E}-11$ & $5.132 \mathrm{E}-11$ & $0.79+0.142 s^{2} S 2 p^{4}\left(\int_{2}^{2} P\right)^{4} P 3 d^{5} P+0.052 s^{2} S 2 p^{4}\left({ }_{2}^{3} P\right){ }^{4} P 3 d^{5} F$ \\
\hline 24 & 120 & $2 s^{2} S 2 p^{4}\left({ }_{2}^{1} D\right)^{2} D 3 p^{3} F_{2}^{\circ}$ & & 6562499 & $7.231 \mathrm{E}-12$ & $7.214 \mathrm{E}-12$ & $0.93+2+2+2$ \\
\hline 24 & 121 & $2 s^{2} S 2 p^{4}\left({ }_{2}^{3} P\right)^{4} P 3 d^{5} D_{1}$ & & 6567306 & $2.491 \mathrm{E}-11$ & $2.493 \mathrm{E}-11$ & $0.76+0.182 s^{2} S 2 p^{4}\left({ }_{2}^{3} P\right)^{4} P 3 d^{5} P$ \\
\hline 24 & 122 & $2 s^{2} S 2 p^{4}\left({ }_{2}^{1} D\right)^{2} D 3 p^{1} P_{1}^{\circ}$ & & 6569219 & 3.644E-13 & 3.634E-13 & $0.58+0.212 s^{2} S 2 p^{4}\left({ }_{2}^{1} D\right)^{2} D 3 p^{3} D^{\circ}+0.072 s^{2} S 2 p^{4}\left({ }_{2}^{3} P\right)^{2} P 3 p^{1} P^{\circ}$ \\
\hline 24 & 123 & $2 s^{2} S 2 p^{4}\left({ }_{2}^{1} D\right)^{2} D 3 p^{3} F_{3}^{\circ}$ & & 6571449 & $5.415 \mathrm{E}-12$ & $5.402 \mathrm{E}-12$ & $0.92+0.032 s^{2} S 2 p^{4}\left({ }_{2}^{1} D\right)^{2} D 3 p^{1} F^{\circ}+0.022 s^{2} S 2 p^{4}\left({ }_{2}^{1} D\right)^{2} D 3 p^{3} D^{\circ}$ \\
\hline 24 & 124 & $2 s^{2} S 2 p^{4}\left({ }_{2}^{3} P\right){ }^{4} P 3 d^{5} F_{5}{ }^{3}$ & & 6573707 & $1.028 \mathrm{E}-10$ & $9.927 \mathrm{E}-11$ & $0.98+0.022 s^{2} S 2 p^{4}\left({ }_{2}^{1} D\right)^{2} D 3 d^{3} G$ \\
\hline 24 & 125 & $2 s^{2} S 2 p^{4}\left({ }_{2}^{3} P\right){ }^{4} P 3 d^{5} D_{0}$ & & 6578733 & $1.366 \mathrm{E}-11$ & $1.372 \mathrm{E}-11$ & $0.94+0.042 s^{2} S 2 p^{4}\left(_{2}^{3} P\right)^{4} P 3 d^{3} P$ \\
\hline 24 & 126 & $2 s^{2} S 2 p^{4}\left({ }_{2}^{\mathrm{T}} D\right)^{2} D 3 p^{3} F_{4}^{\circ}$ & & 6584882 & 2.997E-11 & 2.978E-11 & 0.97 \\
\hline 24 & 127 & $2 s^{2} S 2 p^{4}\left({ }_{2}^{1} D\right)^{2} D 3 p^{1} F_{3}^{\circ}$ & & 6592968 & $3.639 \mathrm{E}-13$ & 3.627E-13 & $0.80+0.122 s^{2} S 2 p^{4}\left({ }_{2}^{1} D\right)^{2} D 3 p^{3} D^{\circ}+0.042 s^{2} S 2 p^{4}\left({ }_{2}^{1} D\right)^{2} D 3 p^{3} F^{\circ}$ \\
\hline 24 & 128 & $2 s^{2} S 2 p^{4}\left(\frac{3}{2} P\right){ }^{4} P 3 d^{5} F_{4}$ & & 6597484 & 7.937E-11 & $7.778 \mathrm{E}-11$ & $0.63+0.242 s^{2} S 2 p^{4}\left(\int_{2}^{3} P\right)^{4} P 3 d^{3} F+0.112 s^{2} S 2 p^{4}\left({ }_{2}^{3} P\right)^{4} P 3 d^{5} D$ \\
\hline 24 & 129 & $2 s^{2} S 2 p^{4}\left(\frac{1}{2} D\right)^{2} D 3 p^{3} D_{2}^{\circ}$ & & 6602451 & $4.349 \mathrm{E}-12$ & 4.334E-12 & $0.87+0.042 s^{2} S 2 p^{4}\left(_{2}^{3} P\right)^{2} P 3 p^{3} P^{\circ}+0.032 s^{2} S 2 p^{4}\left({ }_{2}^{1} D\right)^{2} D 3 p^{3} P^{\circ}$ \\
\hline 24 & 130 & $2 s^{2} S 2 p^{4}\left({ }_{2}^{3} P\right){ }^{4} P 3 d^{5} P_{1}{ }^{2}$ & & 6607339 & $2.801 \mathrm{E}-12$ & 2.799E-12 & $0.79+0.152 s^{2} S 2 p^{4}\left({ }_{2}^{3} P\right){ }^{4} P 3 d^{5} D$ \\
\hline 24 & 131 & $2 s^{2} S 2 p^{4}\left({ }_{2}^{1} D\right)^{2} D 3 p^{3} D_{1}^{\circ}$ & & 6608689 & $1.146 \mathrm{E}-12$ & 1.143E-12 & $0.76+0.182 s^{2} S 2 p^{4}\left({ }_{2}^{1} D\right)^{2} D 3 p^{1} P^{\circ}+0.032 s^{2} S 2 p^{4}\left({ }_{2}^{1} D\right)^{2} D 3 p^{3} P^{\circ}$ \\
\hline 24 & 132 & $2 s^{2} S 2 p^{4}\left({ }_{2}^{3} P\right){ }^{4} P 3 d^{5} F_{3}$ & & 6613816 & $2.452 \mathrm{E}-12$ & $2.448 \mathrm{E}-12$ & $0.75+0.102 s^{2} S 2 p^{4}\left({ }_{2}^{3} P\right){ }^{4} P 3 d^{3} F+0.062 s^{2} S 2 p^{4}\left({ }_{2}^{3} P\right){ }^{4} P 3 d^{5} P$ \\
\hline 24 & 133 & $2 s^{2} S 2 p^{4}\left({ }_{2}^{1} D\right)^{2} D 3 p^{3} D_{3}^{\circ}$ & & 6613959 & 2.044E-12 & $2.037 \mathrm{E}-12$ & $0.83+0.132 s^{2} S 2 p^{4}\left({ }_{2}^{1} D\right)^{2} D 3 p^{1} F^{\circ}$ \\
\hline 24 & 134 & $2 s^{2} S 2 p^{4}\left({ }_{2}^{4} P\right)^{2} P 3 s^{3} P_{1}{ }^{3}$ & & 6615268 & $6.262 \mathrm{E}-13$ & $6.259 \mathrm{E}-13$ & $\left.\left.0.43+0.392 s^{2} S 2 p^{4}{ }_{2}^{2} P\right)^{2} P 3 s^{1} P+0.152 s^{2} S 2 p^{4}{ }_{0}^{1} S\right)^{2} S 3 s^{3} S$ \\
\hline 24 & 135 & $2 s^{2} S 2 p^{4}\left({ }_{2}^{3} P\right){ }^{4} P 3 d d^{5} P_{2}$ & & 6619467 & $1.802 \mathrm{E}-12$ & $1.801 \mathrm{E}-12$ & $\left.0.55+0.362 s^{2} S 2 p^{4}\left({ }_{2}^{3} P\right){ }^{4} P 3 d^{5} F+0.032 s^{2} S 2 p^{4}{ }_{2}^{3} P\right)^{4} P 3 d^{3} D$ \\
\hline 24 & 136 & $2 s^{2} S 2 p^{4}\left({ }_{2}^{1} D\right)^{2} D 3 p{ }^{1} D_{2}^{\circ}$ & & 6620487 & $4.677 \mathrm{E}-13$ & $4.661 \mathrm{E}-13$ & $0.54+0.342 s^{2} S 2 p^{4}\left({ }_{2}^{1} D\right)^{2} D 3 p^{3} P^{\circ}+0.062 s^{2} S 2 p^{4}\left({ }_{2}^{1} D\right)^{2} D 3 p^{3} D^{\circ}$ \\
\hline 24 & 137 & $2 s^{2} S 2 p^{4}\left({ }_{2}^{3} P\right)^{2} P 3 s^{3} P_{0}$ & & 6624301 & $1.666 \mathrm{E}-12$ & $1.665 \mathrm{E}-12$ & $0.60+0.362 s^{2} S 2 p^{4}\left({ }_{0}^{1} S\right)^{2} S 3 s^{1} S$ \\
\hline 24 & 138 & $2 s^{2} S 2 p^{4}\left({ }_{2}^{1} D\right)^{2} D 3 p^{3} P_{1}^{\circ}$ & & 6629157 & 7.717E-13 & $7.688 \mathrm{E}-13$ & $0.66+0.112 s^{2} S 2 p^{4}\left({ }_{2}^{3} P\right)^{4} P 3 p^{3} P^{\circ}+0.092 s^{2} S 2 p^{4}\left({ }_{2}^{3} P\right)^{2} P 3 p^{3} P^{\circ}$ \\
\hline 24 & 139 & $2 s^{2} S 2 p^{4}\left({ }_{2}^{3} P\right){ }^{4} P 3 d^{5} F_{2}$ & & 6629369 & $1.521 \mathrm{E}-11$ & $1.524 \mathrm{E}-11$ & $0.55+0.272 s^{2} S 2 p^{4}\left({ }_{2}^{3} P\right){ }^{4} P 3 d^{5} P+0.152 s^{2} S 2 p^{4}\left({ }_{2}^{2} P\right)^{4} P 3 d^{5} D$ \\
\hline 24 & 140 & $2 s^{2} S 2 p^{4}\left({ }_{2}^{1} D\right)^{2} D 3 p^{3} P_{2}^{\circ}$ & & 6630662 & 5.079E-13 & $5.062 \mathrm{E}-13$ & $\left.0.48+0.402 s^{2} S 2 p^{4}\left({ }_{2}^{1} D\right)^{2} D 3 p^{1} D^{\circ}+0.092 s^{2} S 2 p^{4}{ }_{2}^{3} P\right)^{4} P 3 p^{3} P^{\circ}$ \\
\hline 24 & 141 & $2 s^{2} S 2 p^{4}\left({ }_{2}^{2} P\right){ }^{4} P 3 d^{5} F_{1}^{2}$ & & 6631621 & $8.209 \mathrm{E}-12$ & $8.179 \mathrm{E}-12$ & $0.94+0.032 s^{2} S 2 p^{4}\left({ }_{2}^{3} P\right)^{4} P 3 d^{5} D$ \\
\hline 24 & 142 & $2 s^{2} S 2 p^{4}\left({ }_{2}^{1} D\right)^{2} D 3 p^{3} P_{0}^{\circ}$ & & 6632694 & $7.755 \mathrm{E}-13$ & $7.725 \mathrm{E}-13$ & $0.67+0.162 s^{2} S 2 p^{4}\left({ }_{2}^{3} P\right)^{4} P 3 p^{3} P^{\circ}+0.142 s^{2} S 2 p^{4}\left({ }_{2}^{3} P\right)^{2} P 3 p^{3} P^{\circ}$ \\
\hline 24 & 143 & $2 s^{2} S 2 p^{4}\left({ }_{2}^{3} P\right){ }^{4} P 3 d^{3} F_{4}$ & & 6632913 & $6.497 \mathrm{E}-11$ & $6.406 \mathrm{E}-11$ & $0.73+0.192 s^{2} S 2 p^{4}\left({ }_{2}^{3} P\right)^{4} P 3 d^{5} F+0.062 s^{2} S 2 p^{4}\left({ }_{2}^{3} P\right)^{4} P 3 d^{5} D$ \\
\hline
\end{tabular}




\begin{tabular}{|c|c|c|c|c|c|c|c|}
\hline $\mathrm{z}$ & Key & Level & $E_{\text {NIST }}$ & $E_{\mathrm{MCDHF} / \mathrm{RCI}}$ & $\tau_{\mathrm{MCDHF} / \mathrm{RCI}}^{l}$ & $\tau_{\mathrm{MCDHF} / \mathrm{RCI}}^{v}$ & $L S$-composition \\
\hline 24 & 144 & $2 s^{2} S 2 p^{4}\left({ }_{2}^{3} P\right){ }^{4} P 3 d^{5} P_{3}$ & & 6640029 & $1.601 \mathrm{E}-12$ & $1.600 \mathrm{E}-12$ & $0.69+0.102 s^{2} S 2 p^{4}\left({ }_{3}^{3} P\right)^{4} P 3 d^{5} D+0.072 s^{2} S 2 p^{4}\left({ }_{3}^{3} P\right)^{4} P 3 d^{5} F$ \\
\hline 24 & 145 & $2 s^{2} S 2 p^{4}\left({ }_{2}^{2} P\right)^{4} P 3 d^{3} F_{3}$ & & 6649620 & $9.454 \mathrm{E}-13$ & $9.437 \mathrm{E}-13$ & $0.62+0.152 s^{2} S 2 p^{4}\left({ }_{2}^{3} P\right)^{4} P 3 d^{5} P+0.132 s^{2} S 2 p^{4}\left({ }_{2}^{3} P\right)^{4} P 3 d^{3} D$ \\
\hline 24 & 146 & $2 s^{2} S 2 p^{4}\left({ }_{2}^{3} P\right){ }^{4} P 3 d^{3} P_{0}$ & & 6656360 & 3.873E-13 & $3.869 \mathrm{E}-13$ & $\left.0.83+0.092 s^{2} S 2 p^{4}\left({ }_{2}^{1} D\right)^{2} D 3 d^{3} P+0.052 s^{2} S 2 p^{4}{ }_{2}^{3} P\right)^{4} P 3 d^{5} D$ \\
\hline 24 & 147 & $2 s^{2} S 2 p^{4}\left({ }_{2}^{3} P\right){ }^{4} P 3 d^{3} P_{1}$ & & 6660032 & $2.614 \mathrm{E}-13$ & $2.612 \mathrm{E}-13$ & $0.67+0.172 s^{2} S 2 p^{4}\left({ }_{2}^{3} P\right)^{4} P 3 d^{3} D+0.062 s^{2} S 2 p^{4}\left({ }_{2}^{1} D\right)^{2} D 3 d^{3} P$ \\
\hline 24 & 148 & $2 s^{2} S 2 p^{4}\left({ }_{2}^{3} P\right){ }^{4} P 3 d^{3} D_{2}$ & & 6660886 & $2.447 \mathrm{E}-13$ & $2.444 \mathrm{E}-13$ & $\left.\left.0.39+0.322 s^{2} S 2 p^{4}{ }_{2}^{3} P\right)^{4} P 3 d^{3} F+0.172 s^{2} S 2 p^{4}{ }_{2}^{3} P\right){ }^{4} P 3 d^{3} P$ \\
\hline 24 & 149 & $2 s^{2} S 2 p^{4}\left({ }_{2}^{3} P\right)^{4} P 3 d^{3} F_{2}$ & & 6683722 & $4.950 \mathrm{E}-13$ & $4.946 \mathrm{E}-13$ & $0.59+0.242 s^{2} S 2 p^{4}\left({ }_{2}^{3} P\right){ }^{4} P 3 d^{3} P+0.102 s^{2} S 2 p^{4}\left({ }_{2}^{3} P\right)^{4} P 3 d^{3} D$ \\
\hline 24 & 150 & $2 s^{2} S 2 p^{4}\left(3_{2}^{3} P\right)^{4} P 3 d^{3} D_{1}$ & & 6694200 & $1.868 \mathrm{E}-13$ & $1.867 \mathrm{E}-13$ & $0.67+0.172 s^{2} S 2 p^{4}\left(3_{2}^{3} P\right)^{4} P 3 d^{3} P+0.082 s^{2} S 2 p^{4}\left({ }_{2}^{4} D\right)^{2} D 3 d^{3} D$ \\
\hline 24 & 151 & $2 s^{2} S 2 p^{4}\left({ }_{2}^{3} P\right){ }^{4} P 3 d^{3} D_{3}$ & & 6695430 & $1.343 \mathrm{E}-13$ & $1.341 \mathrm{E}-13$ & $0.71+0.202 s^{2} S 2 p^{4}\left({ }_{2}^{3} P\right)^{4} P 3 d^{3} F+0.032 s^{2} S 2 p^{4}\left({ }_{2}^{1} D\right)^{2} D 3 d^{3} D$ \\
\hline 24 & 152 & $2 s^{2} S 2 p^{4}\left({ }_{0}^{1} S\right)^{2} S 3 p^{3} P_{0}^{\circ}$ & & 6706906 & $2.150 \mathrm{E}-12$ & 2.147E-12 & $0.68+0.212 s^{2} S 2 p^{4}\left({ }_{2}^{3} P\right)^{2} P 3 p^{3} P^{\circ}+0.042 s^{2} S 2 p^{4}\left({ }_{2}^{1} D\right)^{2} D 3 p^{3} P^{\circ}$ \\
\hline 24 & 153 & $2 s^{2} S 2 p^{4}\left({ }_{2}^{3} P\right){ }^{4} P 3 d^{3} P_{2}$ & & 6711330 & $2.164 \mathrm{E}-13$ & $2.161 \mathrm{E}-13$ & $\left.0.48+0.362 s^{2} S 2 p^{4}\left({ }_{2}^{3} P\right)\right)^{4} P 3 d^{3} D+0.052 s^{2} S 2 p^{4}\left({ }_{2}^{1} D\right)^{2} D 3 d^{3} P$ \\
\hline 24 & 154 & $2 s^{2} S 2 p^{4}\left({ }_{0}^{1} S\right)^{2} S 3 p^{3} P_{1}^{\mathrm{o}}$ & & 6714532 & $1.258 \mathrm{E}-12$ & $1.255 \mathrm{E}-12$ & $\left.0.60+0.162 s^{2} S 2 p^{4}{ }_{2}^{3} P\right)^{2} P 3 p^{3} D^{\circ}+0.132 s^{2} S 2 p^{4}\left({ }_{2}^{3} P\right)^{2} P 3 p^{3} P^{\circ}$ \\
\hline 24 & 155 & $2 s^{2} S 2 p^{4}\left({ }_{0}^{1} S\right)^{2} S 3 p^{3} P_{2}^{\circ}$ & & 6732906 & $1.812 \mathrm{E}-12$ & $1.807 \mathrm{E}-12$ & $0.67+0.252 s^{2} S 2 p^{4}\left({ }_{2}^{3} P\right)^{2} P 3 p^{3} D^{\circ}$ \\
\hline 24 & 156 & $2 s^{2} S 2 p^{4}\left({ }_{0}^{1} S\right)^{2} S 3 p p^{1} P_{1}^{\circ}$ & & 6740804 & $4.285 \mathrm{E}-13$ & $4.271 \mathrm{E}-13$ & $\left.0.60+0.122 s^{2} S 2 p^{4}{ }_{2}^{3} P\right)^{2} P 3 p^{3} P^{\circ}+0.102 s^{2} S 2 p^{4}\left({ }_{2}^{3} P\right)^{2} P 3 p^{3} D^{\circ}$ \\
\hline 24 & 157 & $2 s^{2} S 2 p^{4}\left({ }_{2}^{3} P\right)^{2} P 3 p^{3} P_{2}^{\circ}$ & & 6749432 & 1.789E-12 & $1.785 \mathrm{E}-12$ & $0.54+0.152 s^{2} S 2 p^{4}\left({ }_{2}^{3} P\right)^{2} P 3 p^{3} D^{\circ}+0.112 s^{2} S 2 p^{4}\left({ }_{0}^{1} S\right)^{2} S 3 p^{3} P^{\circ}$ \\
\hline 24 & 158 & $2 s^{2} S 2 p^{4}\left(3_{3}^{2} P\right)^{2} P 3 p^{1} D_{2}^{\circ}$ & & 6765303 & 1.613E-12 & $1.609 \mathrm{E}-12$ & $0.47+0.272 s^{2} S 2 p^{4}\left(_{3}^{3} P\right)^{2} P 3 p^{3} P^{\circ}+0.202 s^{2} S 2 p^{4}\left(_{3}^{3} P\right)^{2} P 3 p^{3} D^{\circ}$ \\
\hline 24 & 159 & $2 s^{2} S 2 p^{4}\left(3_{2}^{3} P\right)^{2} P 3 p^{3} D_{3}^{\circ}$ & & 6767173 & 7.916E-13 & $7.890 \mathrm{E}-13$ & 0.95 \\
\hline 24 & 160 & $2 s^{2} S 2 p^{4}\left({ }_{2}^{3} P\right)^{2} P 3 p{ }^{3} P_{1}^{\mathrm{o}}$ & & 6775622 & $1.796 \mathrm{E}-12$ & $1.791 \mathrm{E}-12$ & $0.39+0.262 s^{2} S 2 p^{4}\left({ }_{2}^{3} P\right)^{2} P 3 p^{3} S^{\circ}+0.172 s^{2} S 2 p^{4}\left({ }_{0}^{1} S\right)^{2} S 3 p^{3} P^{\circ}$ \\
\hline 24 & 161 & $2 s^{2} S 2 p^{4}\left({ }_{2}^{3} P\right)^{2} P 3 p{ }^{1} S_{0}^{\circ}$ & & 6789253 & $4.885 \mathrm{E}-12$ & $4.877 \mathrm{E}-12$ & $\left.0.52+0.302 s^{2} S 2 p^{4}{ }_{2}^{3} P\right)^{2} P 3 p^{3} P^{\circ}+0.092 s^{2} S 2 p^{4}\left({ }_{2}^{1} D\right)^{2} D 3 p^{3} P^{\circ}$ \\
\hline 24 & 162 & $\left.2 s^{2} S 2 p^{4}{ }_{3}^{2} P\right)^{2} P 3 p^{3} D_{1}^{\circ}$ & & 6806218 & $7.777 \mathrm{E}-13$ & $7.751 \mathrm{E}-13$ & $\left.0.57+0.202 s^{2} S 2 p^{4}{ }_{2}^{3} P\right)^{2} P 3 p^{3} S^{\circ}+0.072 s^{2} S 2 p^{4}\left({ }_{0}^{1} S\right)^{2} S 3 p^{3} P^{\circ}$ \\
\hline 24 & 163 & $2 s^{2} S 2 p^{4}\left({ }_{2}^{2} D\right)^{2} D 3 d^{3} G_{3}$ & & 6817033 & 3.101E-11 & 3.071E-11 & 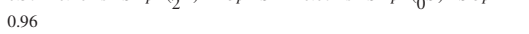 \\
\hline 24 & 164 & $2 s^{2} S 2 p^{4}\left({ }_{2}^{2} D\right)^{2} D 3 d^{3} G_{4}$ & & 6820961 & 3.617E-11 & $3.573 \mathrm{E}-11$ & 0.96 \\
\hline 24 & 165 & $2 s^{2} S 2 p^{4}\left({ }_{2}^{1} D\right)^{2} D 3 d^{3} G_{5}$ & & 6826258 & 3.938E-11 & 3.887E-11 & $0.97+0.022 s^{2} S 2 p^{4}\left({ }_{2}^{3} P\right)^{4} P 3 d^{5} F$ \\
\hline 24 & 166 & $2 s^{2} S 2 p^{4}\left({ }_{2}^{3} P\right)^{2} P 3 p^{3} P_{0}^{\circ}$ & & 6832794 & $6.034 \mathrm{E}-12$ & $6.029 \mathrm{E}-12$ & $0.31+0.412 s^{2} S 2 p^{4}\left({ }_{2}^{3} P\right)^{2} P 3 p^{1} S^{\circ}+0.232 s^{2} S 2 p^{4}\left({ }_{0}^{1} S\right)^{2} S 3 p^{3} P^{\circ}$ \\
\hline 24 & 167 & $2 s^{2} S 2 p^{4}\left({ }_{2}^{3} P\right)^{2} P 3 p^{3} D_{2}^{\circ}$ & & 6835294 & $1.842 \mathrm{E}-12$ & $1.837 \mathrm{E}-12$ & $0.37+0.382 s^{2} S 2 p^{4}\left(\int_{2}^{3} P\right)^{2} P 3 p^{1} D^{\circ}+0.182 s^{2} S 2 p^{4}\left({ }^{1} S\right)^{2} S 3 p^{3} P^{\circ}$ \\
\hline 24 & 168 & $\left.2 s^{2} S 2 p^{4}{ }_{2}^{3} P\right)^{2} P 3 p^{3} S_{1}^{\circ}$ & & 6840496 & $5.954 \mathrm{E}-13$ & $5.931 \mathrm{E}-13$ & $\left.0.44+0.192 s^{2} S 2 p^{4}\left({ }_{0}^{1} S\right)^{2} S 3 p^{1} P^{\circ}+0.182 s^{2} S 2 p^{4}{ }_{2}^{3} P\right)^{2} P 3 p^{3} P^{\circ}$ \\
\hline 24 & 169 & $2 s^{2} S 2 p^{4}\left({ }_{2}^{1} D\right)^{2} D 3 d^{3} F_{2}$ & & 6851799 & $9.653 \mathrm{E}-12$ & $9.655 \mathrm{E}-12$ & $0.90+0.042 s^{2} S 2 p^{4}\left({ }_{2}^{1} D\right)^{2} D 3 d^{1} D$ \\
\hline 24 & 170 & $2 s^{2} S 2 p^{4}\left({ }_{2}^{1} D\right)^{2} D 3 d^{3} S_{1}$ & & 6855243 & $7.226 \mathrm{E}-14$ & $7.220 \mathrm{E}-14$ & 0.94 \\
\hline 24 & 171 & $2 s^{2} S 2 p^{4}\left({ }_{2}^{1} D\right)^{2} D 3 d^{3} F_{3}$ & & 6856396 & $2.480 \mathrm{E}-12$ & $2.478 \mathrm{E}-12$ & $0.92+0.042 s^{2} S 2 p^{4}\left({ }_{2}^{1} D\right)^{2} D 3 d^{3} D$ \\
\hline 24 & 172 & $2 s^{2} S 2 p^{4}\left({ }_{2}^{1} D\right)^{2} D 3 d^{3} F_{4}$ & & 6858503 & $3.019 \mathrm{E}-11$ & $3.006 \mathrm{E}-11$ & $0.64+0.332 s^{2} S 2 p^{4}\left({ }_{2}^{1} D\right)^{2} D 3 d^{1} G$ \\
\hline 24 & 173 & $2 s^{2} S 2 p^{4}\left(1_{1}^{1} D\right)^{2} D 3 d^{3} D_{1}$ & & 6861443 & $1.647 \mathrm{E}-13$ & $1.645 \mathrm{E}-13$ & $0.46+0.252 s^{2} S 2 p^{4}\left(1_{1}^{1} D\right)^{2} D 3 d^{1} P+0.122 s^{2} S 2 p^{4}\left({ }_{1}^{1} D\right)^{2} D 3 d^{3} P$ \\
\hline 24 & 174 & $2 s^{2} S 2 p^{4}\left({ }_{2}^{1} D\right)^{2} D 3 d^{3} P_{0}$ & & 6864694 & $8.556 \mathrm{E}-14$ & $8.545 \mathrm{E}-14$ & $\left.0.71+0.142 s^{2} S 2 p^{4}\left({ }_{2}^{1} D\right)^{2} D 3 d^{1} S+0.072 s^{2} S 2 p^{4}{ }_{2}^{3} P\right)^{4} P 3 d^{3} P$ \\
\hline 24 & 175 & $2 s^{2} S 2 p^{4}\left({ }_{2}^{1} D\right)^{2} D 3 d^{3} D_{2}$ & & 6867498 & $1.324 \mathrm{E}-13$ & $1.322 \mathrm{E}-13$ & $0.81+0.052 s^{2} S 2 p^{4}\left({ }_{2}^{3} P\right)^{4} P 3 d^{3} D+0.042 s^{2} S 2 p^{4}\left({ }_{2}^{1} D\right)^{2} D 3 d^{1} D$ \\
\hline 24 & 176 & $2 s^{2} S 2 p^{4}\left({ }_{2}^{1} D\right)^{2} D 3 d^{1} G_{4}$ & & 6867671 & $2.834 \mathrm{E}-11$ & $2.829 \mathrm{E}-11$ & $0.63+0.332 s^{2} S 2 p^{4}\left({ }_{2}^{1} D\right)^{2} D 3 d^{3} F$ \\
\hline 24 & 177 & $2 s^{2} S 2 p^{4}\left({ }_{2}^{1} D\right)^{2} D 3 d^{3} P_{2}$ & & 6869690 & $7.326 \mathrm{E}-14$ & $7.320 \mathrm{E}-14$ & $0.87+0.062 s^{2} S 2 p^{4}\left({ }_{2}^{3} P\right)^{4} P 3 d^{3} P+0.022 s^{2} S 2 p^{4}\left({ }_{2}^{1} D\right)^{2} D 3 d^{3} D$ \\
\hline 24 & 178 & $2 s^{2} S 2 p^{4}\left({ }_{2}^{1} D\right)^{2} D 3 d^{3} D_{3}$ & & 6869895 & $1.236 \mathrm{E}-13$ & $1.235 \mathrm{E}-13$ & $0.87+0.042 s^{2} S 2 p^{4}\left(3_{2}^{3} P\right)^{4} P 3 d^{3} D+0.032 s^{2} S 2 p^{4}\left({ }_{2}^{2} D\right)^{2} D 3 d^{3} F$ \\
\hline 24 & 179 & $2 s^{2} S 2 p^{4}\left({ }_{2}^{1} D\right)^{2} D 3 d^{3} P_{1}$ & & 6873924 & 7.490E-14 & 7.483E-14 & $\left.0.72+0.102 s^{2} S 2 p^{4}{ }_{2}^{3} P\right)^{4} P 3 d^{3} P+0.092 s^{2} S 2 p^{4}\left({ }_{2}^{1} D\right)^{2} D 3 d^{3} D$ \\
\hline 24 & 180 & $2 s^{2} S 2 p^{4}\left(\int_{1}^{1} D\right)^{2} D 3 d^{1} P_{1}$ & & 6889694 & $1.555 \mathrm{E}-13$ & $1.554 \mathrm{E}-13$ & $0.61+0.242 s^{2} S 2 p^{4}\left(\int_{1}^{1} D\right)^{2} D 3 d^{3} D+0.052 s^{2} S 2 p^{4}\left(3_{2}^{2} P\right)^{2} P 3 d^{1} P$ \\
\hline 24 & 181 & $2 s^{2} S 2 p^{4}\left({ }_{2}^{1} D\right)^{2} D 3 d^{1} D_{2}$ & & 6892388 & 3.453E-13 & $3.446 \mathrm{E}-13$ & $0.76+0.062 s^{2} S 2 p^{4}\left({ }_{2}^{1} D\right)^{2} D 3 d^{3} F+0.052 s^{2} S 2 p^{4}\left({ }_{2}^{3} P\right)^{2} P 3 d^{1} D$ \\
\hline 24 & 182 & $2 s^{2} S 2 p^{4}\left({ }_{2}^{1} D\right)^{2} D 3 d^{1} F_{3}$ & & 6899128 & 1.125E-12 & $1.125 \mathrm{E}-12$ & 0.92 \\
\hline 24 & 183 & $2 s^{2} S 2 p^{4}\left({ }_{2}^{3} P\right)^{2} P 3 p{ }^{1} P_{1}^{\circ}$ & & 6902386 & $1.502 \mathrm{E}-12$ & $1.496 \mathrm{E}-12$ & $0.79+0.062 s^{2} S 2 p^{4}\left({ }_{2}^{1} D\right)^{2} D 3 p^{1} P^{\circ}+0.042 s^{2} S 2 p^{4}\left({ }_{0}^{1} S\right)^{2} S 3 p^{3} P^{\circ}$ \\
\hline 24 & 184 & $2 s^{2} S 2 p^{4}\left({ }_{2}^{1} D\right)^{2} D 3 d^{1} S_{0}$ & & 6908434 & $7.788 \mathrm{E}-14$ & $7.782 \mathrm{E}-14$ & $0.81+0.122 s^{2} S 2 p^{4}\left({ }_{2}^{1} D\right)^{2} D 3 d^{3} P+0.052 s^{2} S 2 p^{4}\left({ }_{2}^{3} P\right)^{4} P 3 d^{3} P$ \\
\hline 24 & 185 & $2 s^{2} S 2 p^{4}\left(1_{0}^{1} S\right)^{2} S 3 d^{3} D_{1}$ & & 6975119 & $4.384 \mathrm{E}-12$ & 4.385E-12 & $0.60+0.302 s^{2} S 2 p^{4}\left(3_{2}^{3} P\right)^{2} P 3 d^{3} D+0.052 s^{2} S 2 p^{4}\left(c_{2}^{2} D\right)^{2} D 3 d^{3} D$ \\
\hline 24 & 186 & $2 s^{2} S 2 p^{4}\left({ }_{0}^{1} S\right)^{2} S 3 d^{3} D_{2}$ & & 6980671 & $2.060 \mathrm{E}-12$ & $2.060 \mathrm{E}-12$ & $0.65+0.172 s^{2} S 2 p^{4}\left(3_{2}^{3} P\right)^{2} P 3 d^{3} D+0.092 s^{2} S 2 p^{4}\left(_{2}^{3} P\right)^{2} P 3 d^{3} F$ \\
\hline 24 & 187 & $2 s^{2} S 2 p^{4}\left({ }_{0}^{1} S\right)^{2} S 3 d^{3} D_{3}$ & & 6984180 & $3.184 \mathrm{E}-13$ & $3.180 \mathrm{E}-13$ & $\left.0.77+0.082 s^{2} S 2 p^{4}\left({ }_{2}^{3} P\right)^{2} P 3 d^{3} F+0.062 s^{2} S 2 p^{4}{ }_{2}^{3} P\right)^{2} P 3 d^{3} D$ \\
\hline 24 & 188 & $2 s^{2} S 2 p^{4}\left(3_{2}^{3} P\right)^{2} P 3 d^{3} D_{3}$ & & 7000457 & $4.685 \mathrm{E}-13$ & $4.681 \mathrm{E}-13$ & $0.79+0.102 s^{2} S 2 p^{4}\left({ }_{2}^{3} P\right)^{2} P 3 d^{3} F+0.042 s^{2} S 2 p^{4}\left({ }_{2}^{1} D\right)^{2} D 3 d^{1} F$ \\
\hline 24 & 189 & $2 s^{2} S 2 p^{4}\left({ }_{0}^{1} S\right)^{2} S 3 d^{1} D_{2}$ & & 7005687 & $2.482 \mathrm{E}-13$ & $2.478 \mathrm{E}-13$ & $\left.0.37+0.372 s^{2} S 2 p^{4}\left({ }_{2}^{3} P\right)^{2} P 3 d^{3} D+0.082 s^{2} S 2 p^{4}{ }_{2}^{3} P\right)^{2} P 3 d^{3} F$ \\
\hline 24 & 190 & $2 s^{2} S 2 p^{4}\left({ }_{2}^{3} P\right)^{2} P 3 d^{3} D_{2}$ & & 7015684 & 3.014E-13 & 3.010E-13 & $0.25+0.312 s^{2} S 2 p^{4}\left({ }_{2}^{2} P\right)^{2} P 3 d^{3} P+0.212 s^{2} S 2 p^{4}\left({ }_{0}^{1} S\right)^{2} S 3 d^{1} D$ \\
\hline 24 & 191 & $2 s^{2} S 2 p^{4}\left({ }_{2}^{2} P\right)^{2} P 3 d^{1} F_{3}$ & & 7024712 & $8.806 \mathrm{E}-12$ & $8.875 \mathrm{E}-12$ & $0.55+0.402 s^{2} S 2 p^{4}\left({ }_{2}^{2} P\right)^{2} P 3 d^{3} F+0.022 s^{2} S 2 p^{4}\left({ }_{2}^{1} D\right)^{2} D 3 d^{3} G$ \\
\hline 24 & 192 & $\left.2 s^{2} S 2 p^{4}{ }_{3}^{3} P\right)^{2} P 3 d^{3} F_{4}$ & & 7024879 & $8.724 \mathrm{E}-12$ & $8.825 \mathrm{E}-12$ & $0.95+25$ \\
\hline 24 & 193 & $2 s^{2} S 2 p^{4}\left({ }_{2}^{2} P\right)^{2} P 3 d^{3} D_{1}$ & & 7027980 & $1.340 \mathrm{E}-13$ & $1.338 \mathrm{E}-13$ & $0.40+0.342 s^{2} S 2 p^{4}\left({ }_{2}^{3} P\right)^{2} P 3 d^{3} P+0.162 s^{2} S 2 p^{4}\left(1_{0}^{1} S\right)^{2} S 3 d^{3} D$ \\
\hline 24 & 194 & $2 s^{2} S 2 p^{4}\left({ }_{2}^{2} P\right)^{2} P 3 d^{3} P_{0}$ & & 7044366 & $2.474 \mathrm{E}-13$ & $2.471 \mathrm{E}-13$ & $0.92+0.042 s^{2} S 2 p^{4}\left({ }_{2}^{1} D\right)^{2} D 3 d^{3} P$ \\
\hline 24 & 195 & $2 s^{2} S 2 p^{4}\left({ }_{2}^{3} P\right)^{2} P 3 d^{3} P_{1}$ & & 7056342 & $1.523 \mathrm{E}-13$ & $1.521 \mathrm{E}-13$ & $0.53+0.172 s^{2} S 2 p^{4}\left({ }_{2}^{2} P\right)^{2} P 3 d^{1} P+0.142 s^{2} S 2 p^{4}\left({ }_{2}^{3} P\right)^{2} P 3 d^{3} D$ \\
\hline 24 & 196 & $2 s^{2} S 2 p^{4}\left({ }_{2}^{3} P\right)^{2} P 3 d^{3} F_{2}$ & & 7059512 & $1.921 \mathrm{E}-13$ & $1.919 \mathrm{E}-13$ & $0.47+0.212 s^{2} S 2 p^{4}\left({ }_{2}^{3} P\right)^{2} P 3 d^{1} D+0.192 s^{2} S 2 p^{4}\left({ }_{2}^{3} P\right)^{2} P 3 d^{3} P$ \\
\hline 24 & 197 & $2 s^{2} S 2 p^{4}\left({ }_{2}^{3} P\right)^{2} P 3 d^{3} F_{3}$ & & 7087264 & $9.168 \mathrm{E}-12$ & $9.236 \mathrm{E}-12$ & $0.39+0.352 s^{2} S 2 p^{4}\left({ }_{2}^{3} P\right)^{2} P 3 d^{1} F+0.172 s^{2} S 2 p^{4}\left({ }_{0}^{1} S\right)^{2} S 3 d^{3} D$ \\
\hline 24 & 198 & $2 s^{2} S 2 p^{4}\left({ }_{2}^{3} P\right)^{2} P 3 d^{3} P_{2}$ & & 7099319 & $9.692 \mathrm{E}-13$ & $9.682 \mathrm{E}-13$ & $0.41+0.262 s^{2} S 2 p^{4}\left(1_{0}^{1} S\right)^{2} S 3 d^{1} D+0.122 s^{2} S 2 p^{4}\left({ }_{2}^{3} P\right)^{2} P 3 d^{3} F$ \\
\hline 24 & 199 & $2 s^{2} S 2 p^{4}\left({ }_{2}^{3} P\right)^{2} P 3 d^{1} P_{1}$ & & 7122885 & $8.010 \mathrm{E}-14$ & $8.001 \mathrm{E}-14$ & $\left.0.69+0.092 s^{2} S 2 p^{4}\left({ }_{0}^{1} S\right)^{2} S 3 d^{3} D+0.072 s^{2} S 2 p^{4}{ }_{2}^{3} P\right)^{2} P 3 d^{3} P$ \\
\hline 24 & 200 & $2 s^{2} S 2 p^{4}\left({ }_{2}^{3} P\right)^{2} P 3 d^{1} D_{2}$ & & 7132737 & $5.356 \mathrm{E}-14$ & 5.349E-14 & $\left.0.62+0.122 s^{2} S 2 p^{4}{ }_{2}^{3} P\right)^{2} P 3 d^{3} F+0.112 s^{2} S 2 p^{4}\left({ }_{0}^{1} S\right)^{2} S 3 d^{1} D$ \\
\hline
\end{tabular}


Table 2

Transition wavelengths $\lambda$ (in $\AA$ ), transition rates $A$ (in s ${ }^{-1}$ ), weighted oscillator strengths $g f$, and line strengths $S$ (in a.u.) between the lowest 156 (179, 184, 196, 200, 200, 200) states of the $n \leq 3$ configurations in Ar XI (K XII, Ca XIII, Sc XIV, Ti XV, V XVI, Cr XVII) listed in Table 1. Transitions with the branching fraction (BF) $\geq 10^{-5}$ are presented.

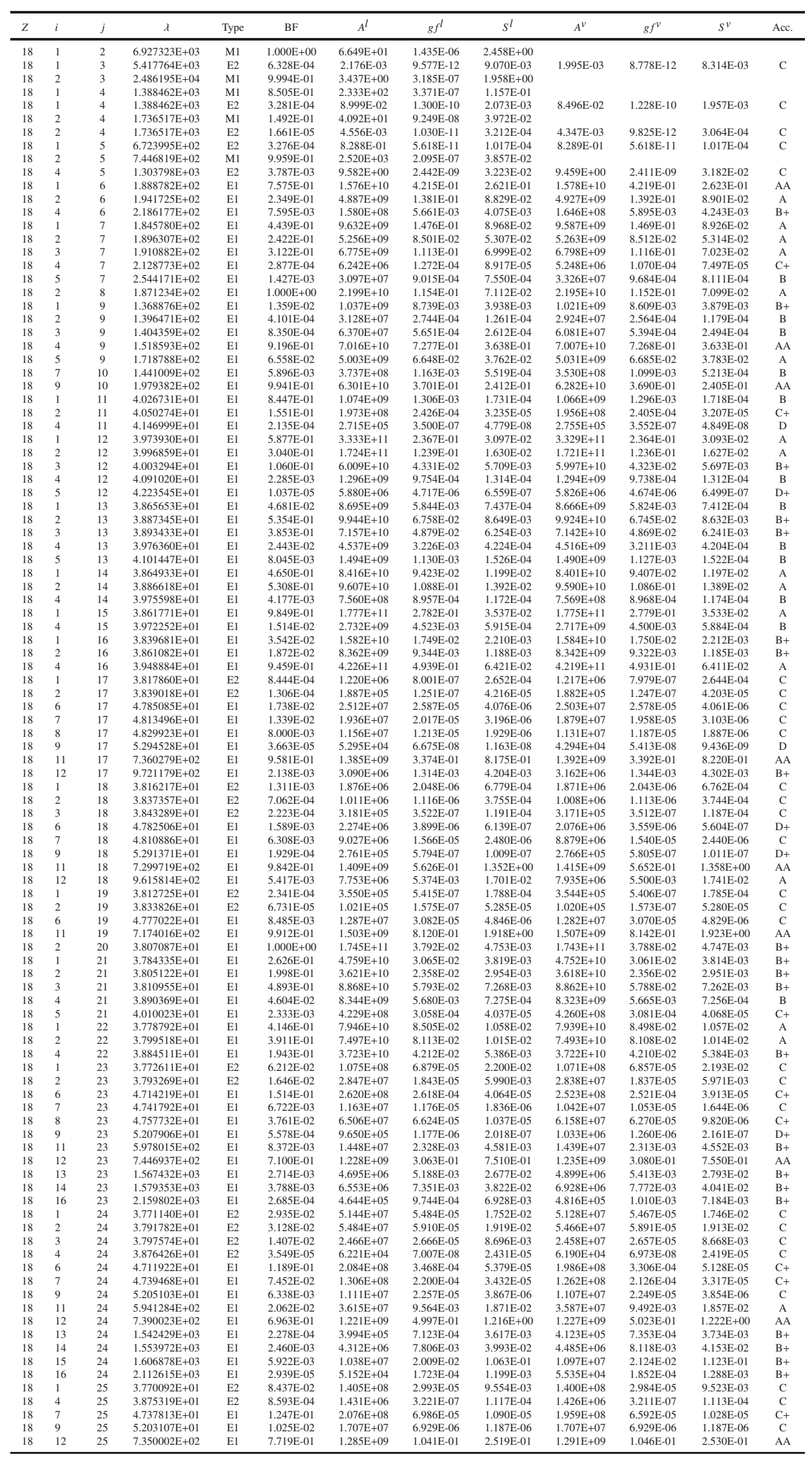




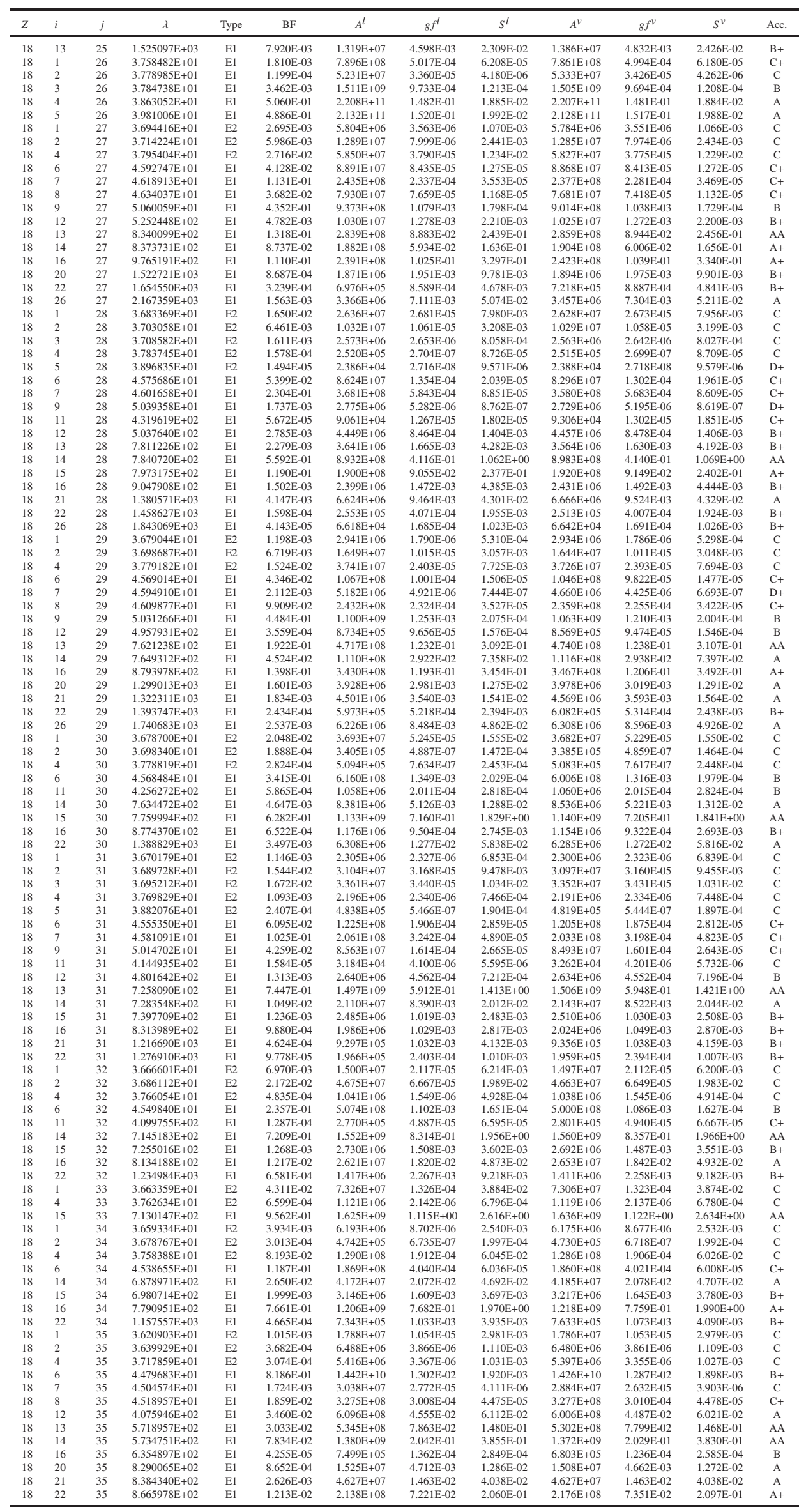




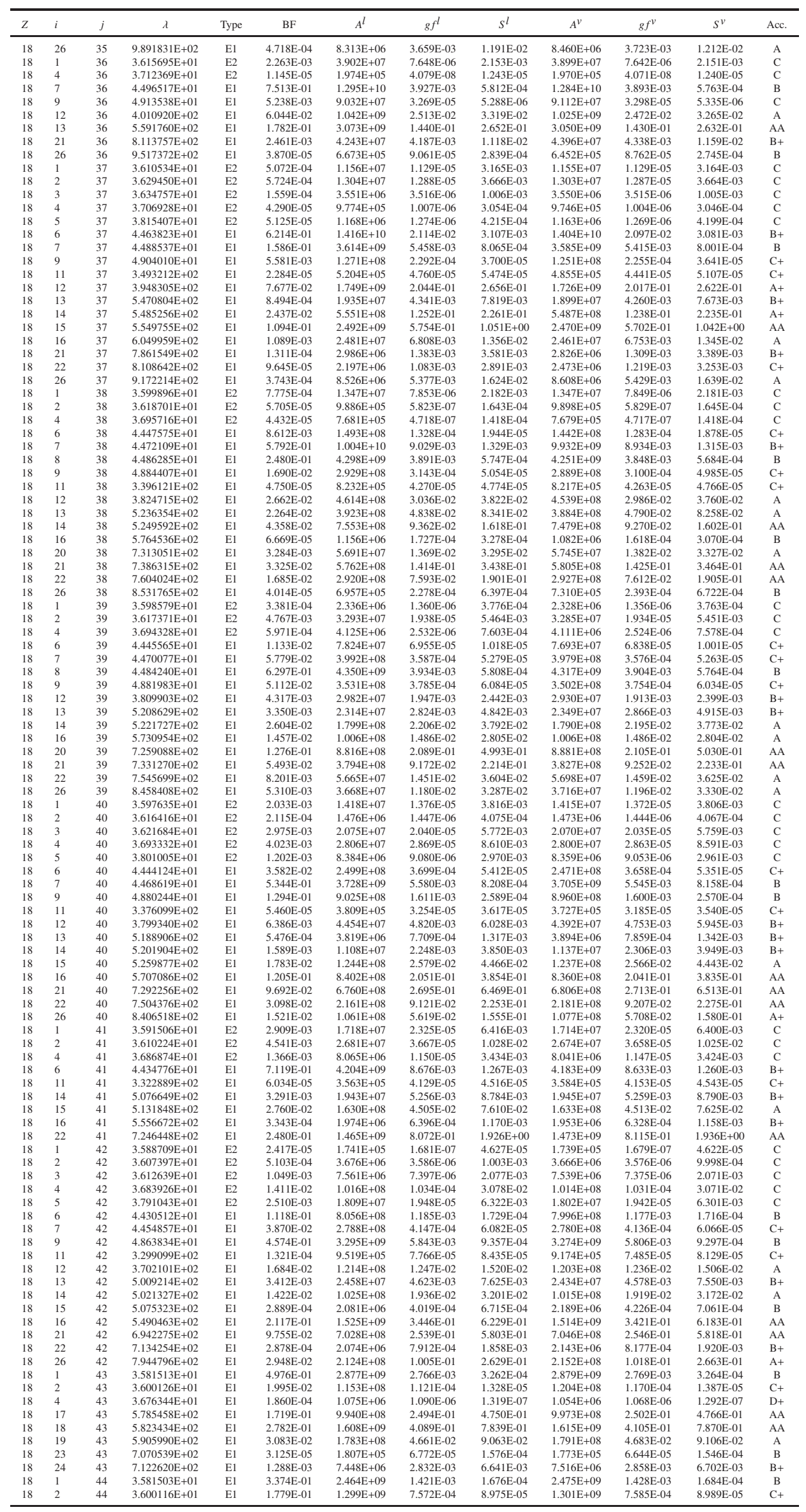




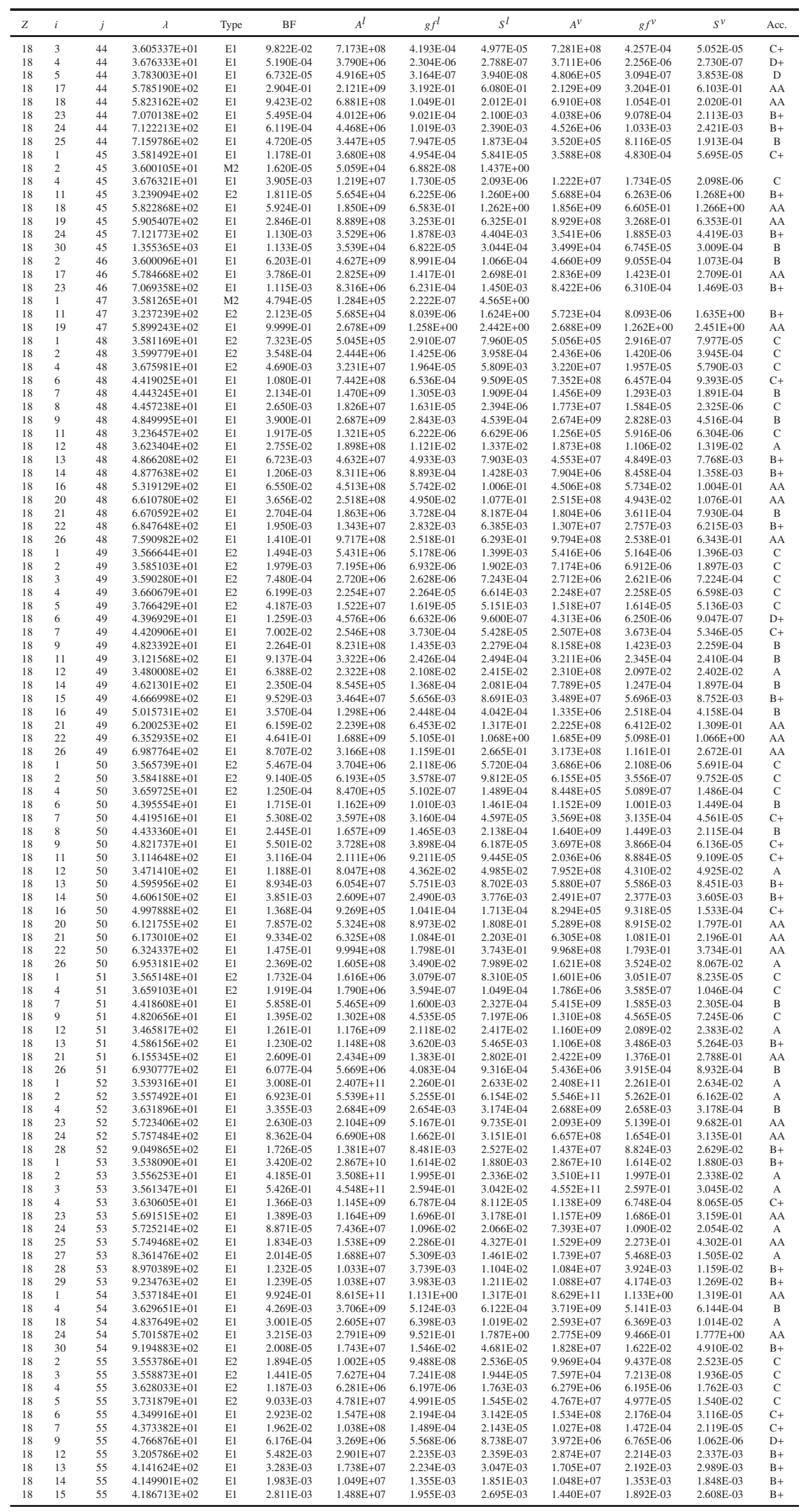




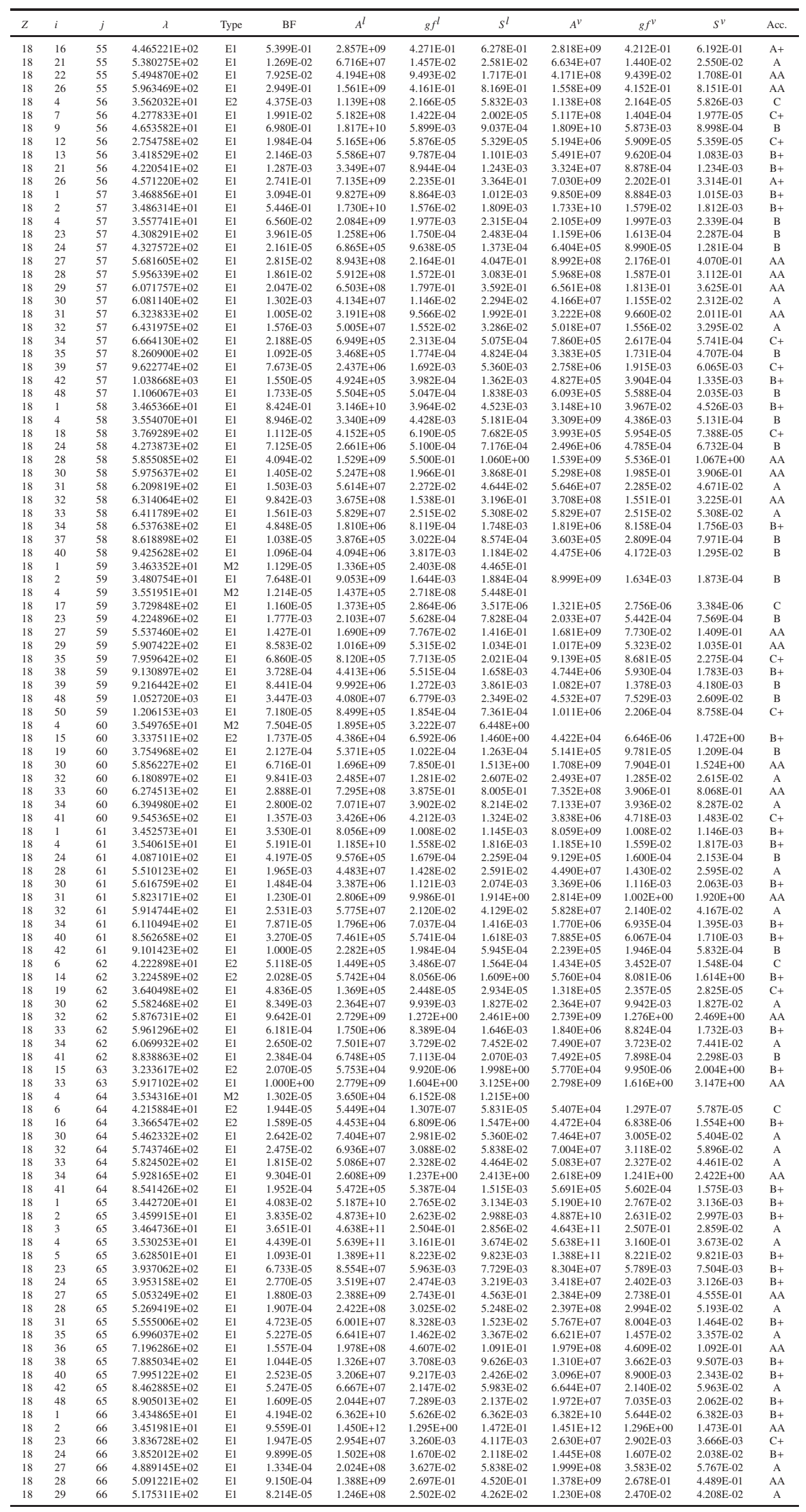




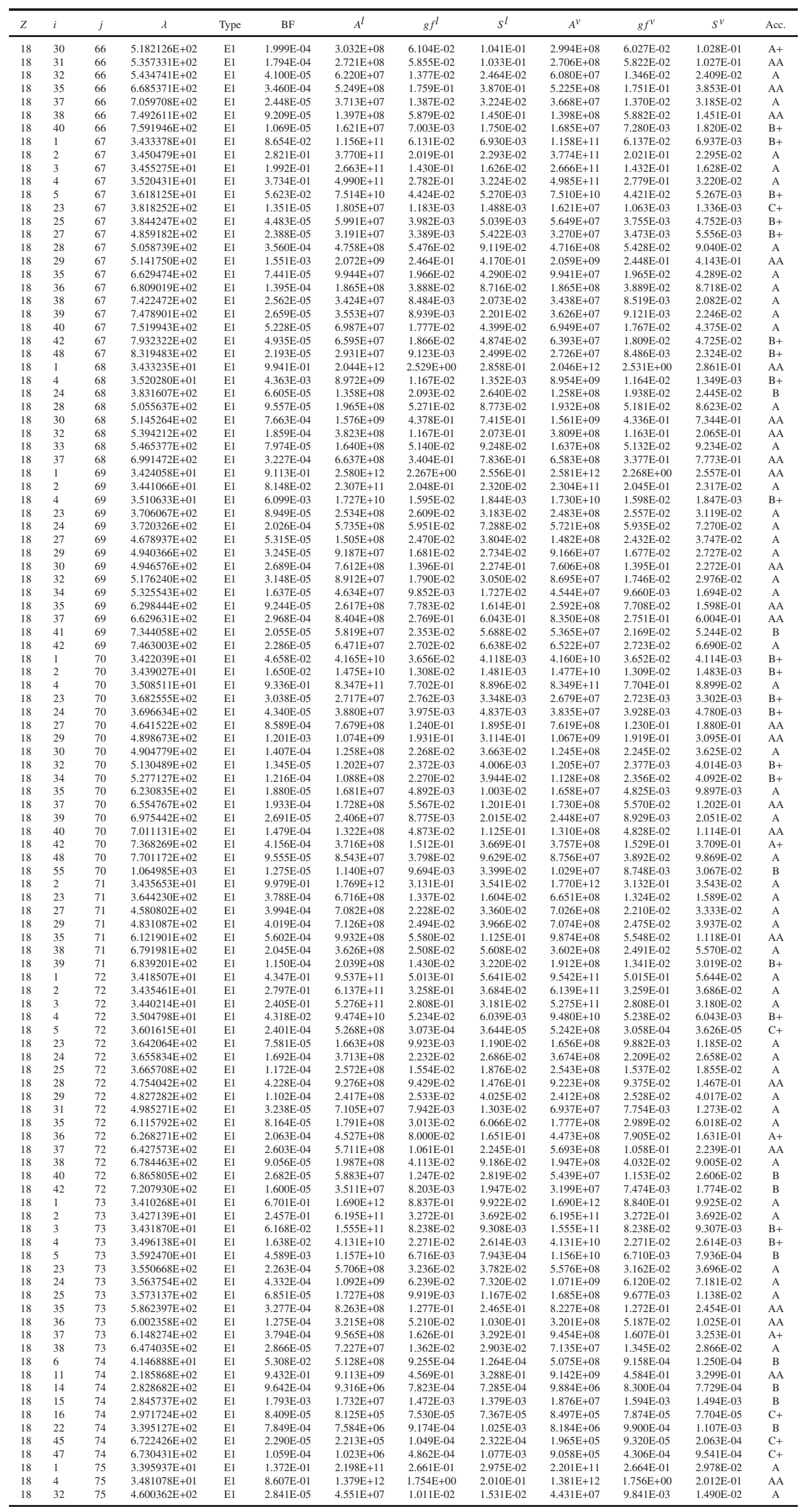




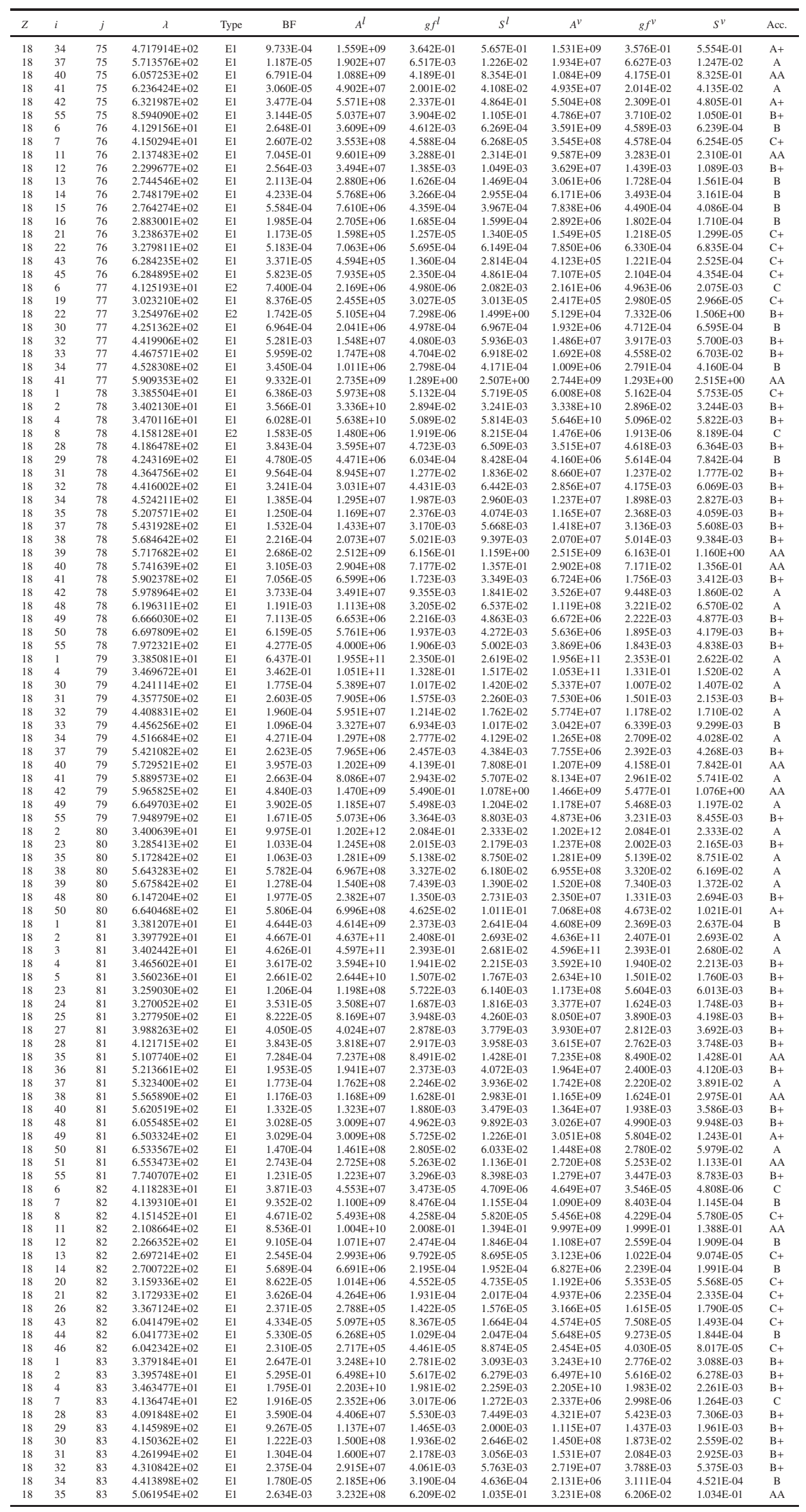




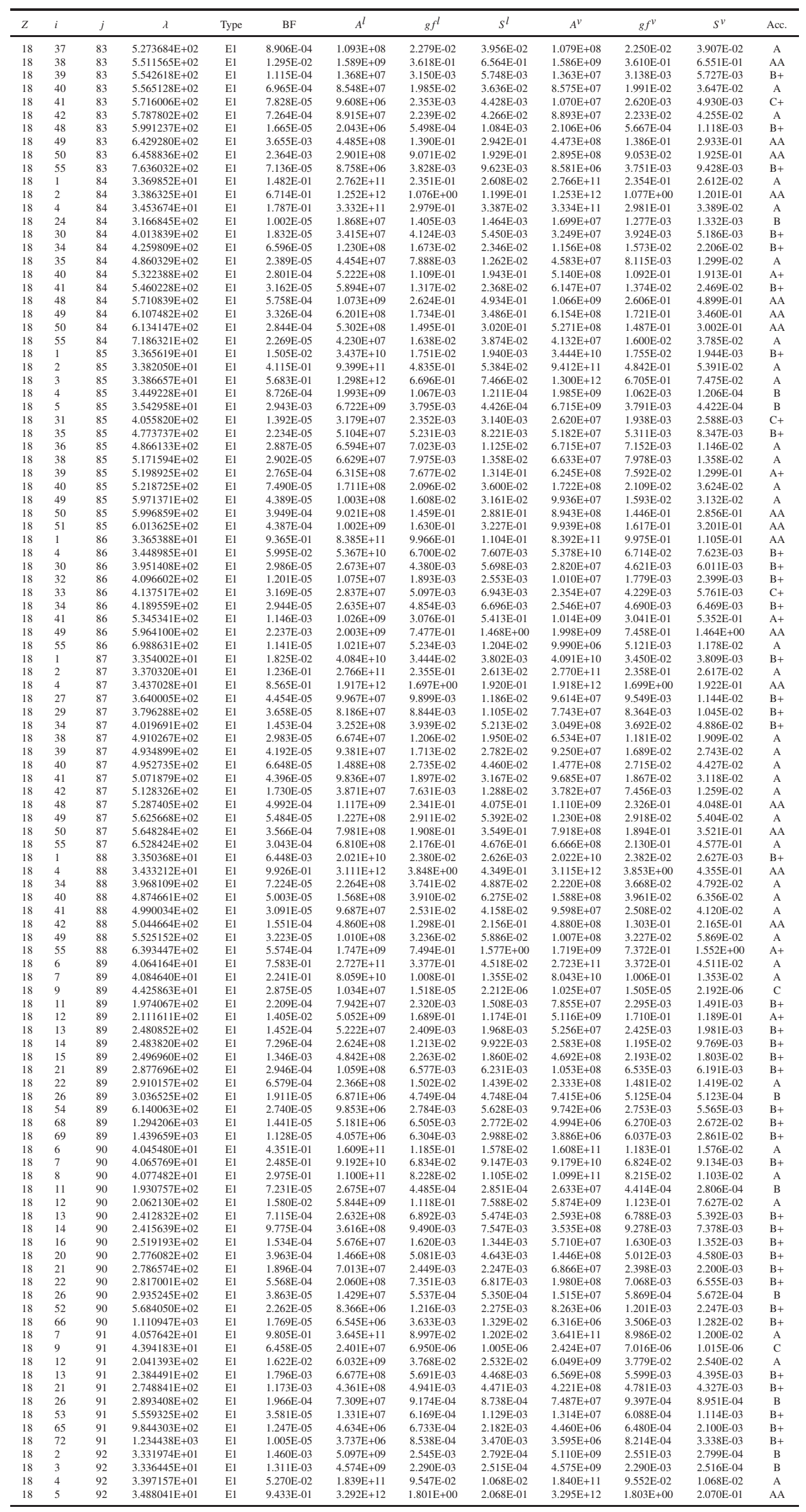




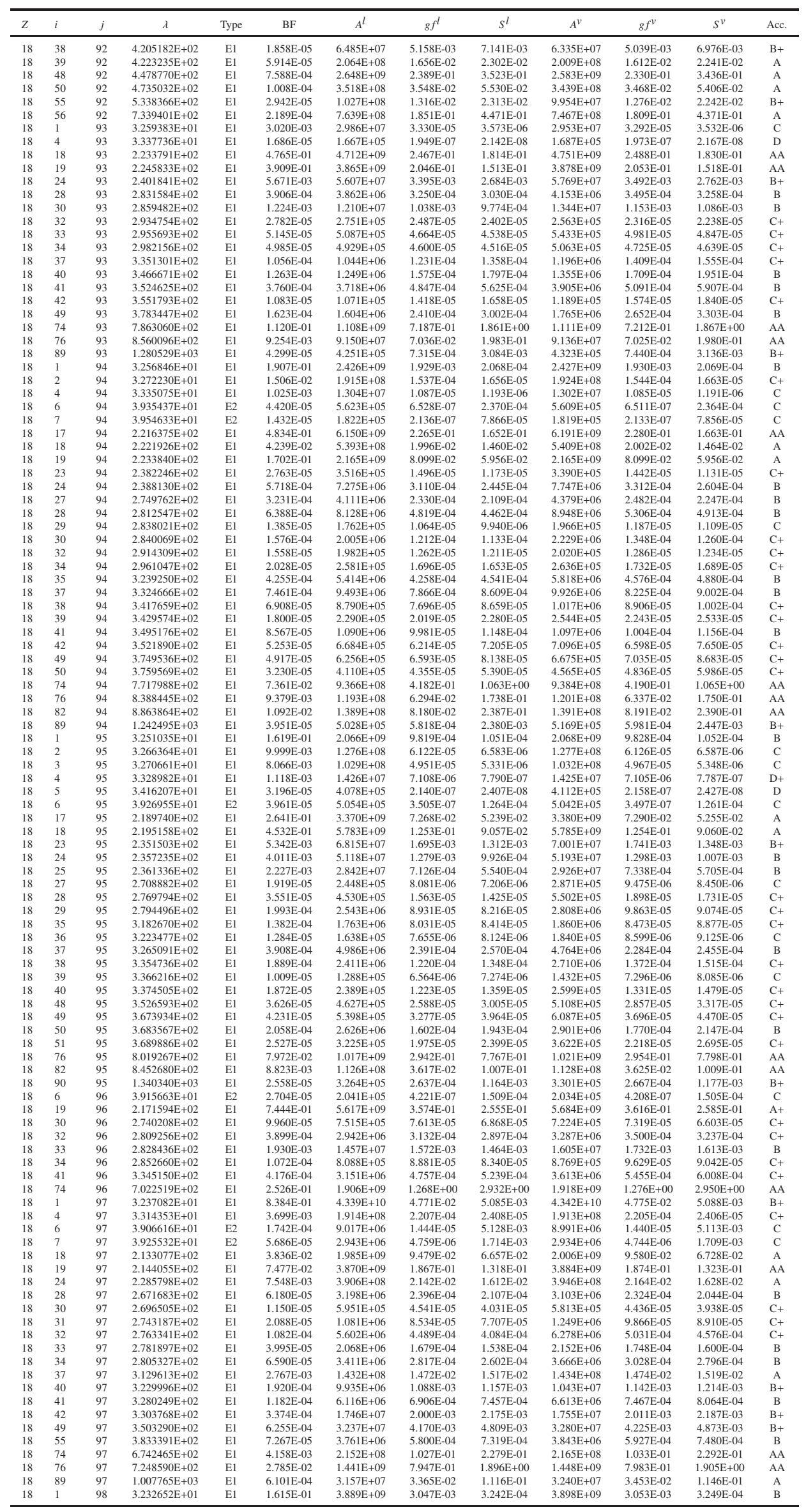




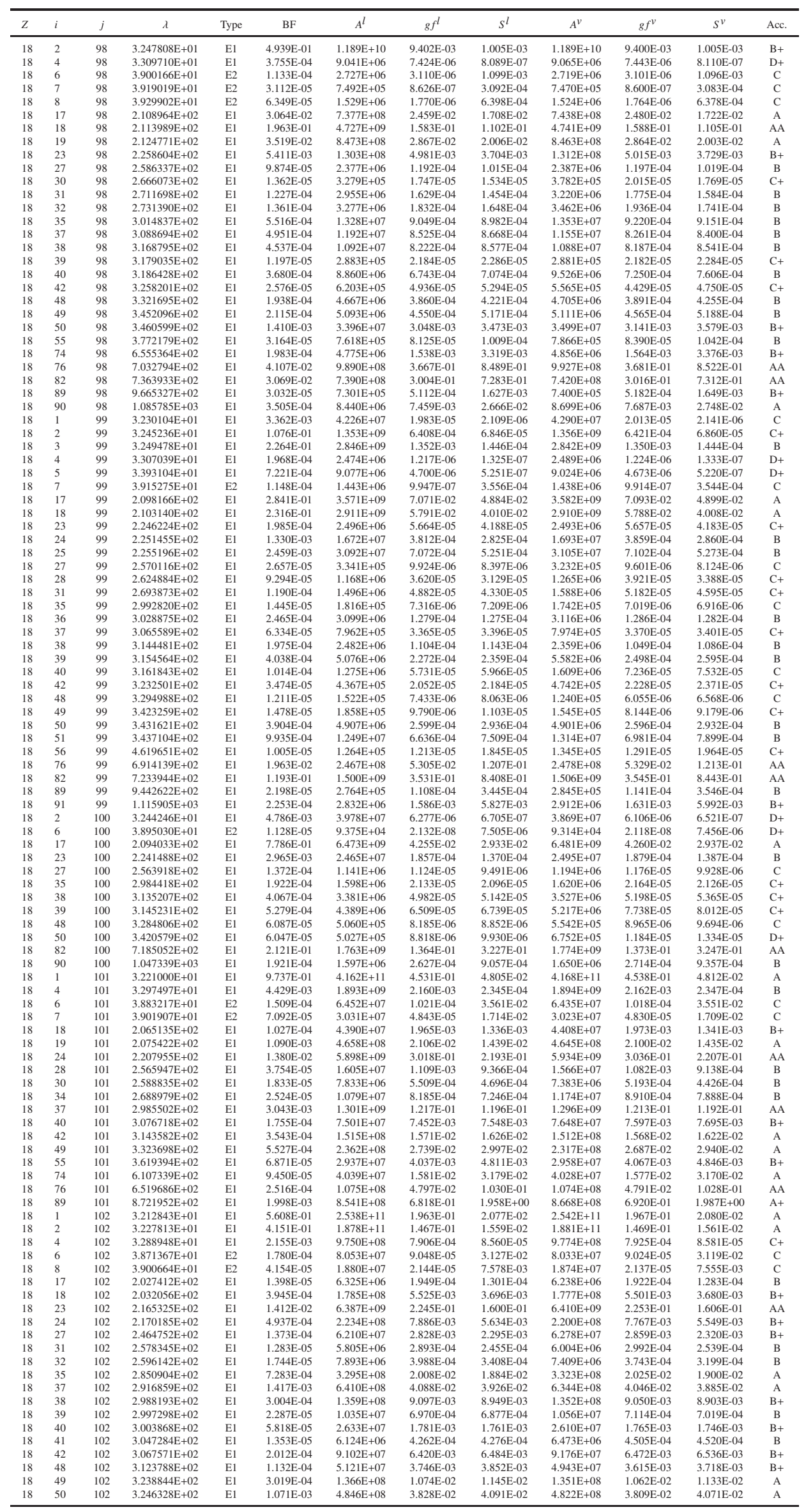




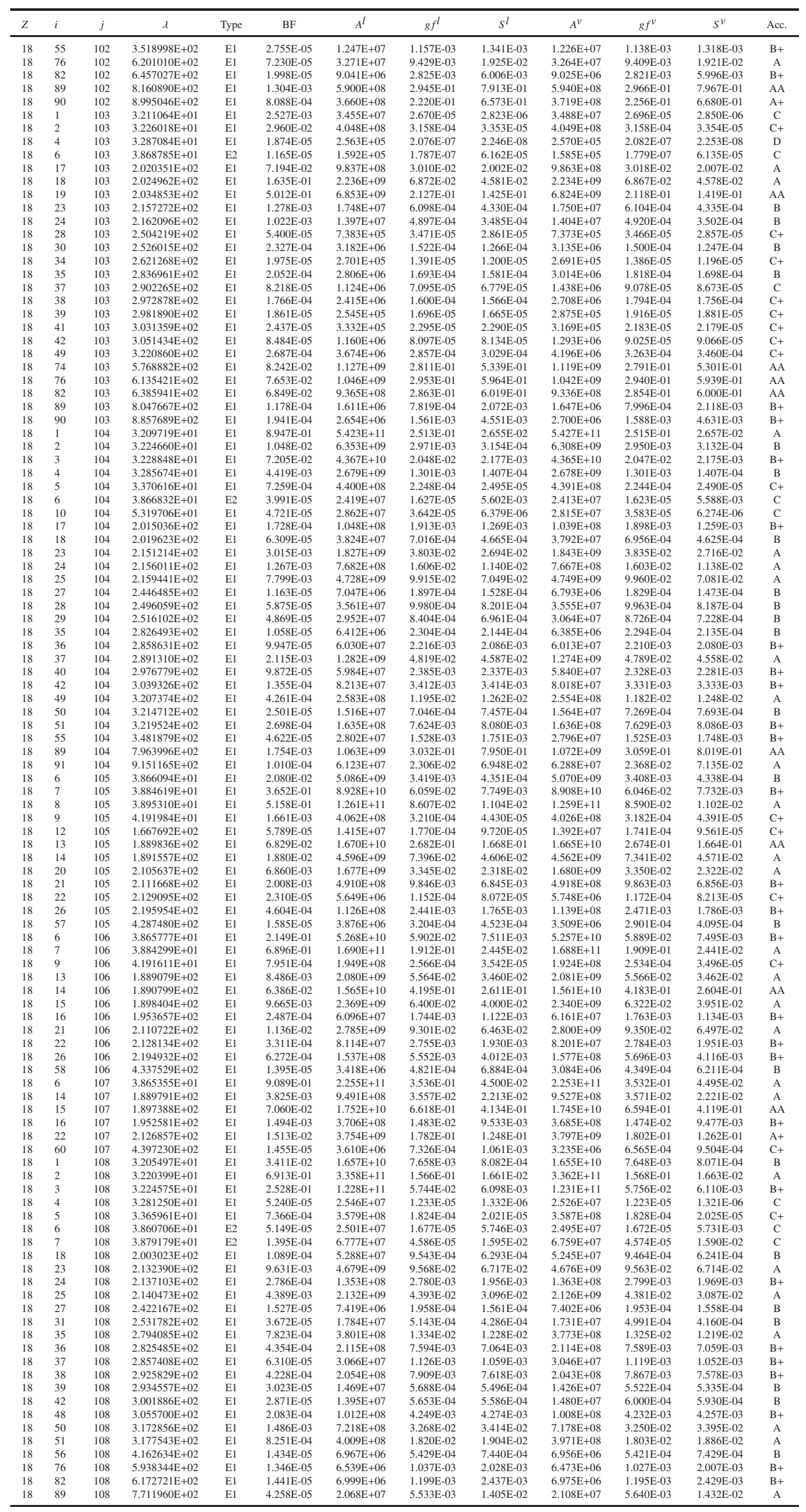




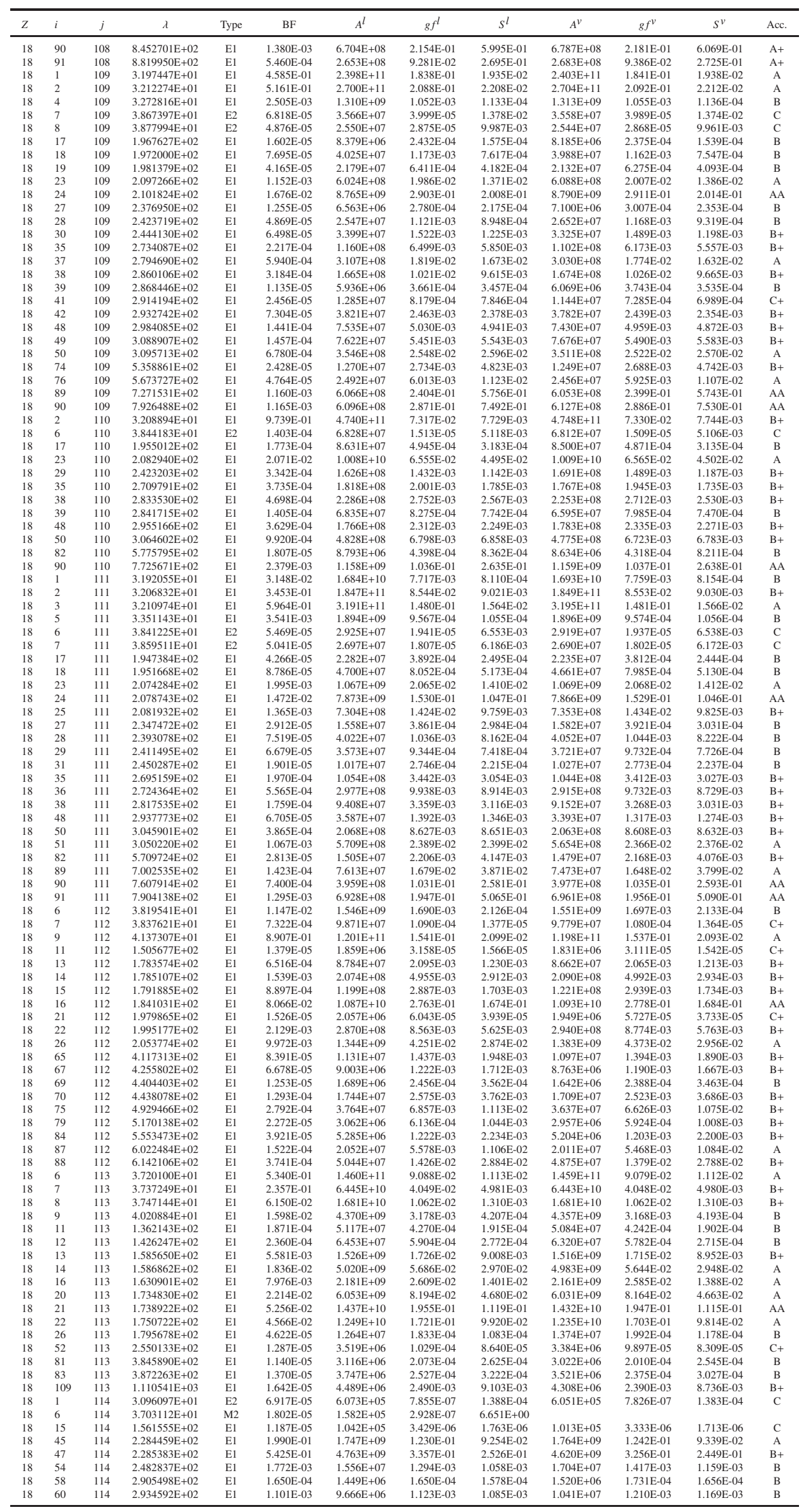




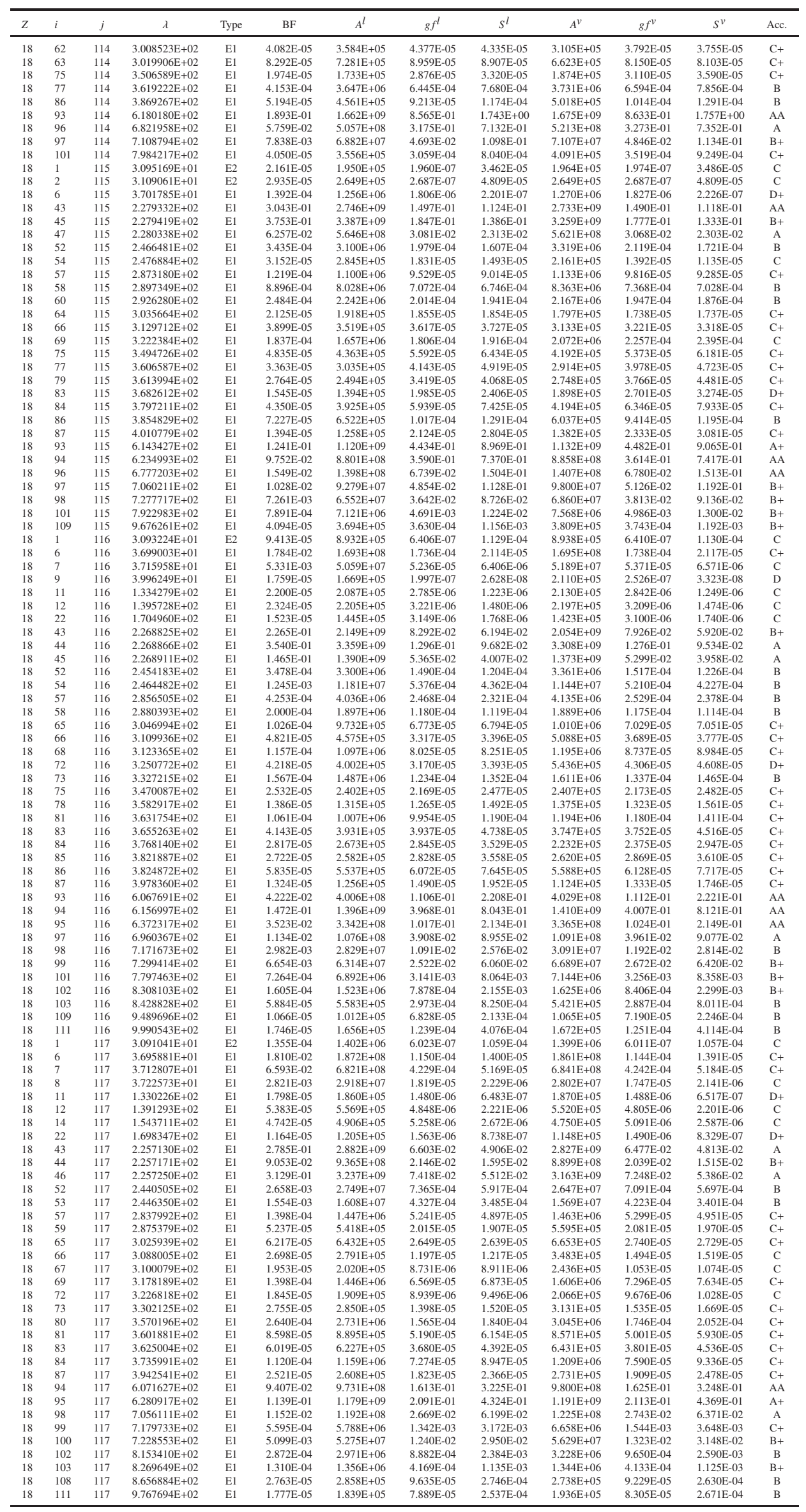




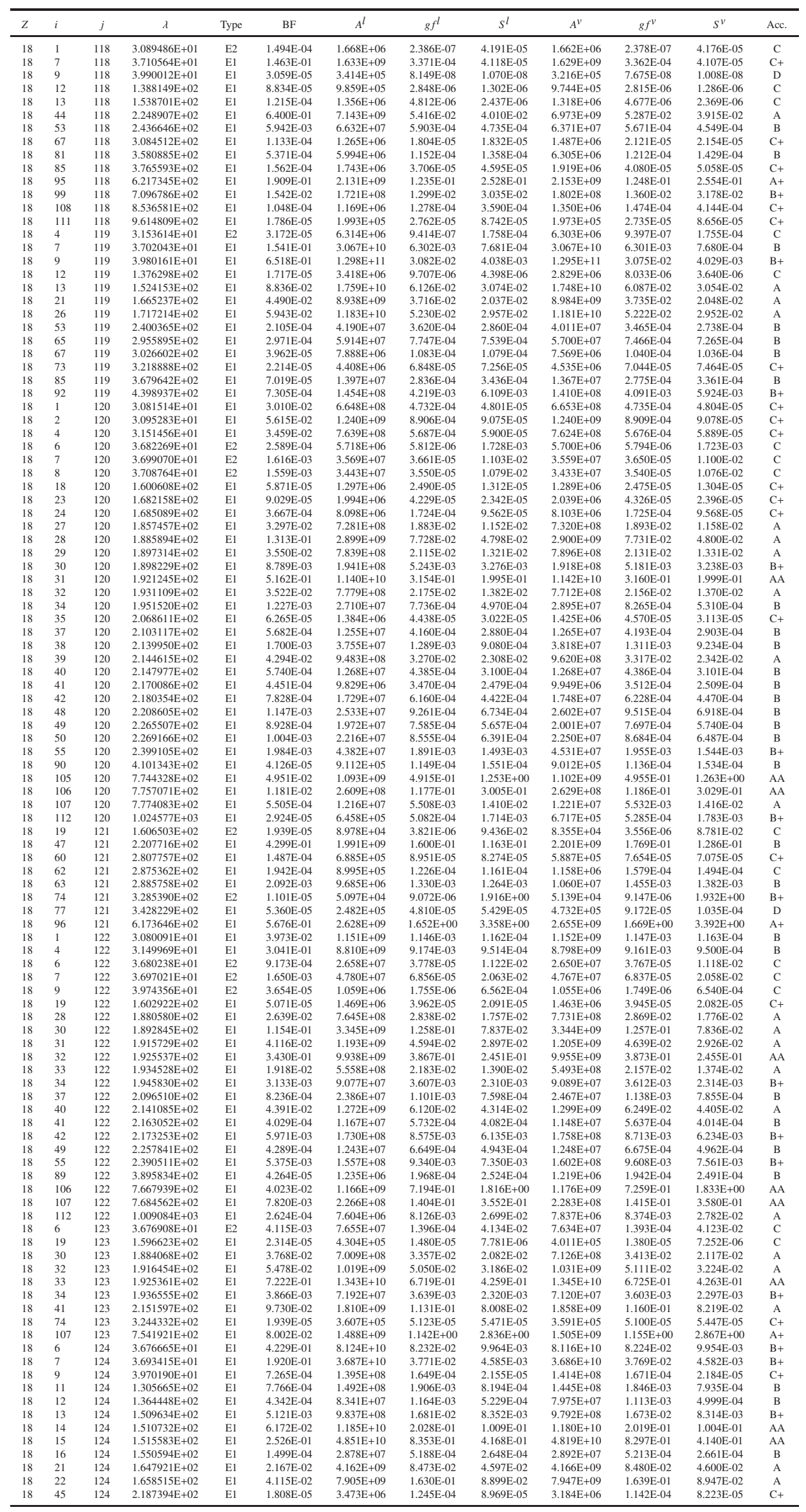




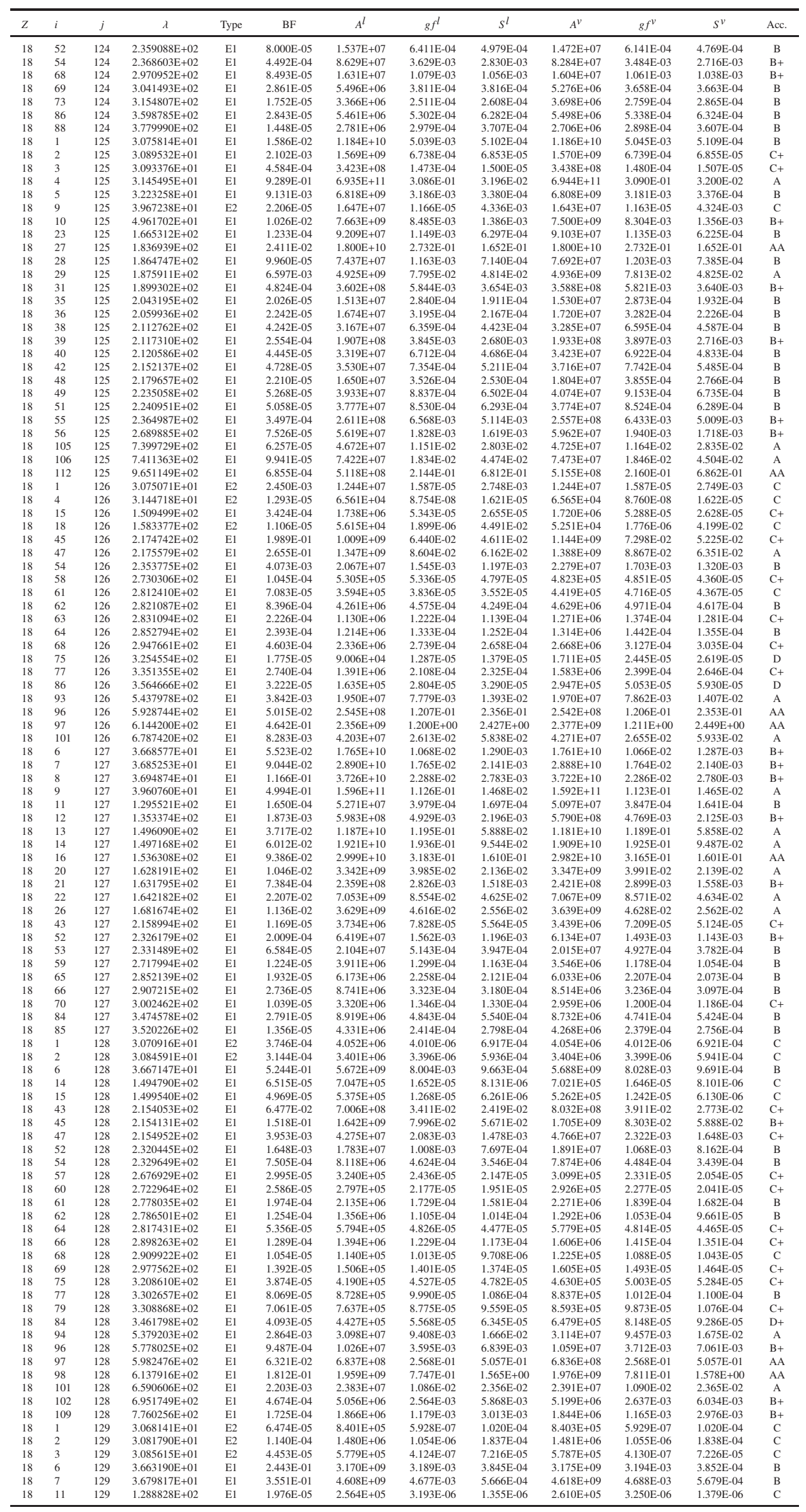




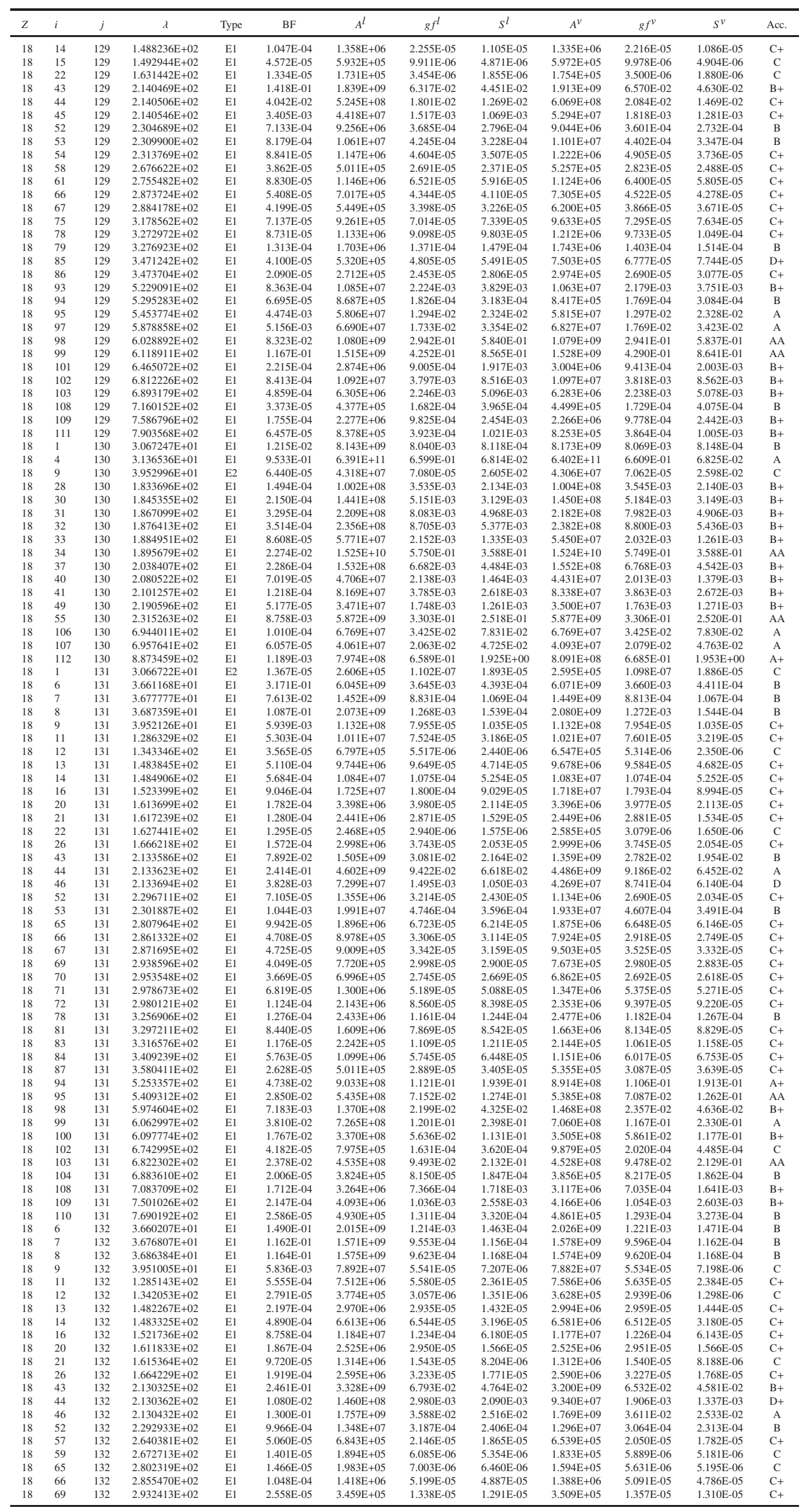




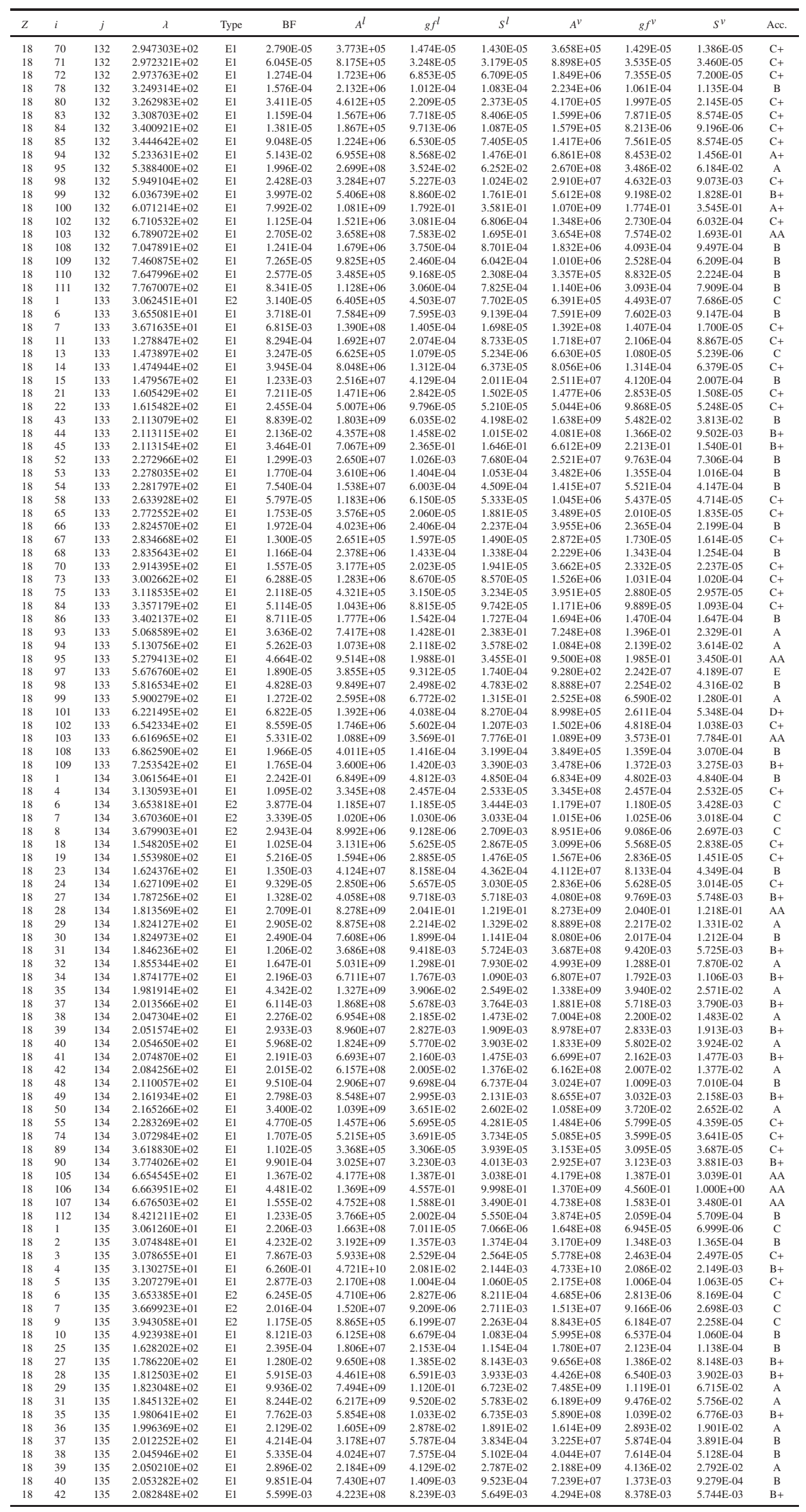




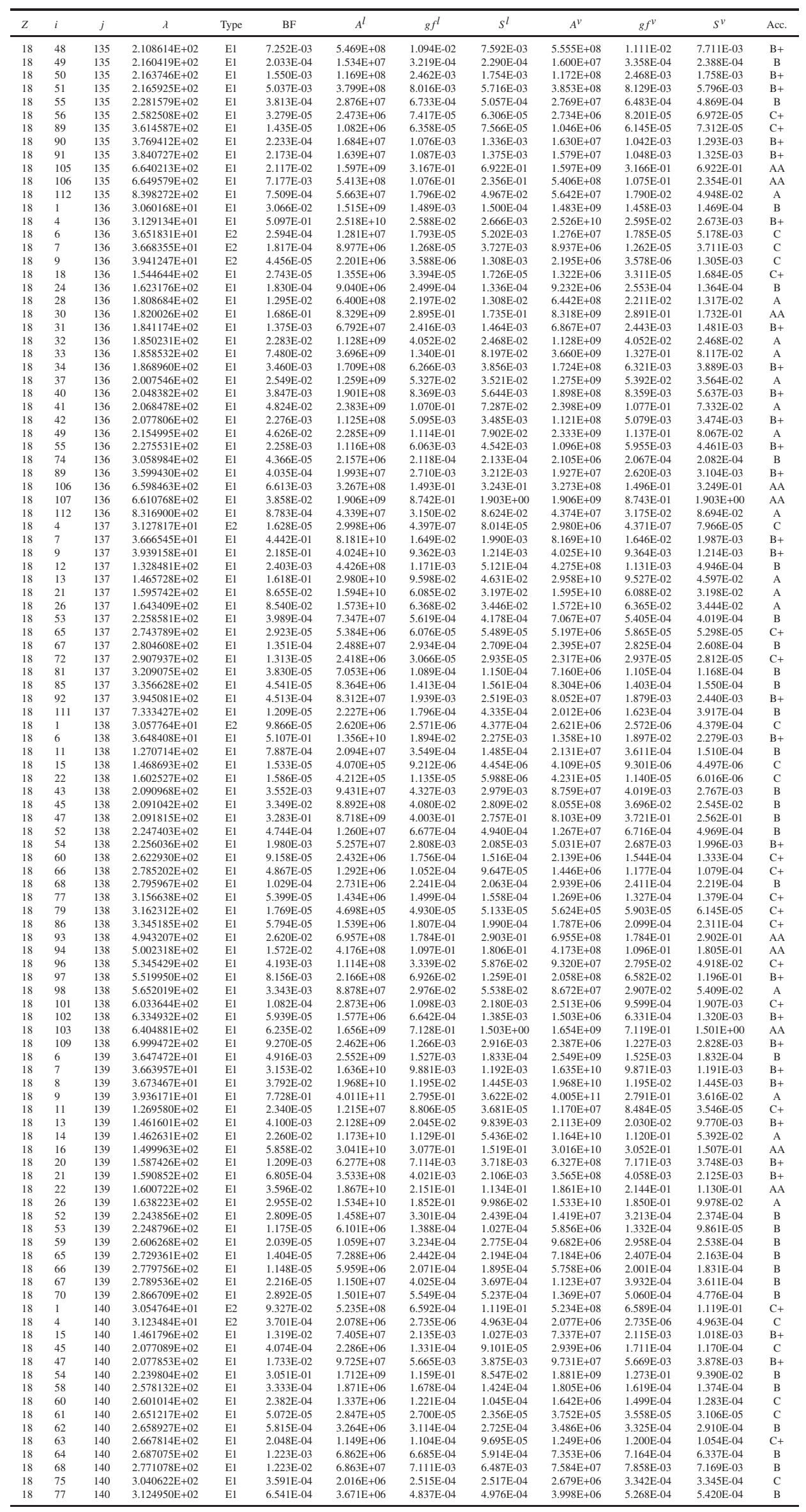




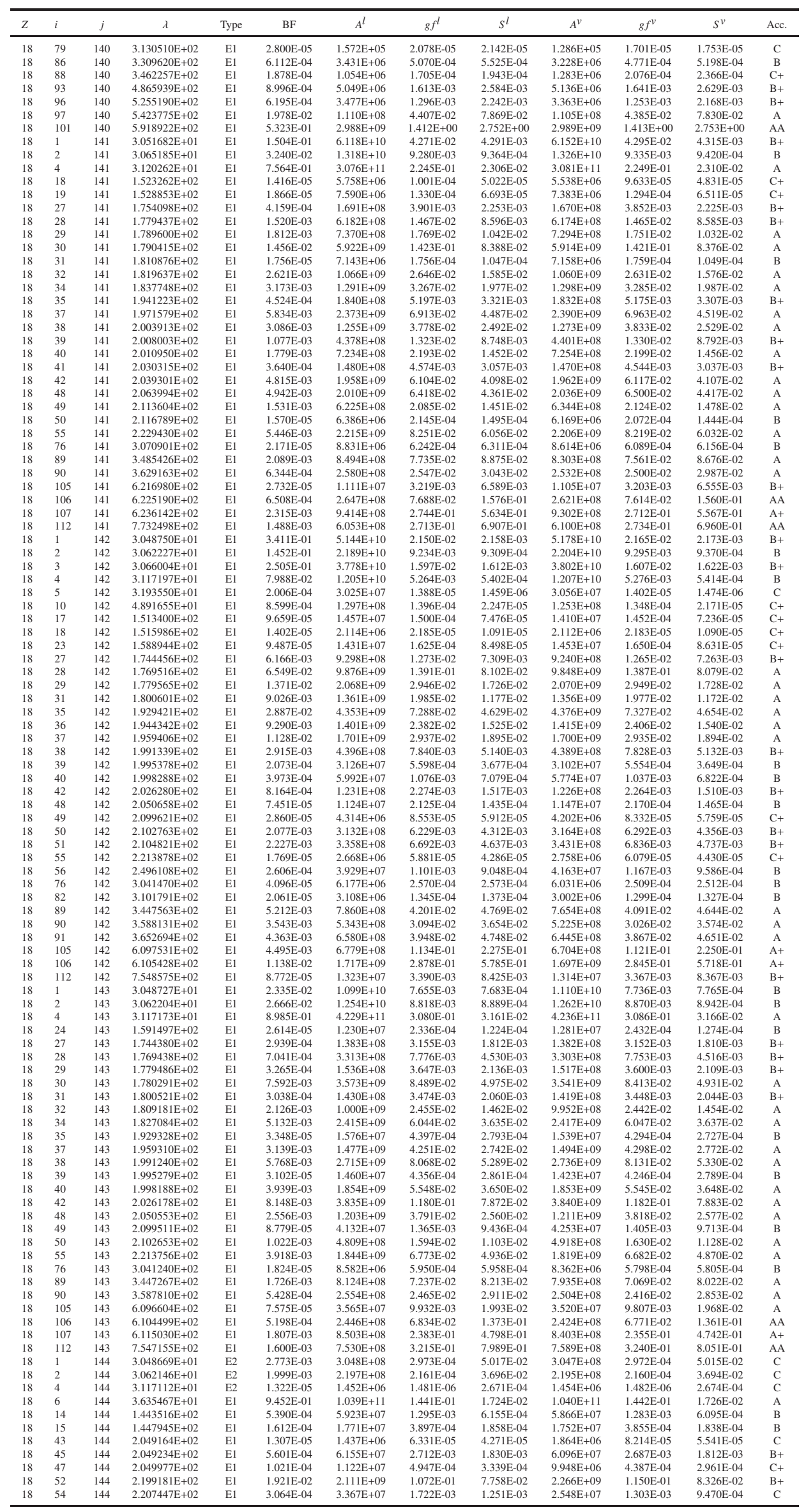




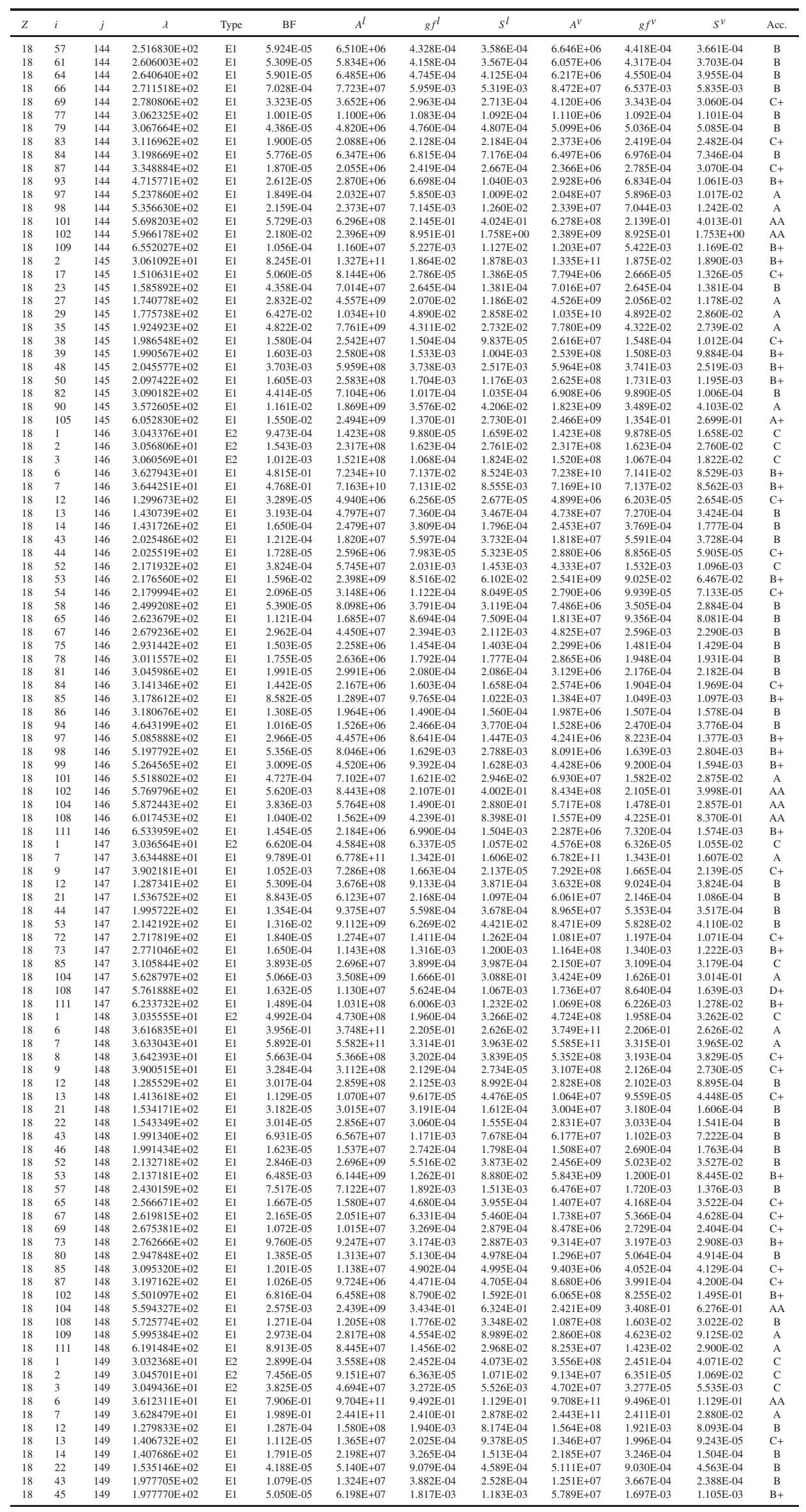




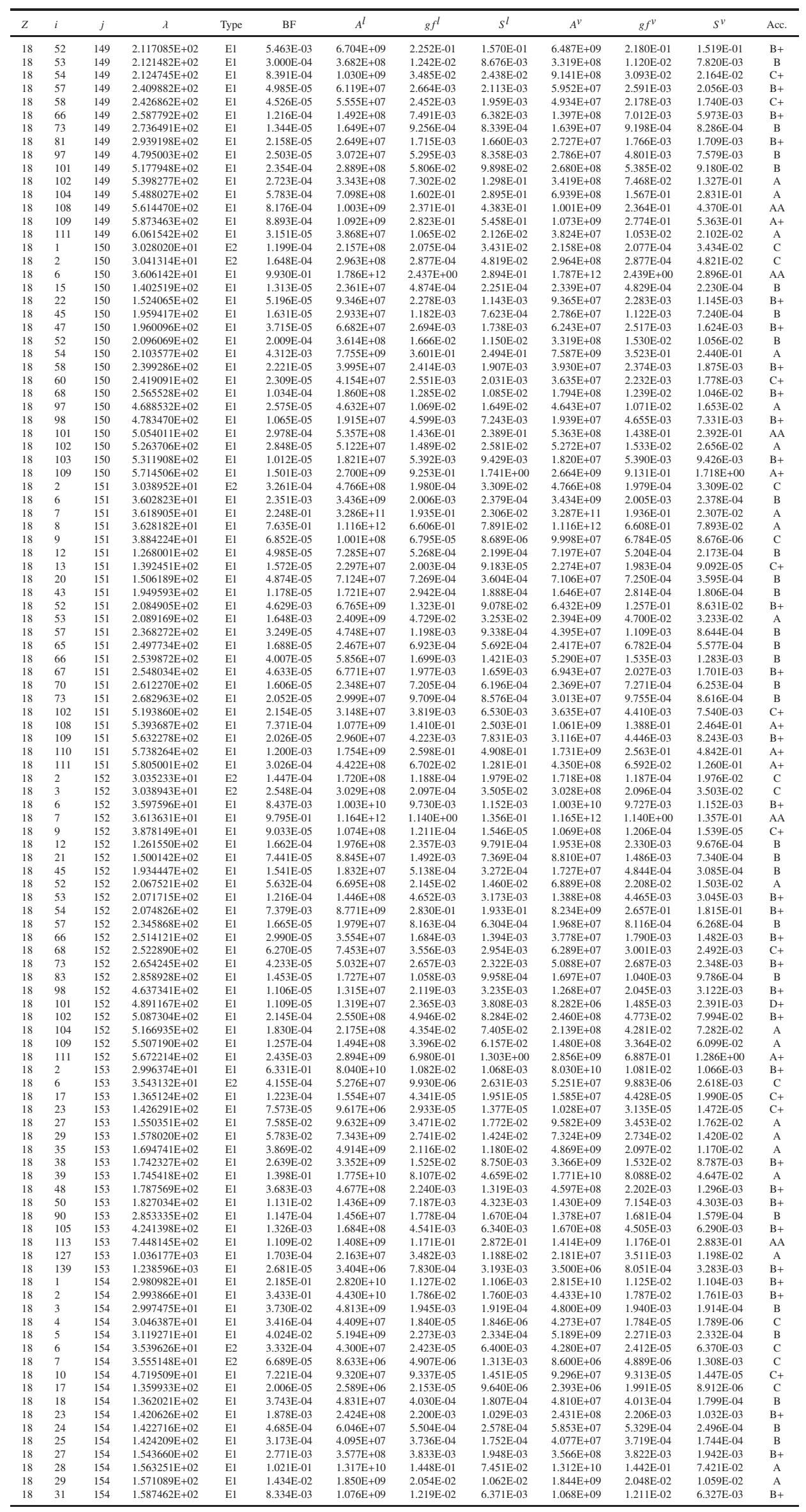




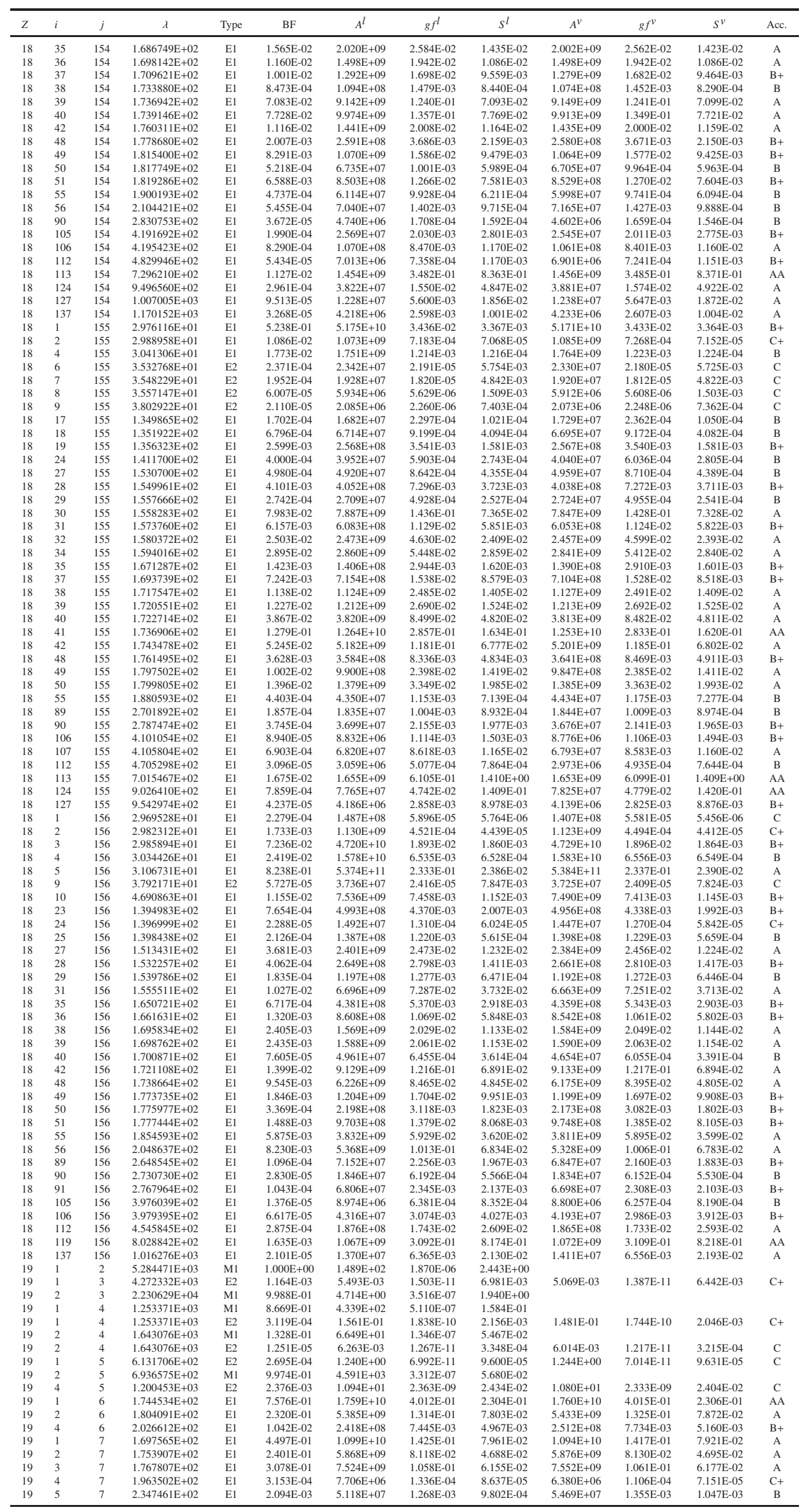




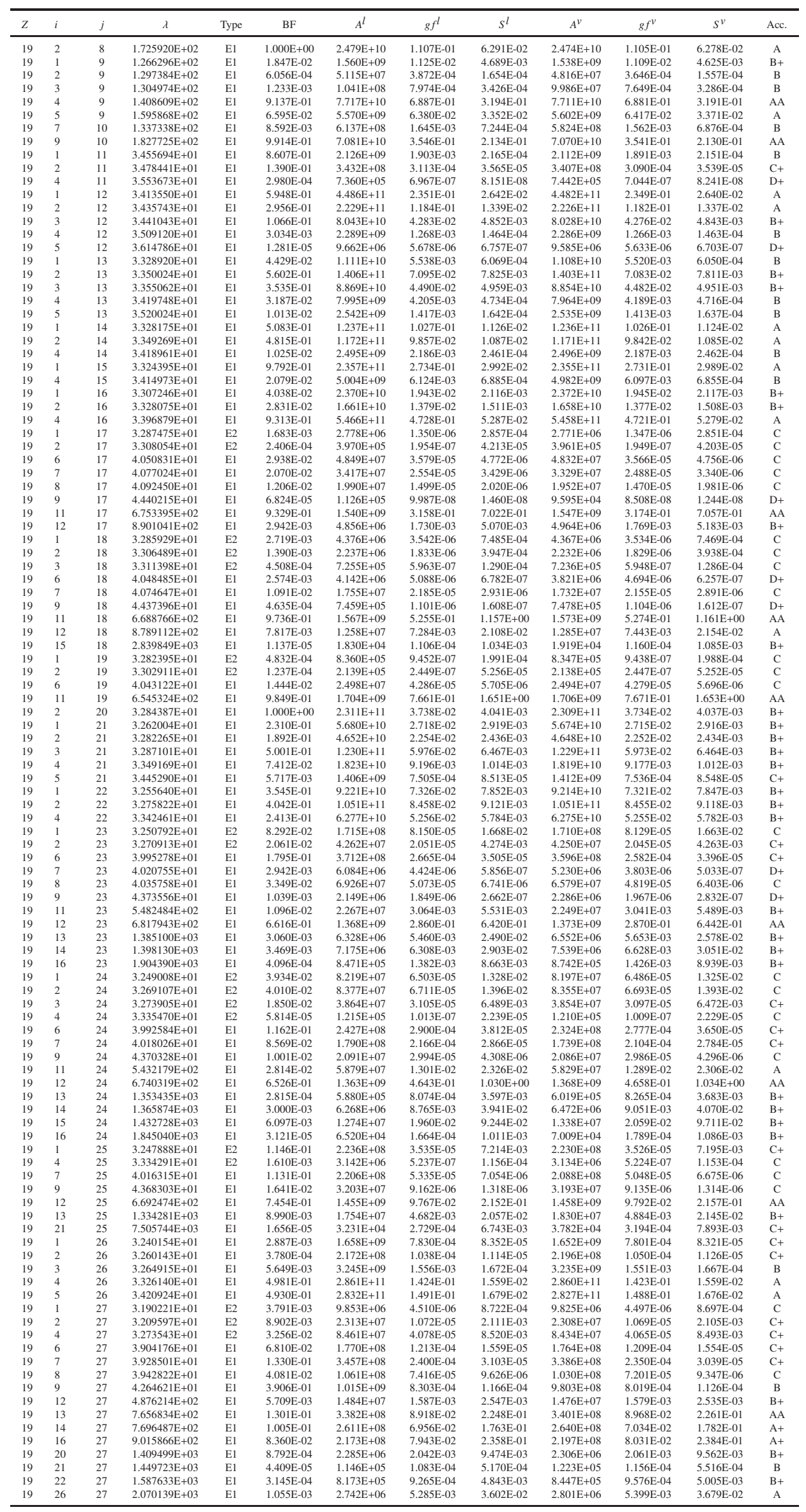




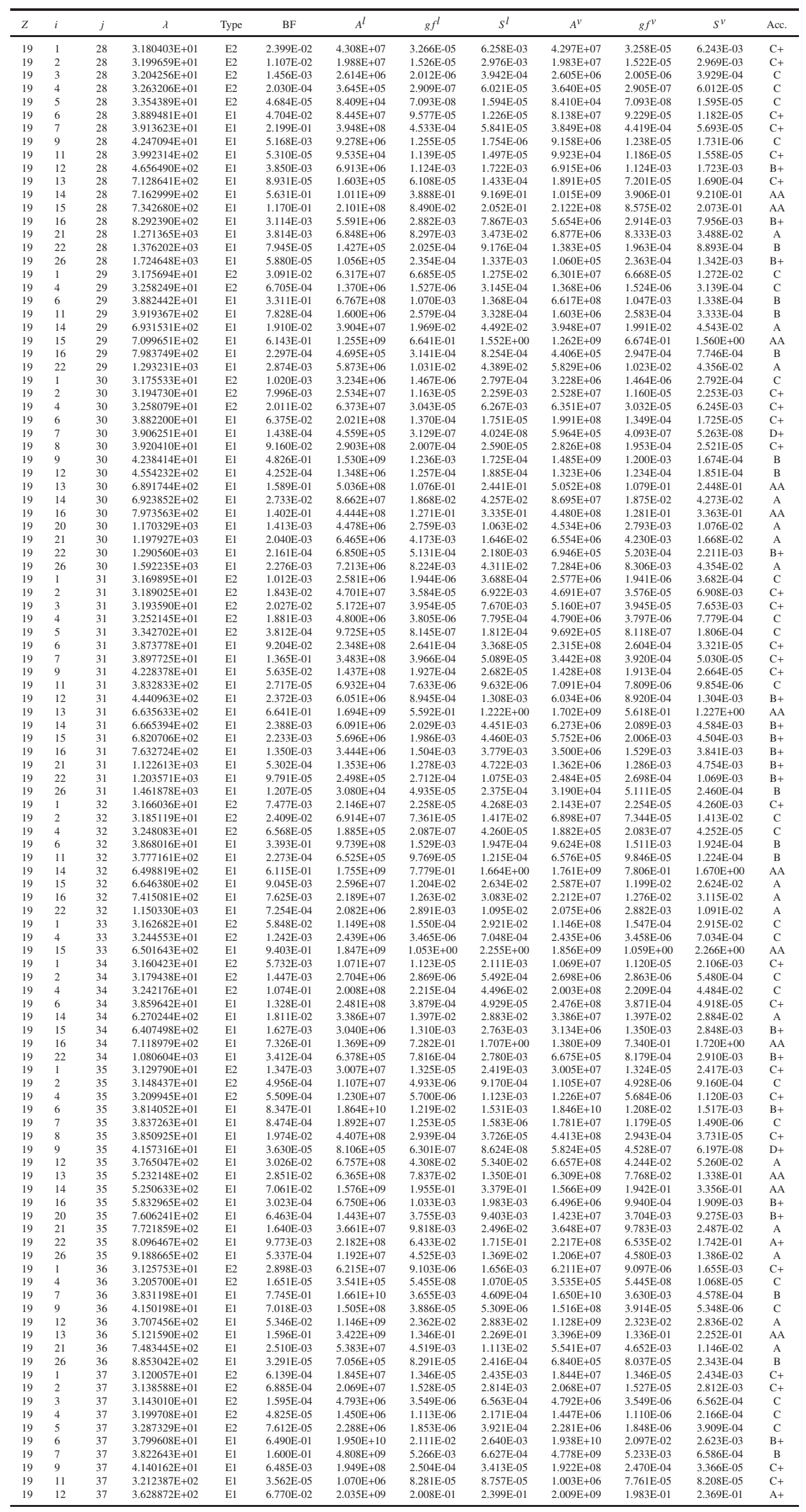




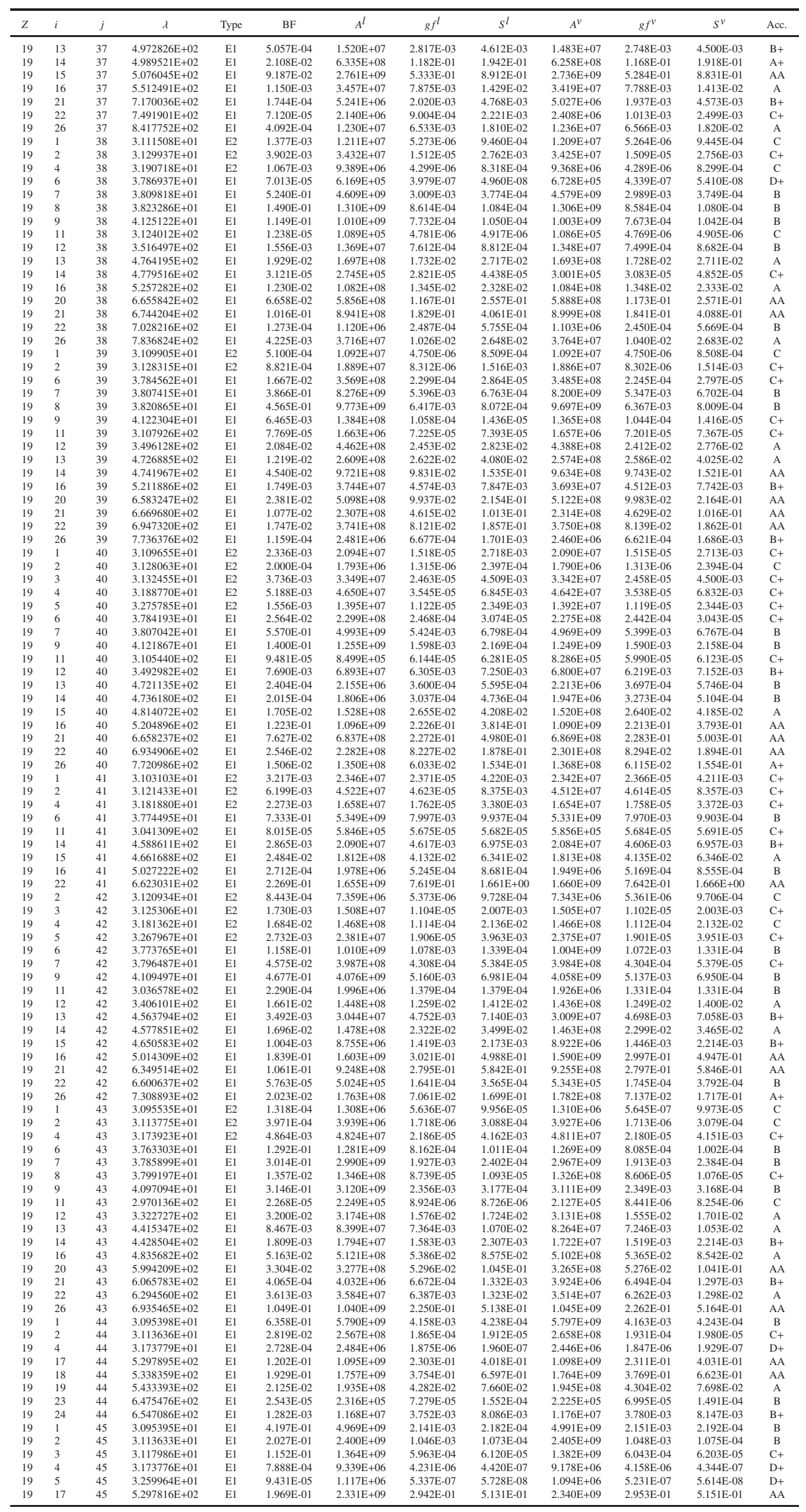




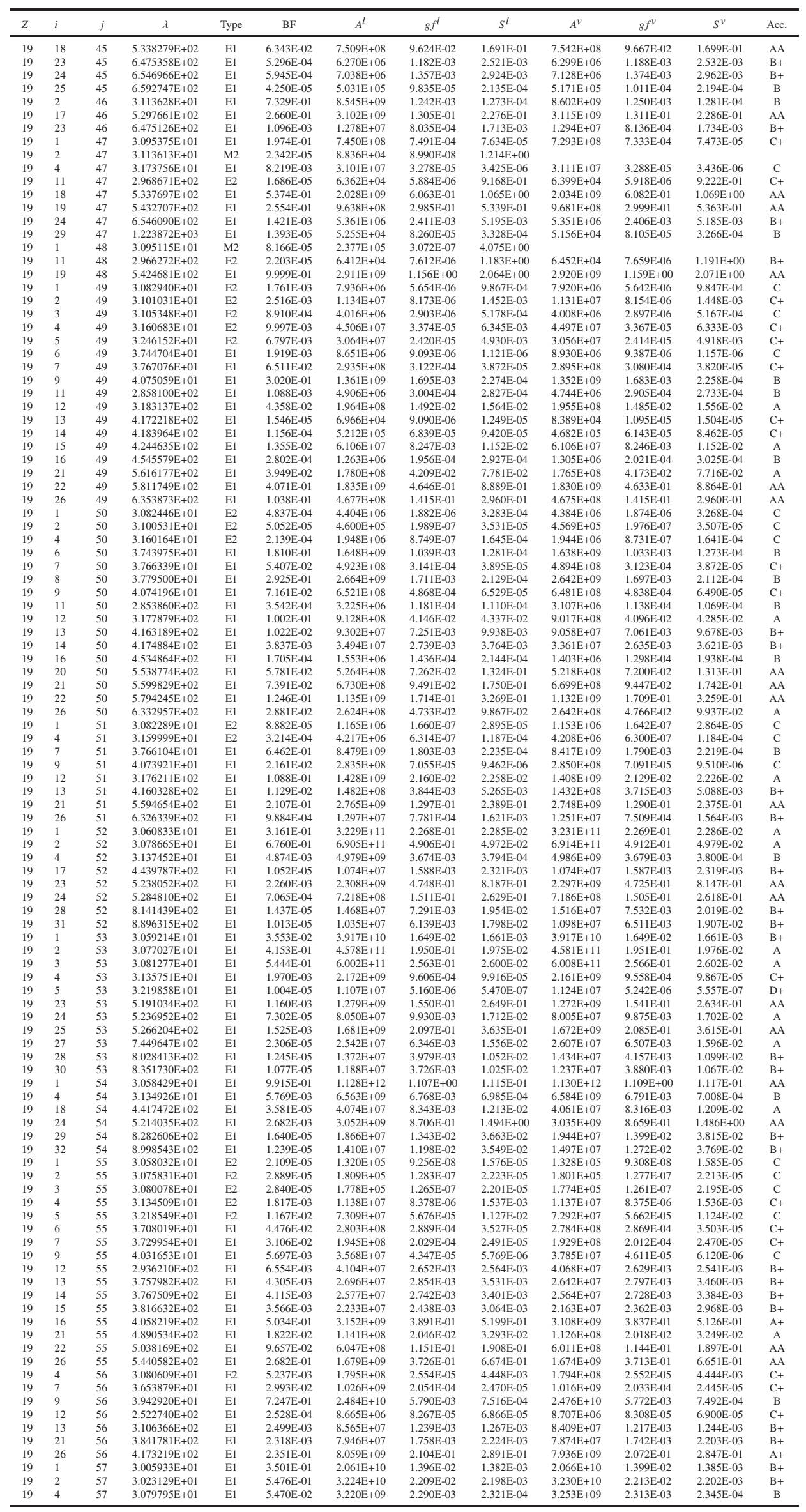




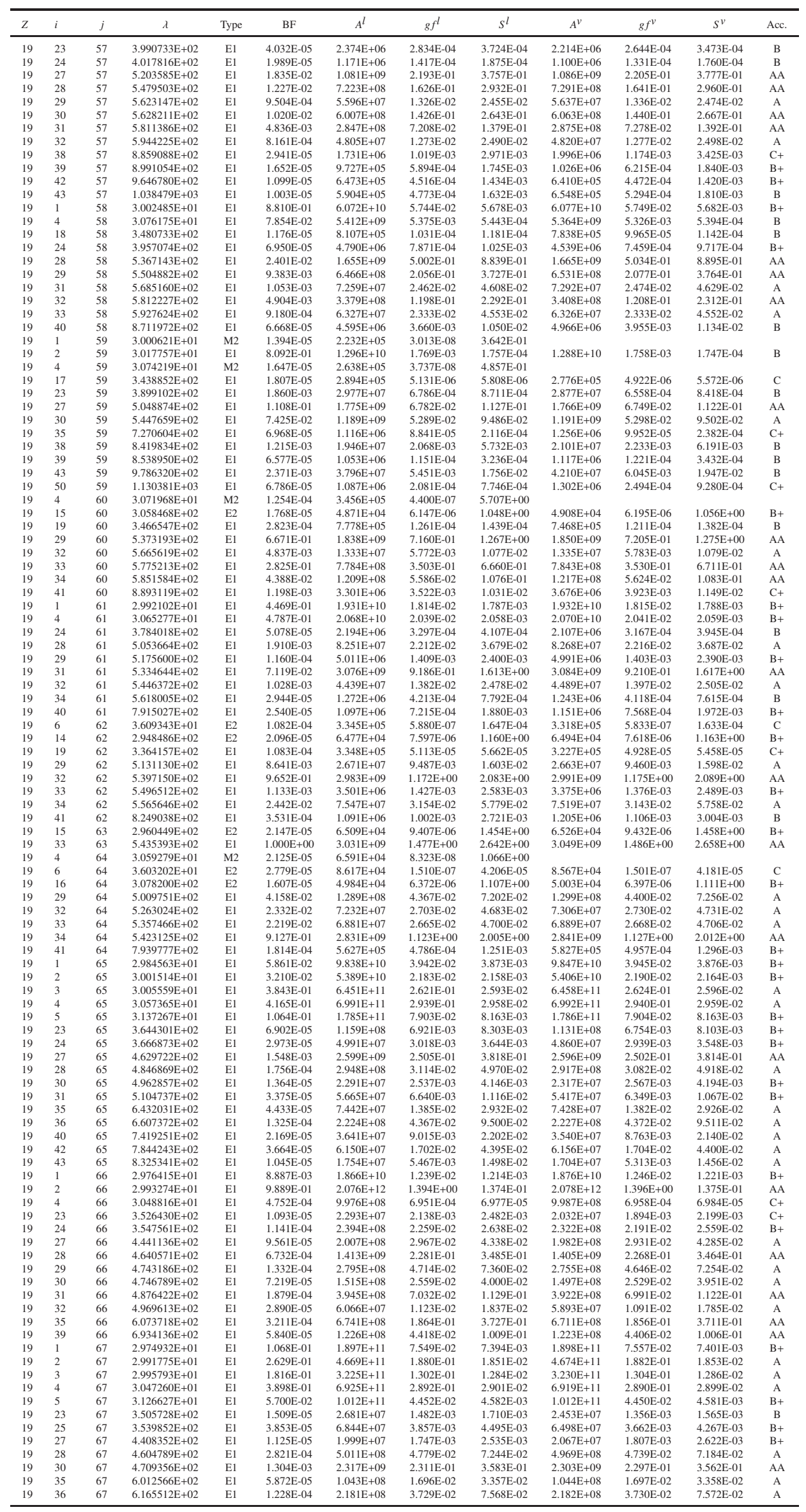




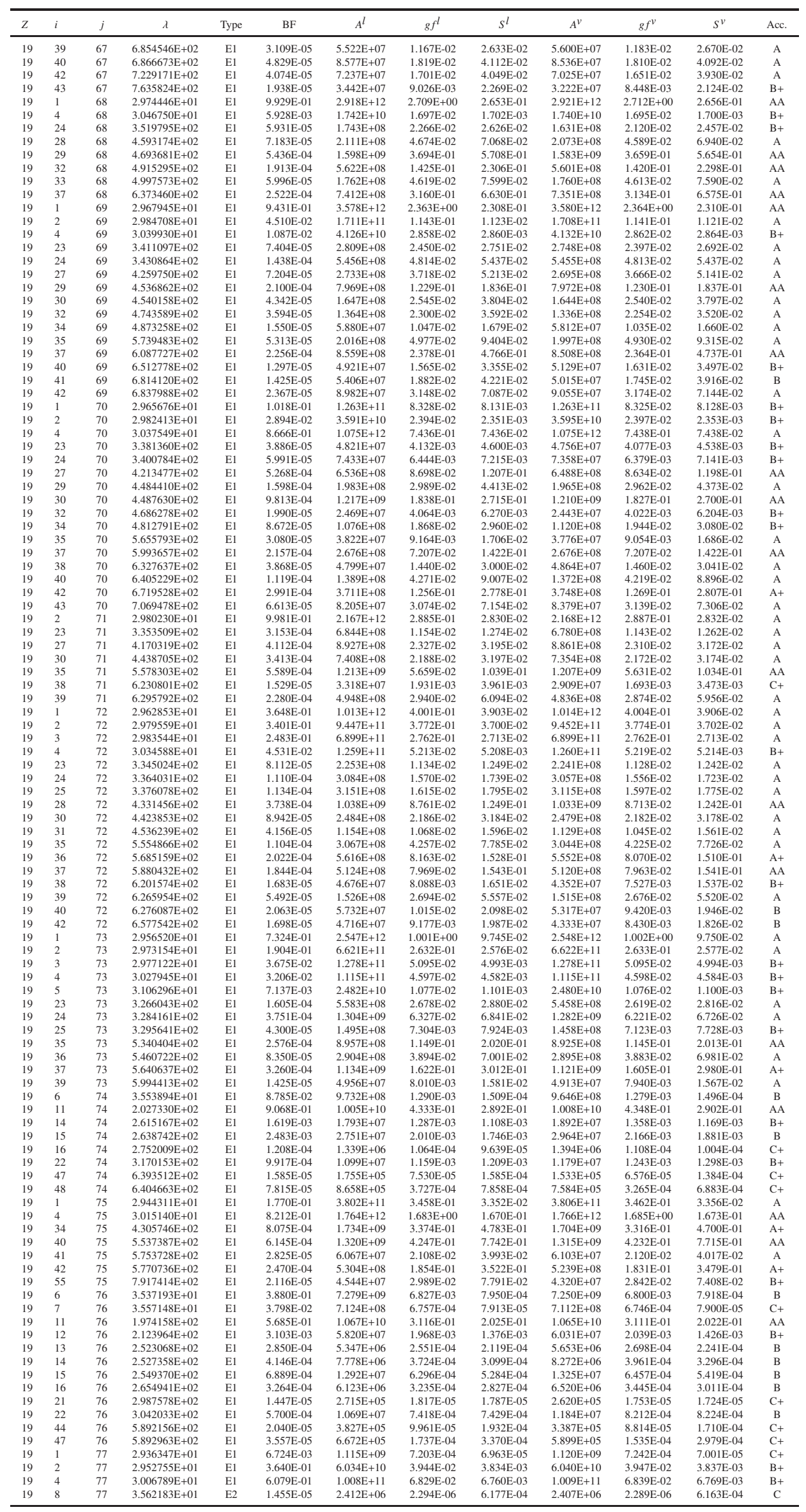




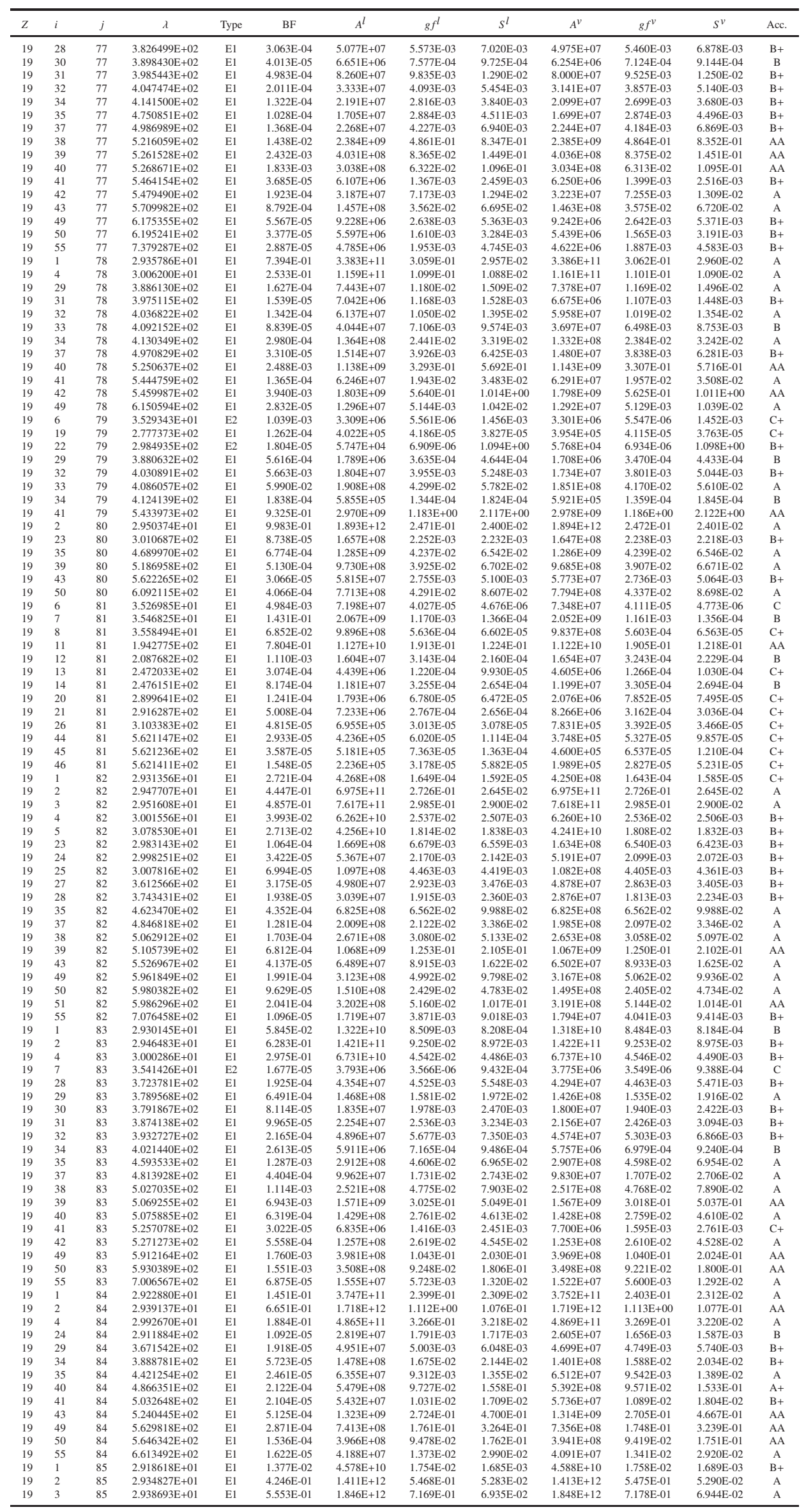




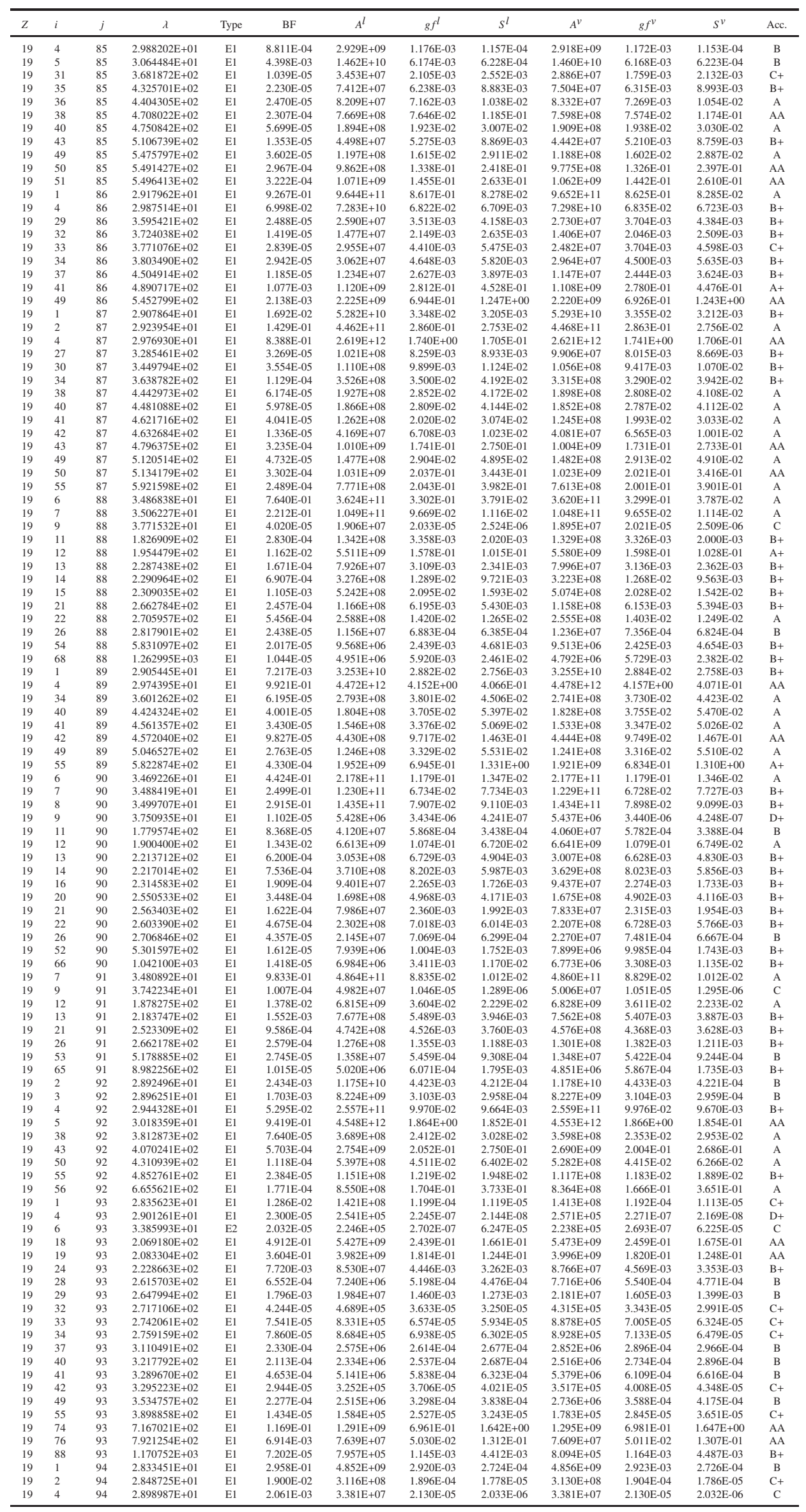




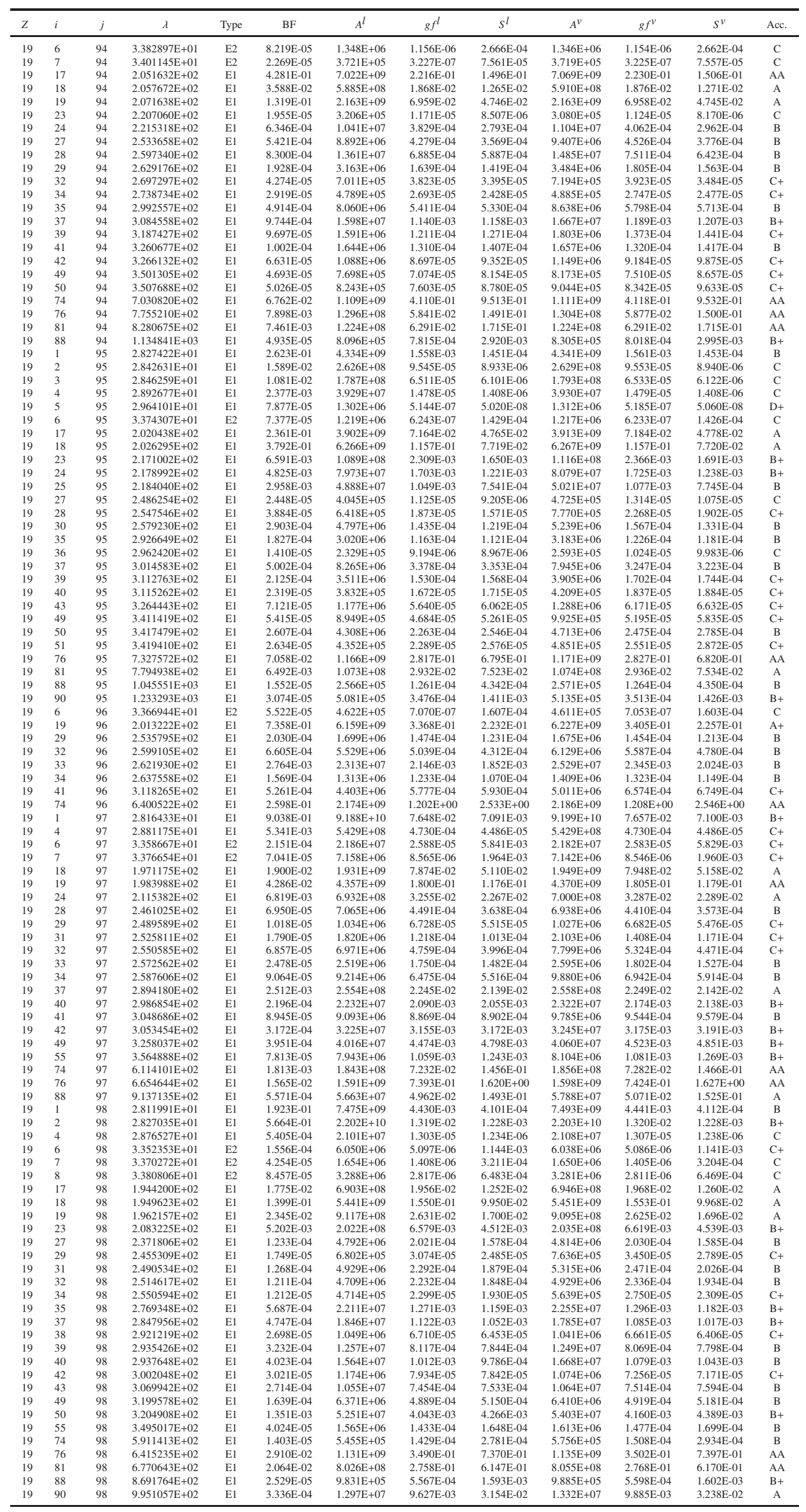




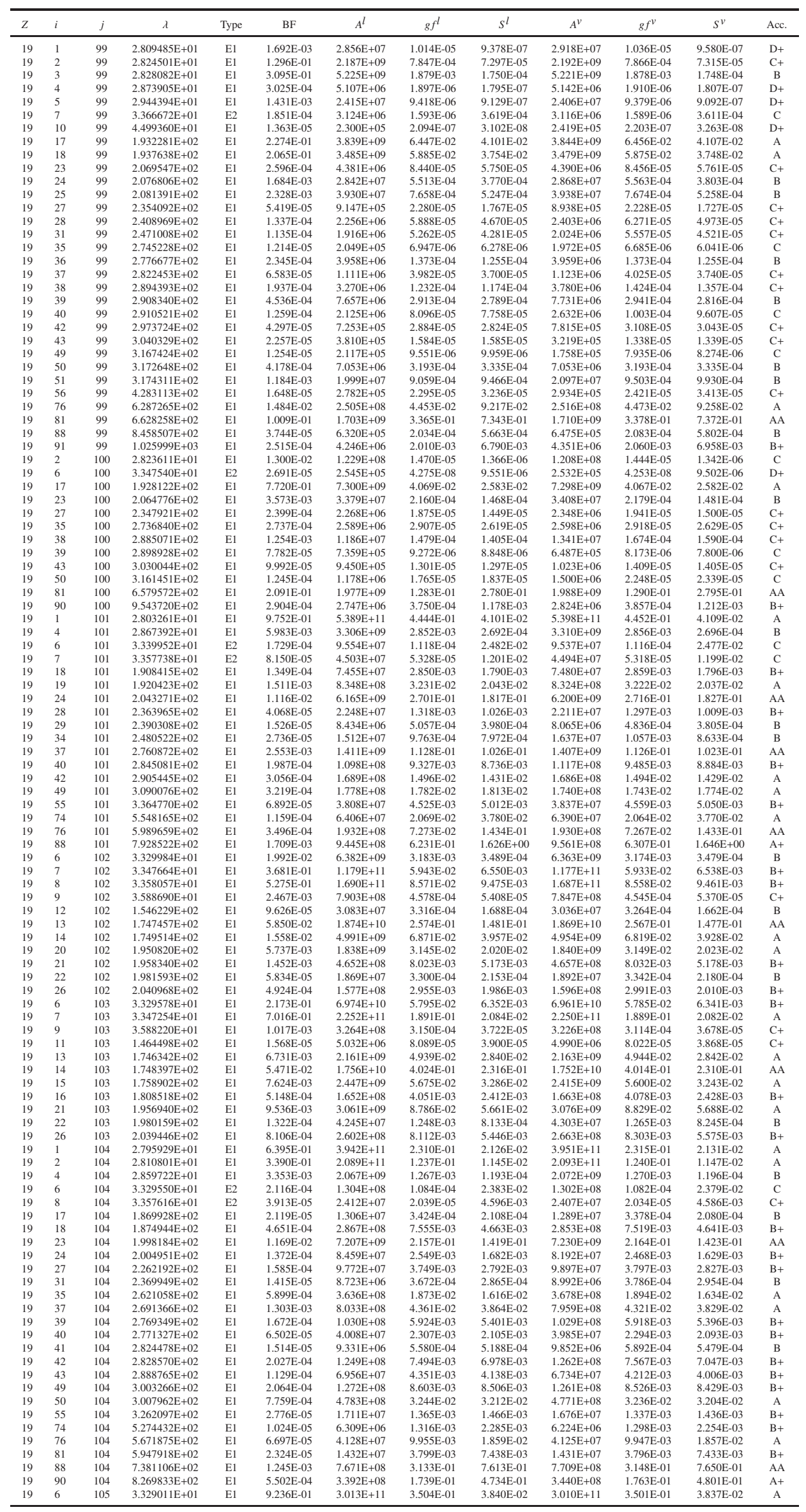




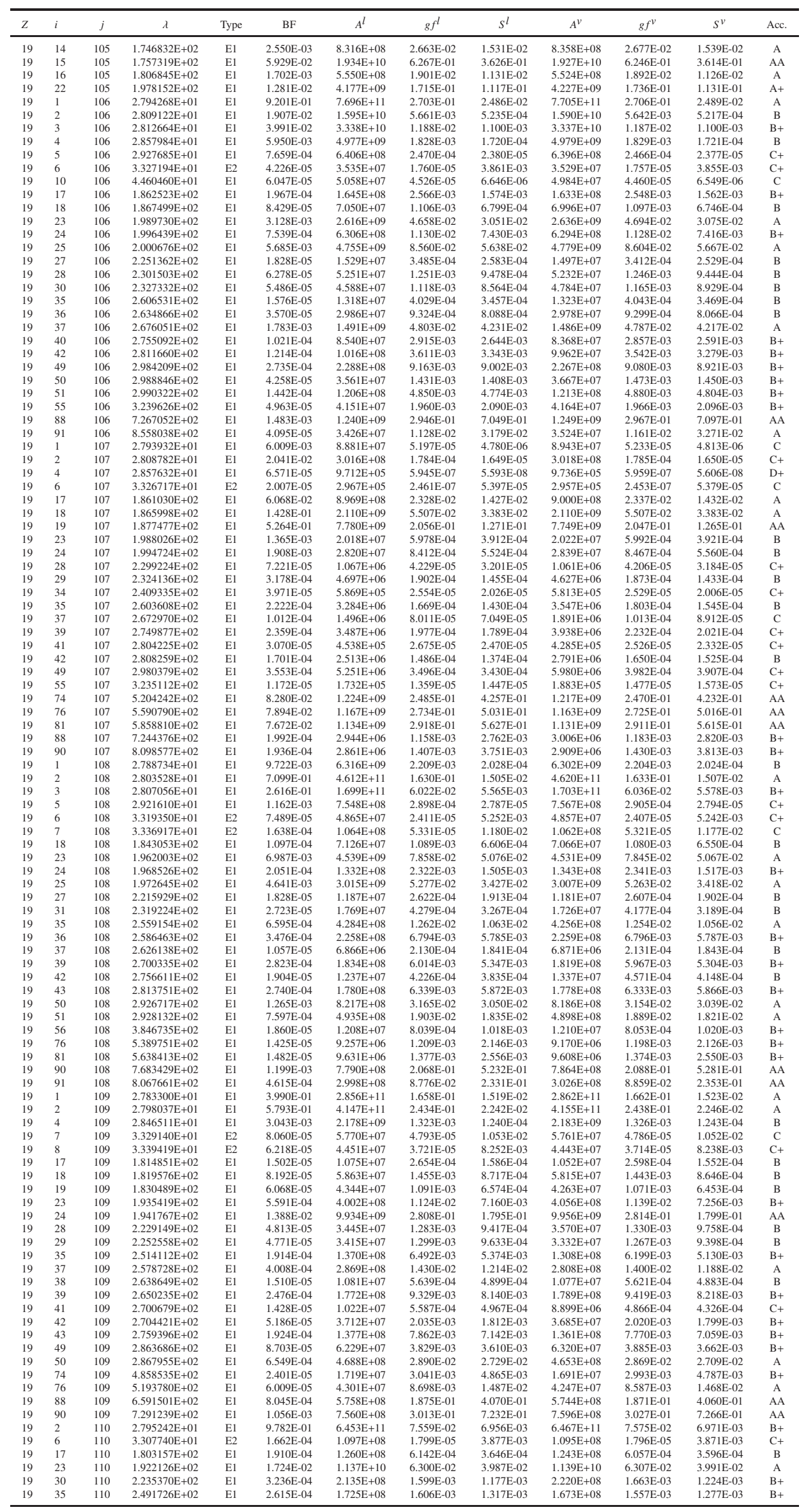




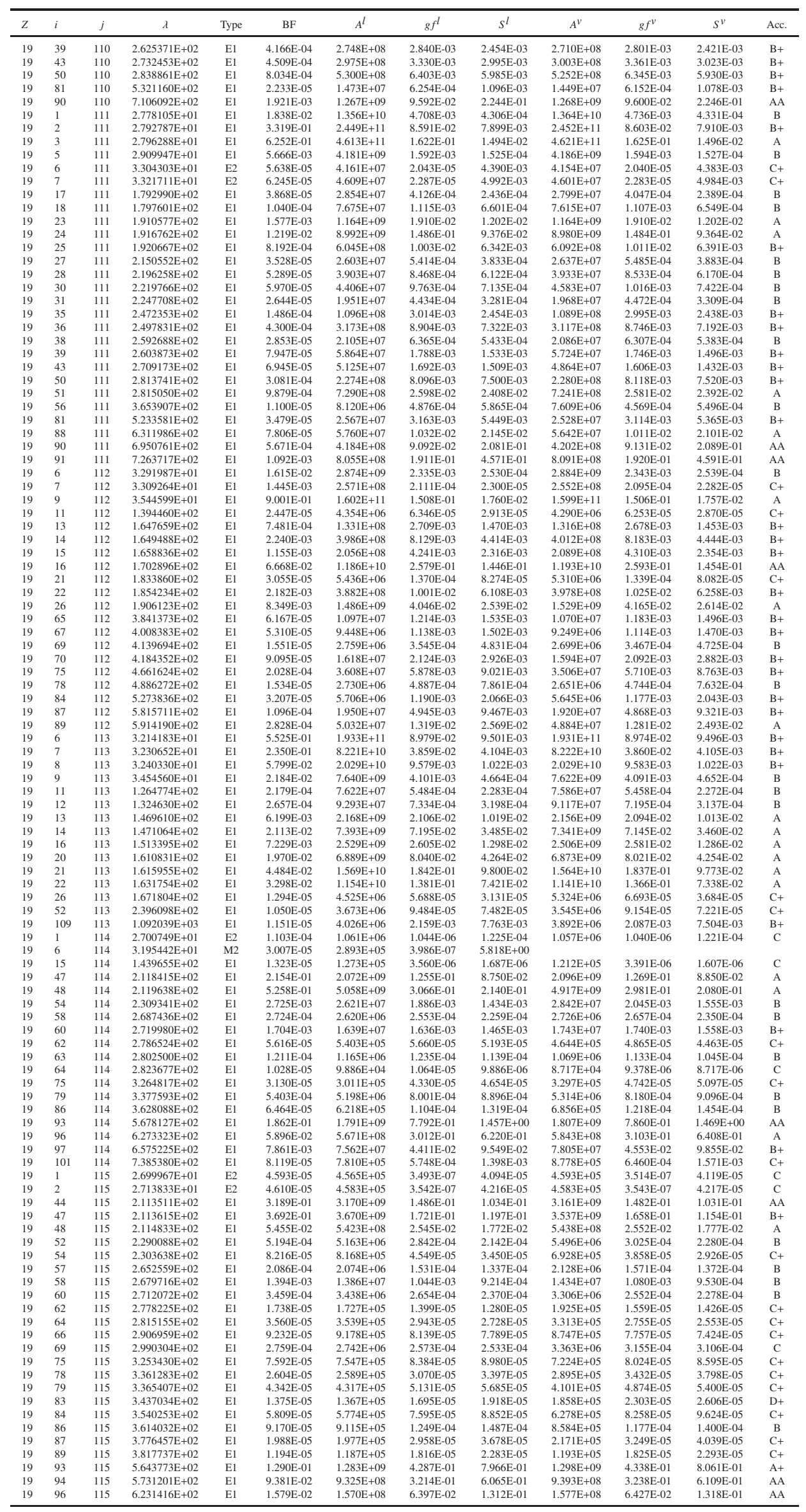




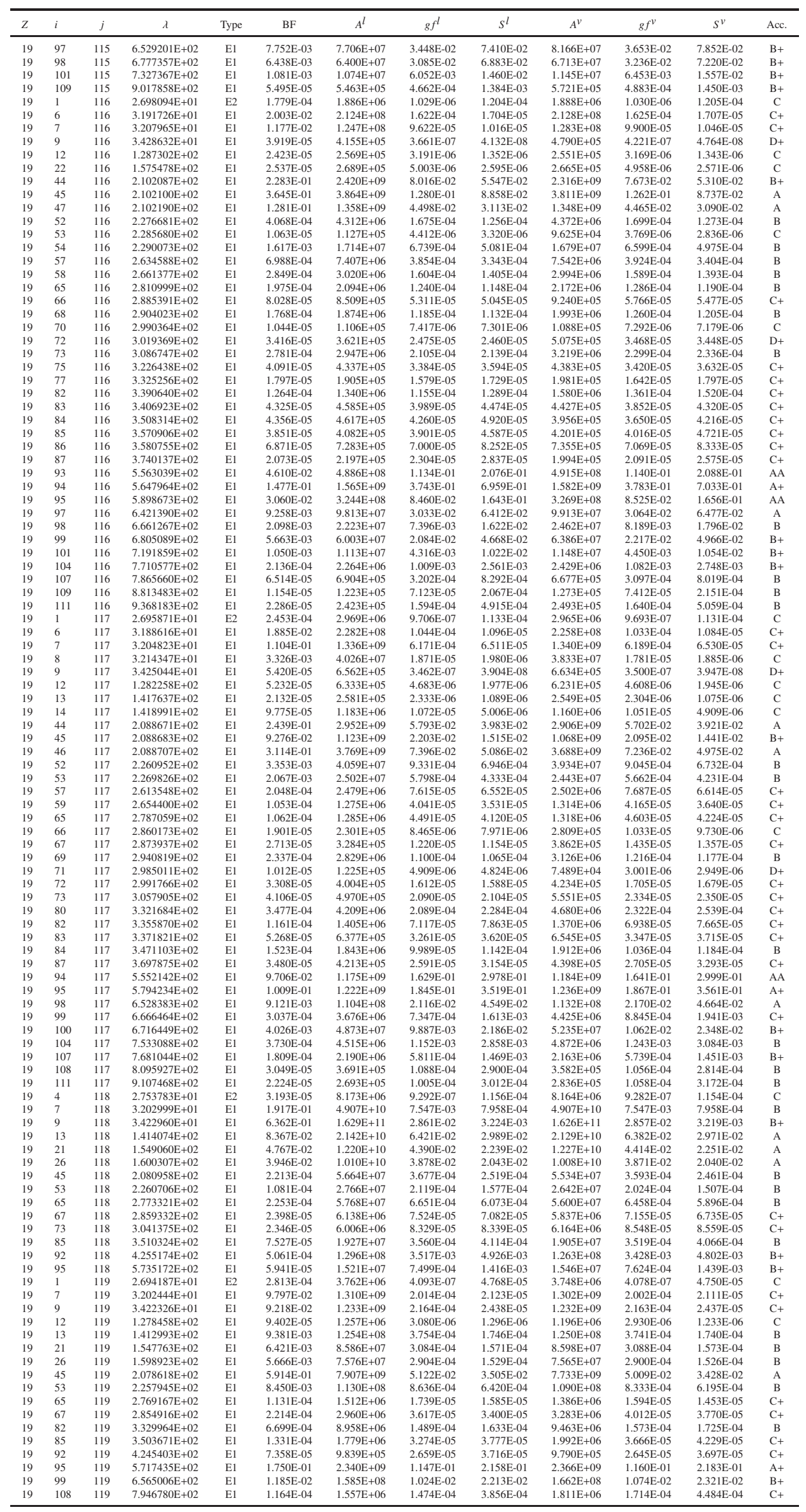




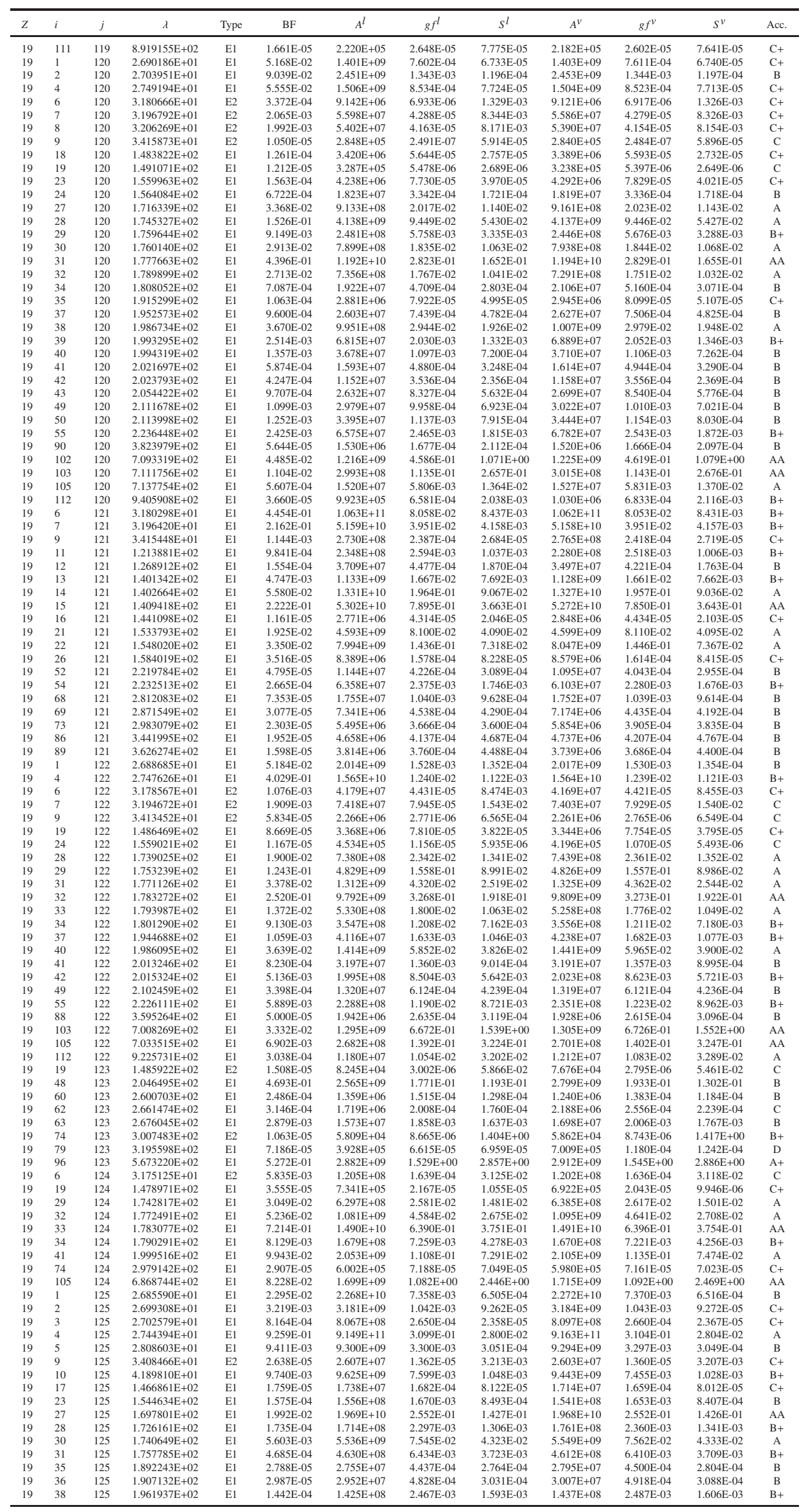




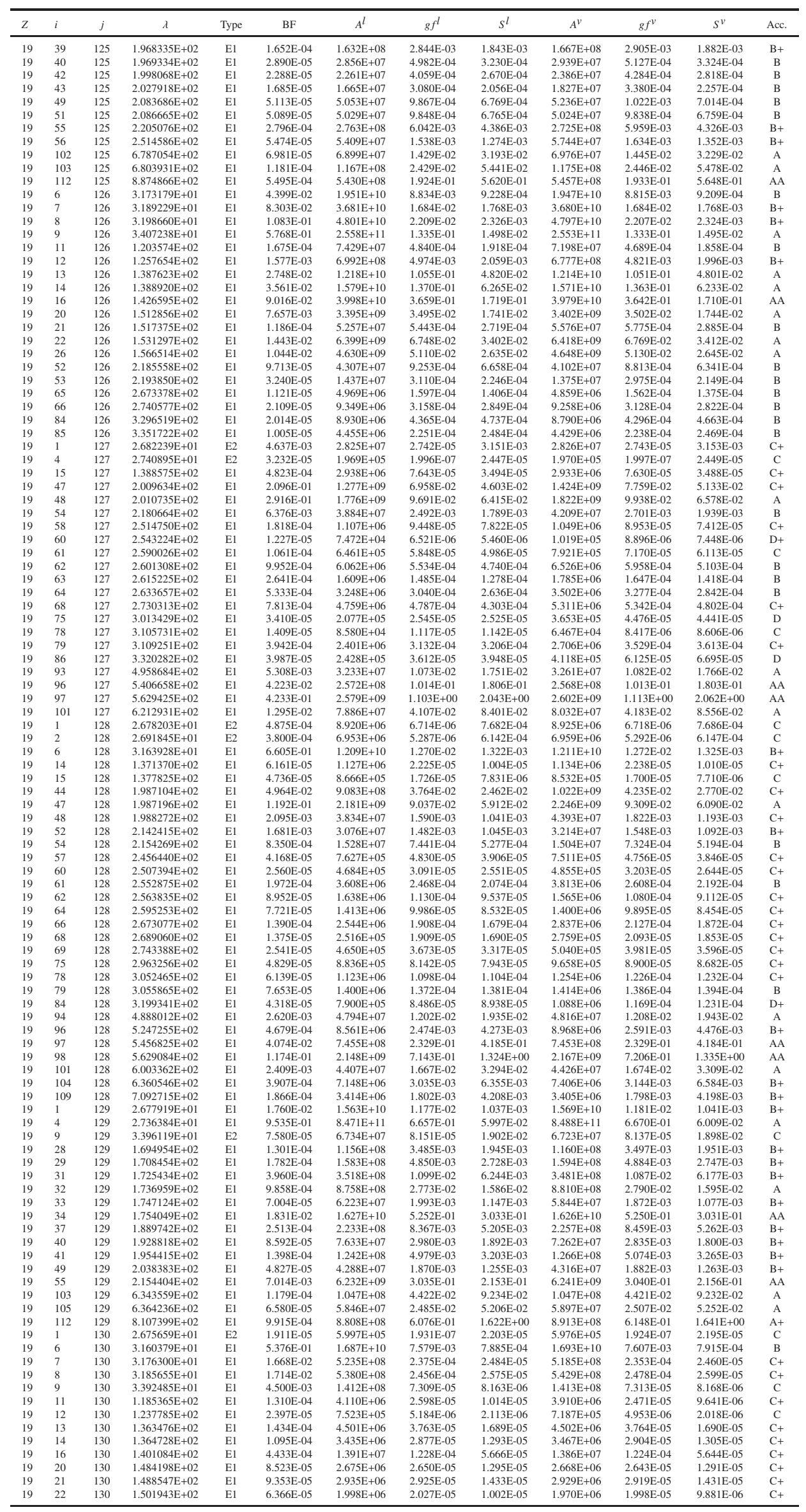




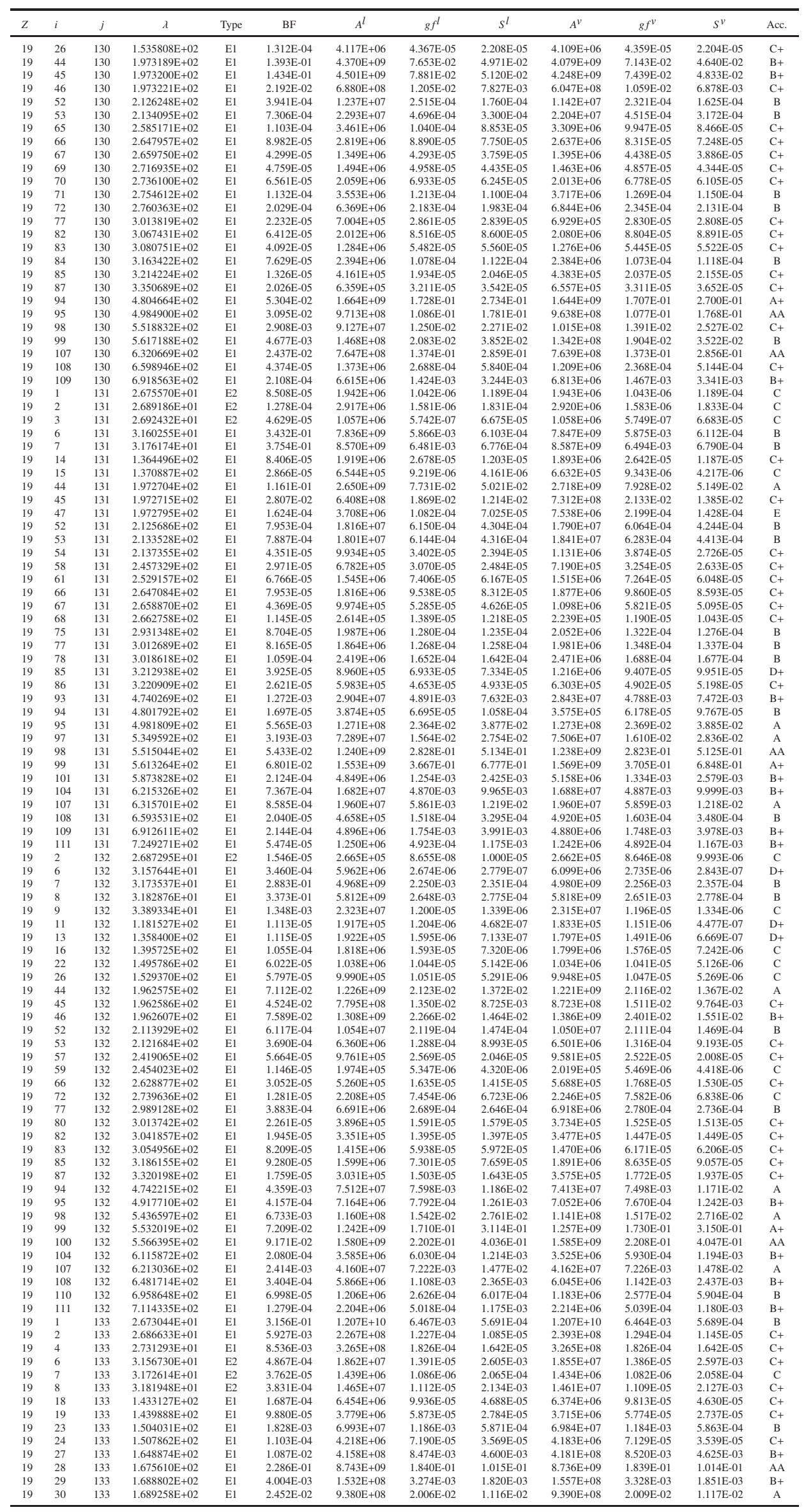




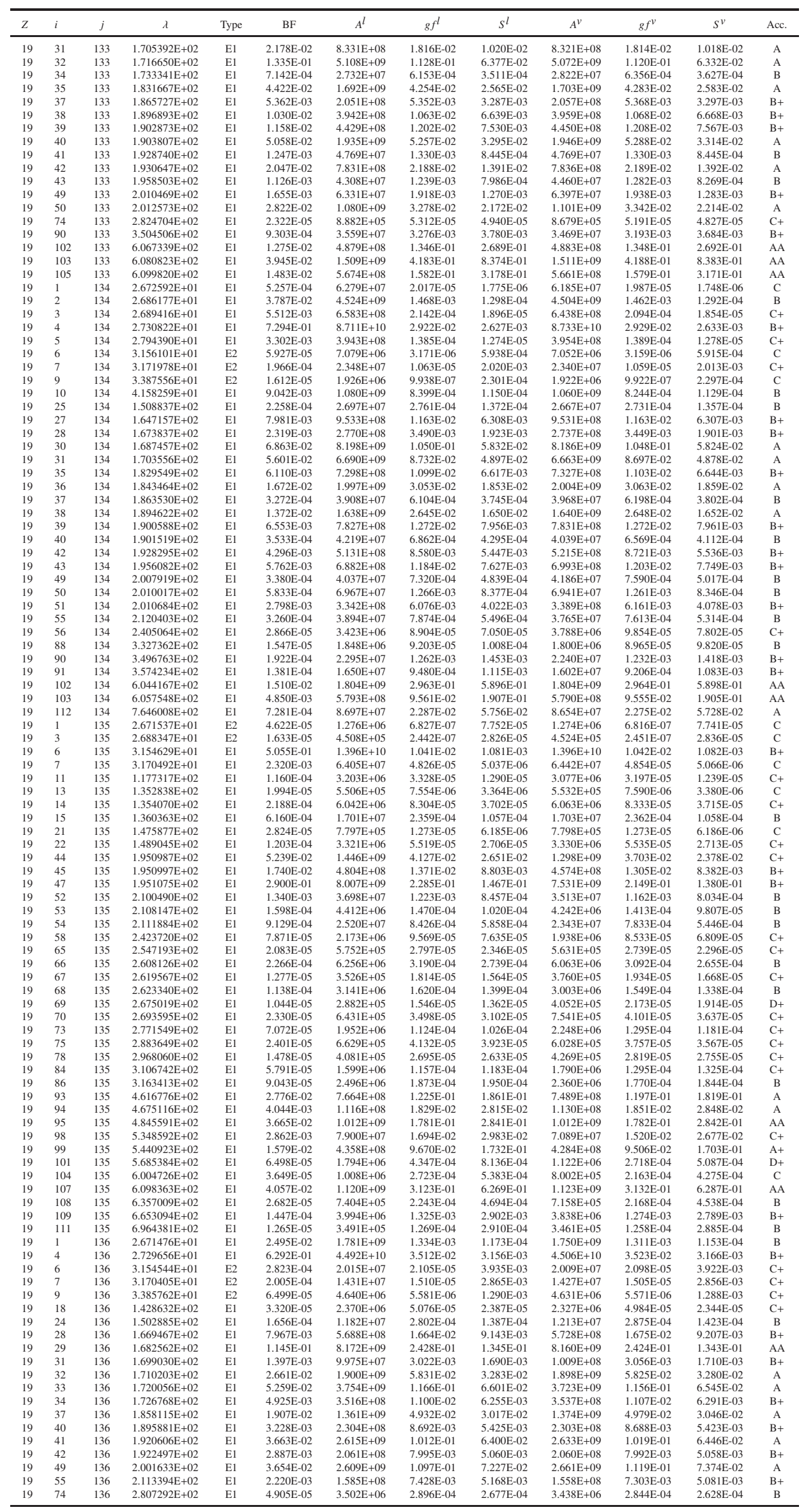




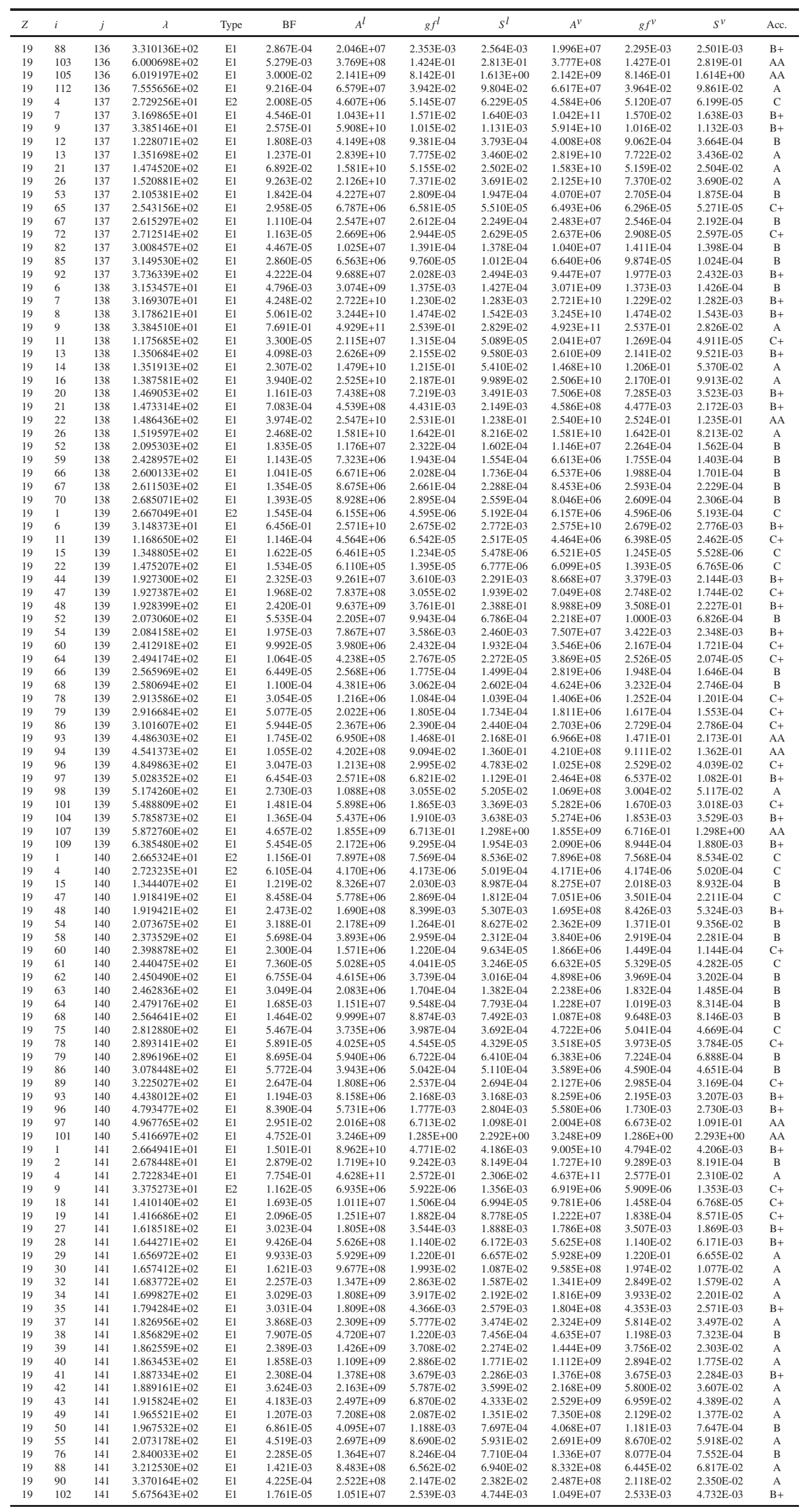




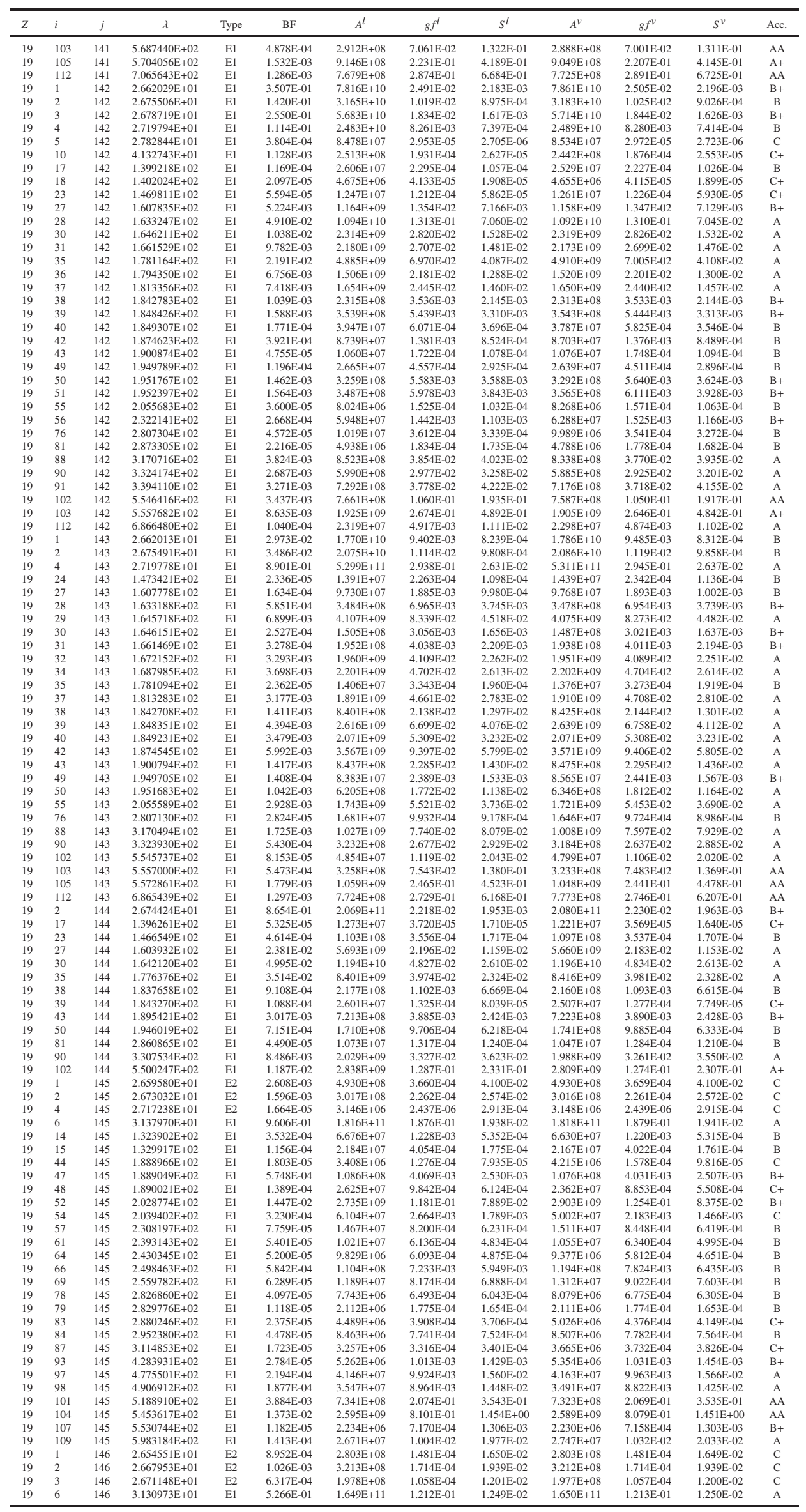




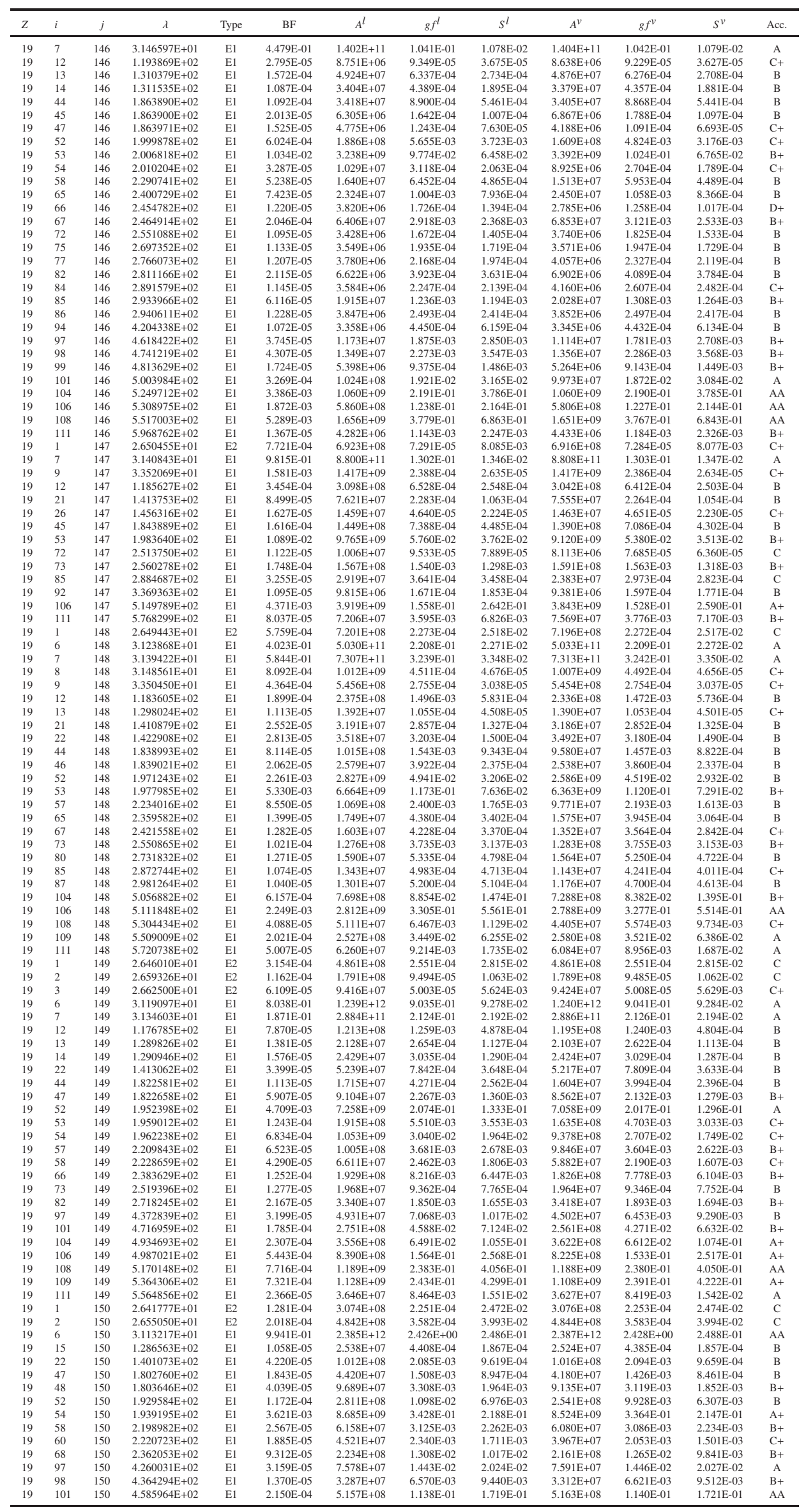




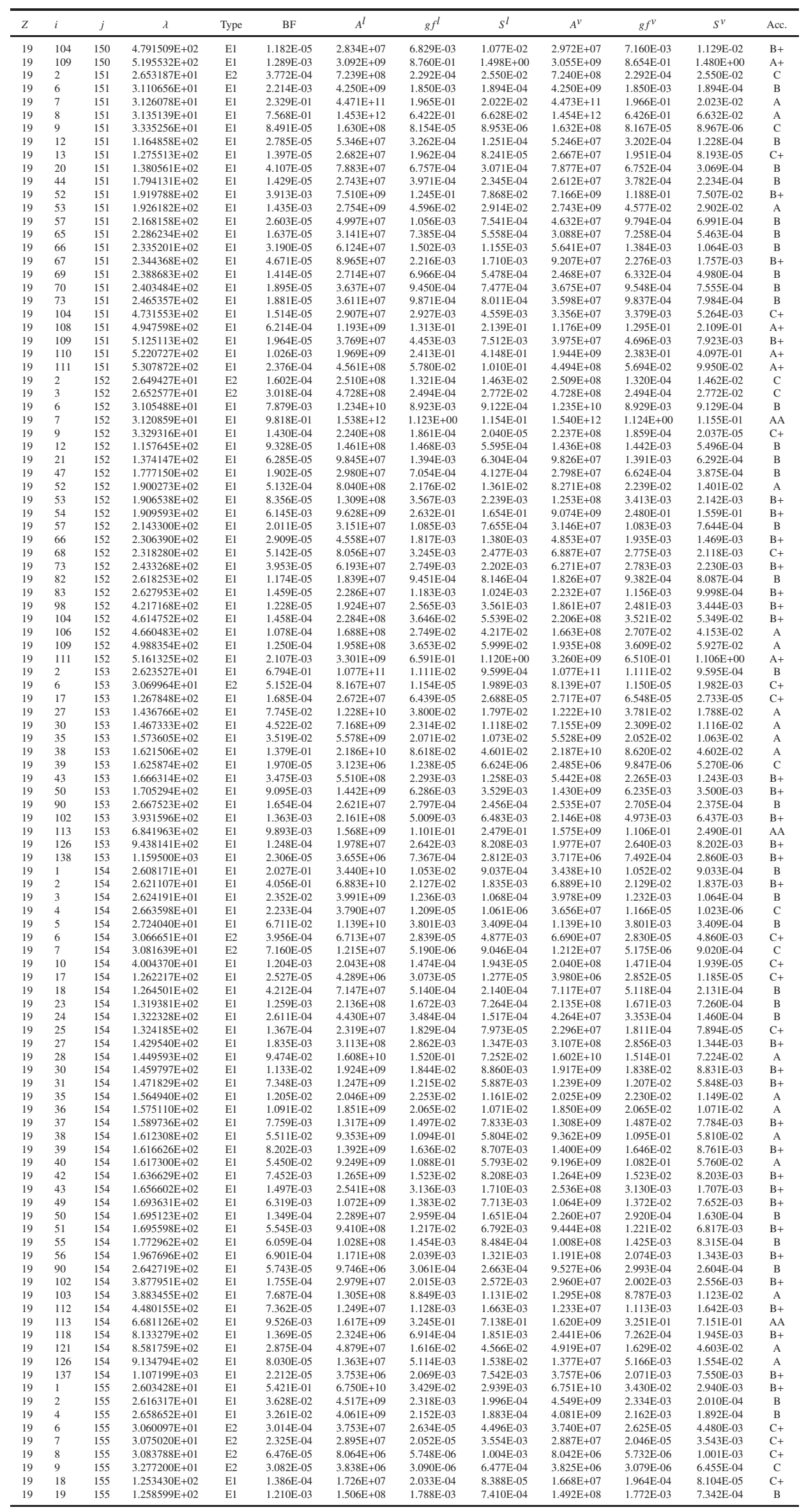




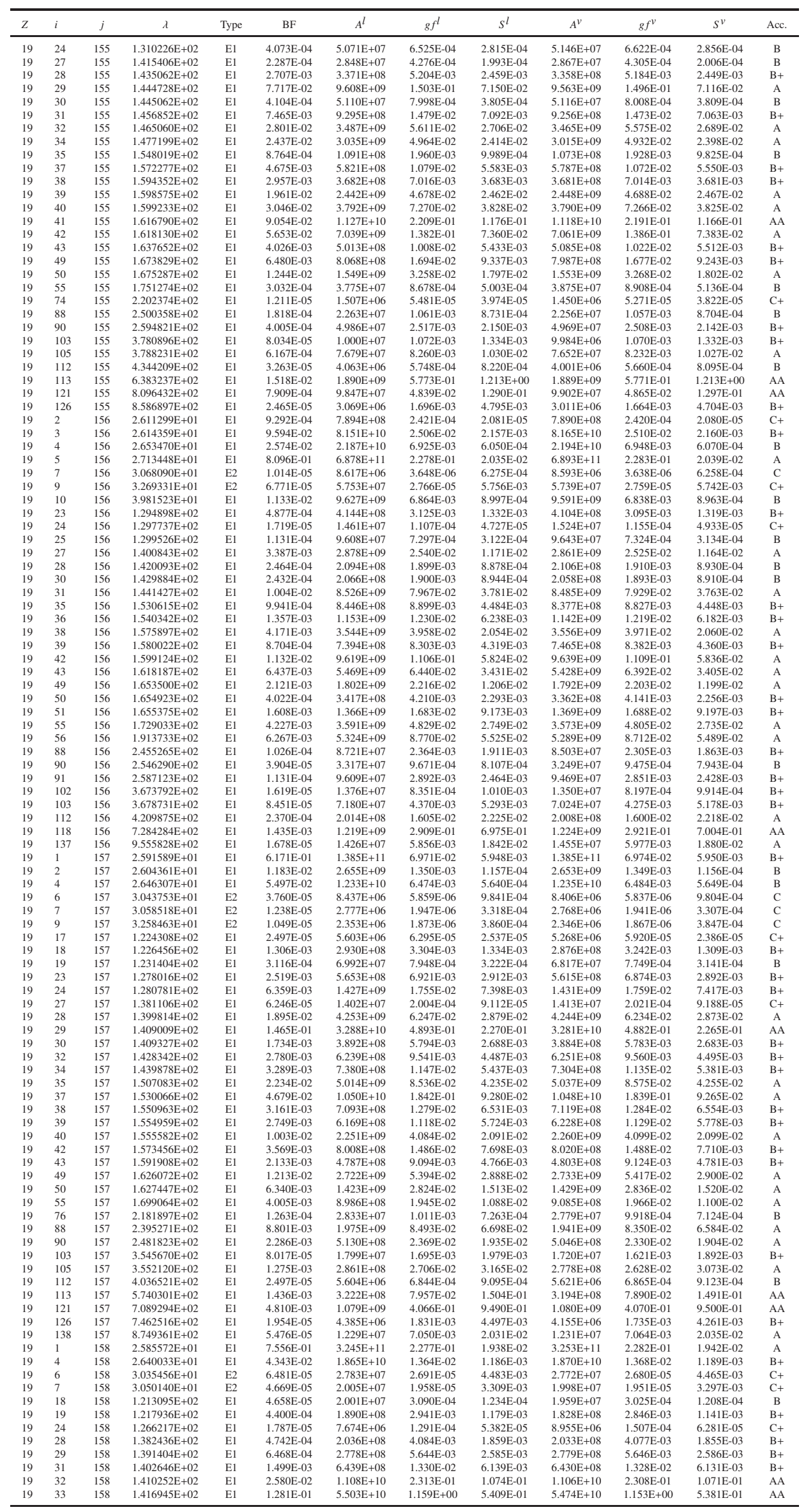




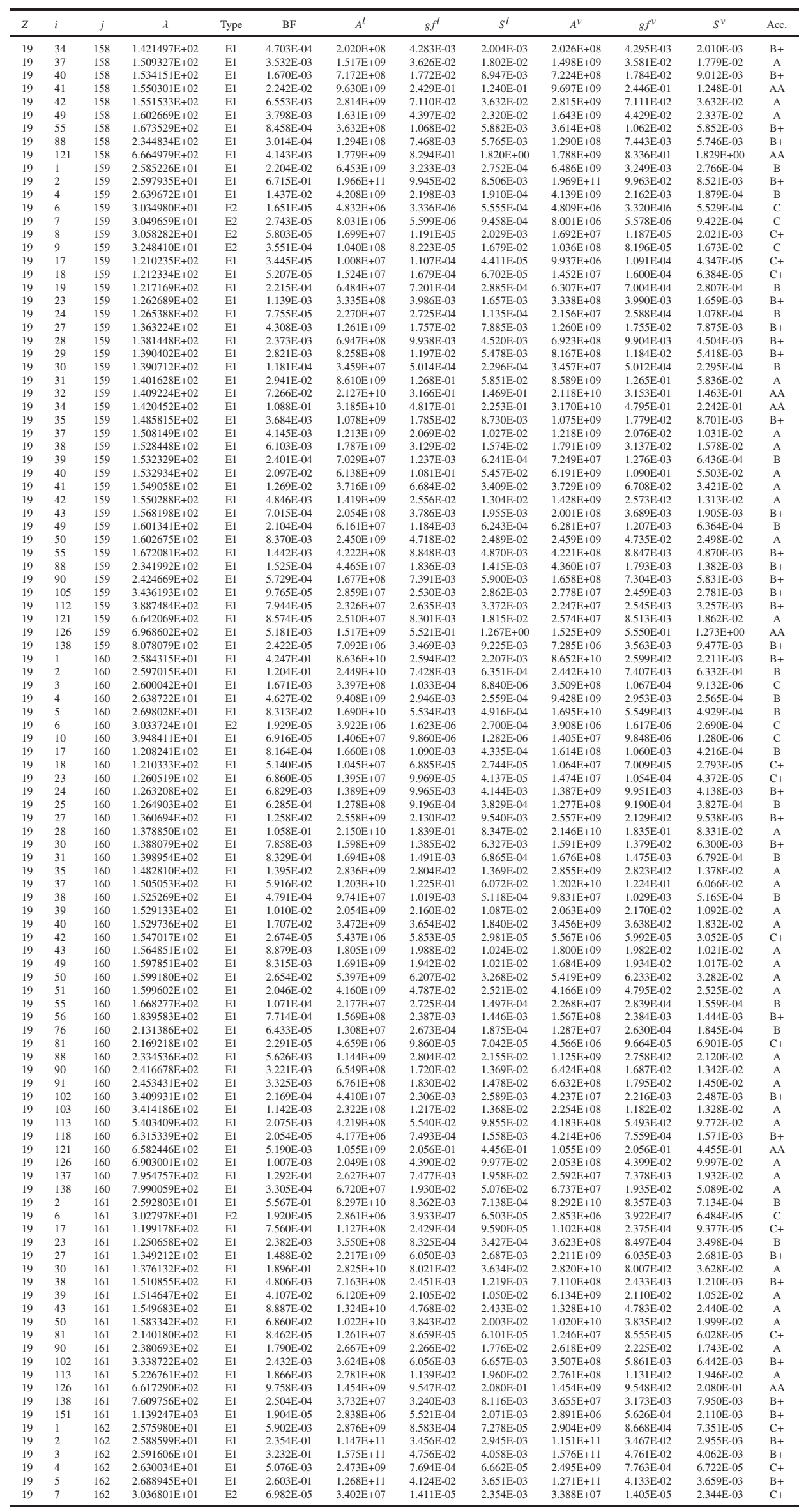




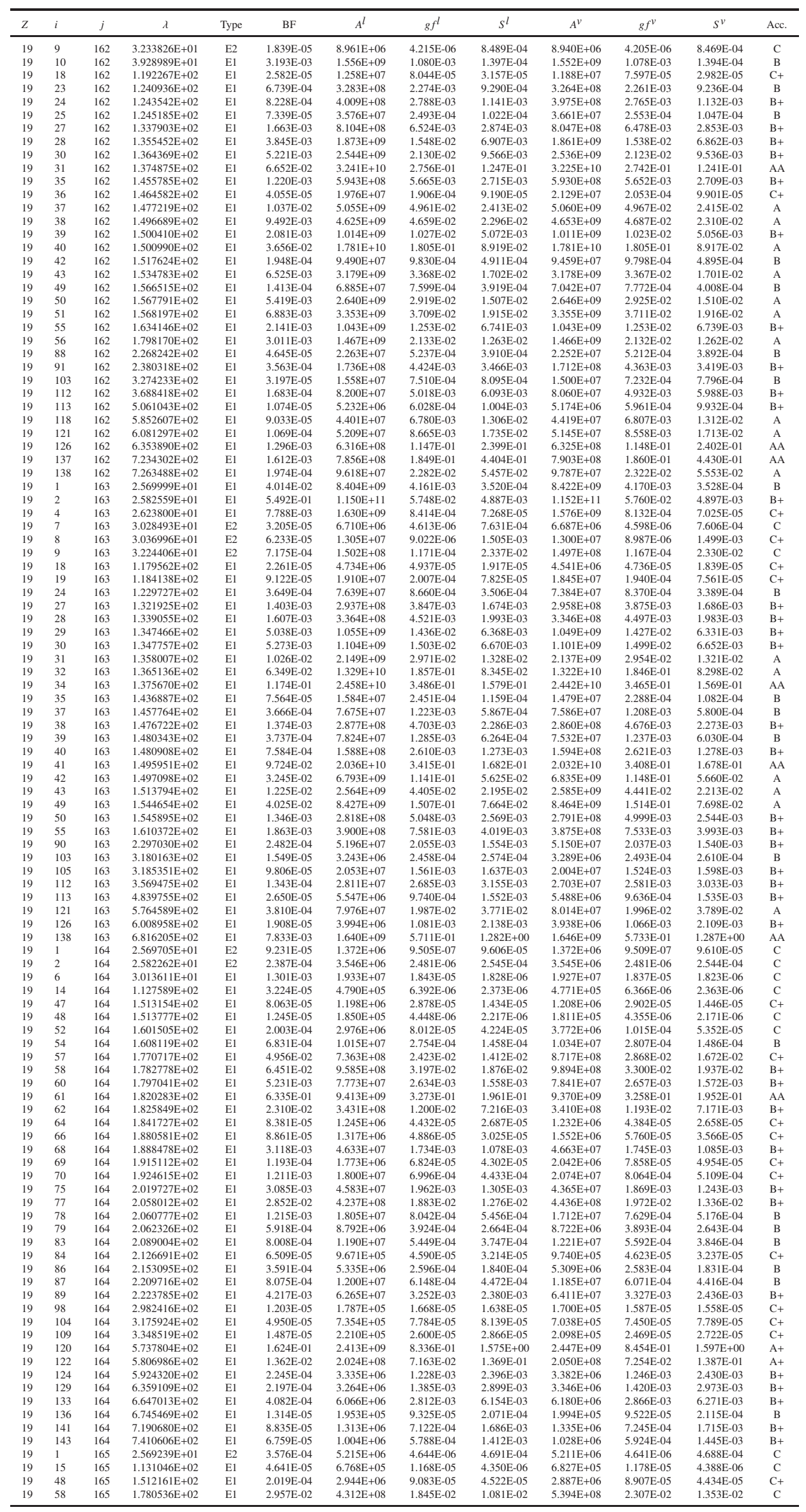




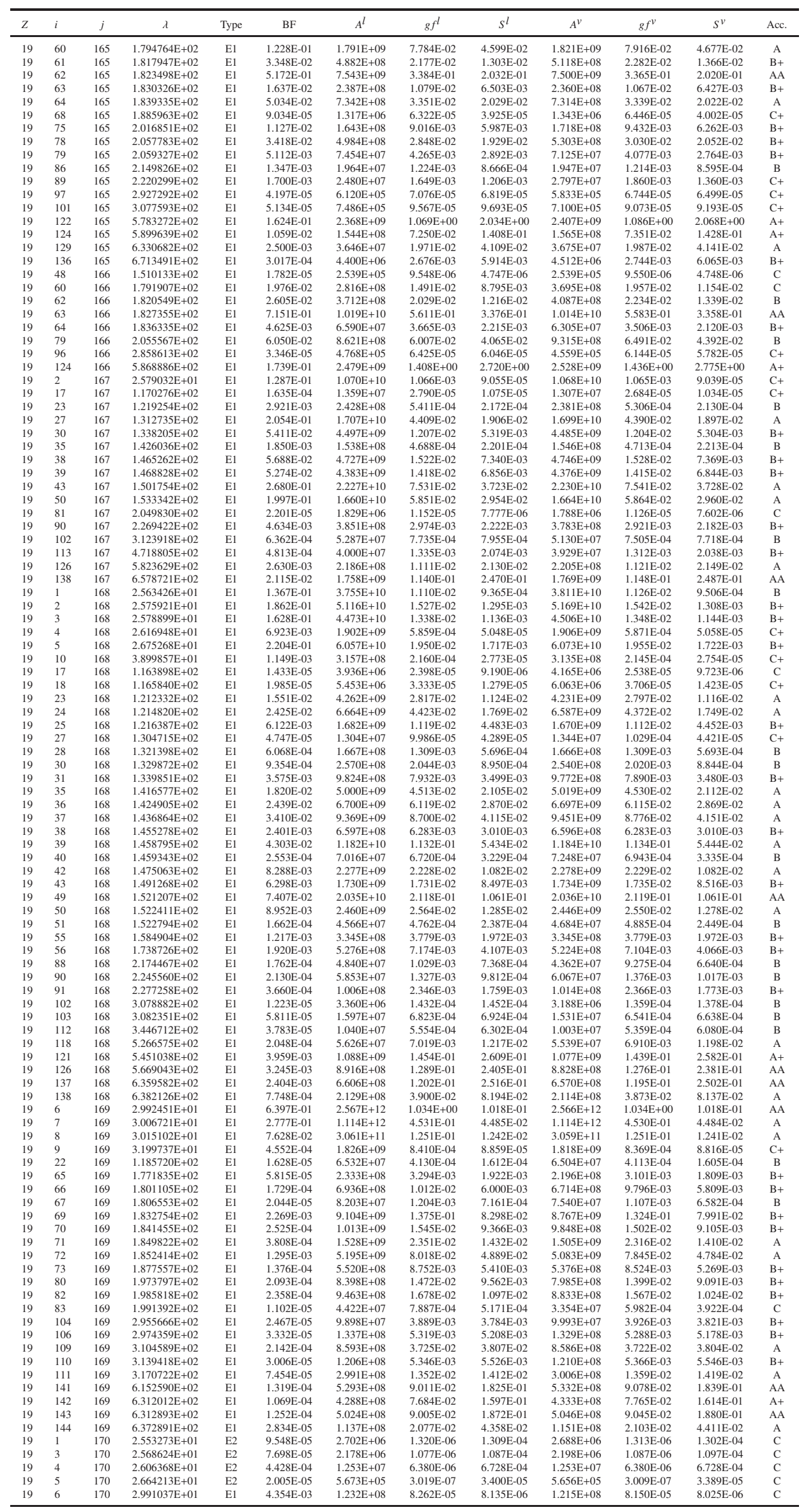




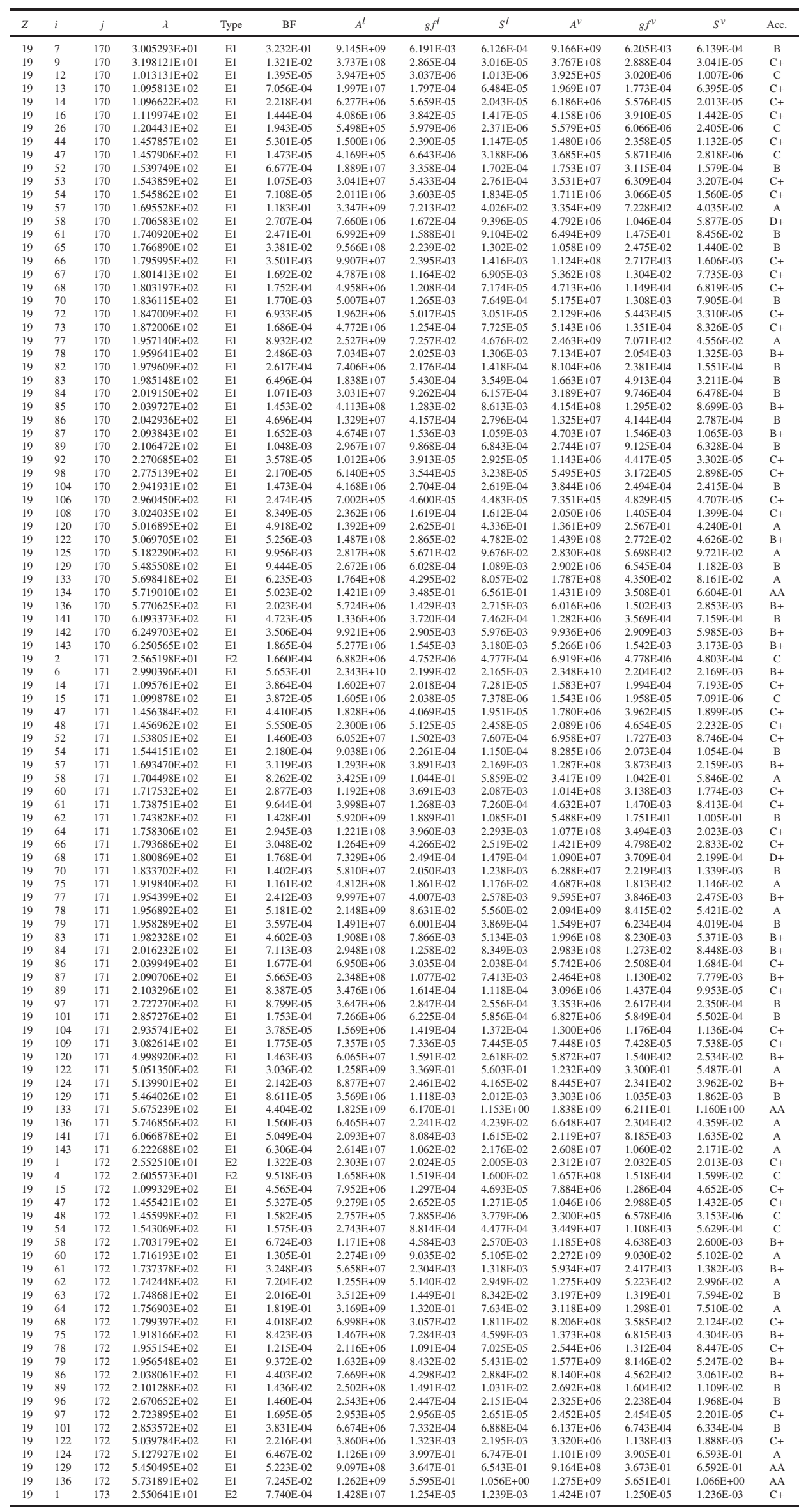




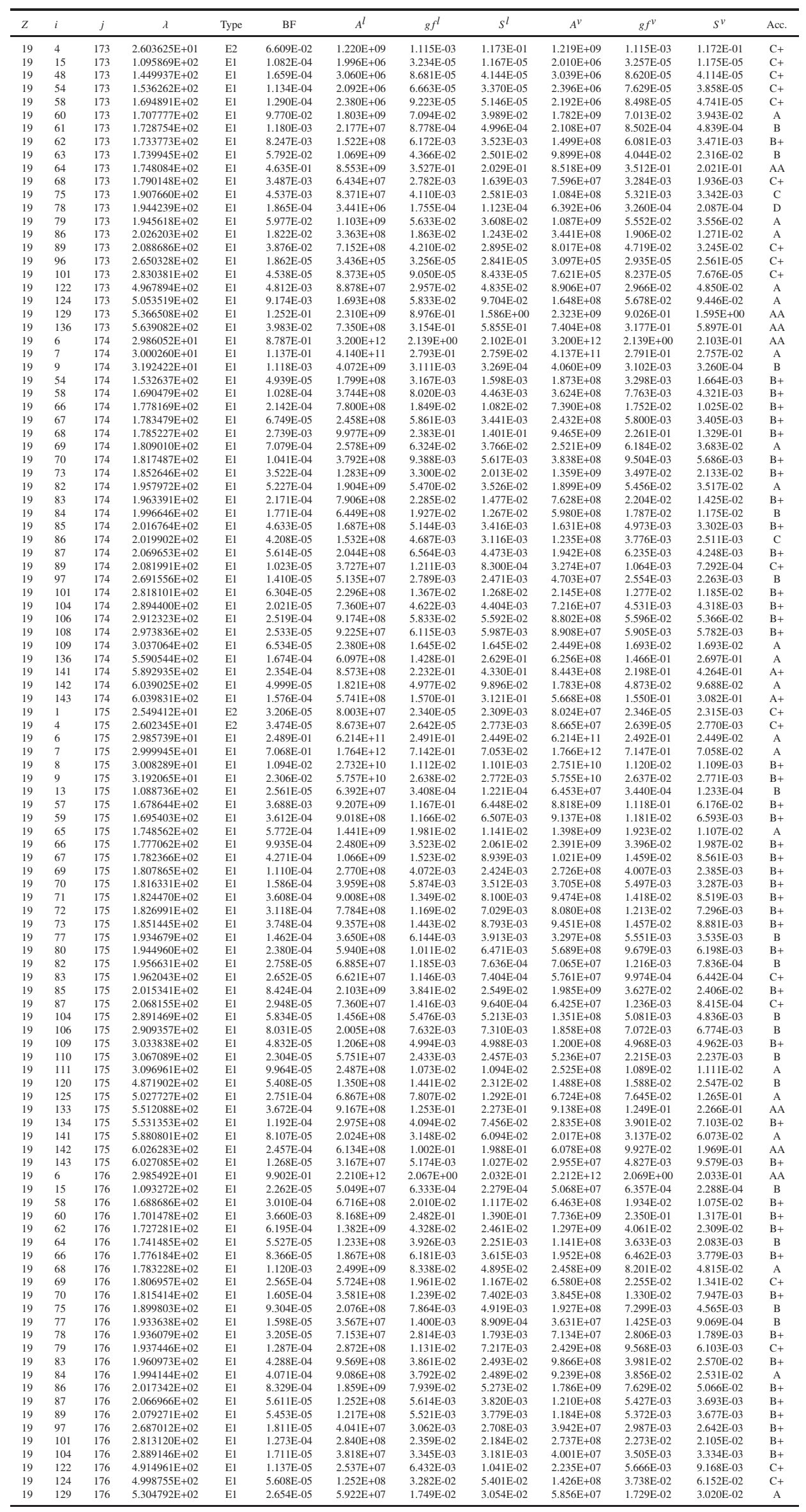




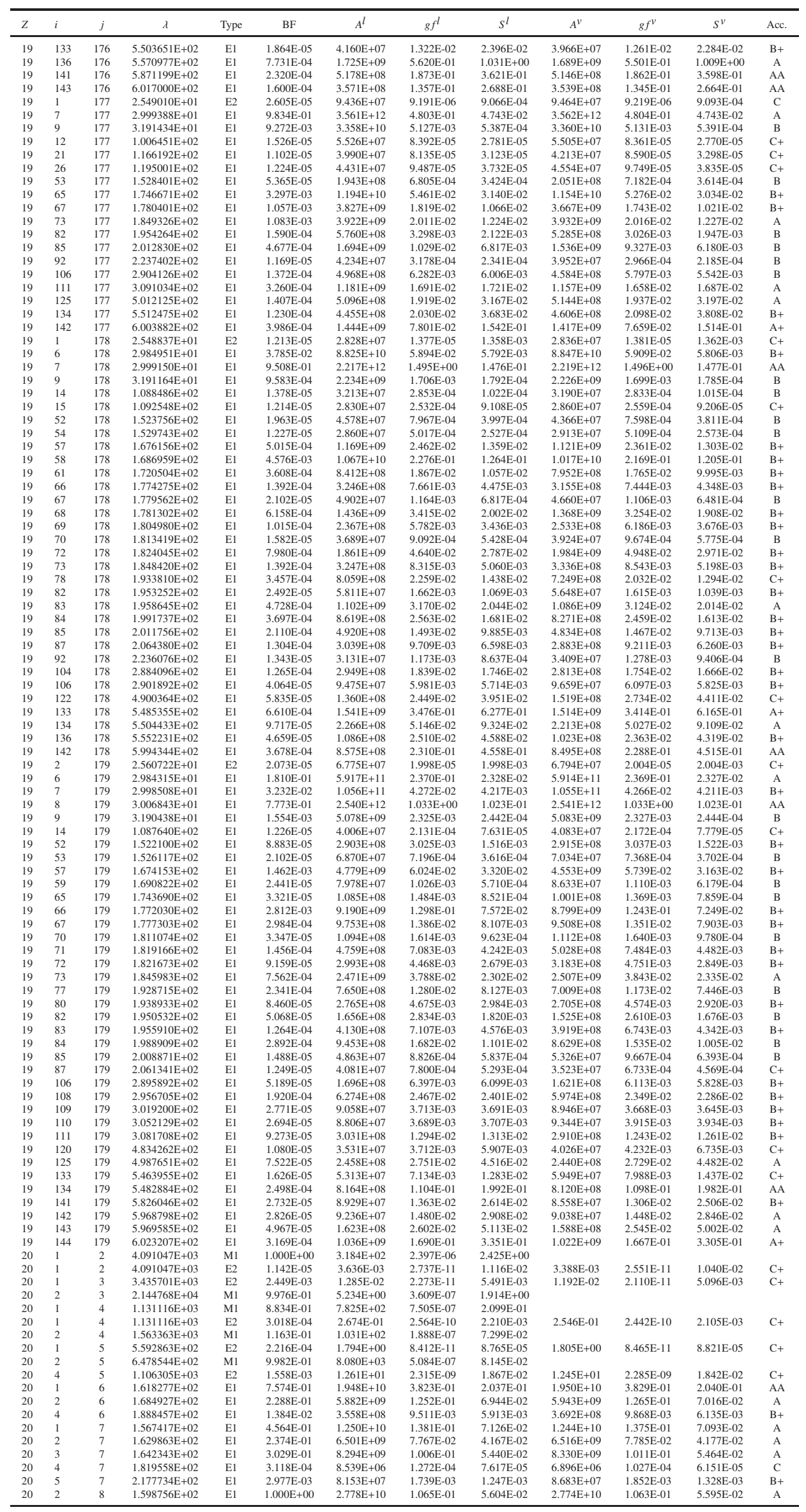




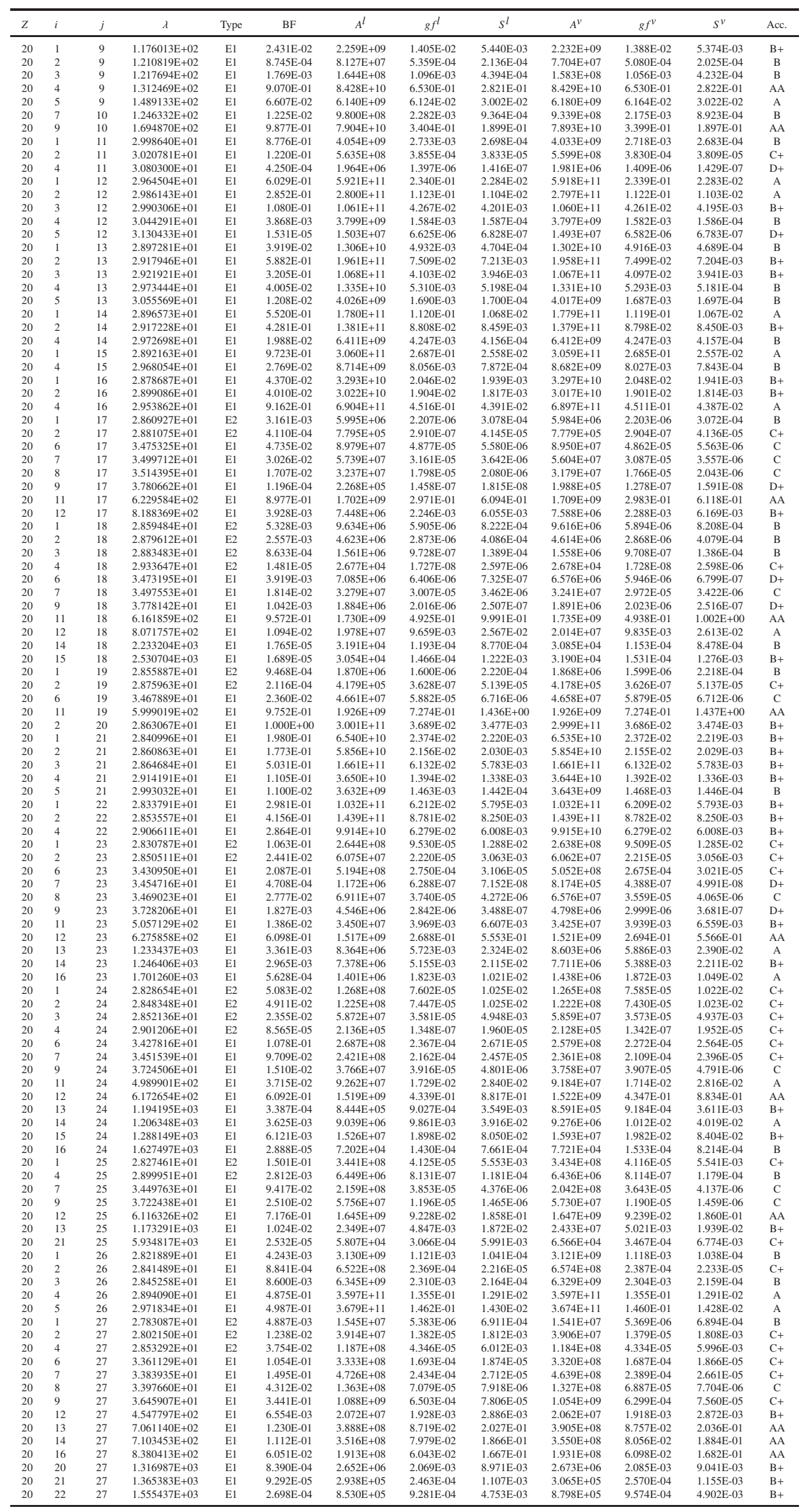




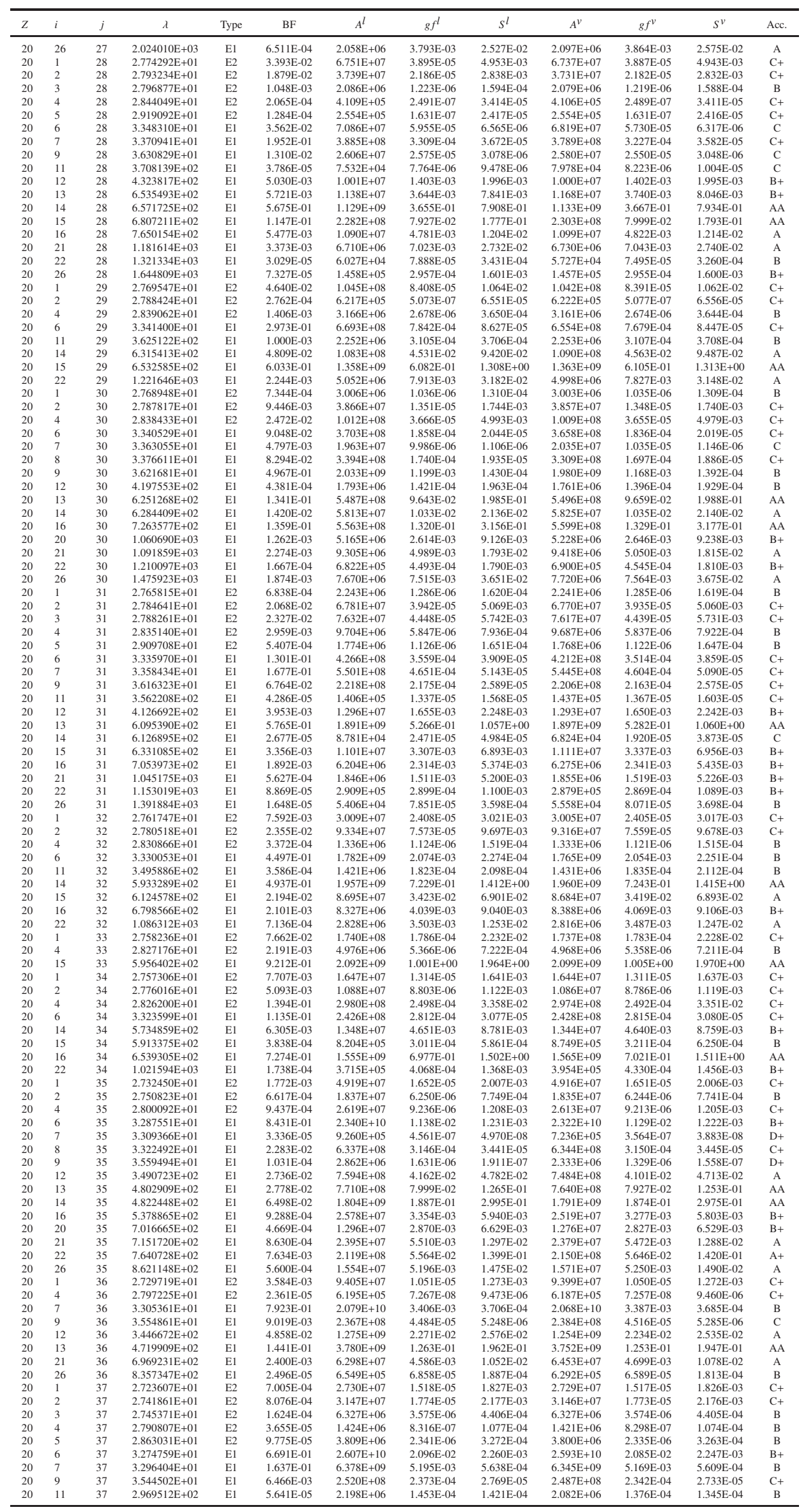




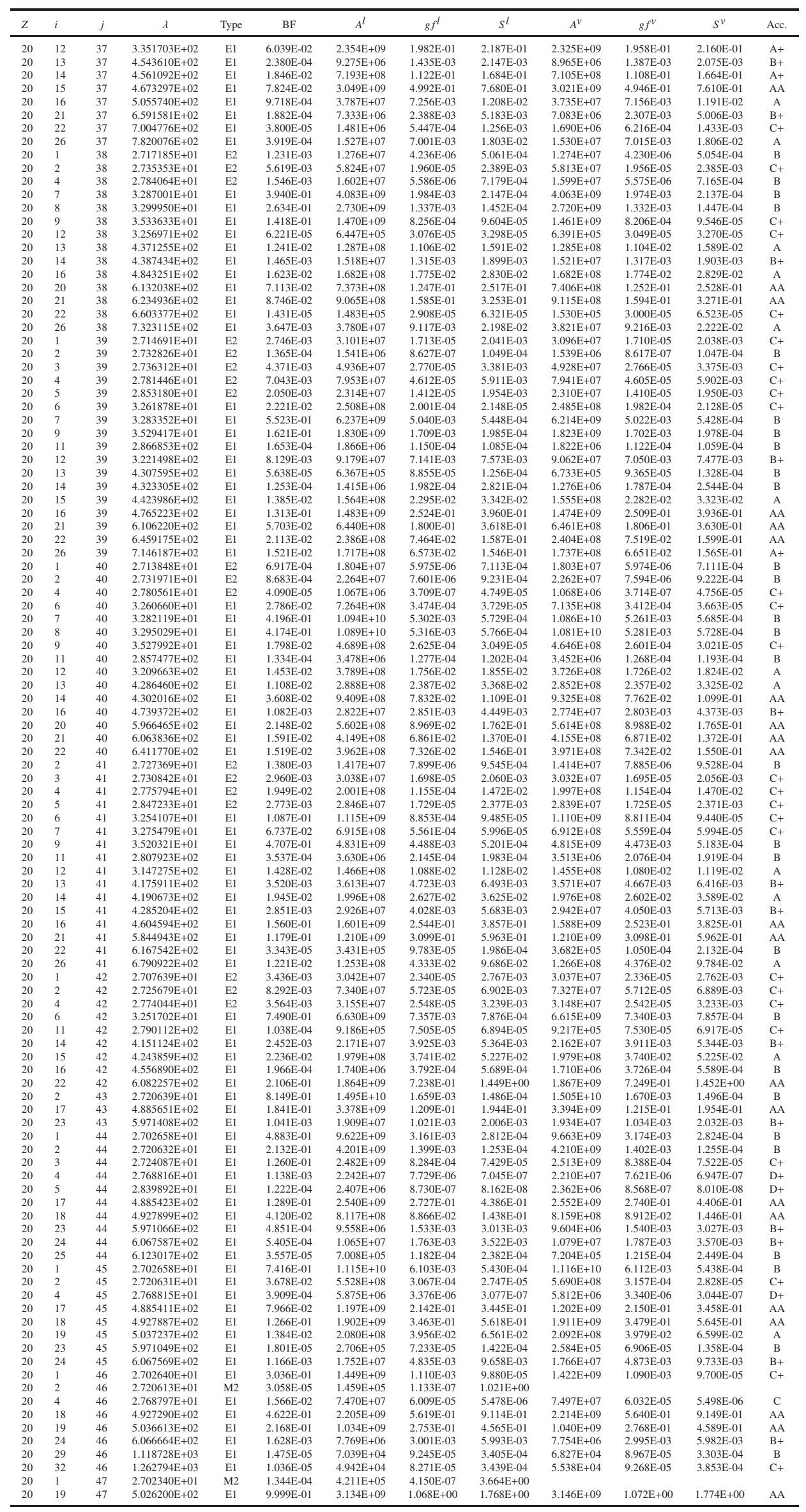




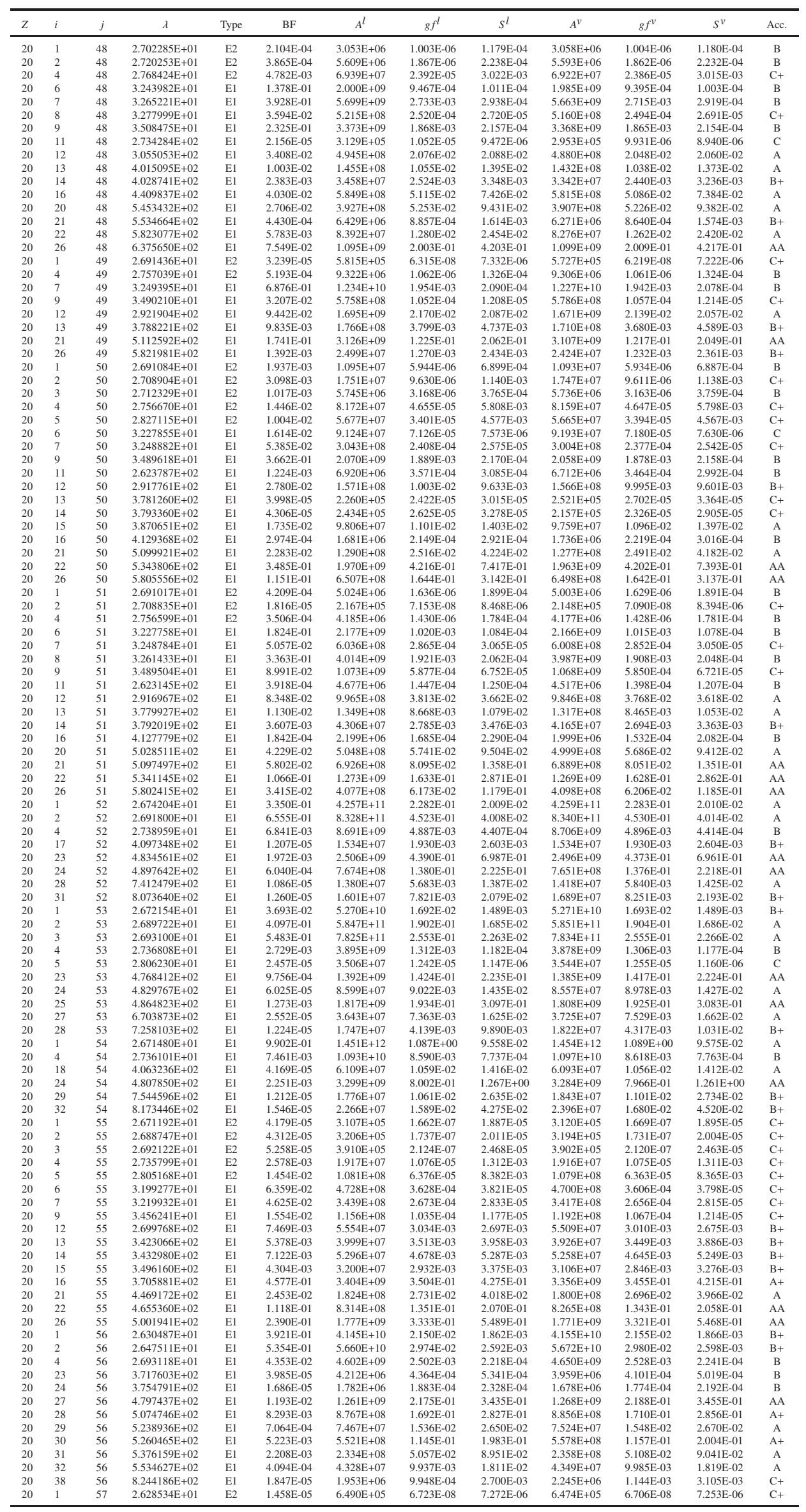




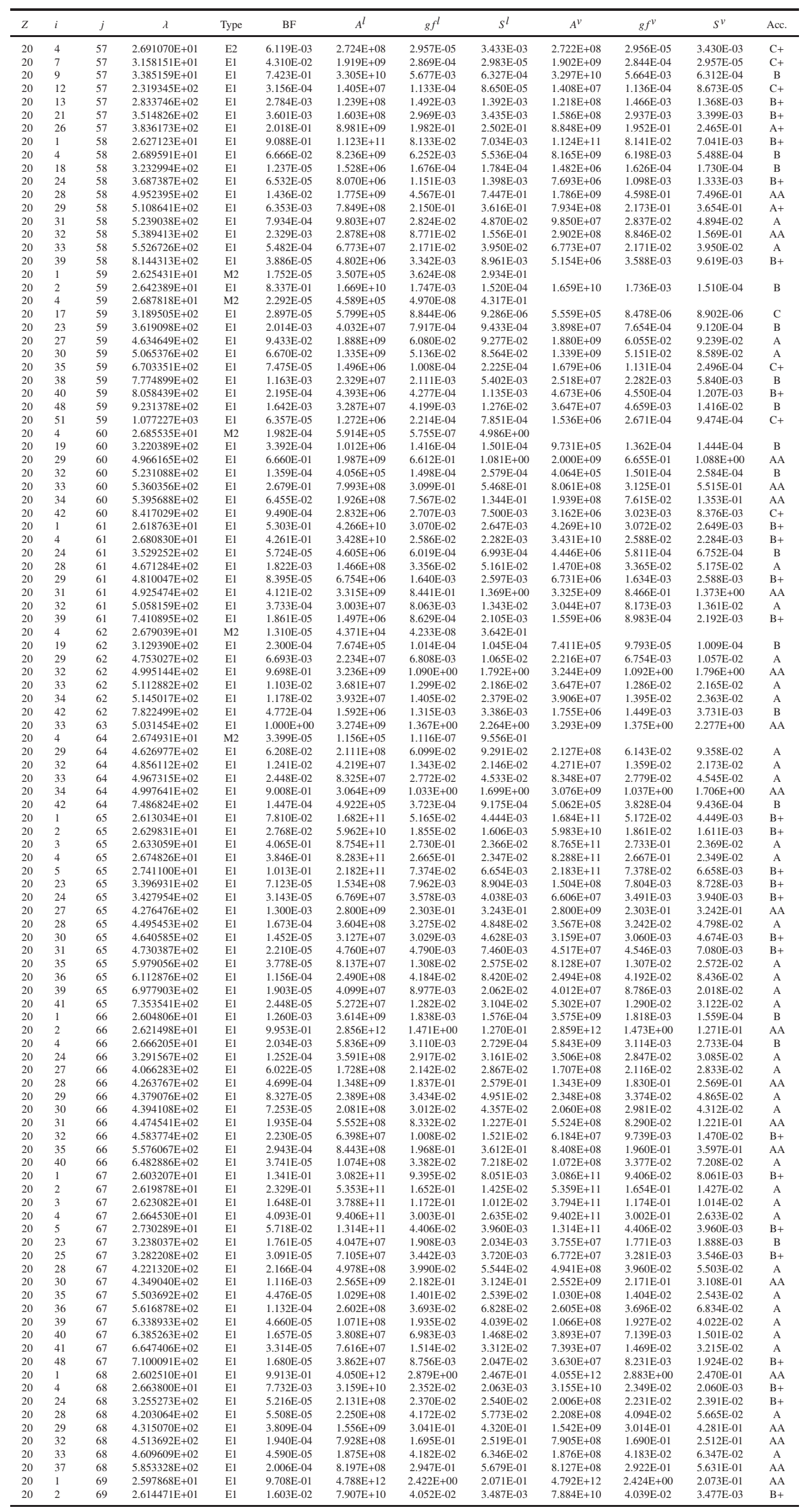




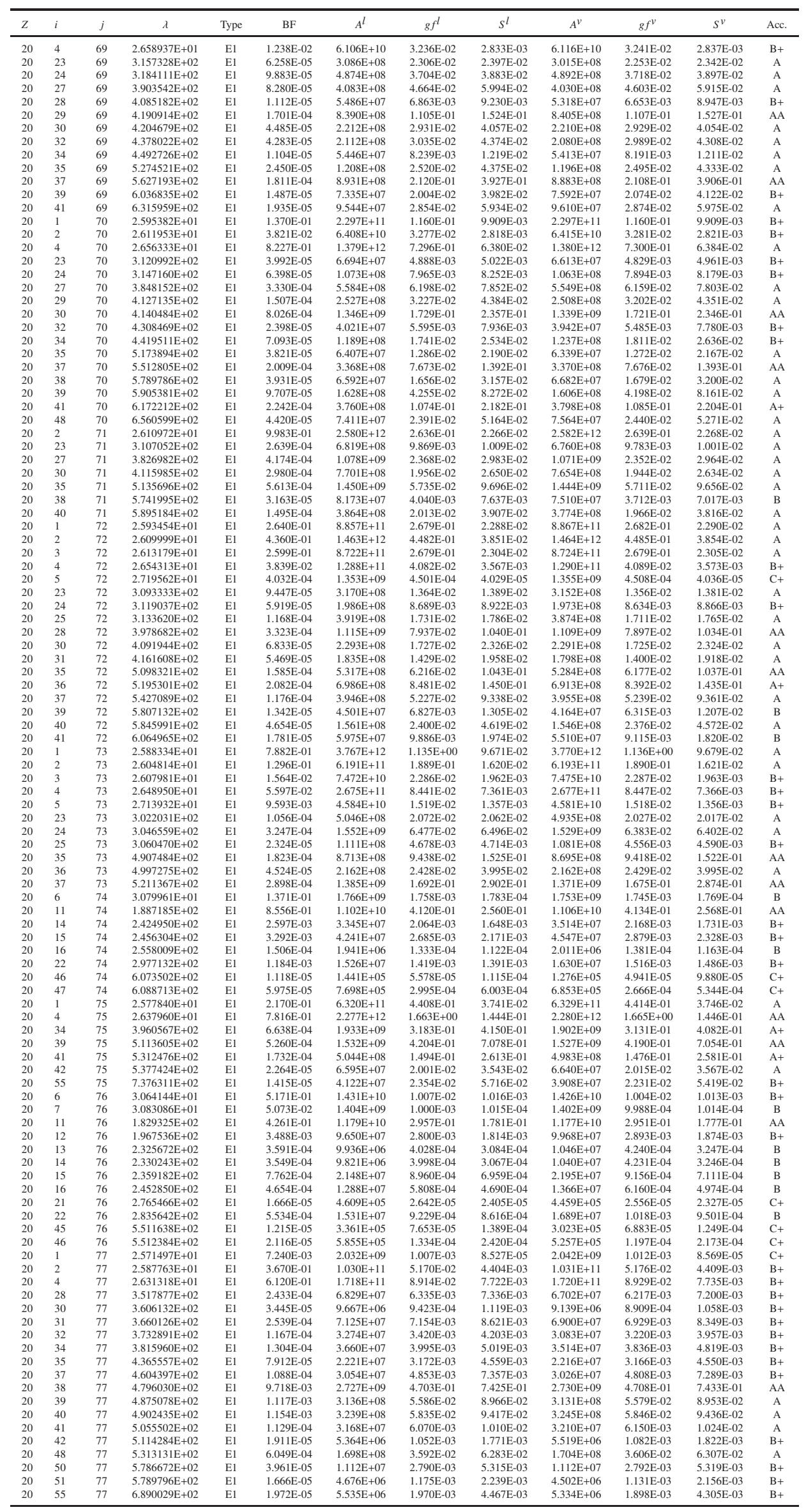




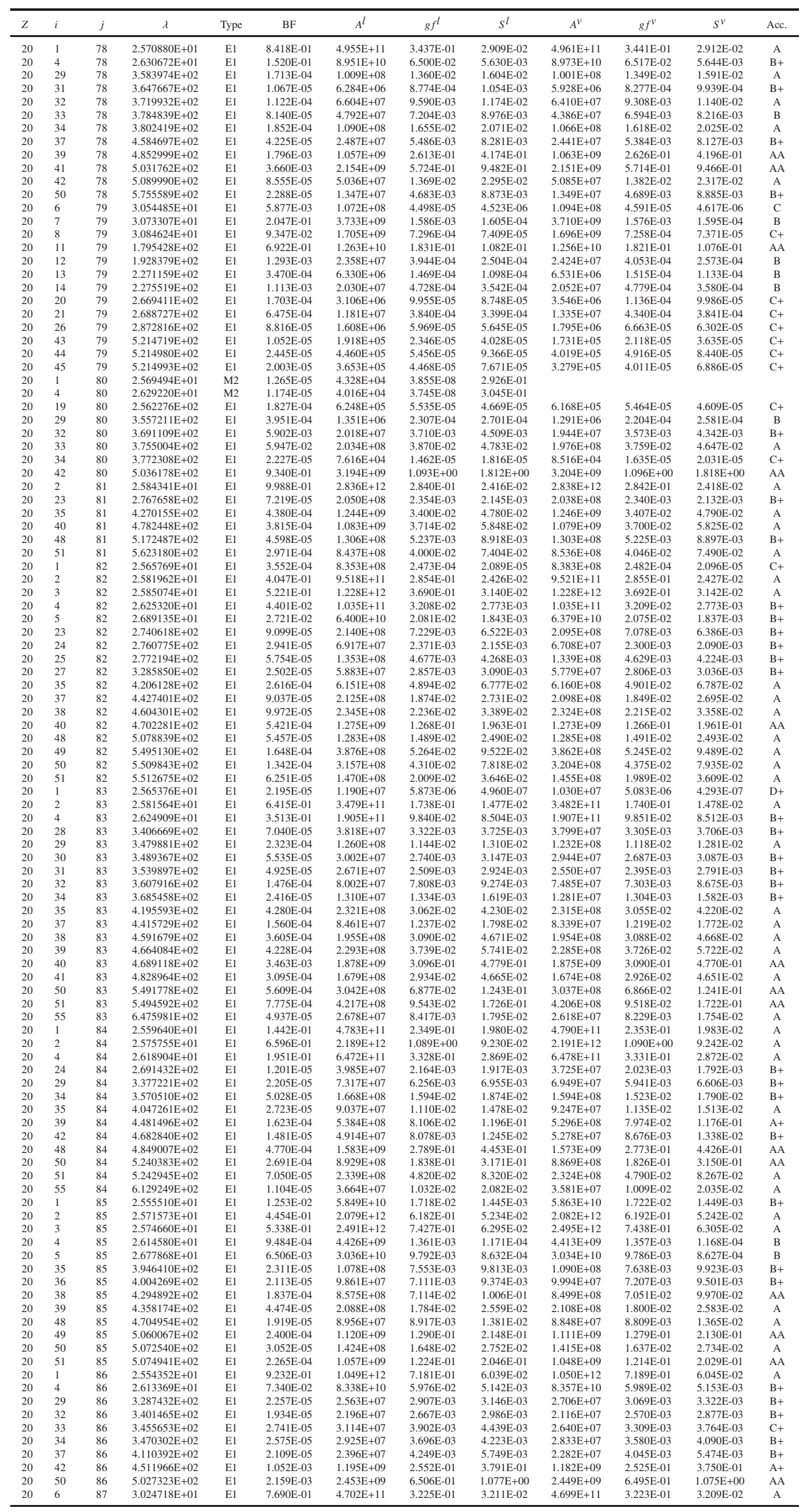




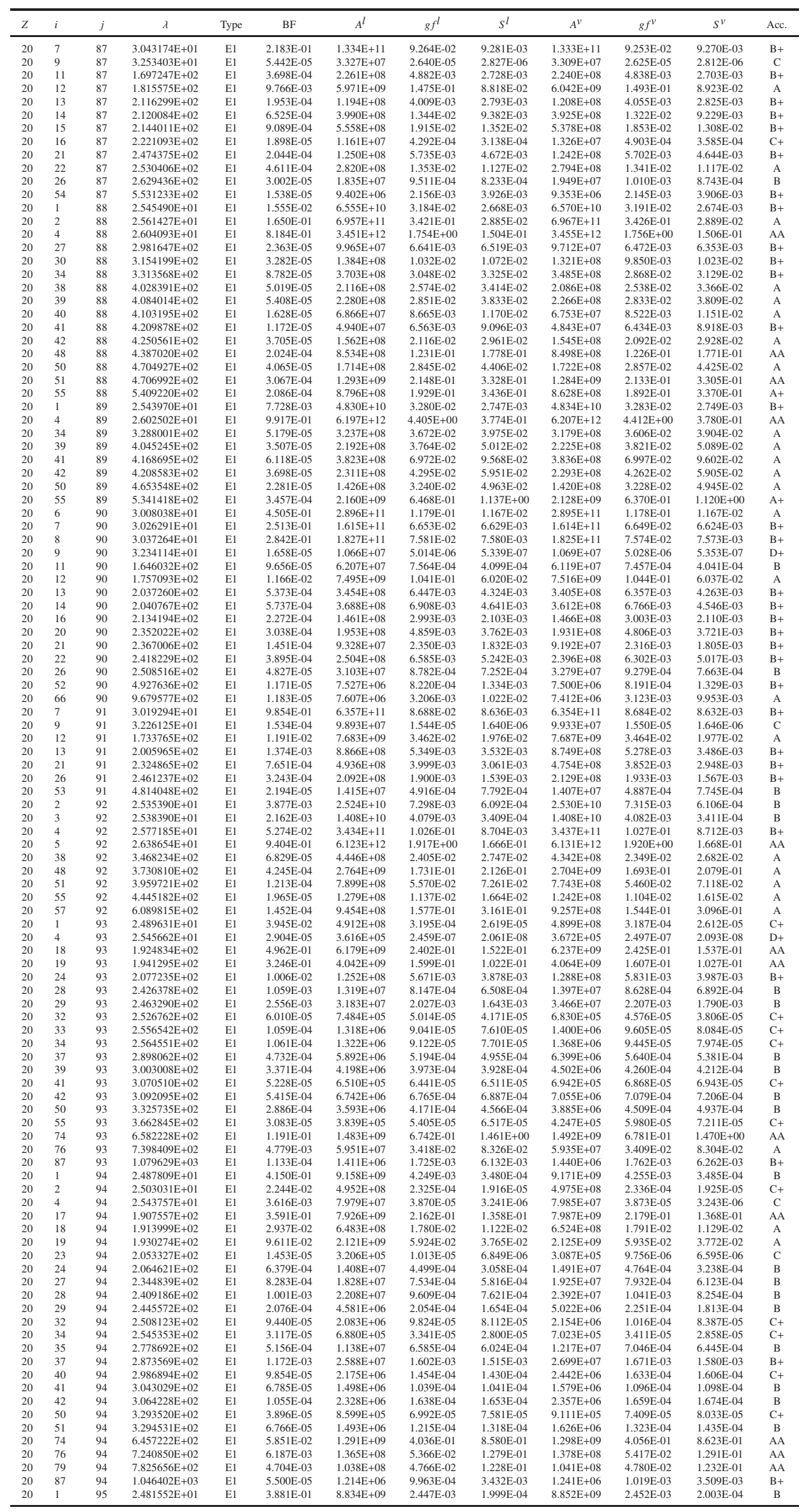




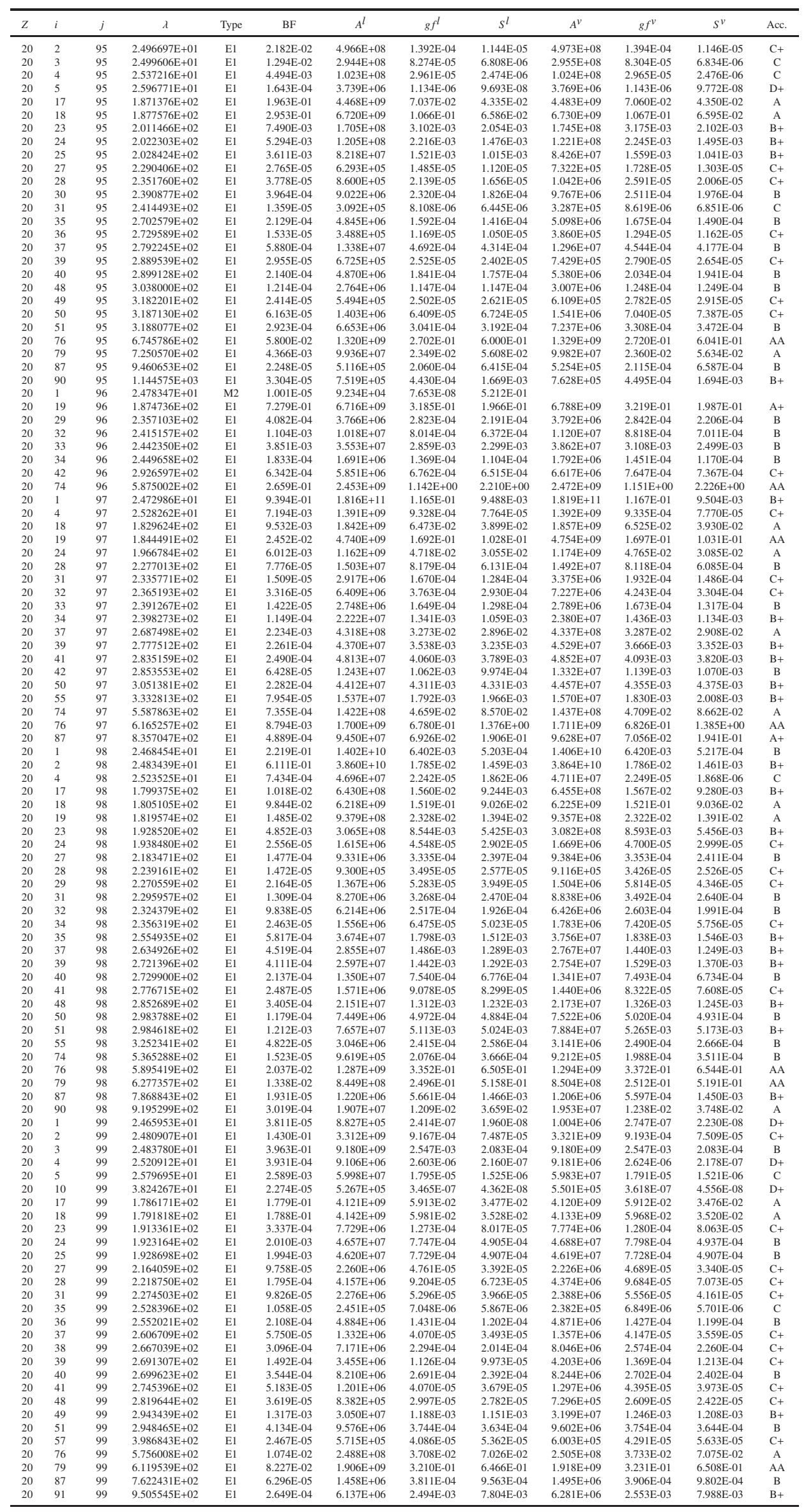




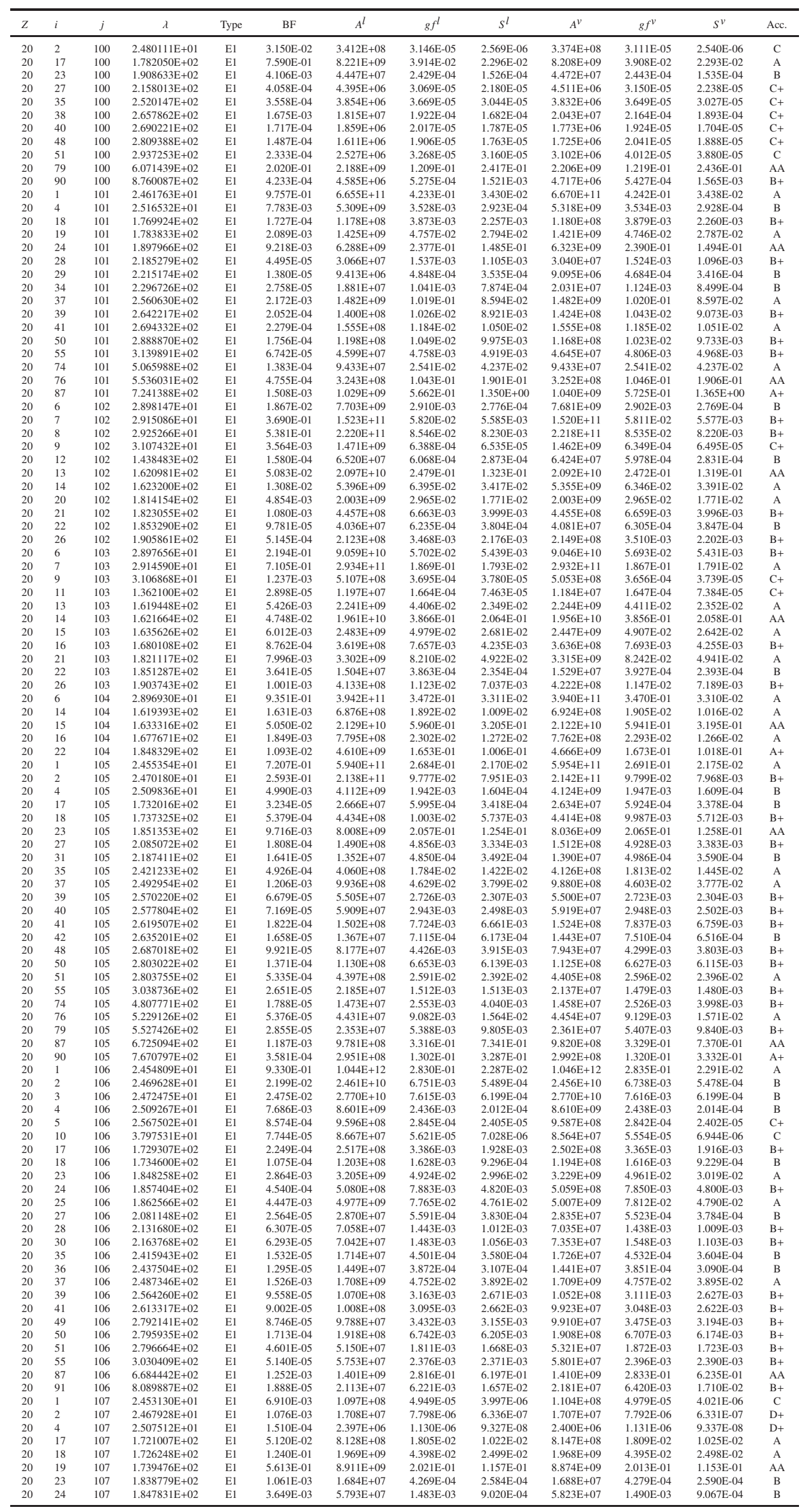




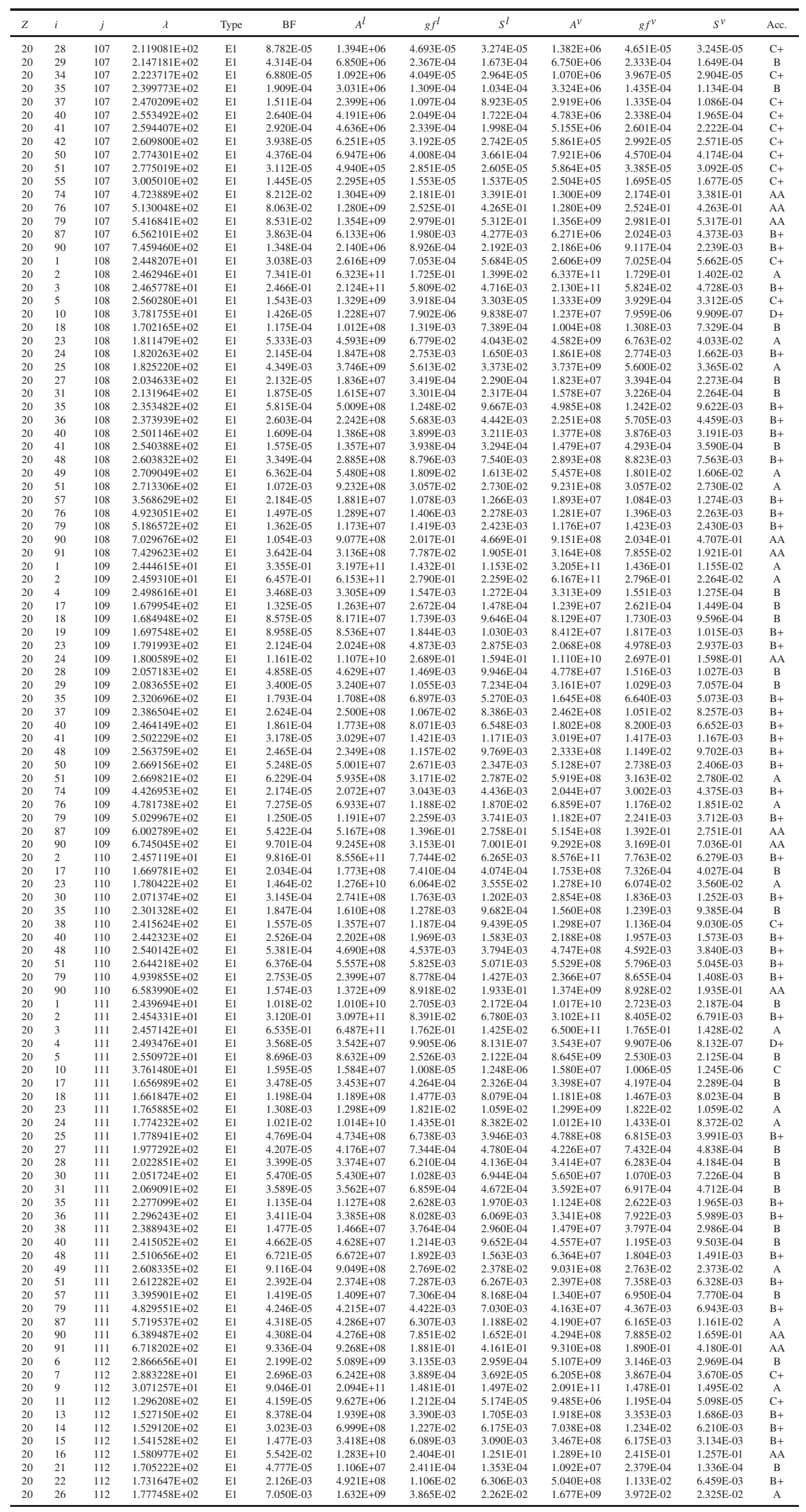




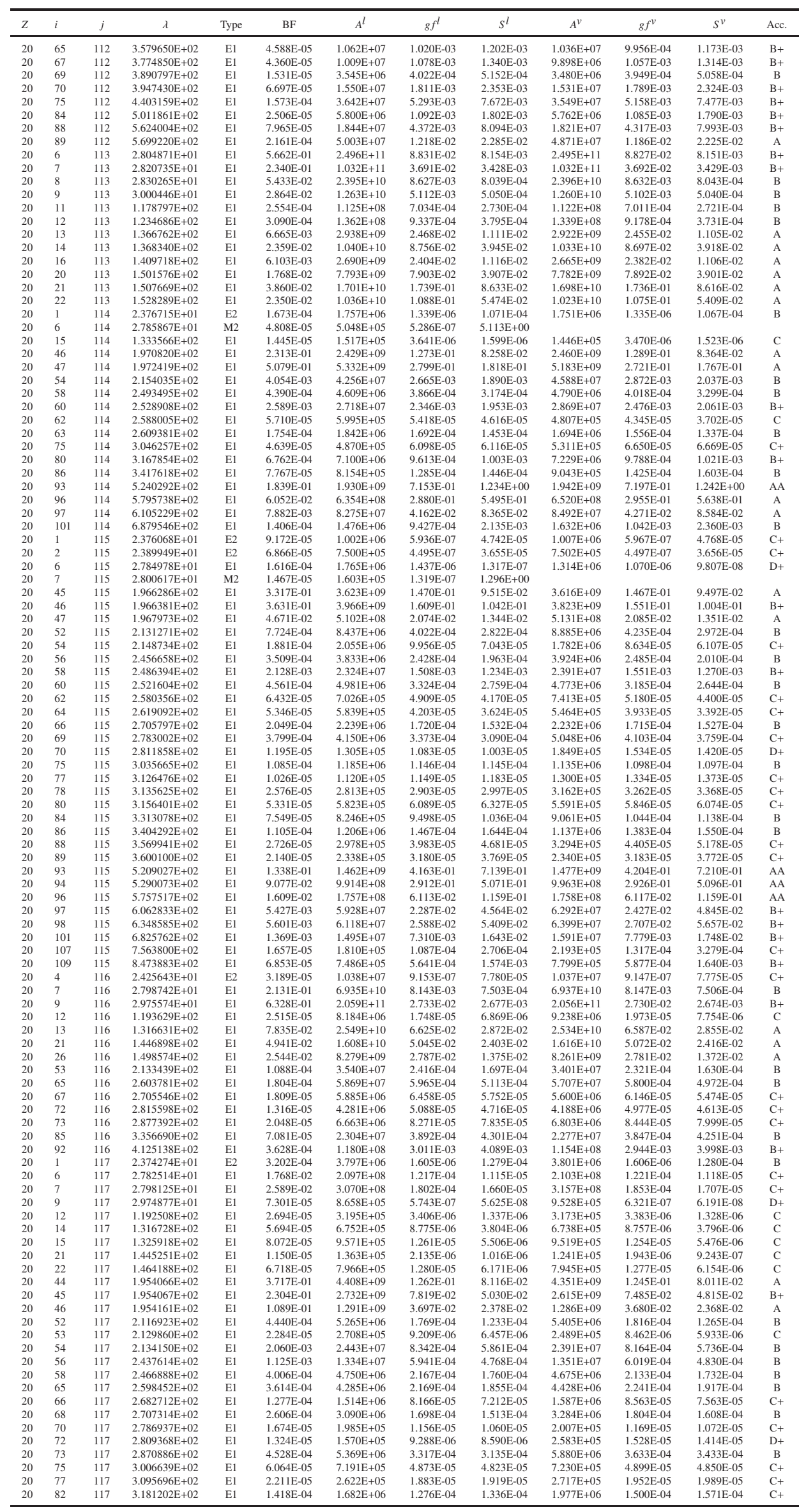




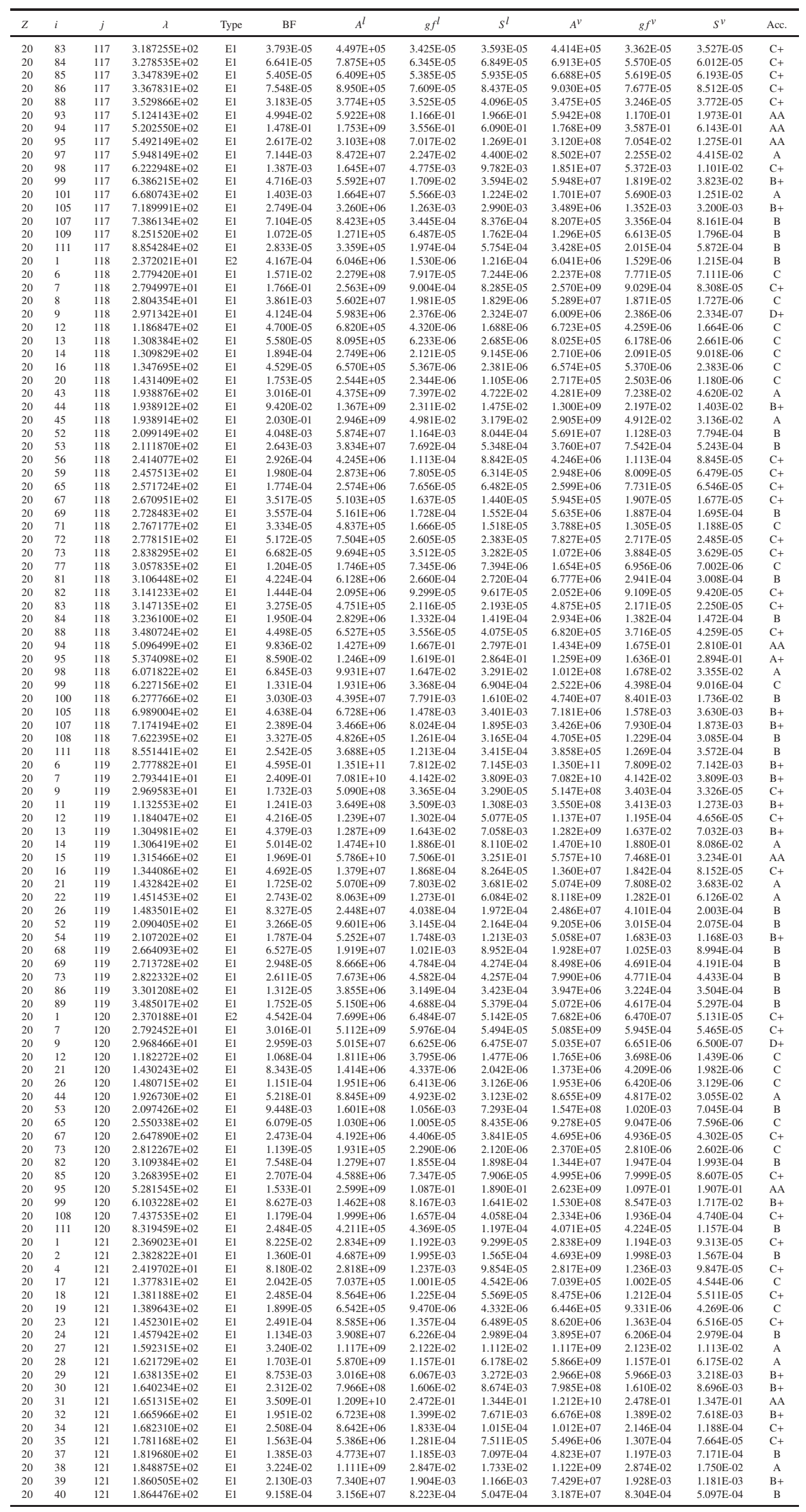




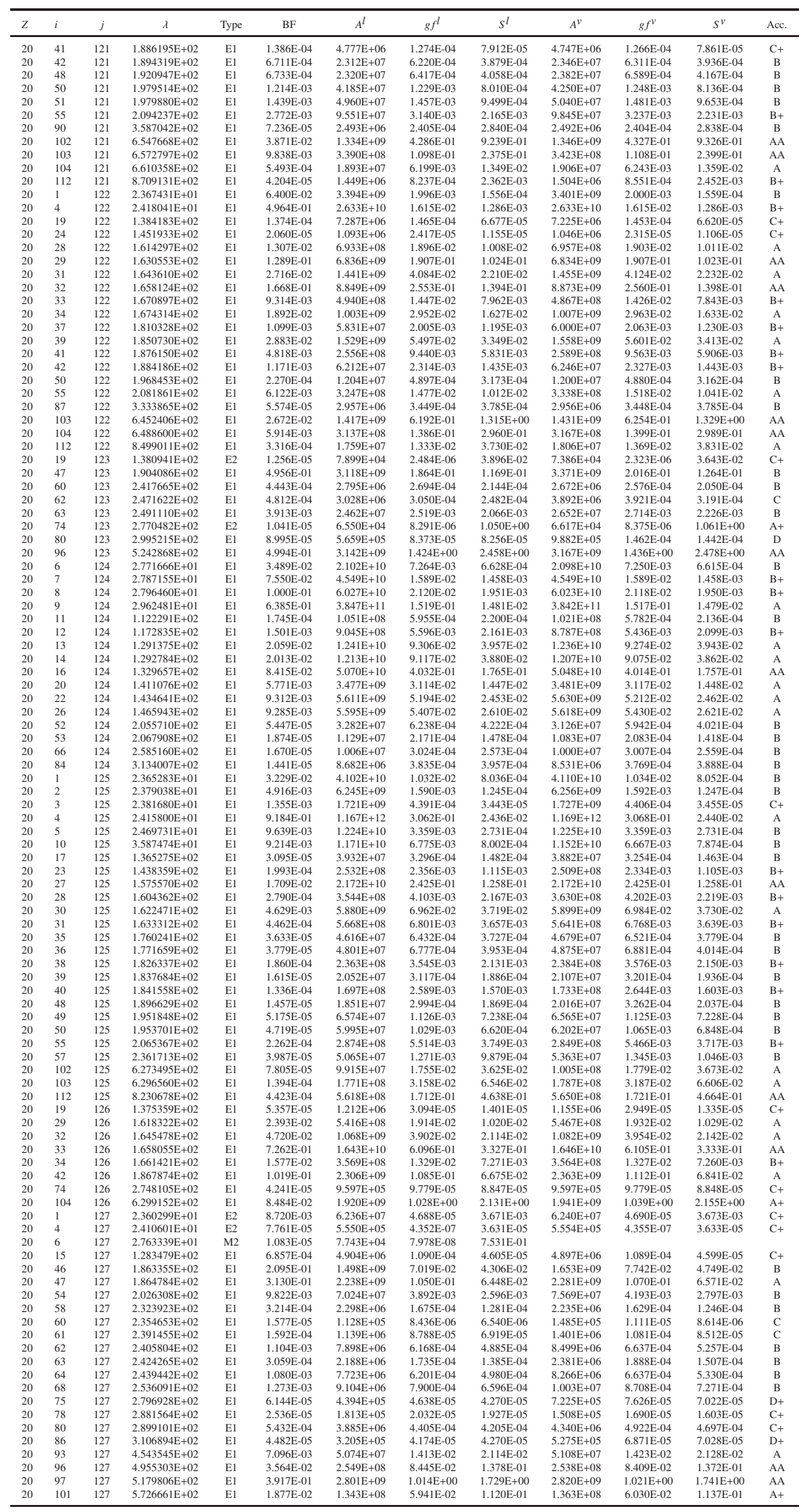




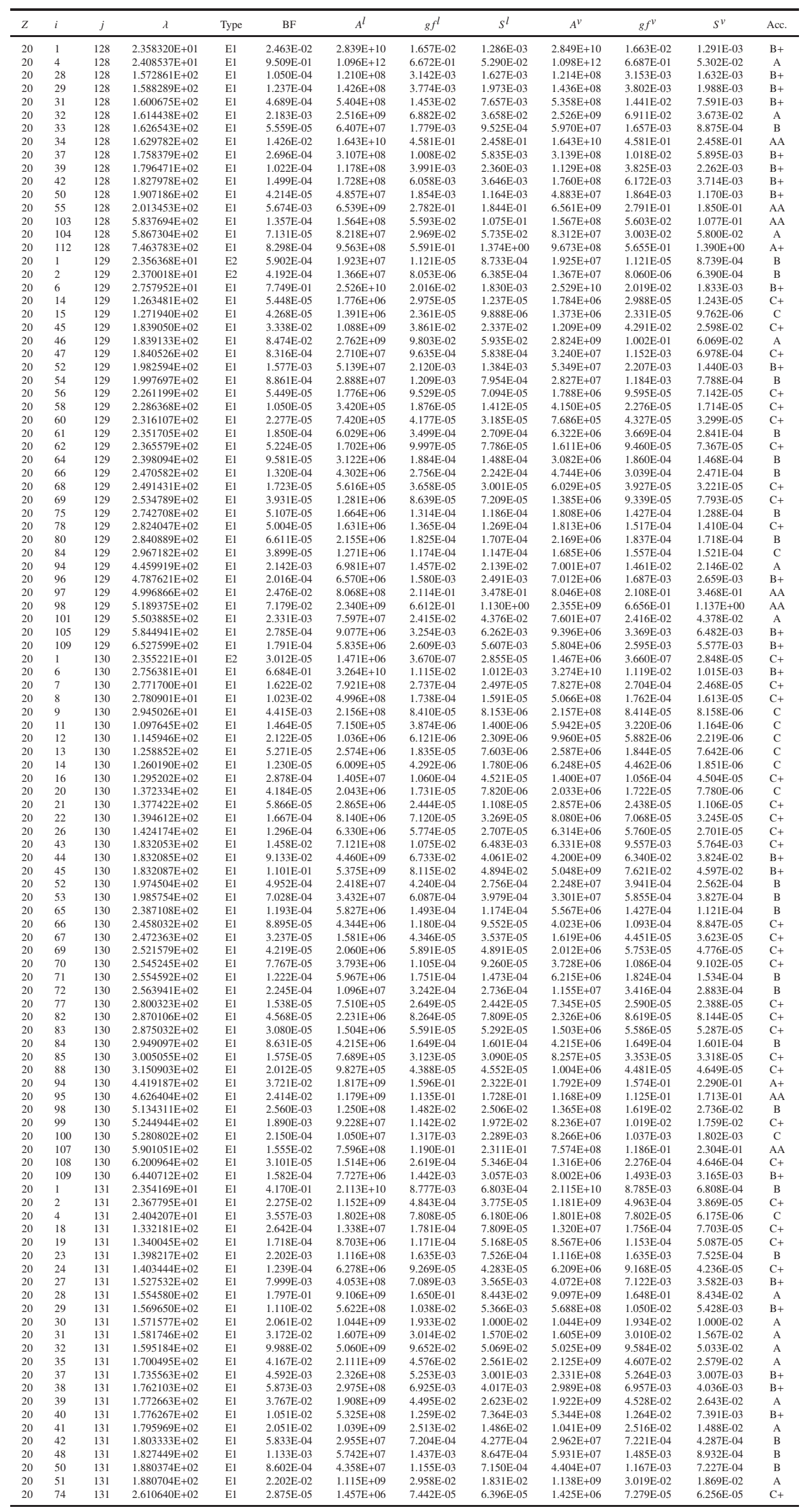




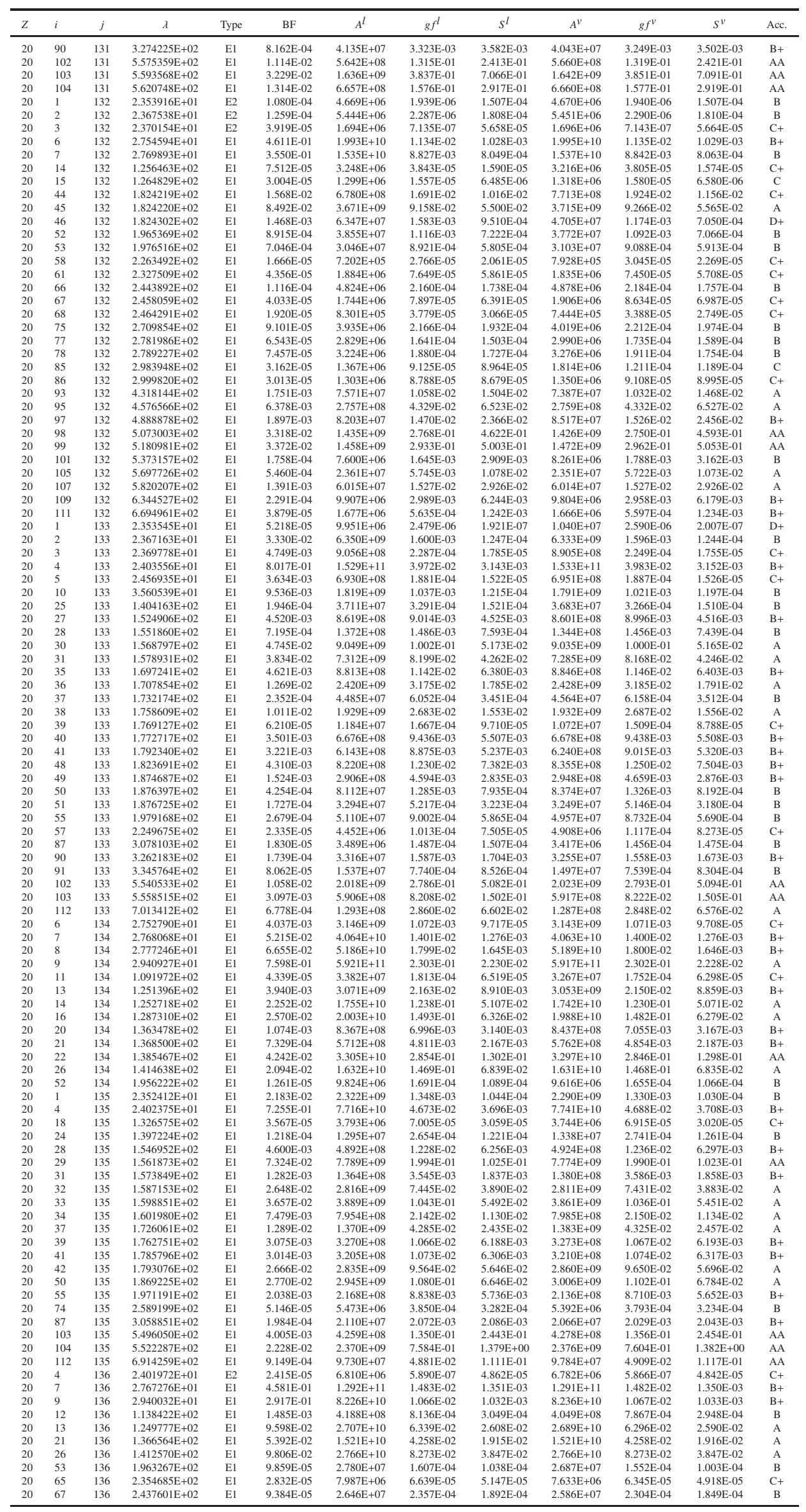




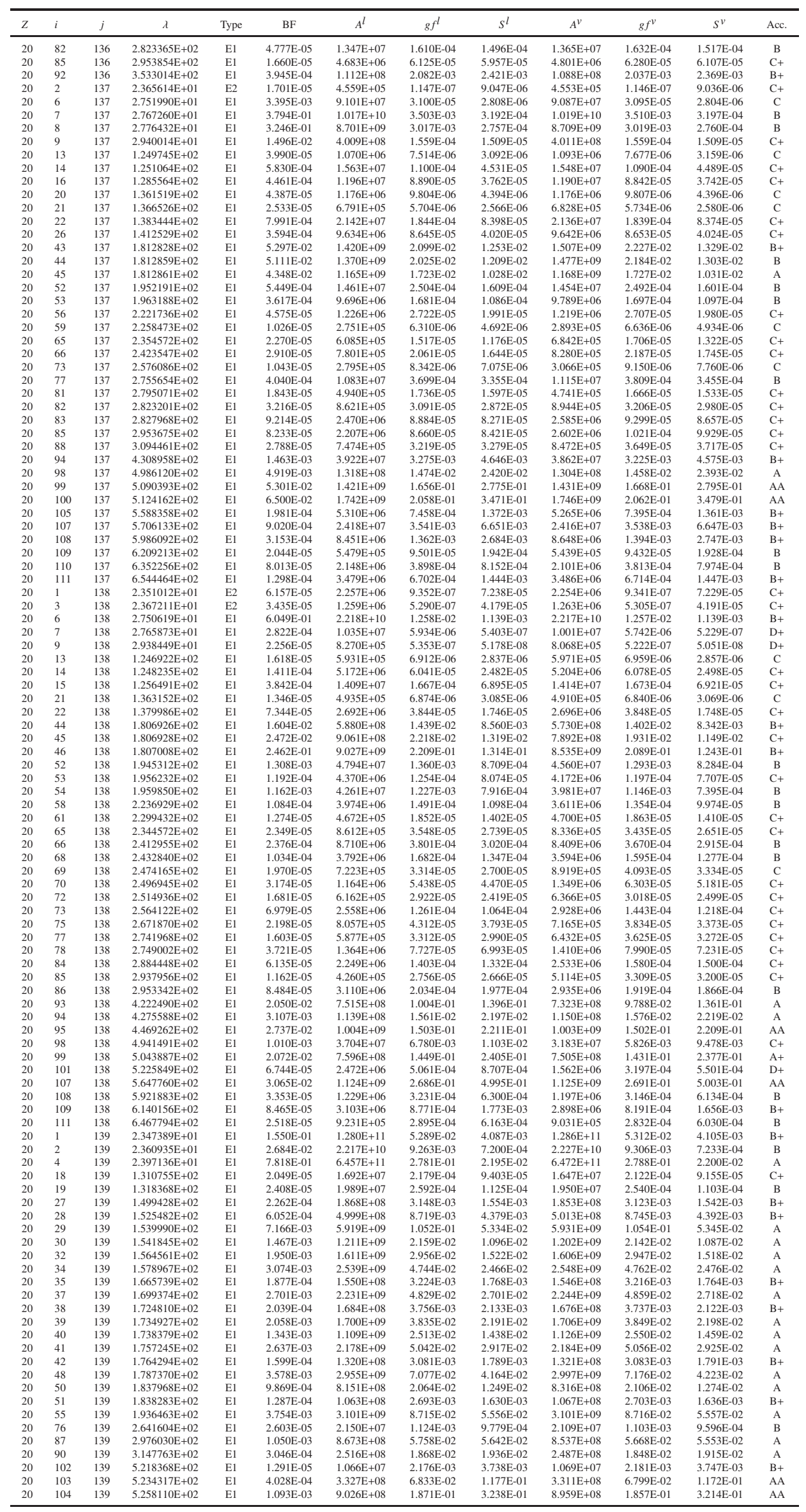




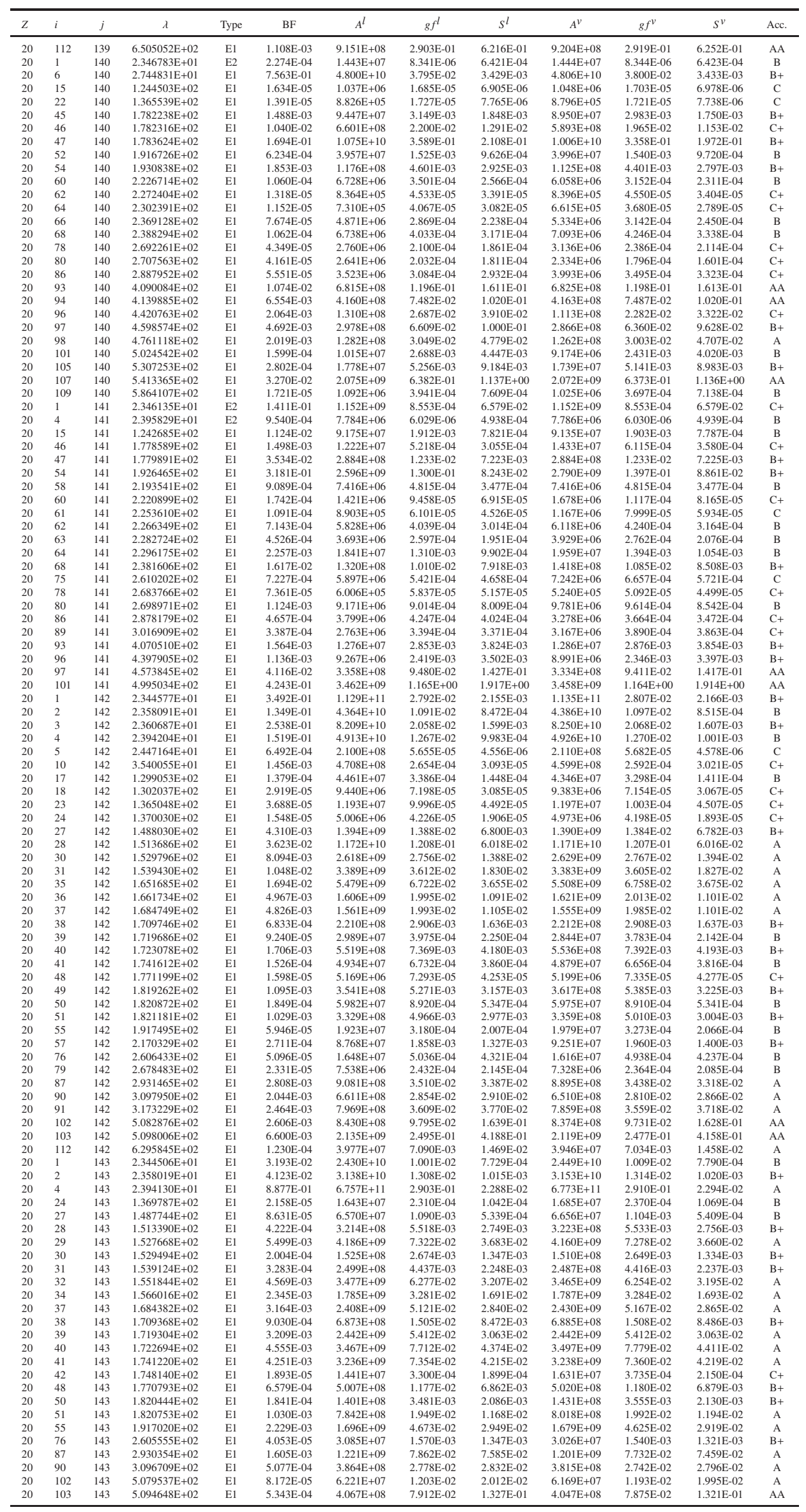




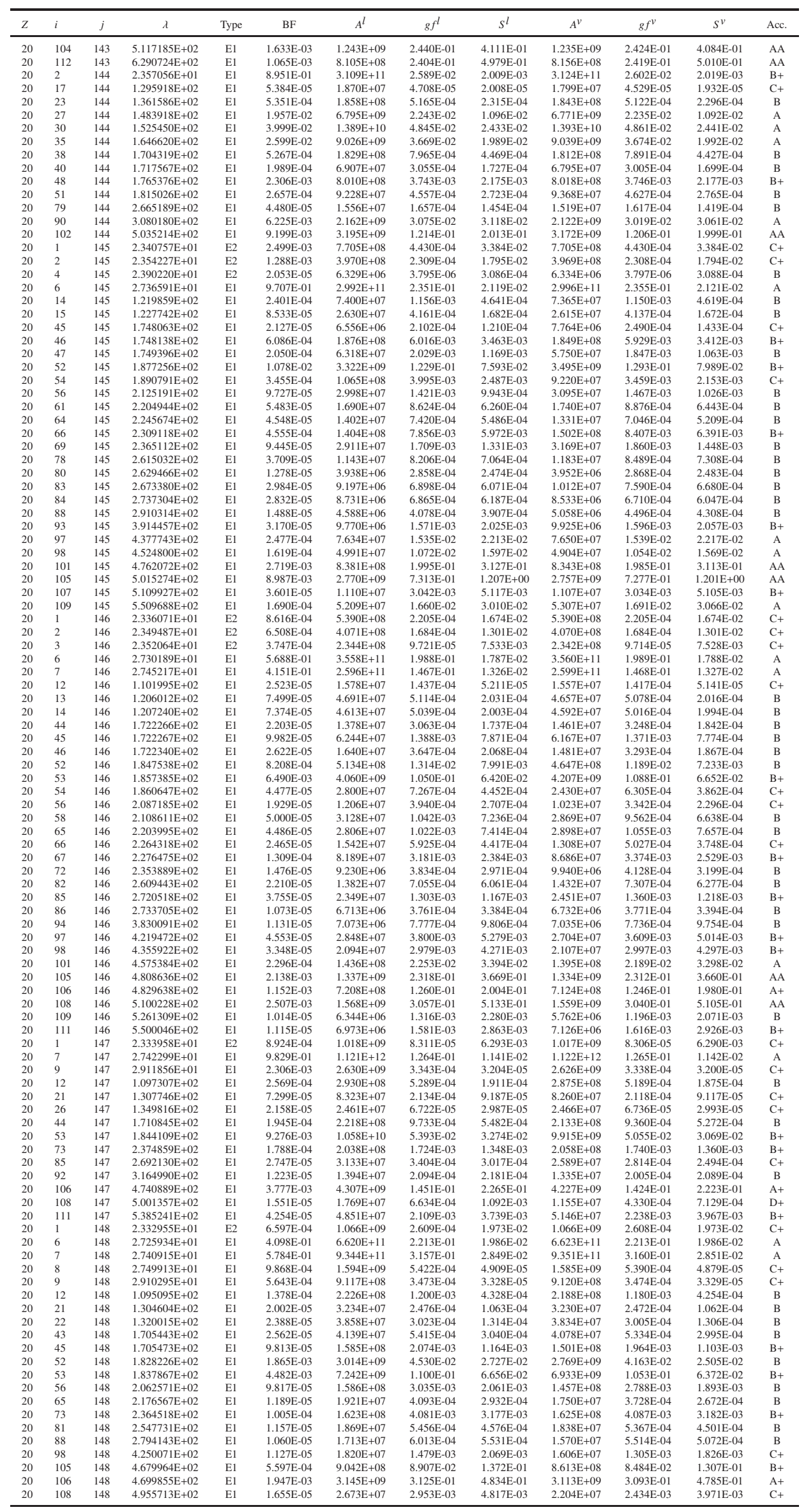




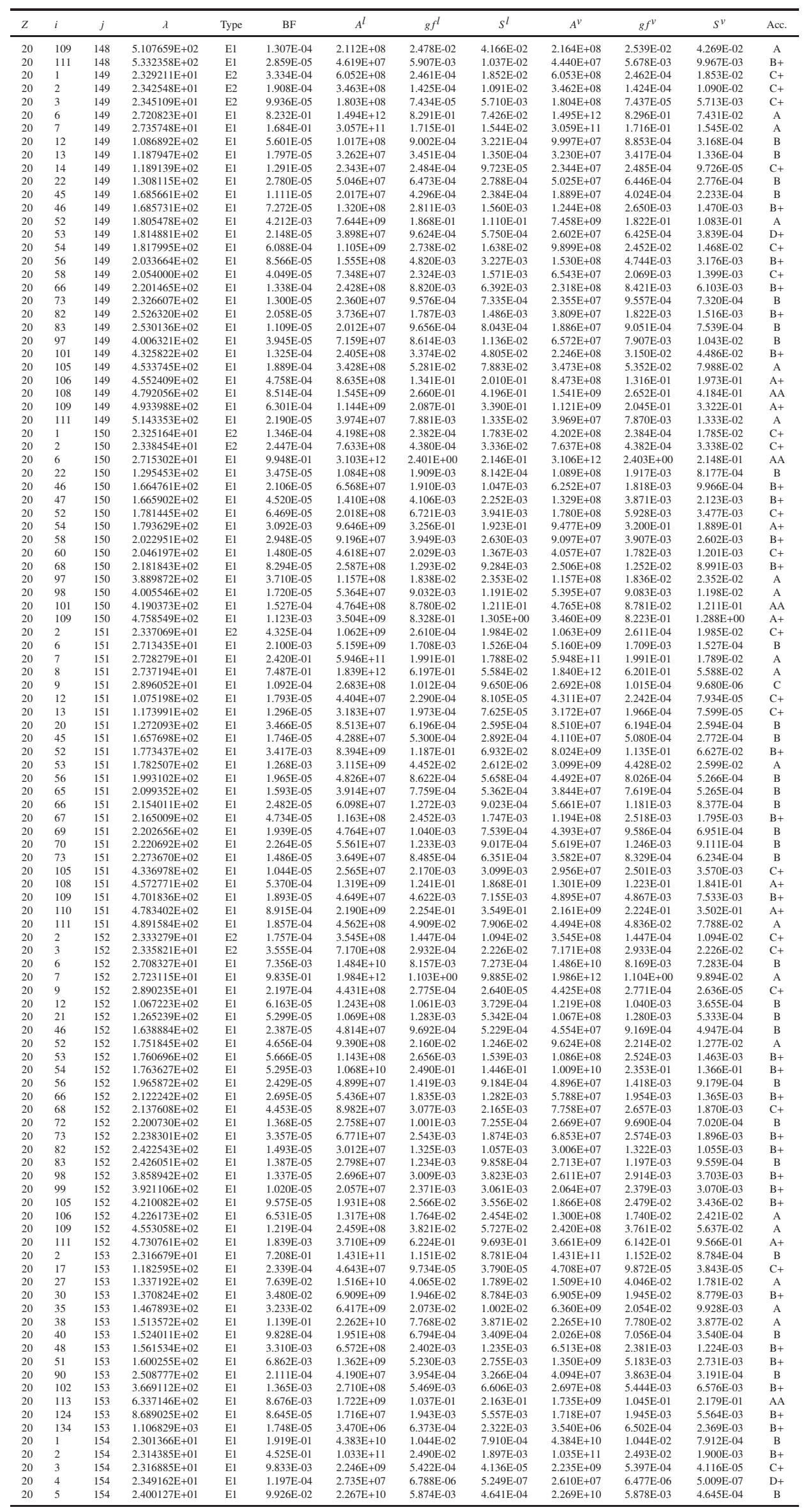




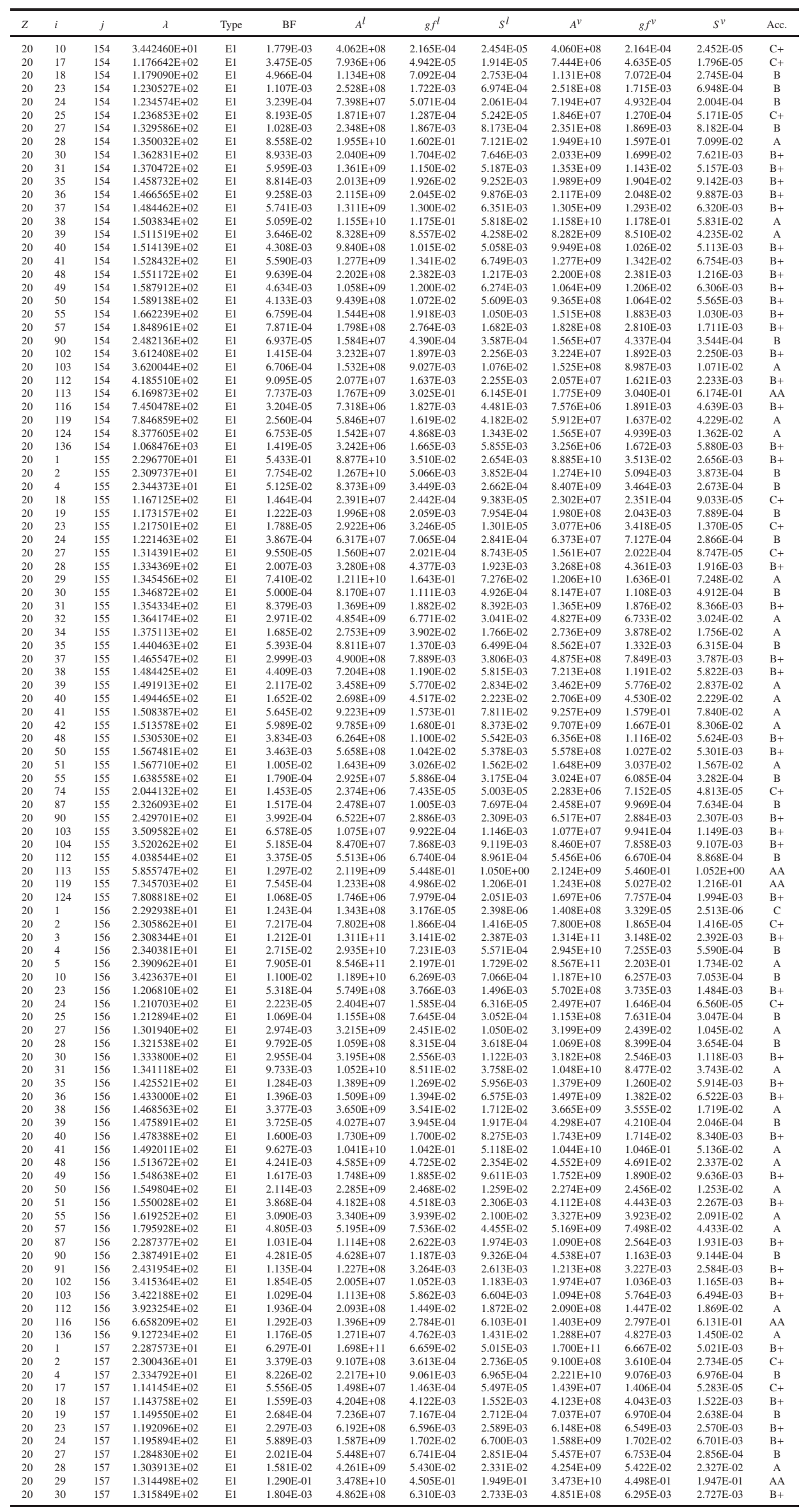




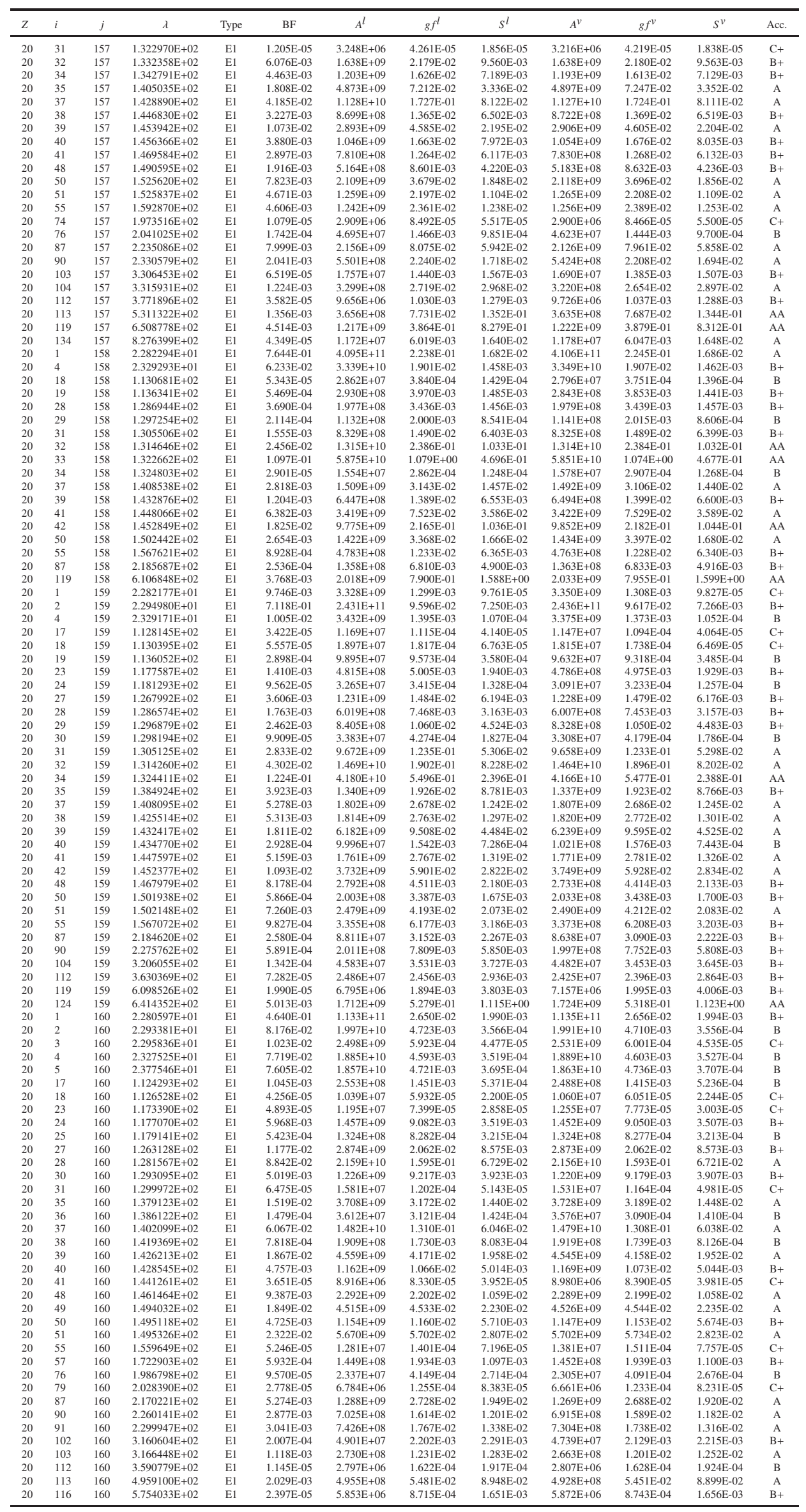




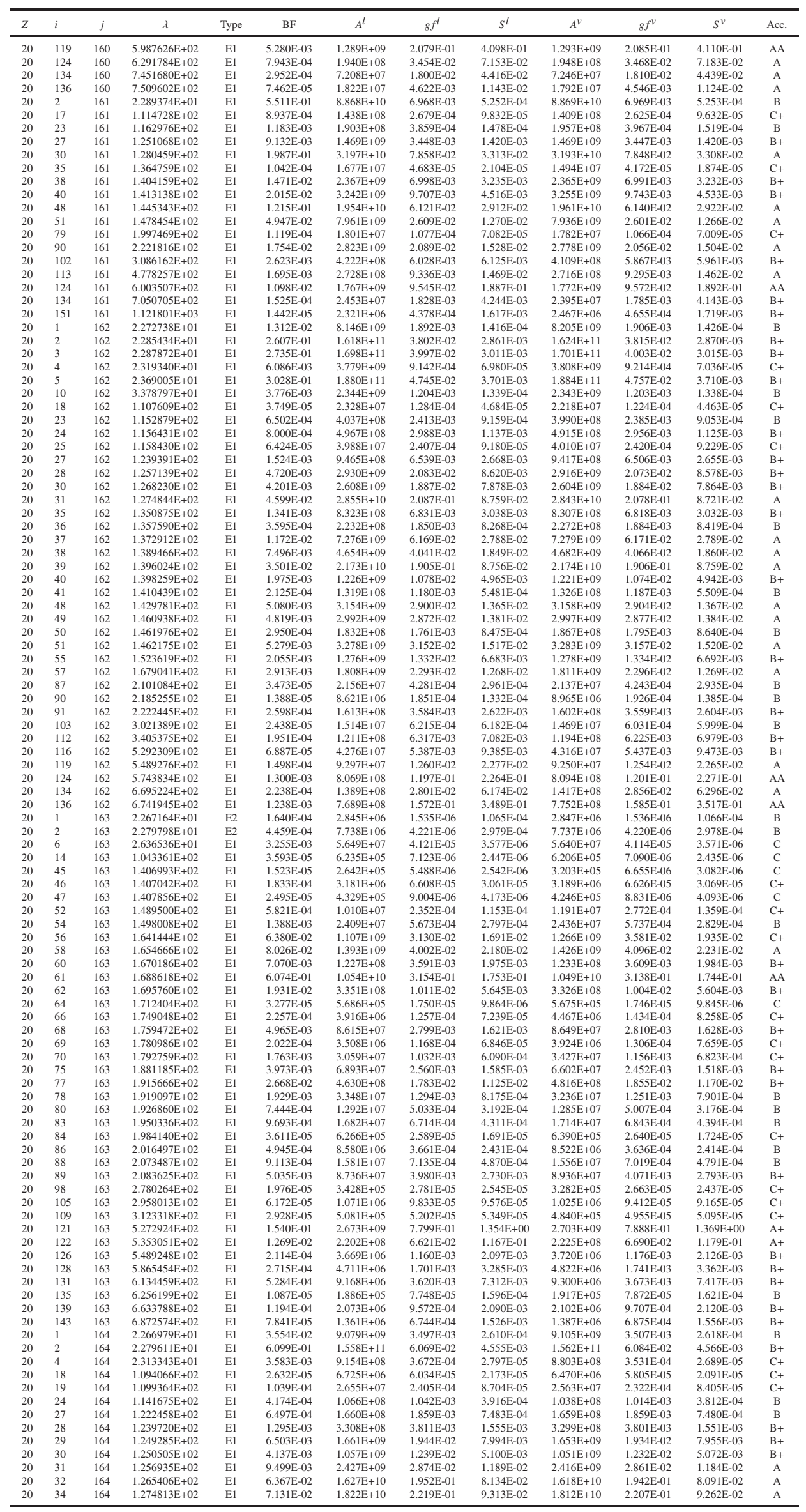




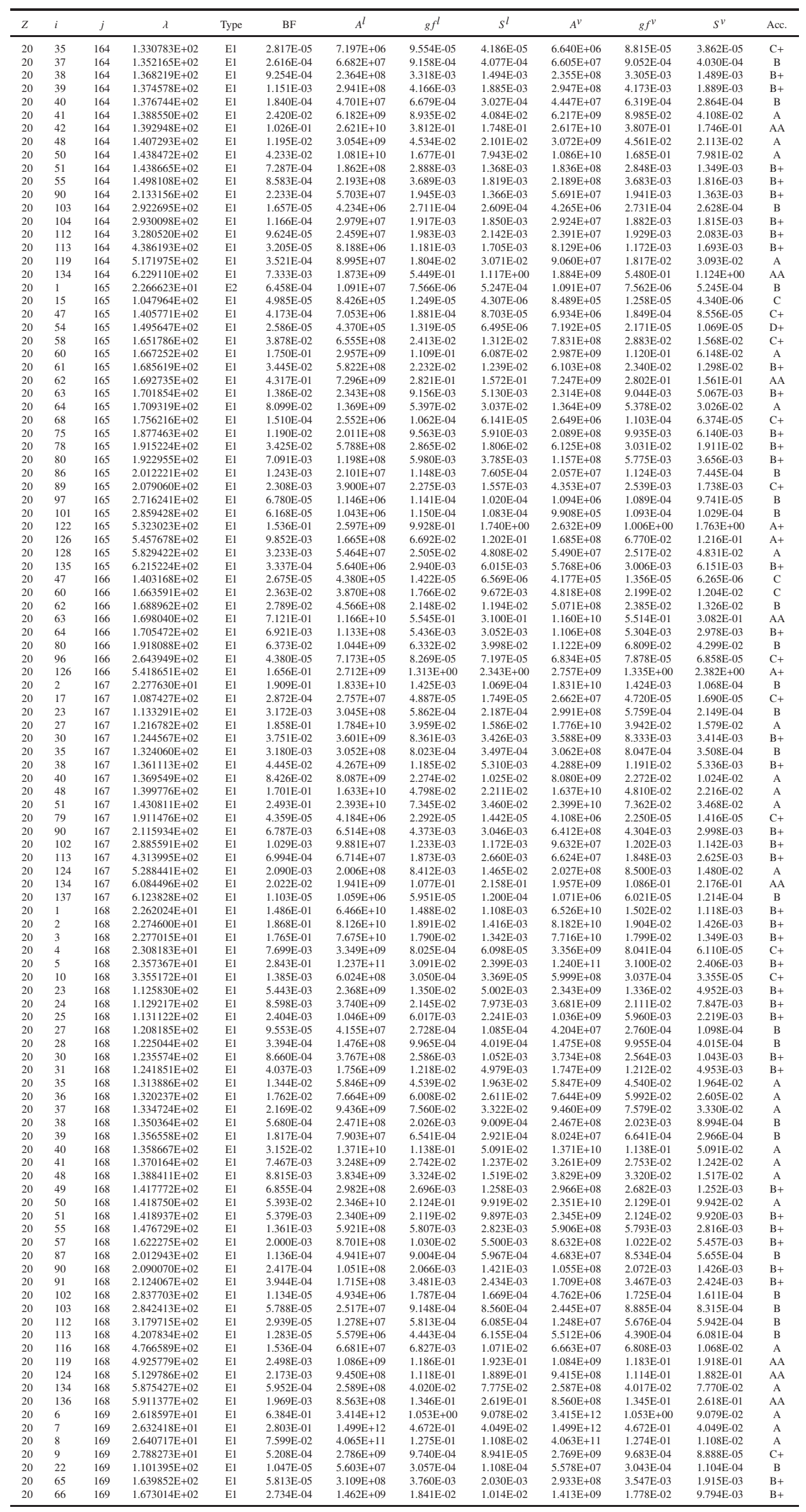




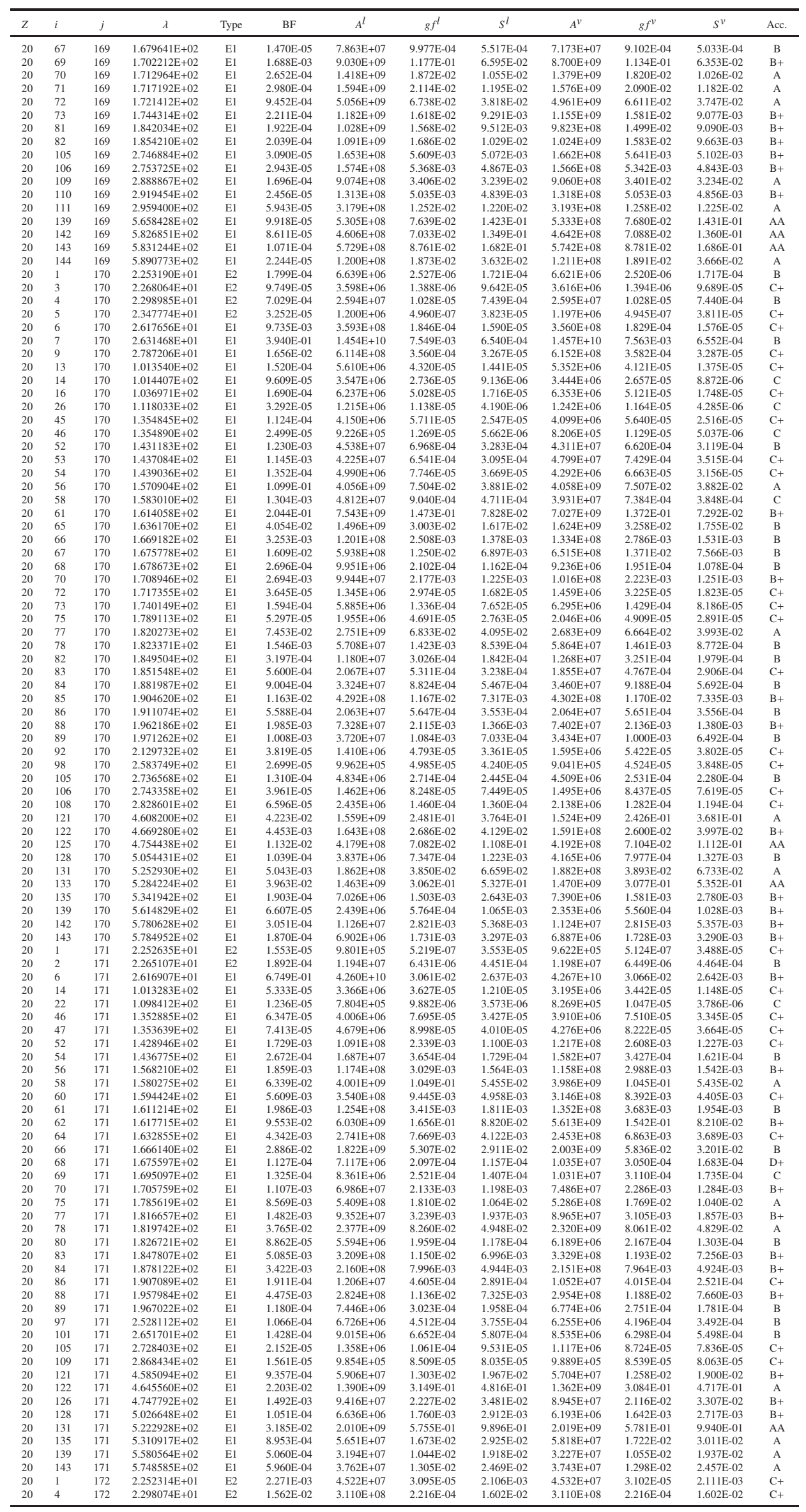




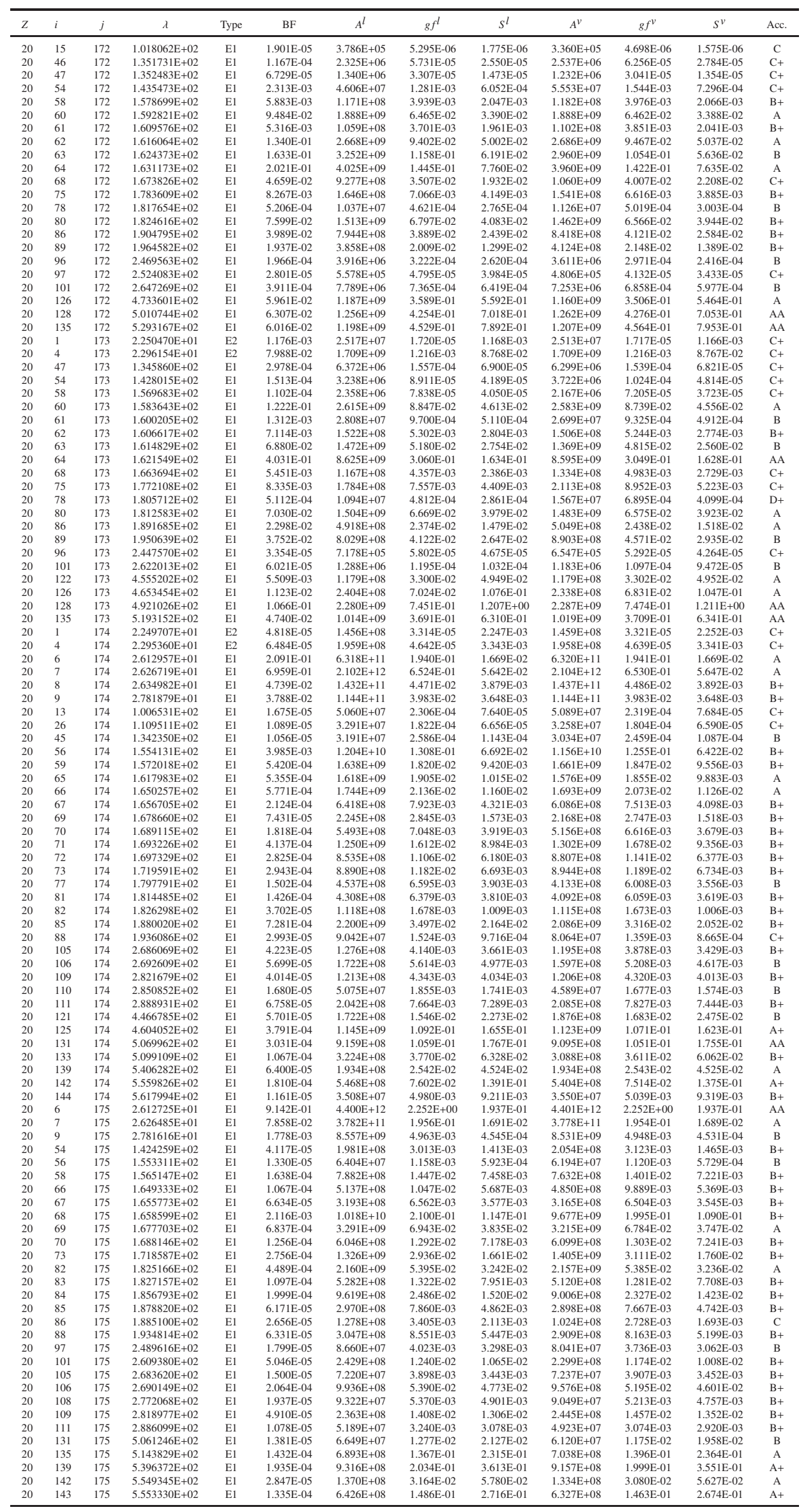




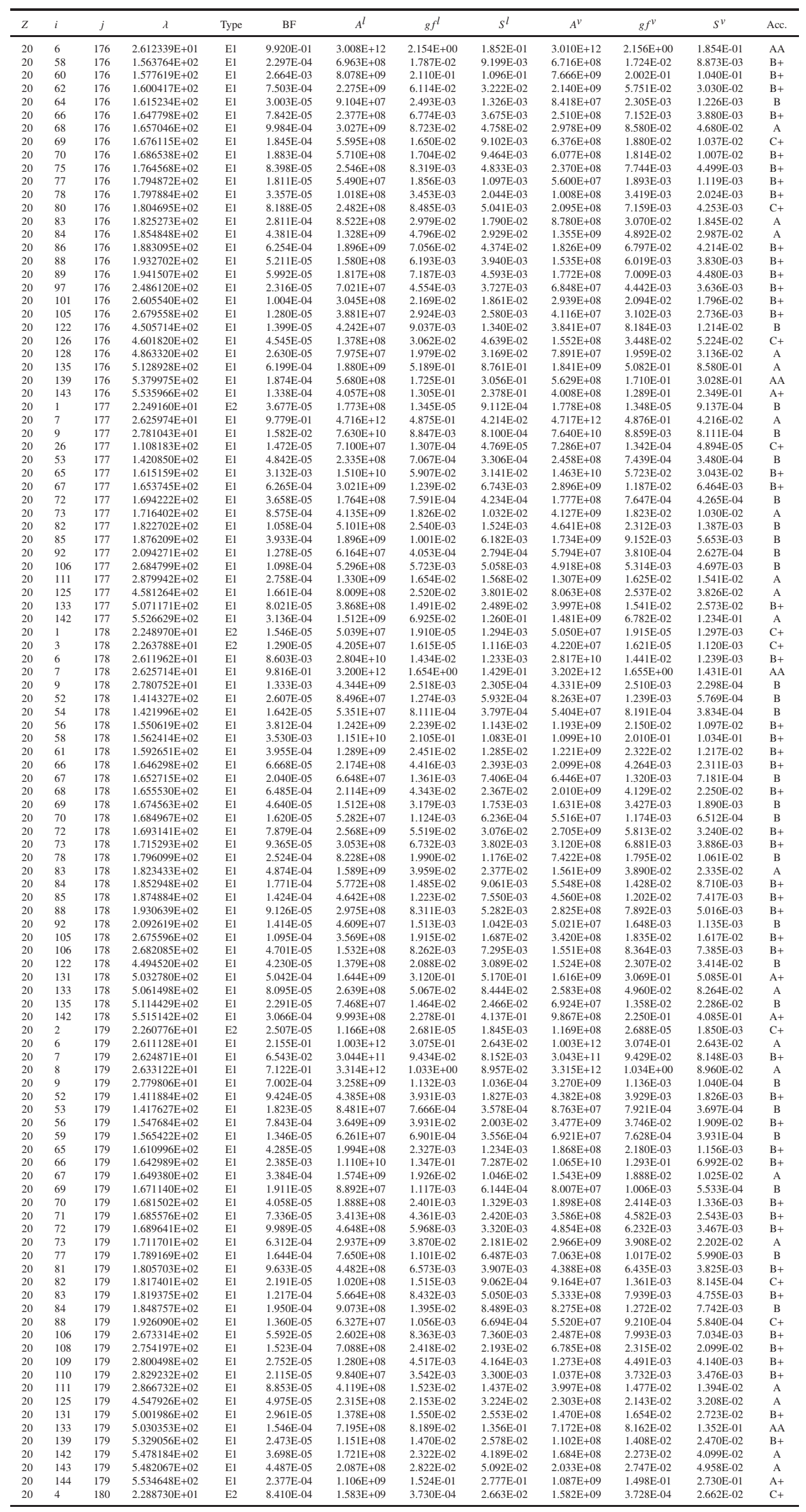




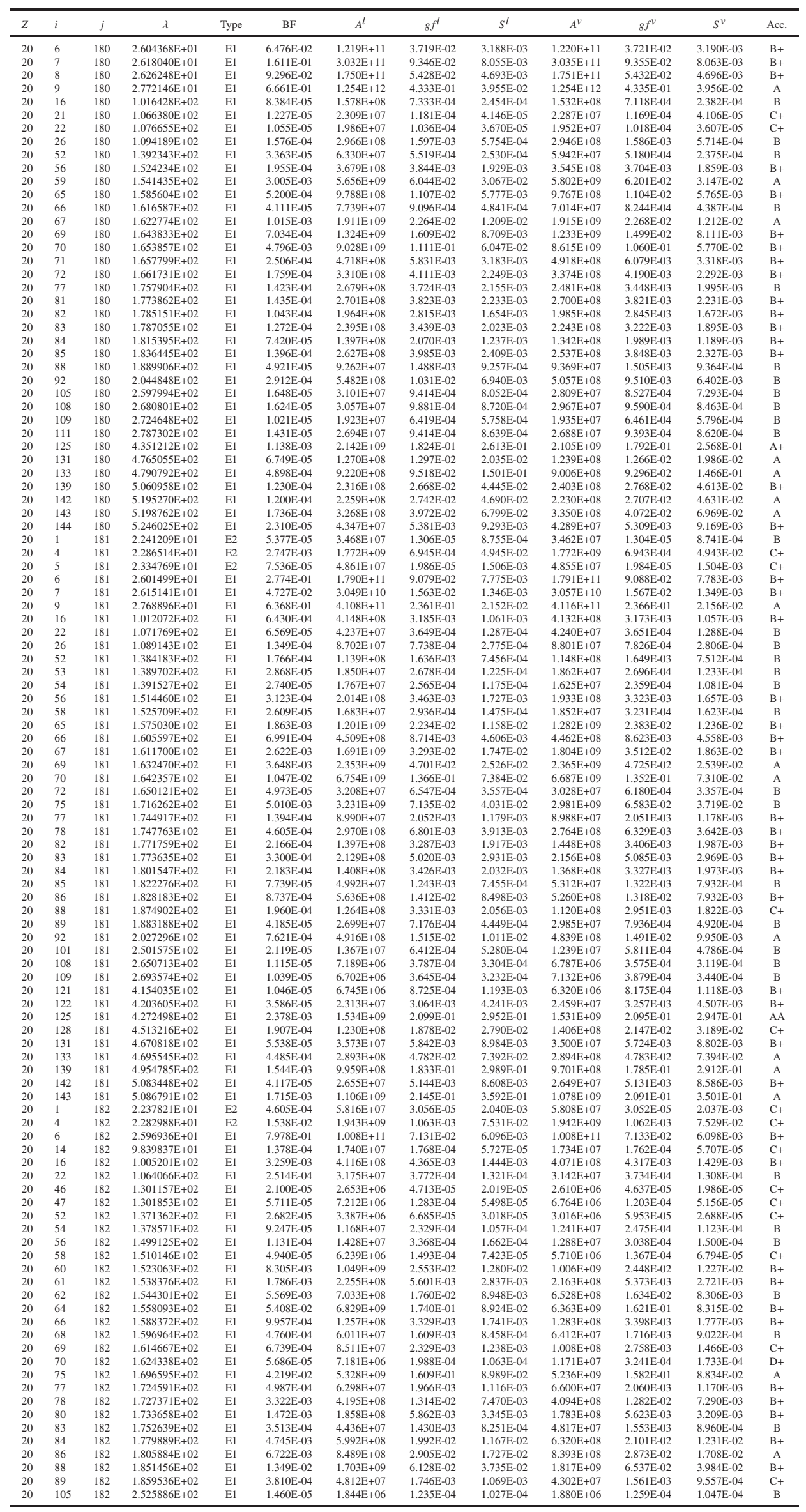




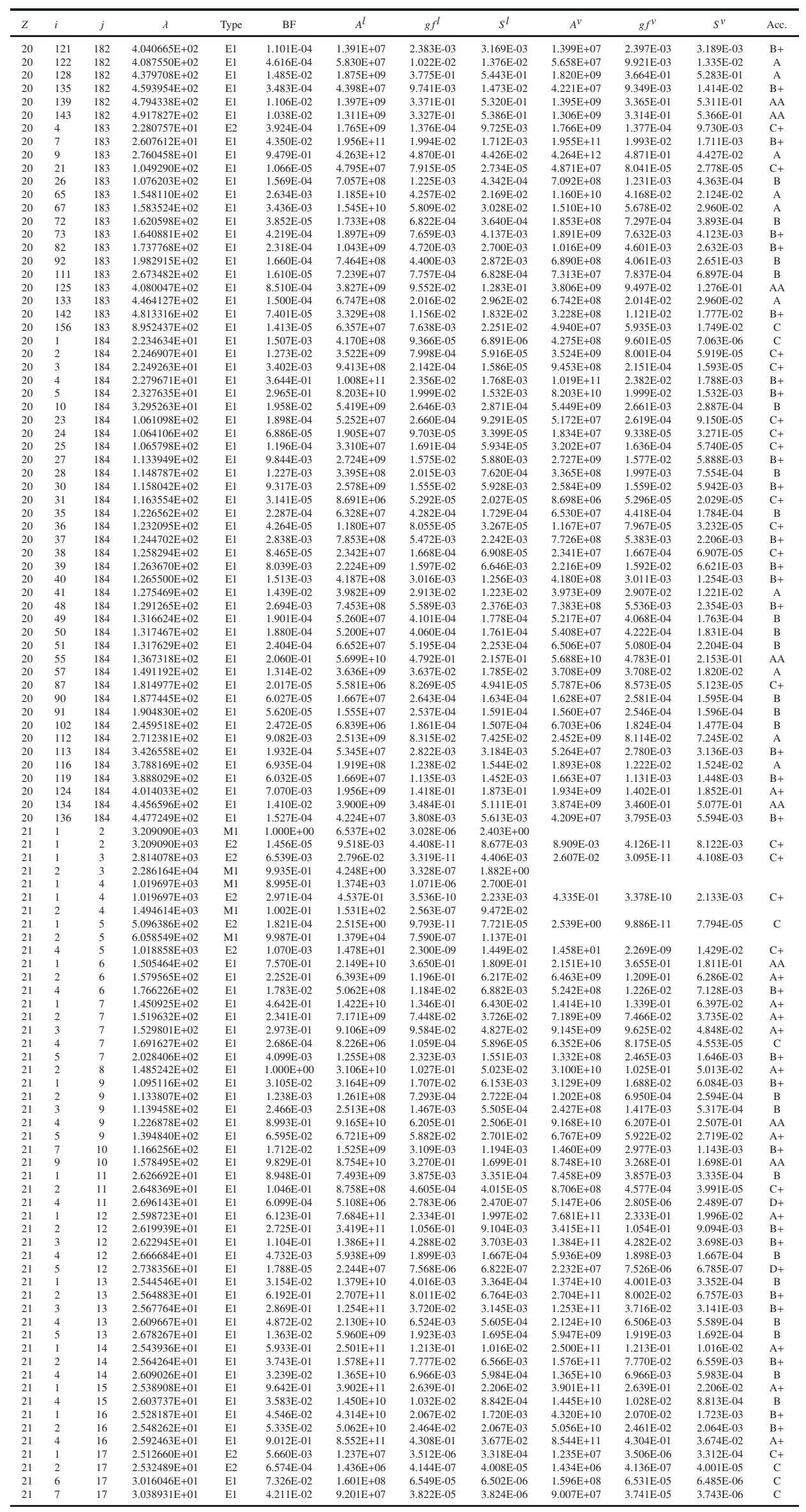




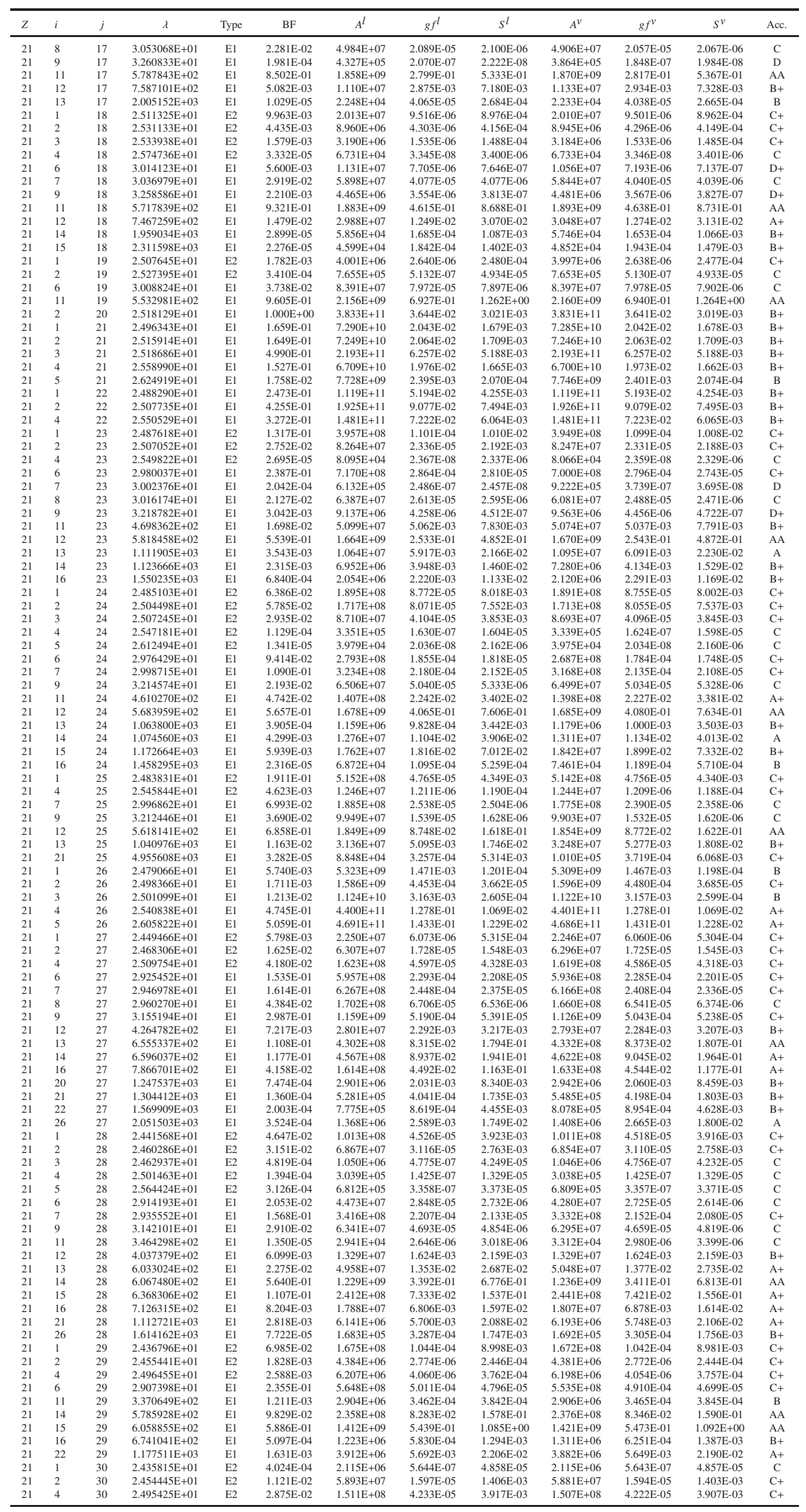




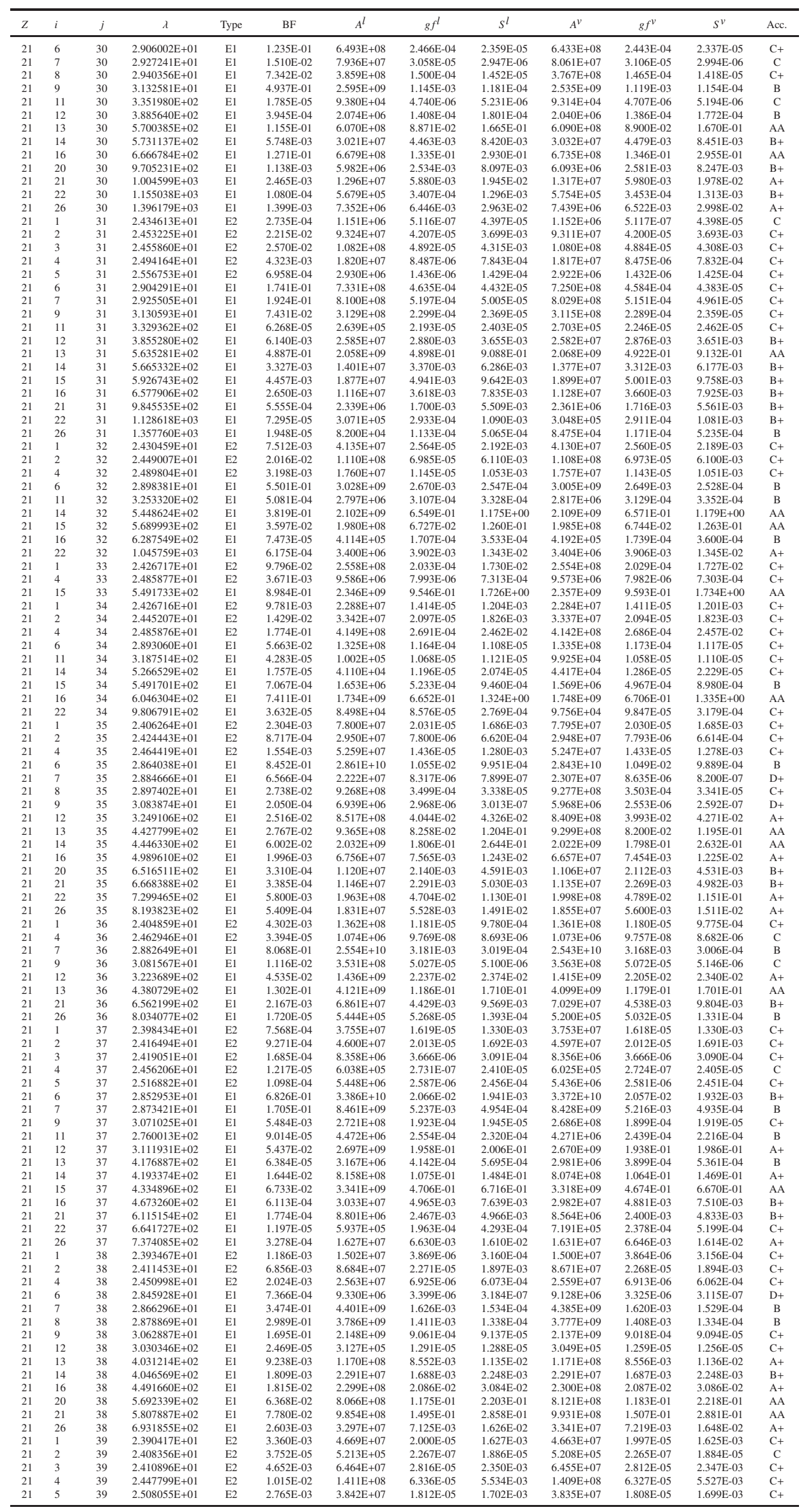




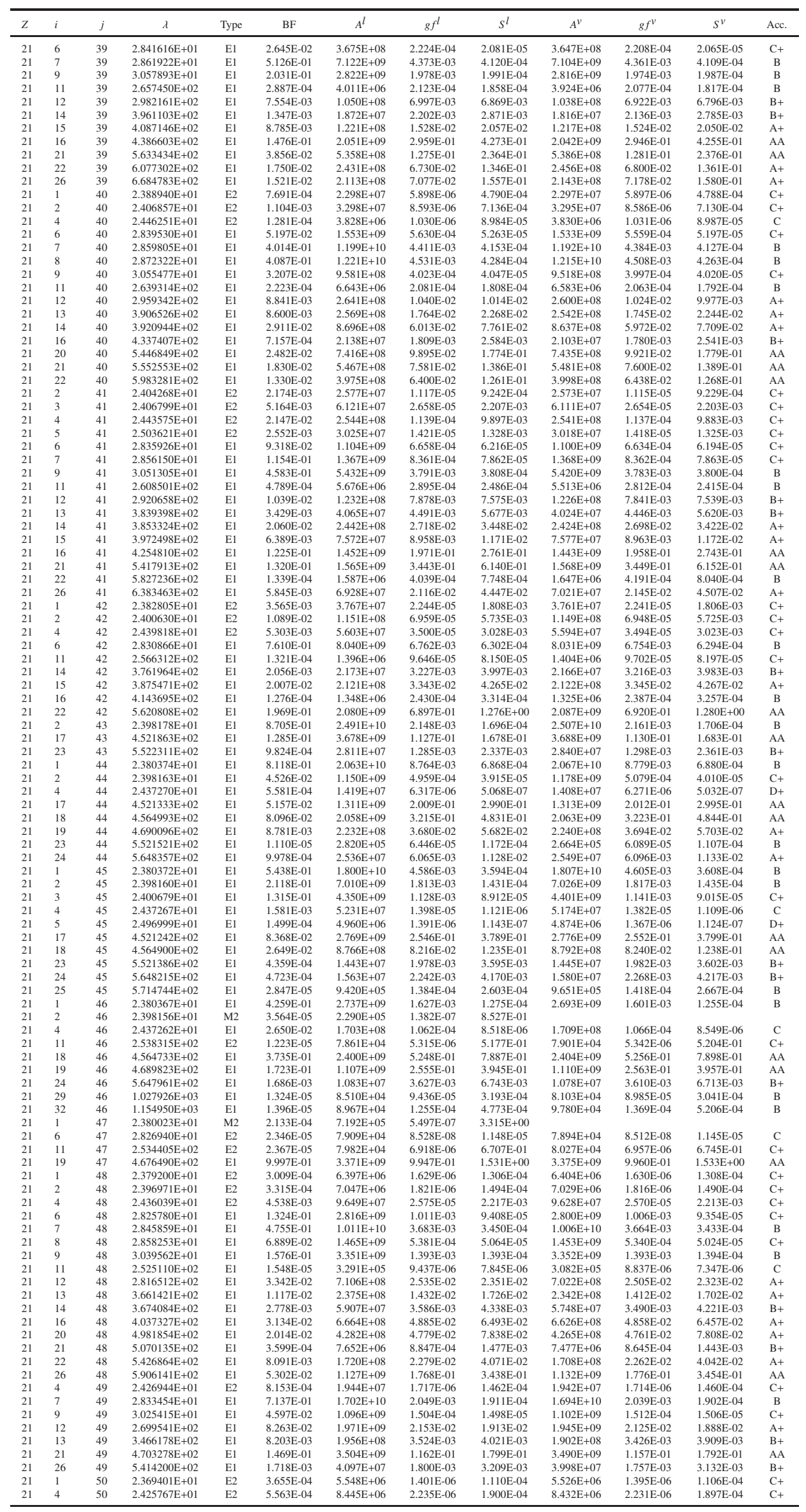




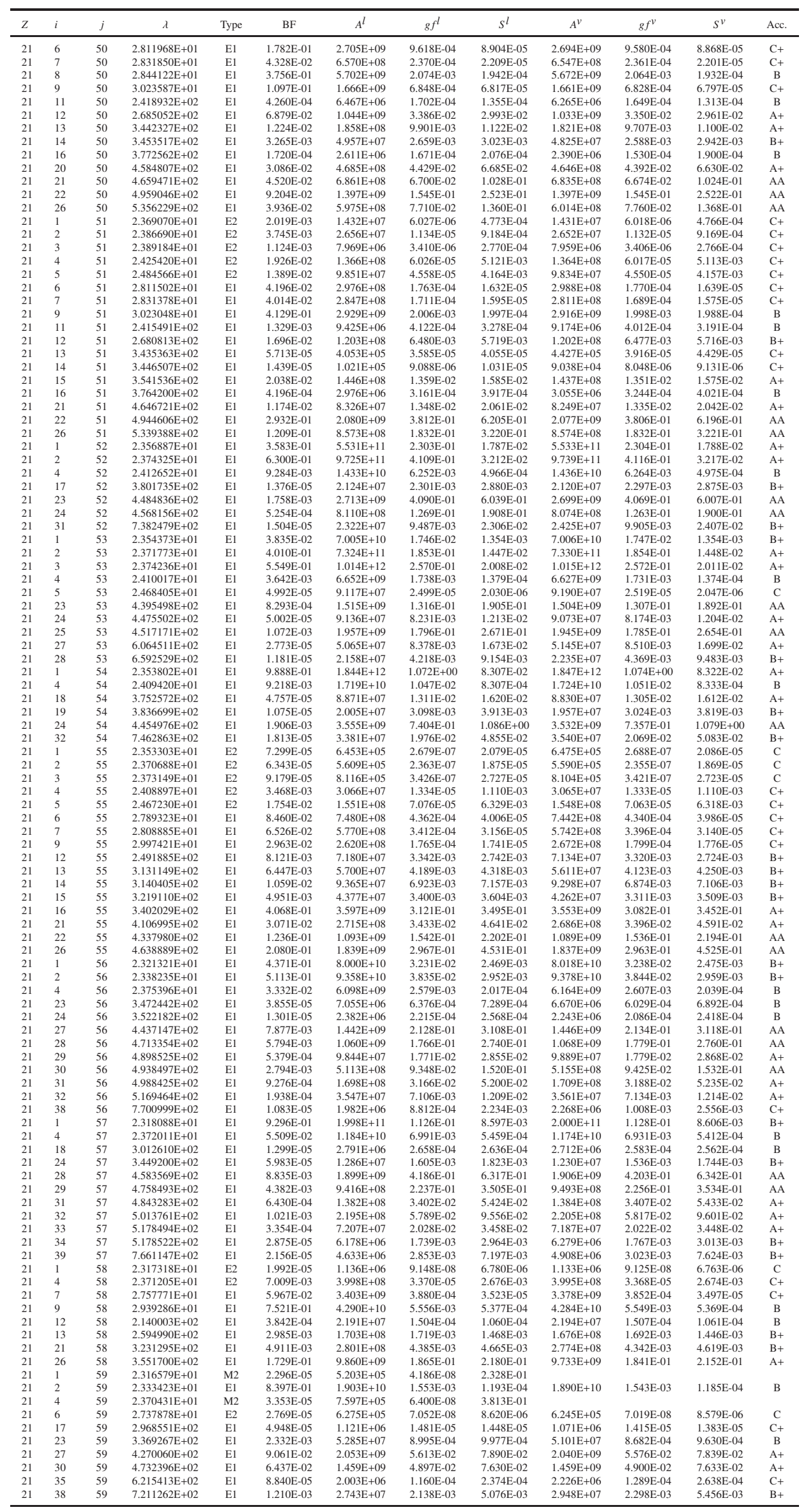




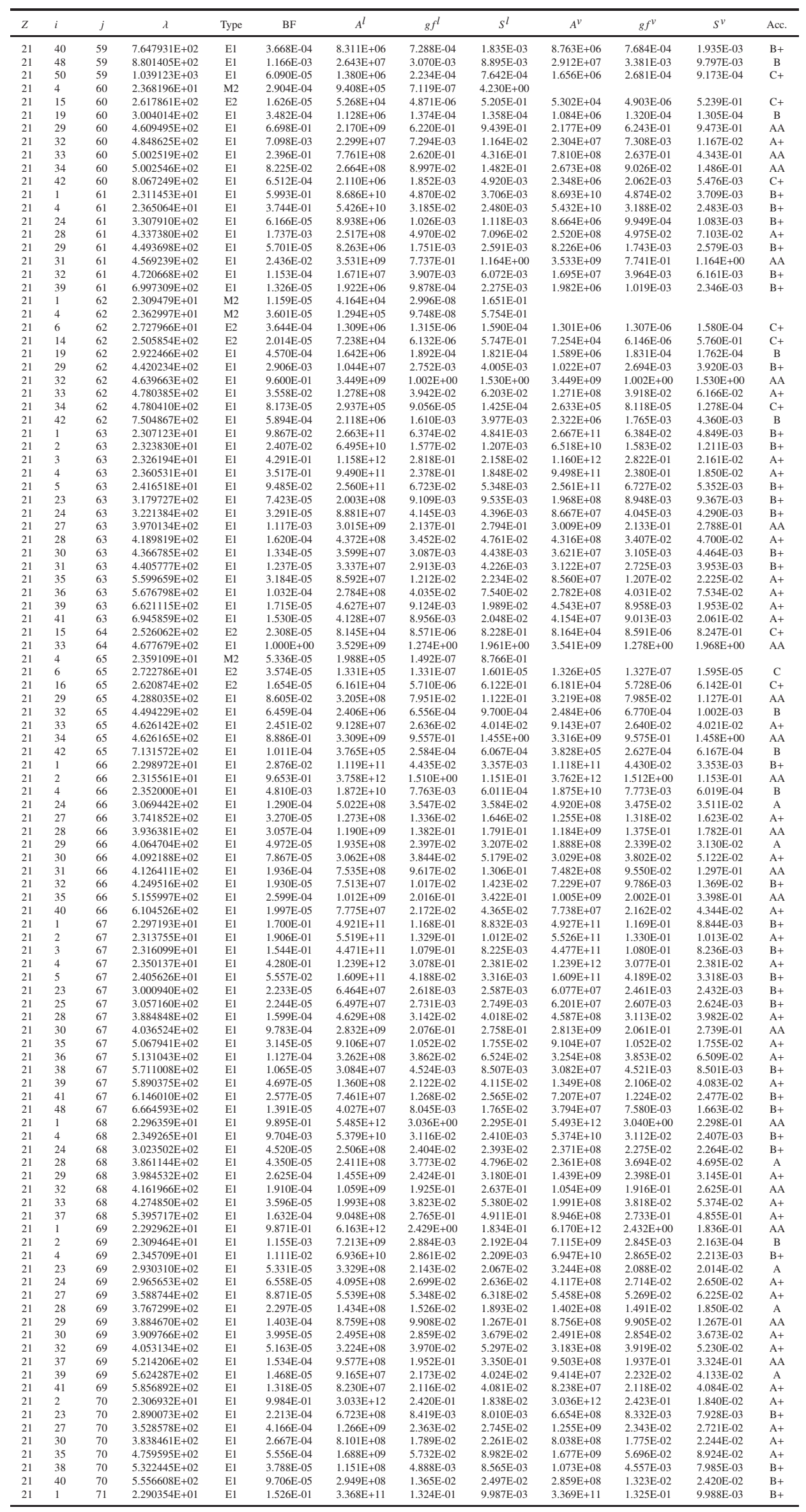




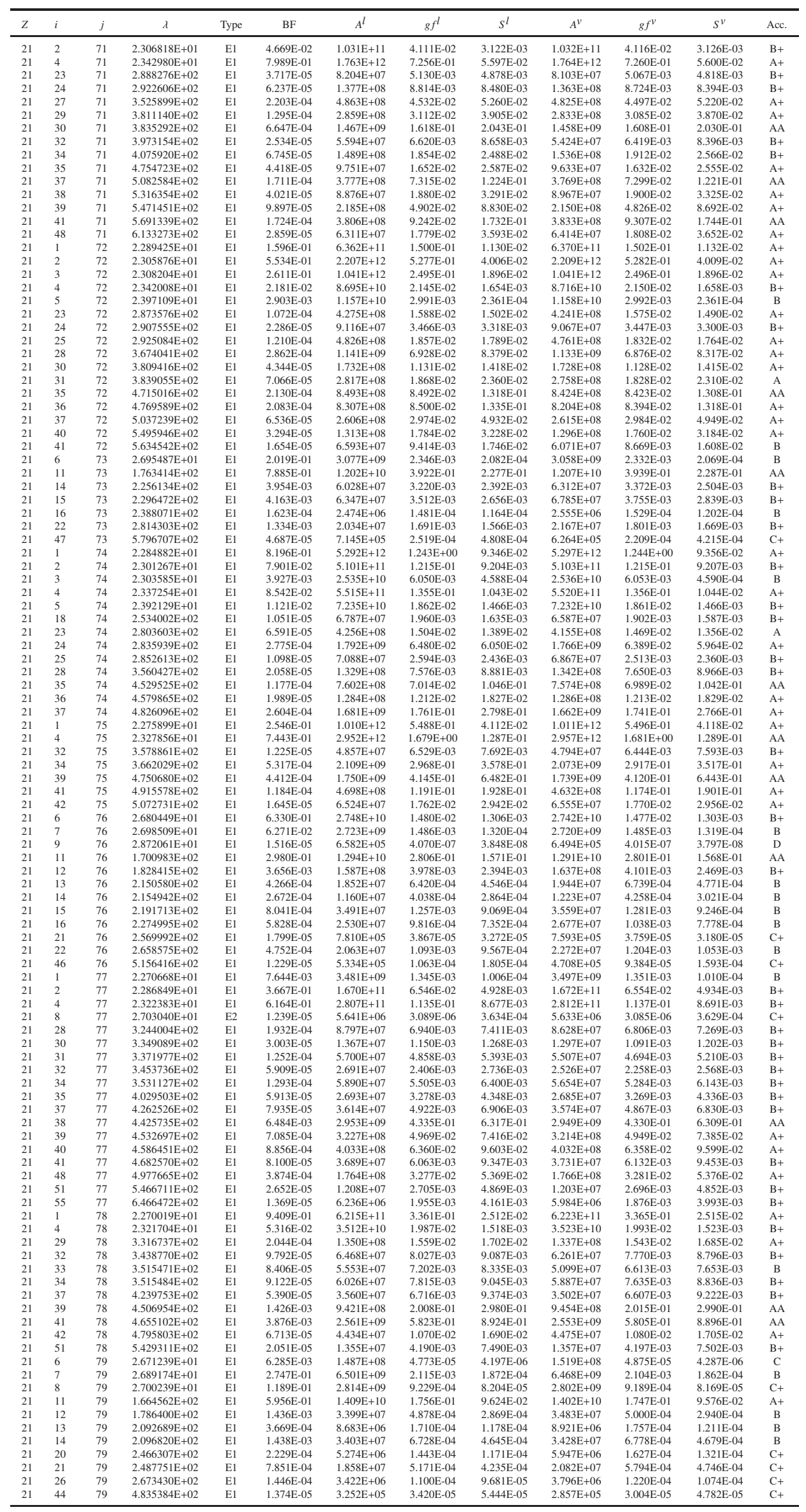




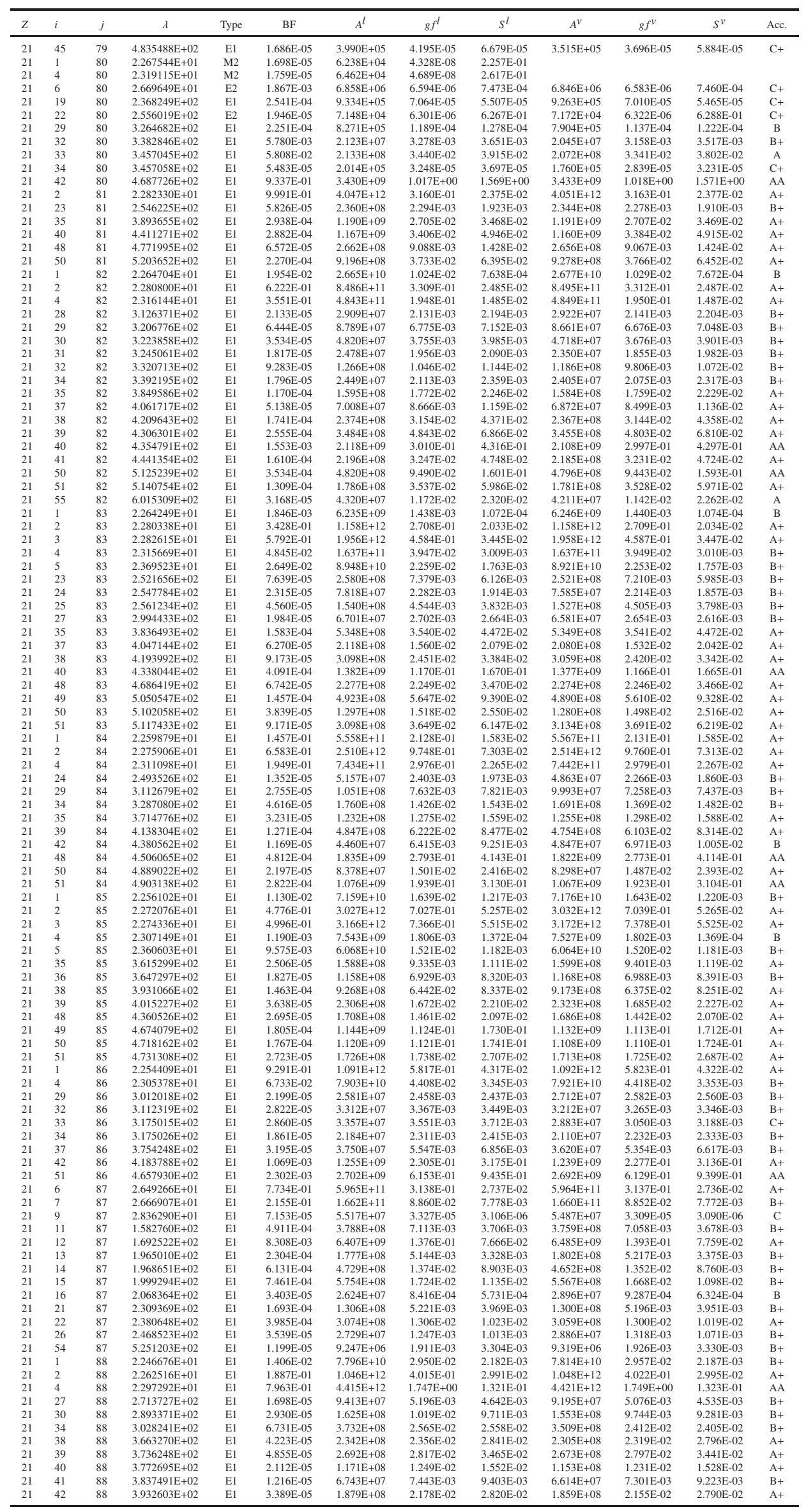




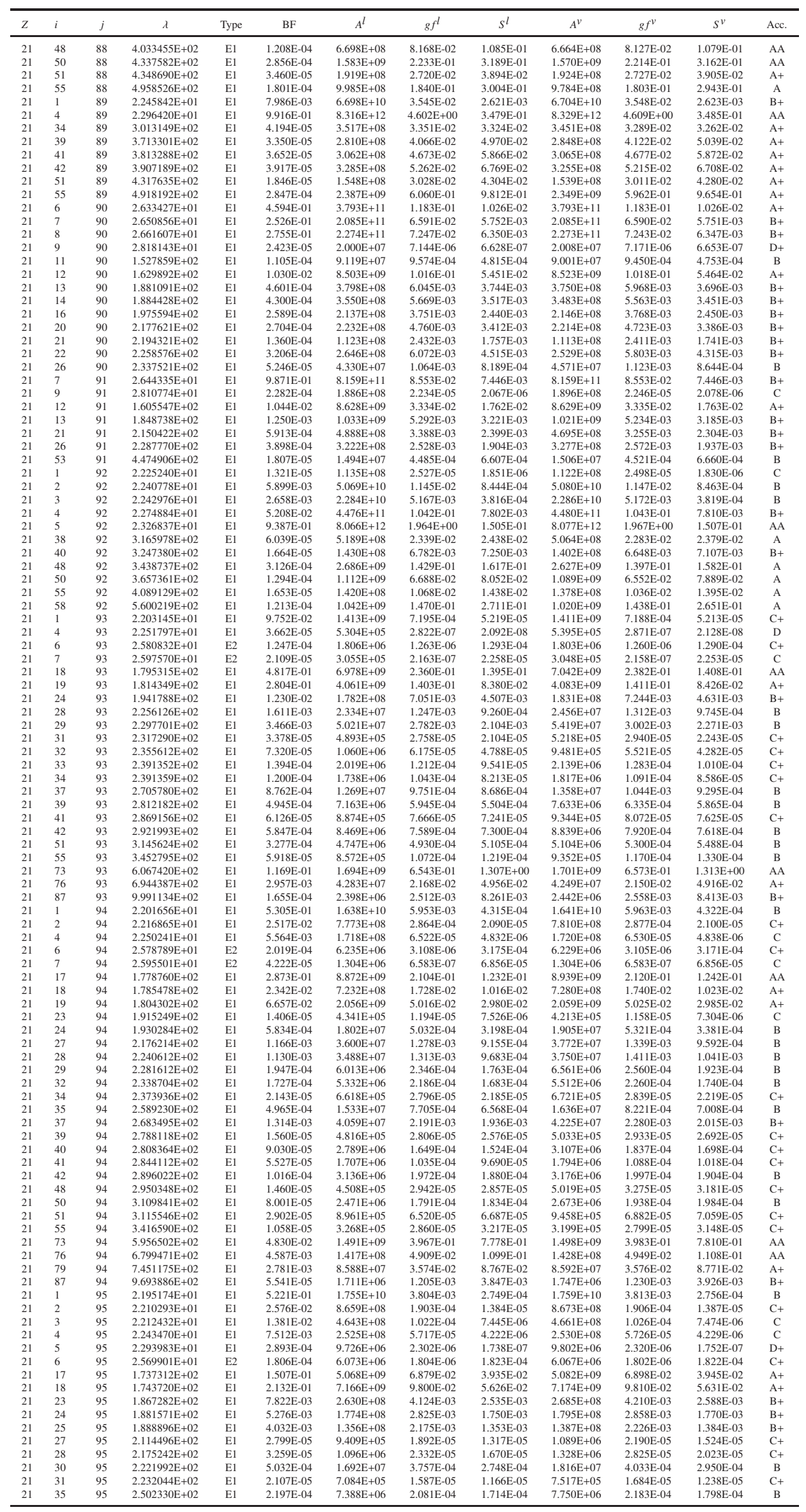




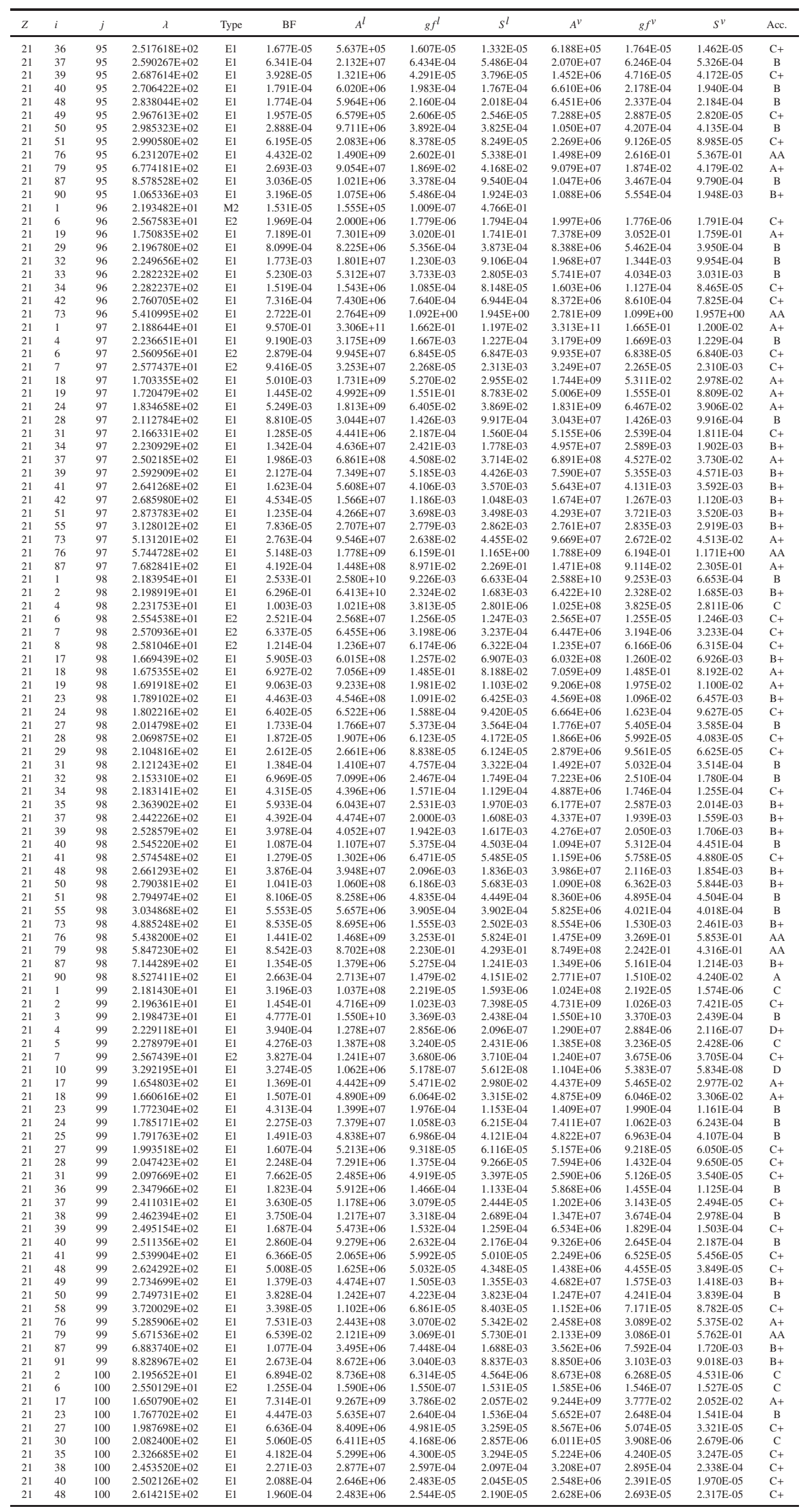




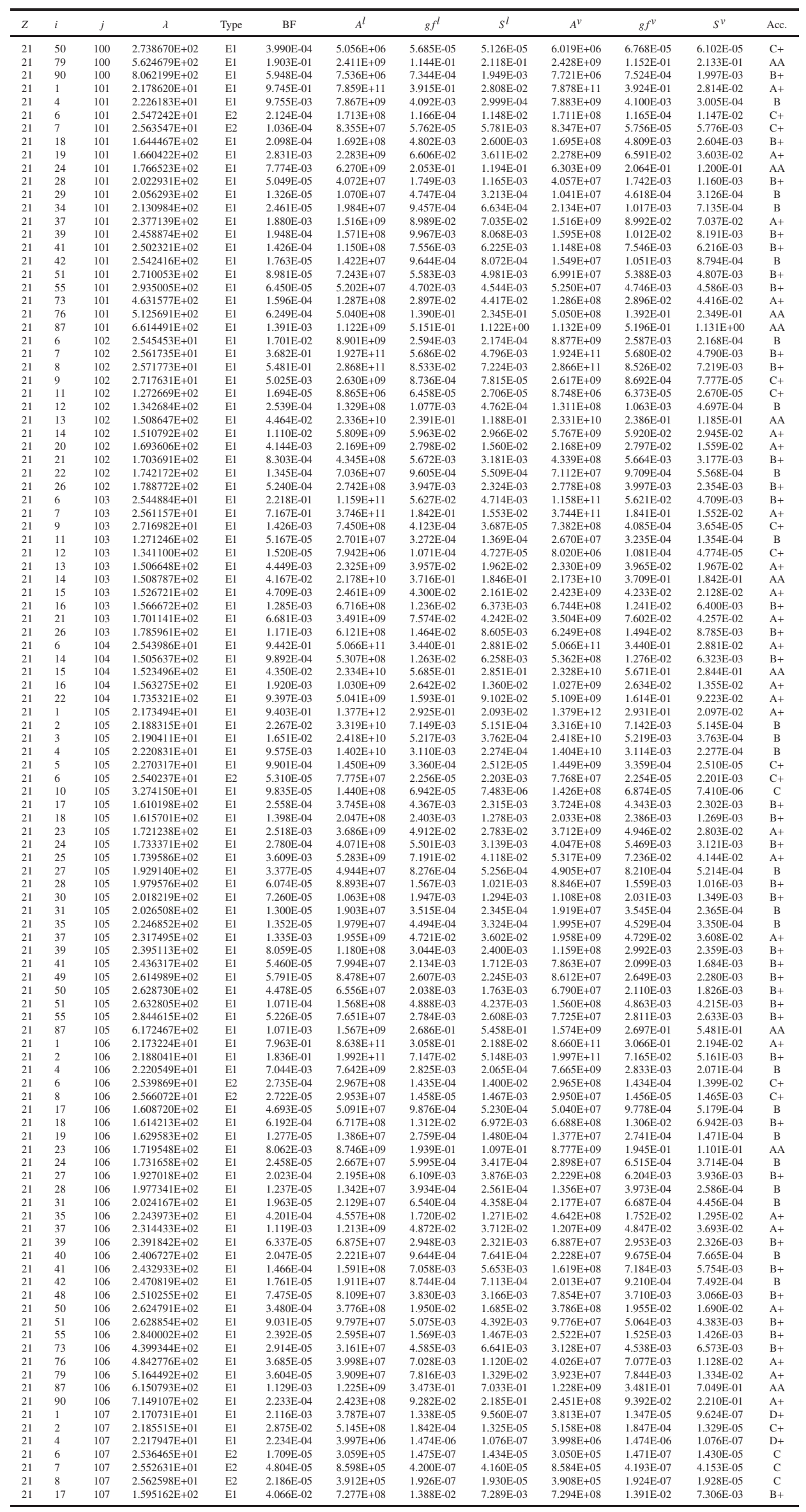




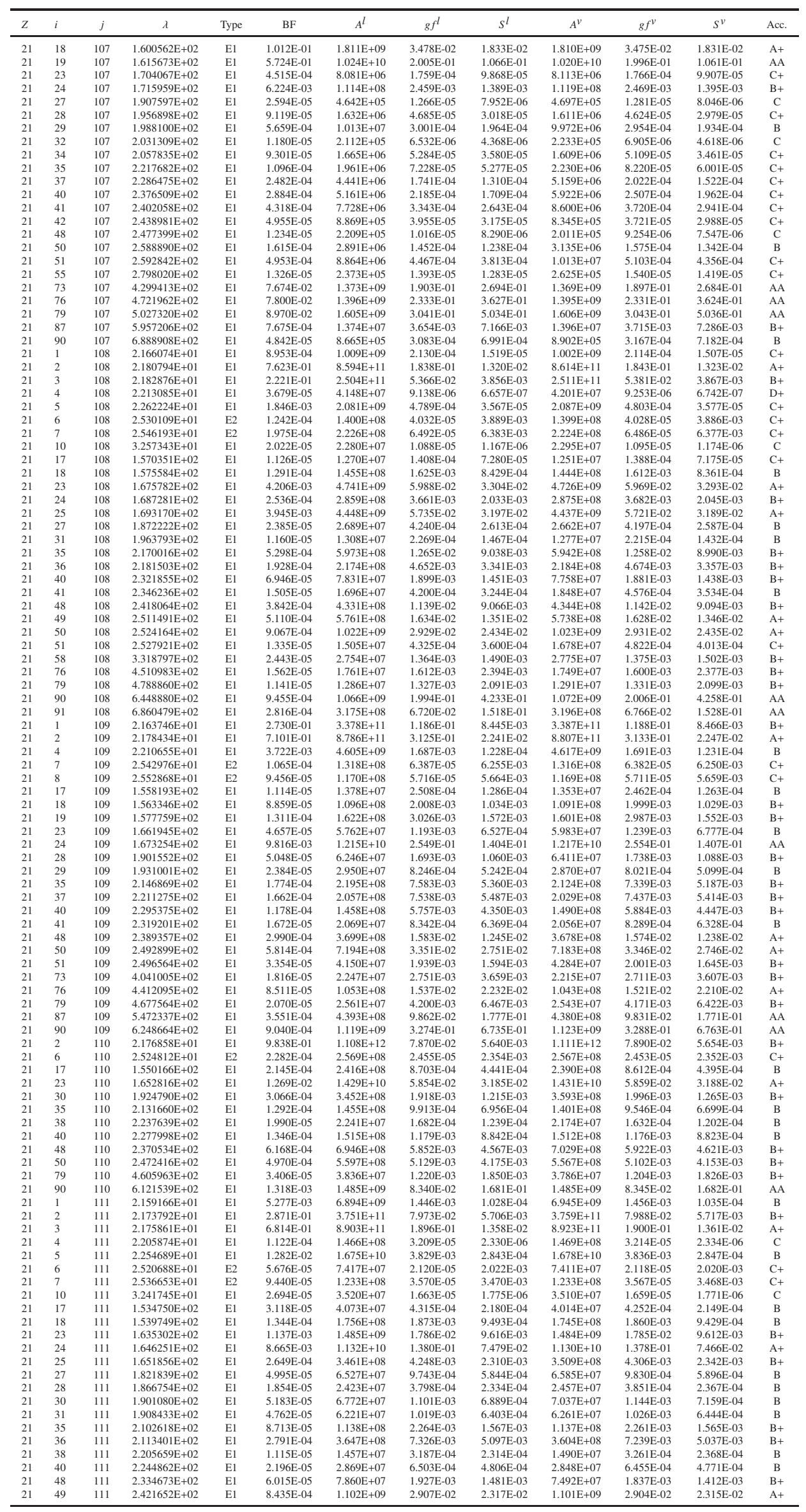




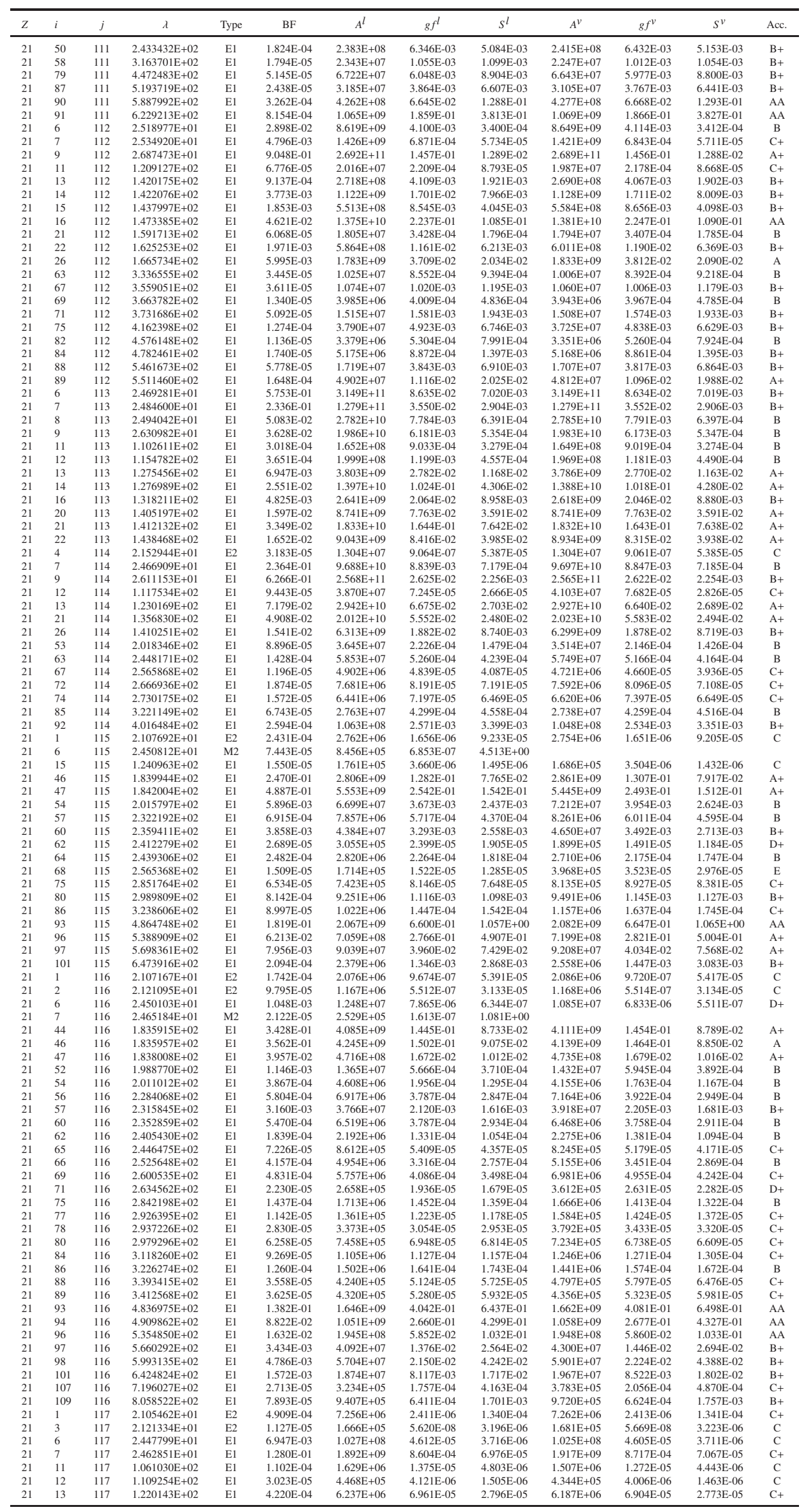




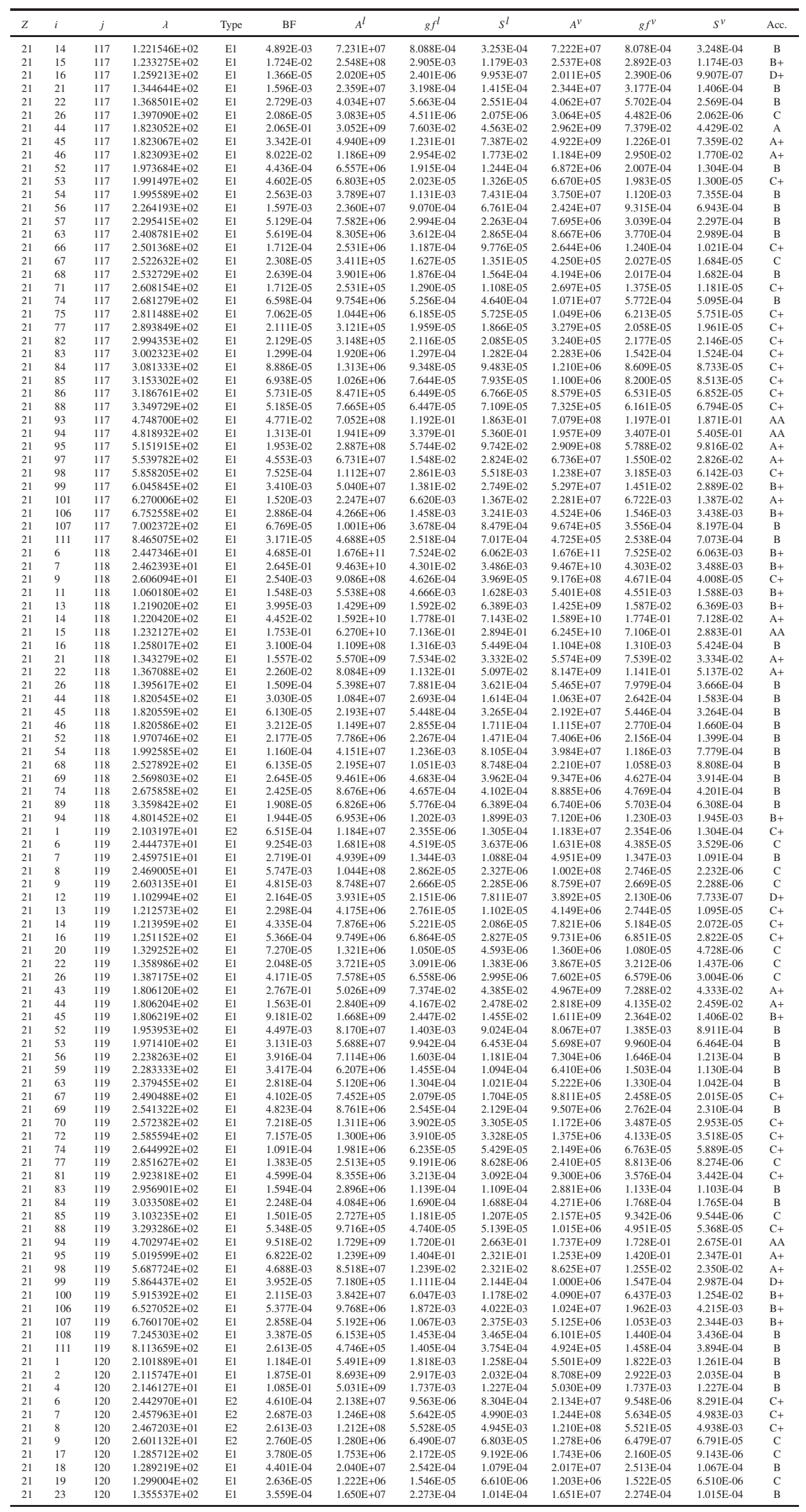




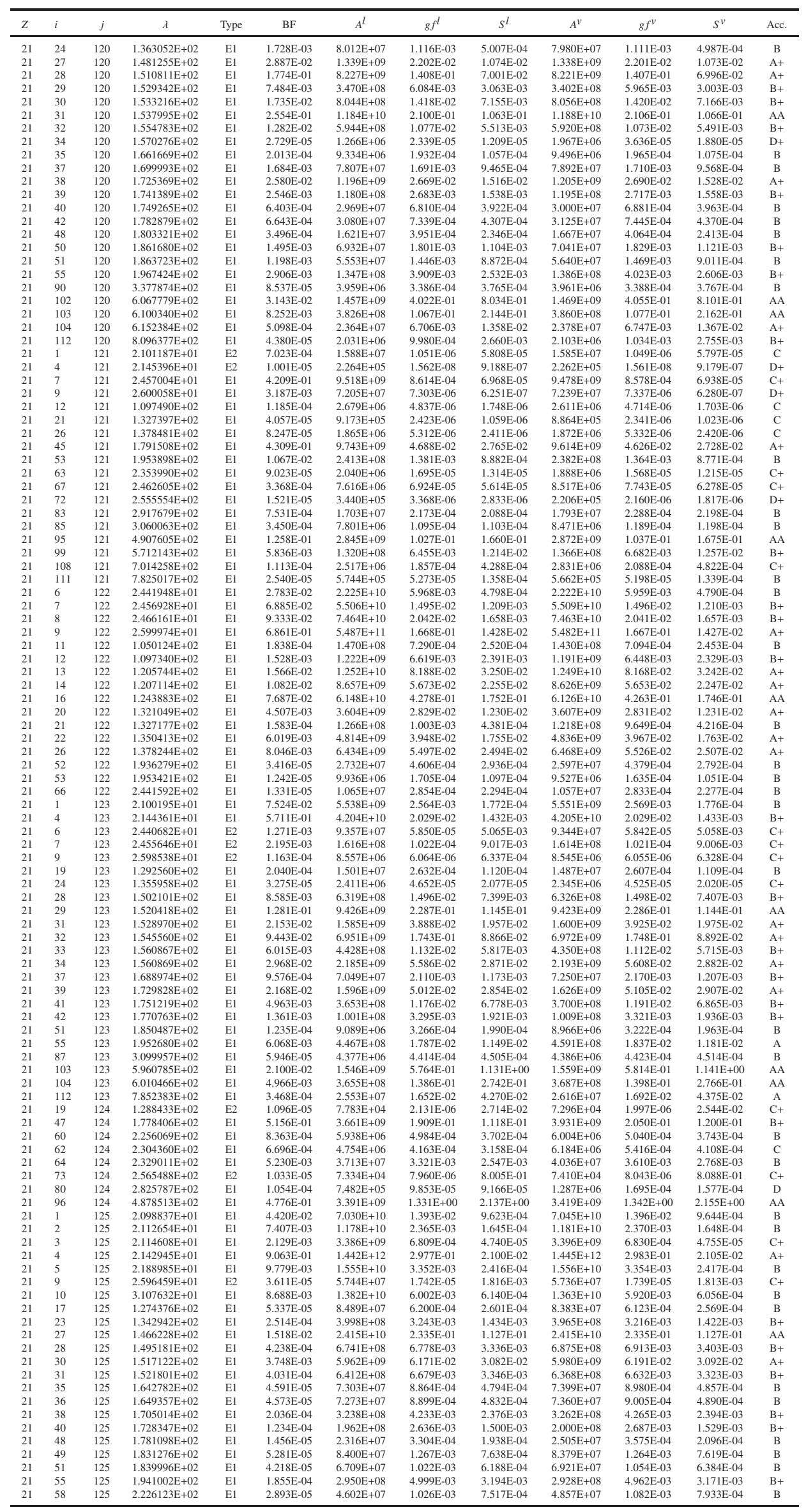




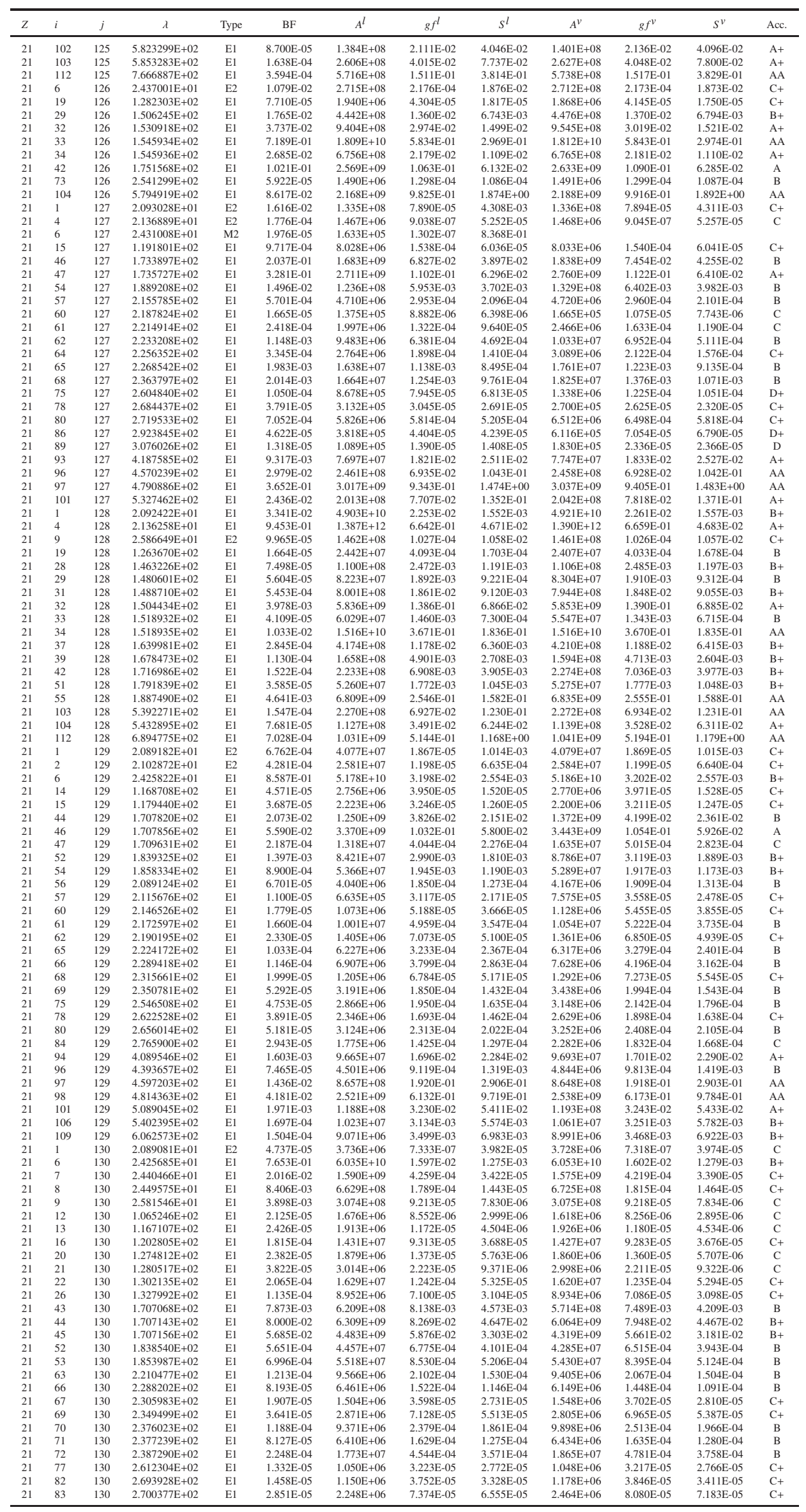




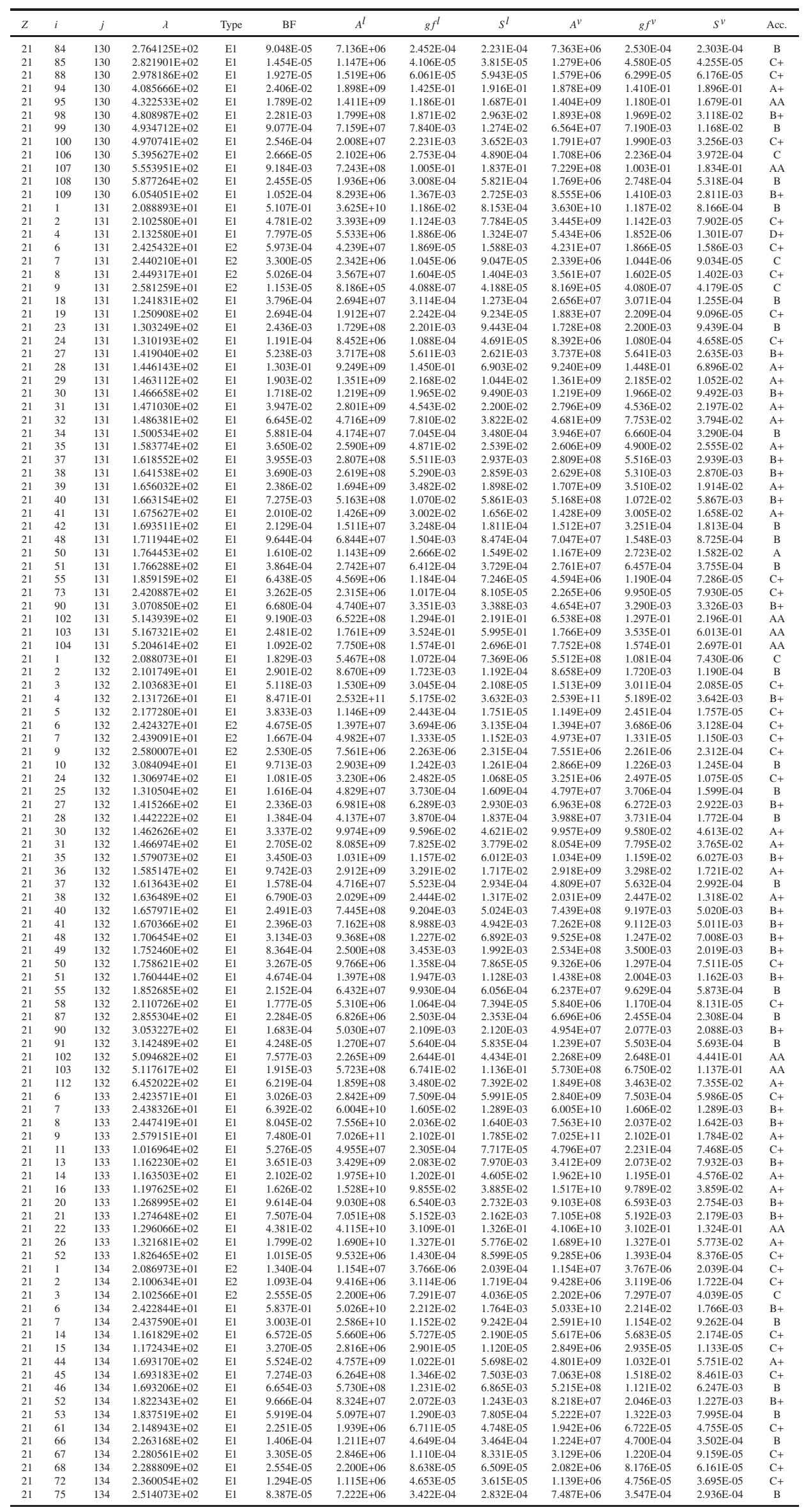




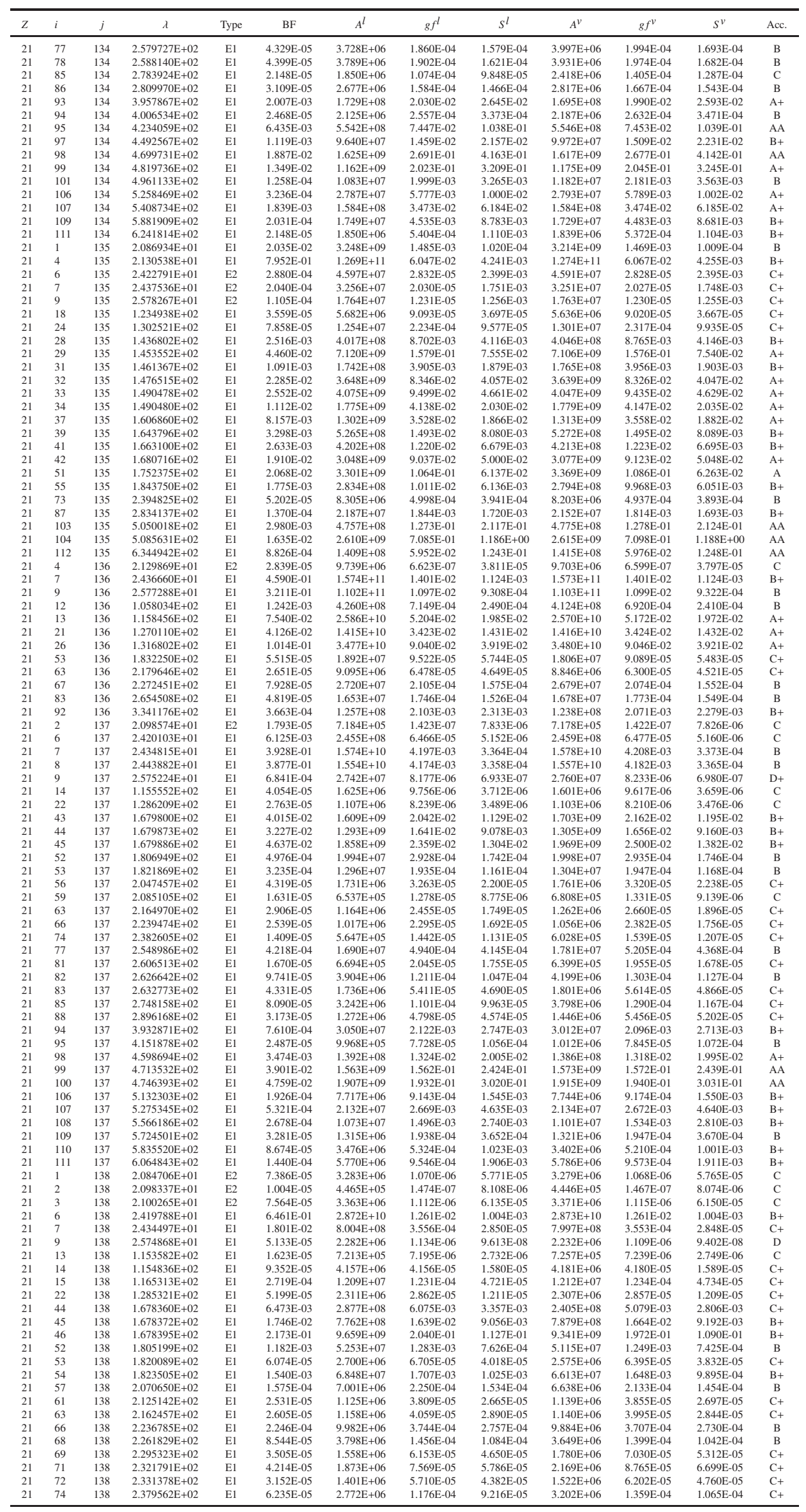




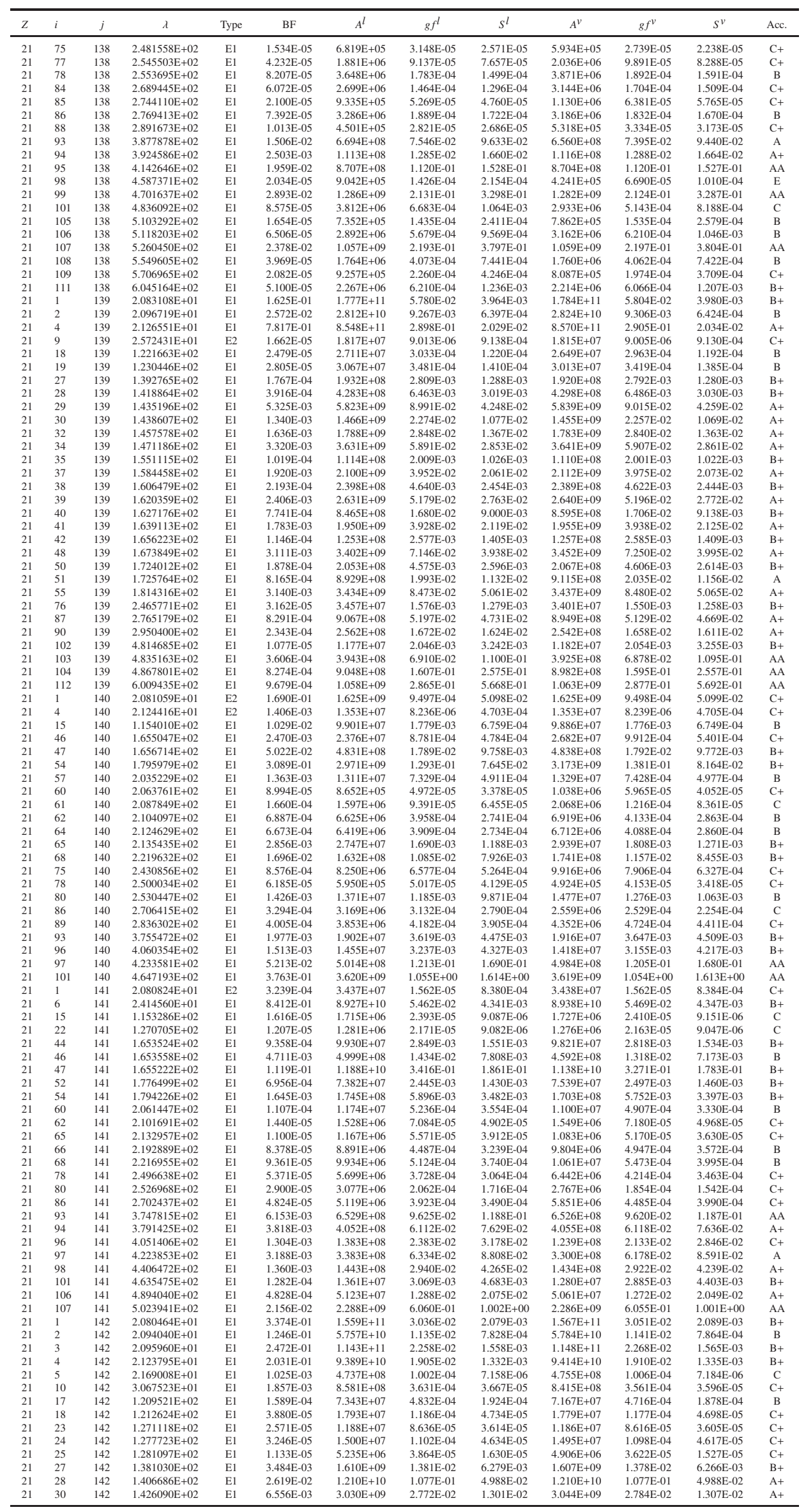




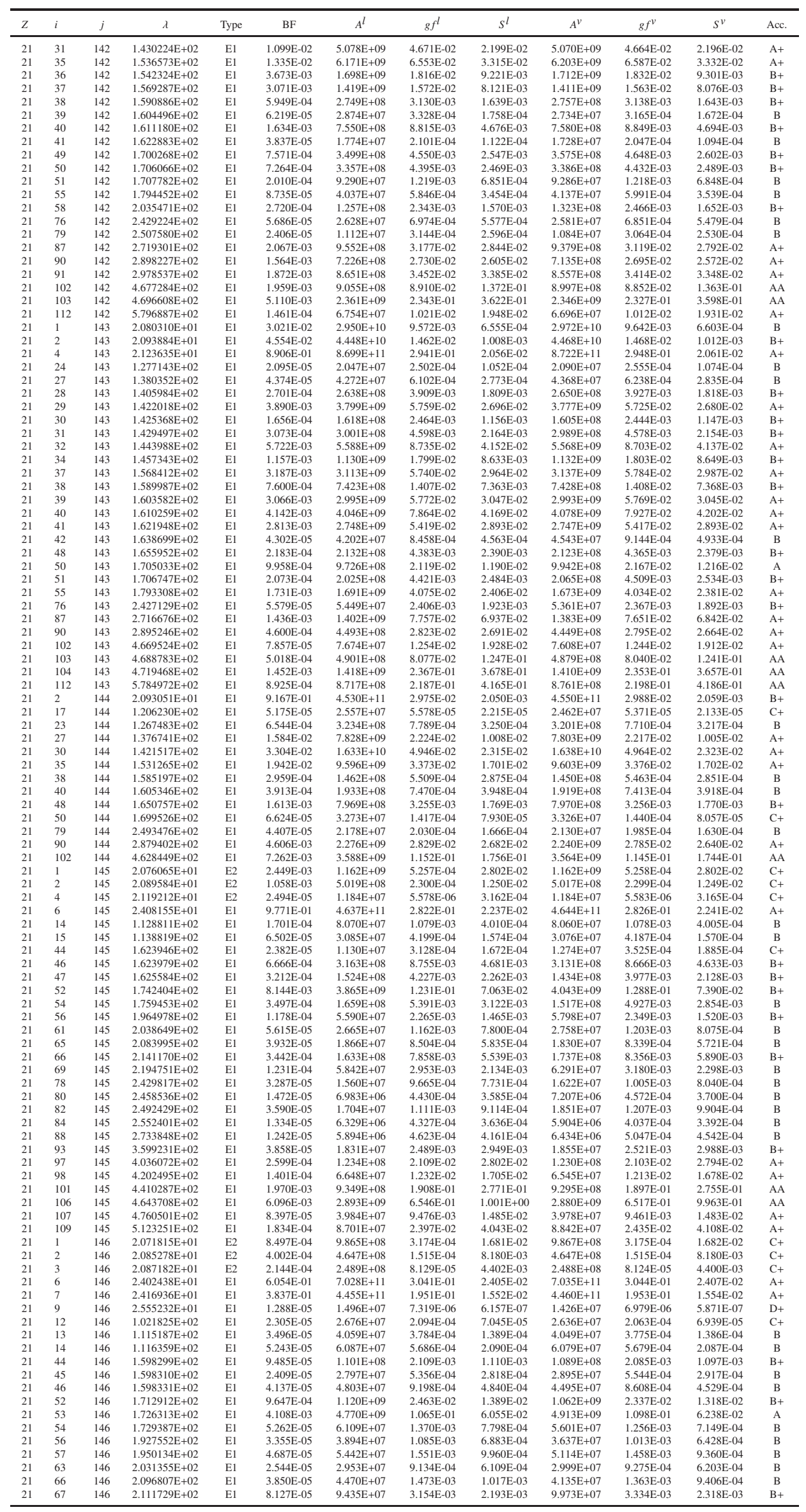




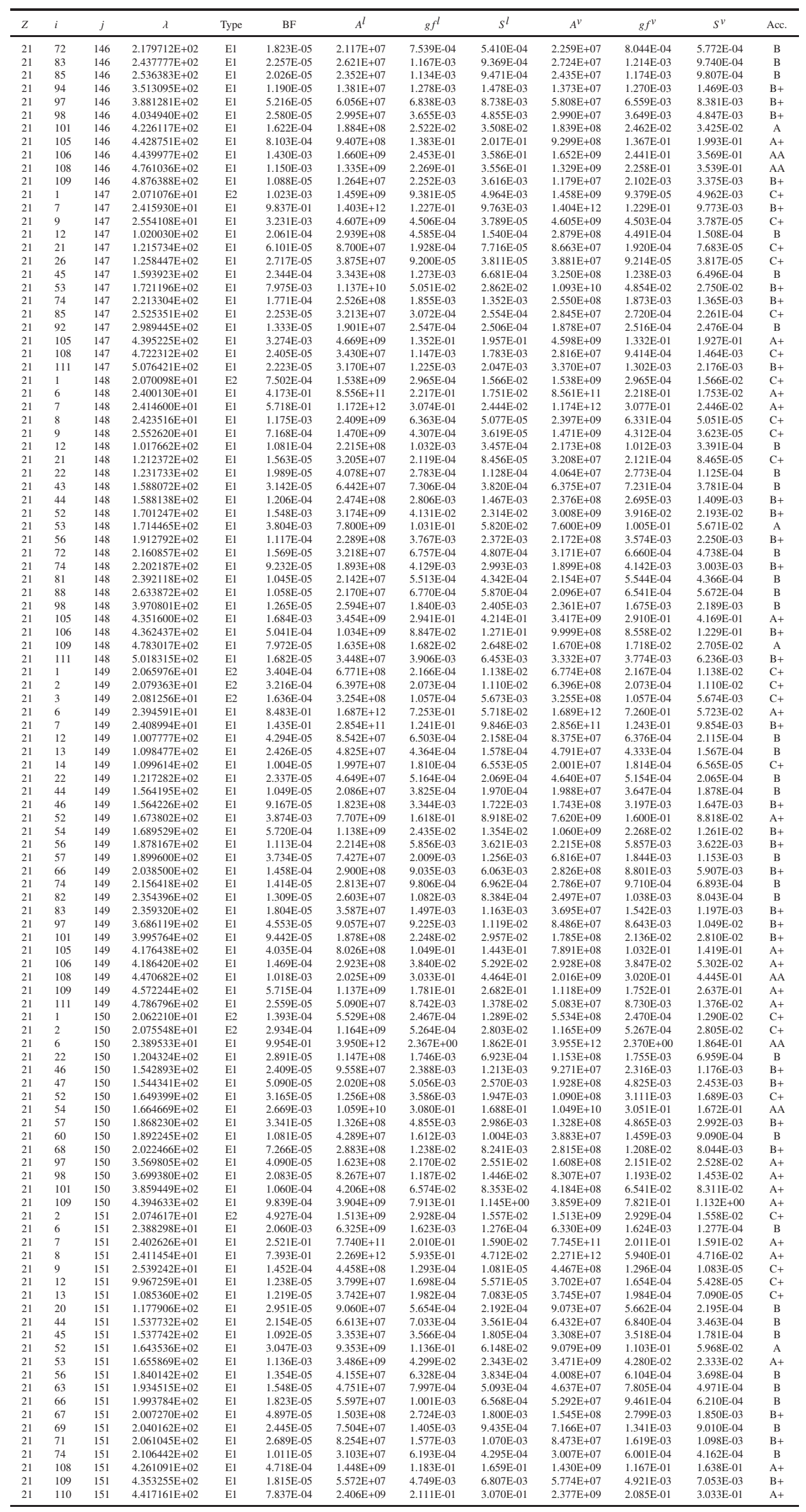




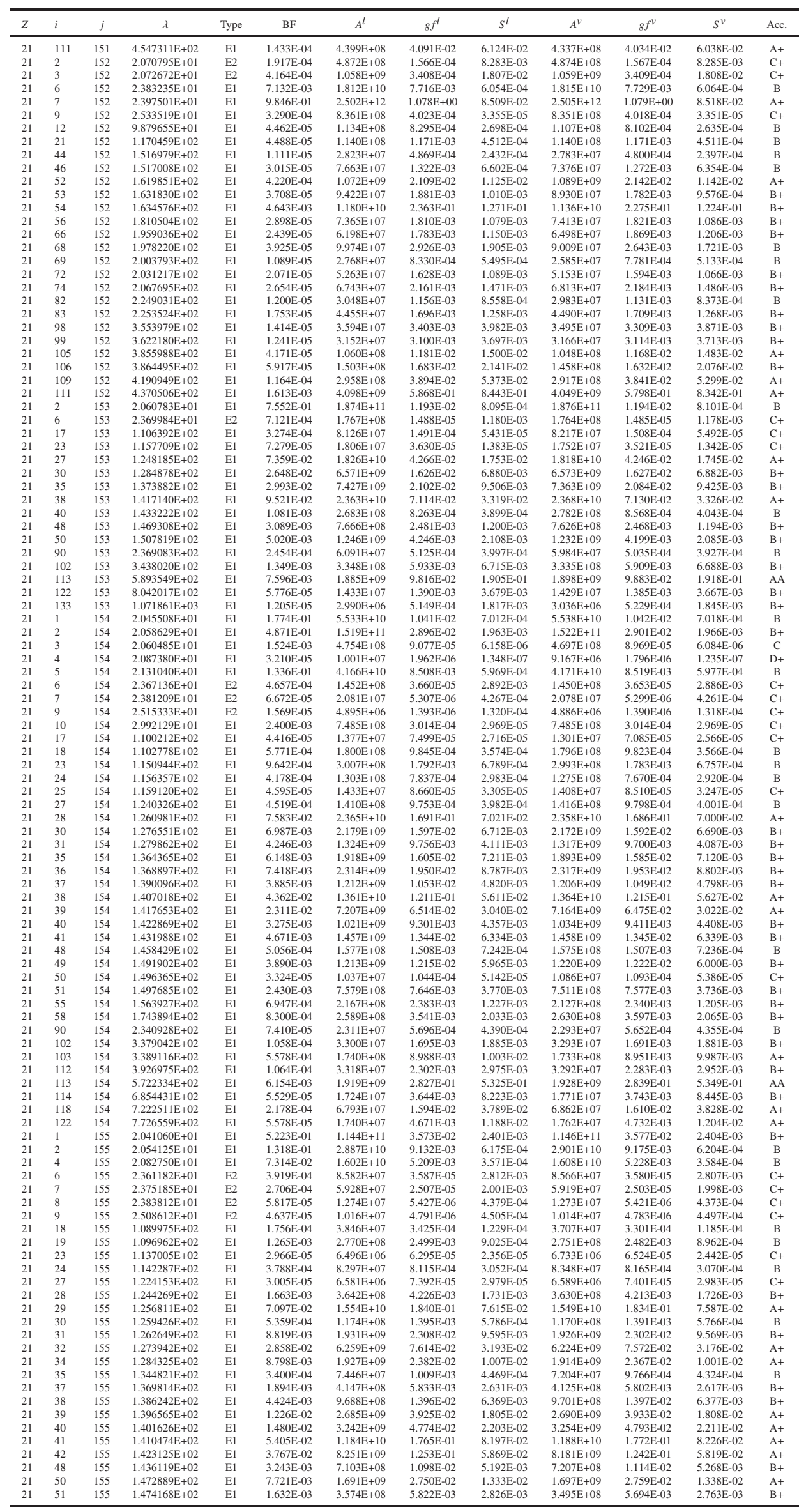

133 


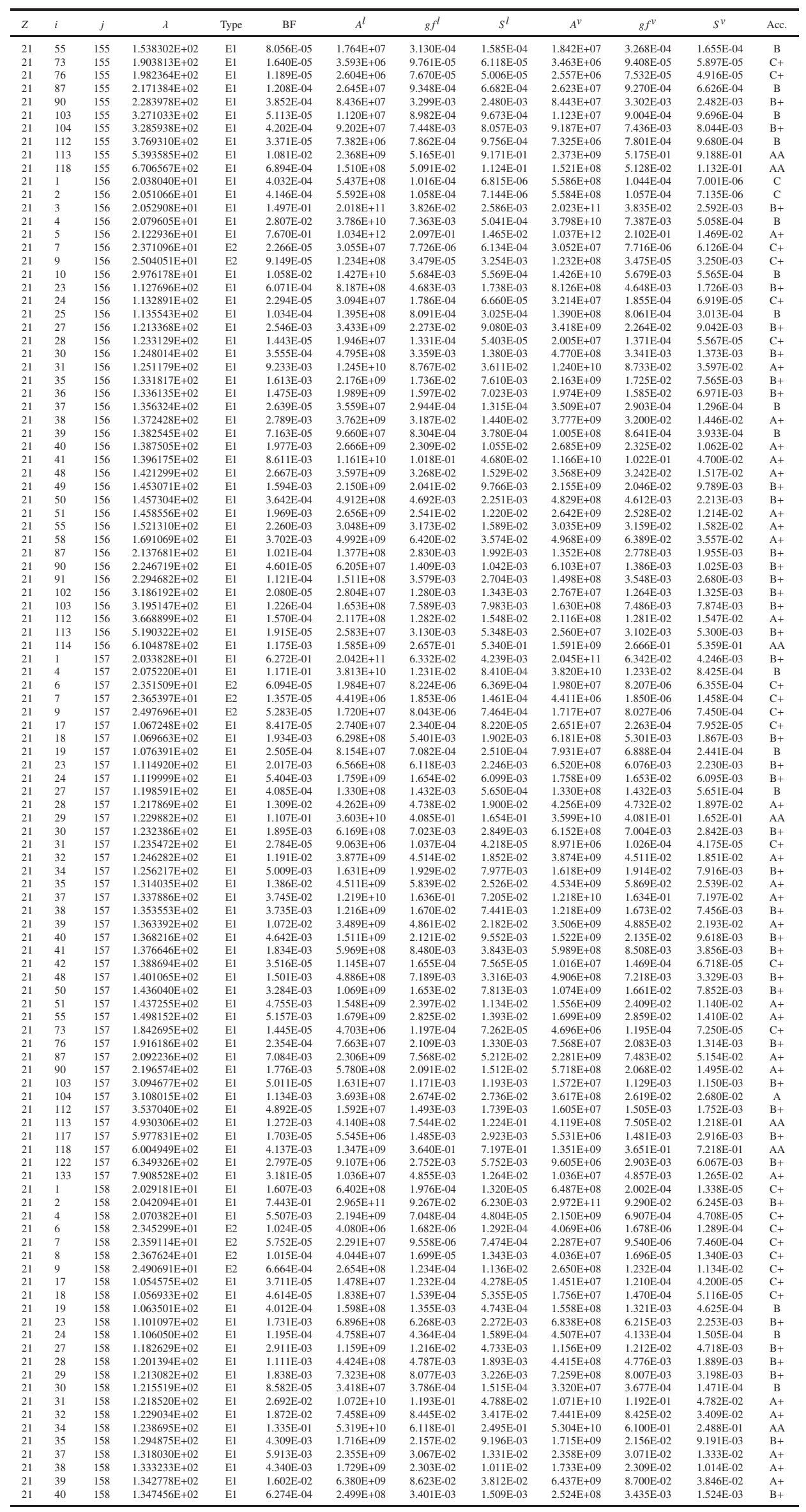




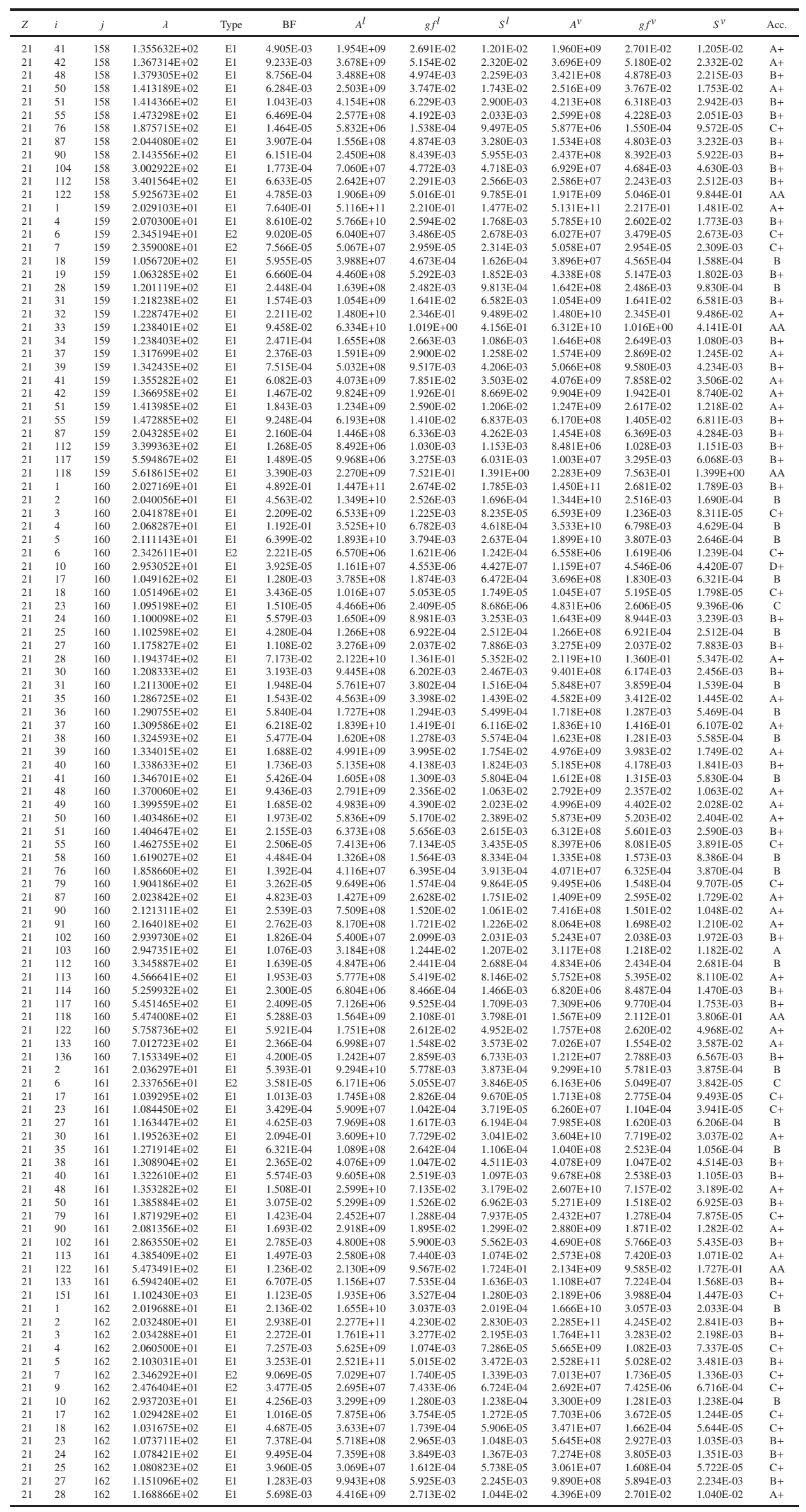




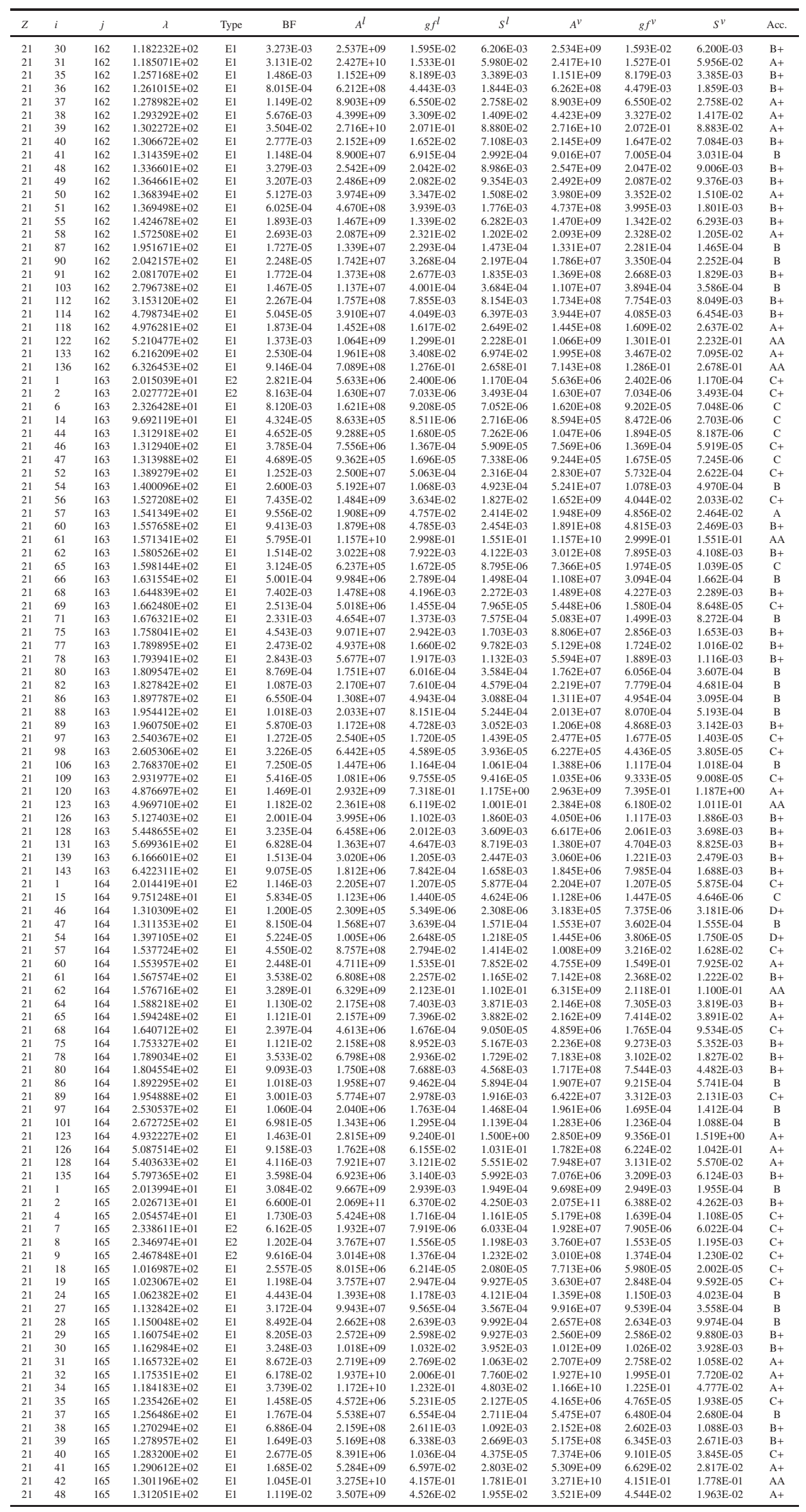




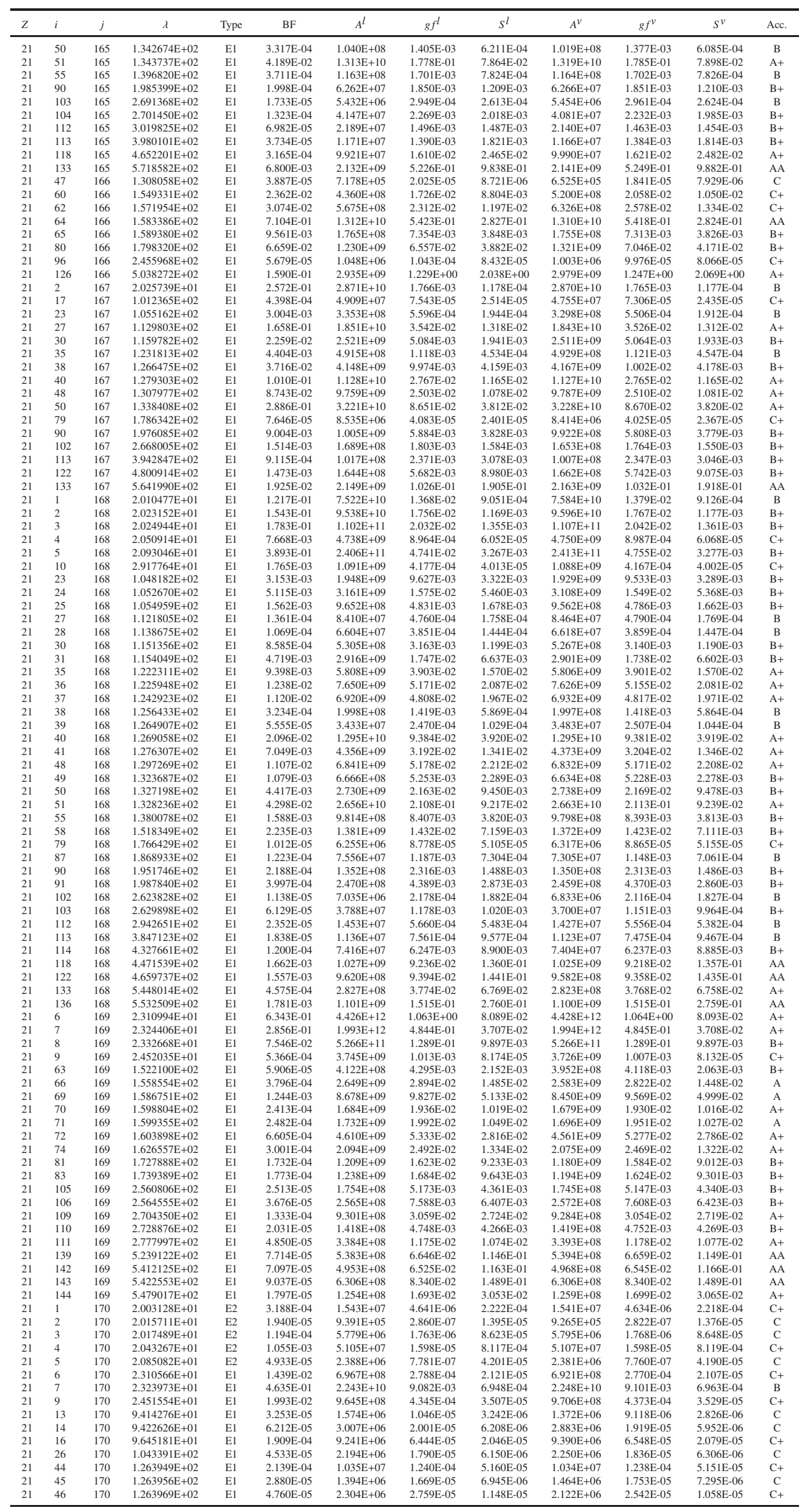




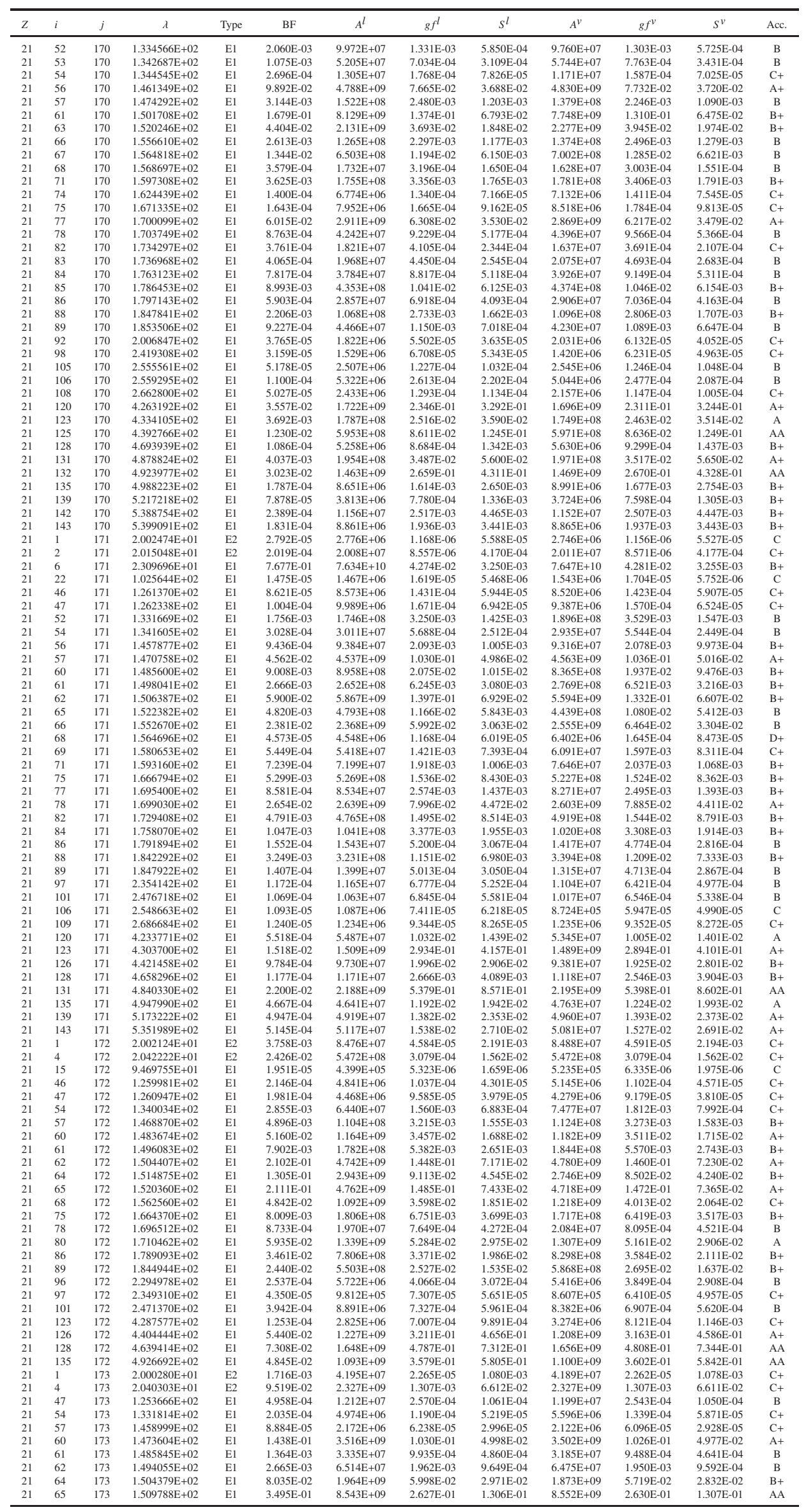




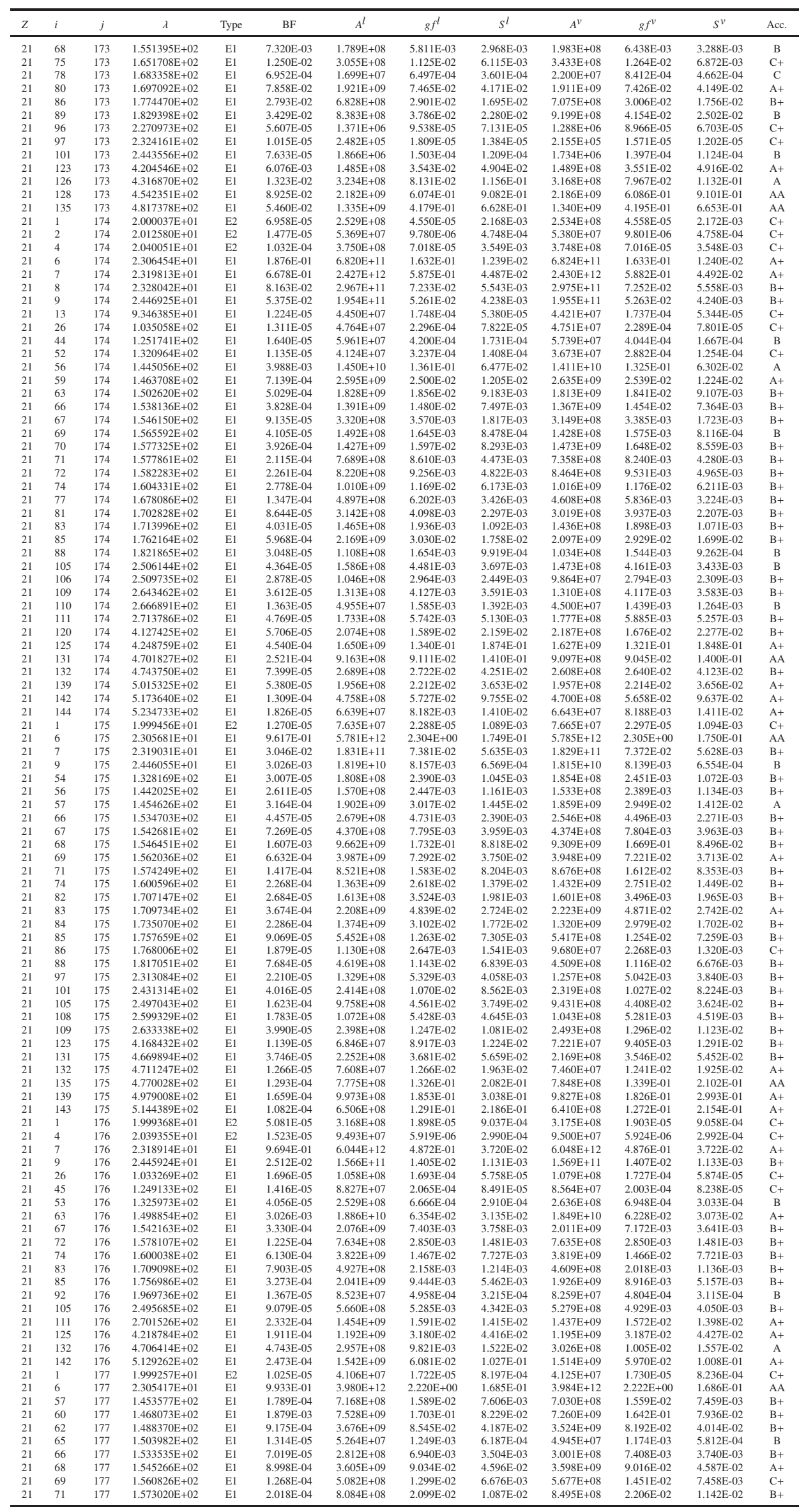

139 


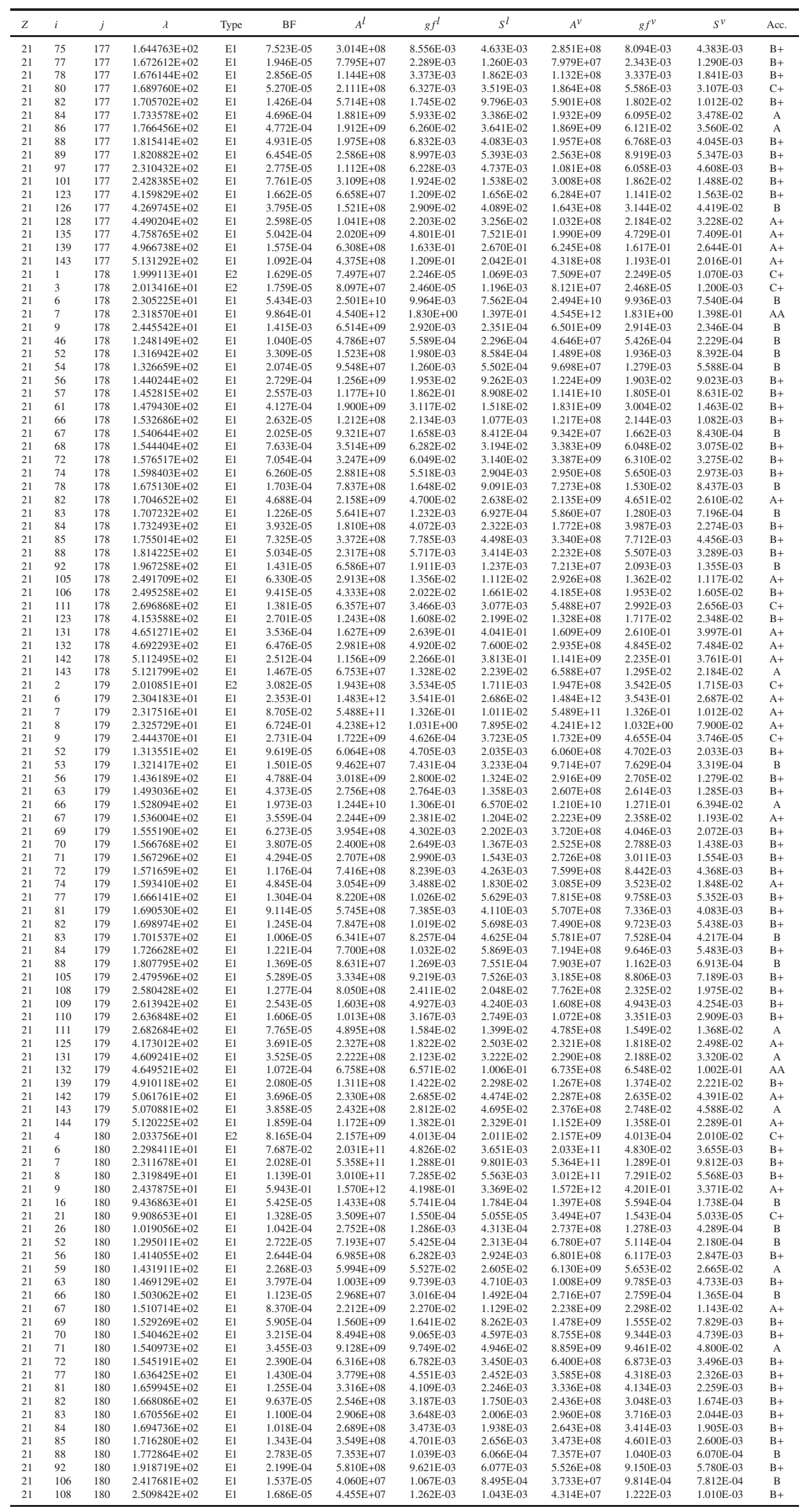




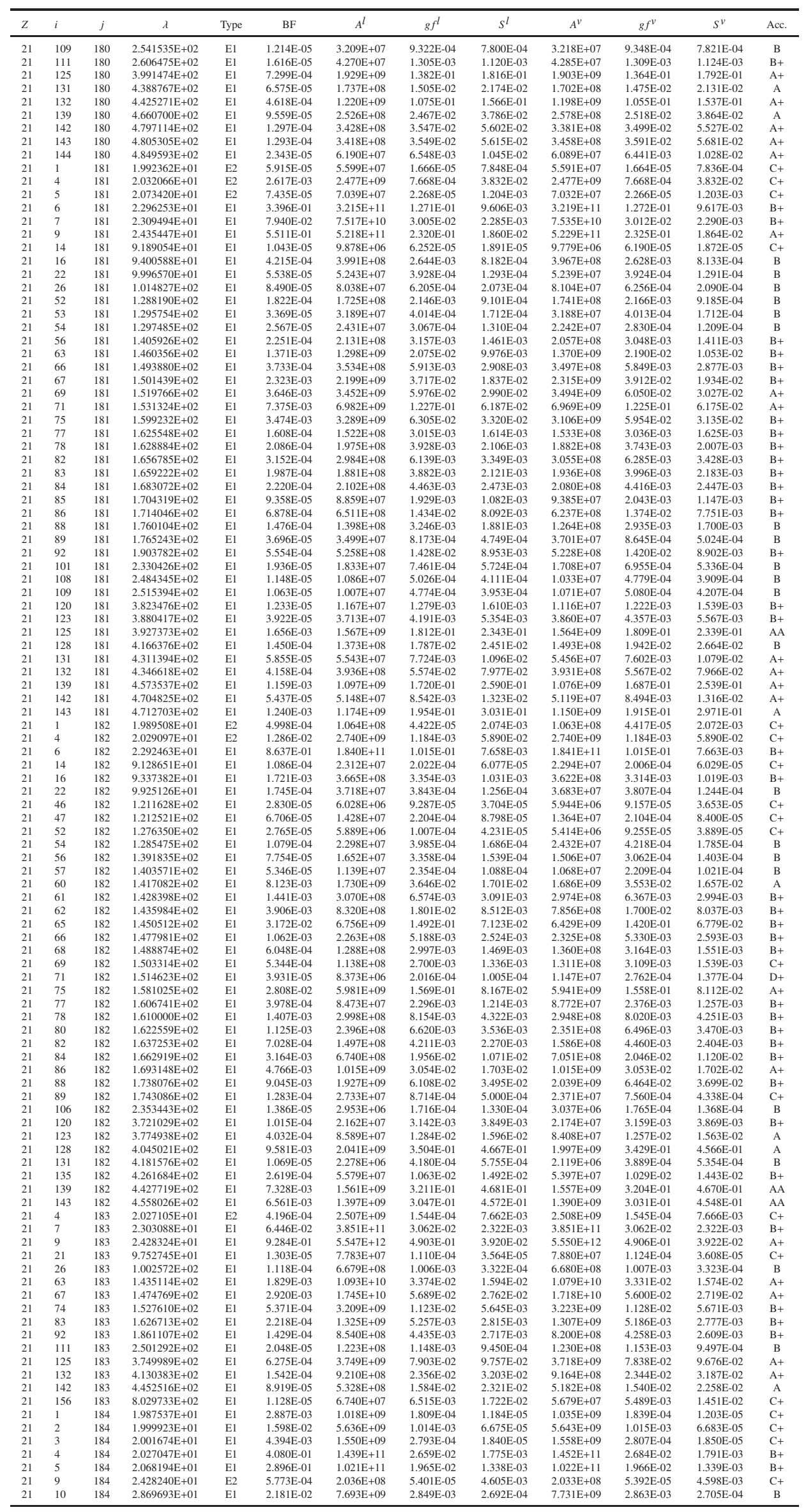




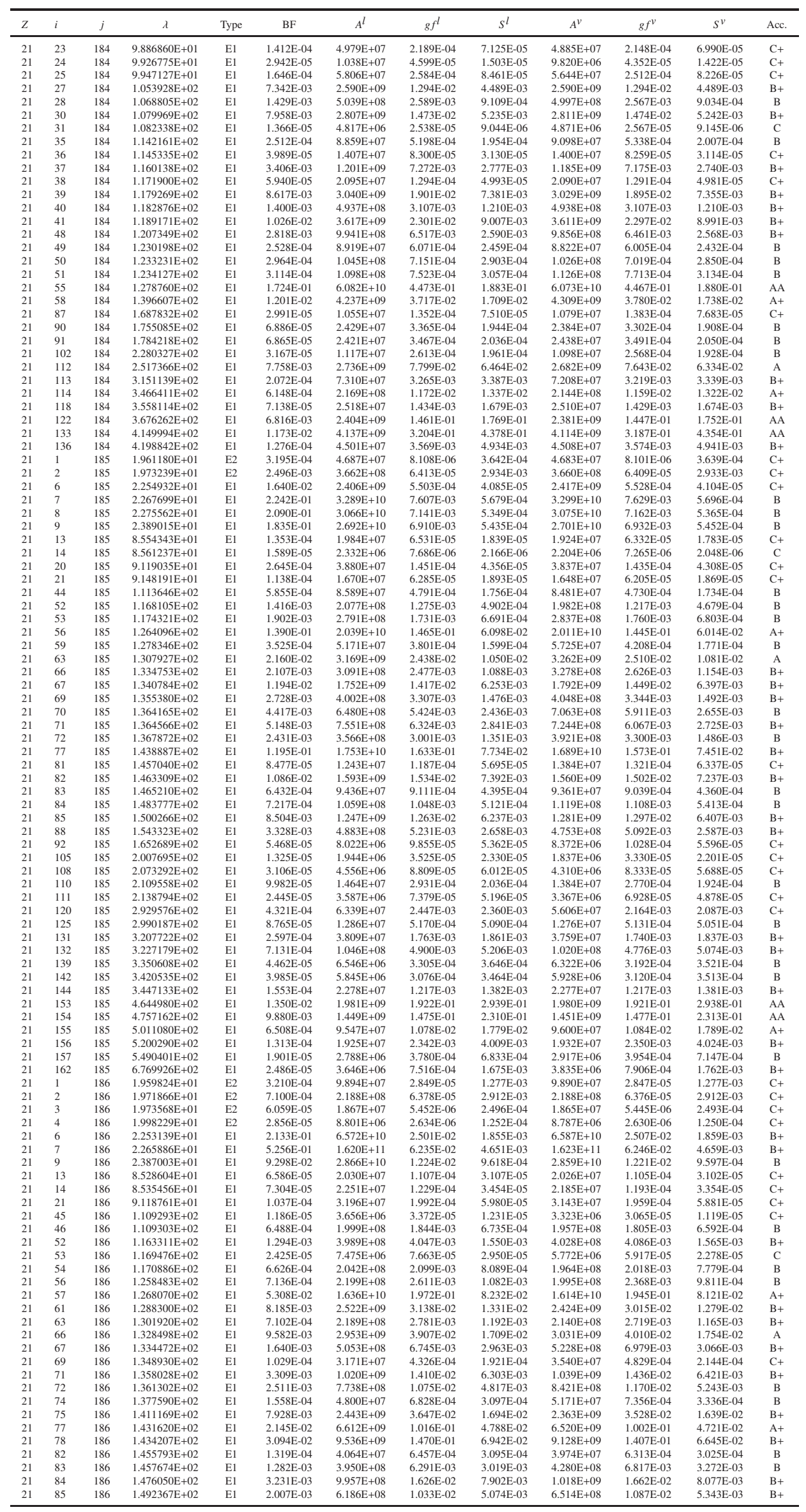




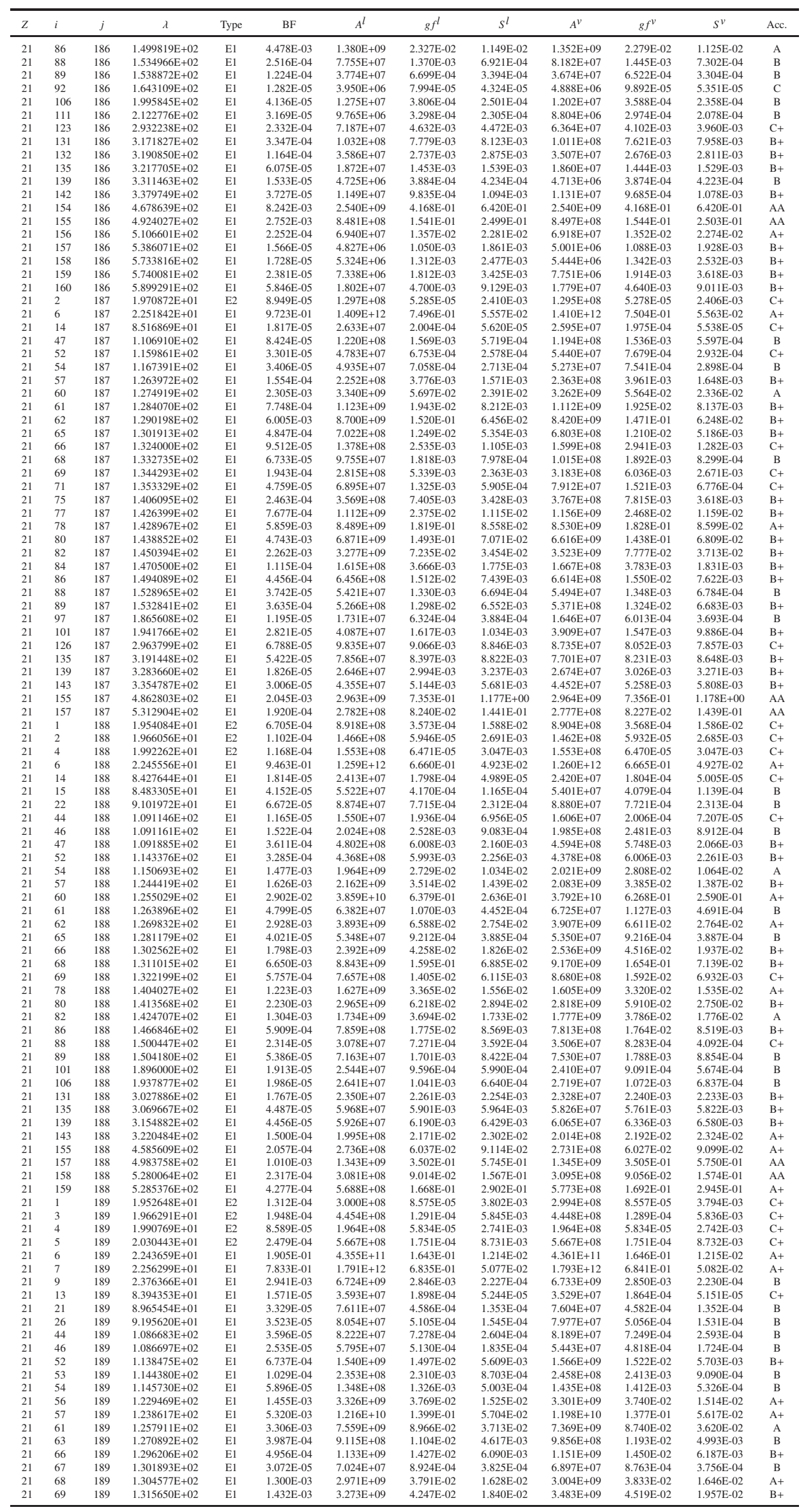




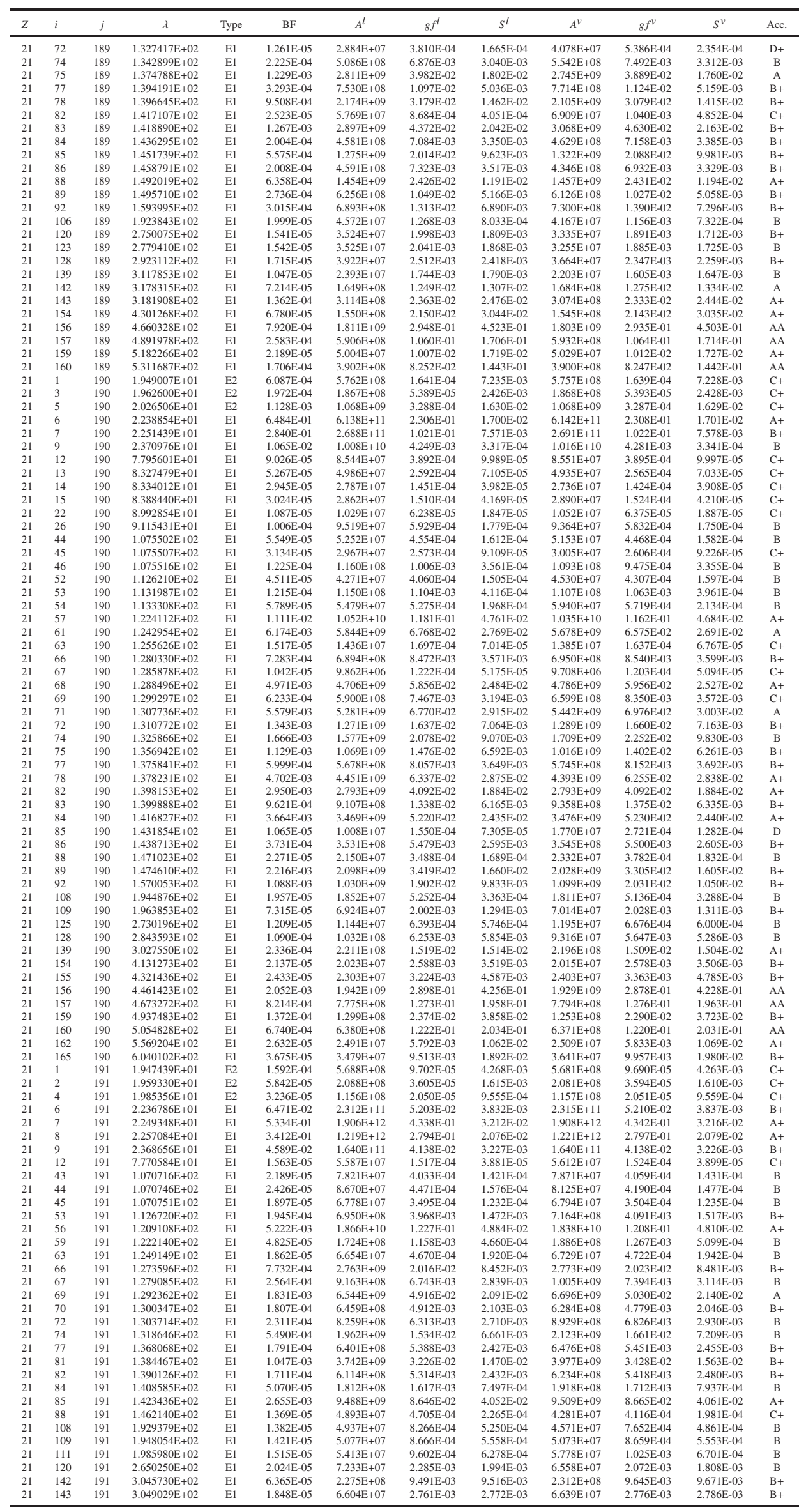




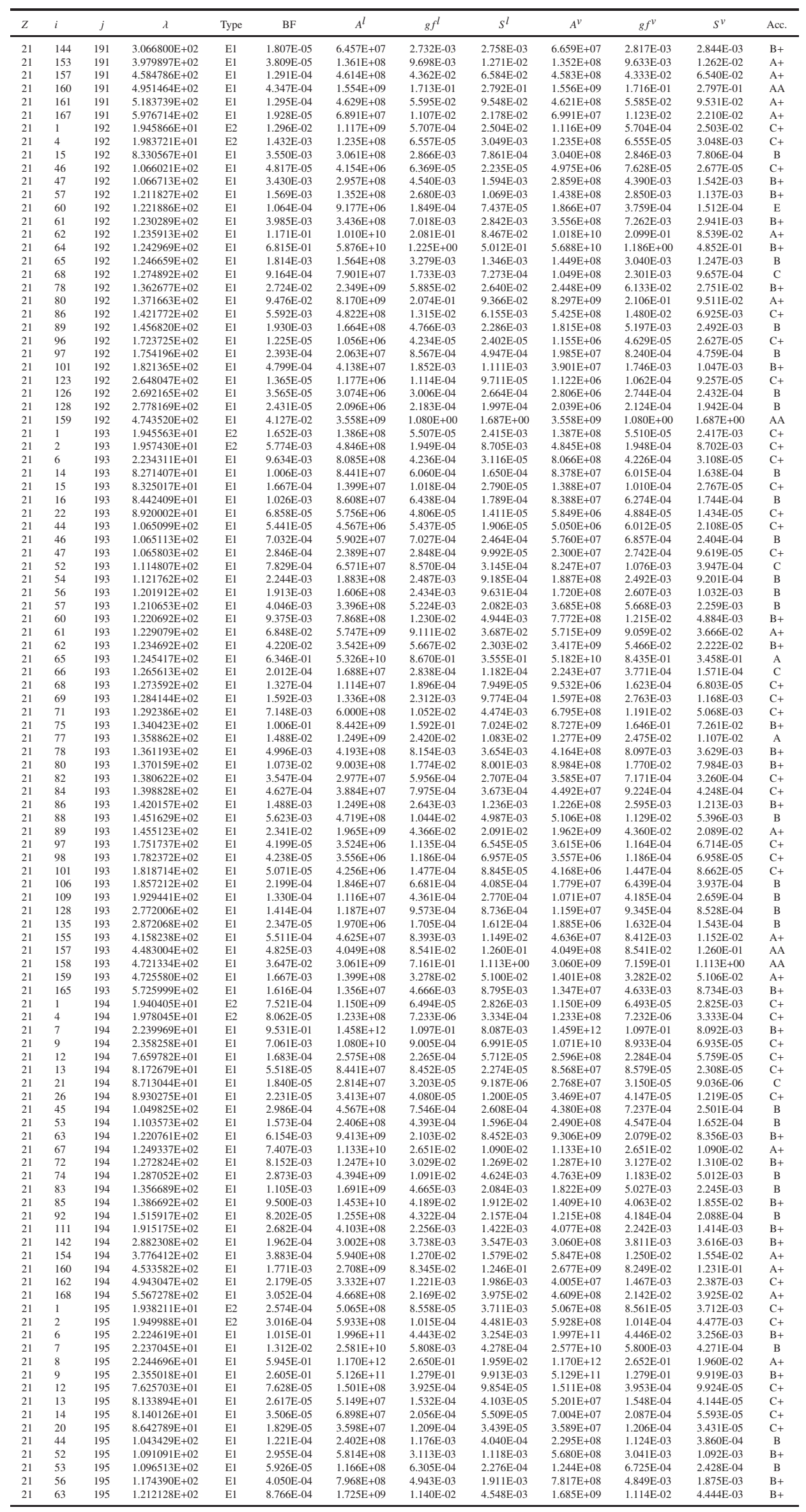




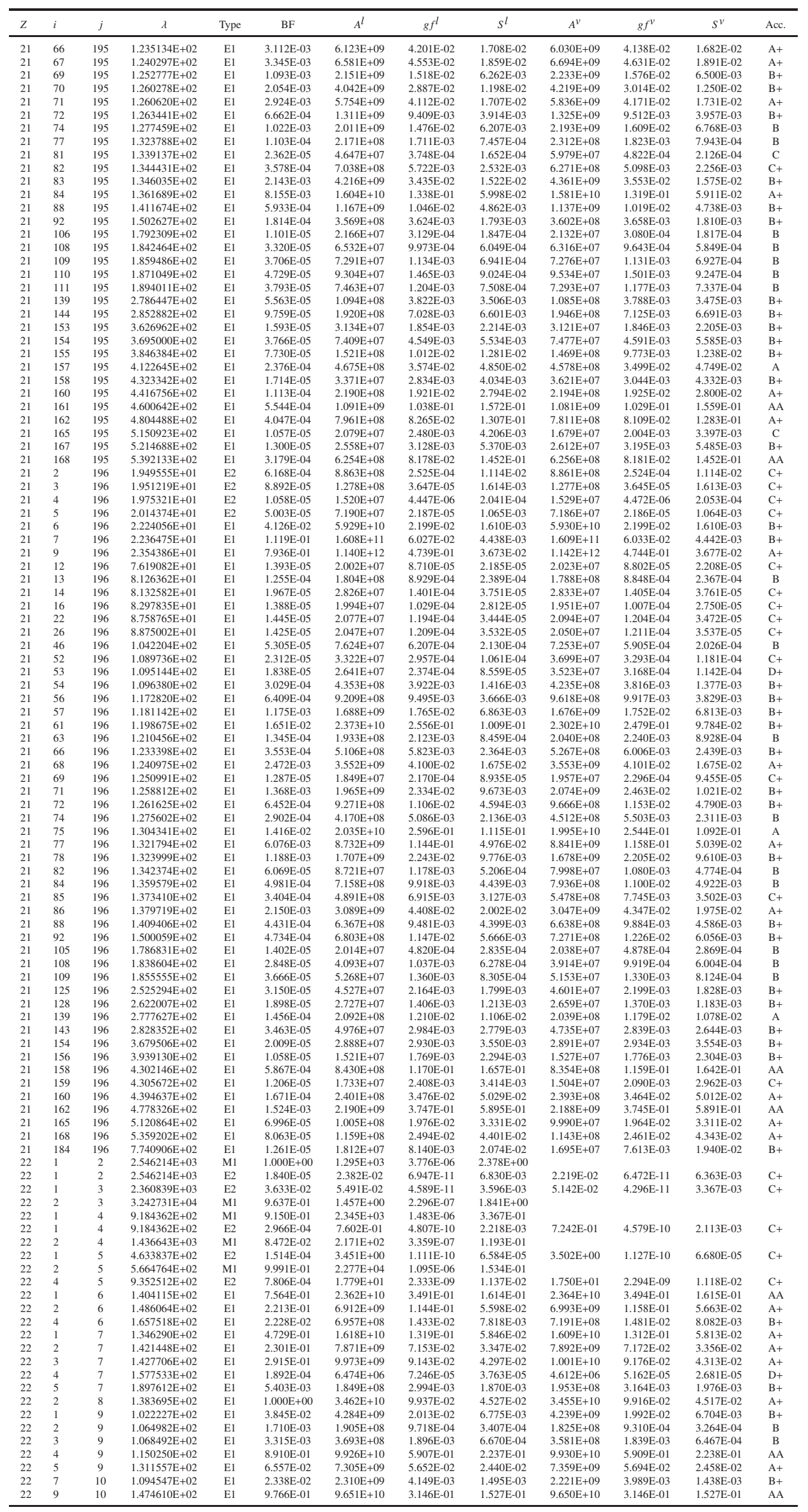




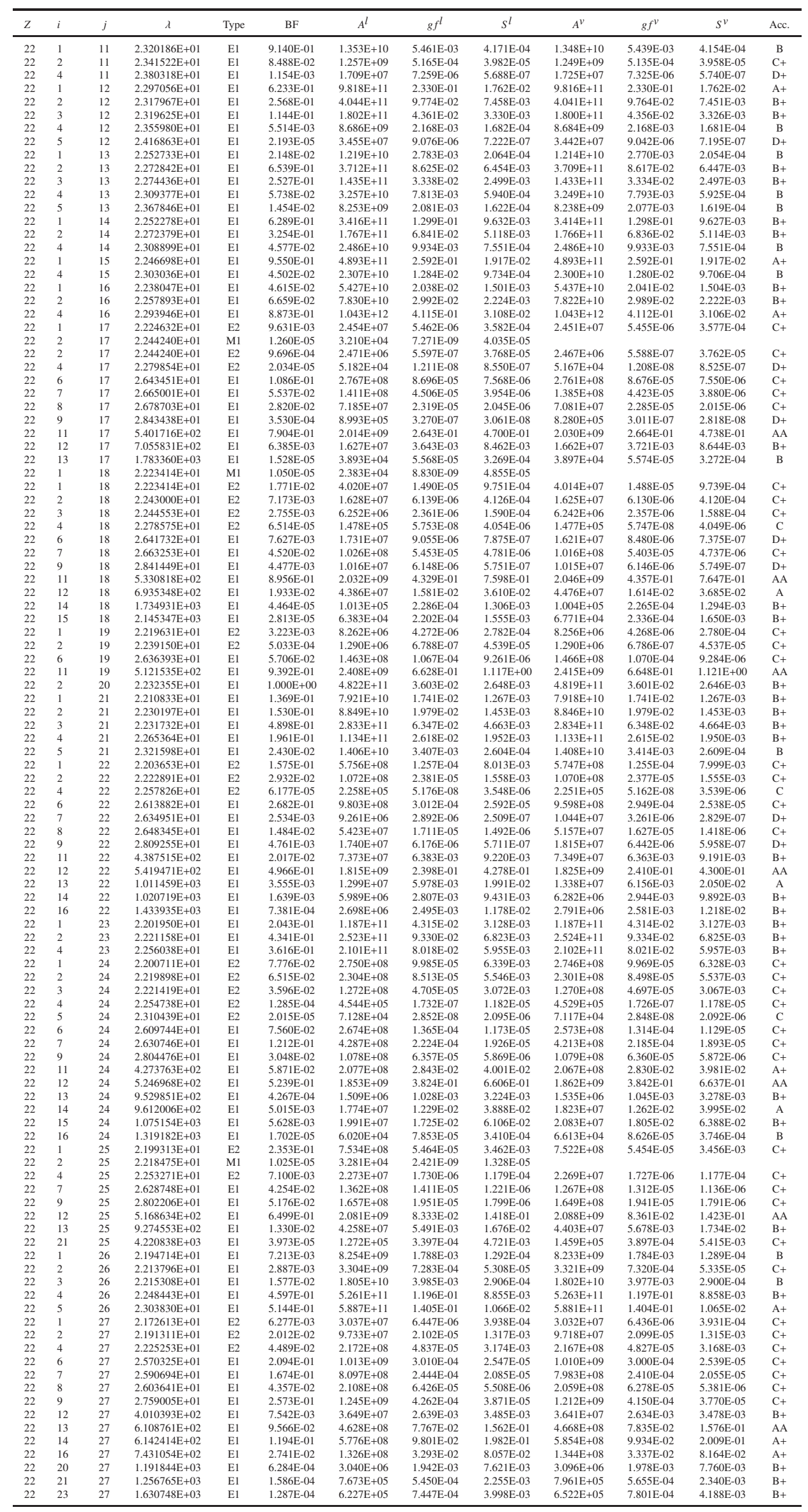




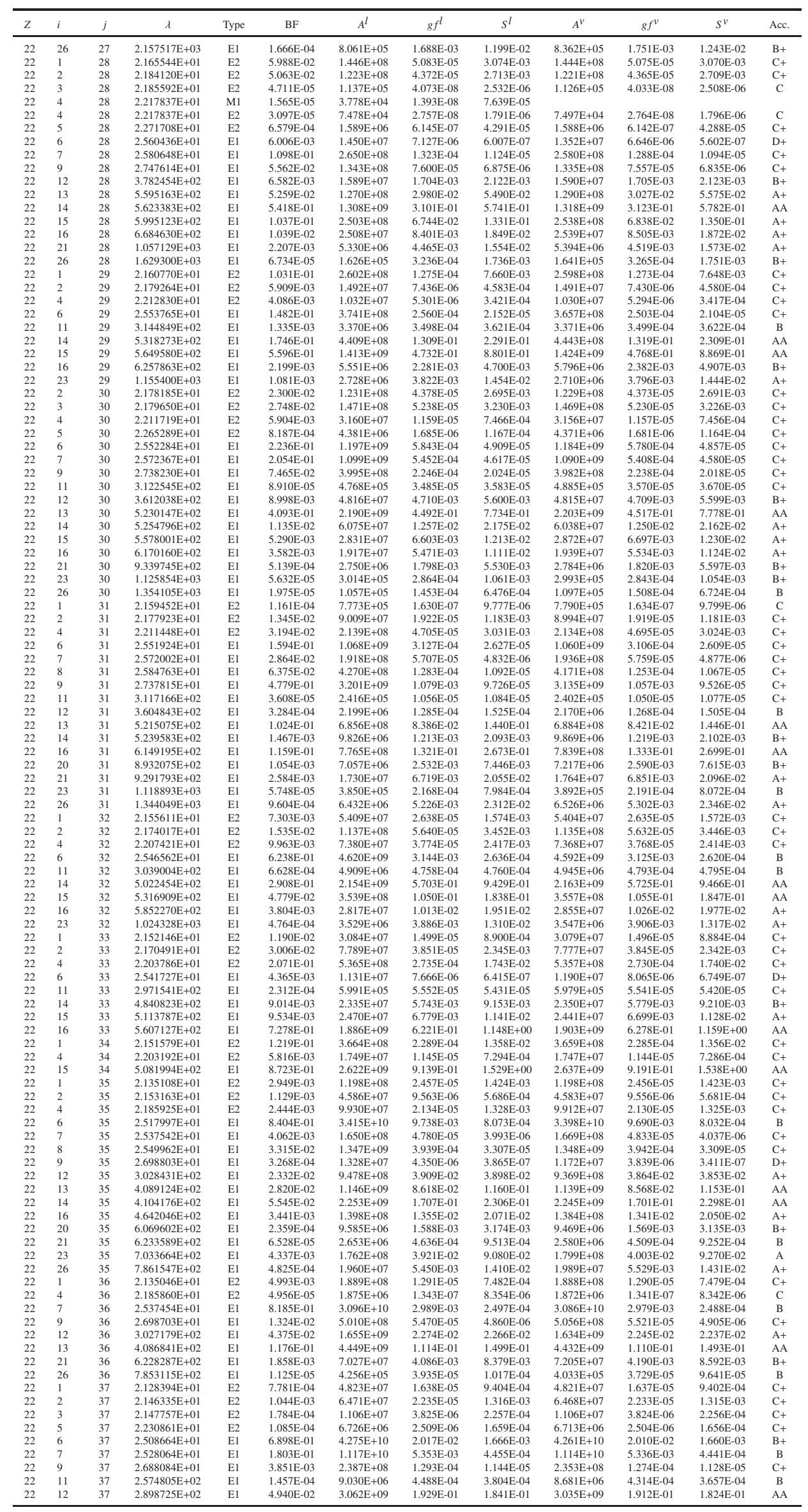




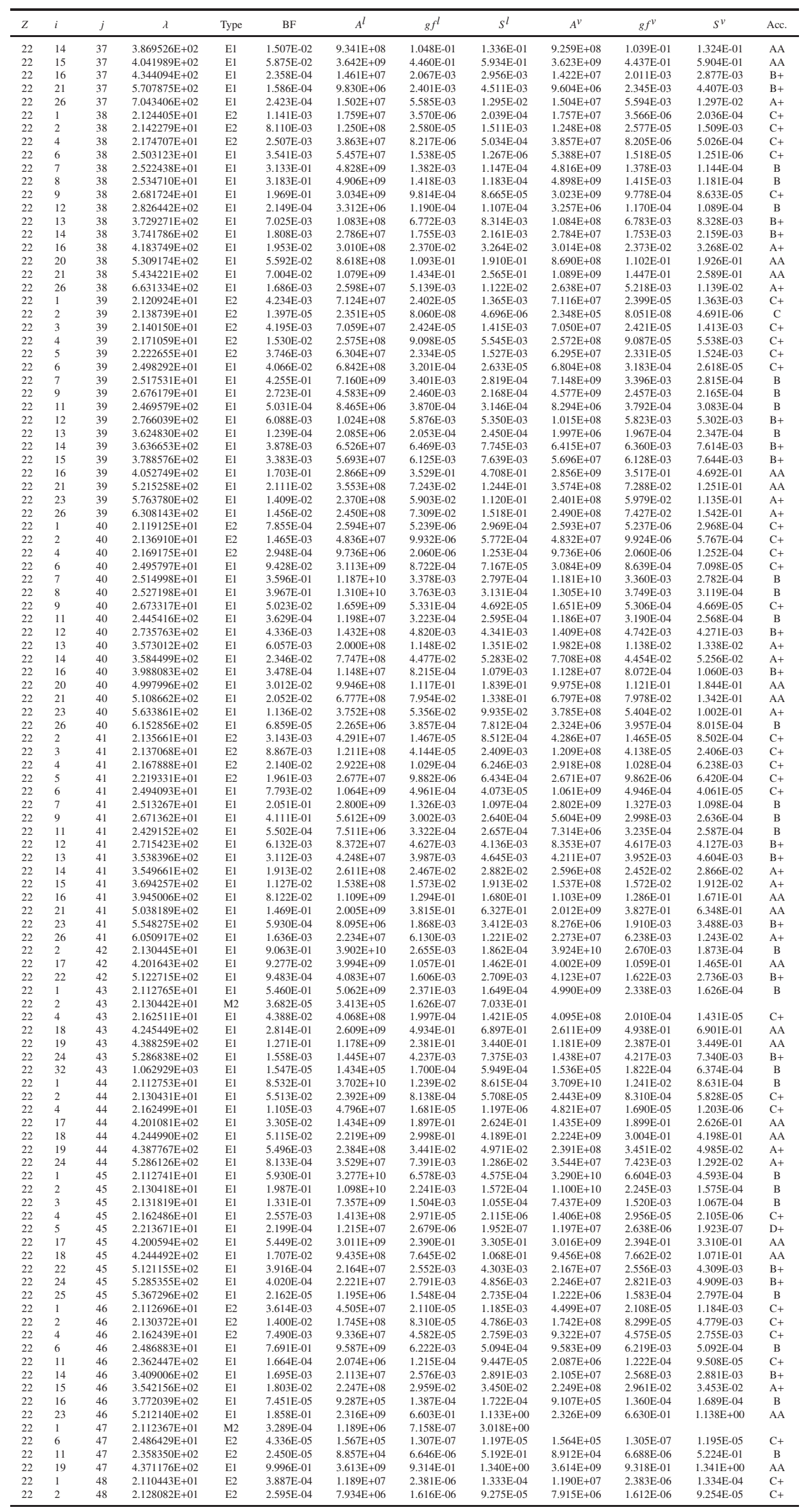




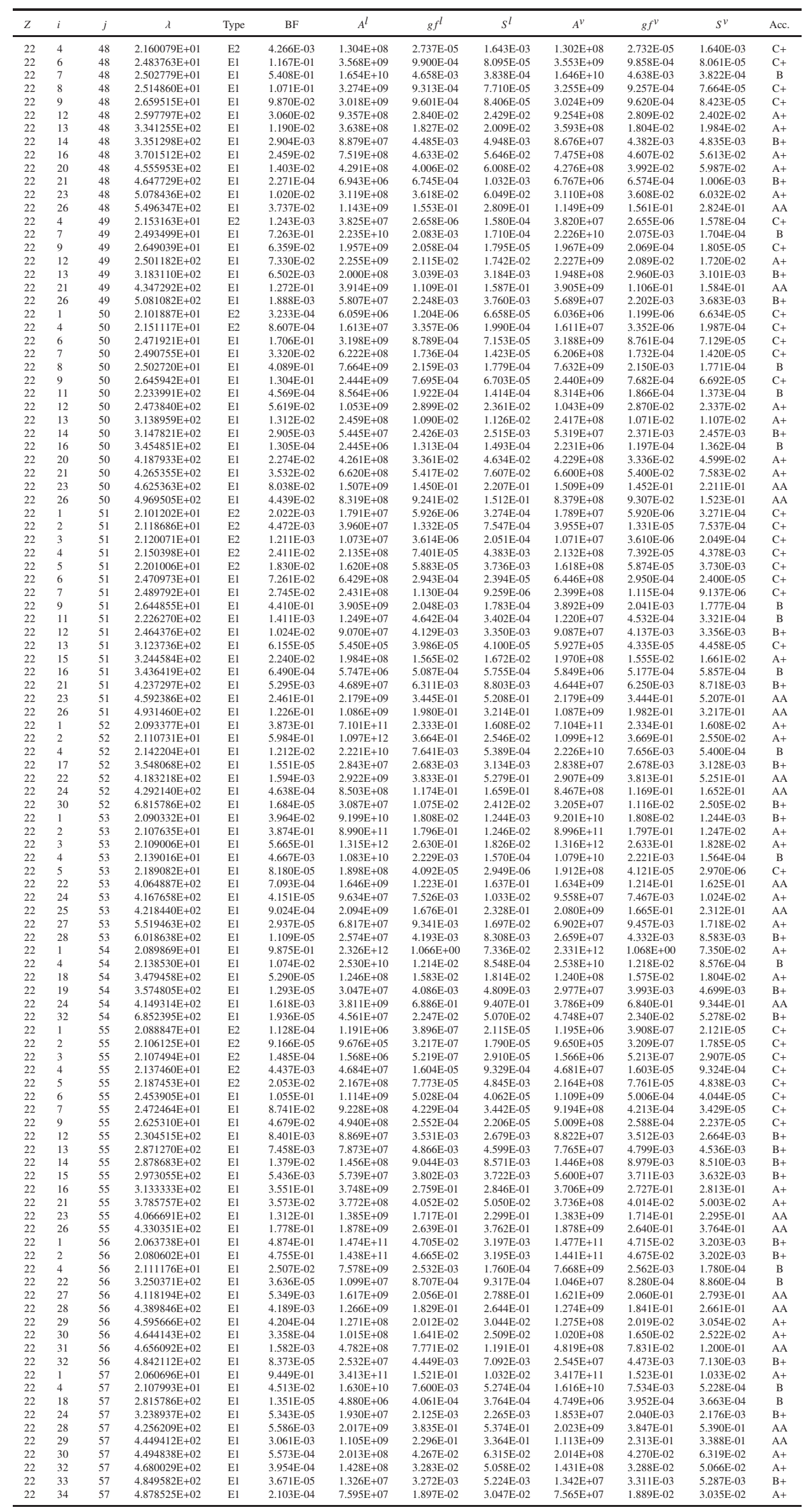

150 


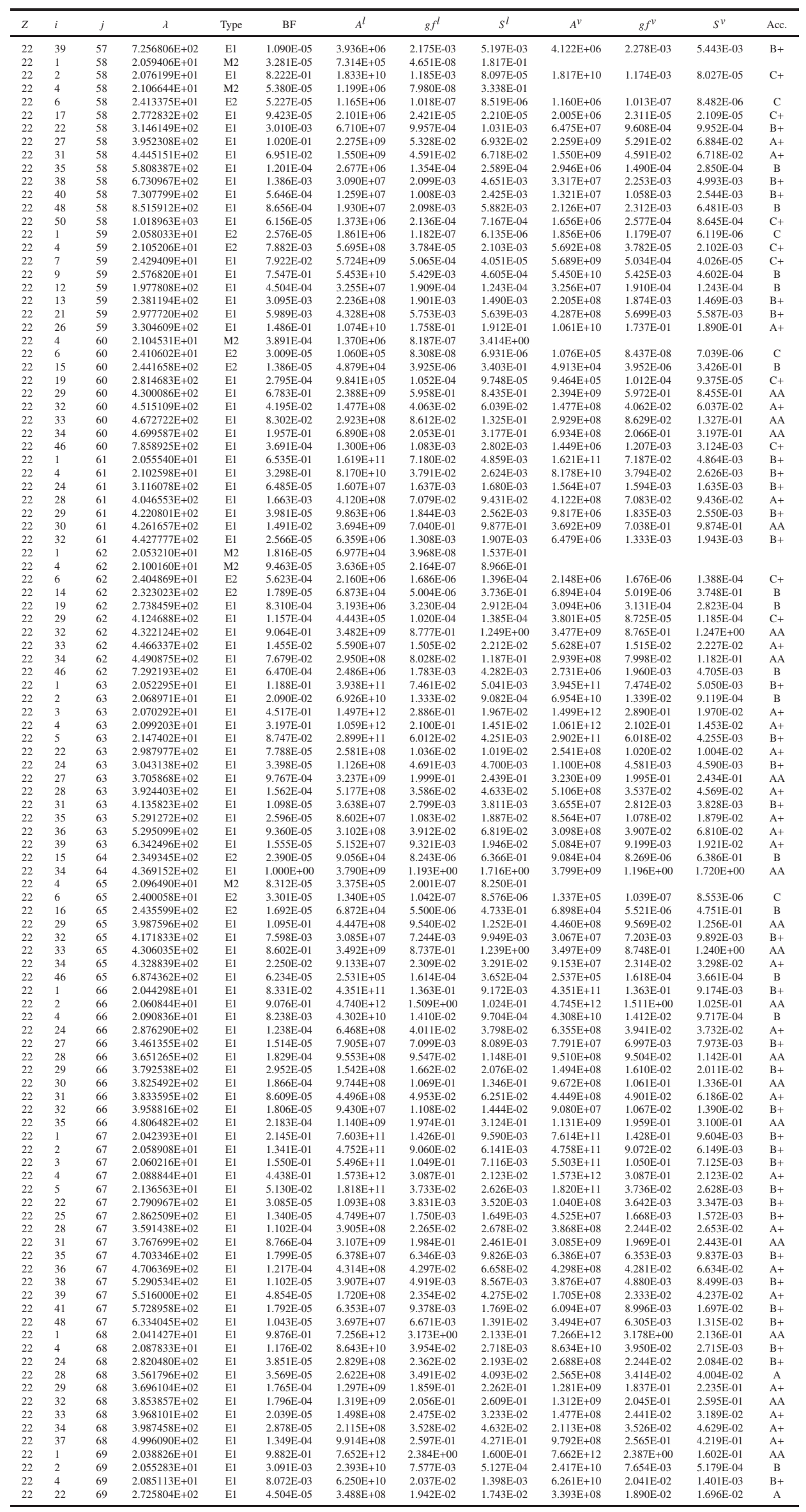




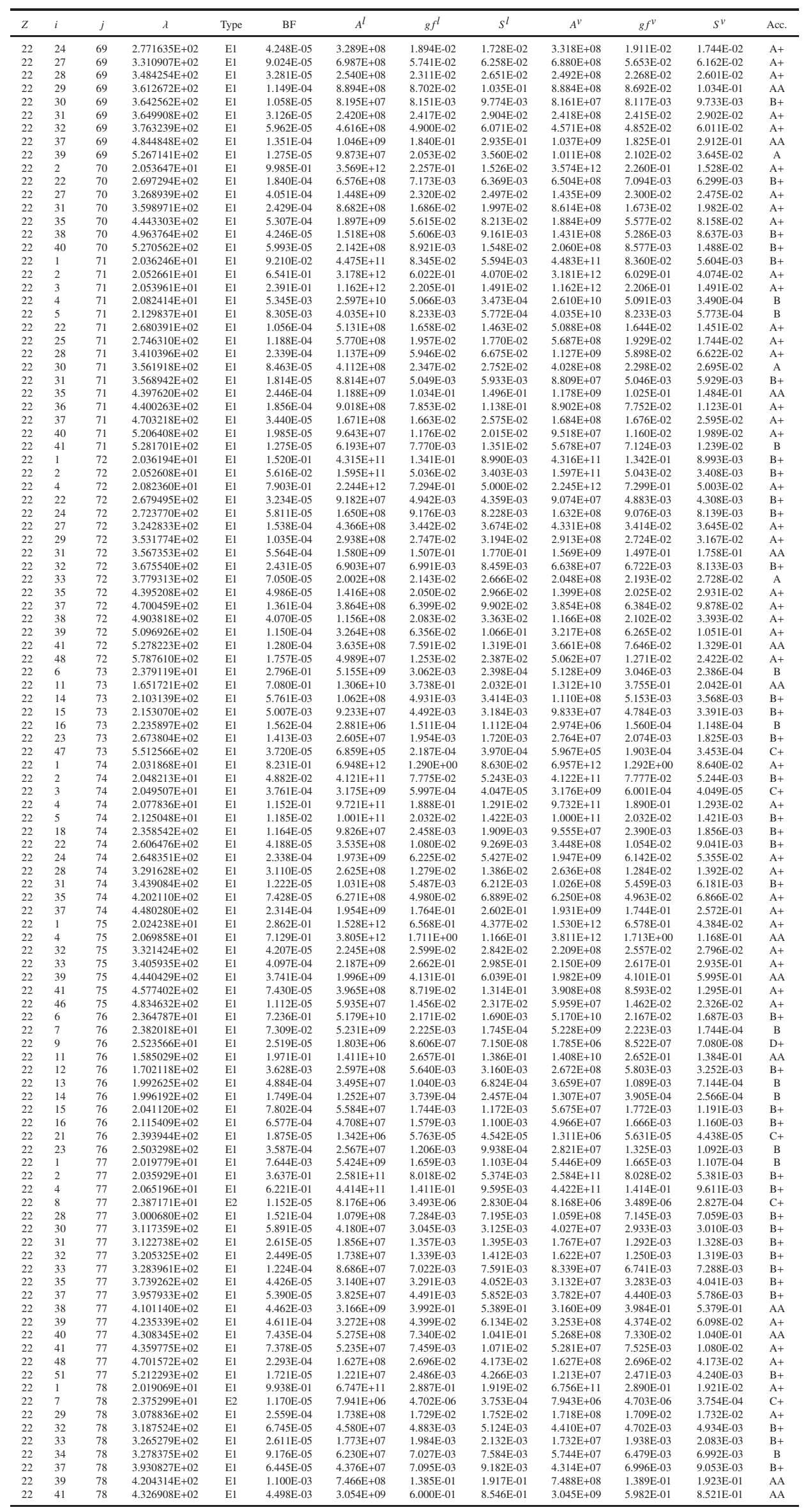

152 


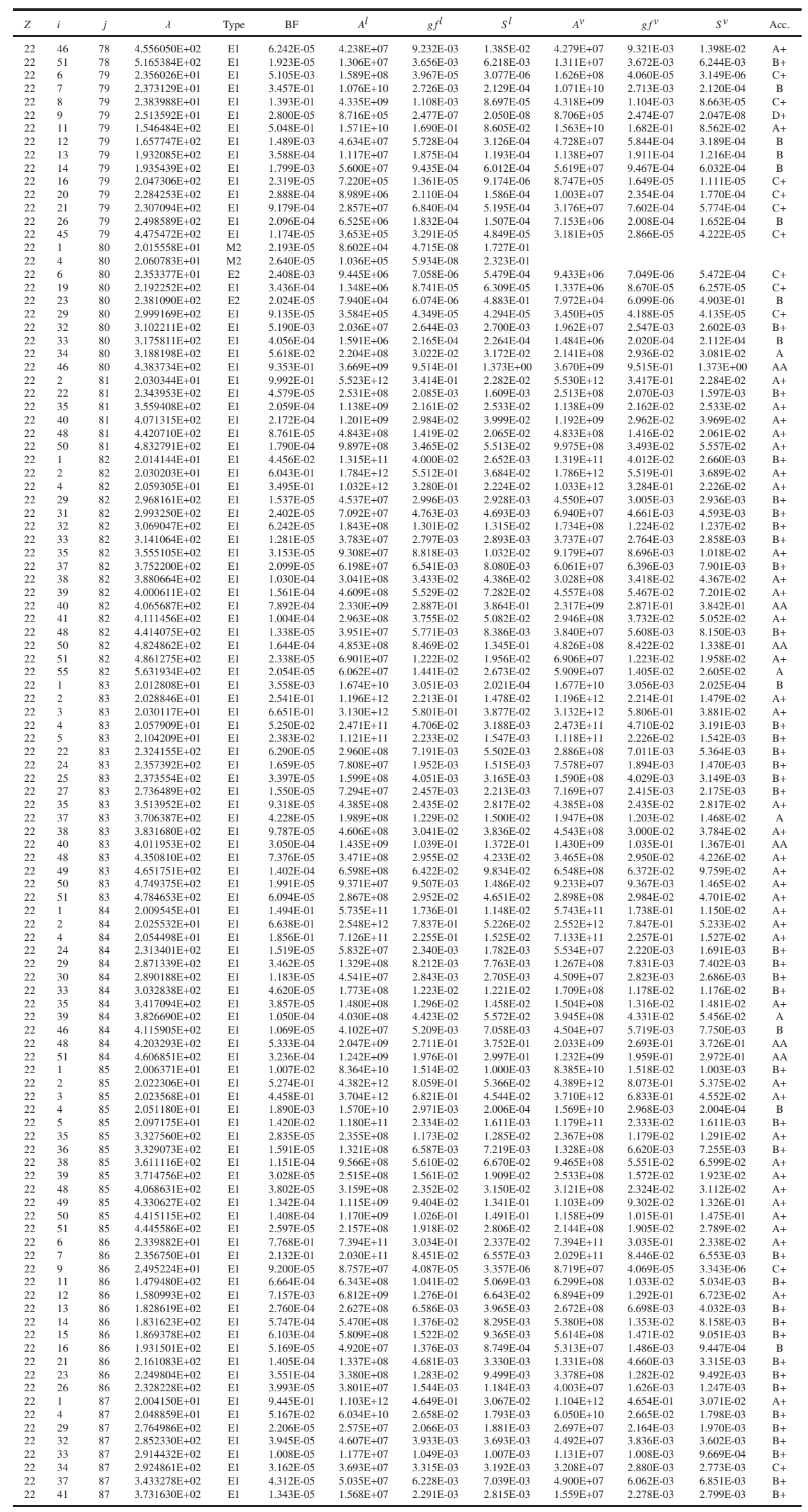




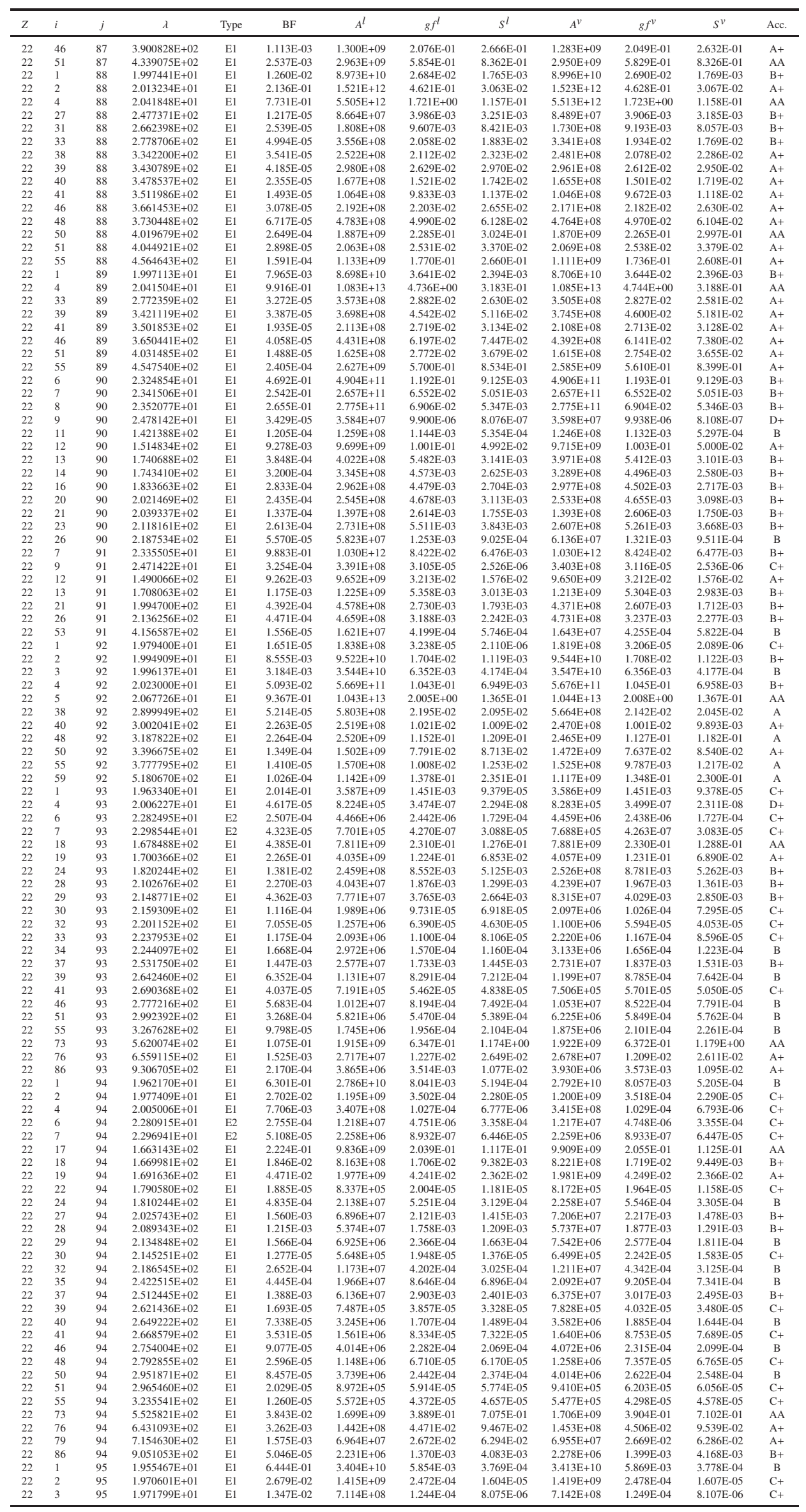




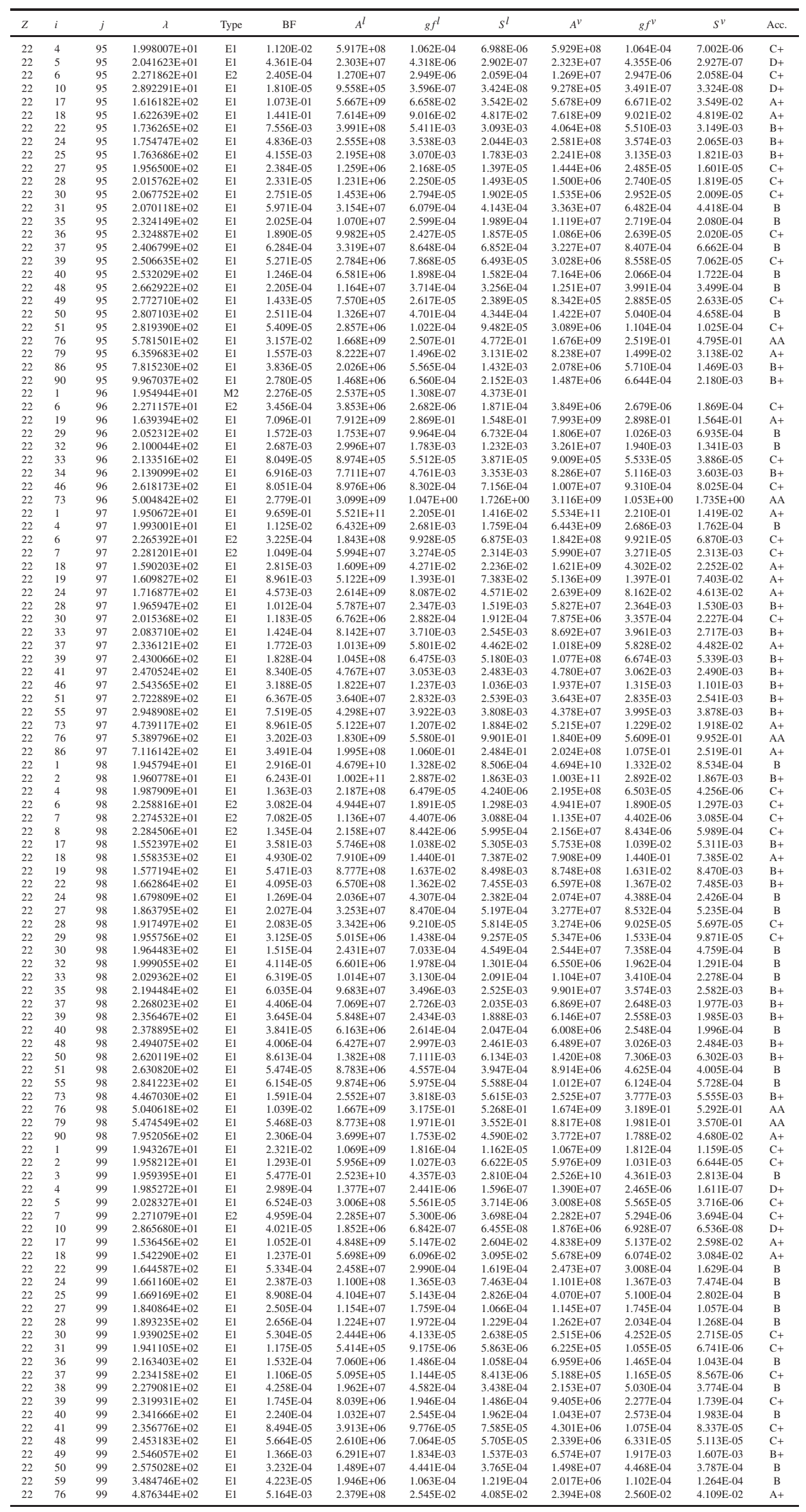




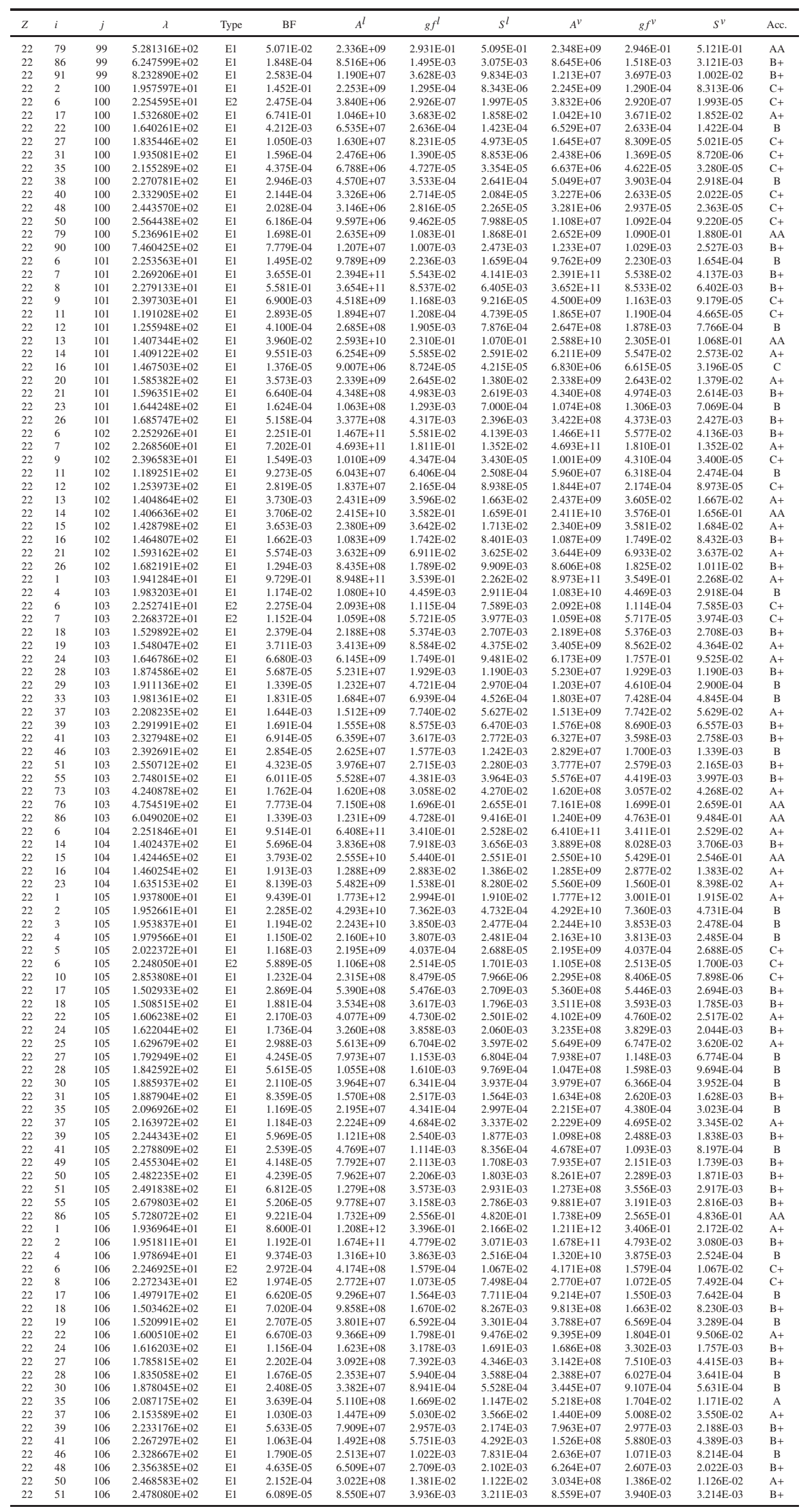




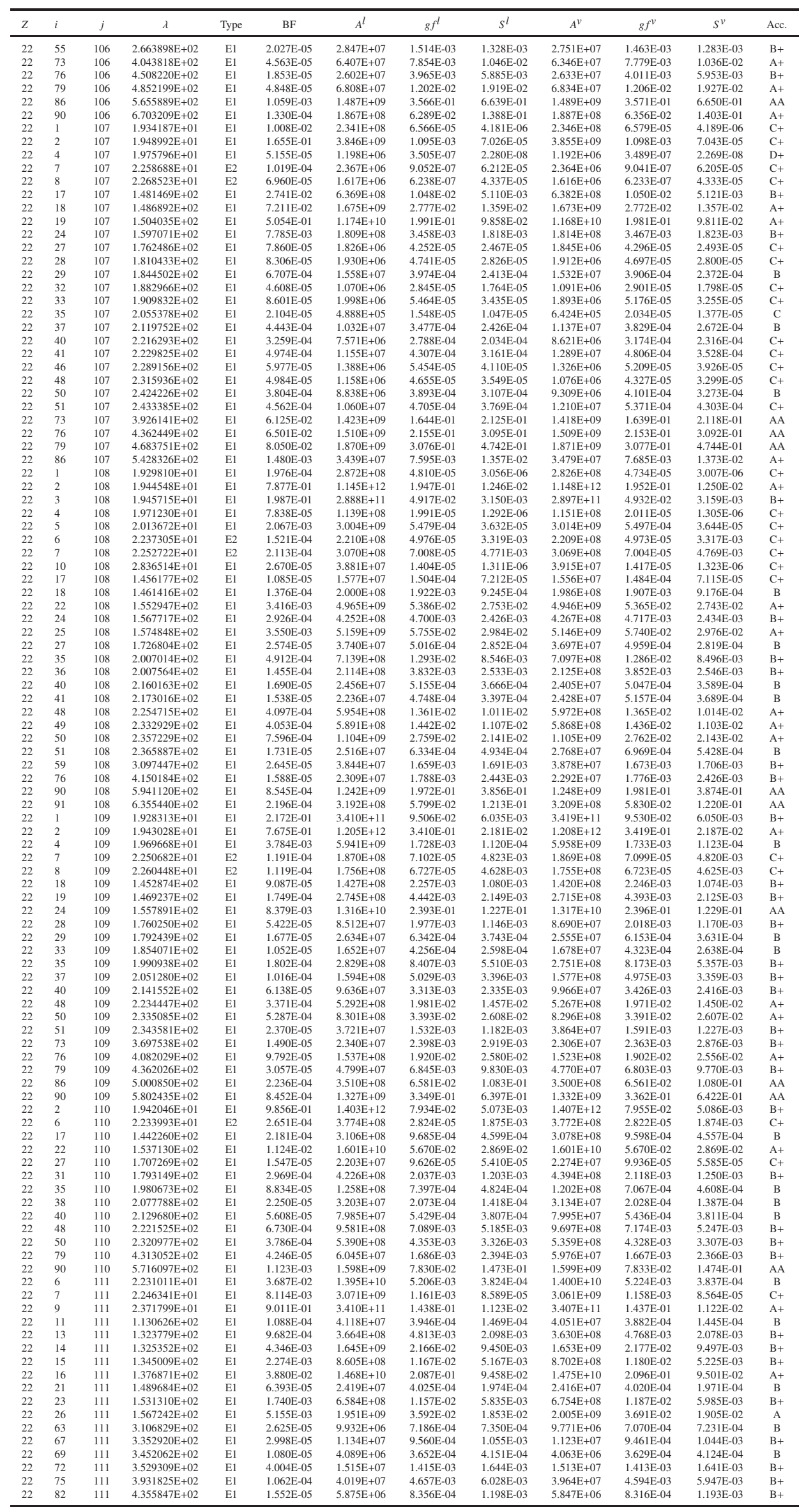




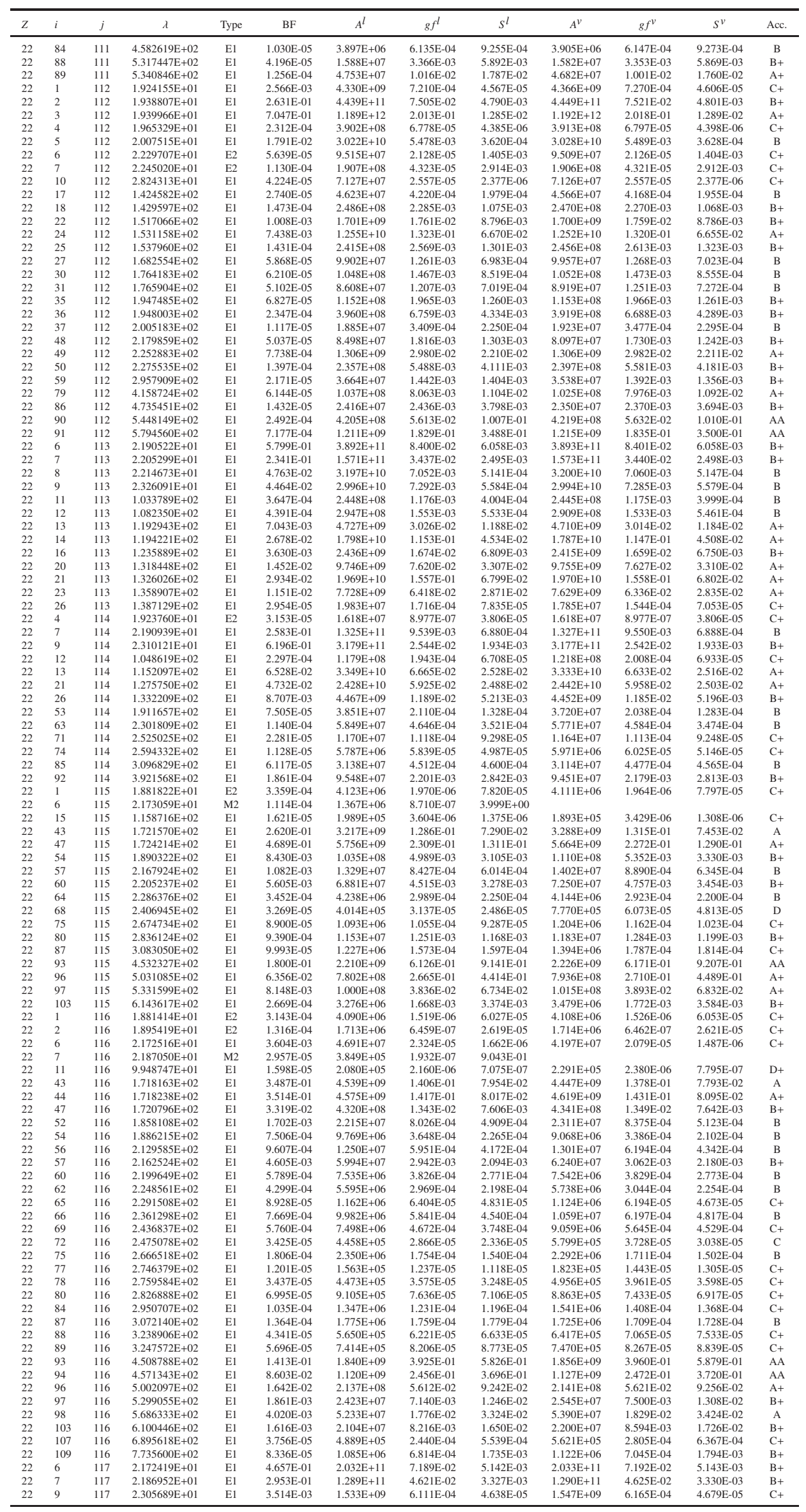




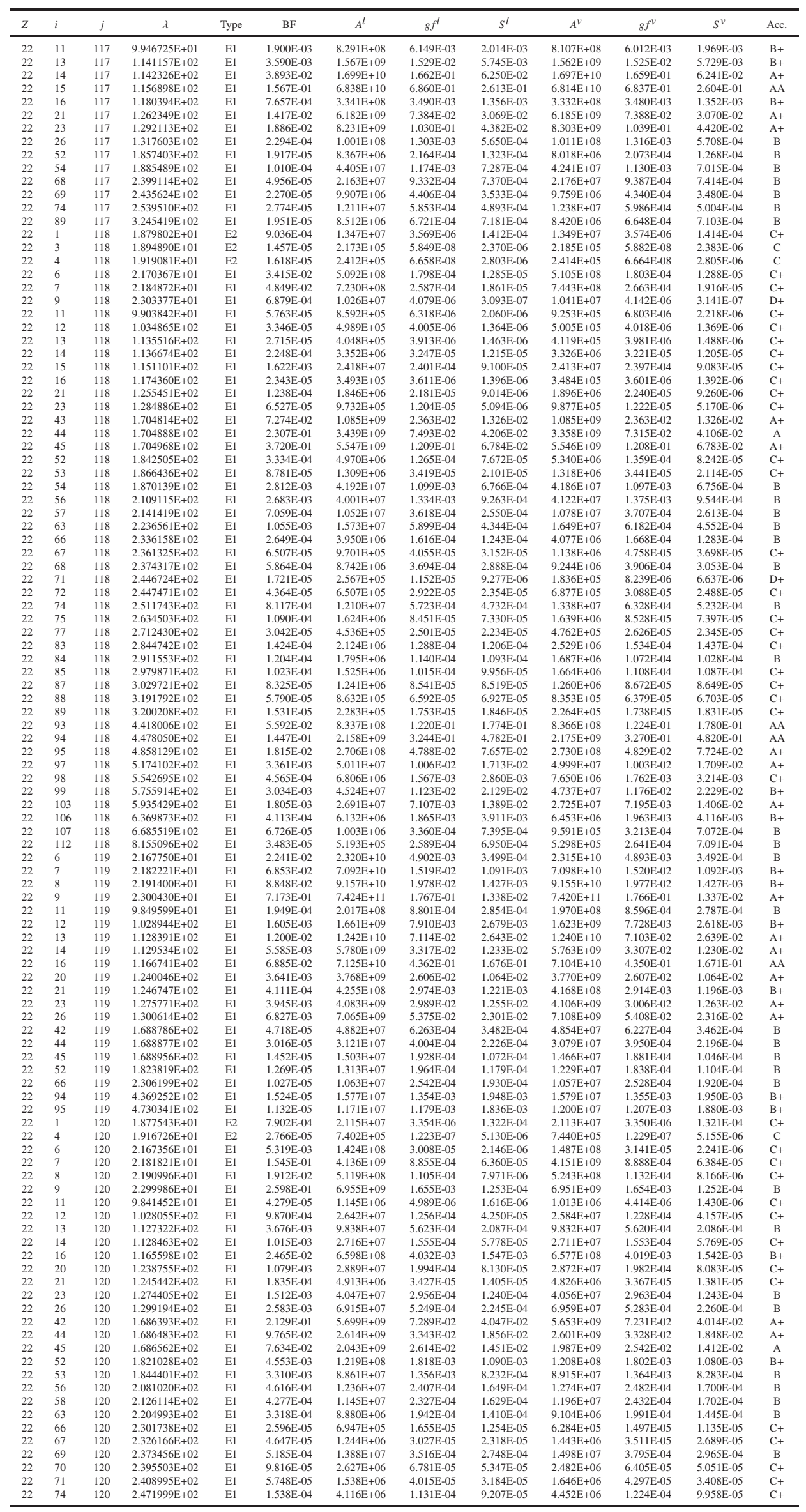




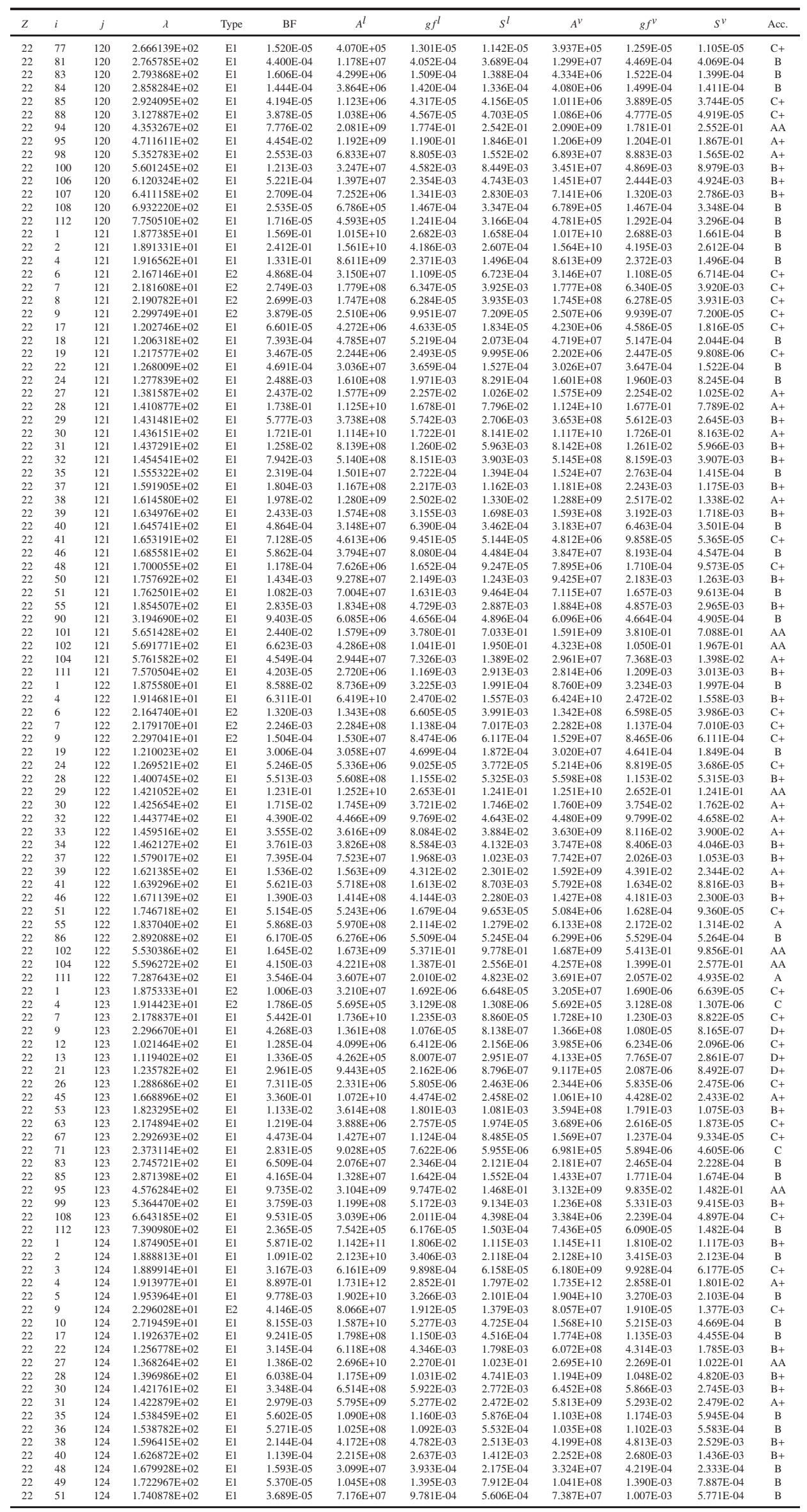




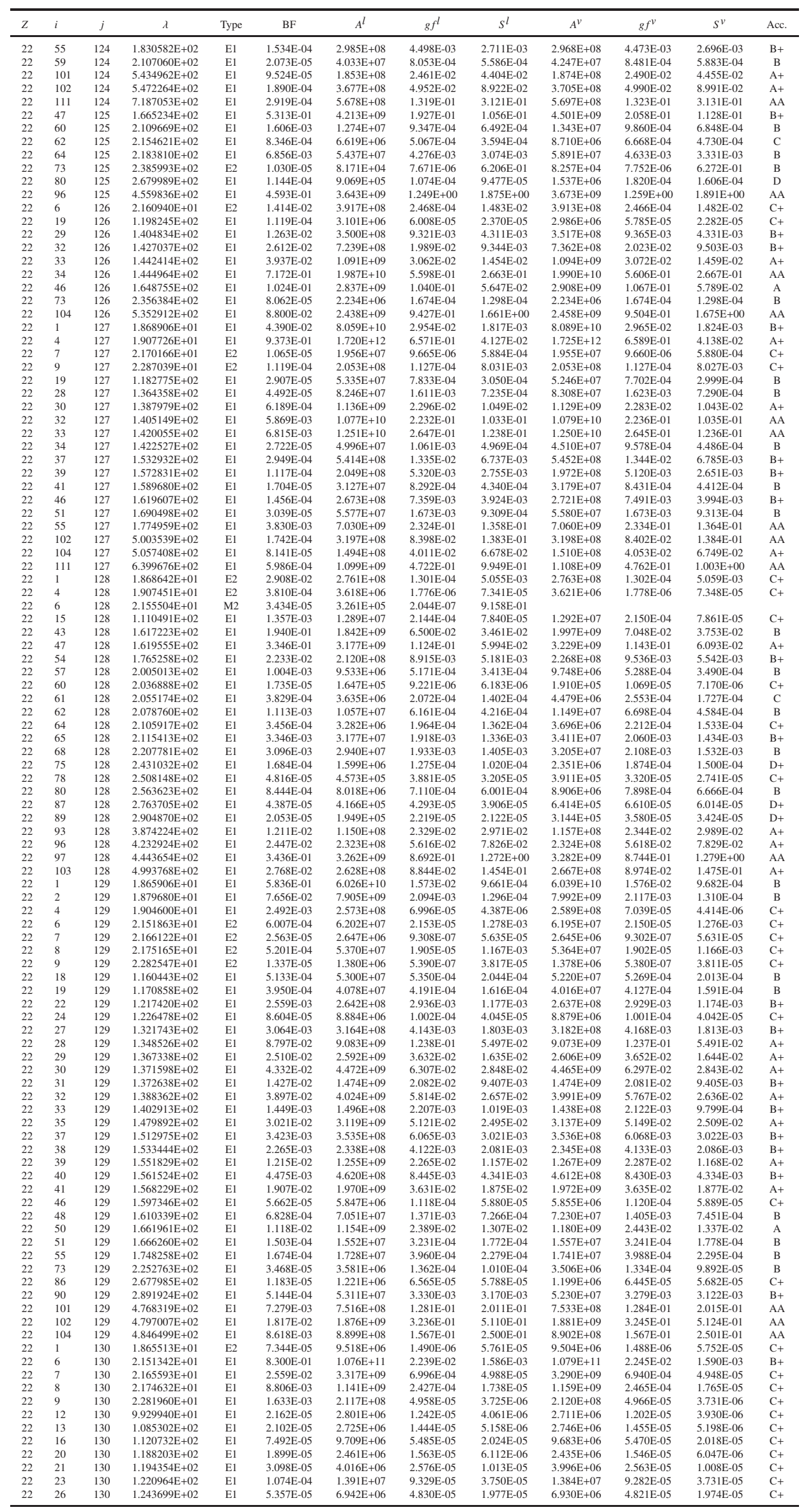




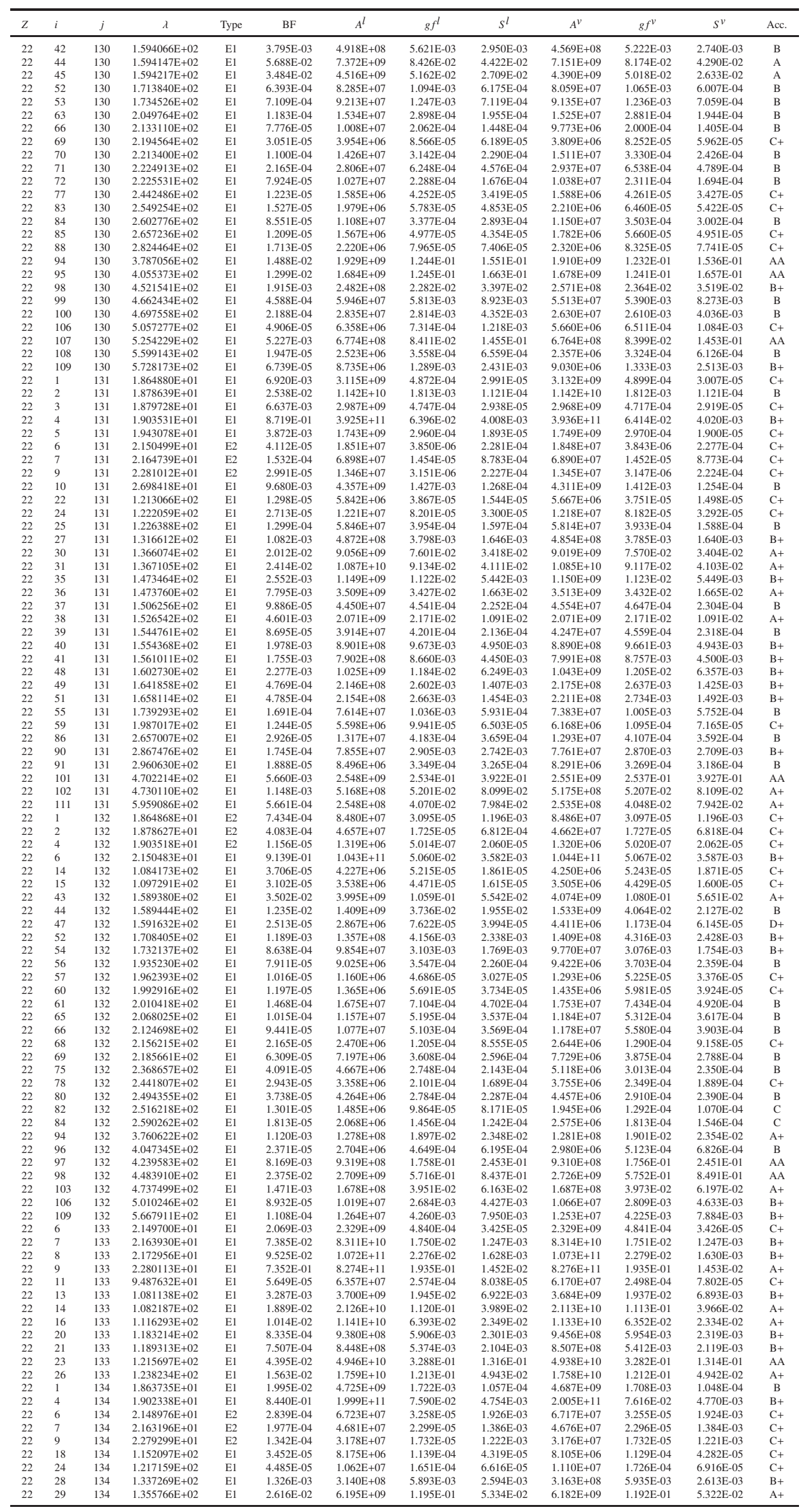




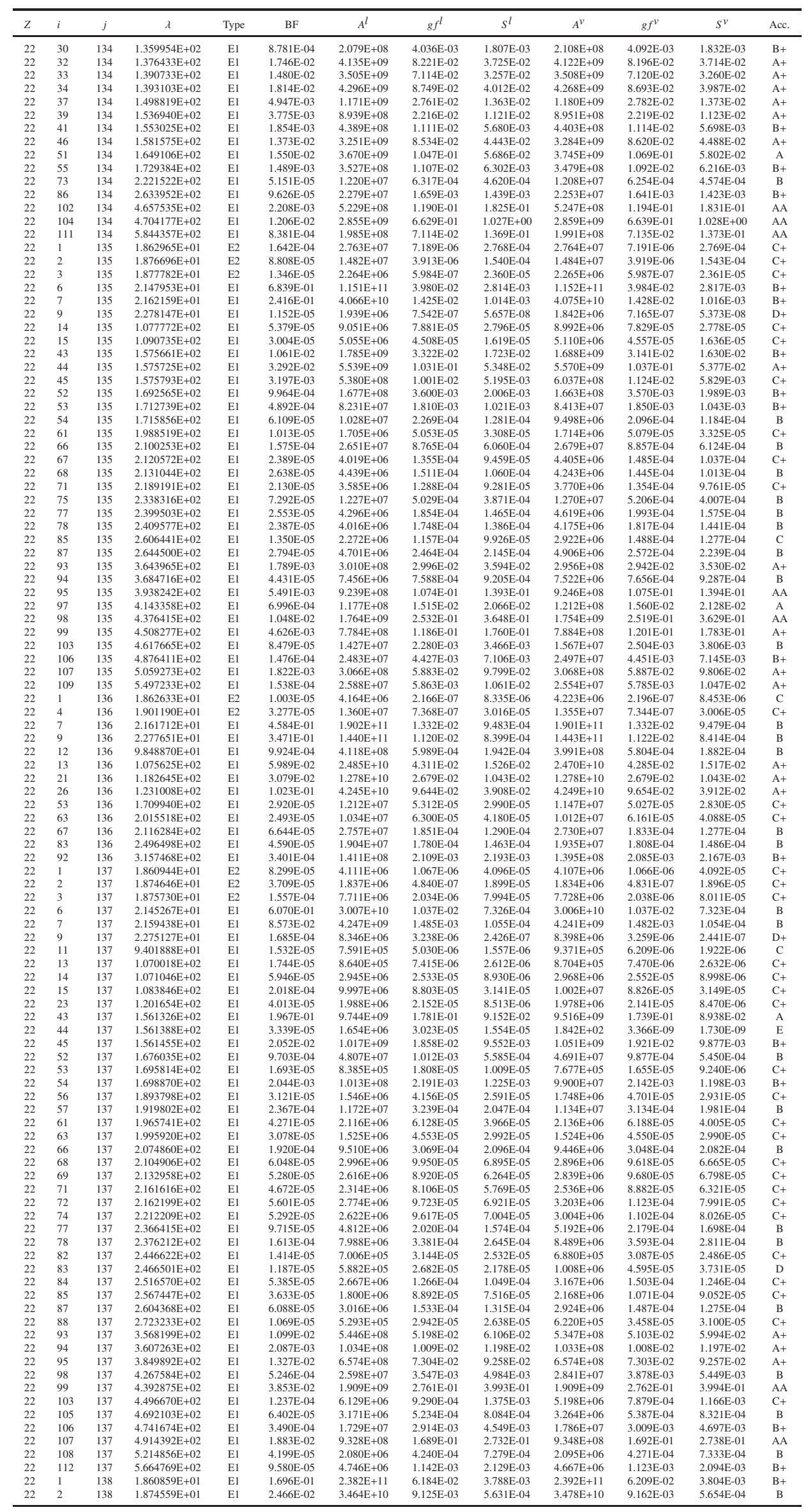




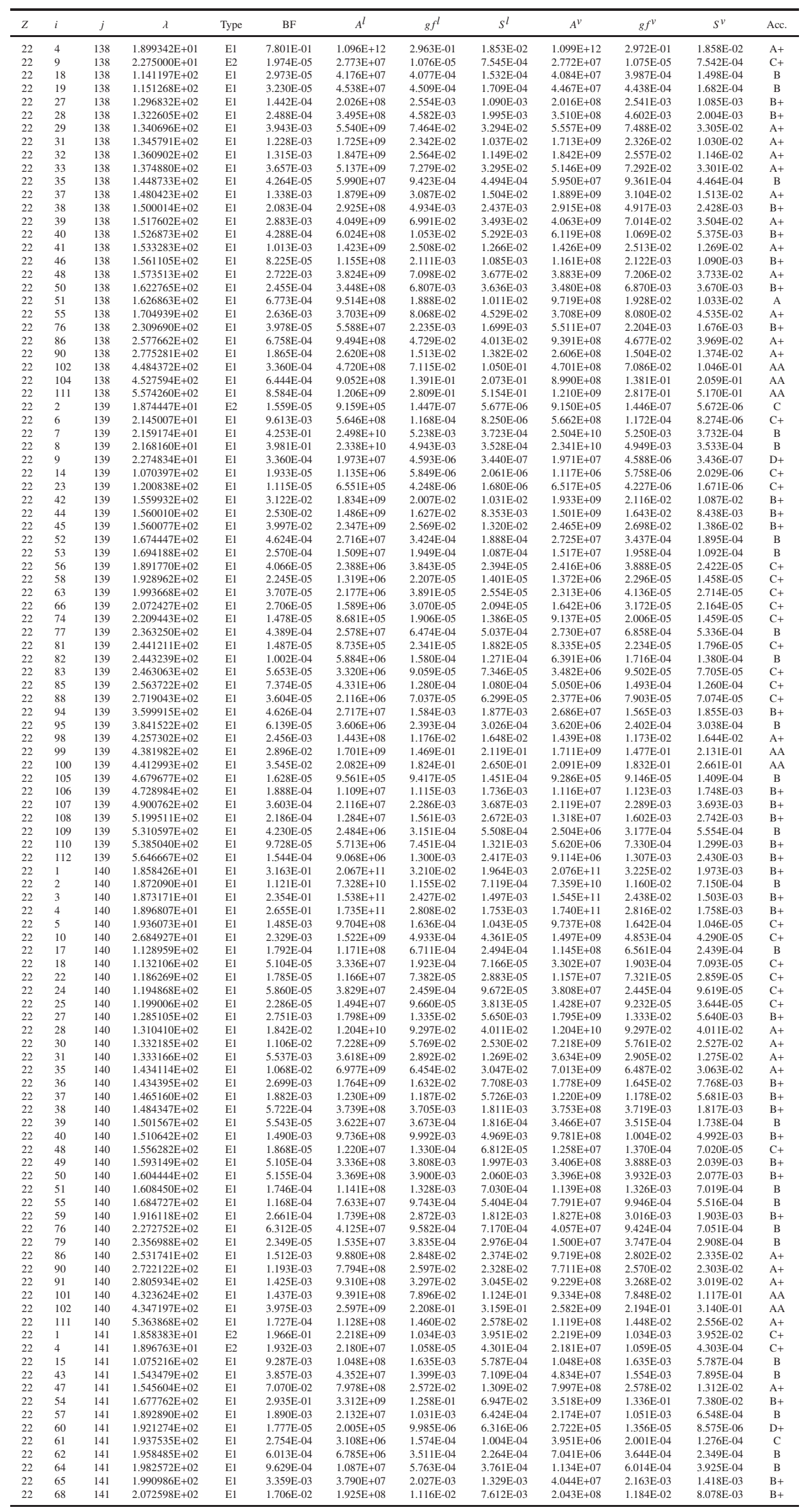




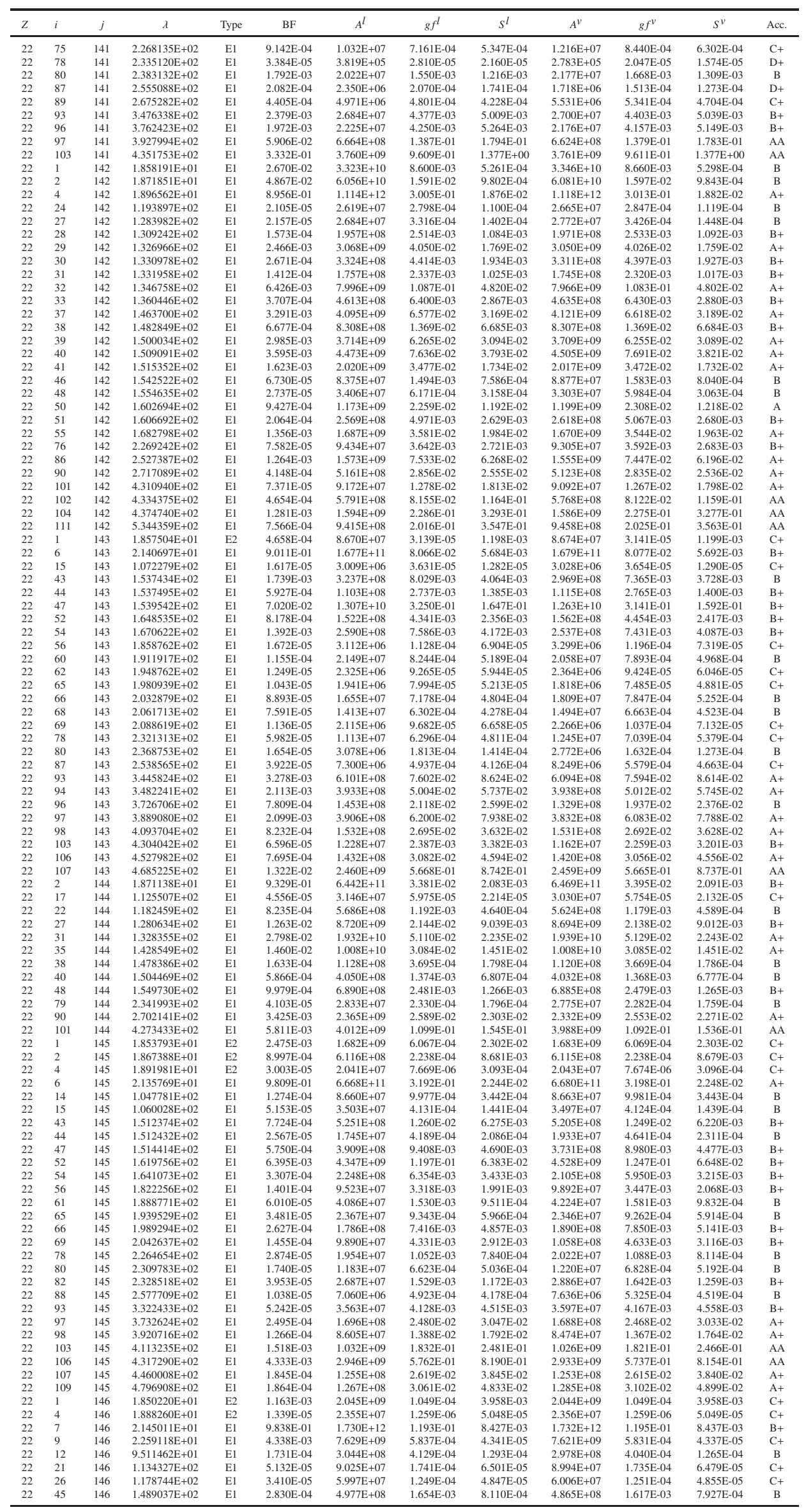




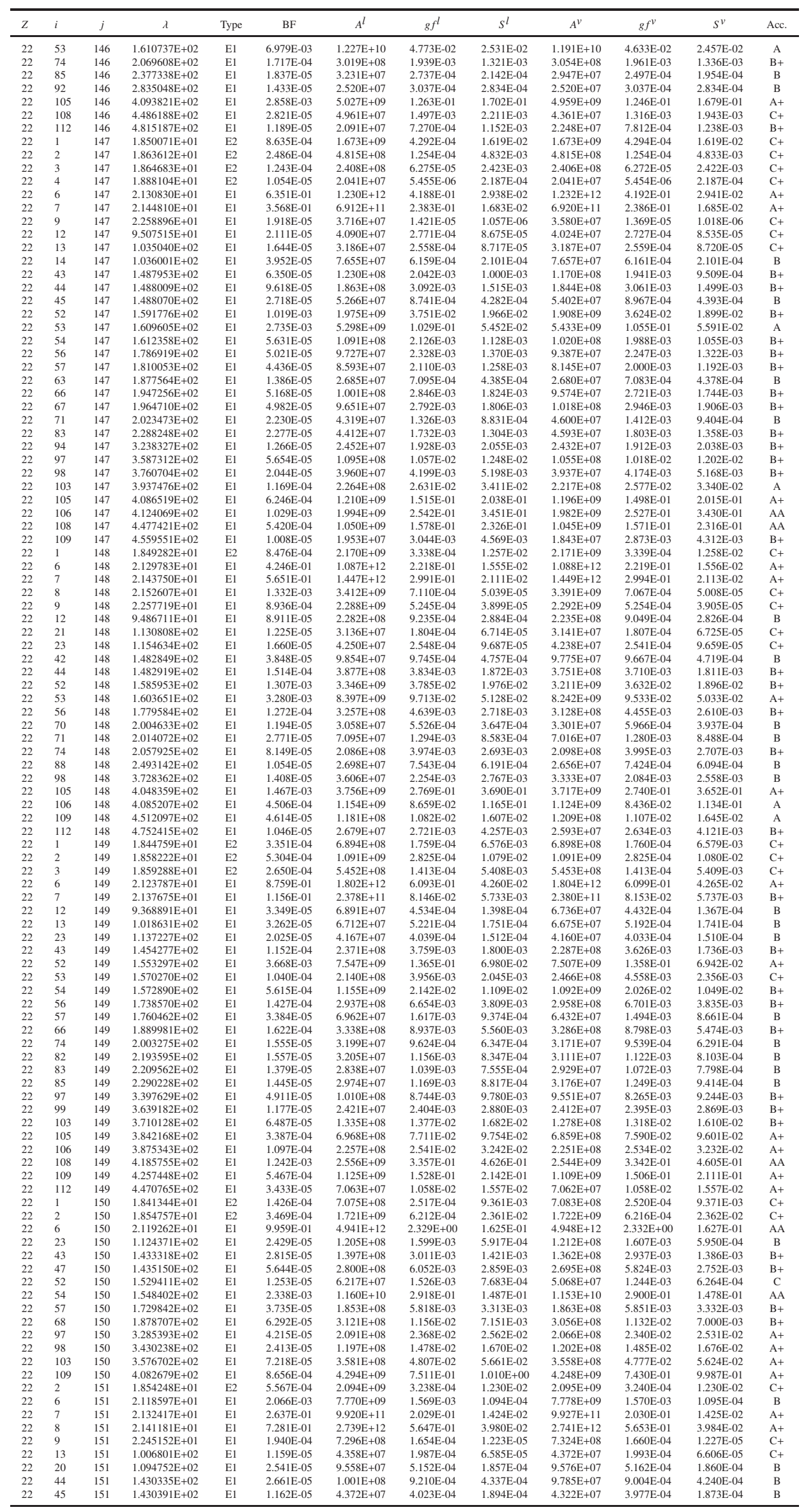




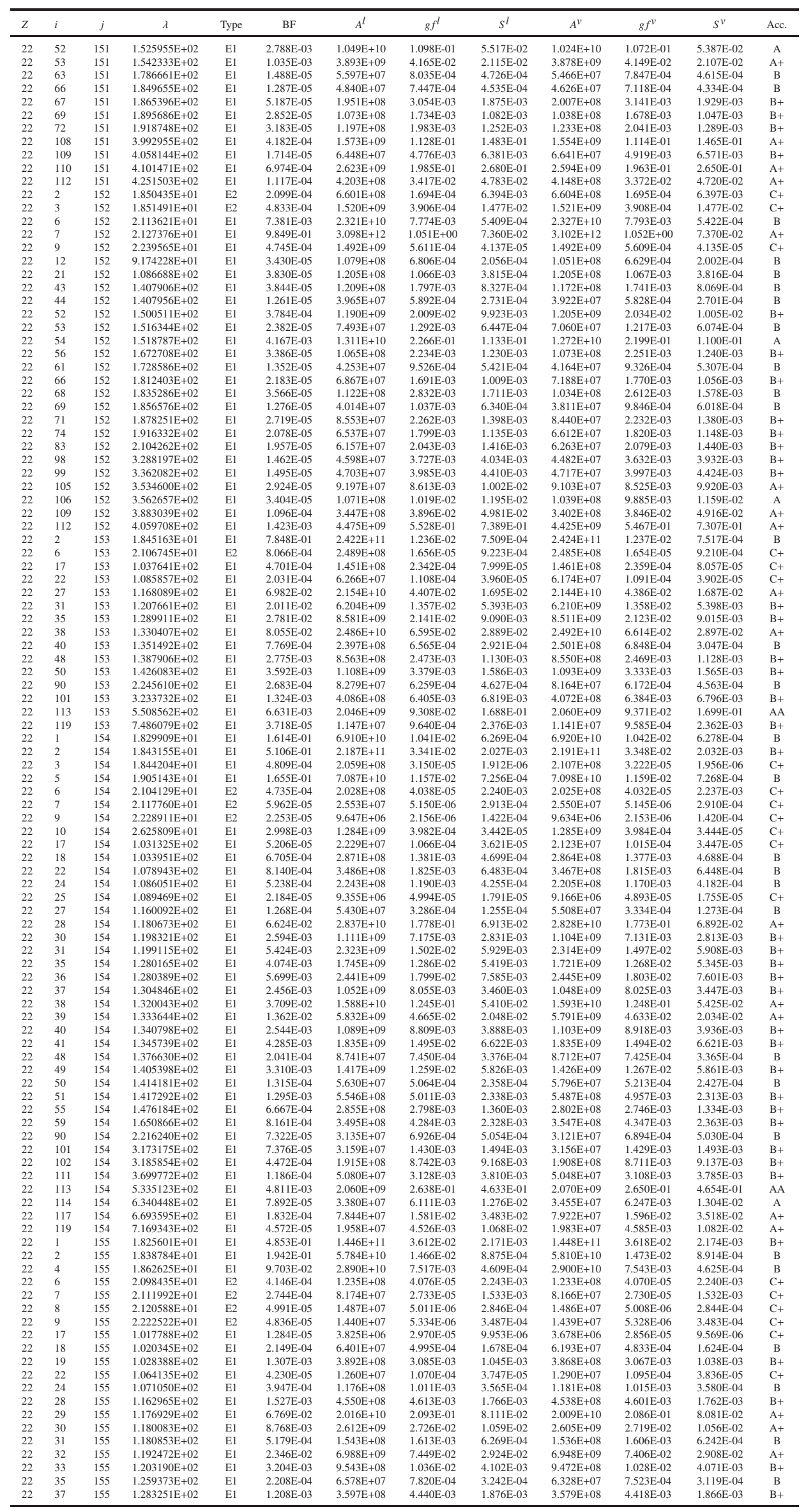




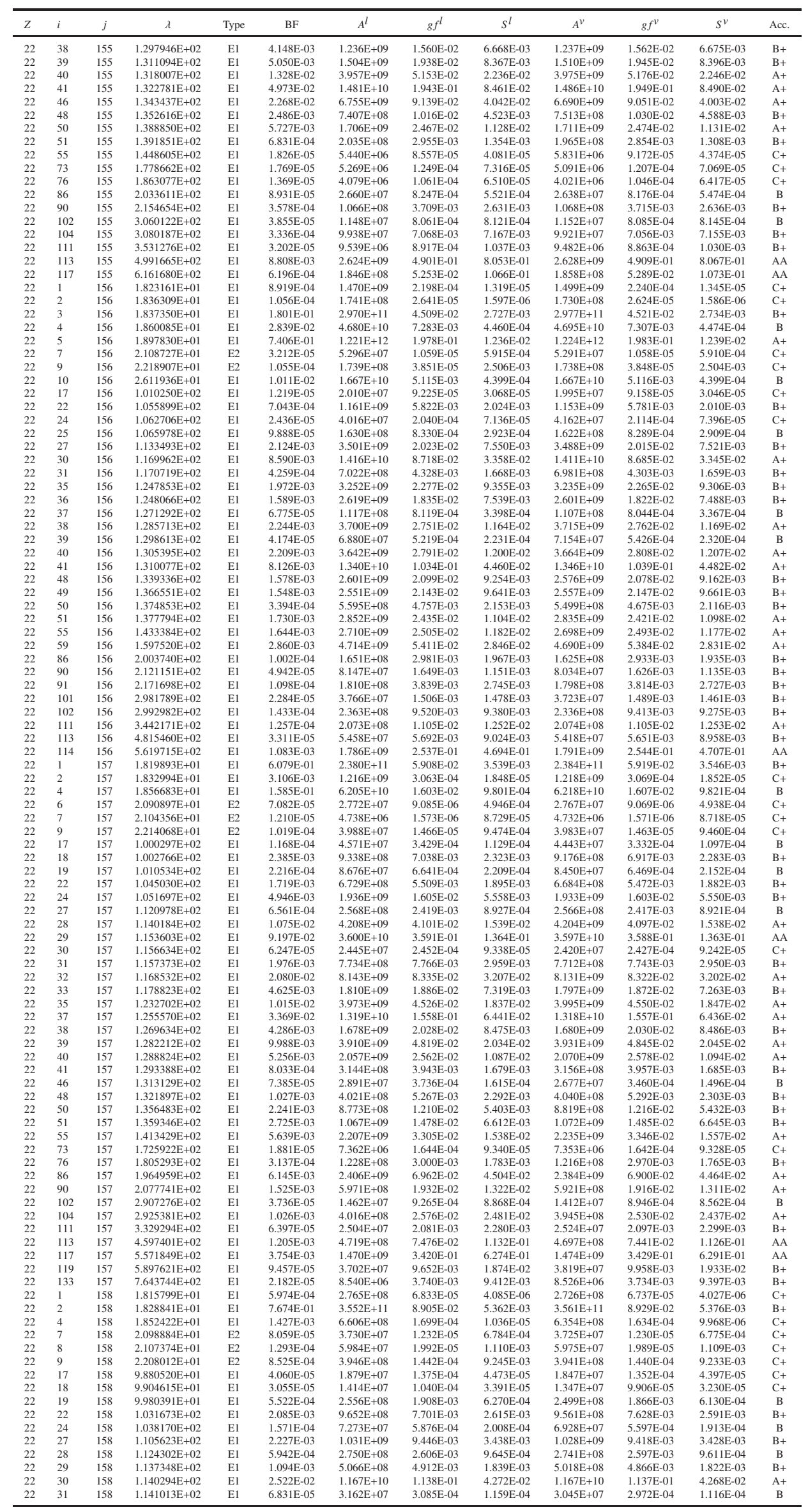




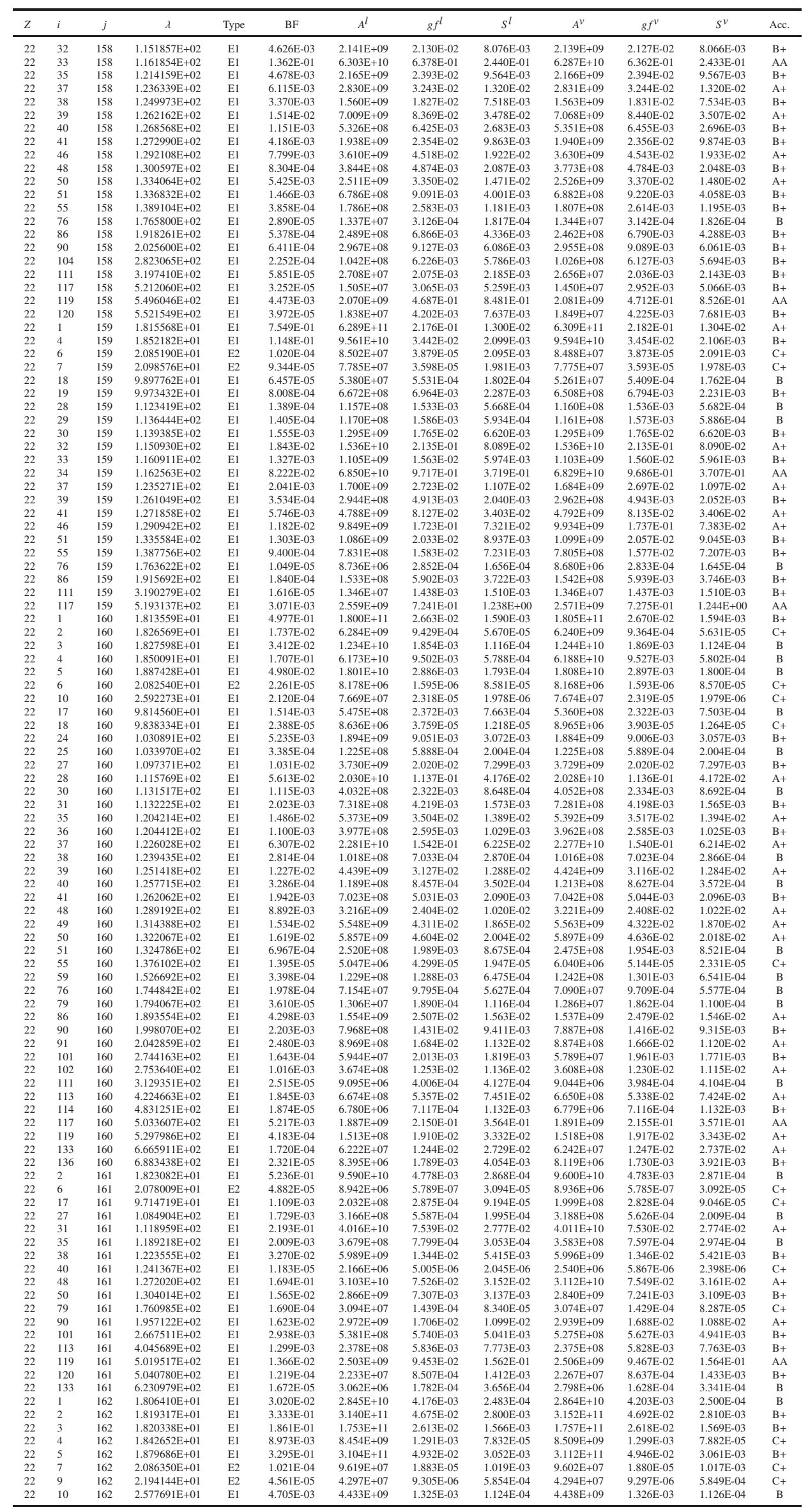




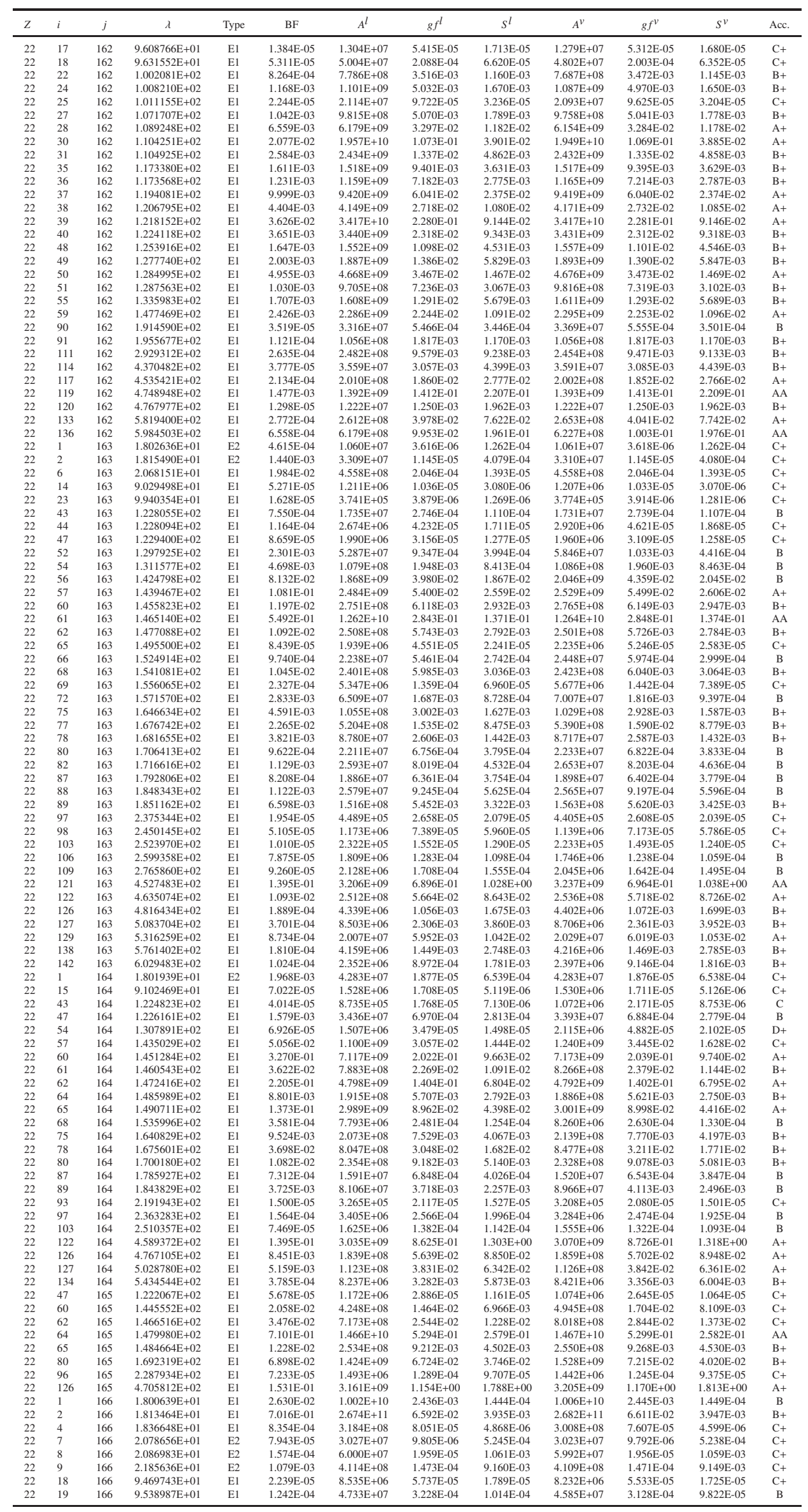




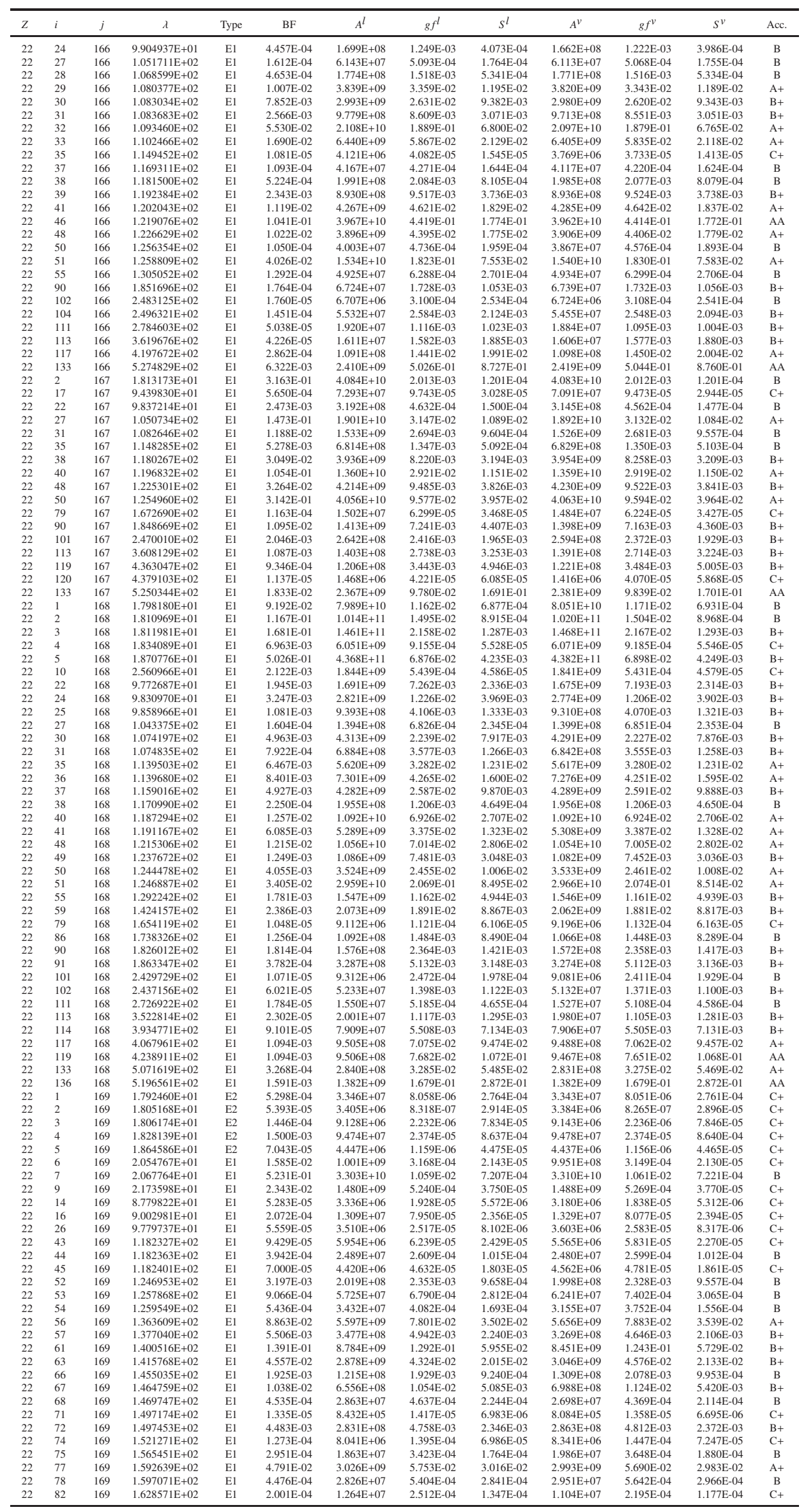




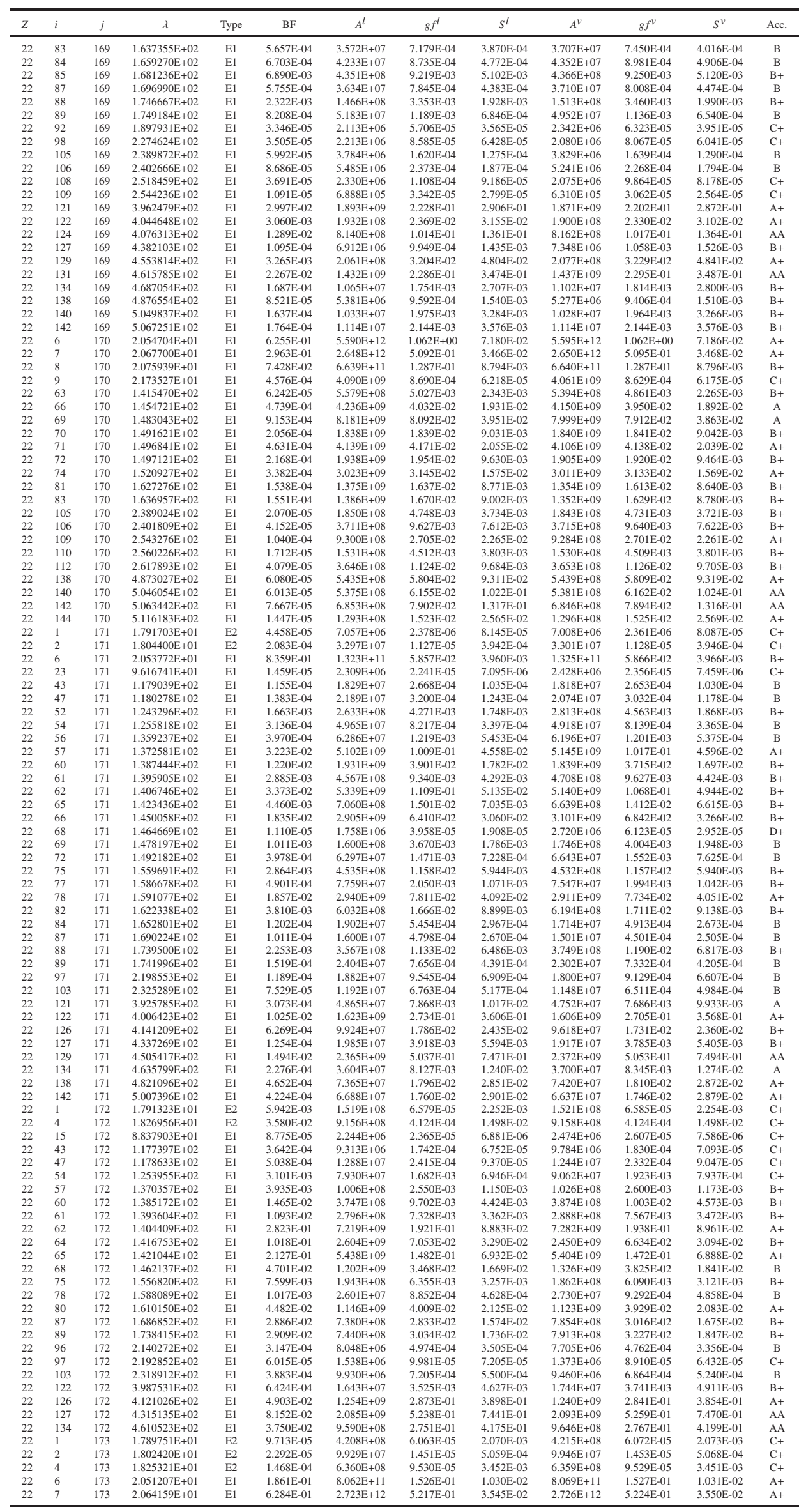




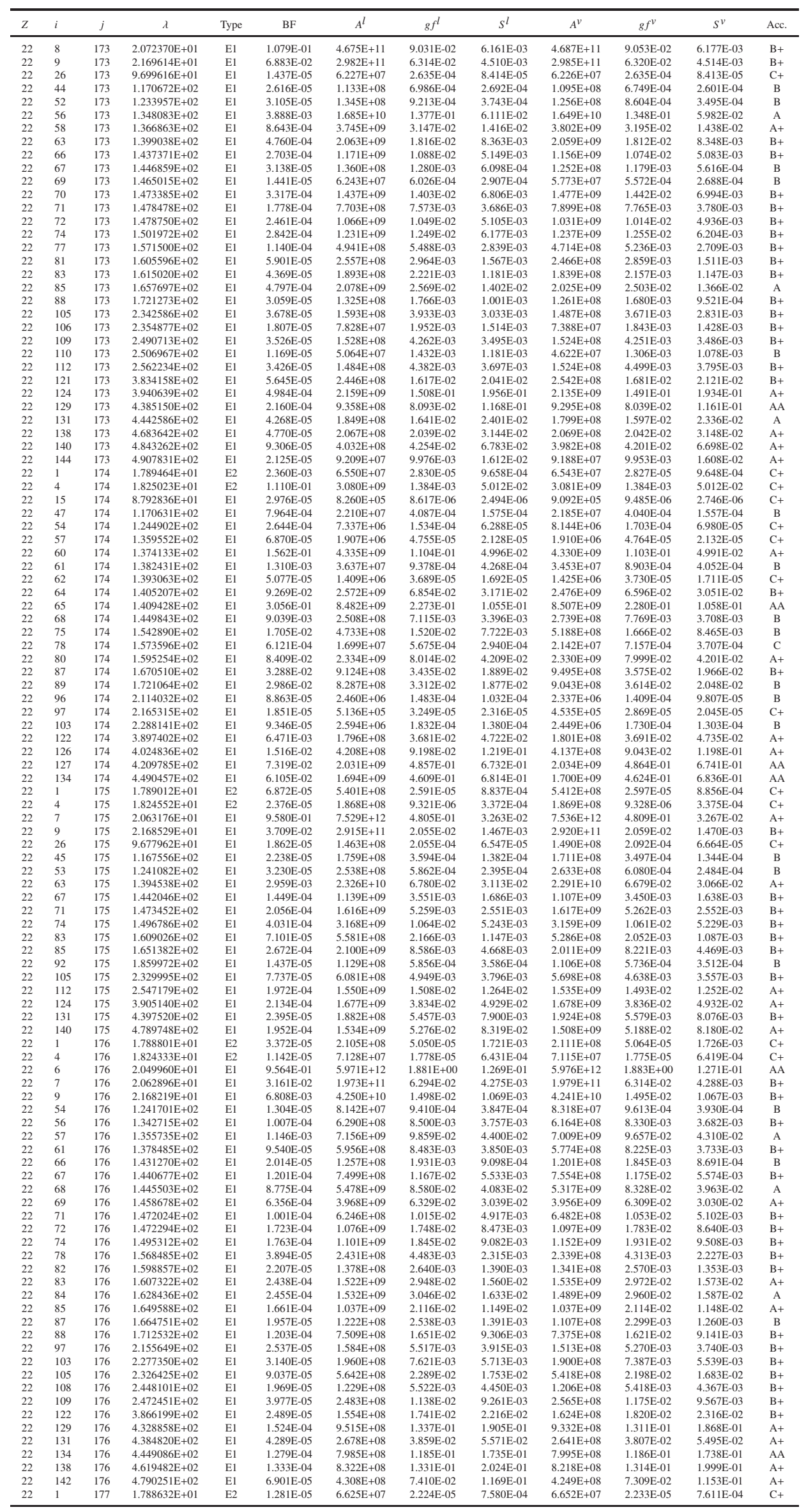




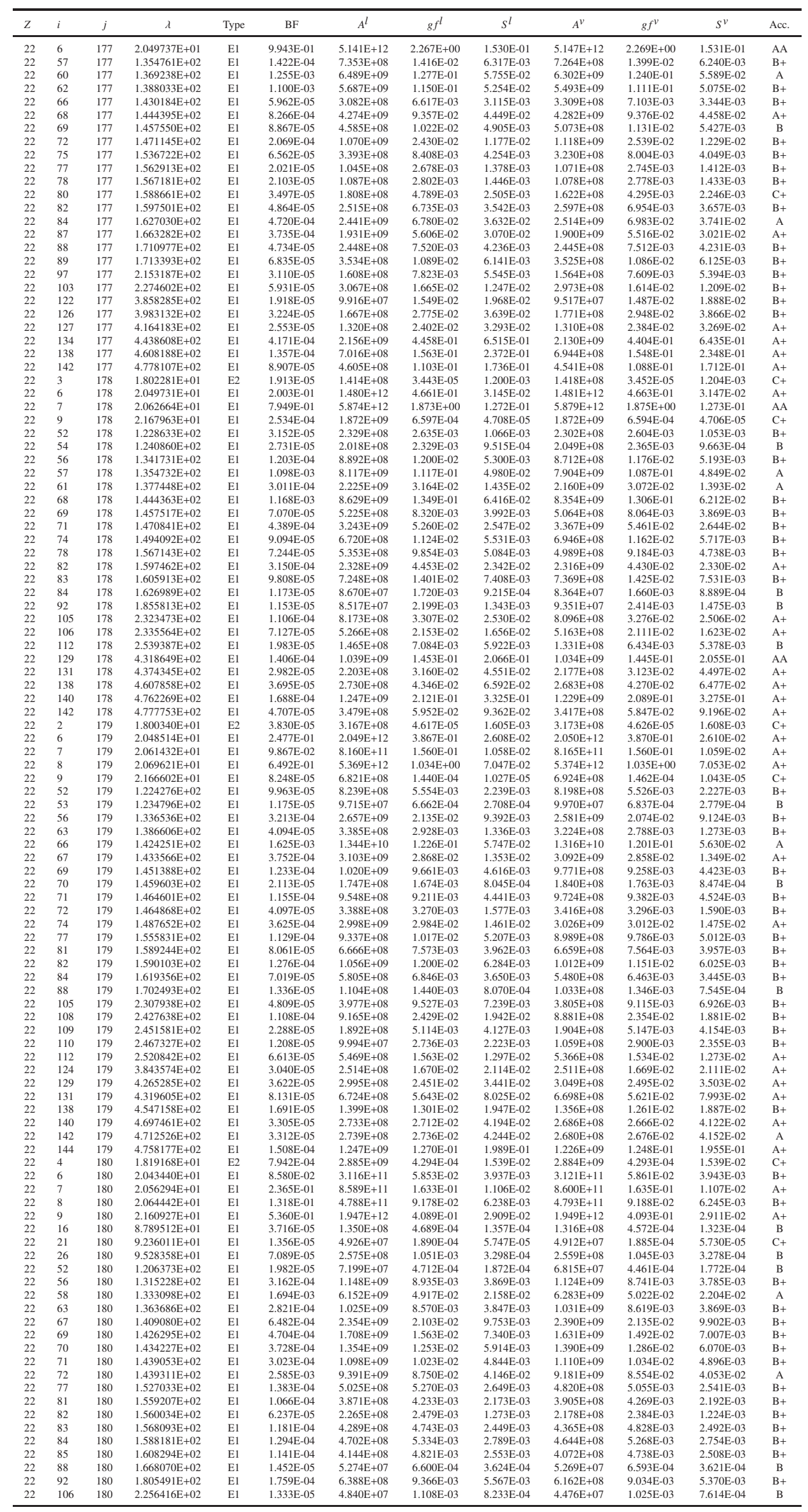




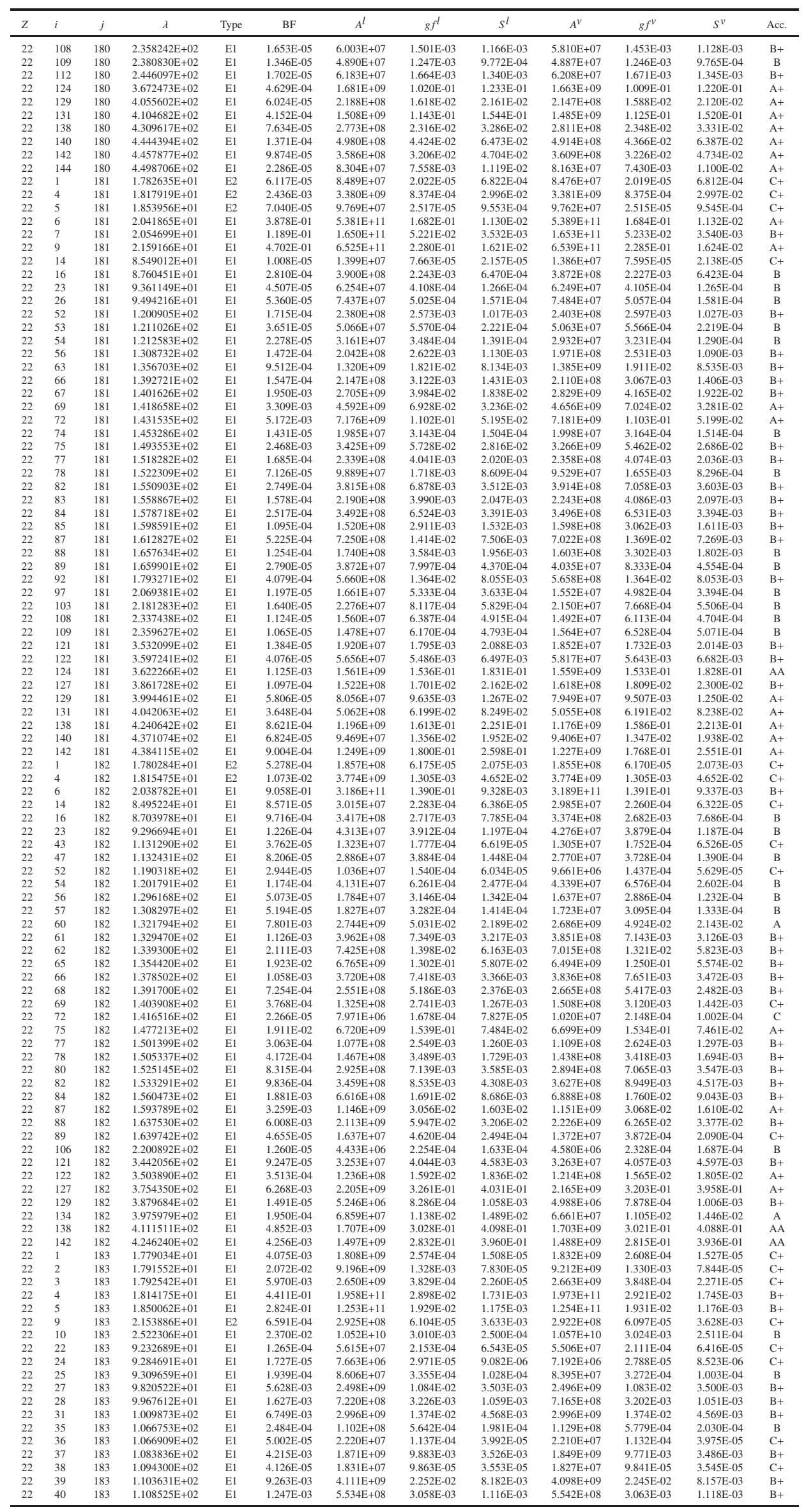




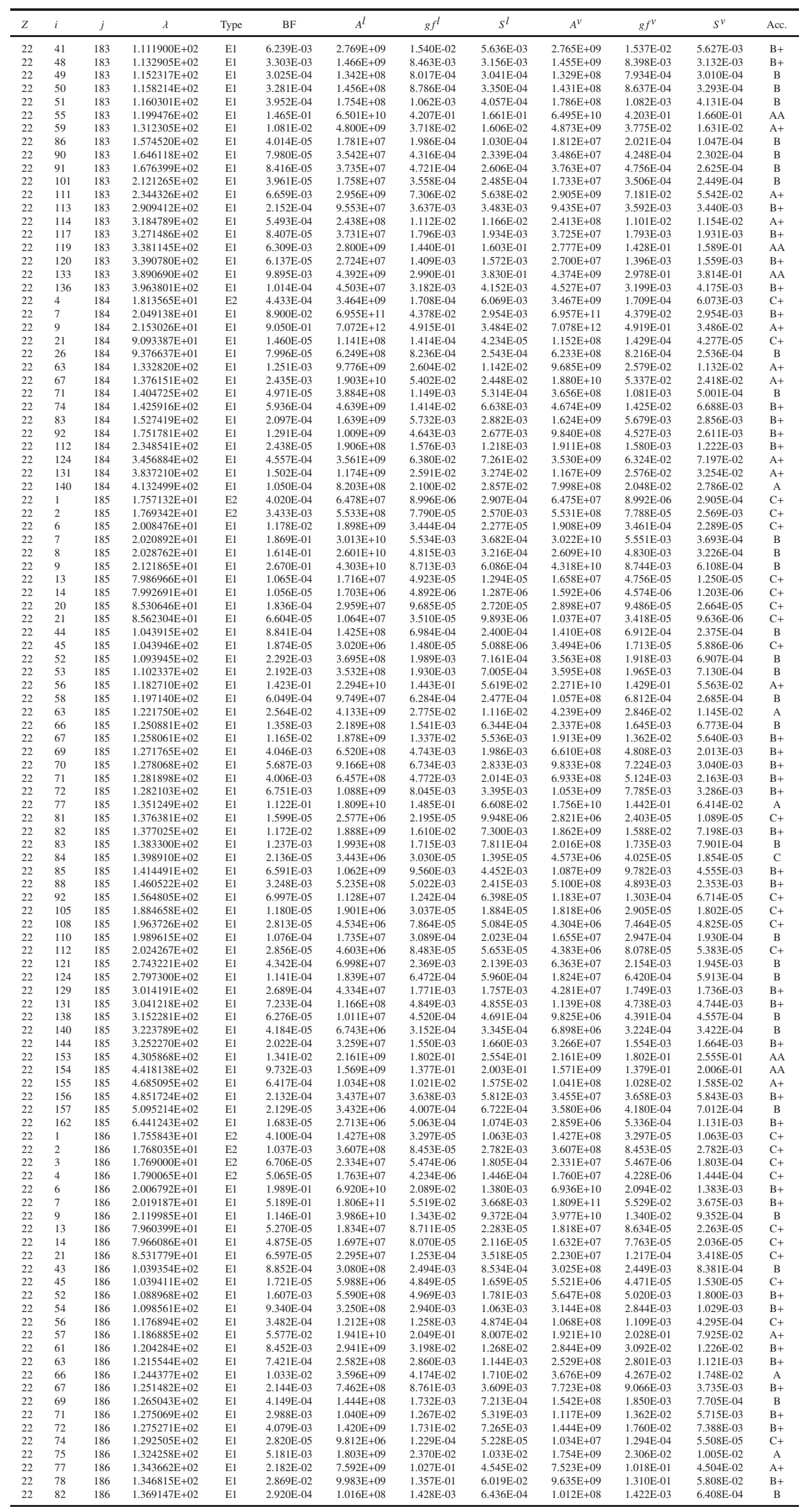




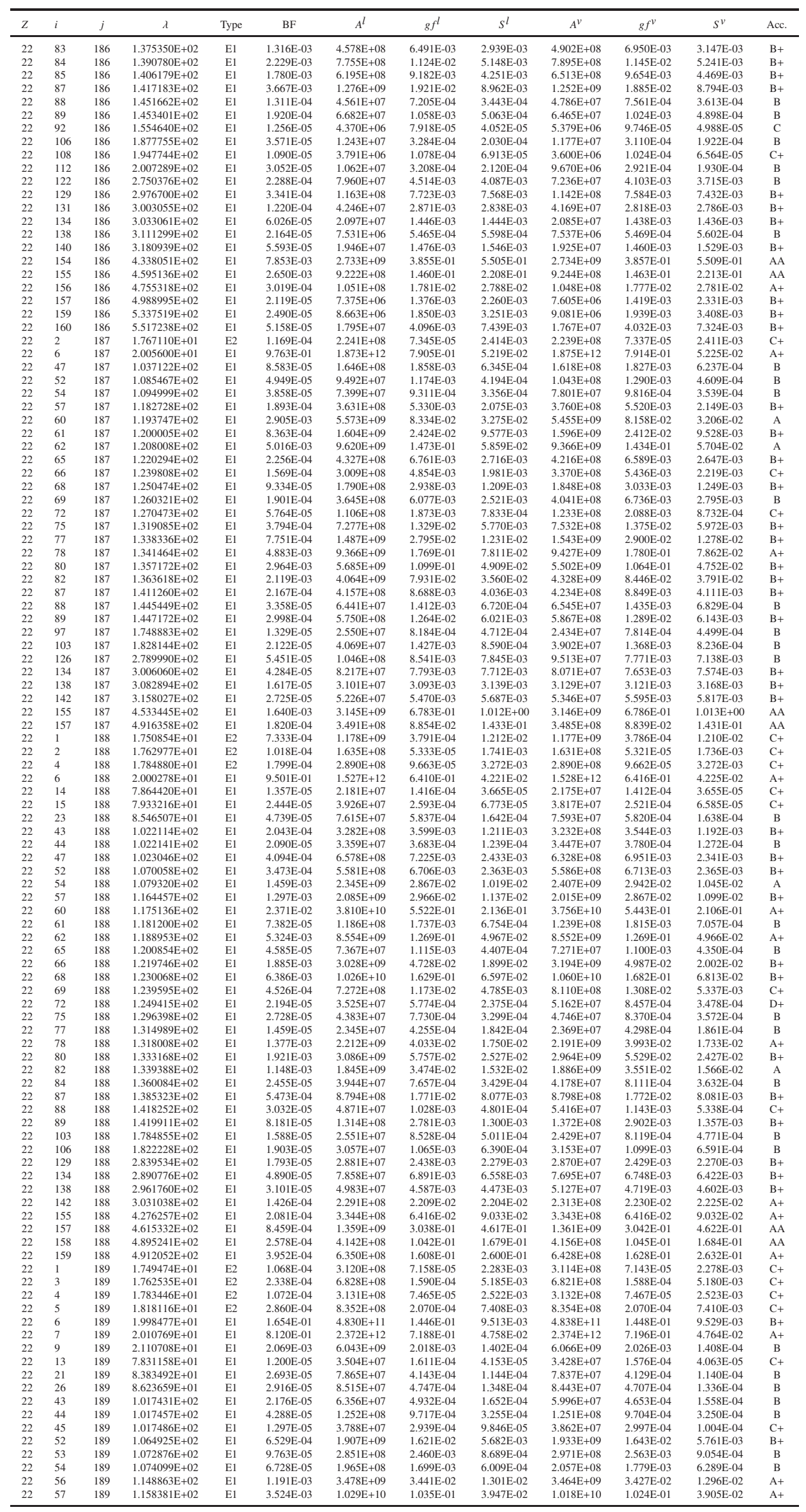




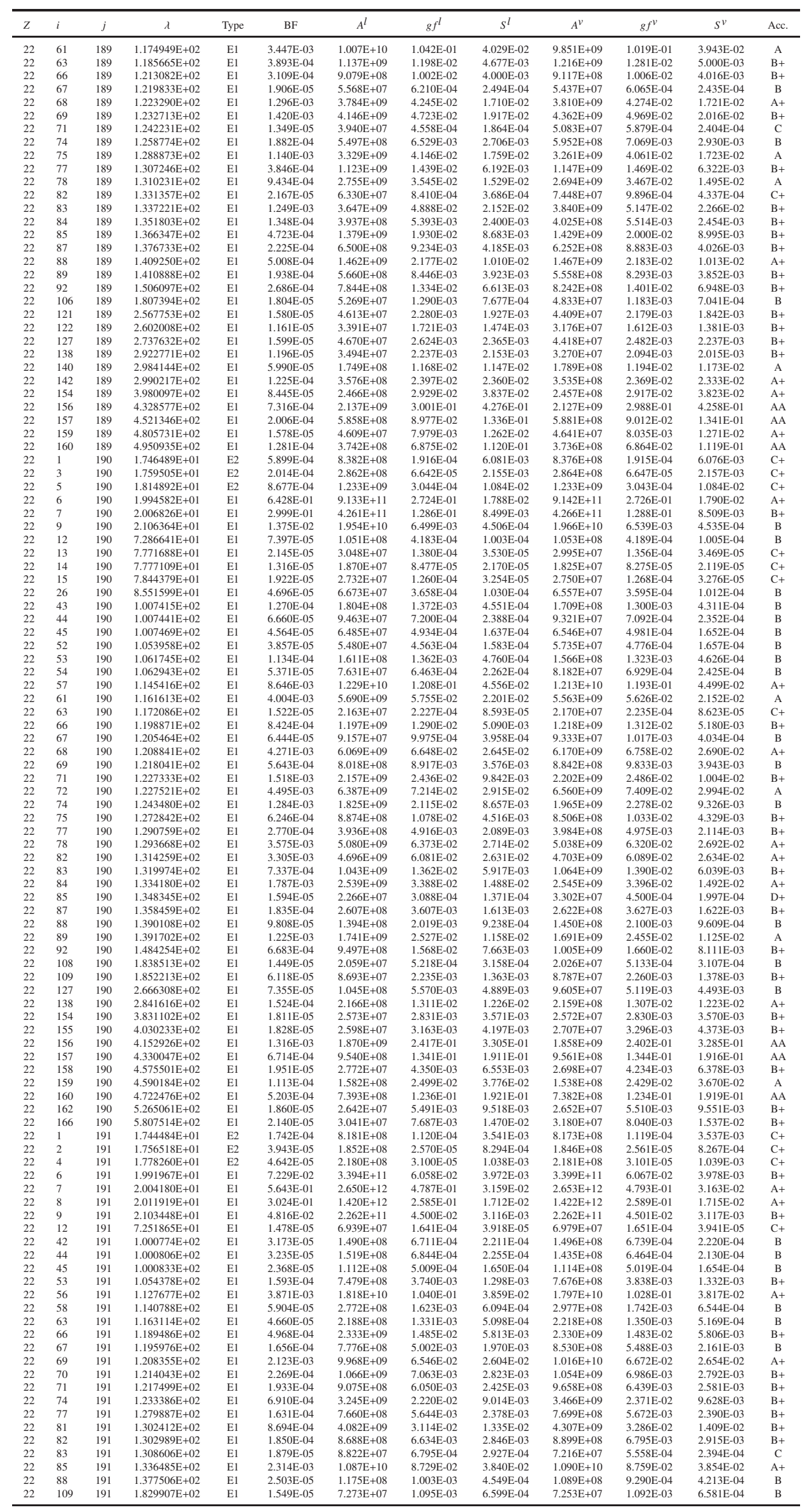




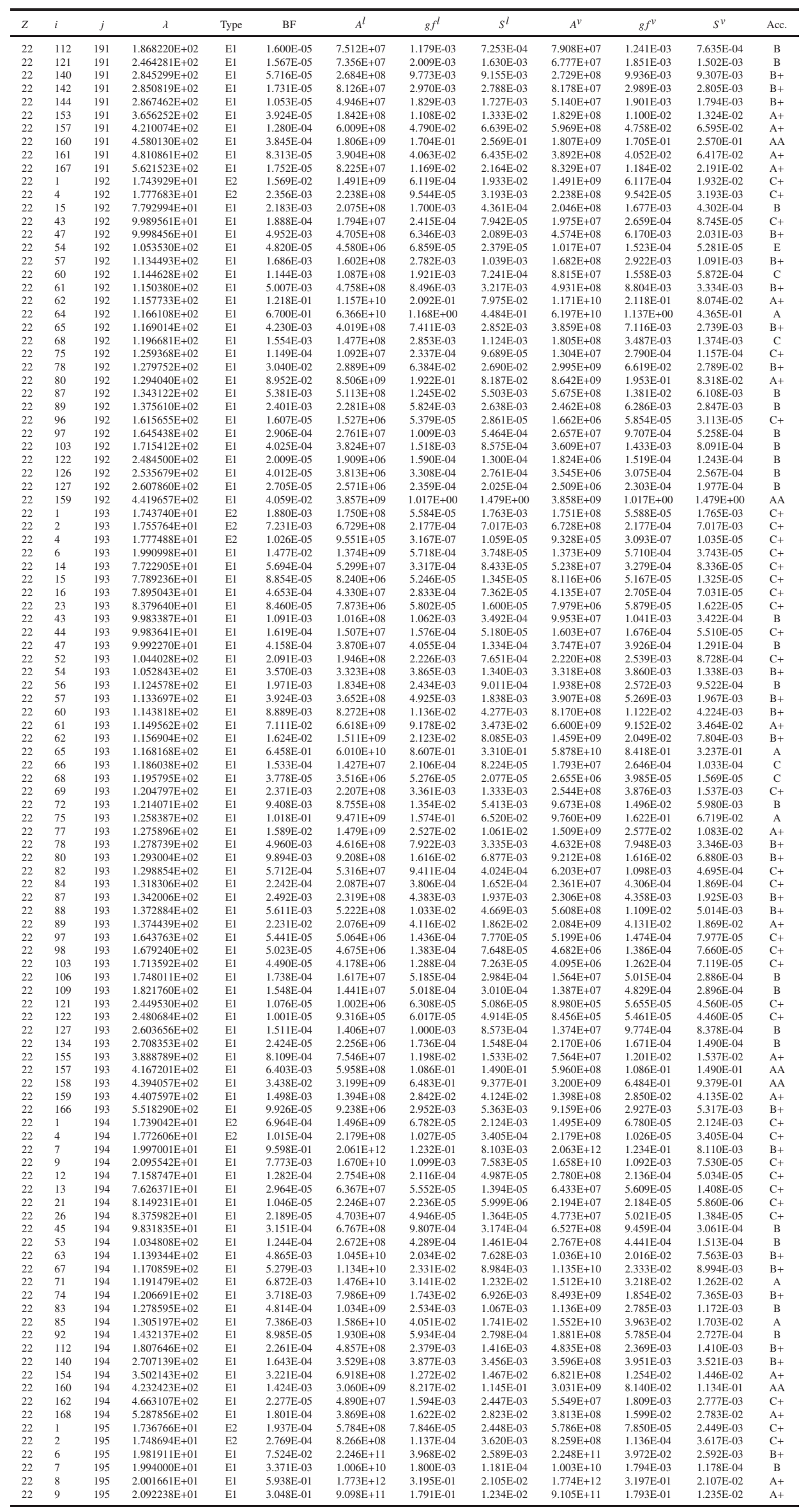




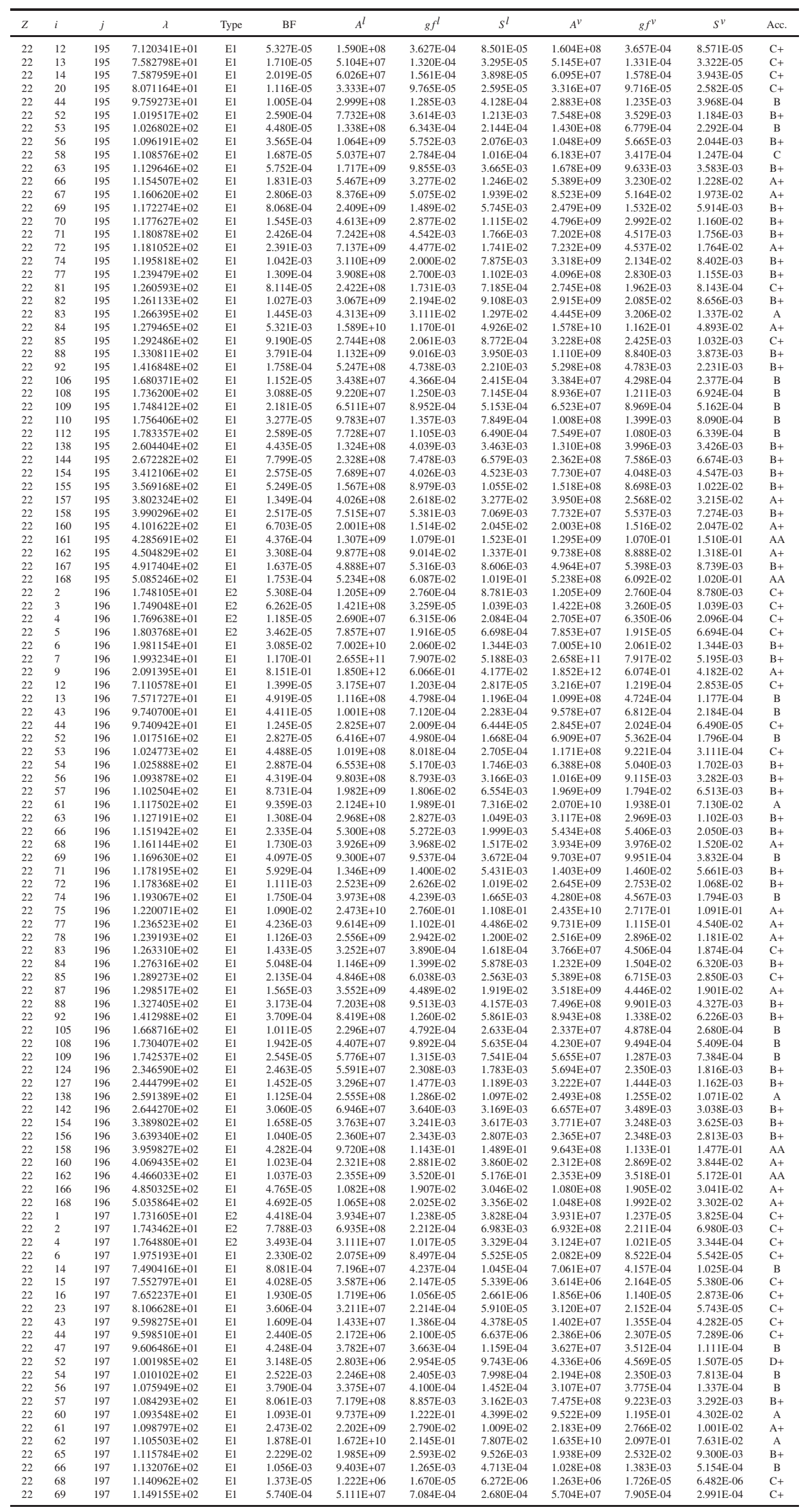

180 


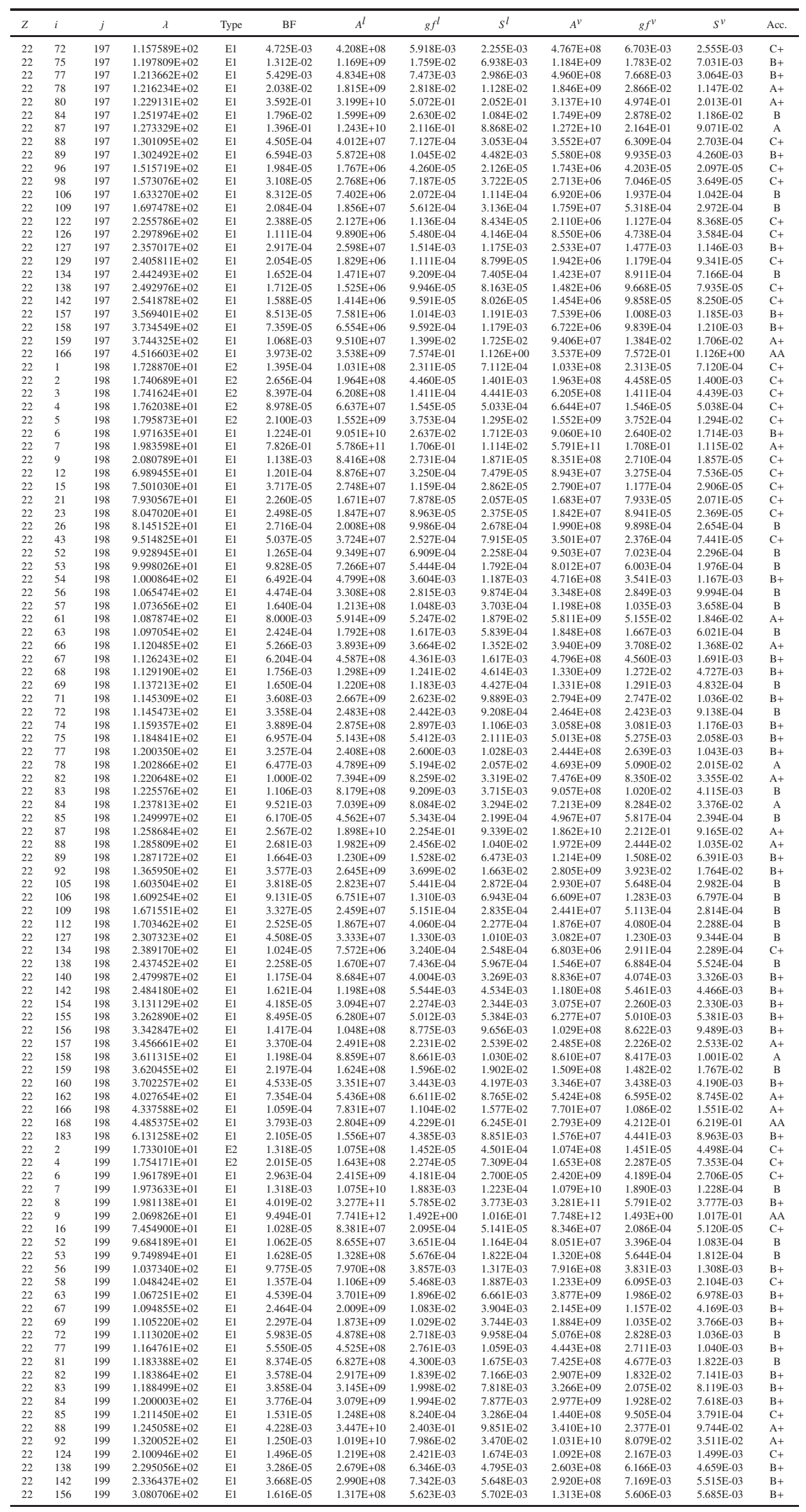




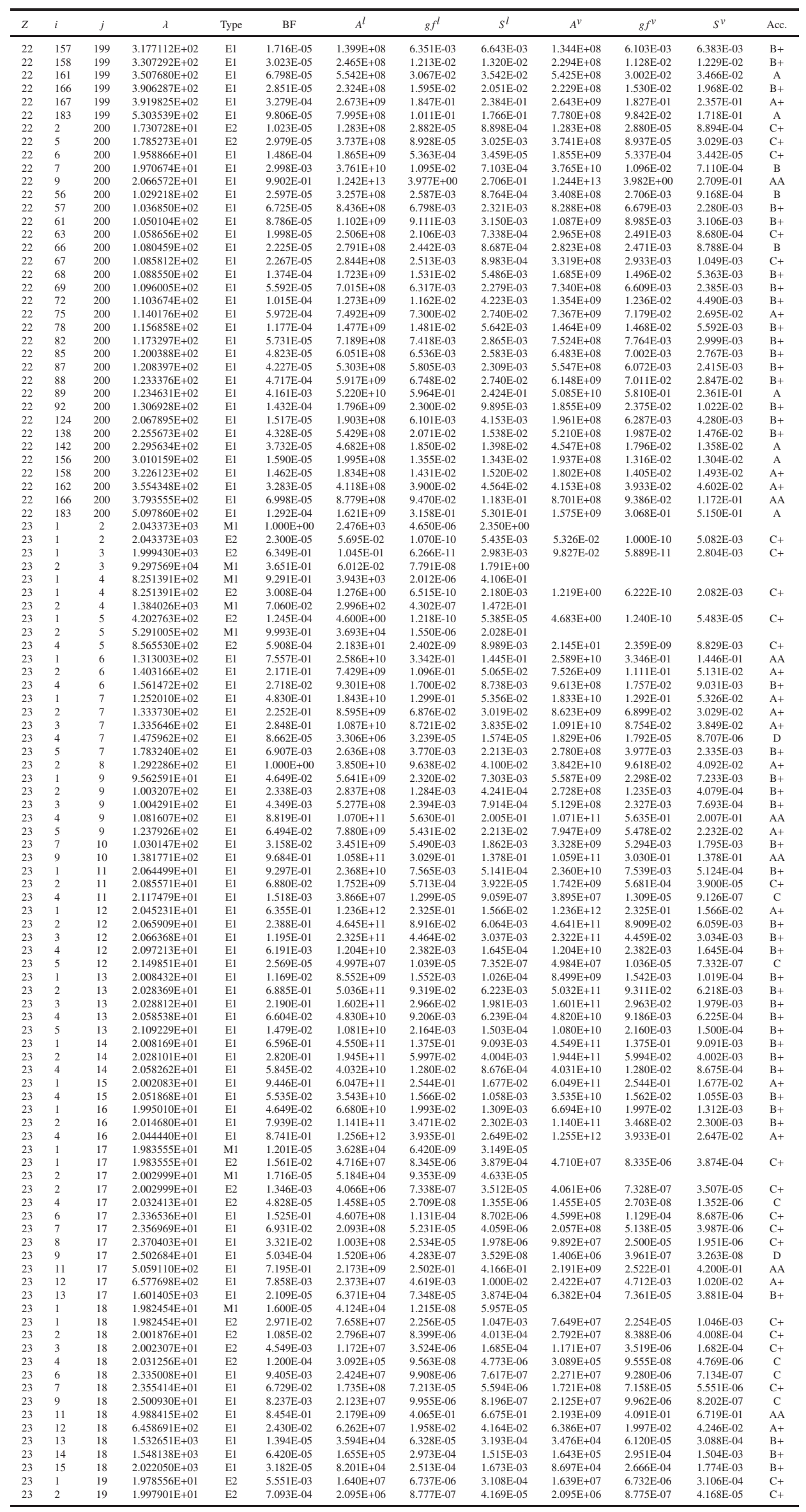




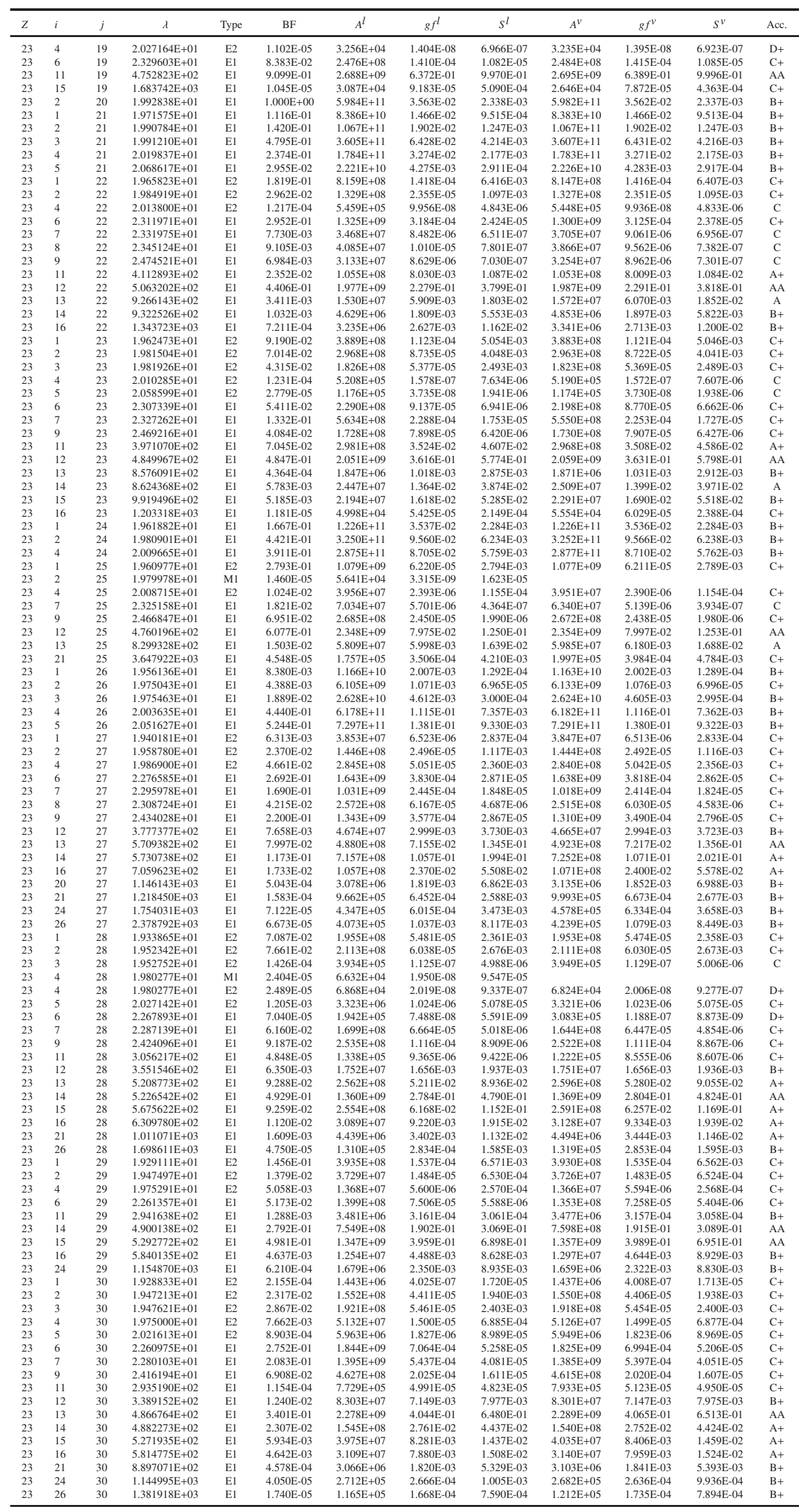




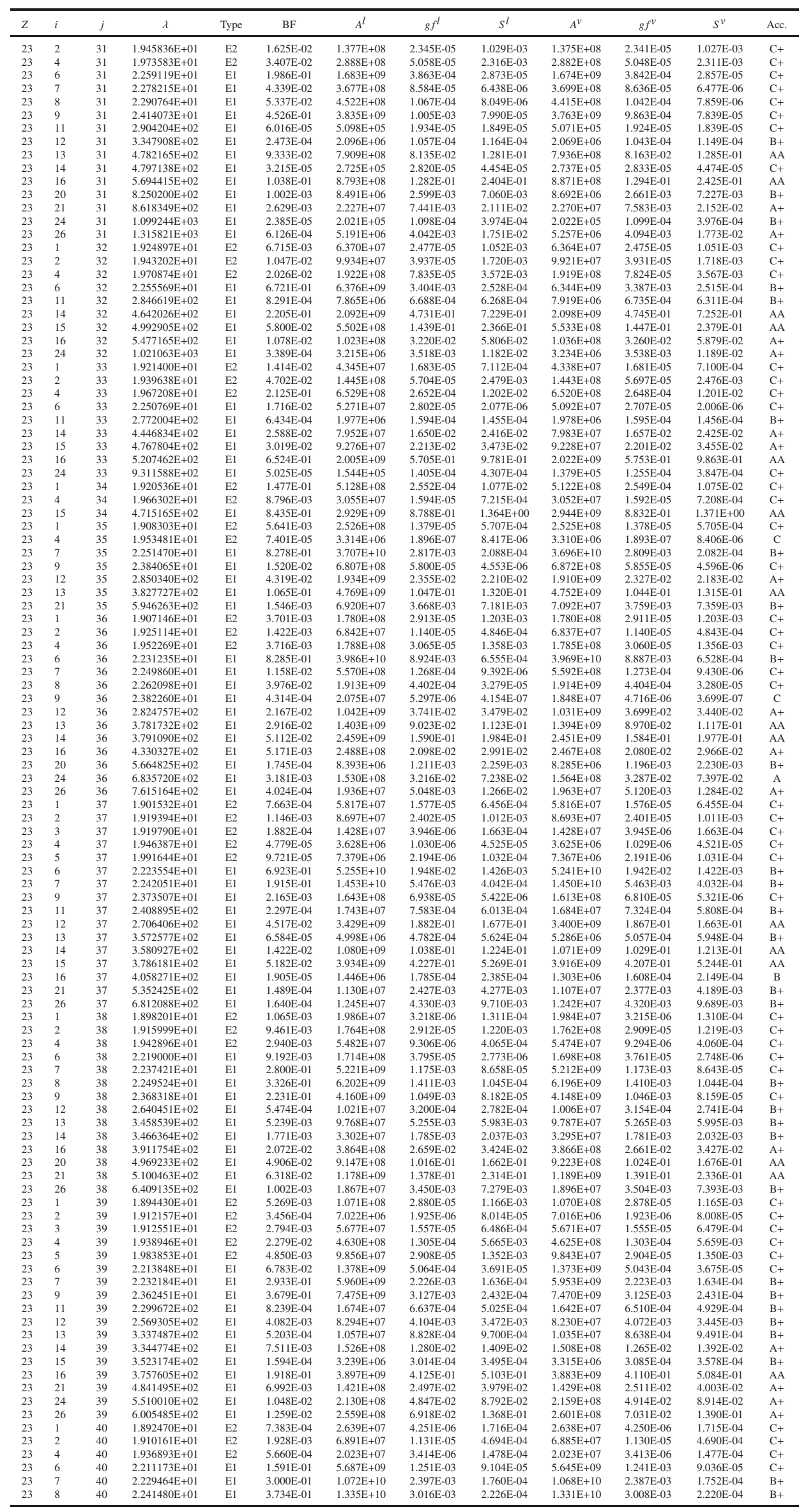




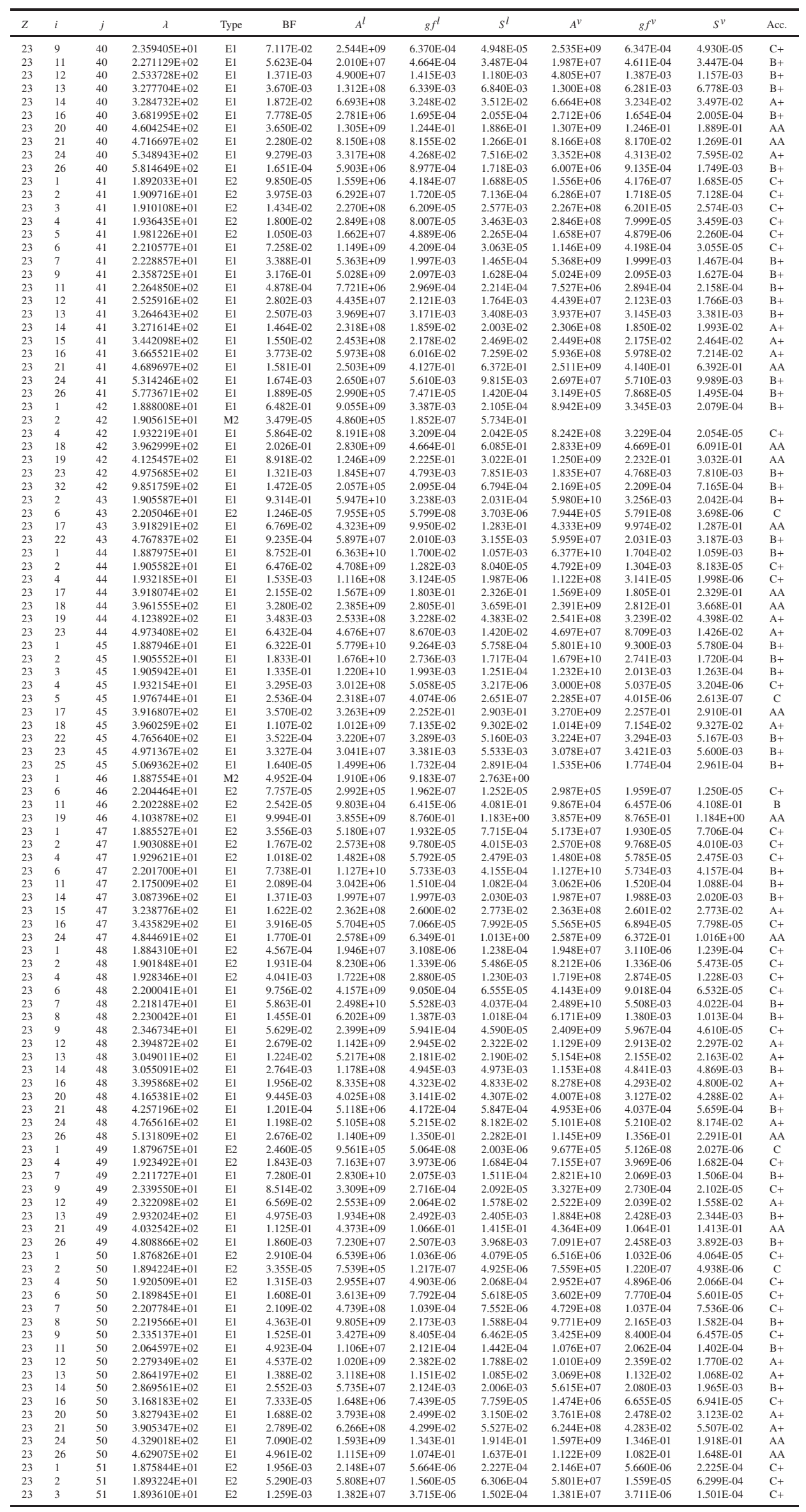




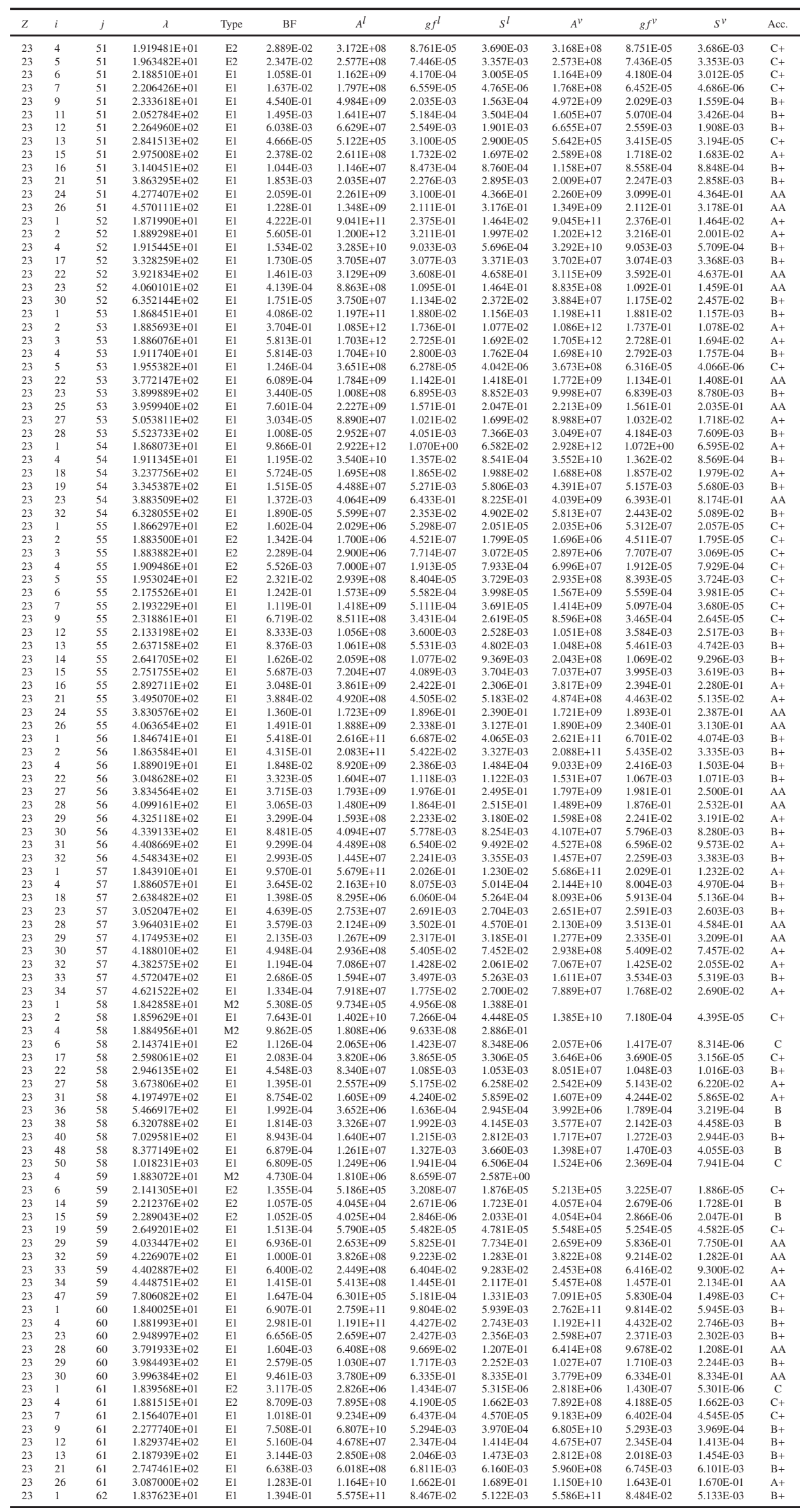




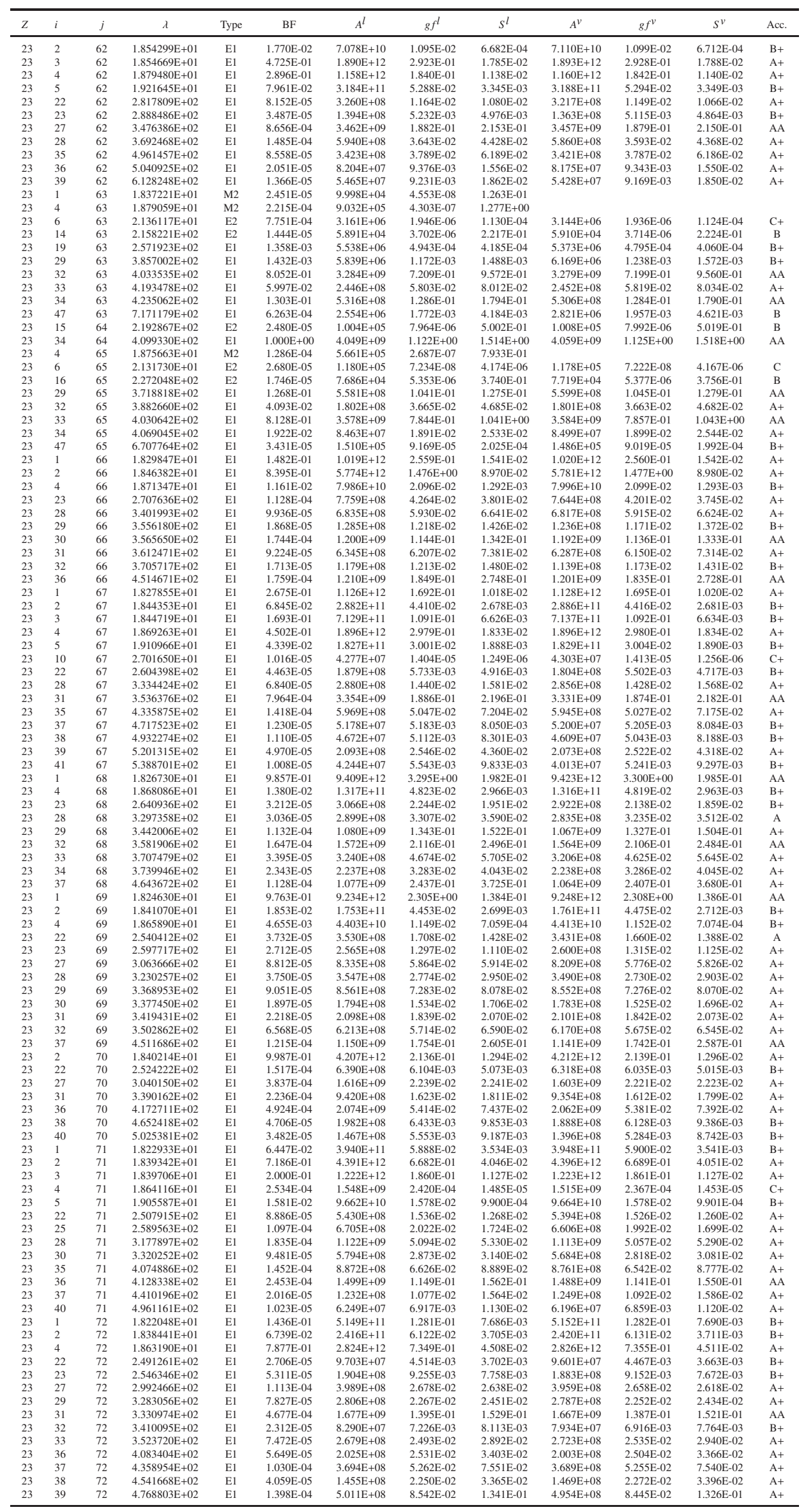




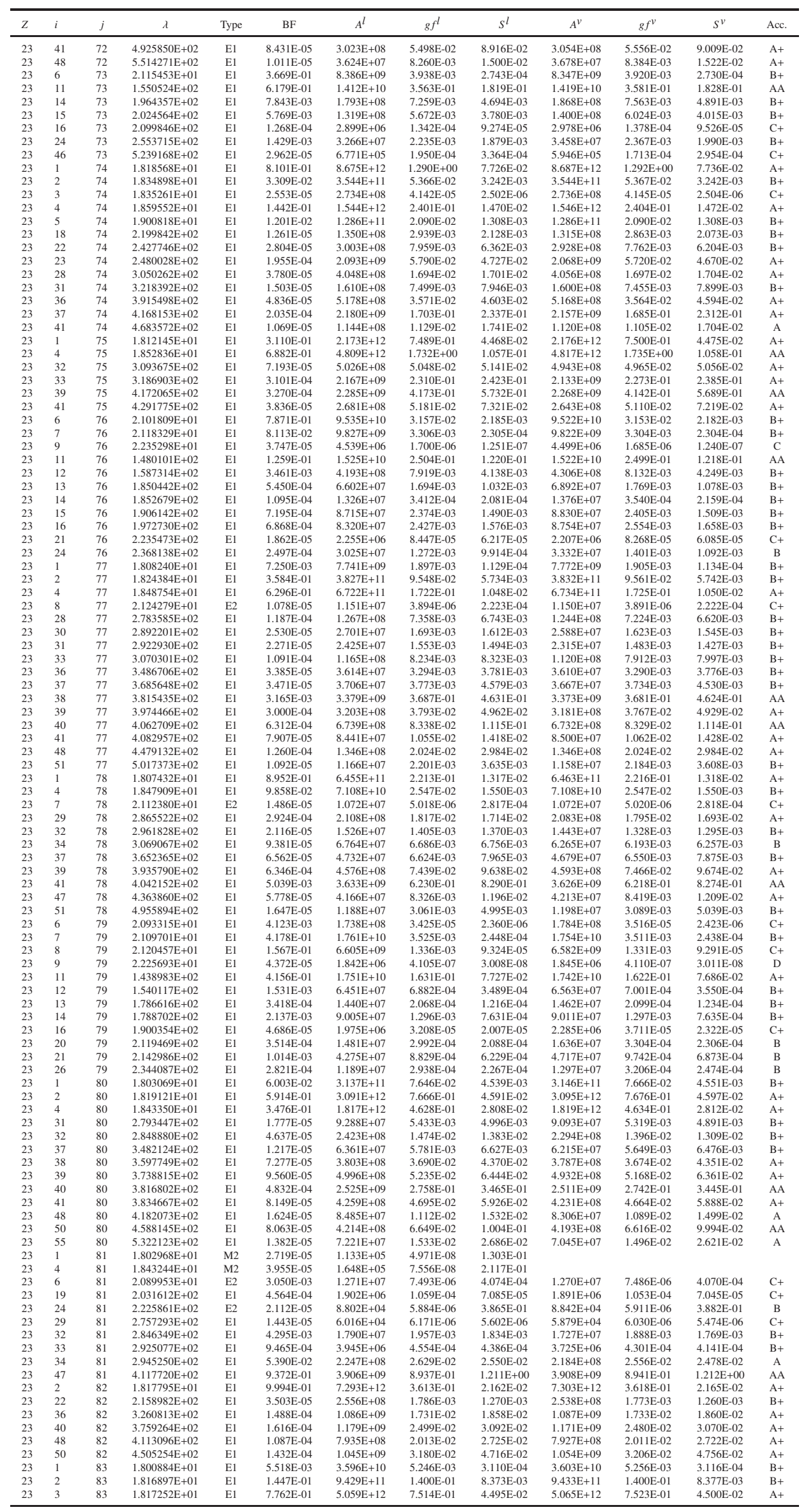




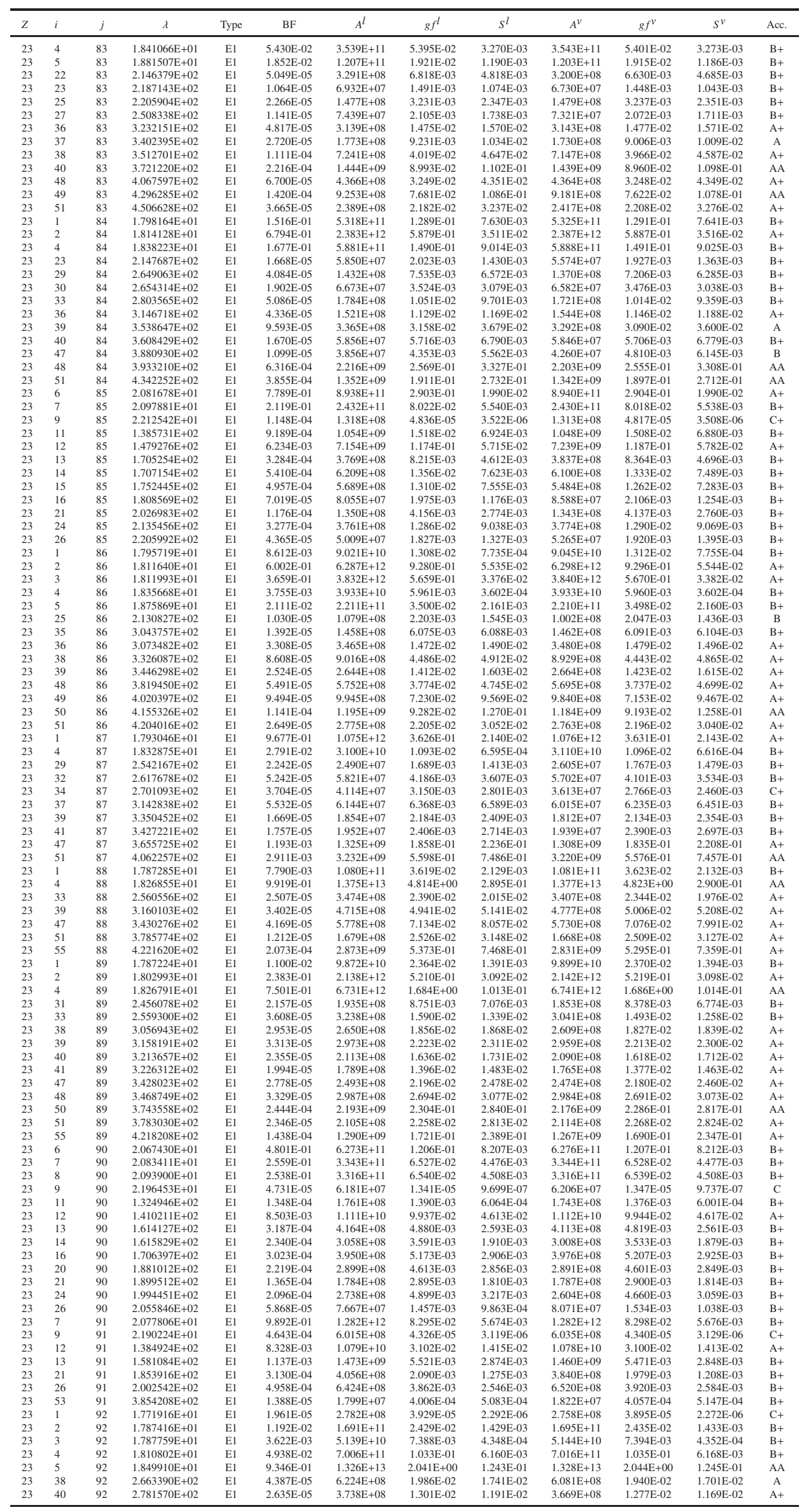




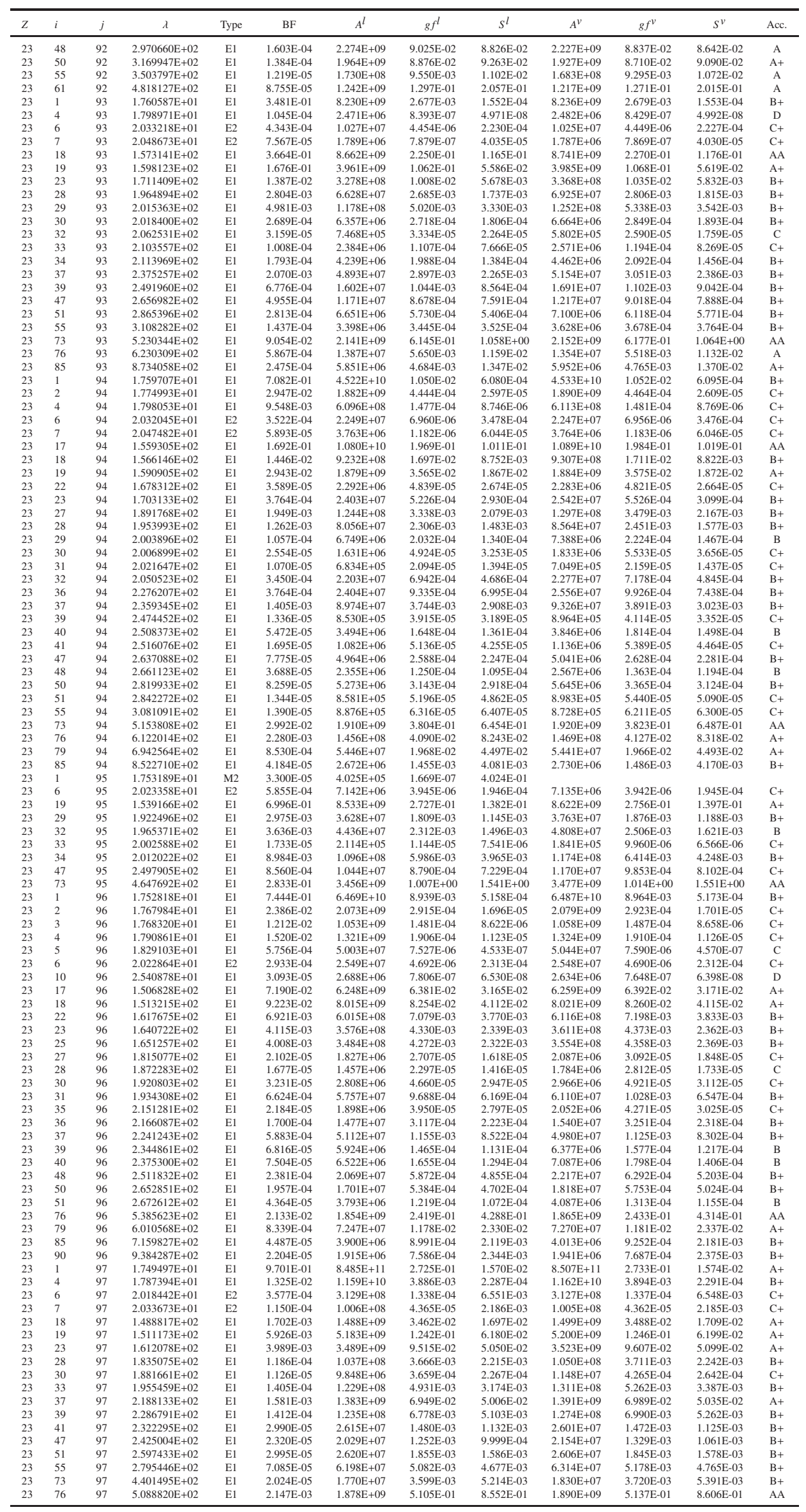




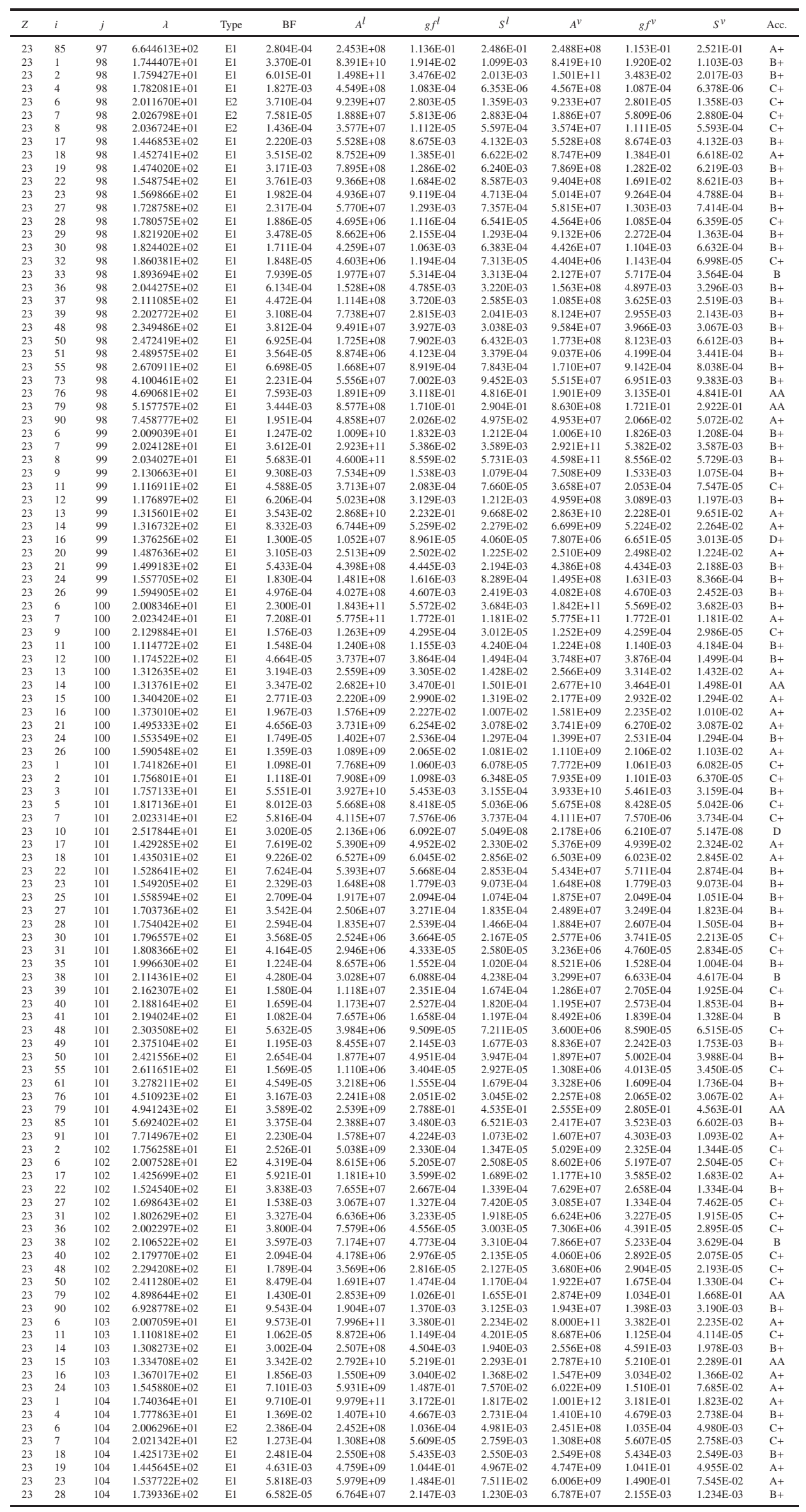




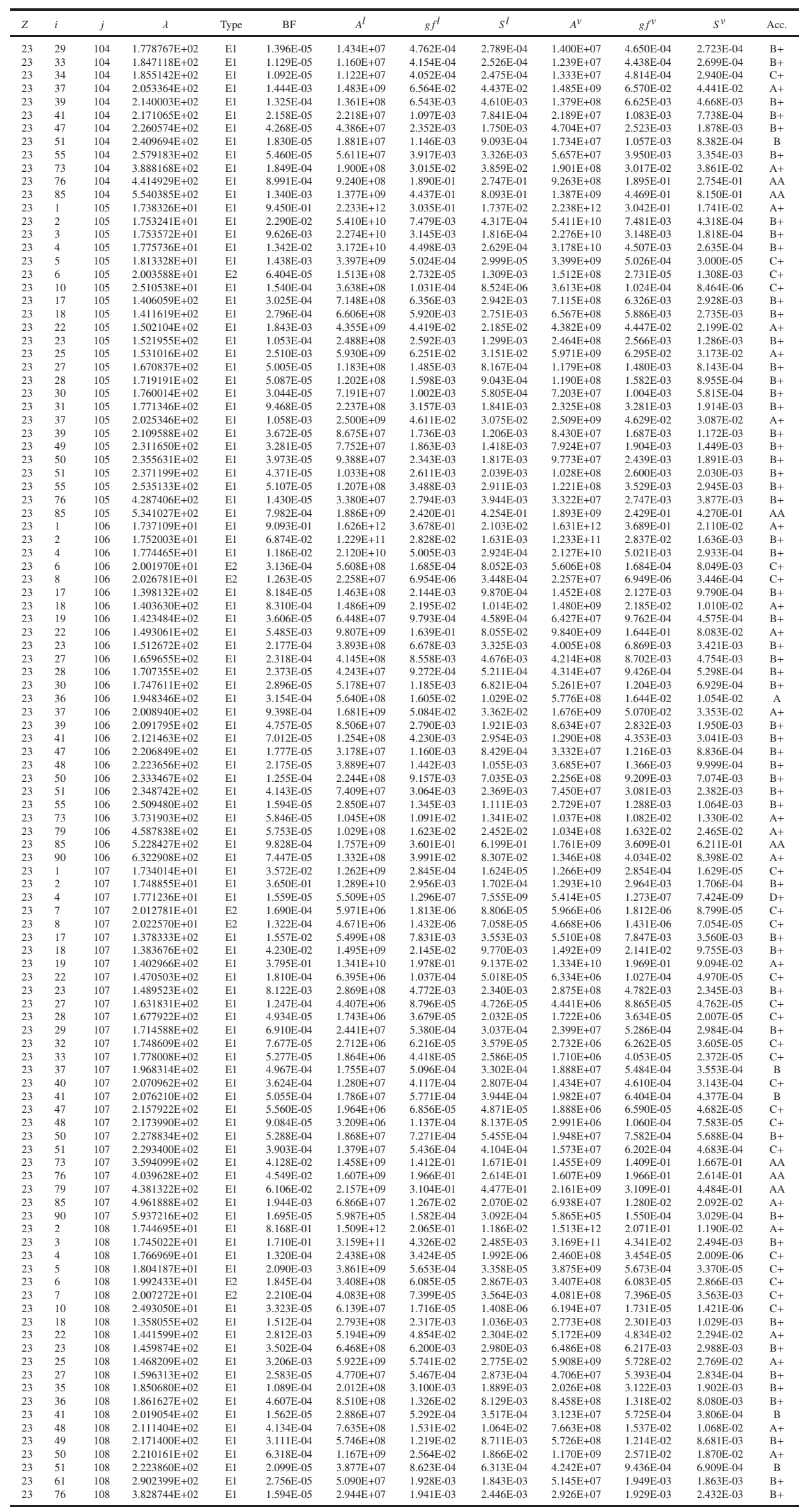

192 


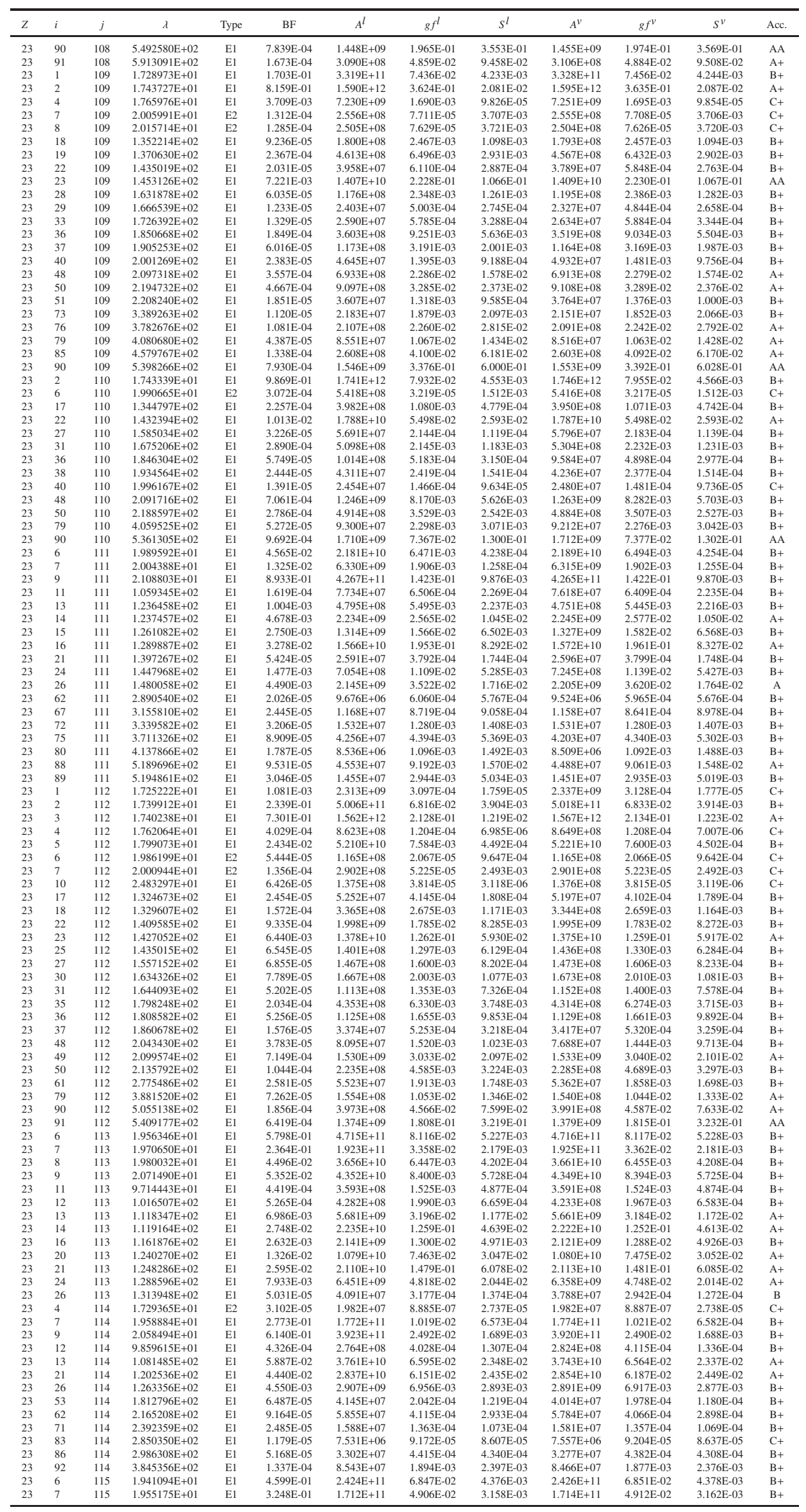




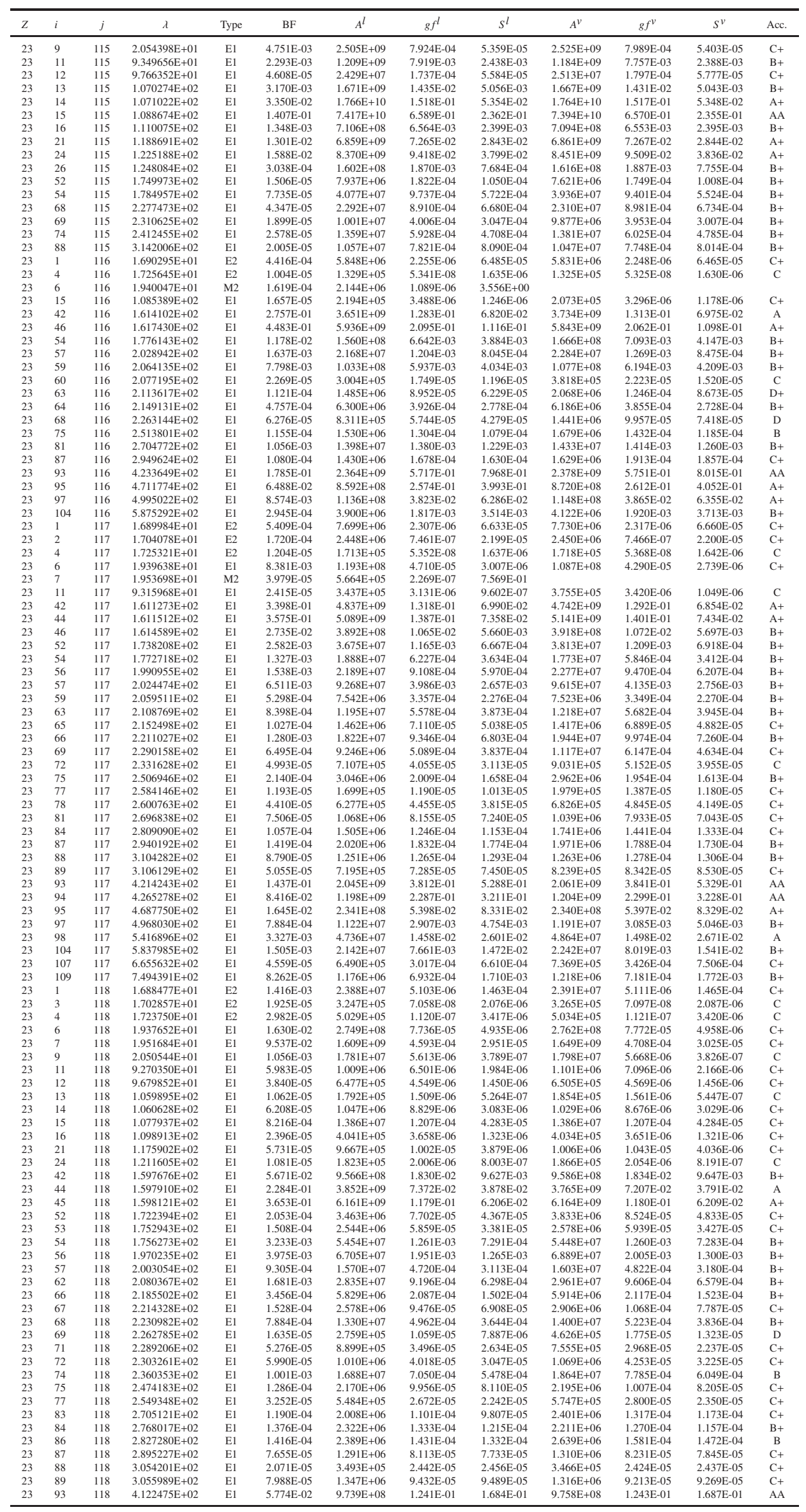




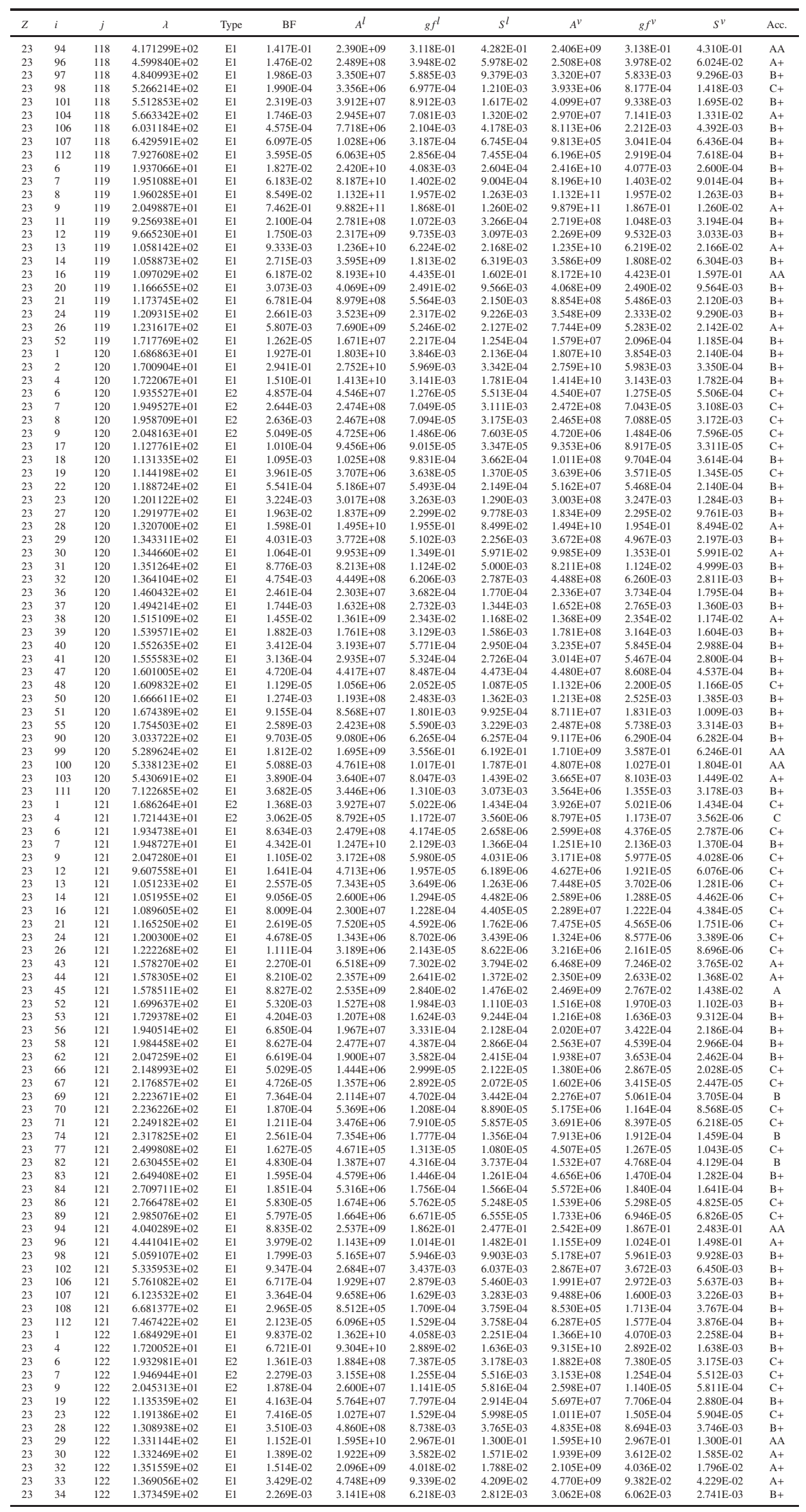




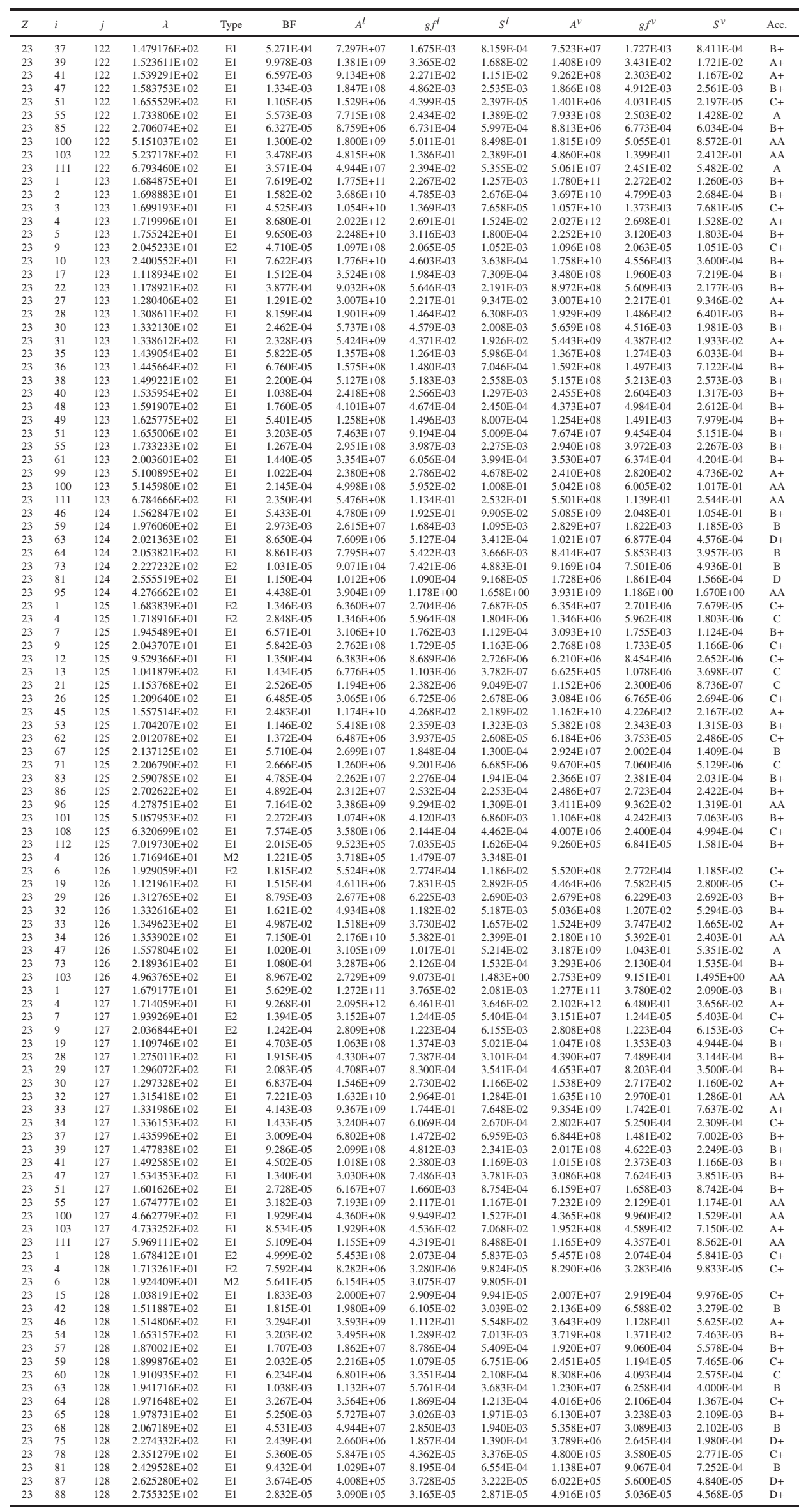




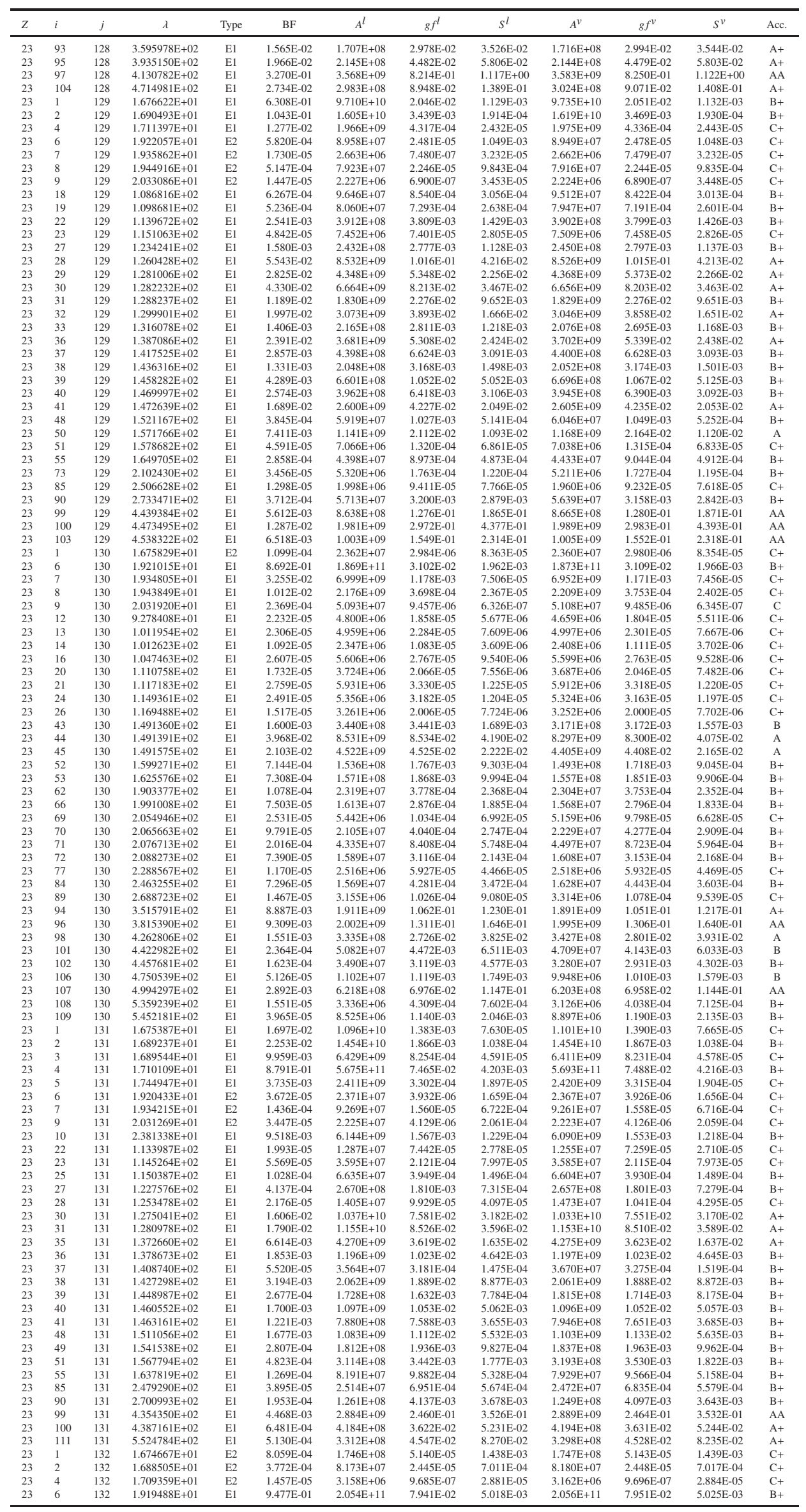




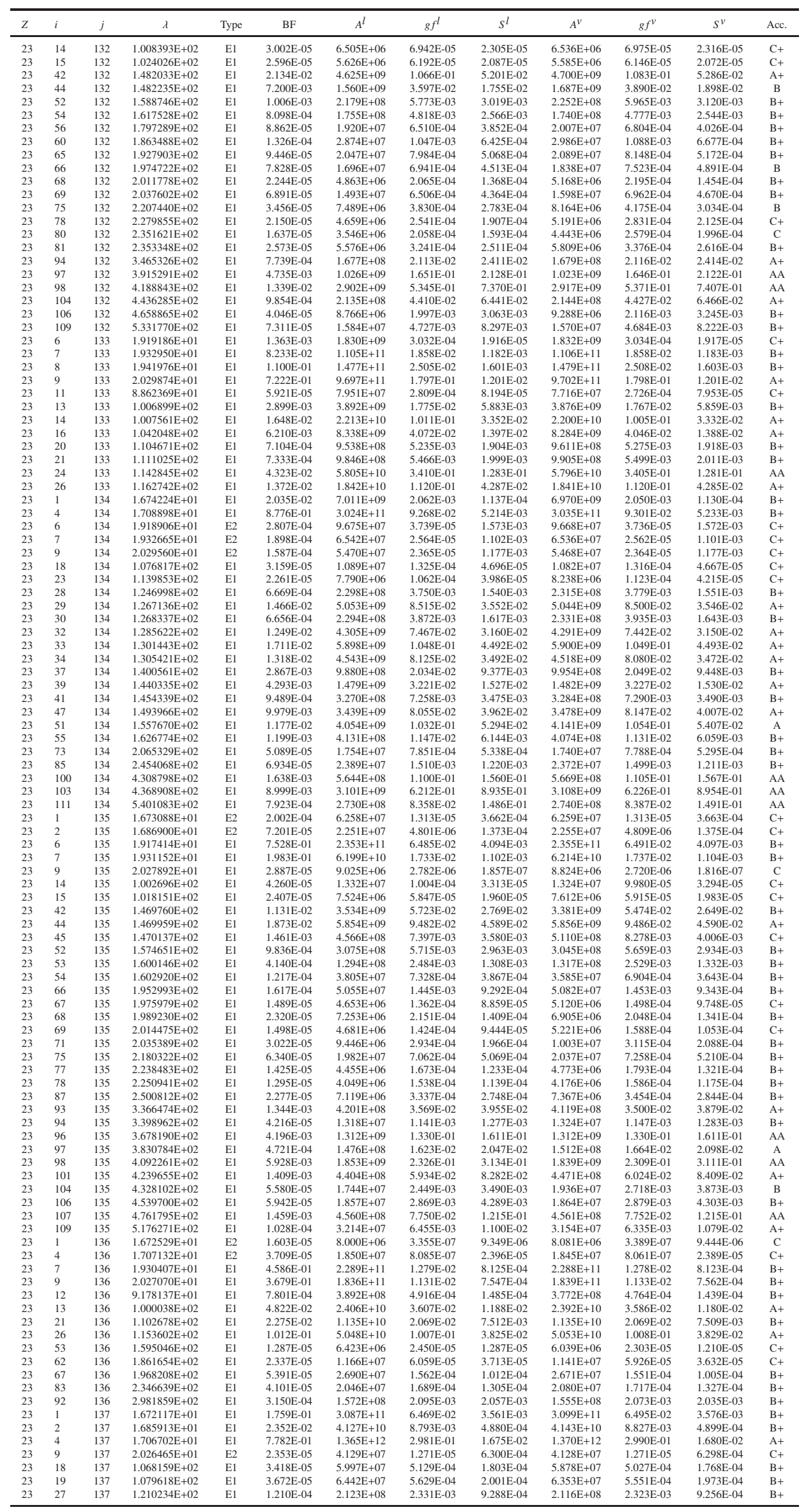




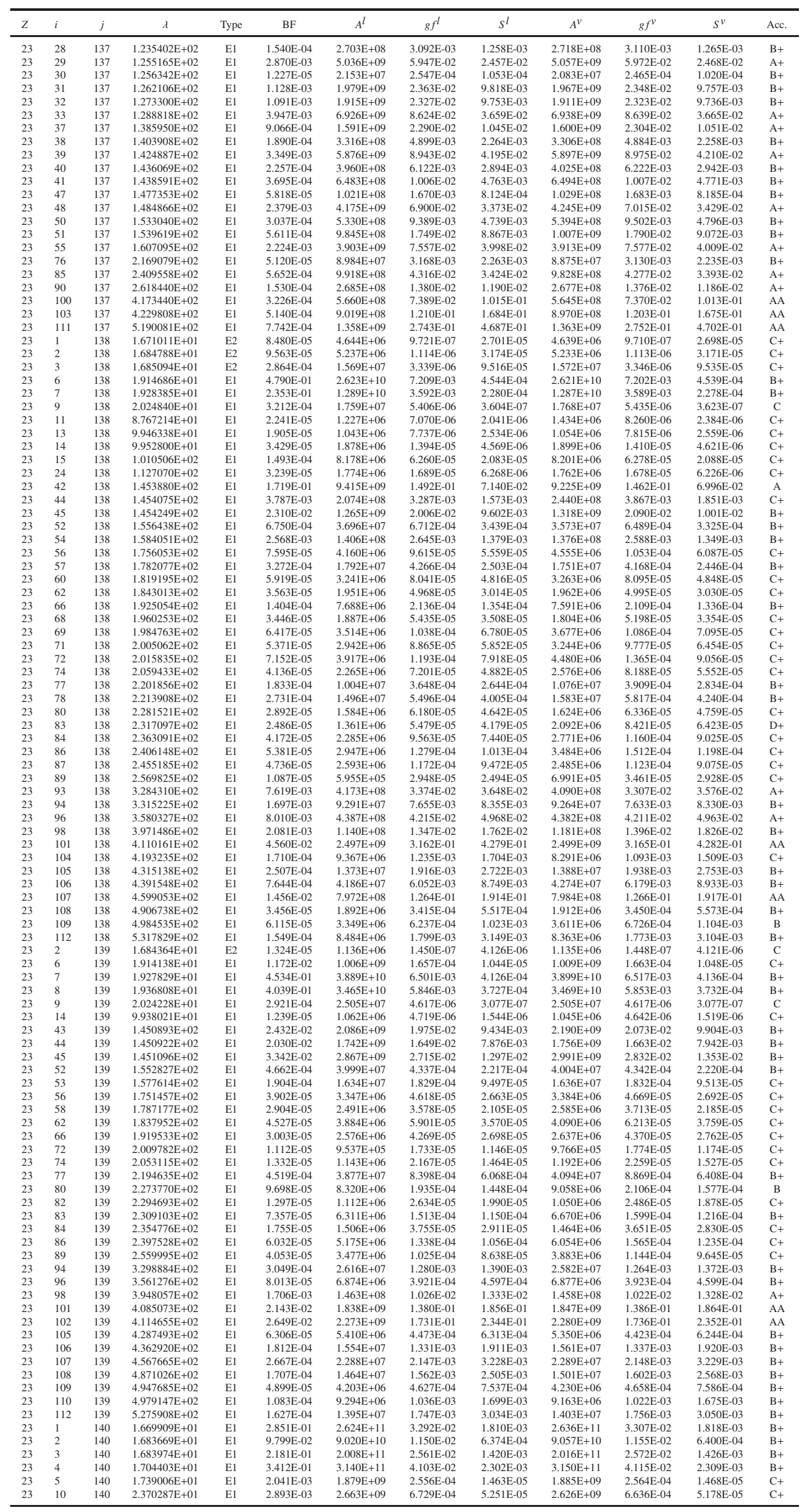




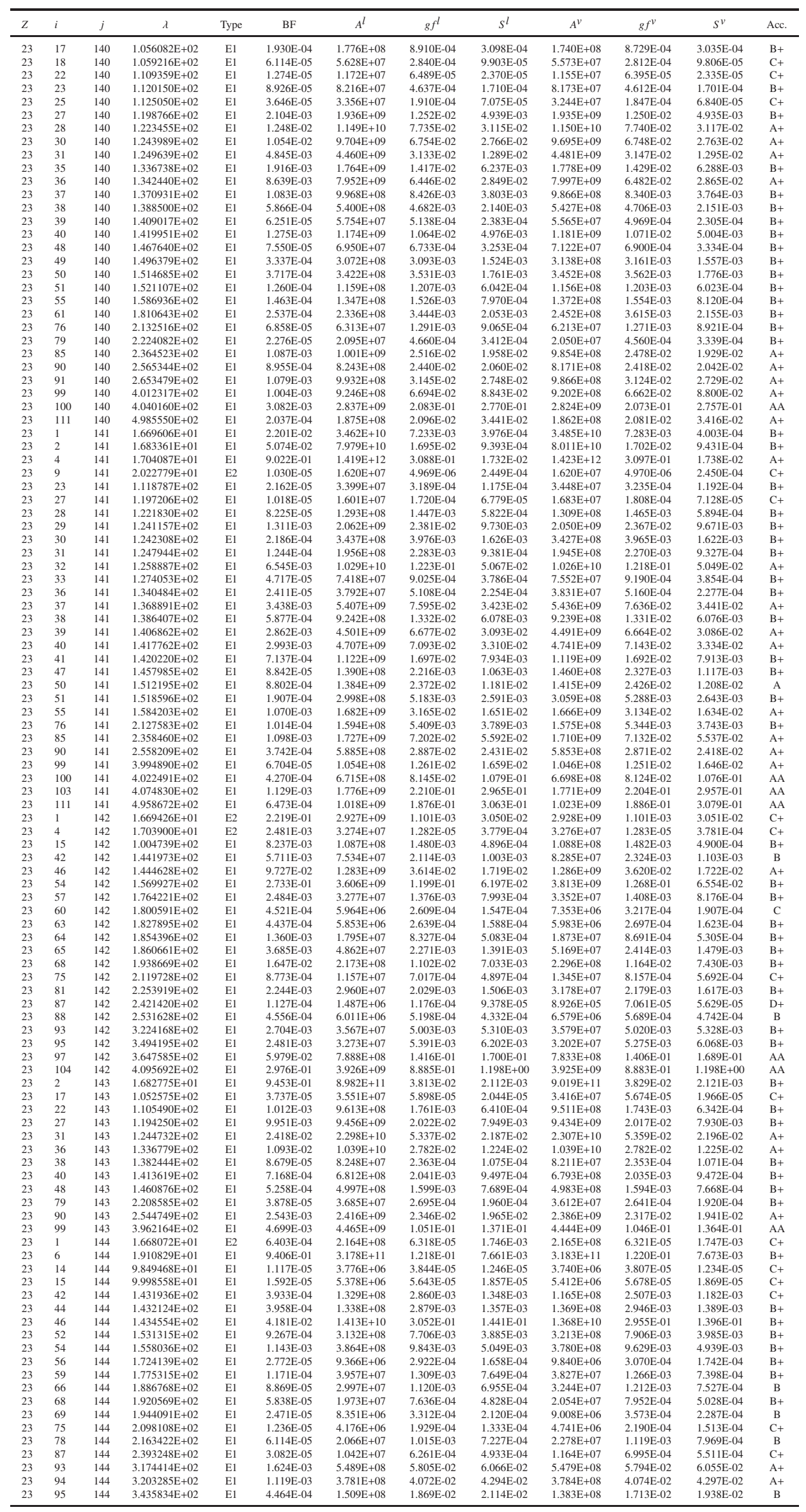




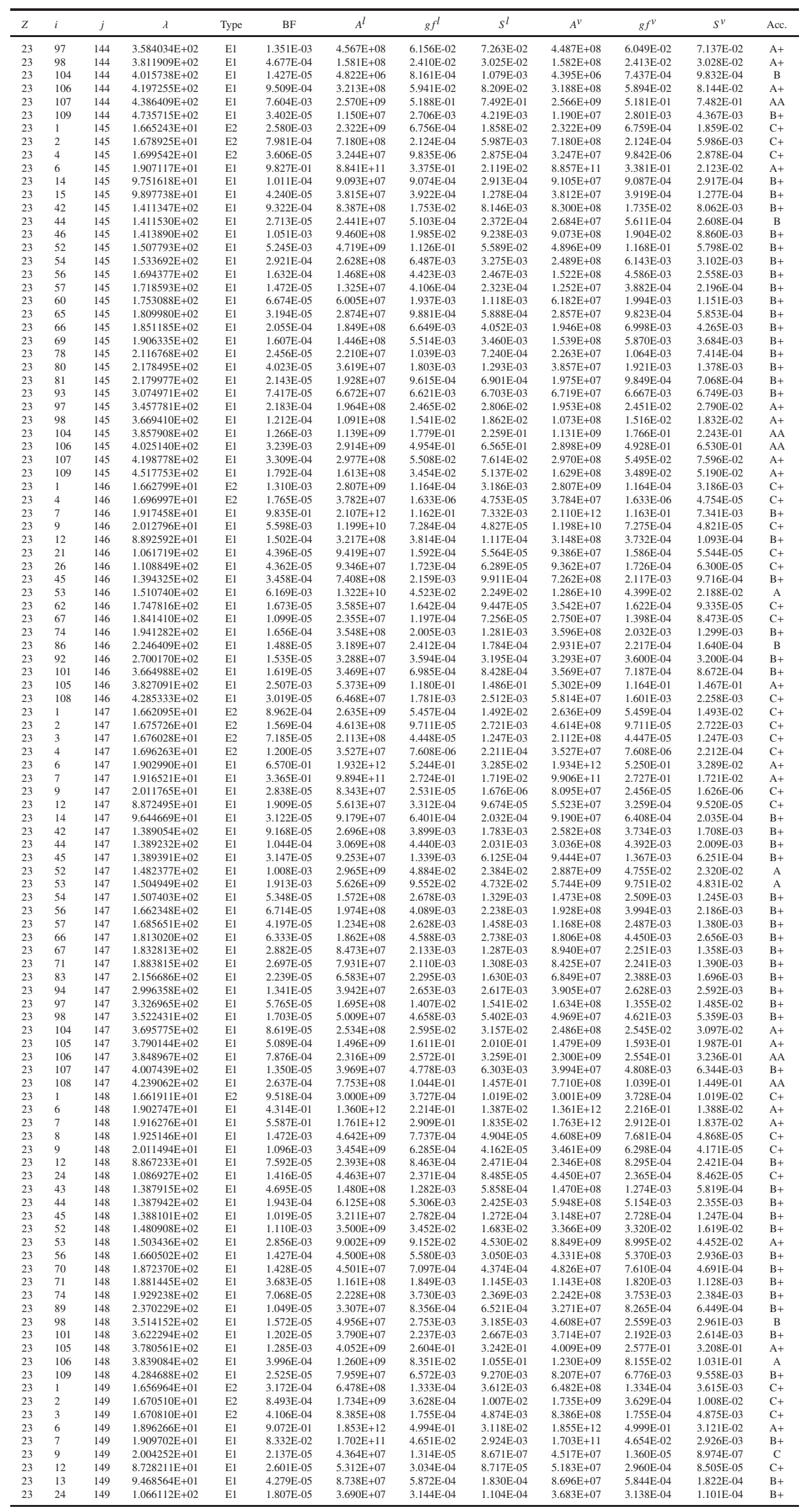




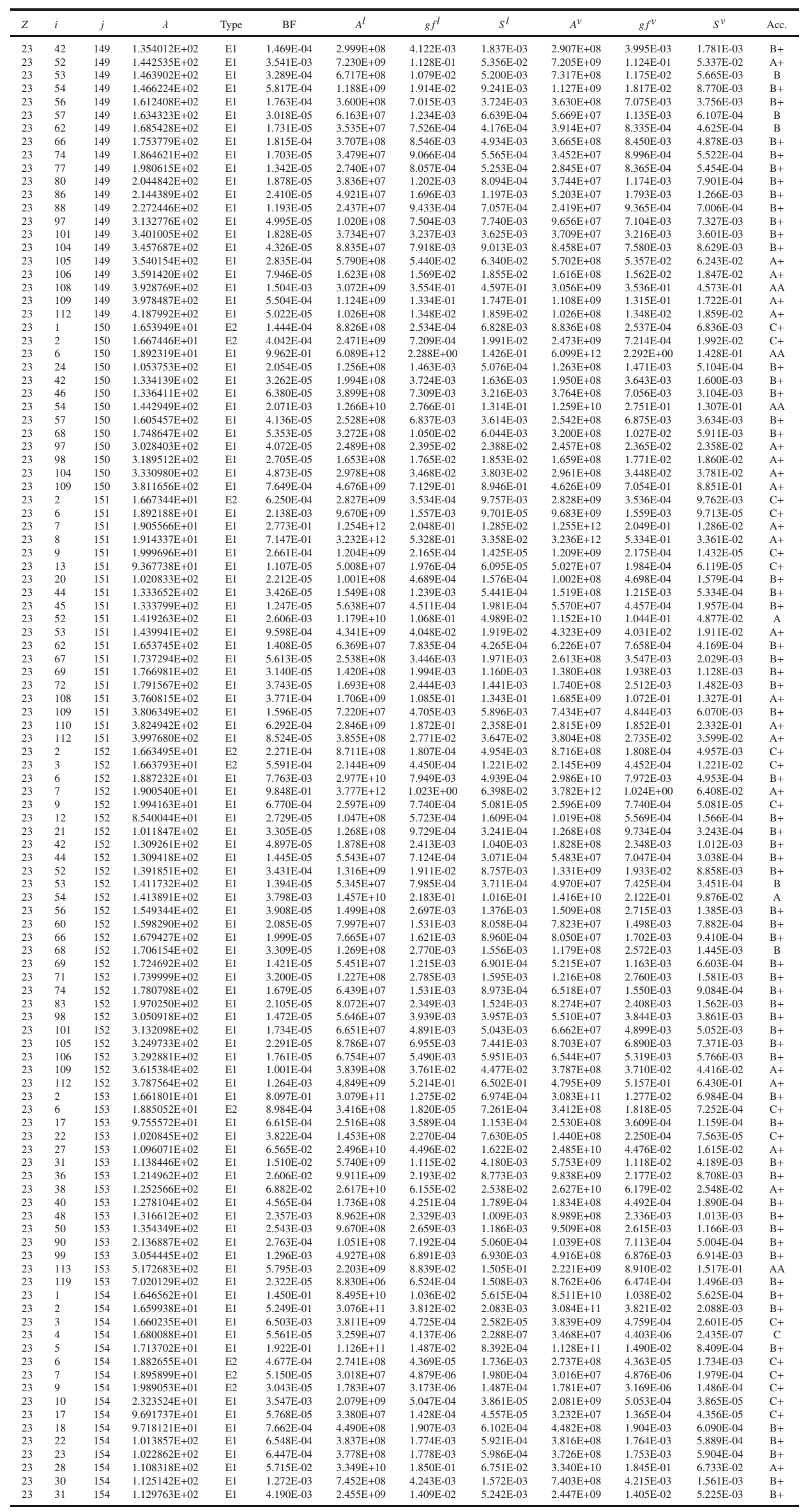




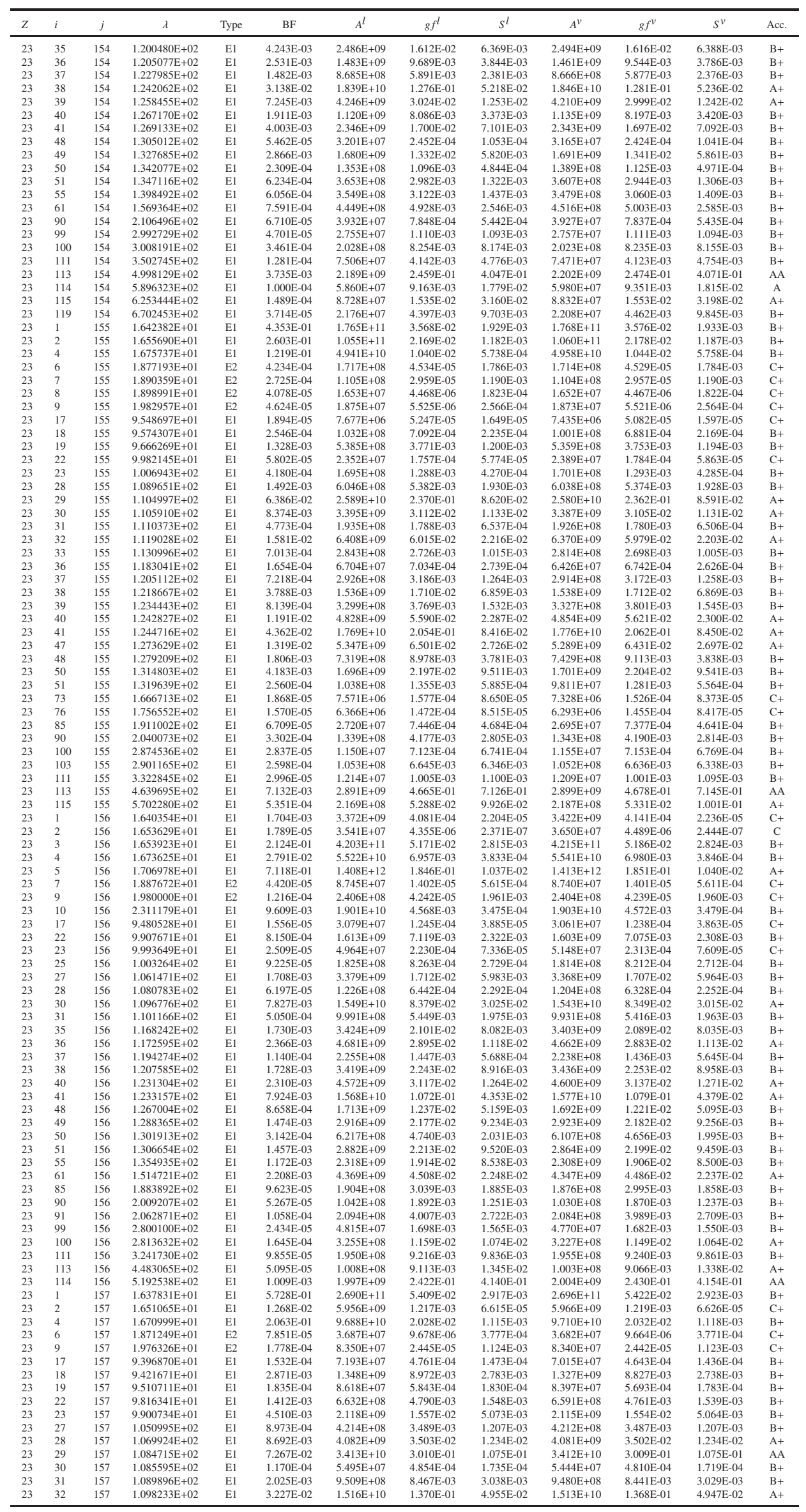




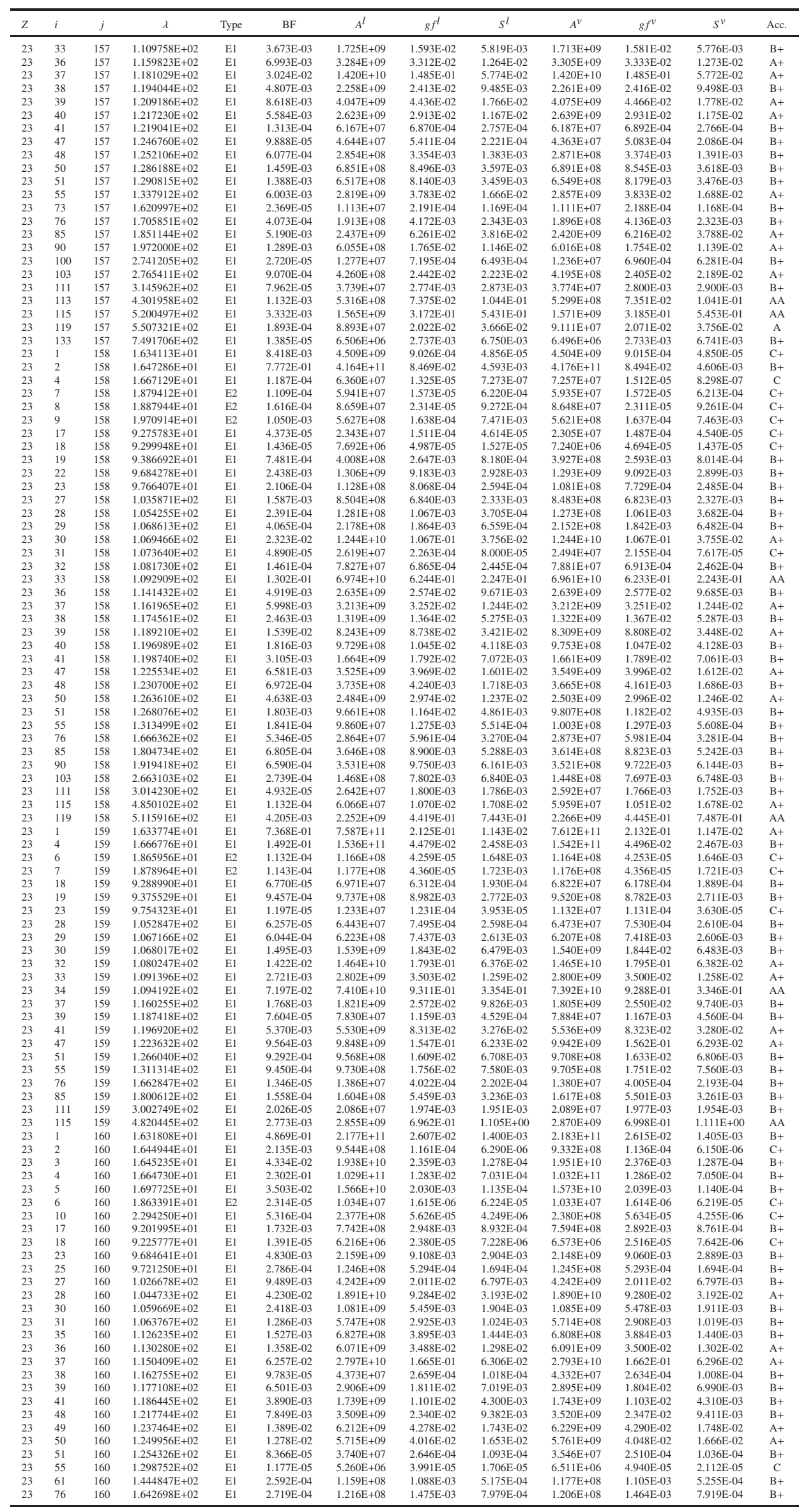




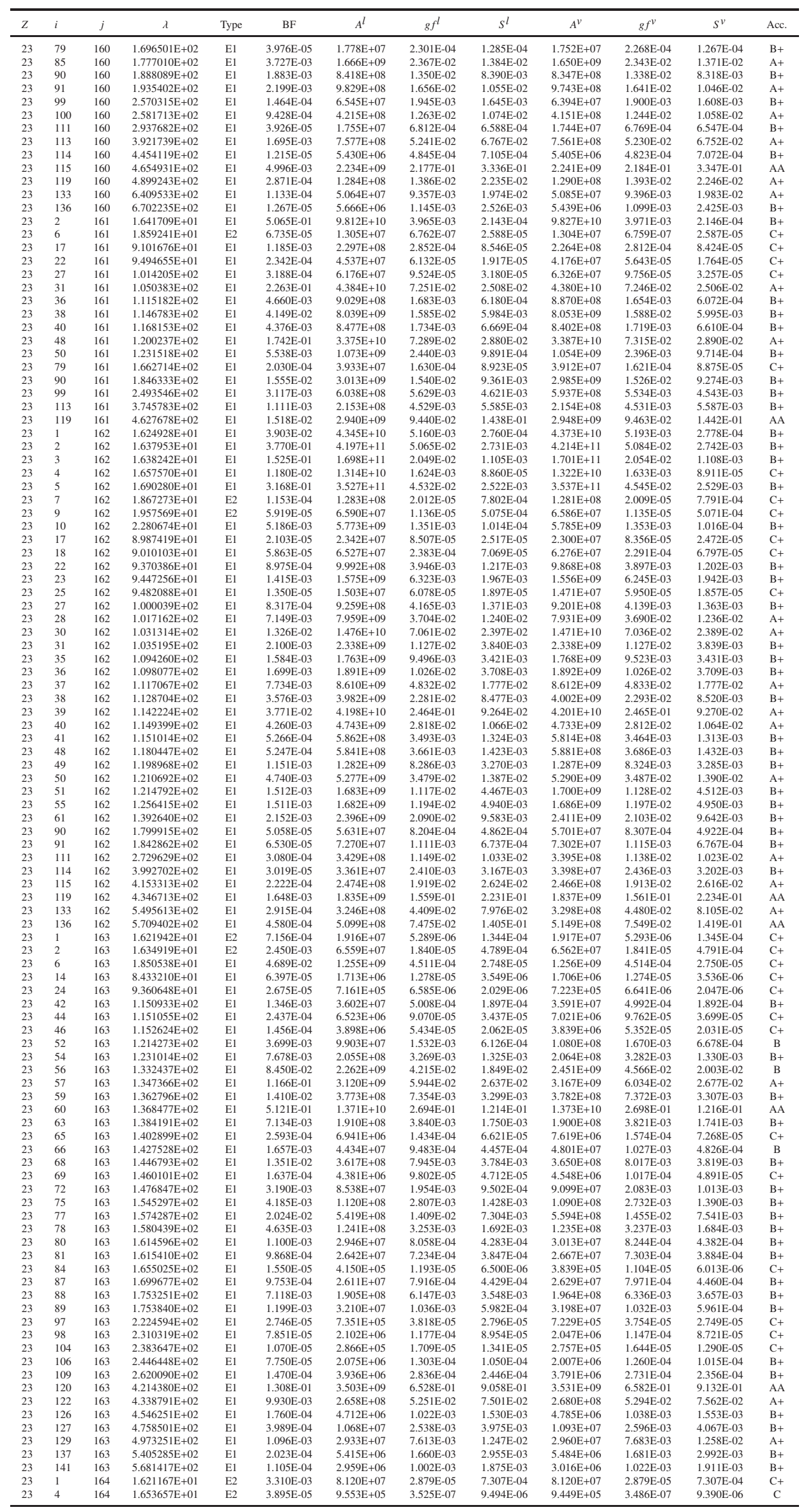




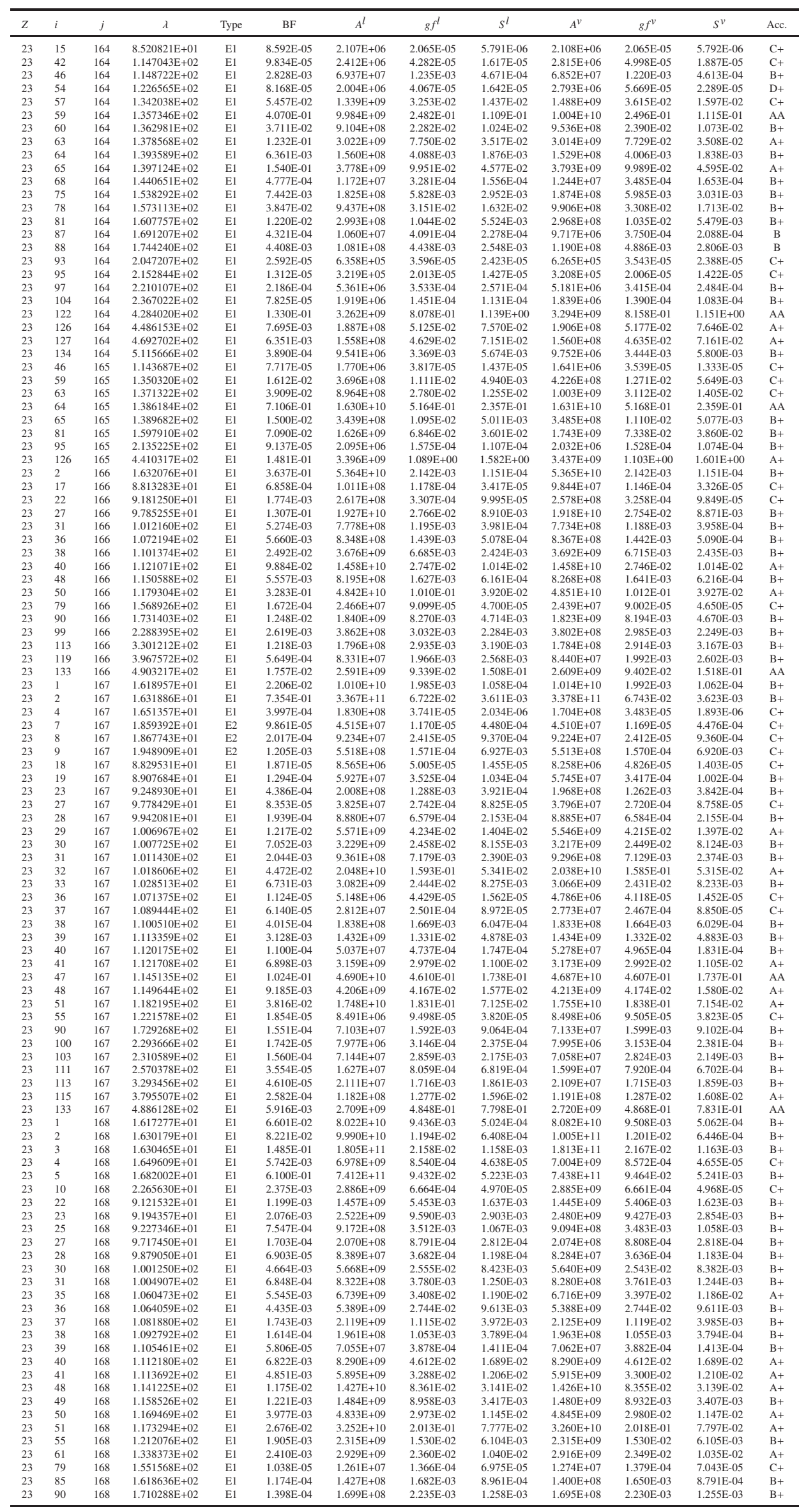




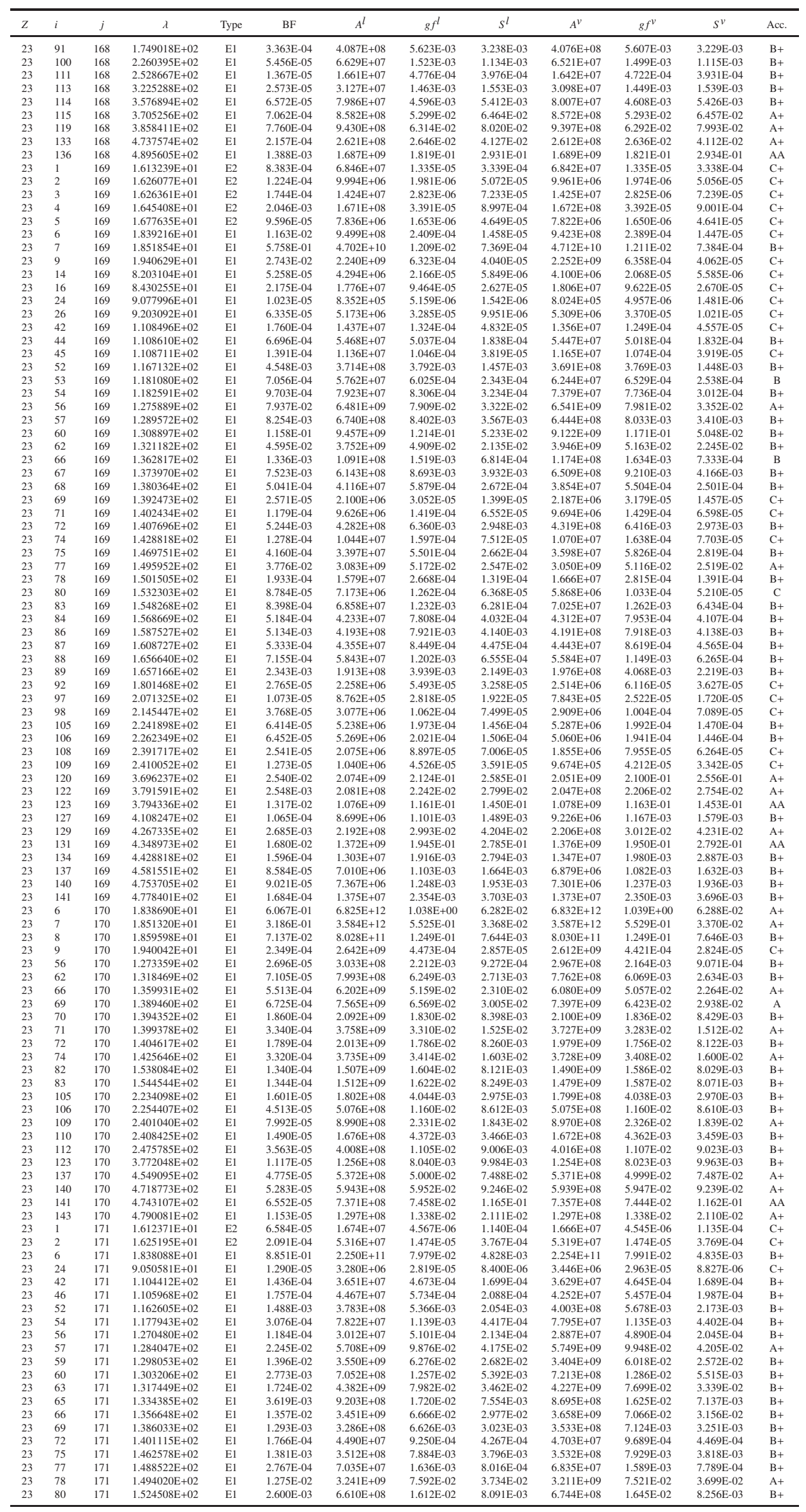




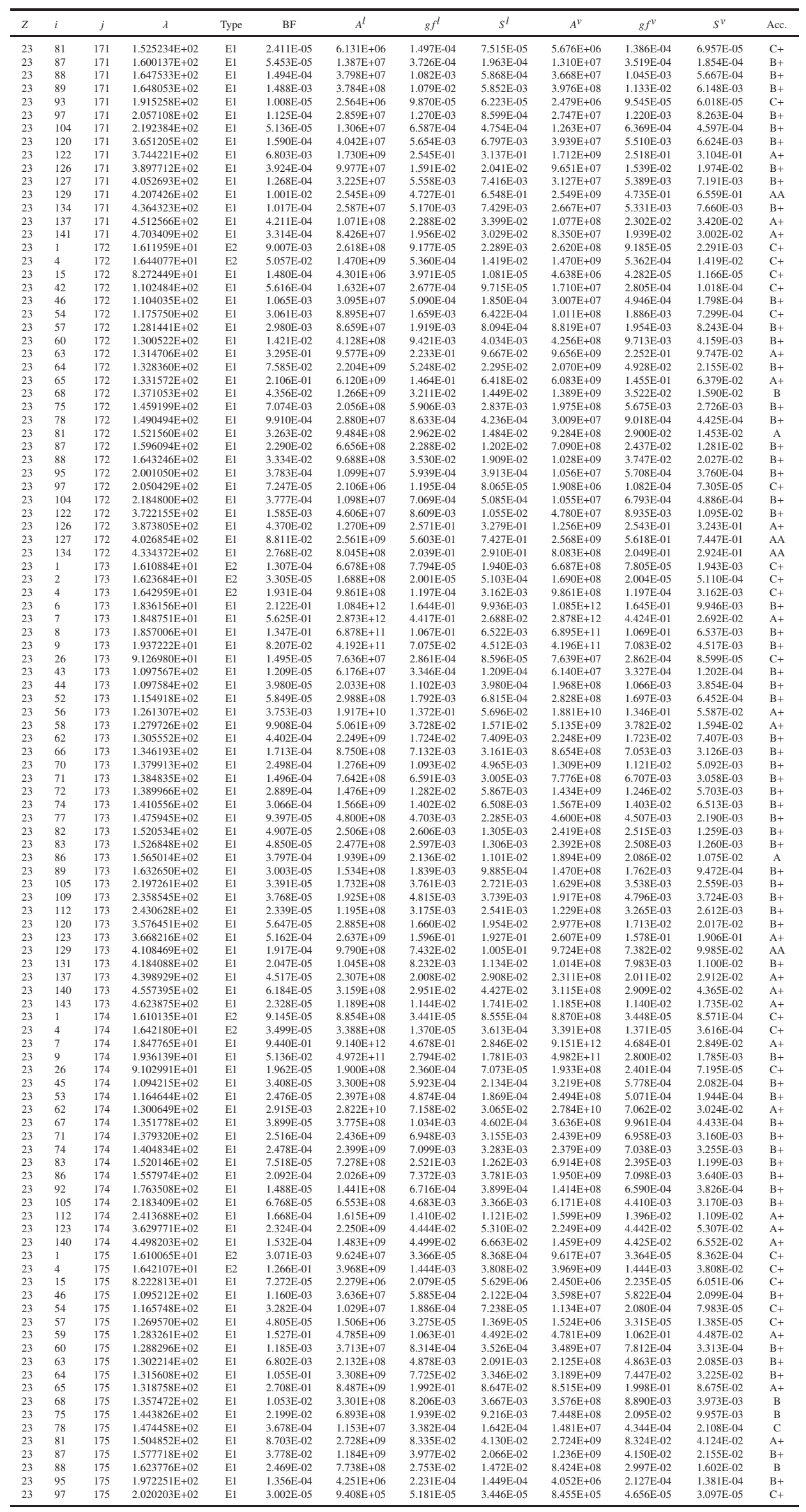




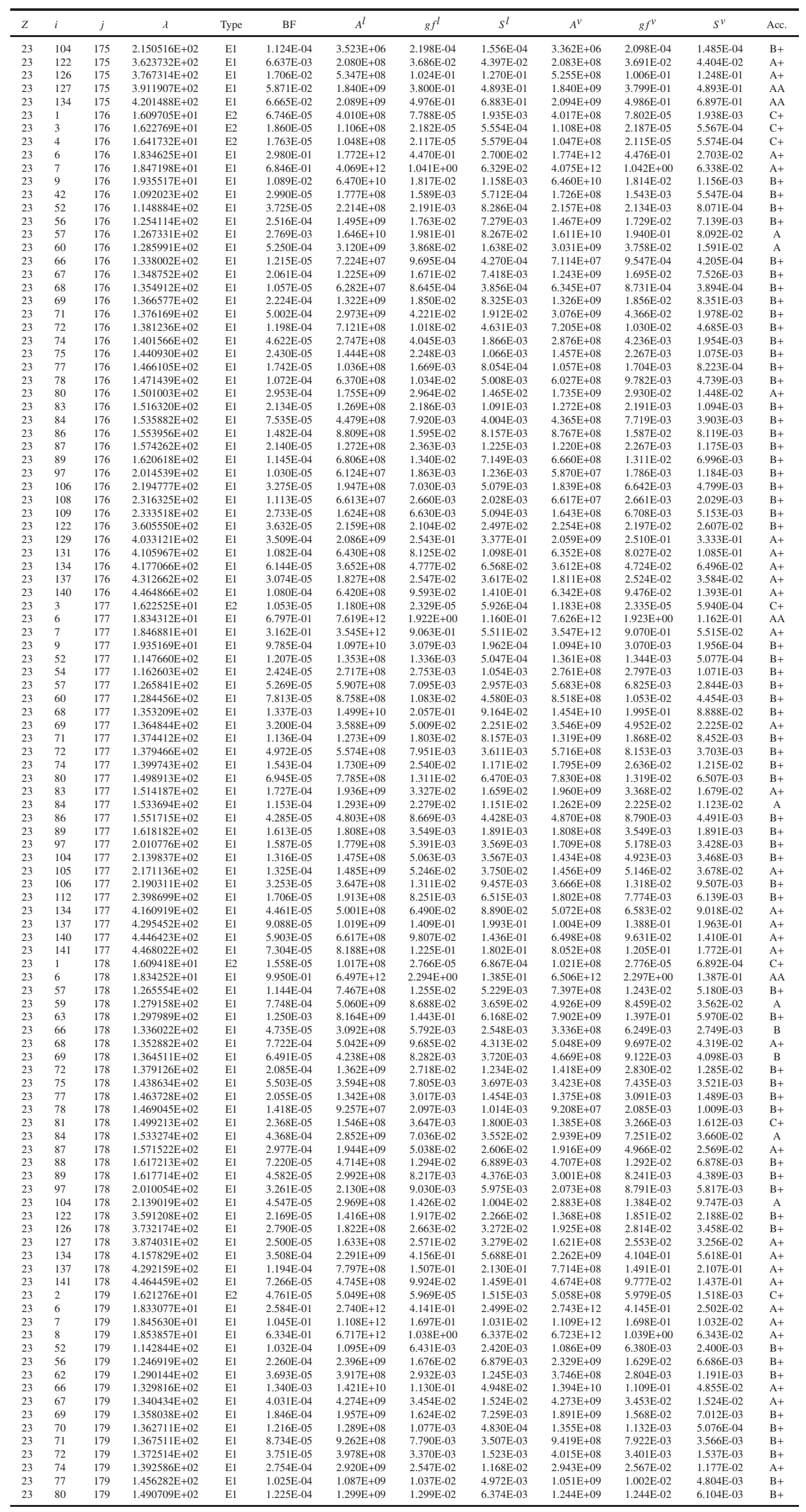




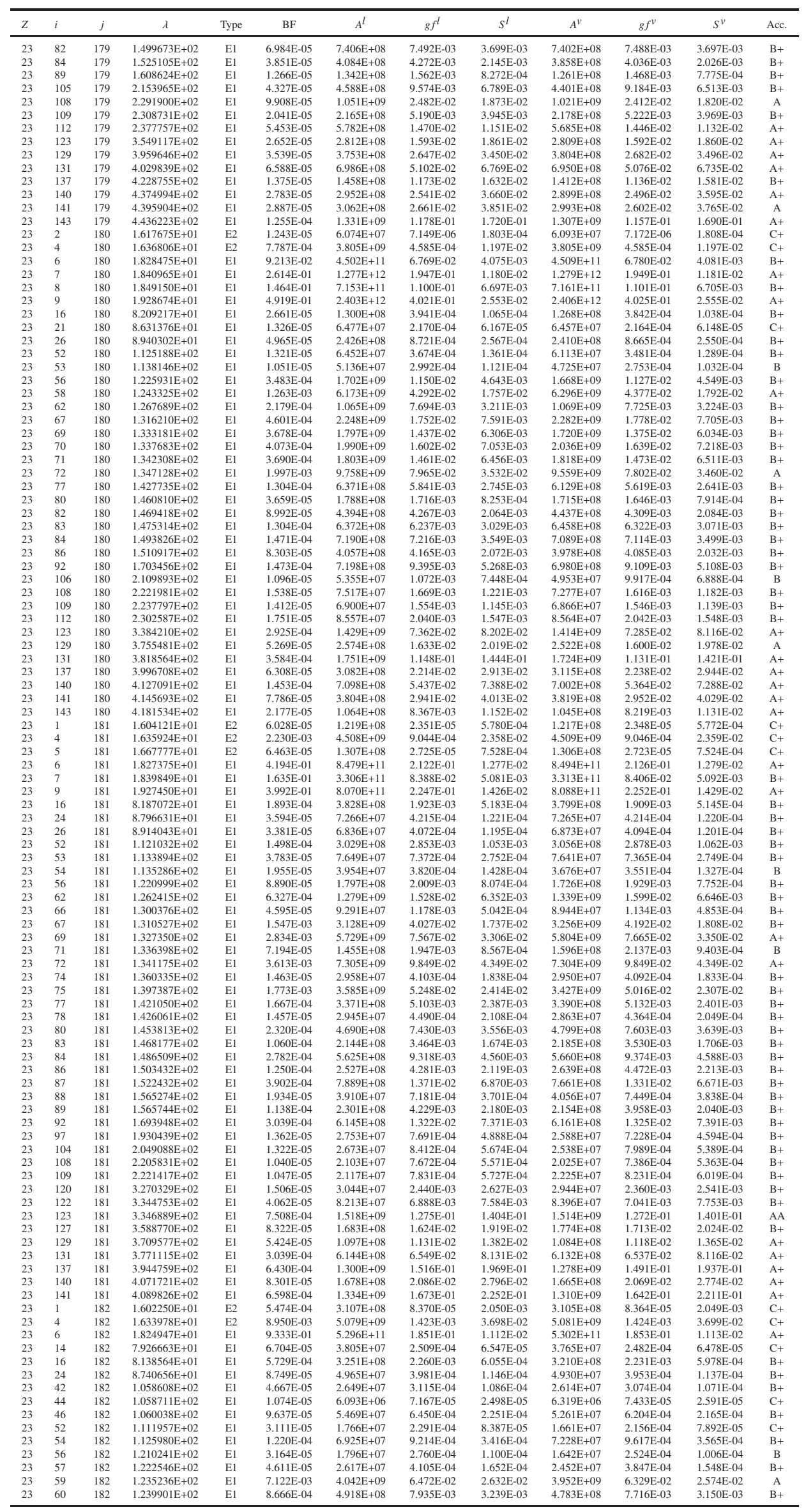




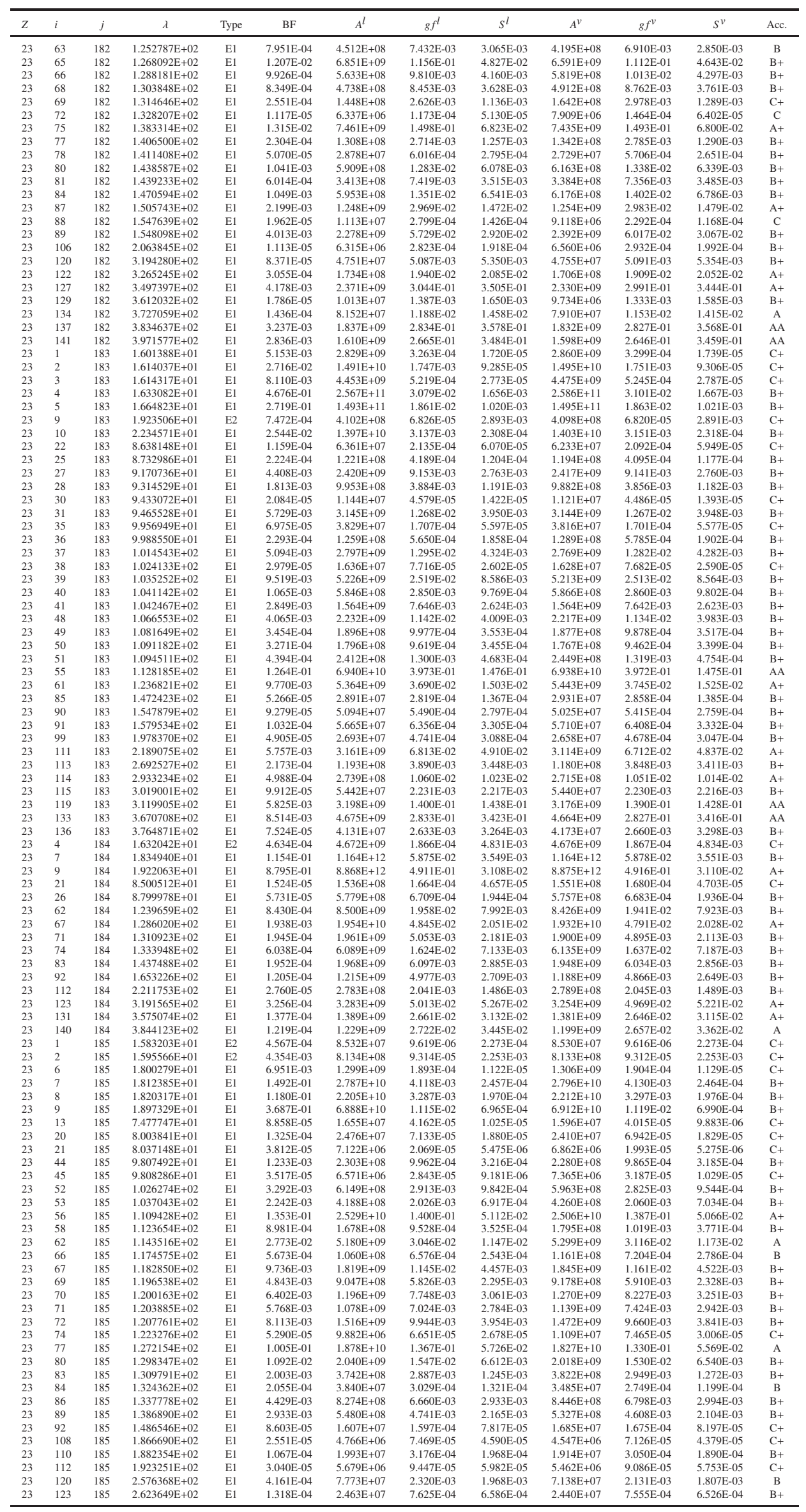




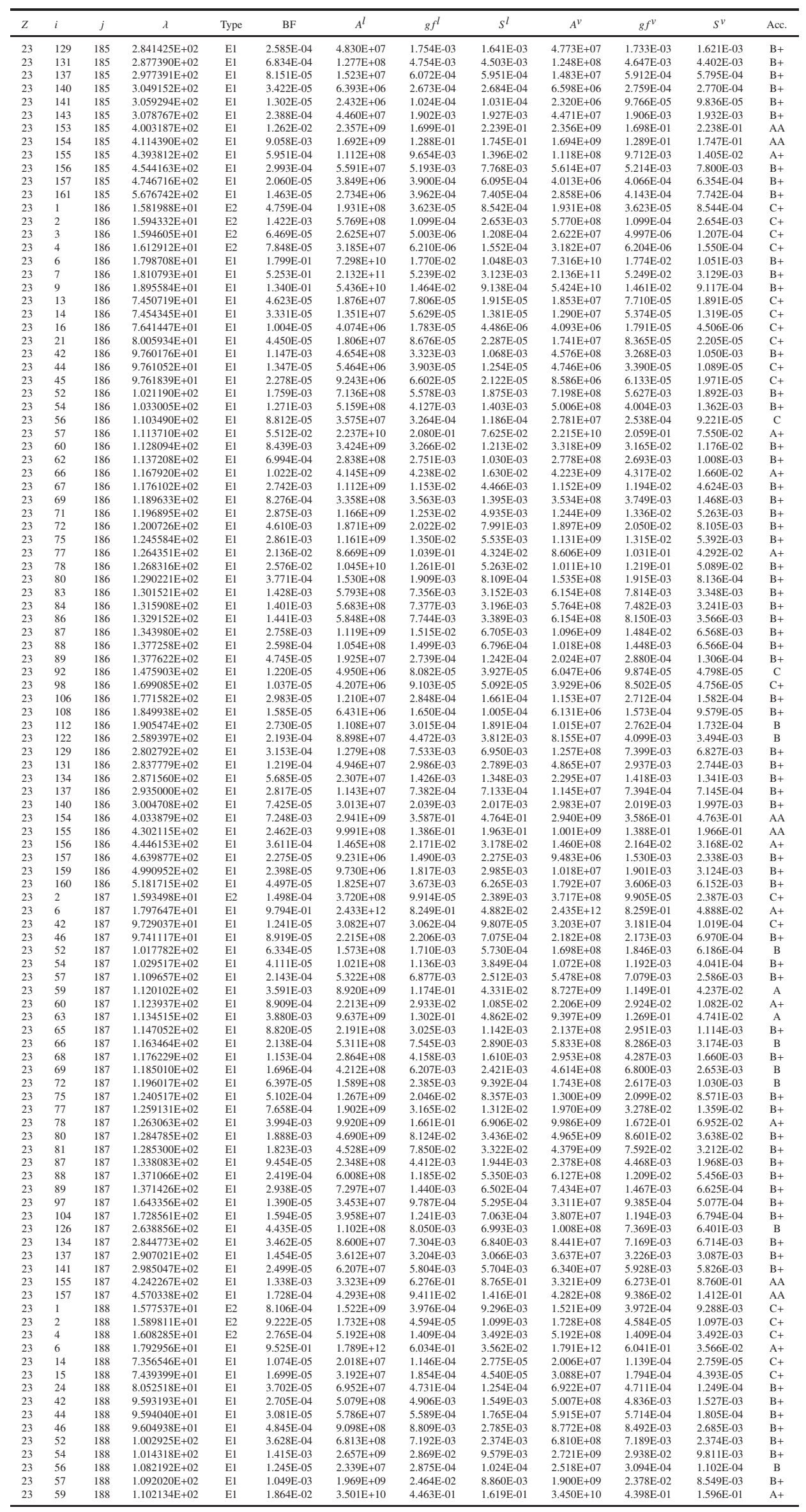




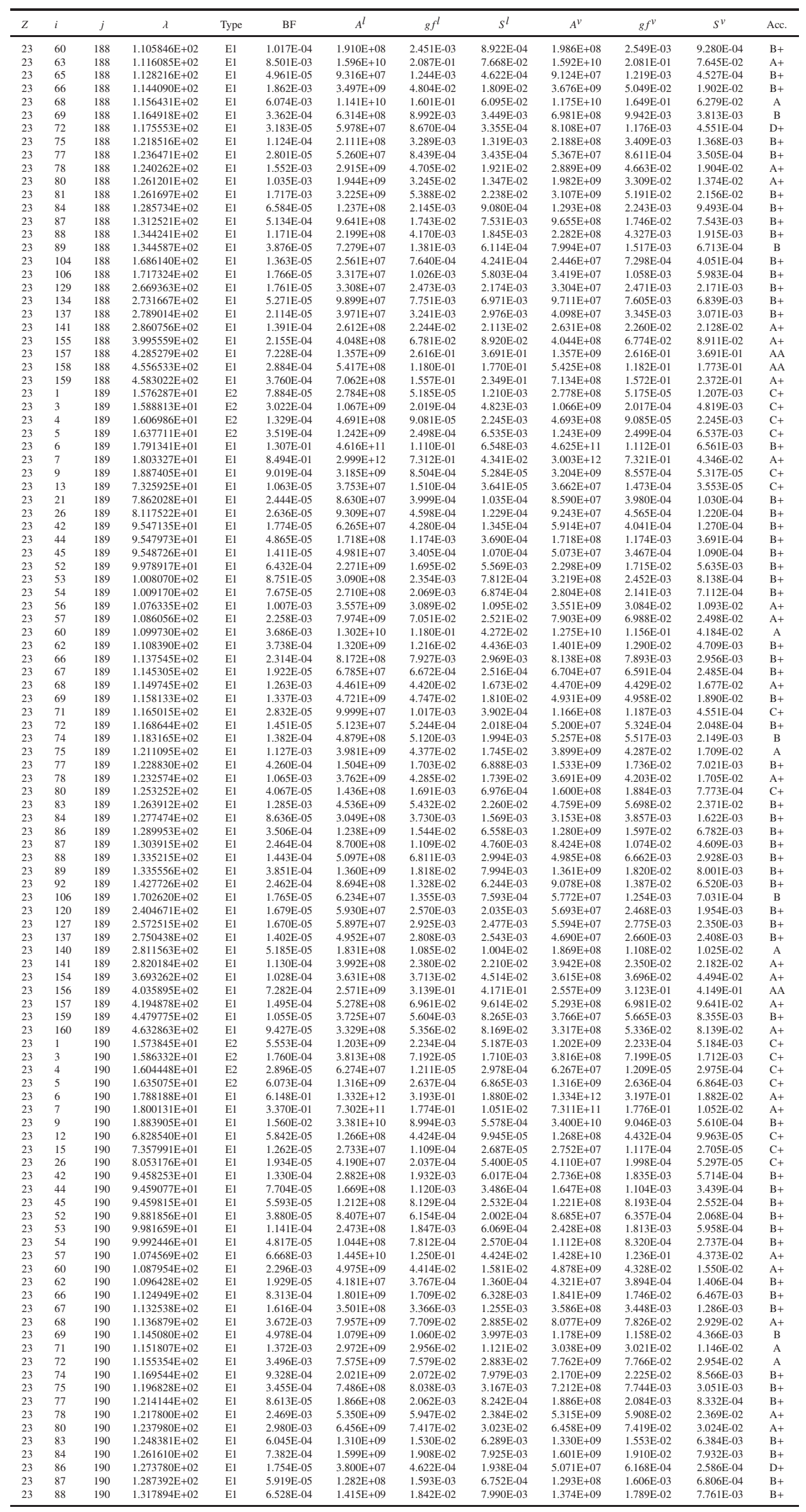




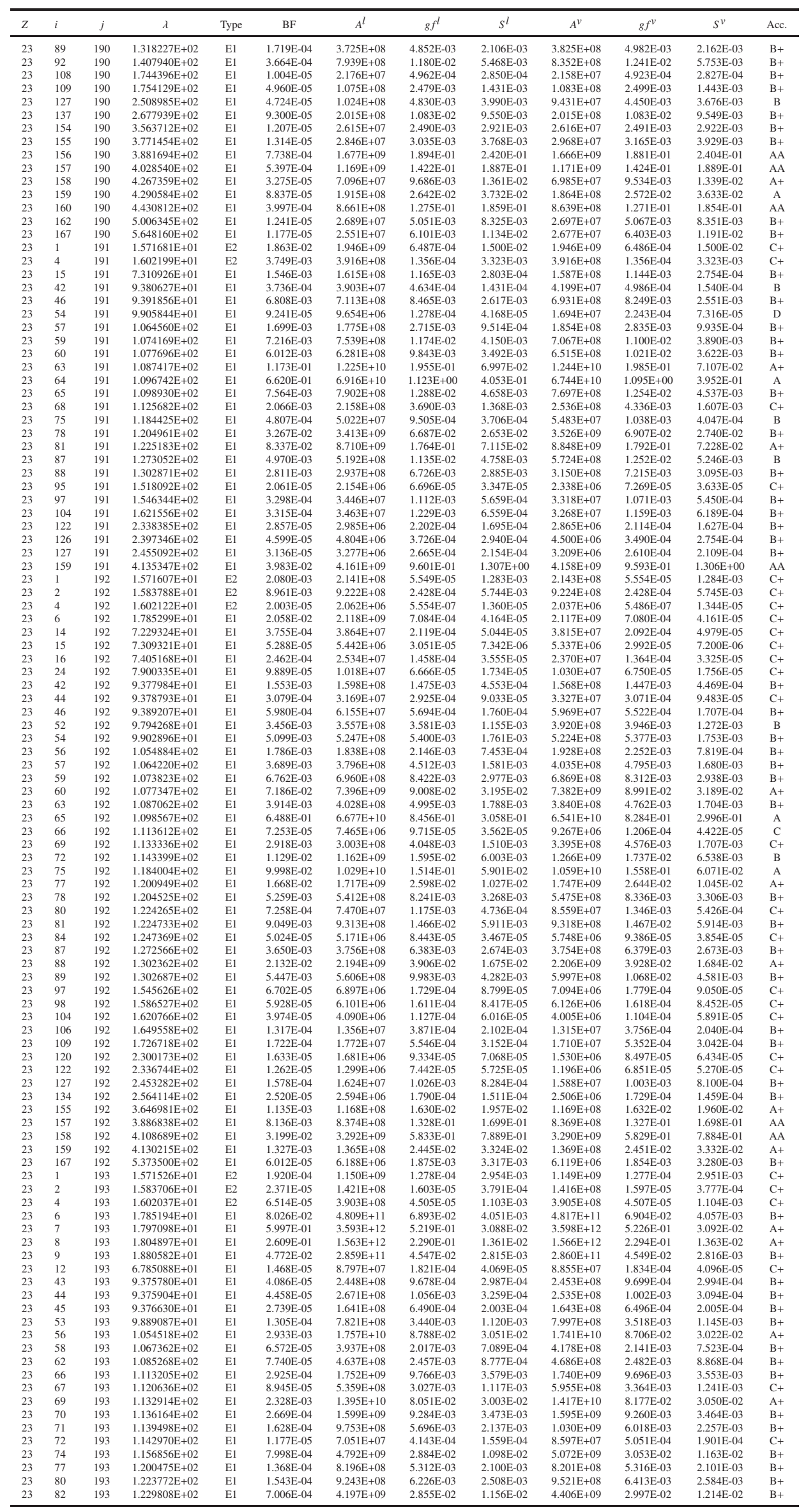




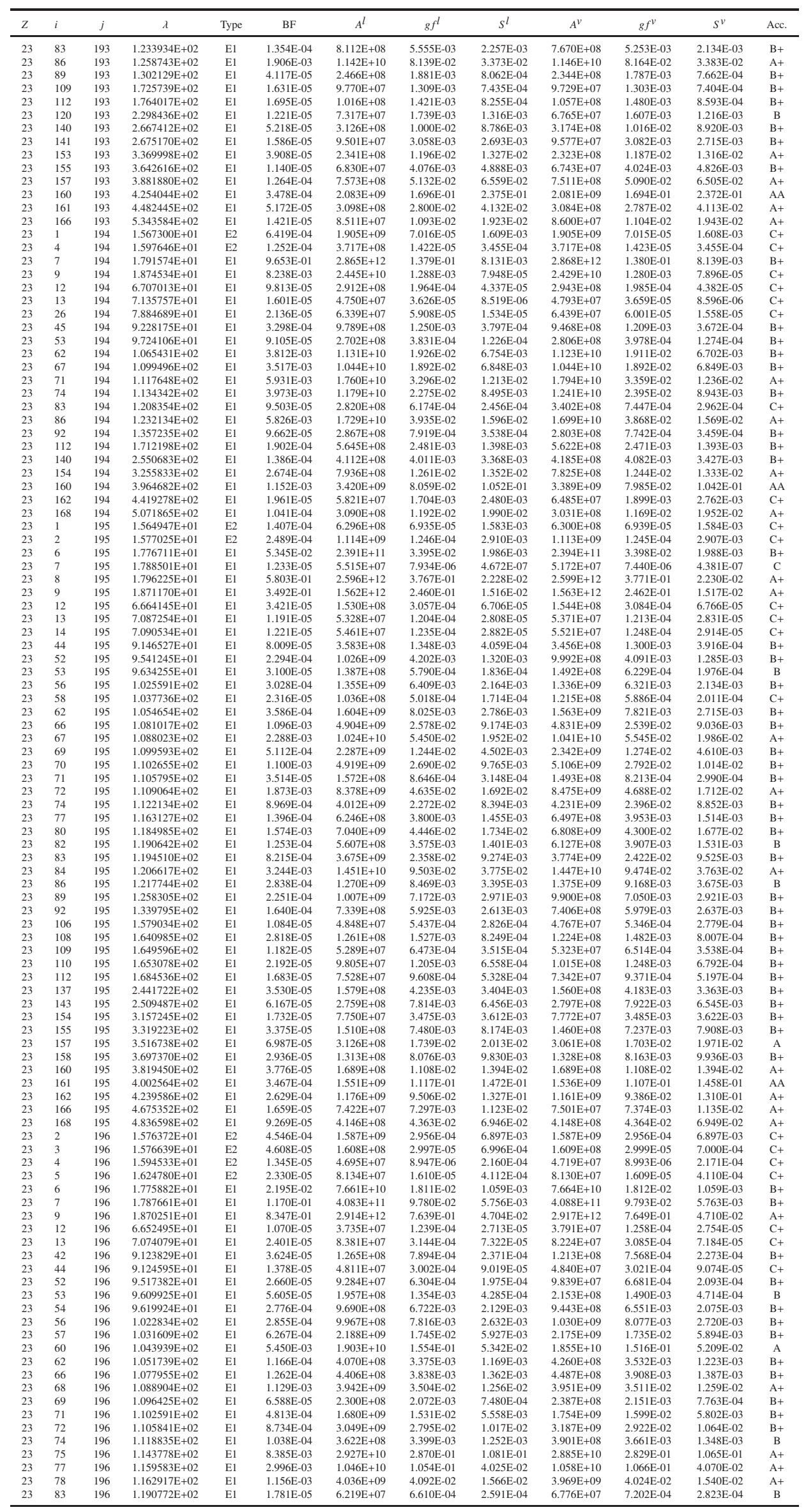




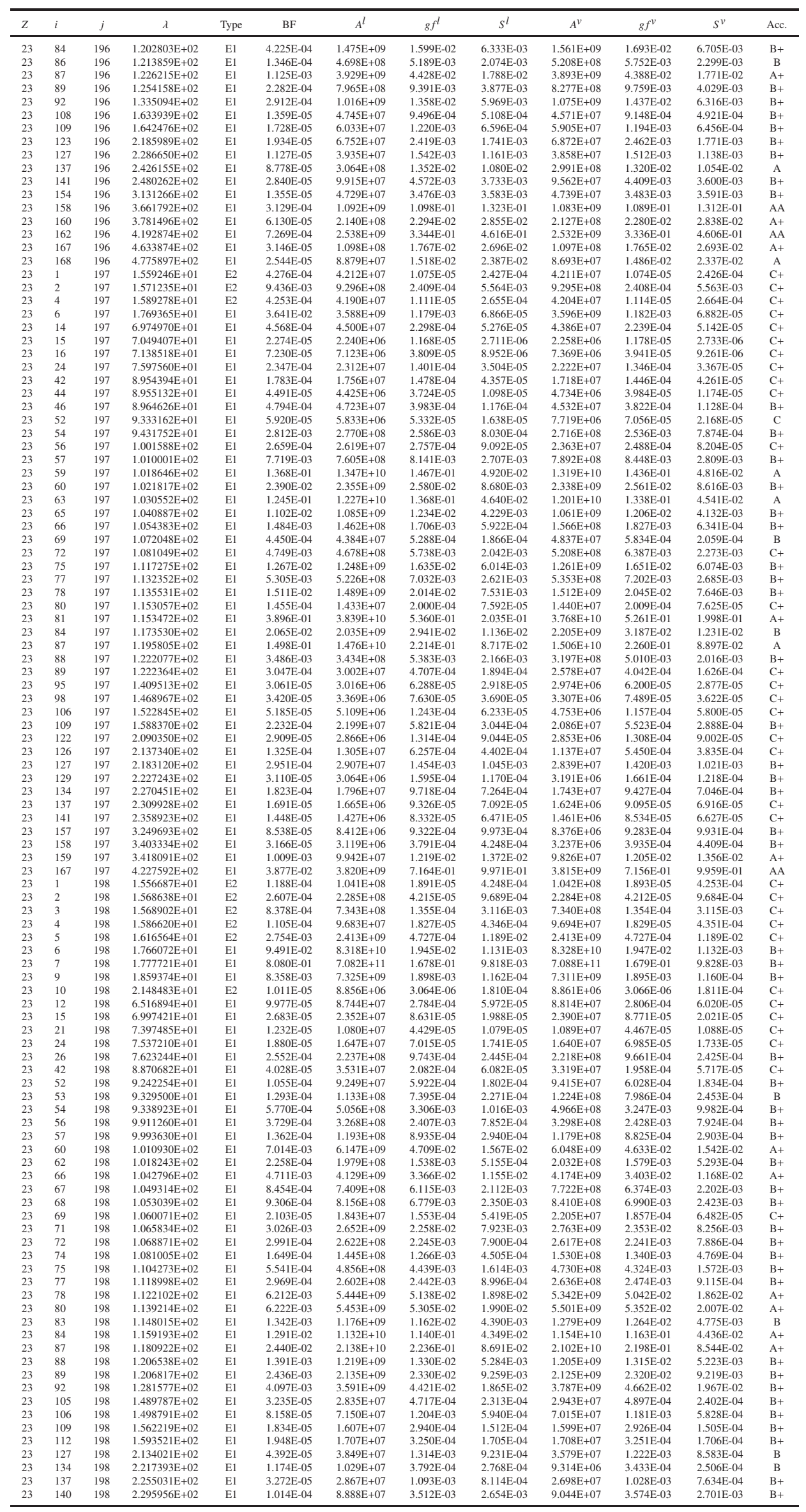




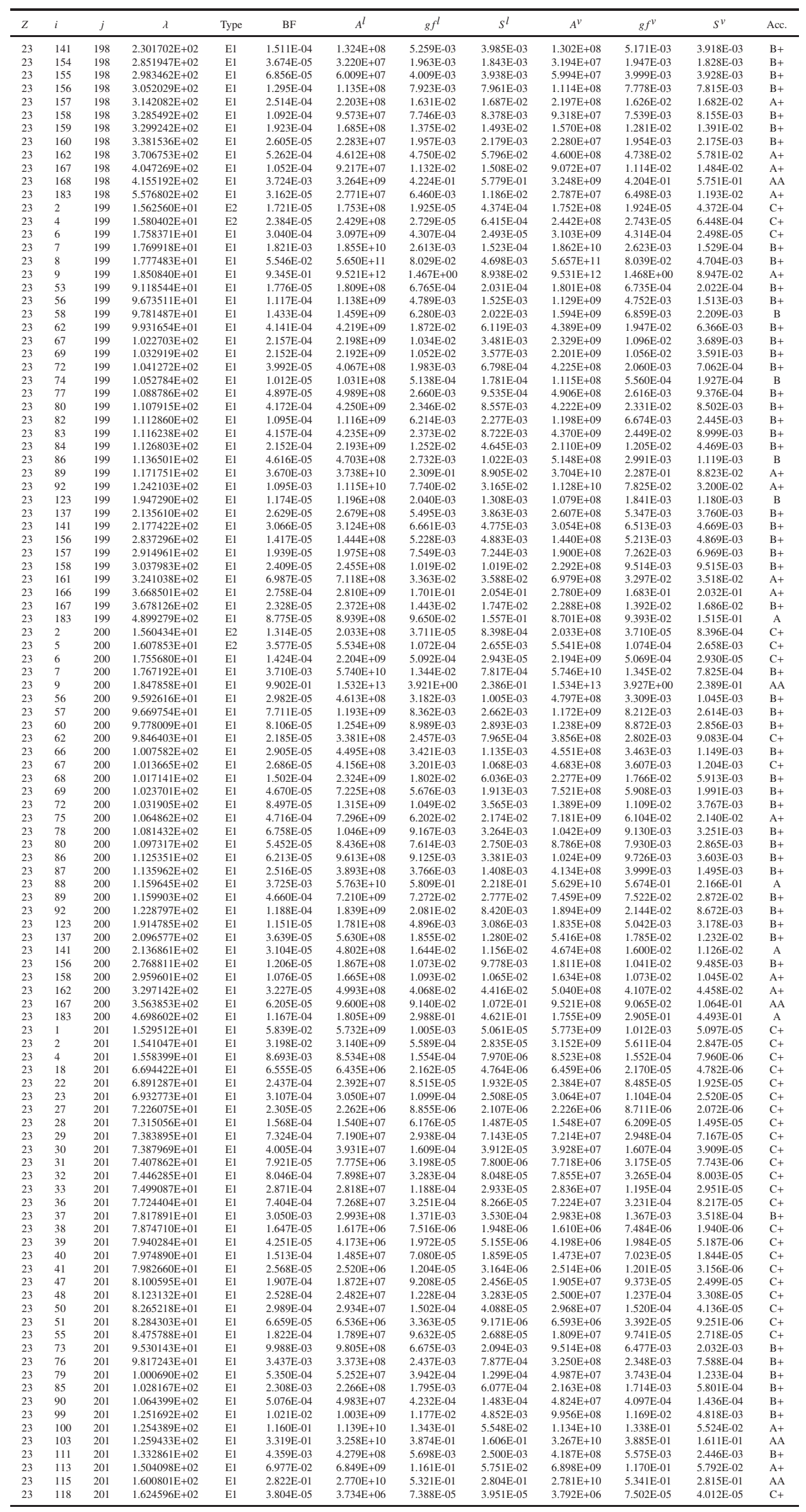




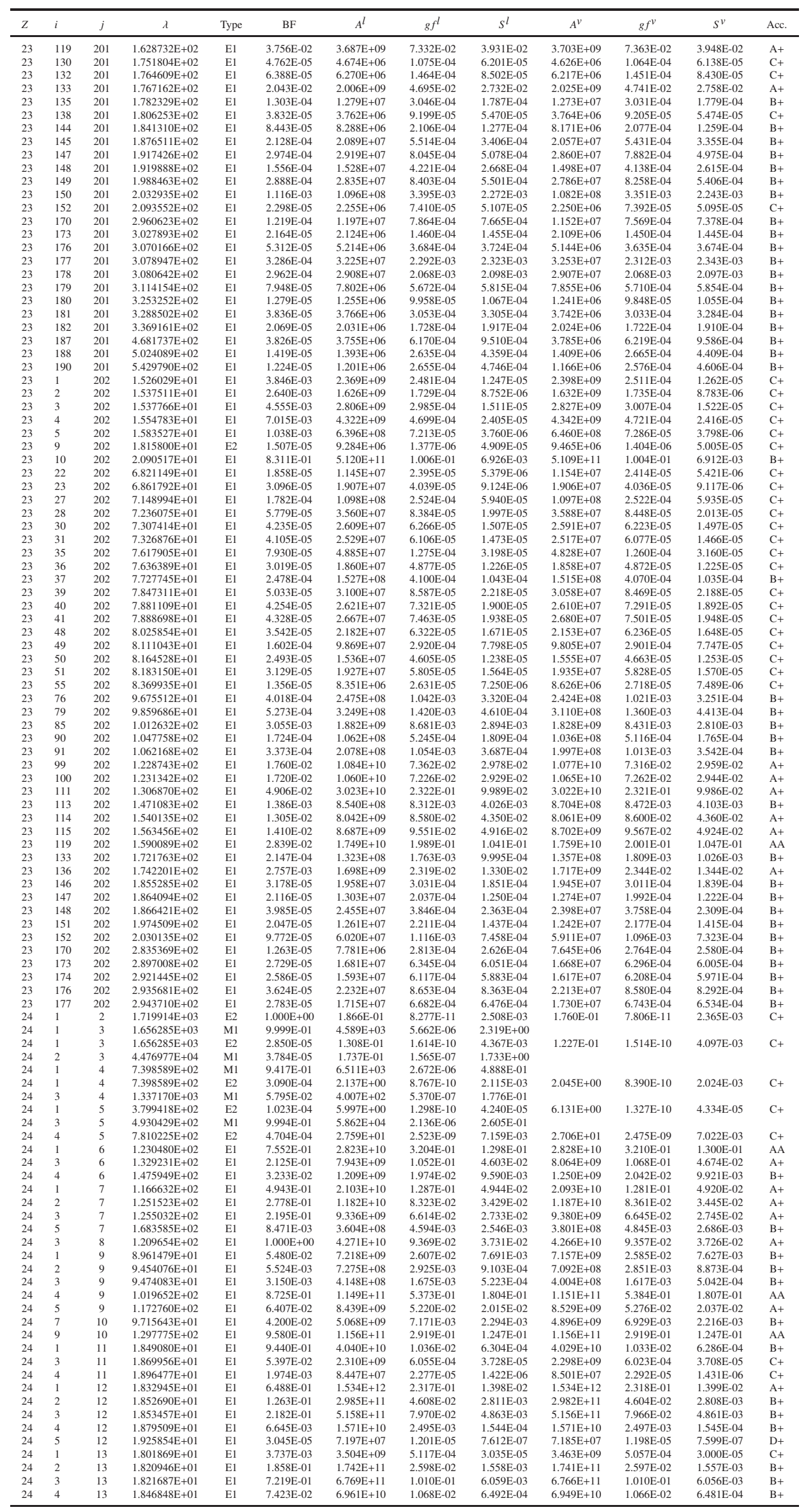




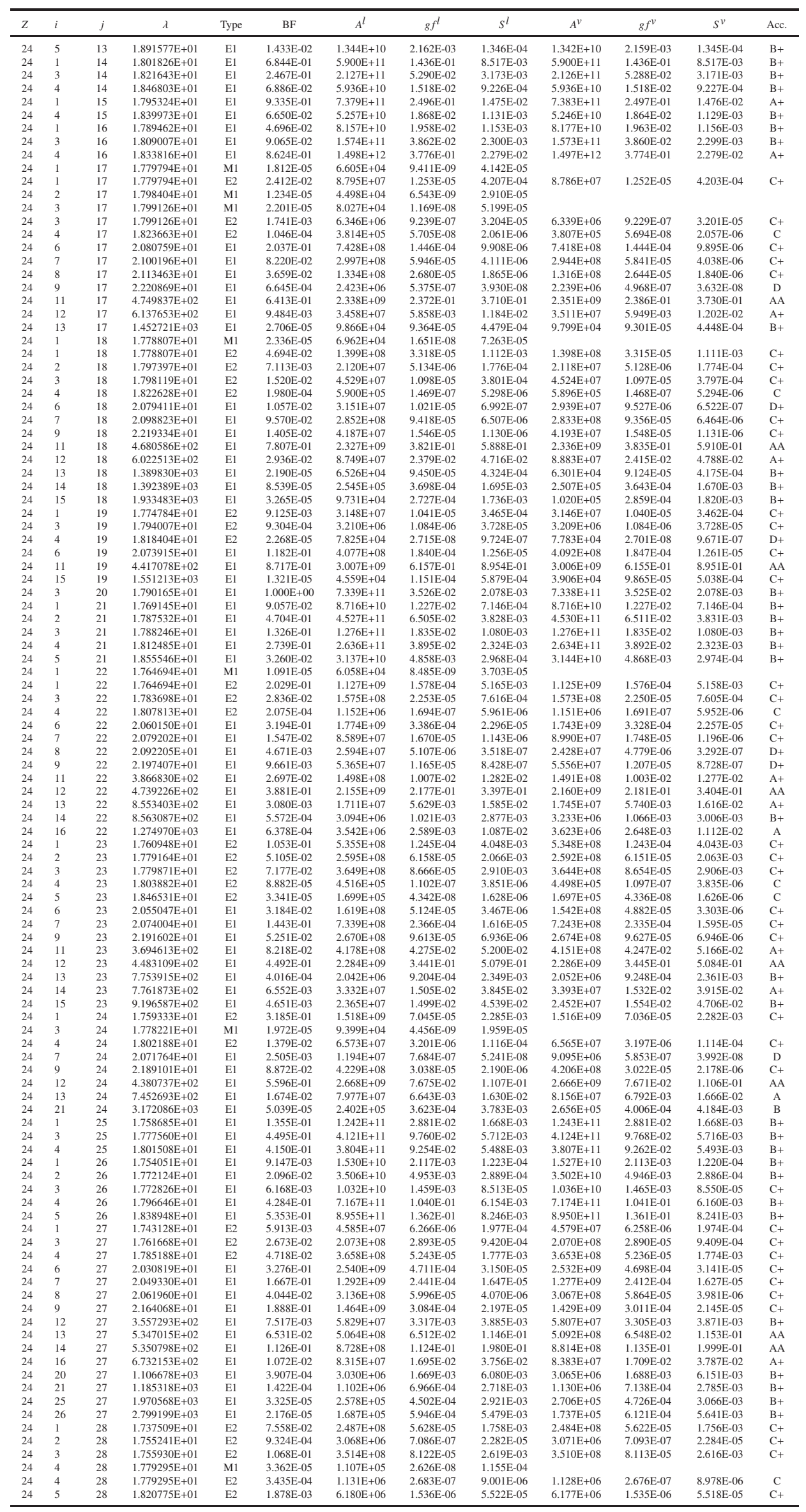




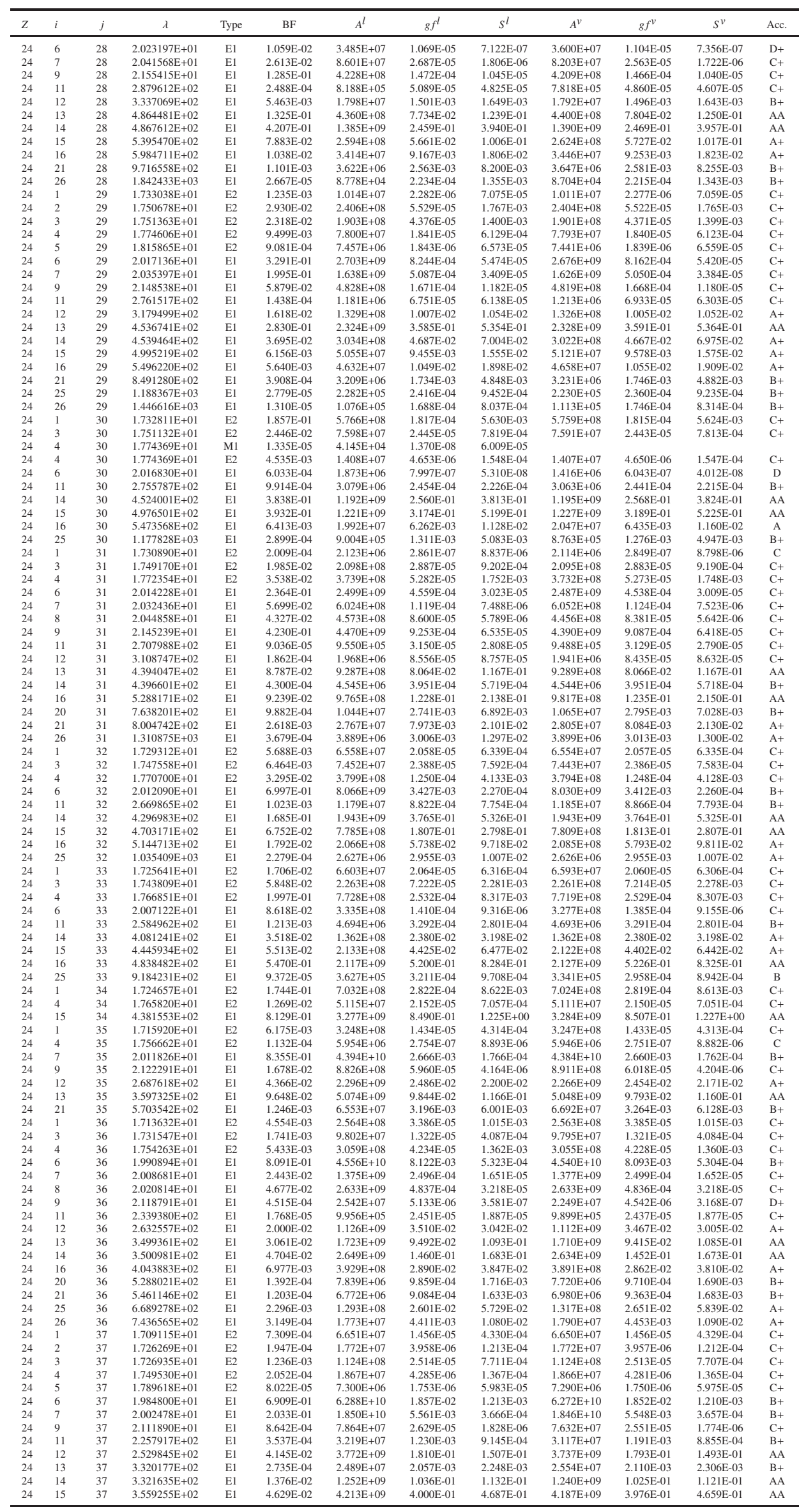




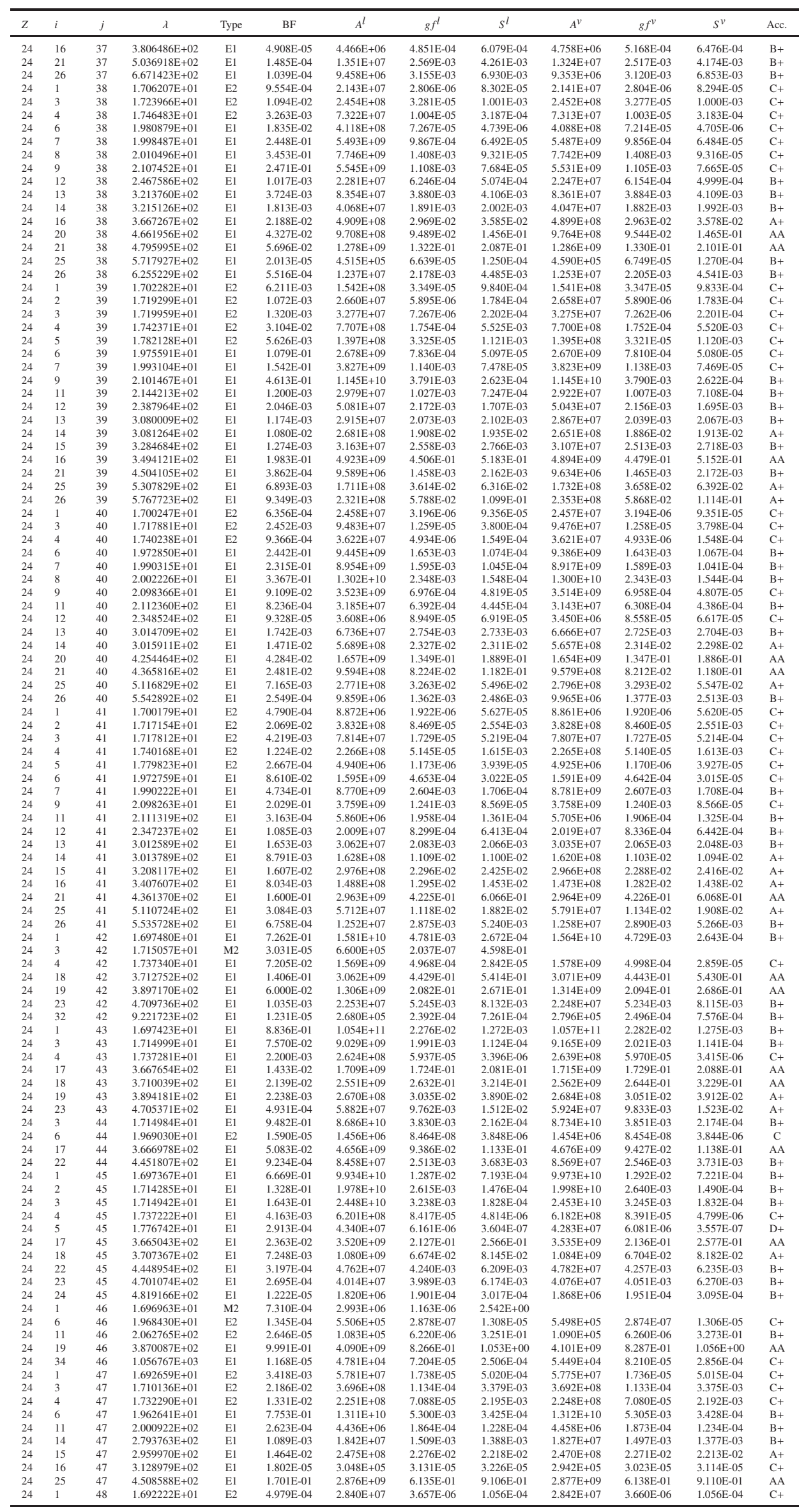




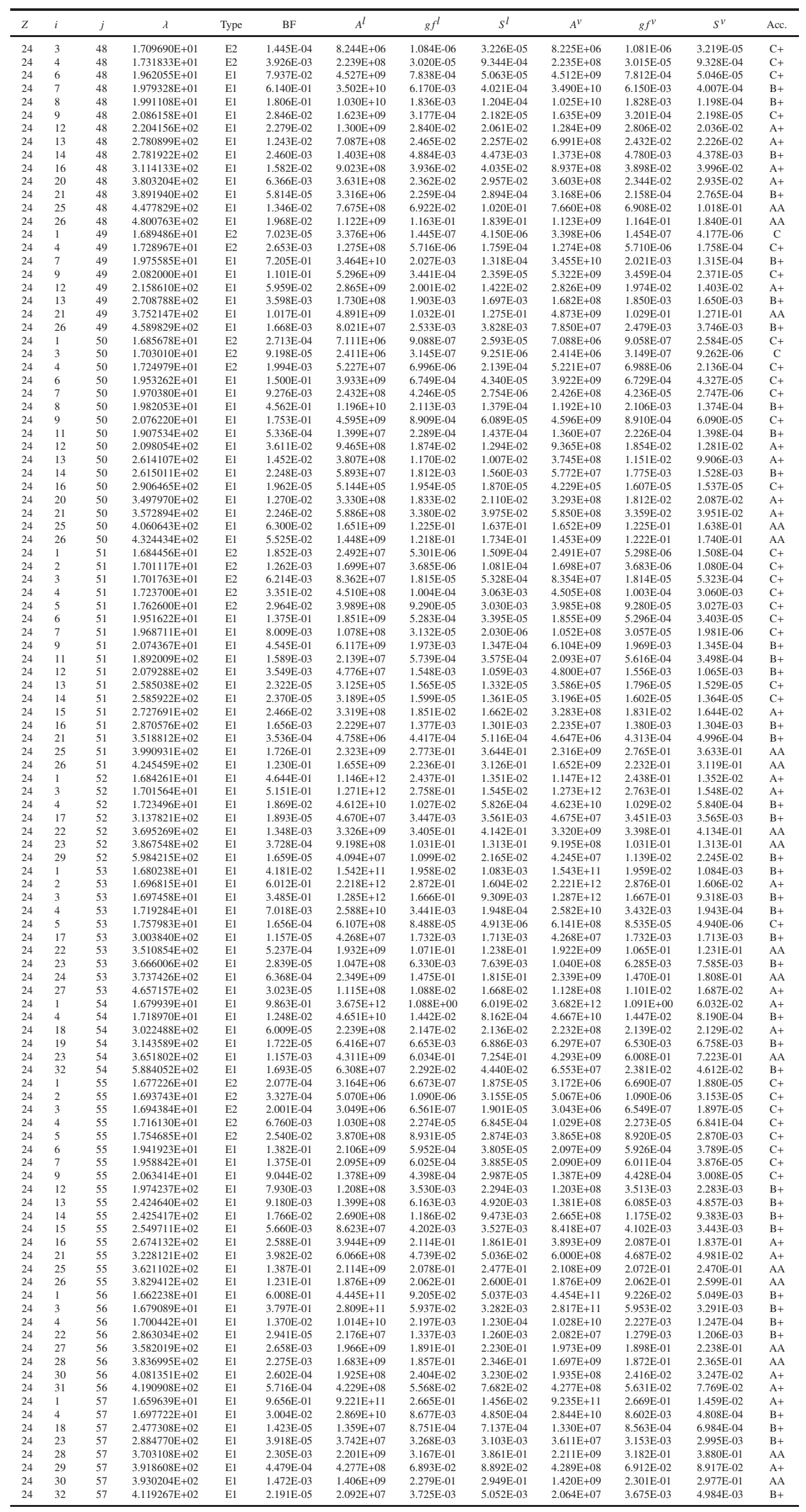




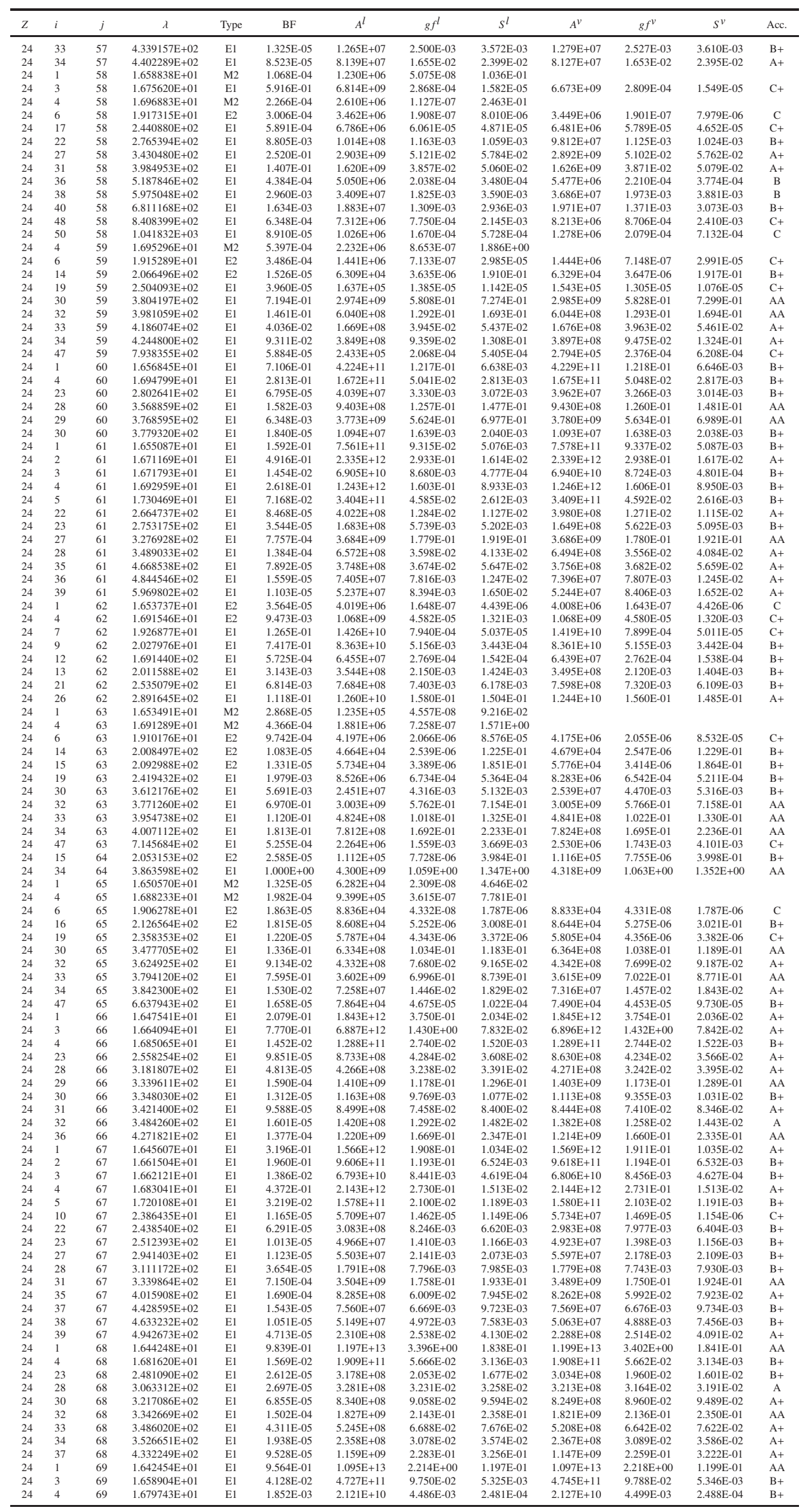




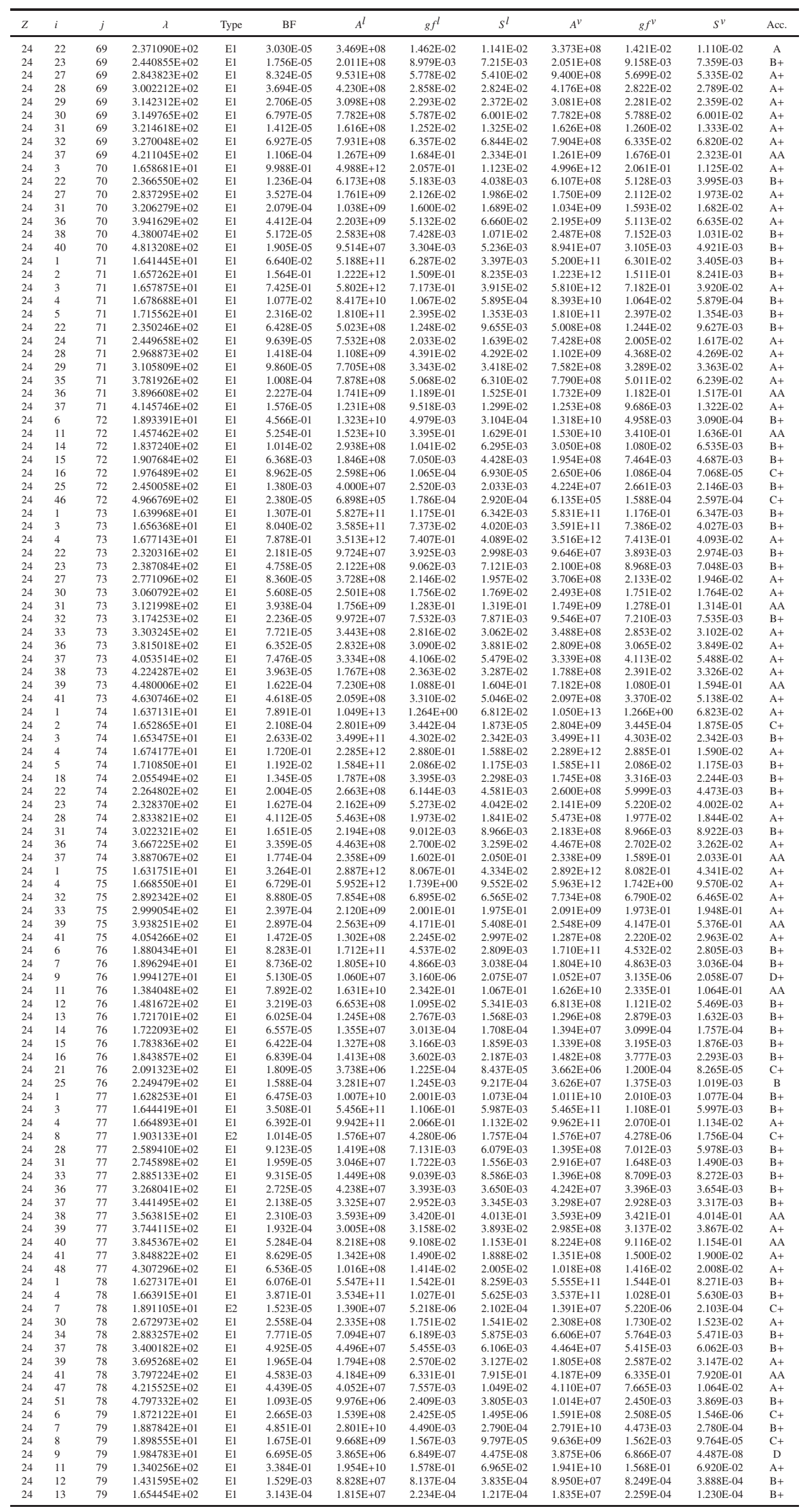




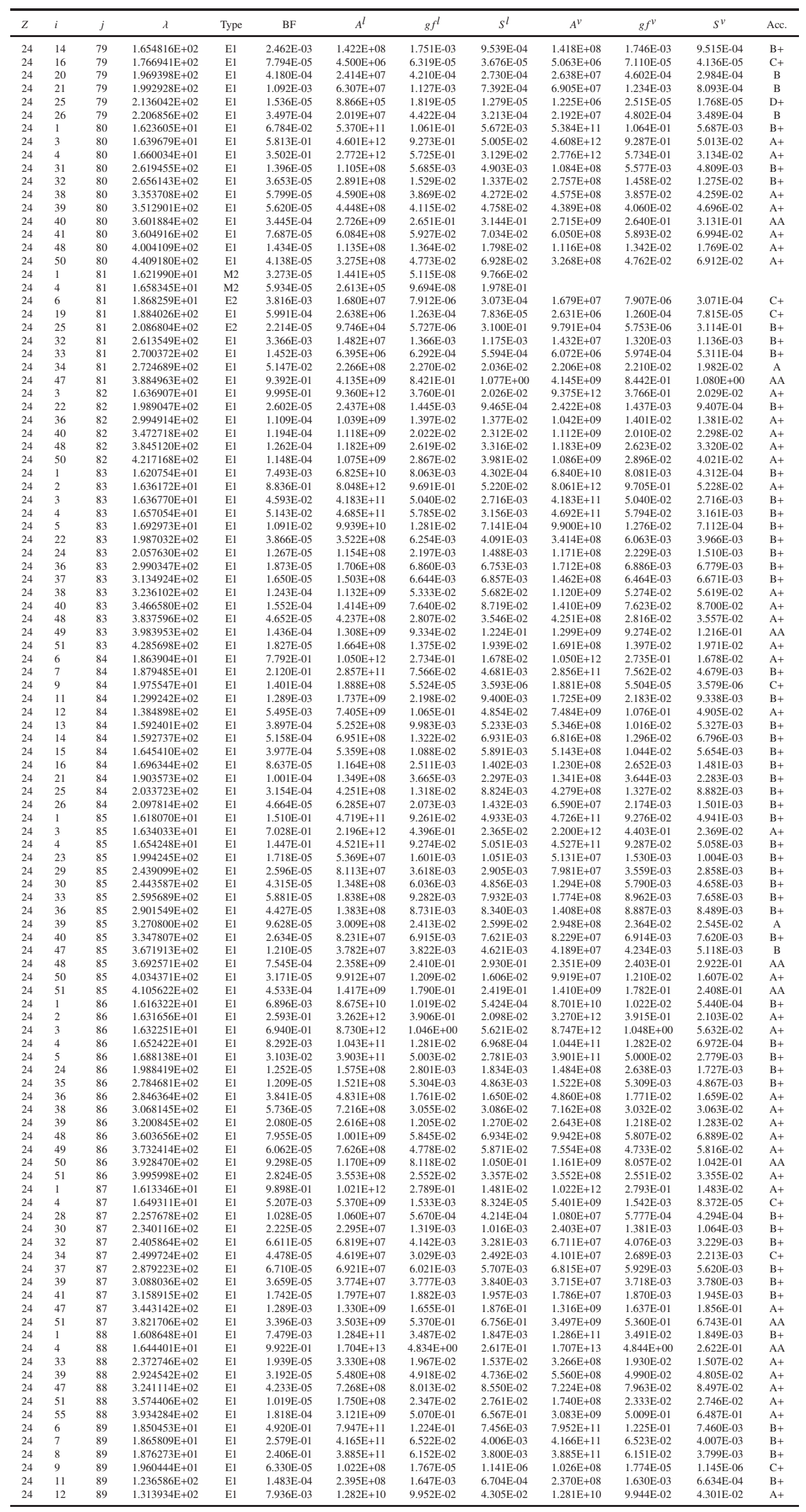




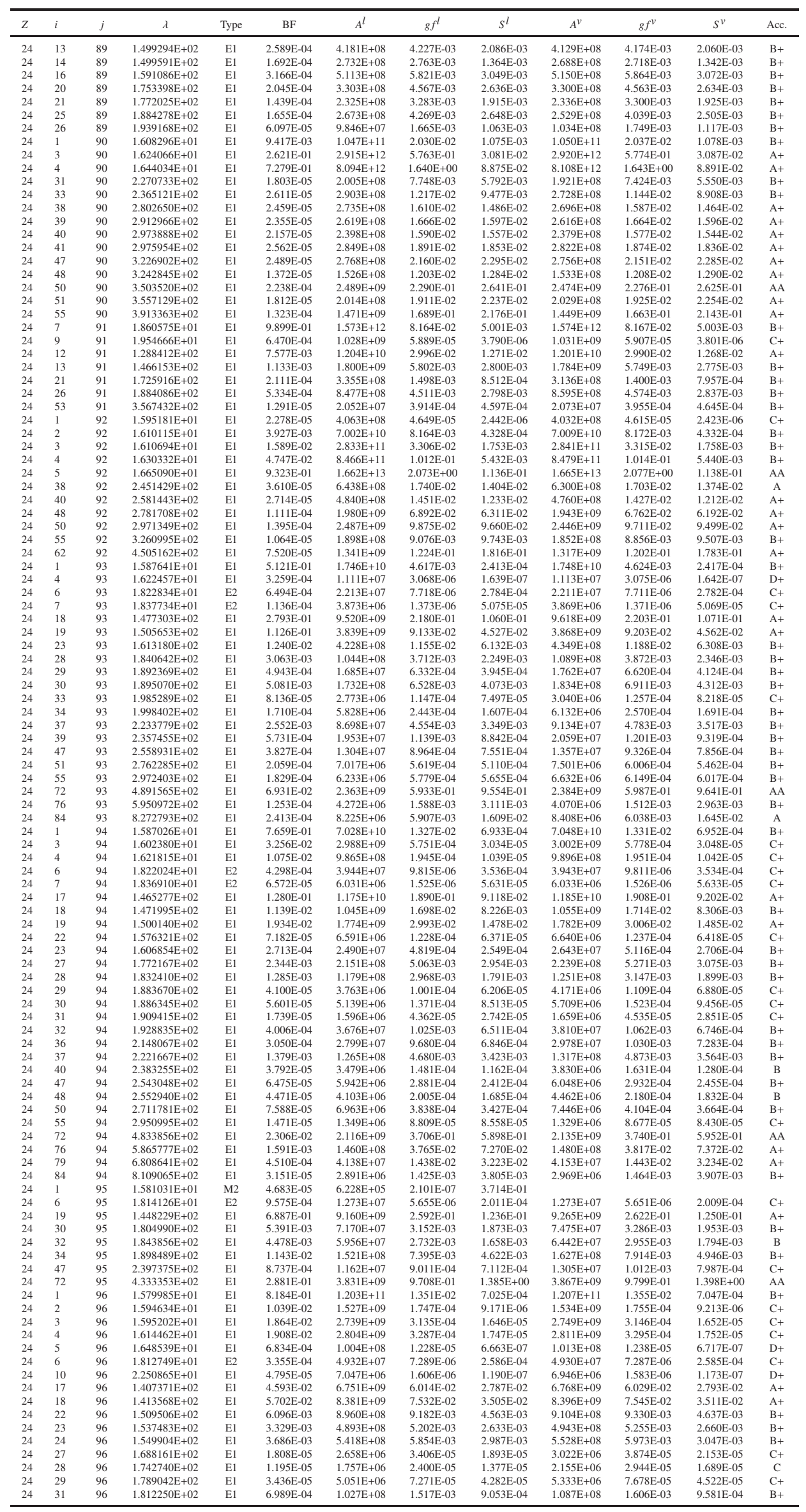




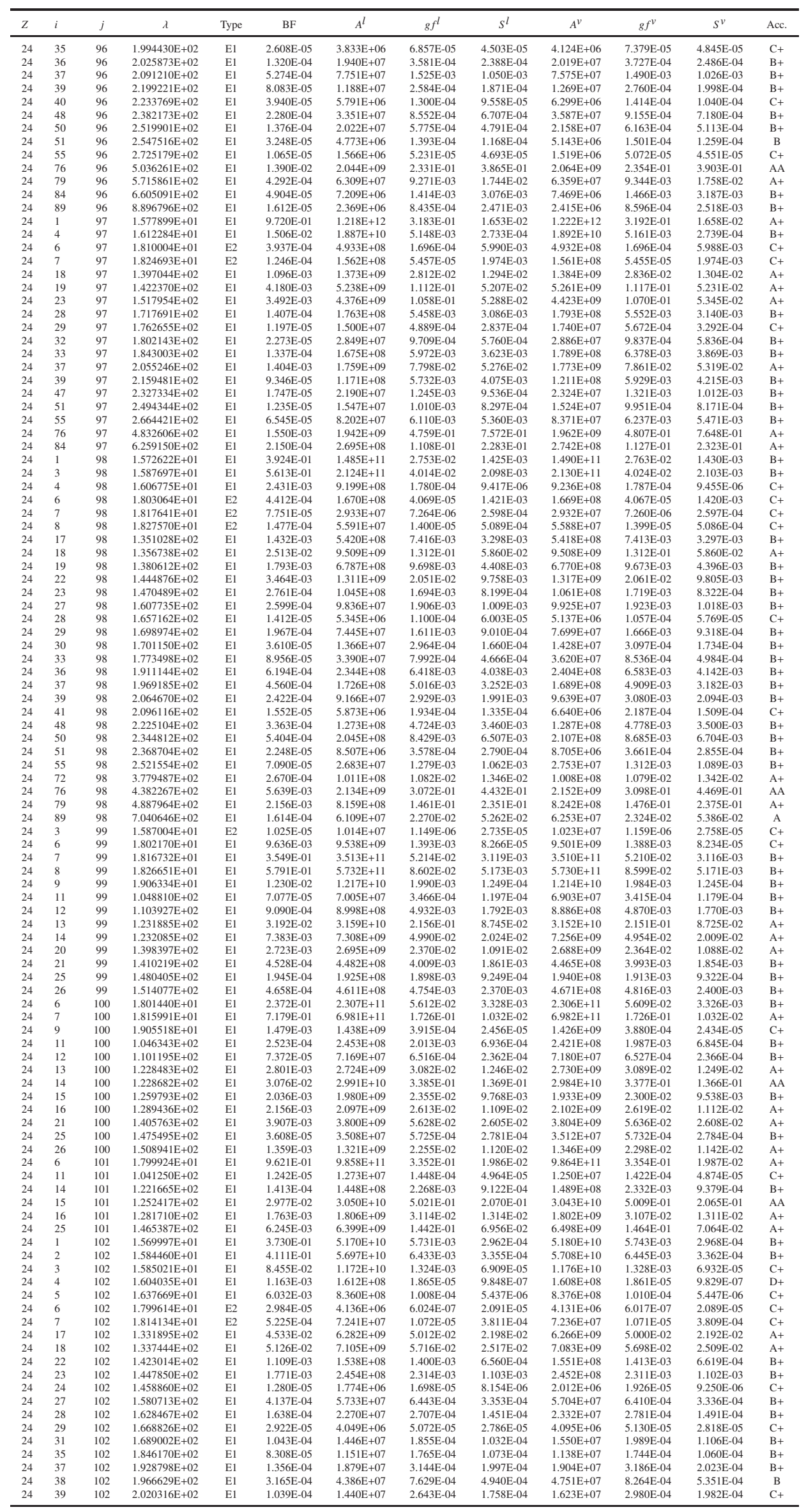




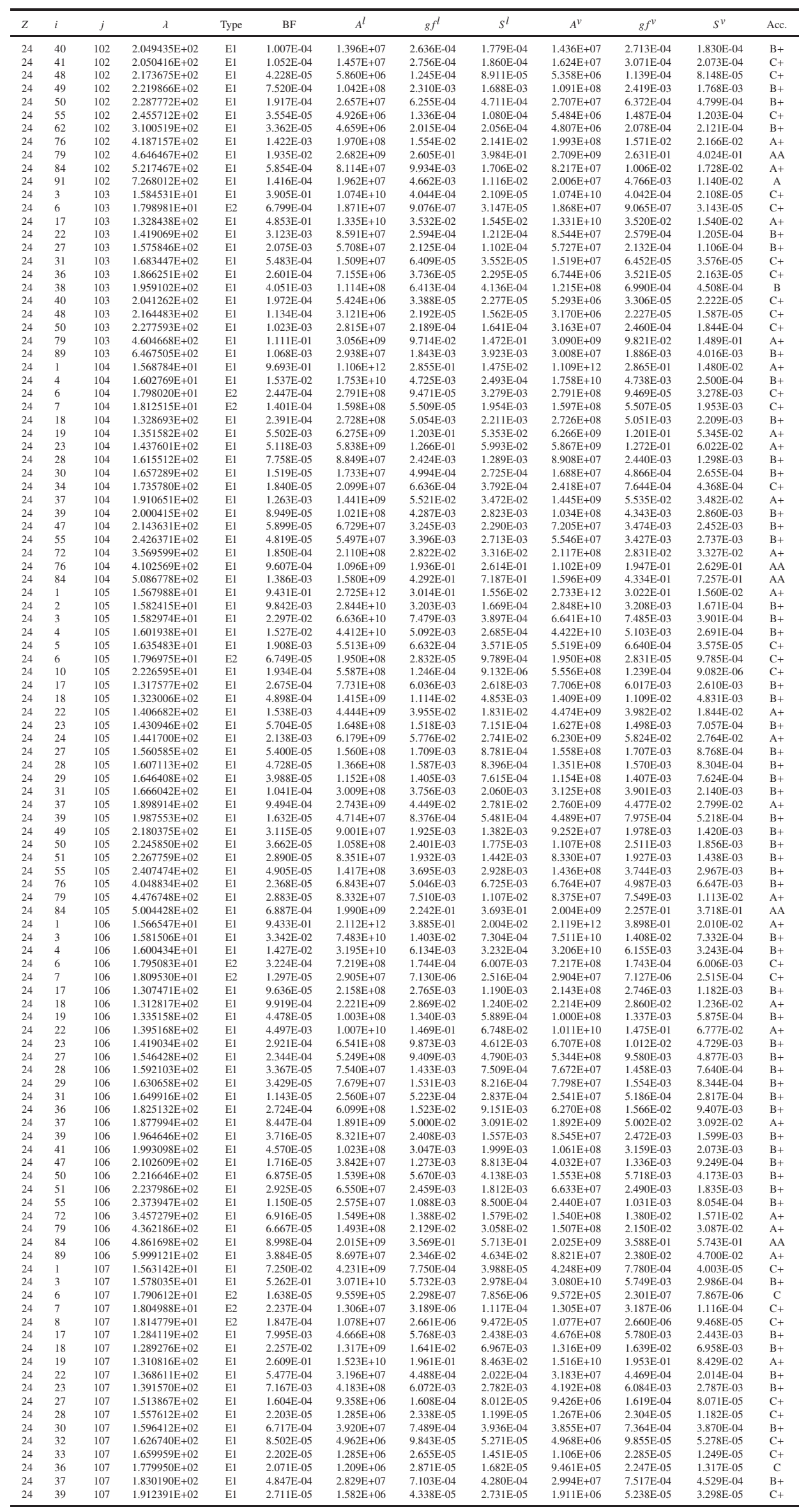




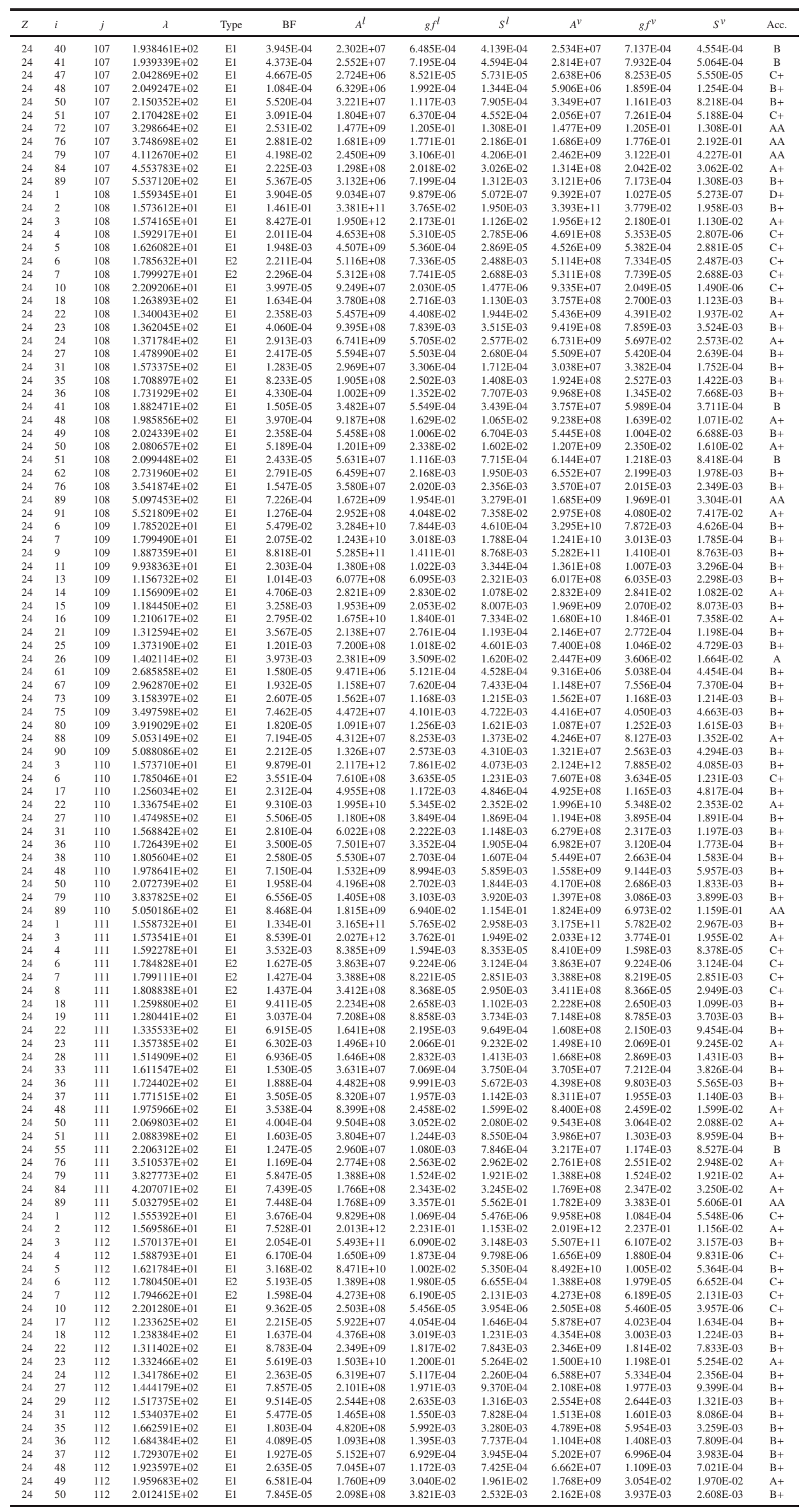




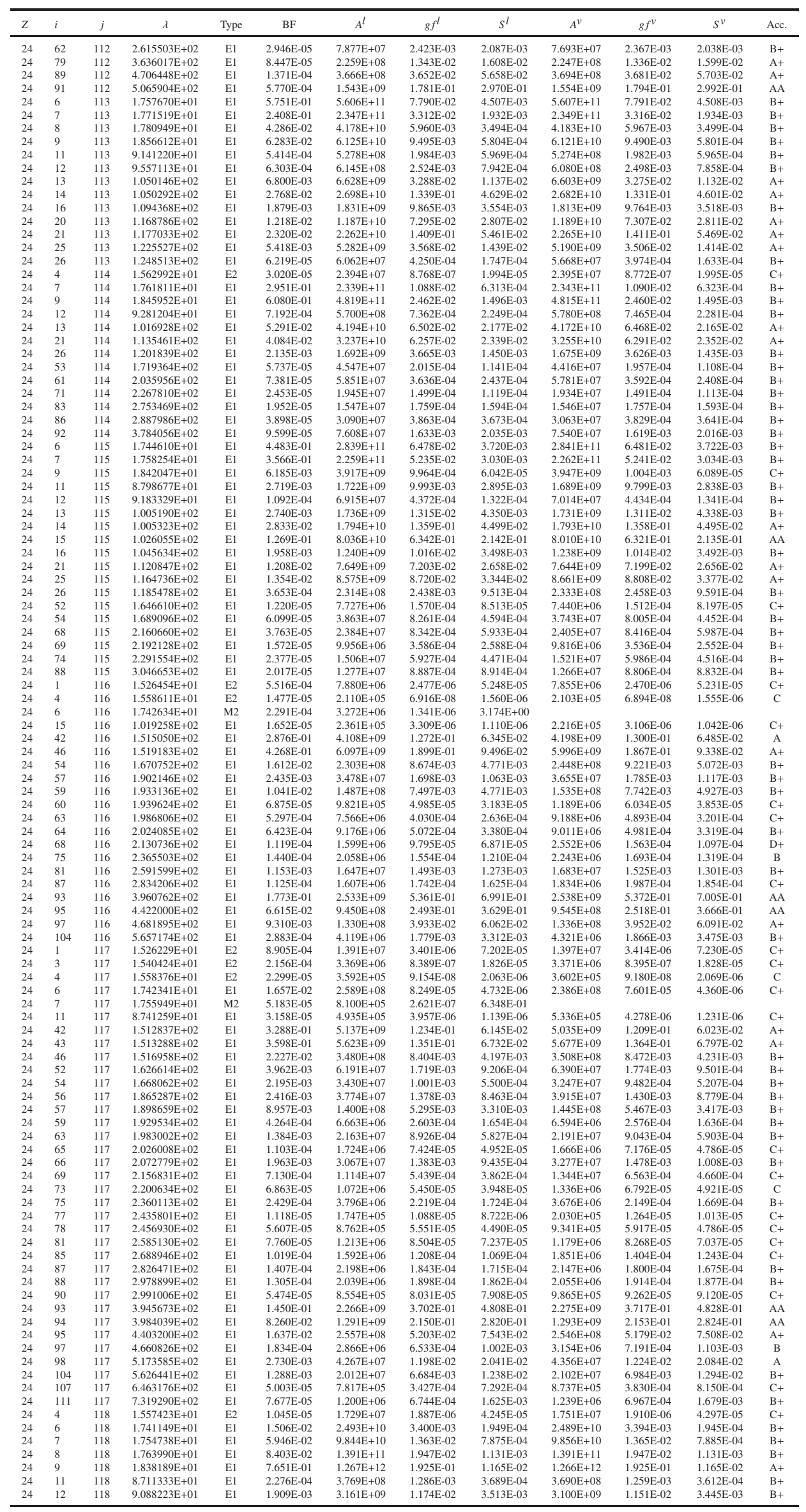




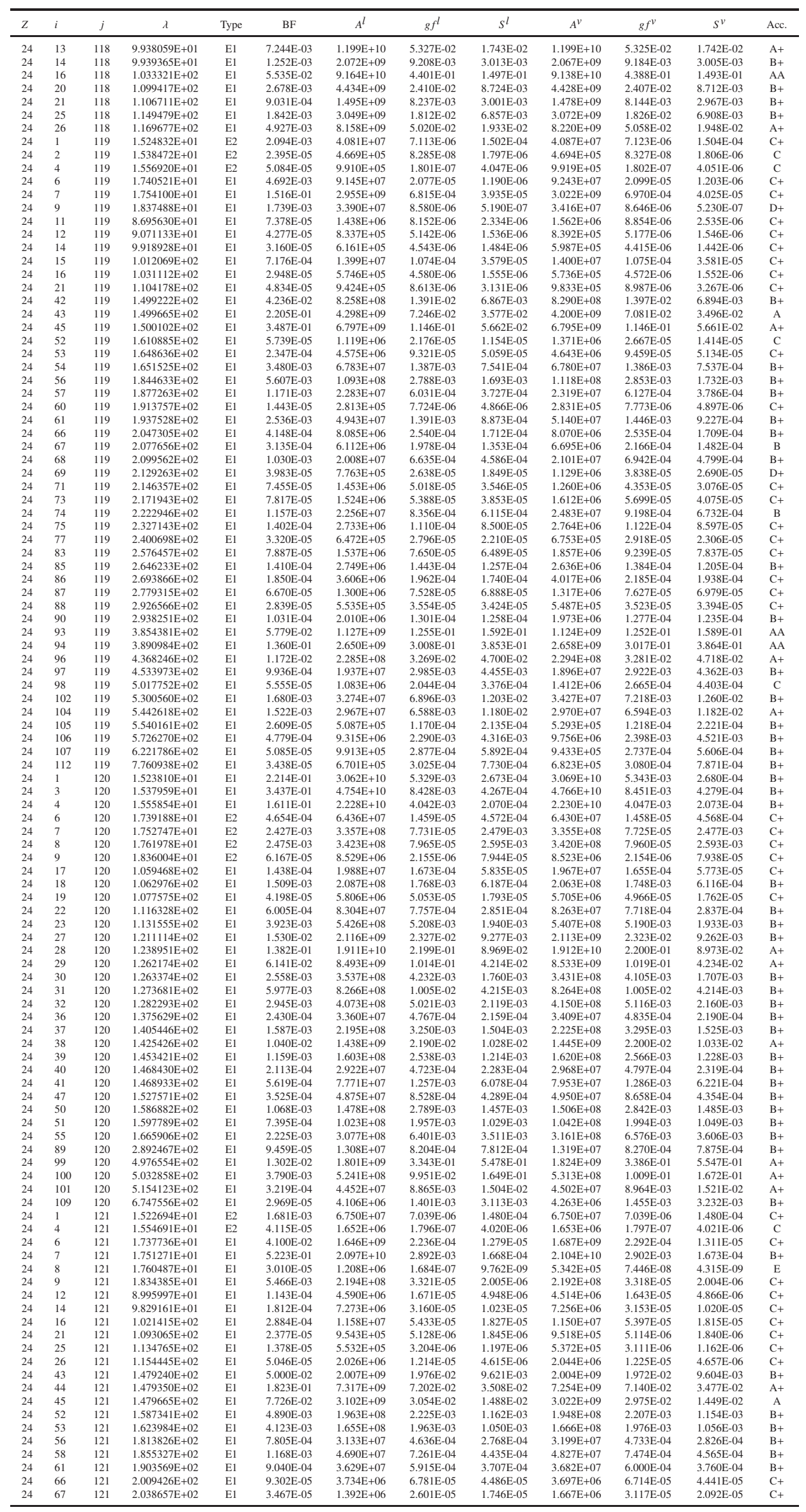




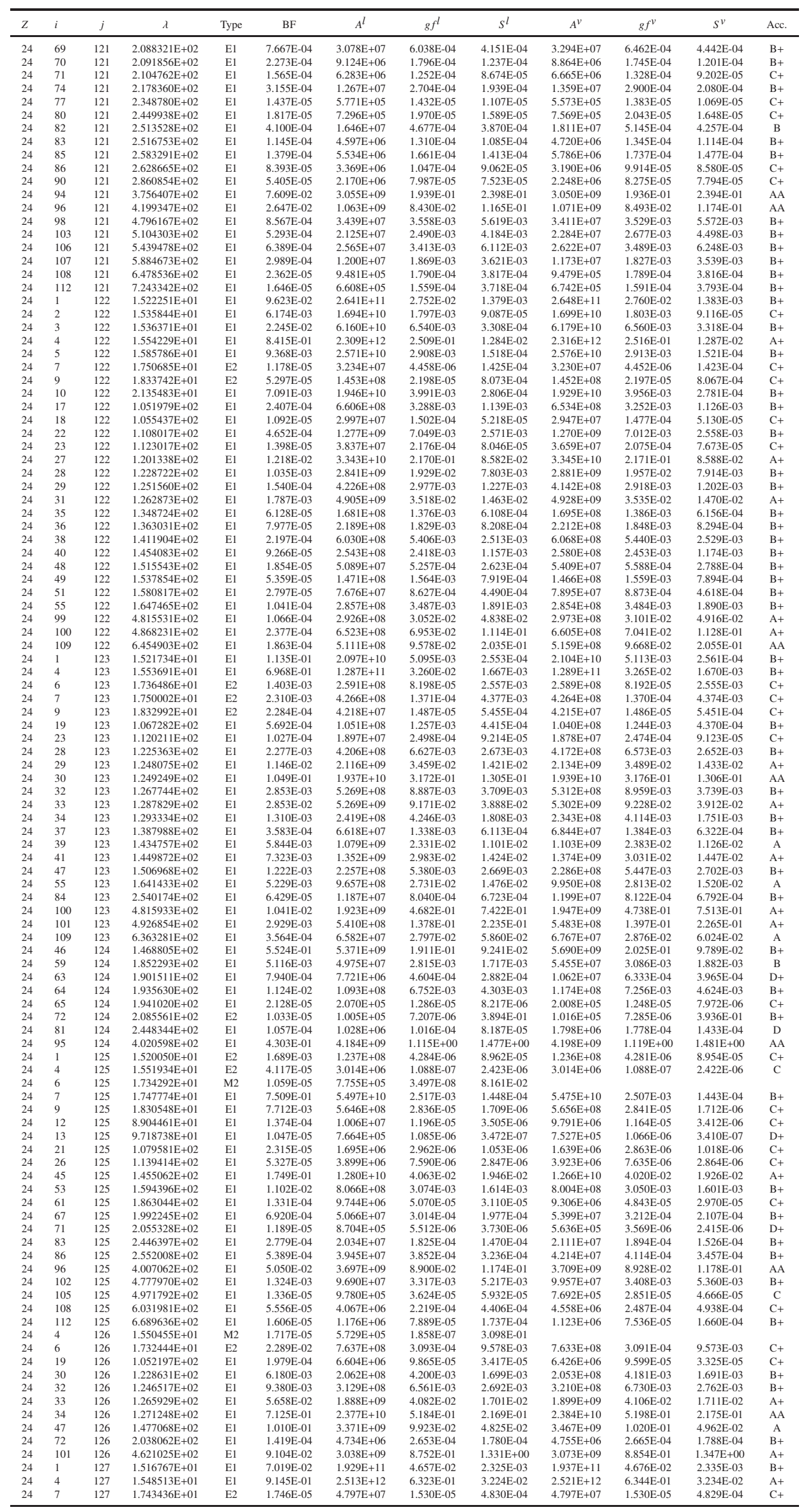




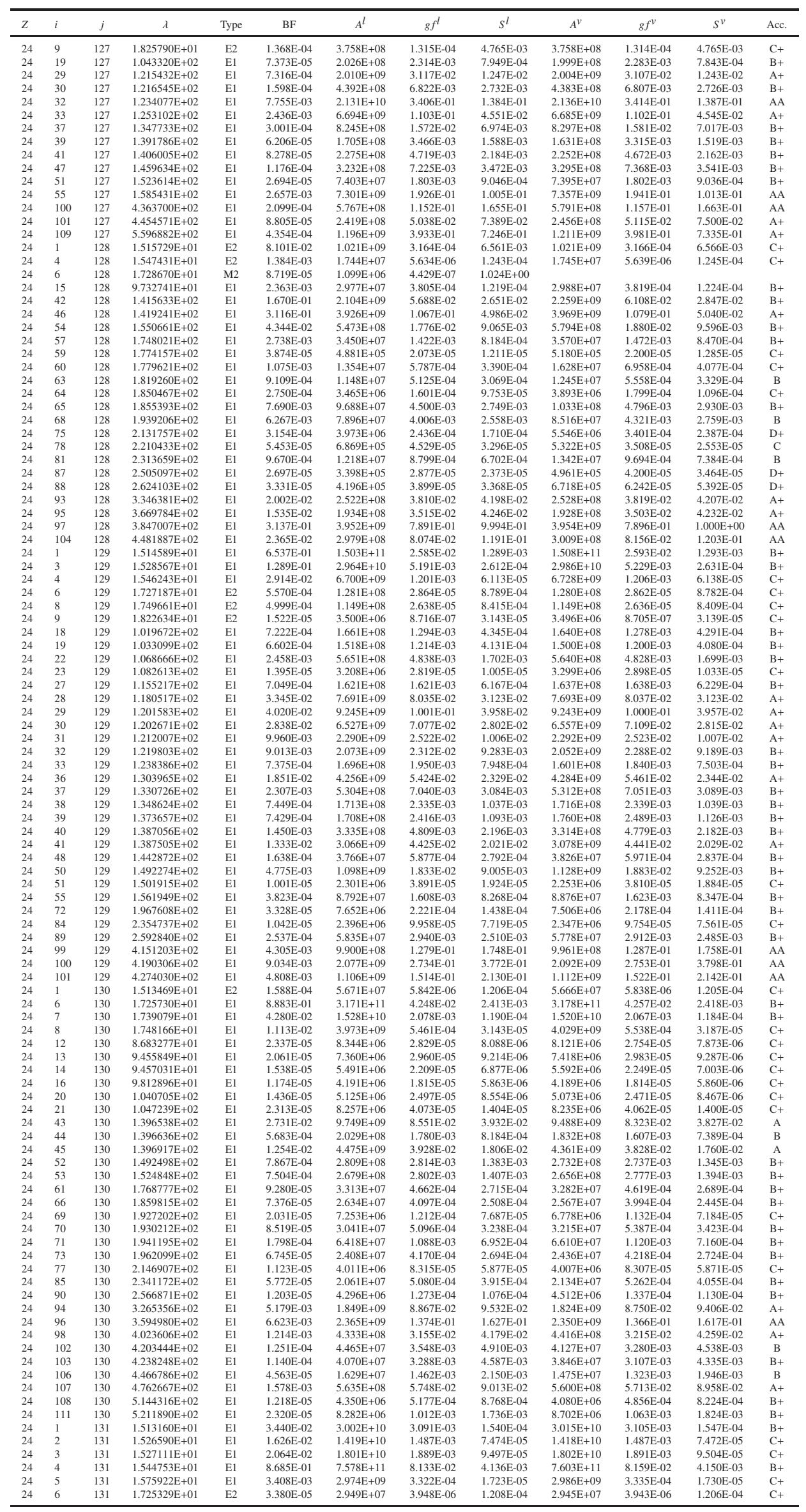




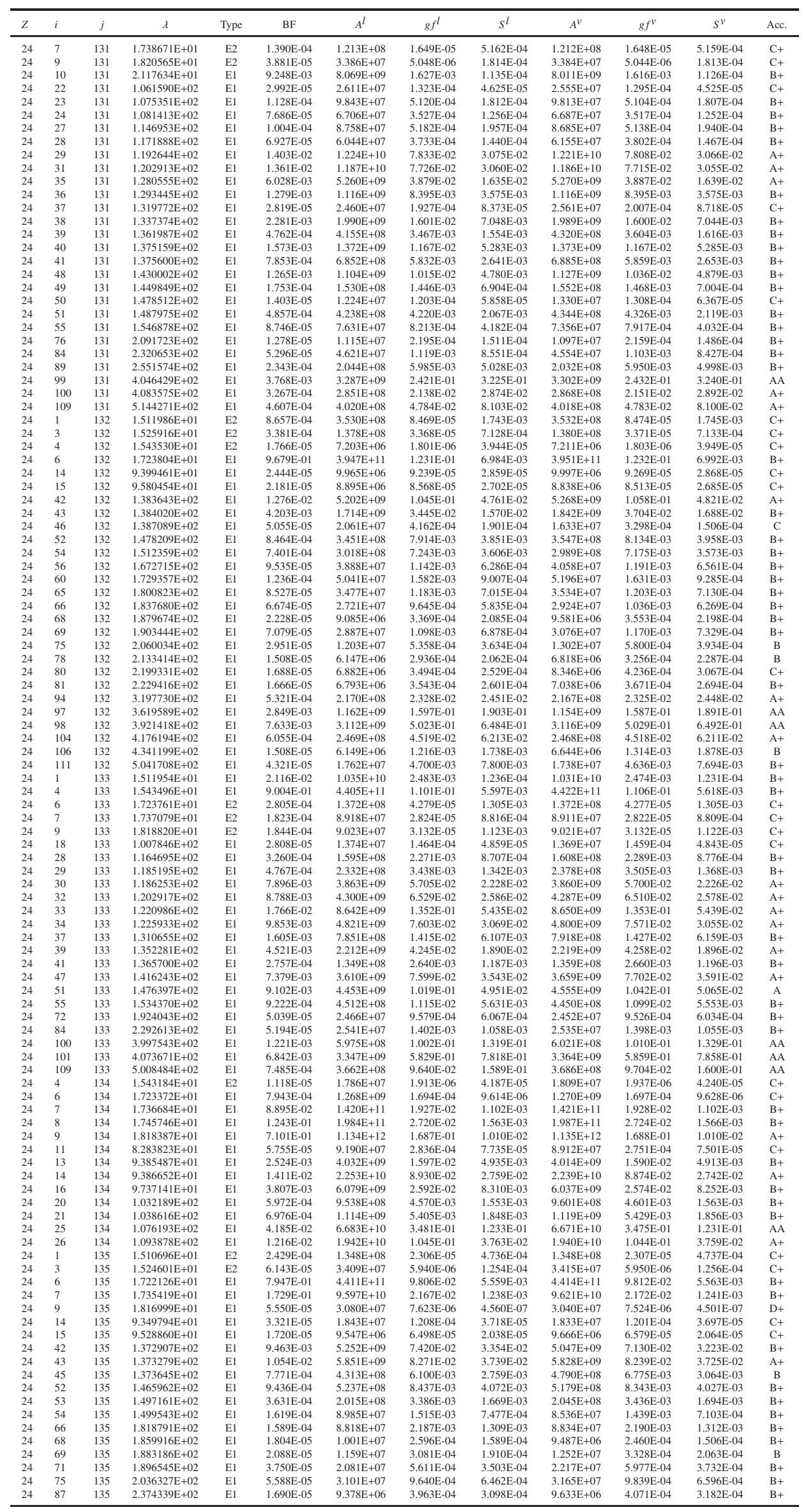




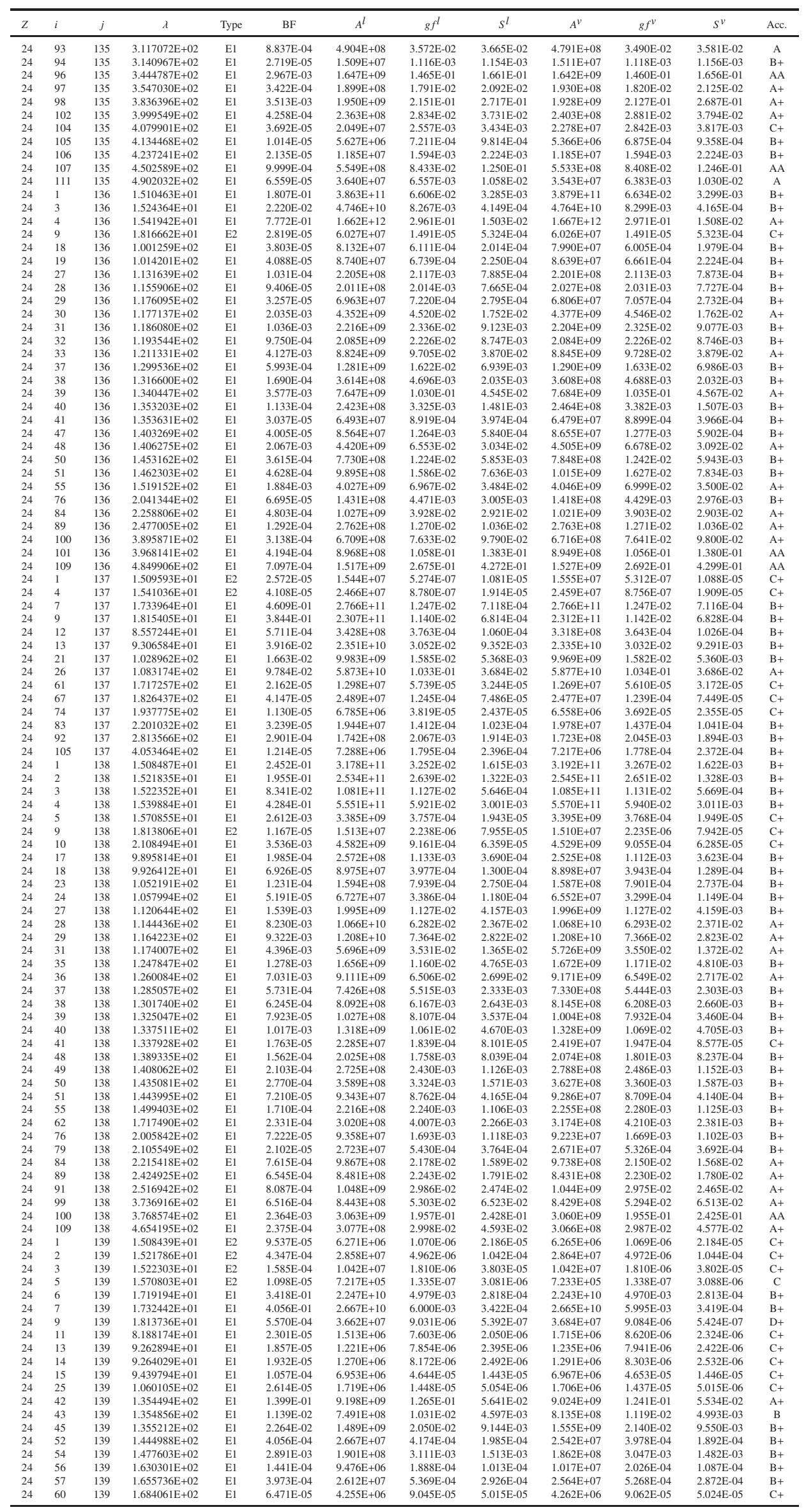




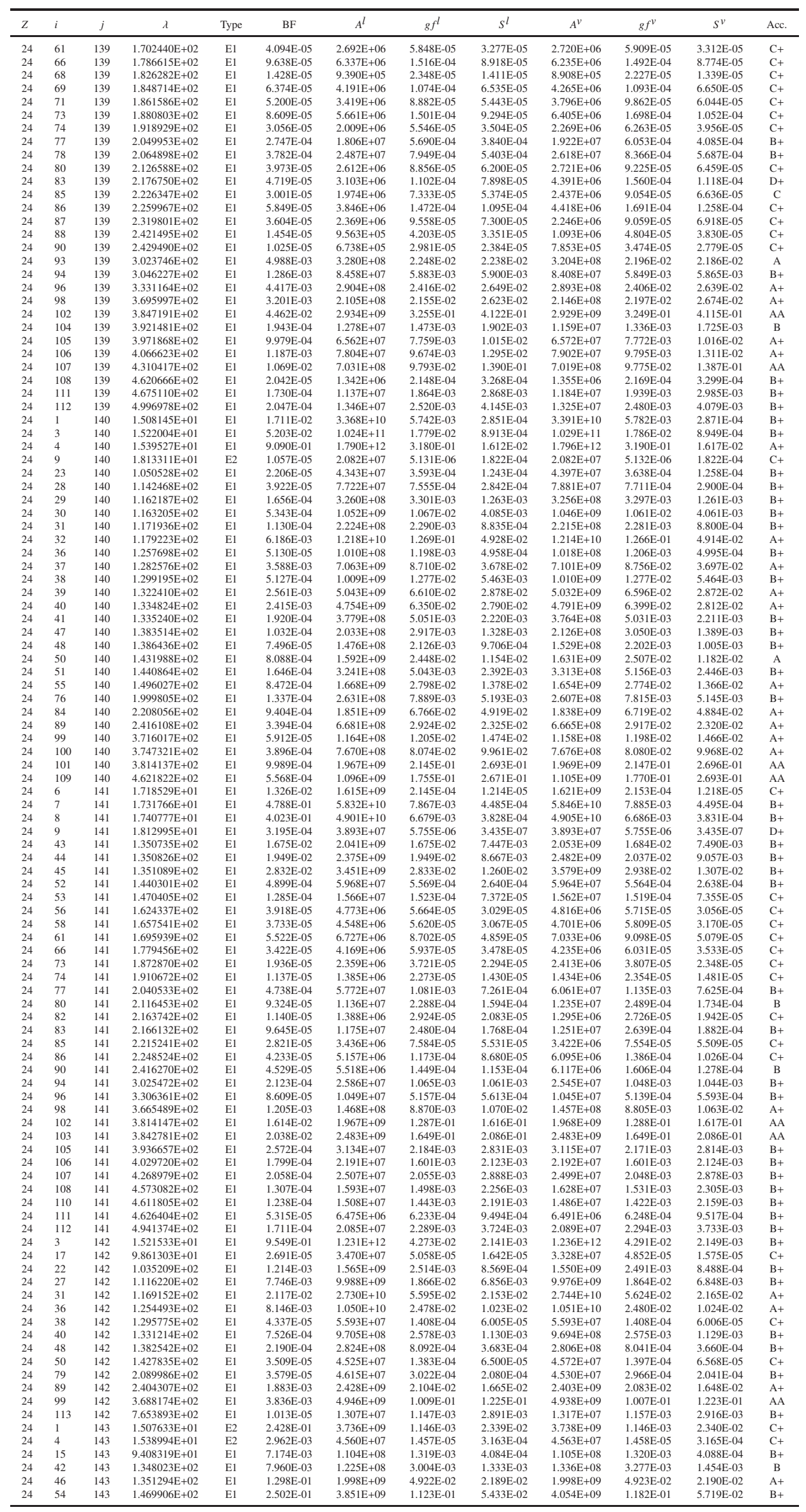




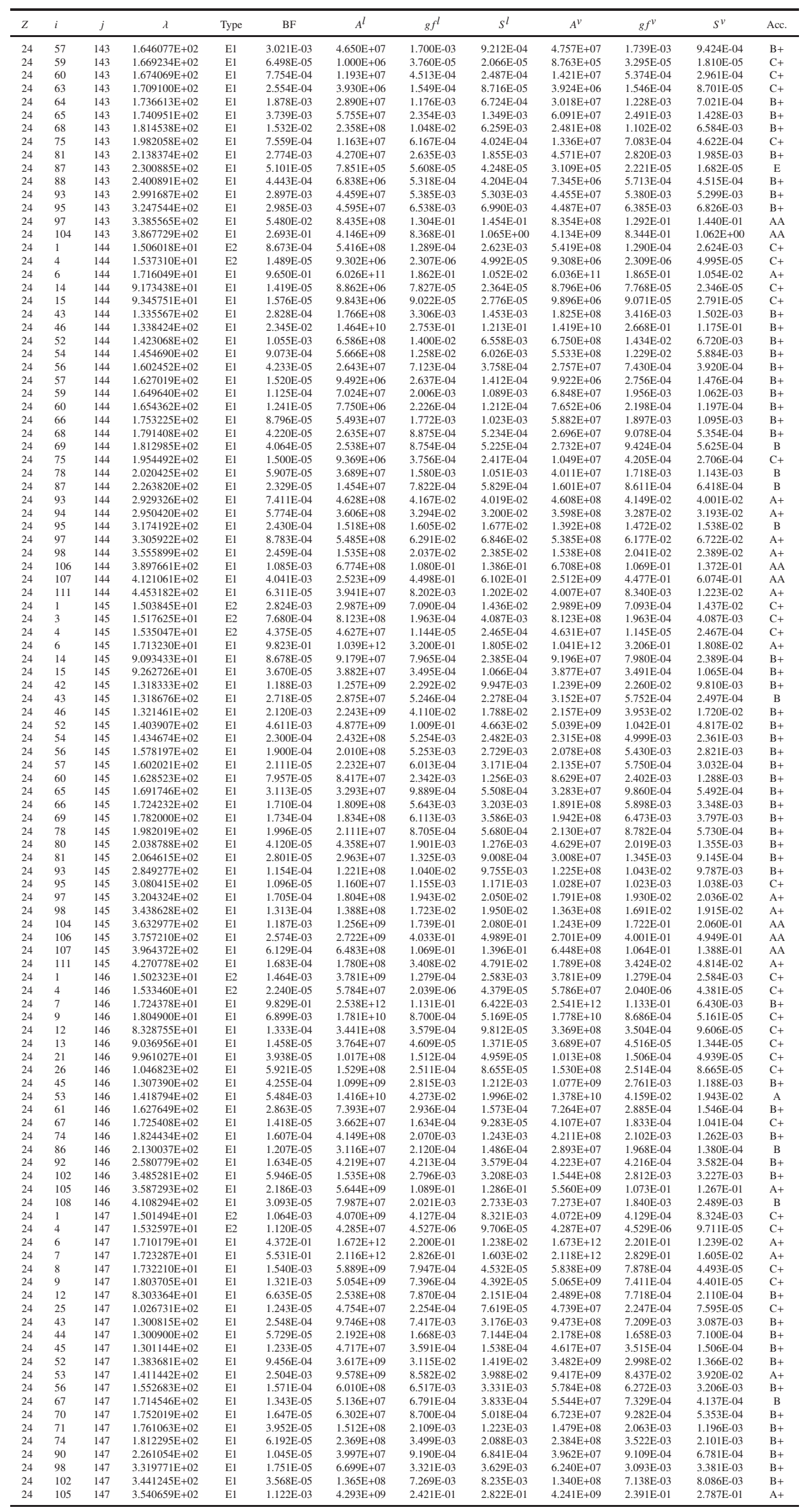




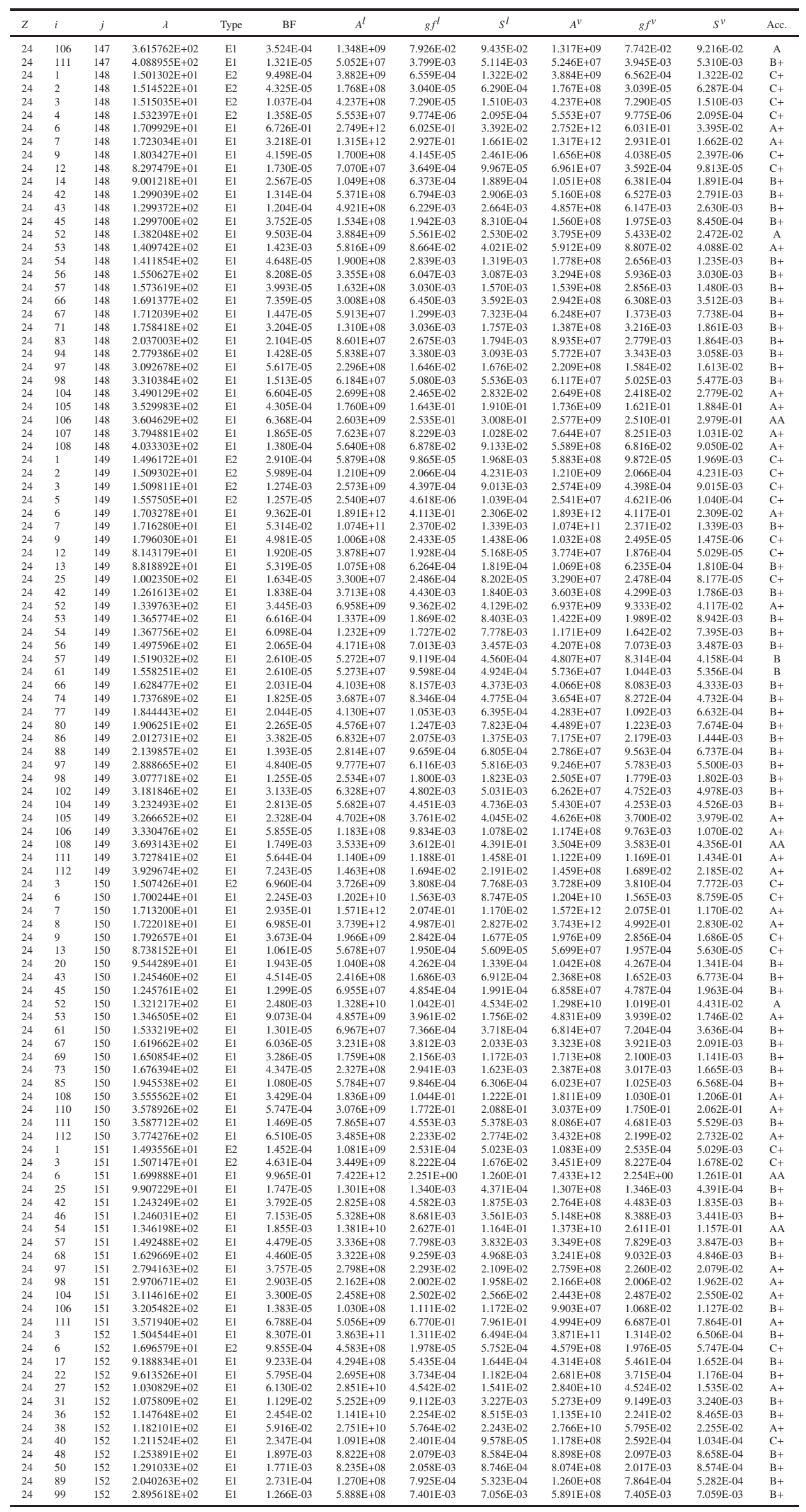




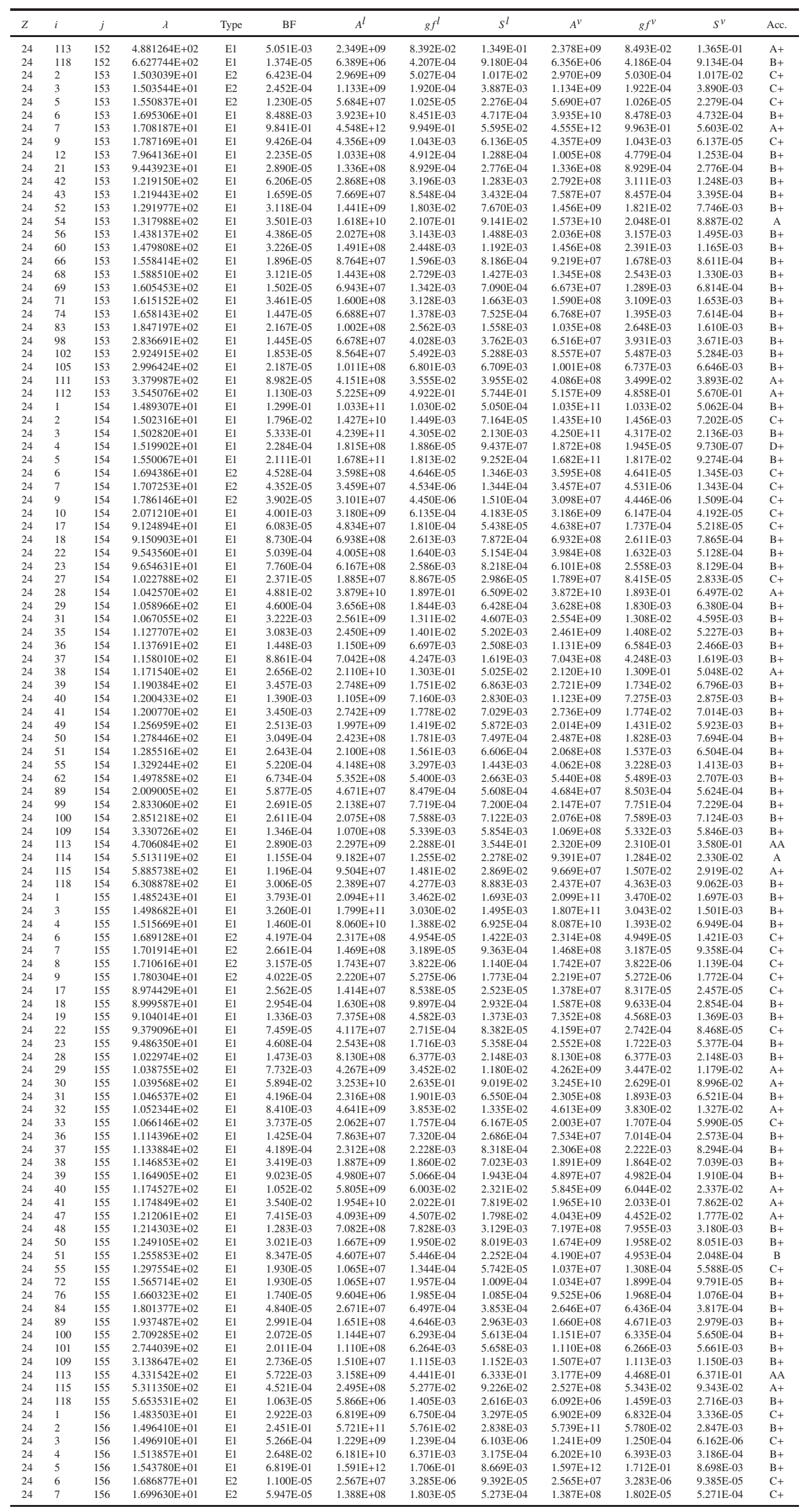




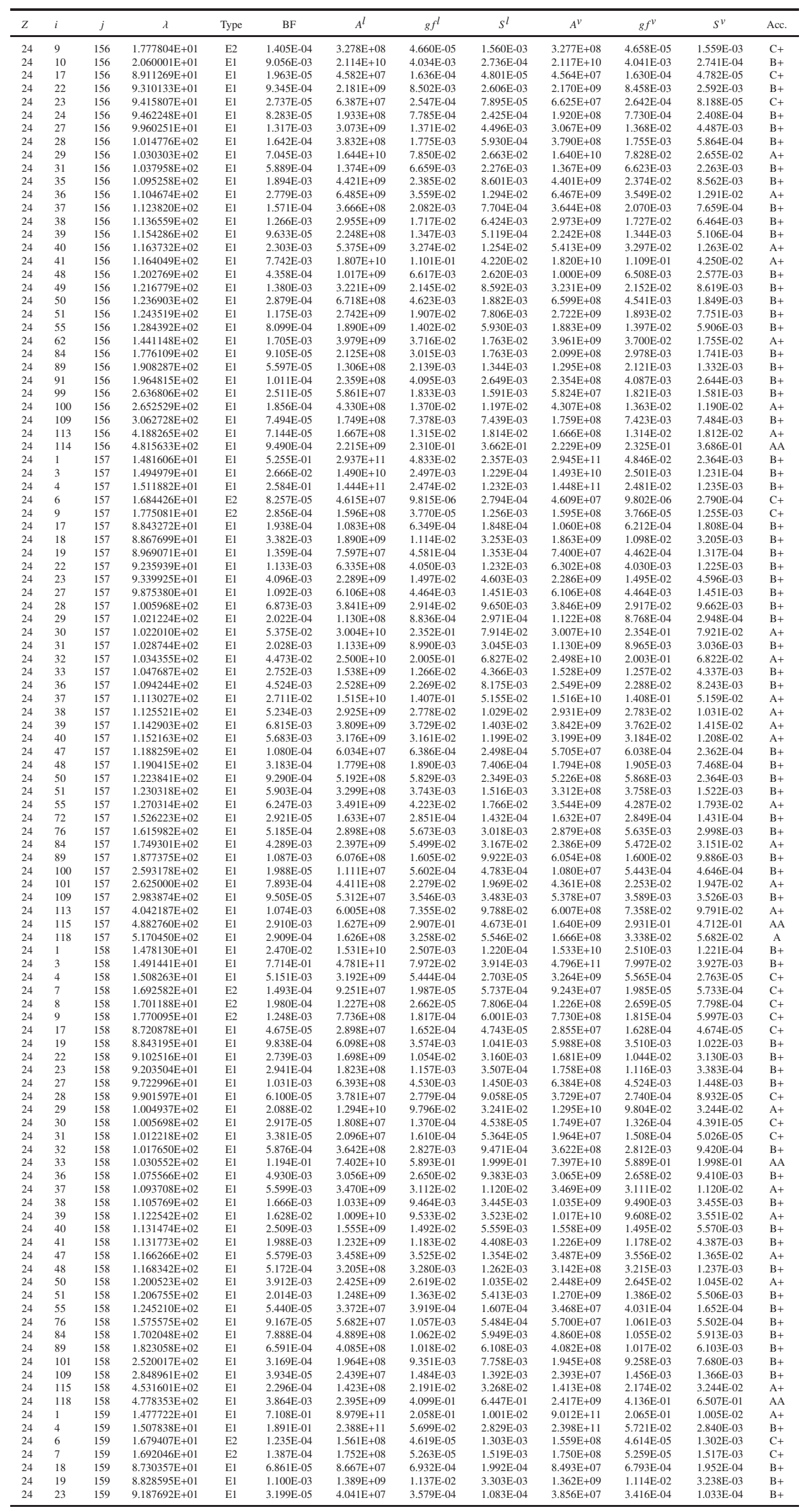




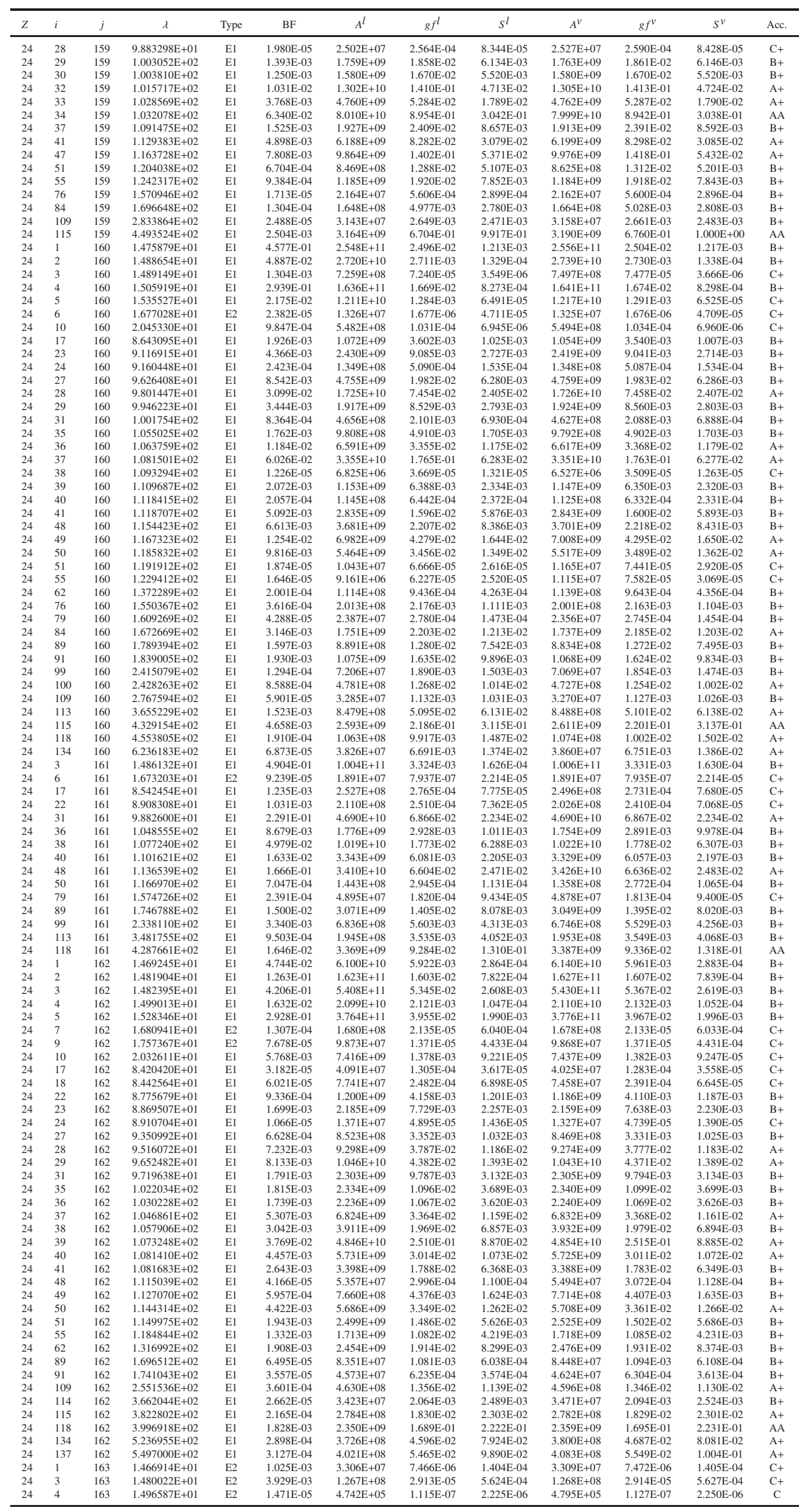




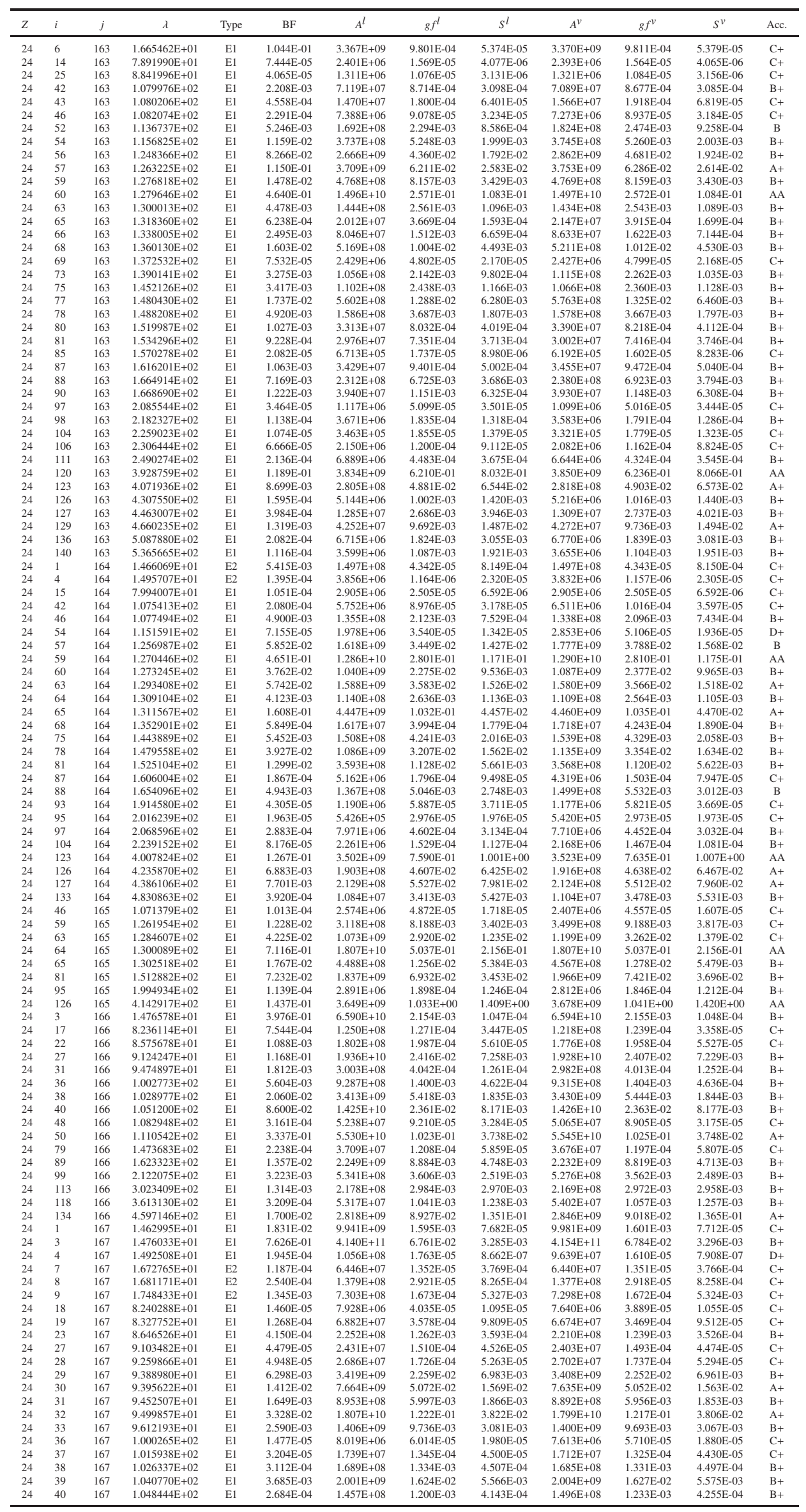




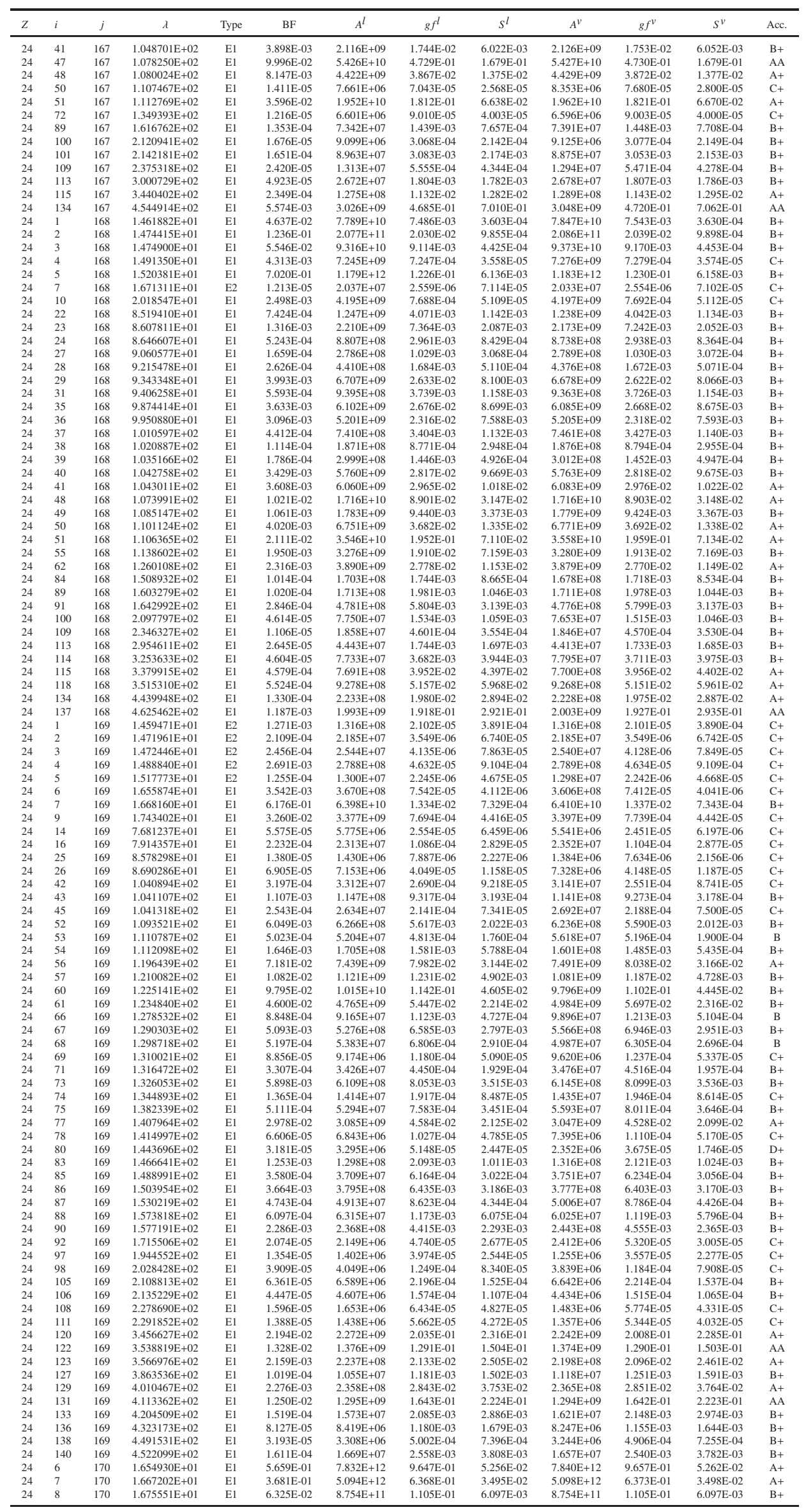




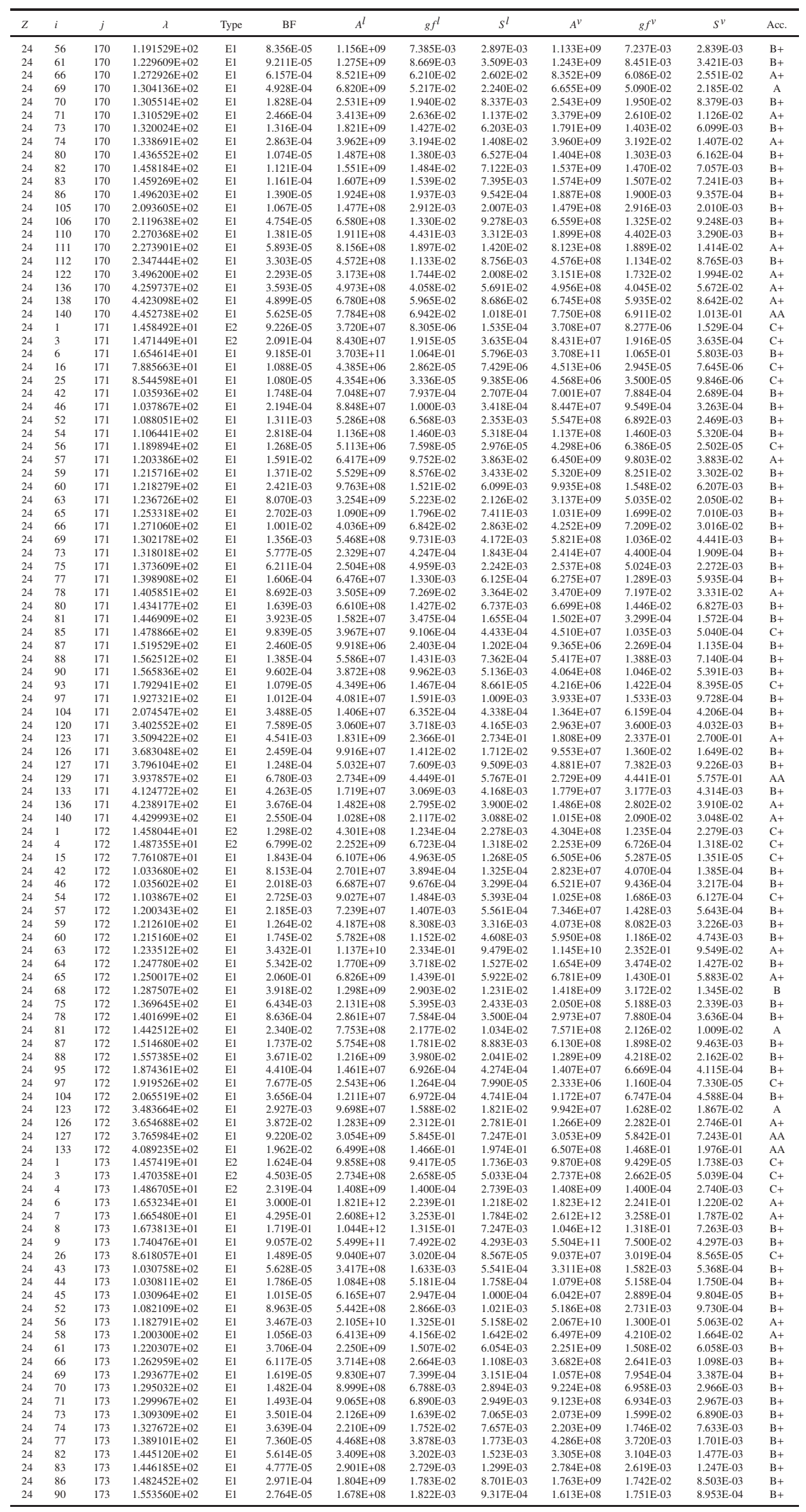




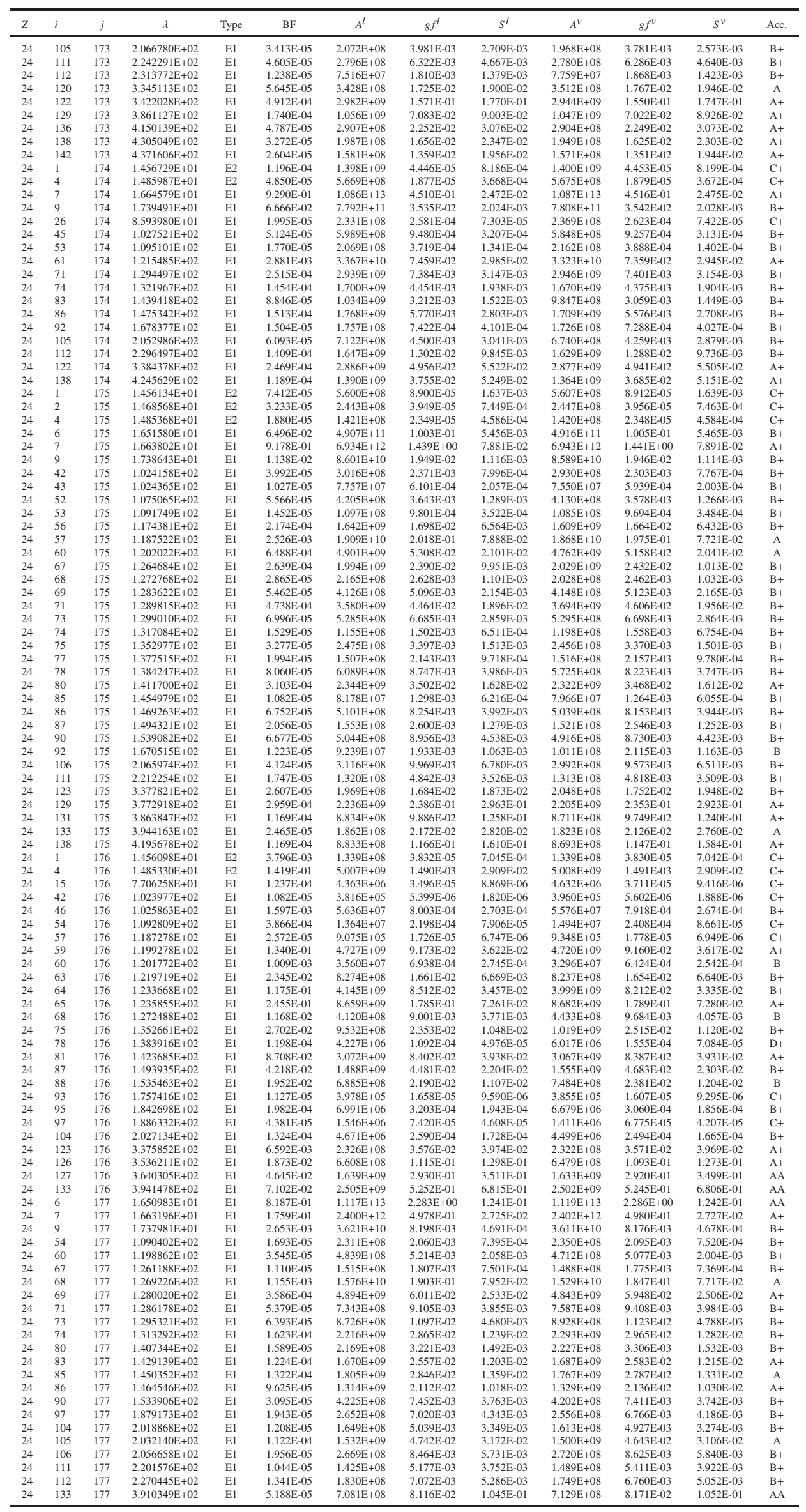




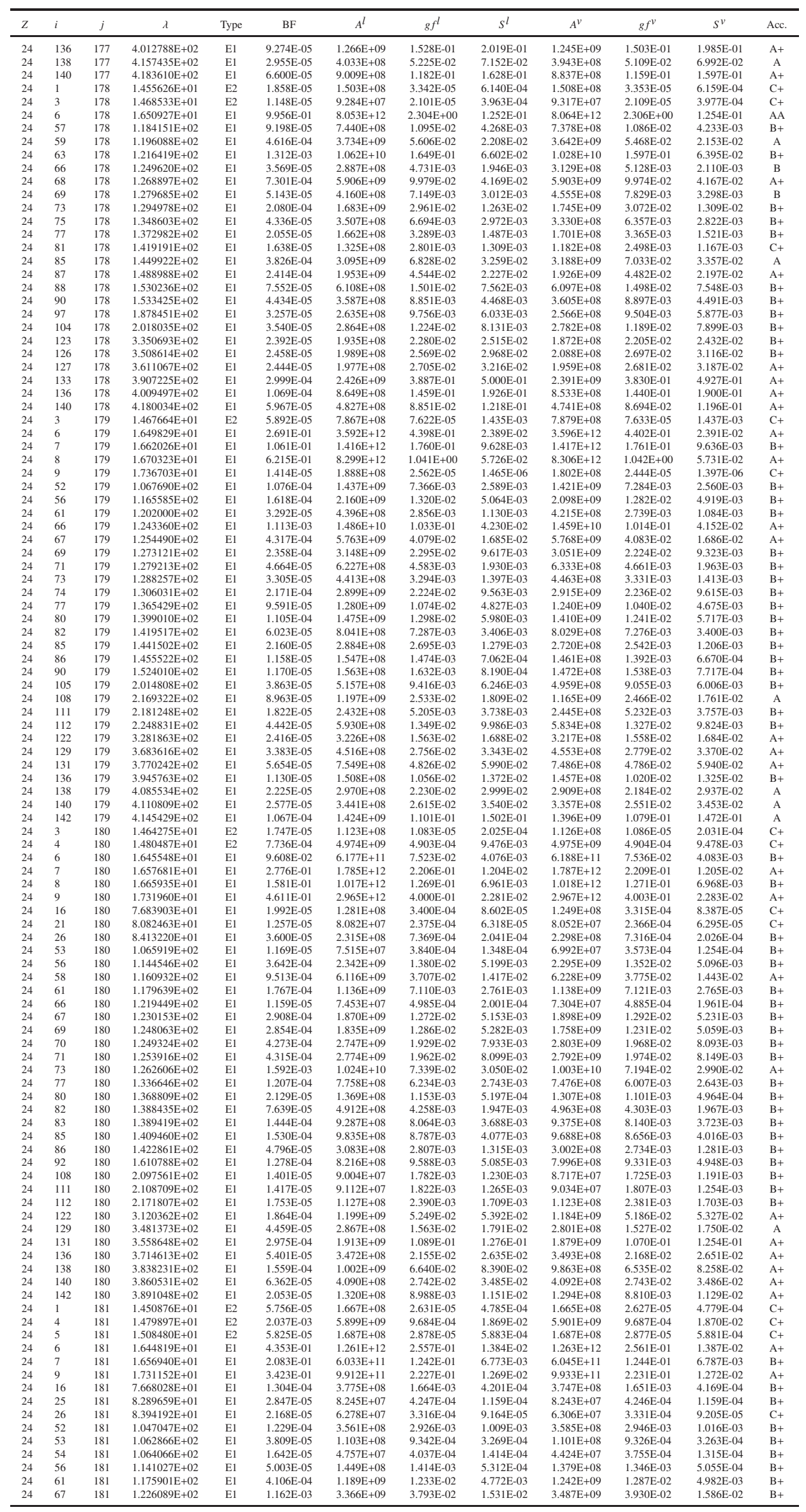




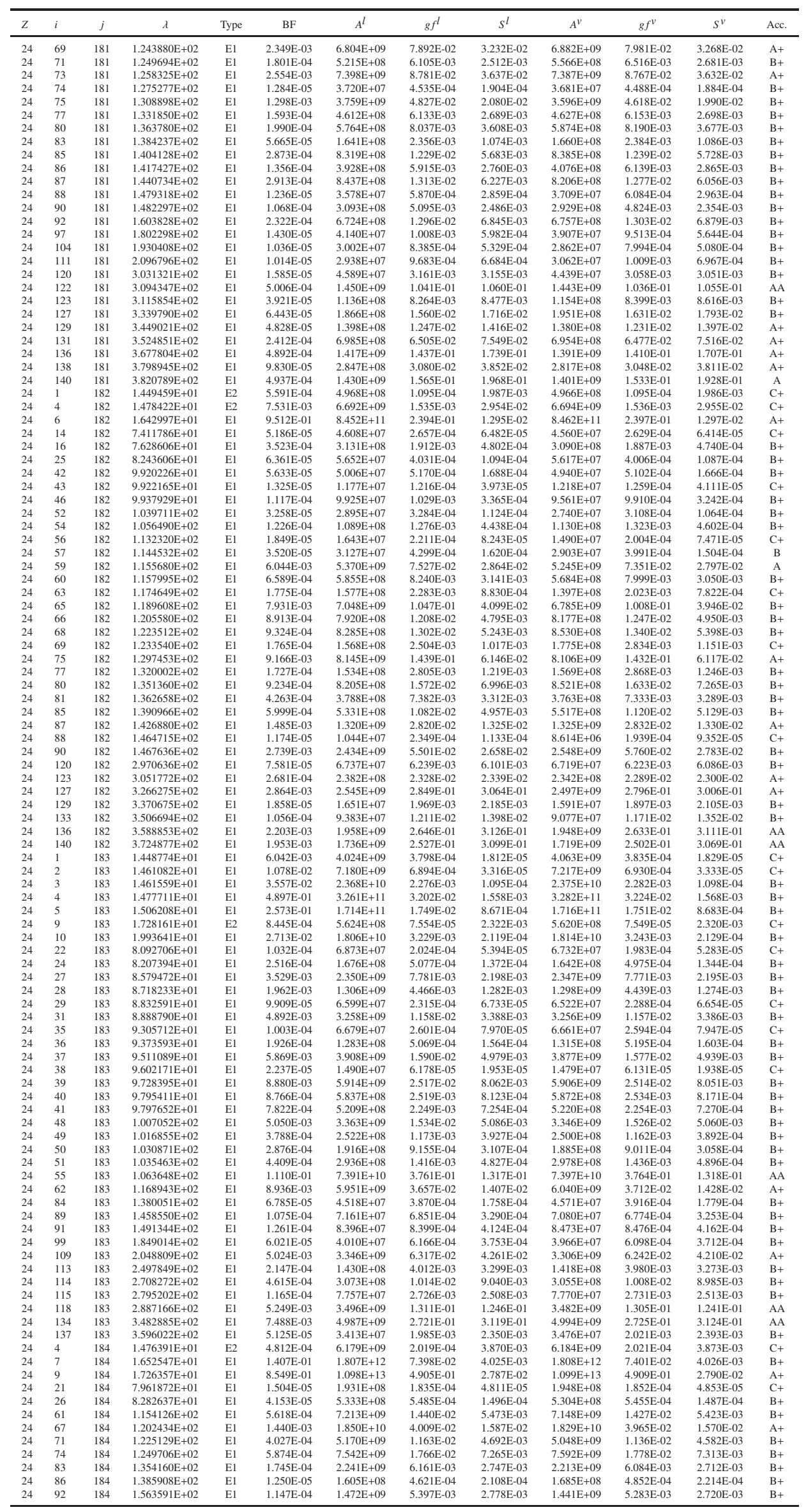




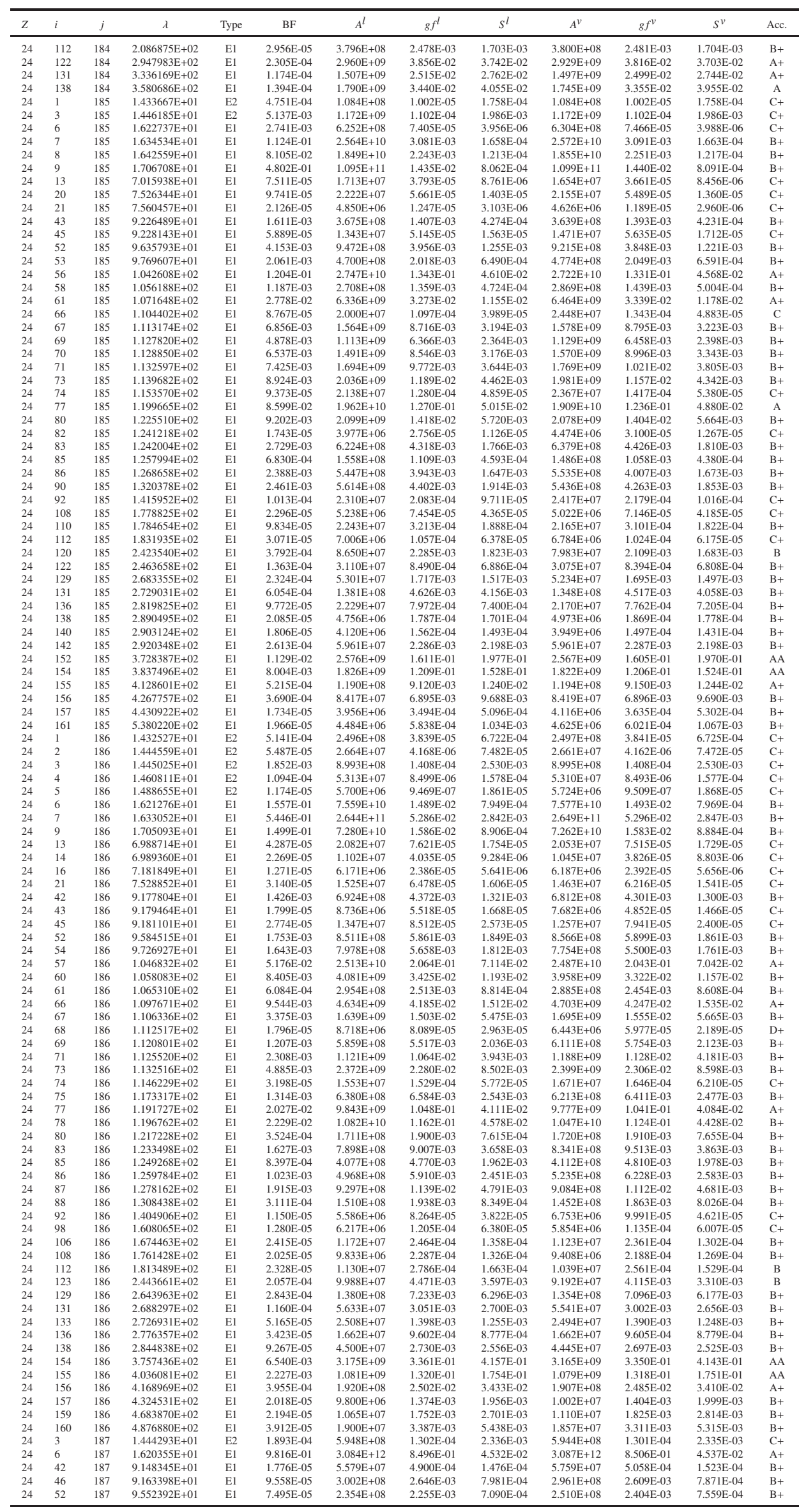




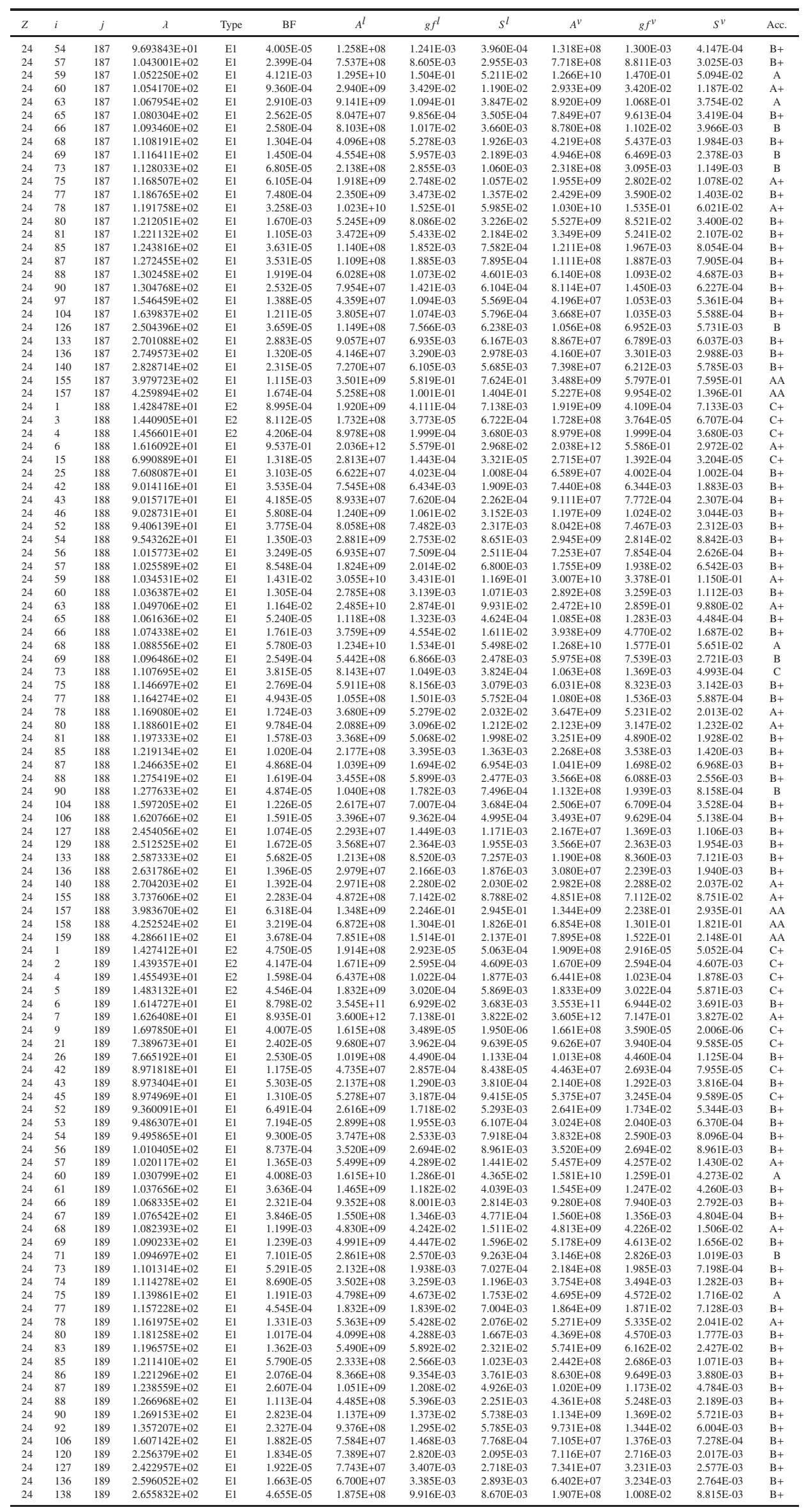




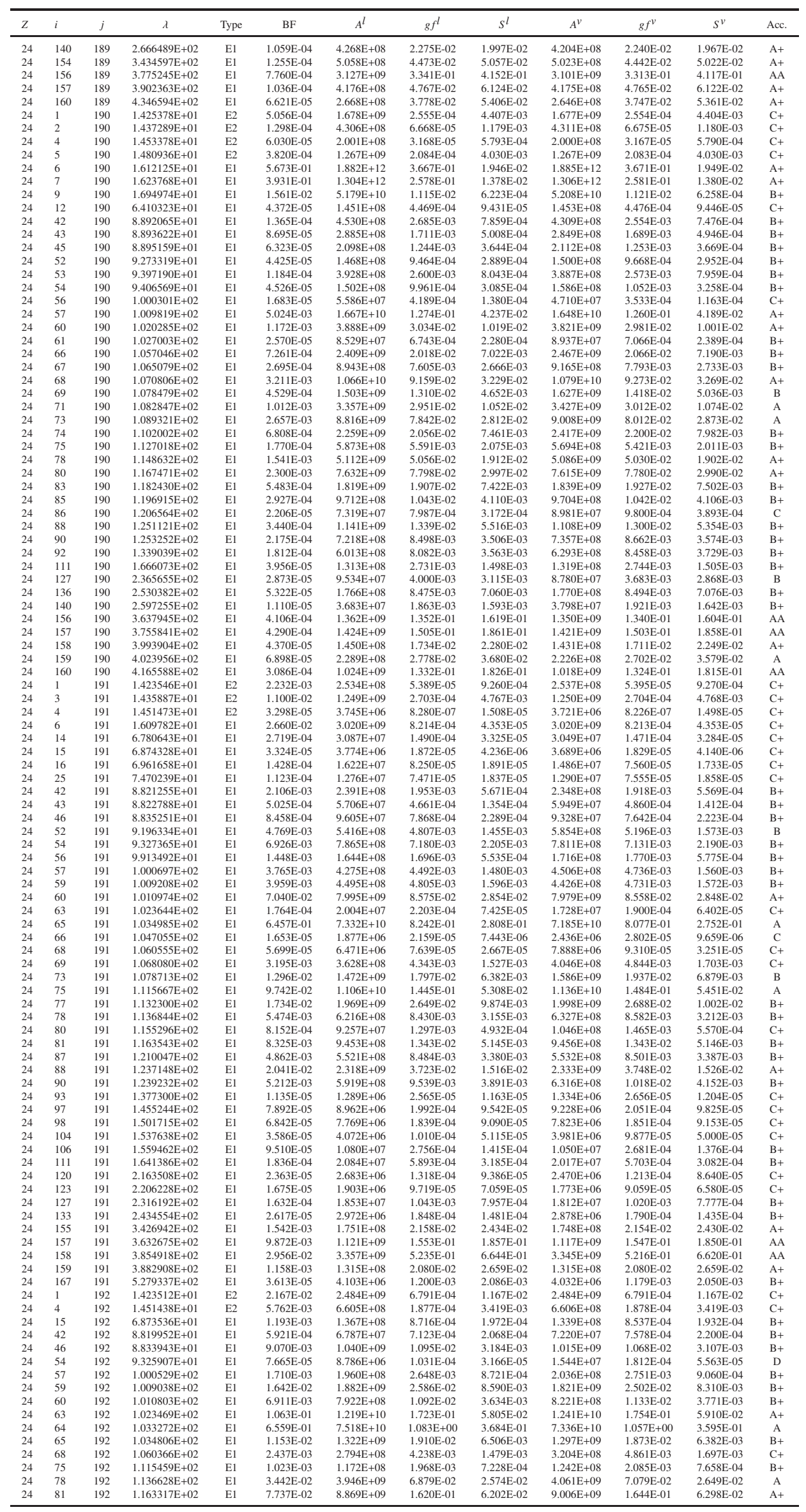




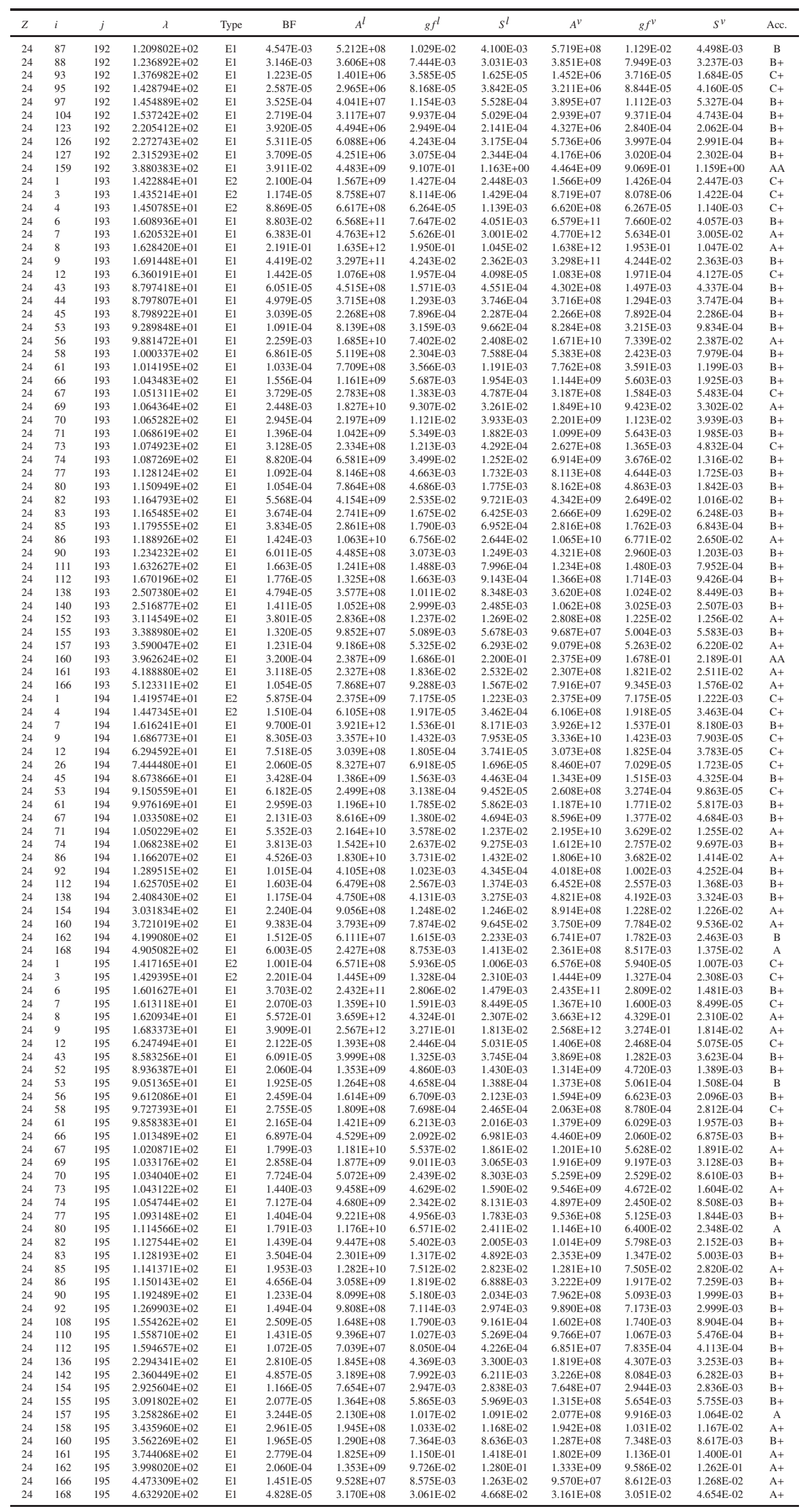




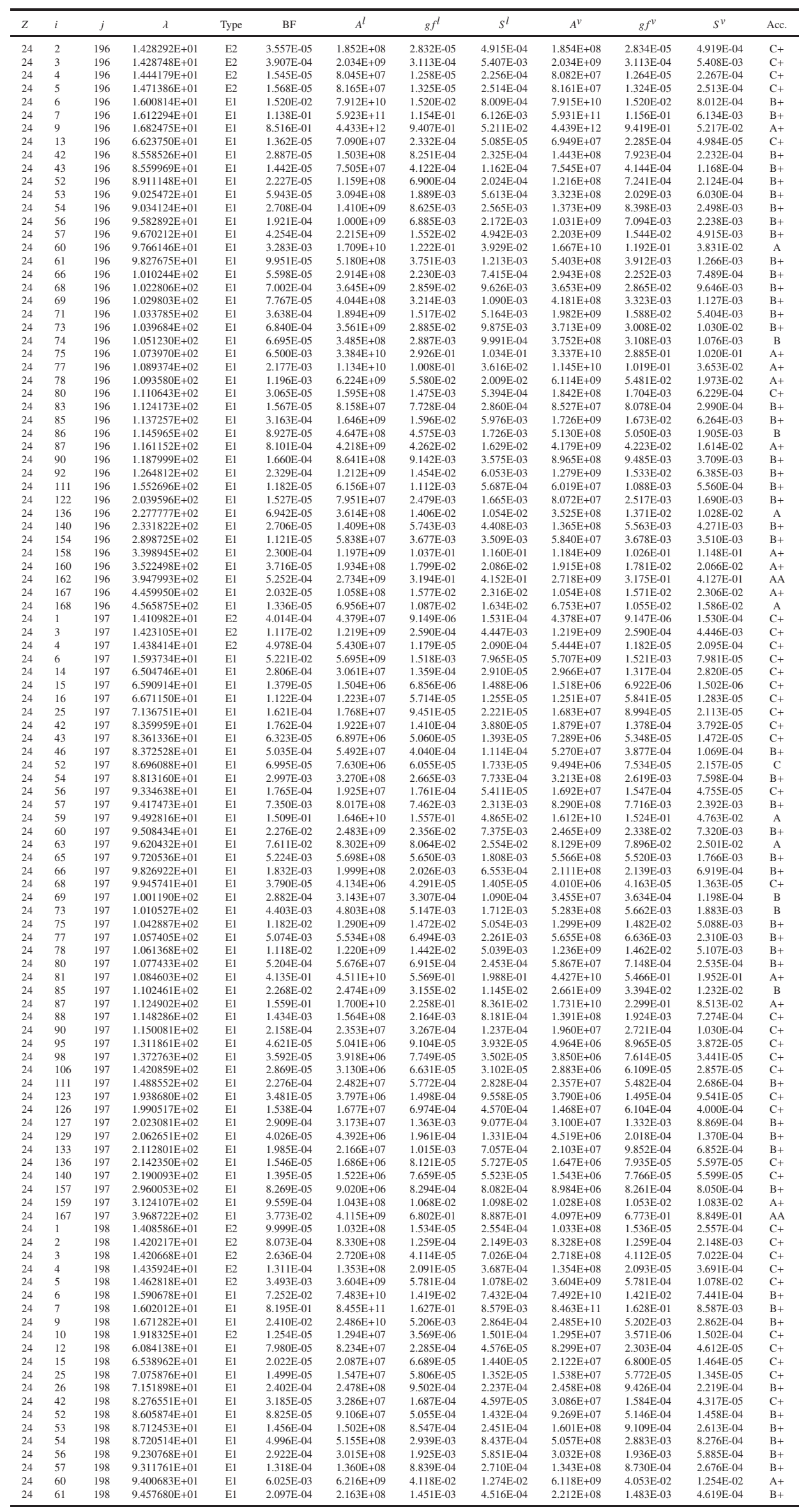




\begin{tabular}{|c|c|c|c|}
\hline Z & $i$ & $j$ & $\lambda$ \\
\hline 24 & 66 & 198 & $9.711875 \mathrm{E}+01$ \\
\hline 24 & 67 & 198 & $9.779646 \mathrm{E}+01$ \\
\hline 24 & 68 & 198 & $9.827912 \mathrm{E}+01$ \\
\hline 24 & 71 & 198 & $9.929244 \mathrm{E}+01$ \\
\hline 24 & 73 & 198 & $9.983651 \mathrm{E}+01$ \\
\hline 24 & 74 & 198 & $1.009007 \mathrm{E}+02$ \\
\hline 24 & 75 & 198 & $1.029939 \mathrm{E}+02$ \\
\hline 24 & 77 & 198 & $1.044097 \mathrm{E}+02$ \\
\hline 24 & 78 & 198 & $1.047960 \mathrm{E}+02$ \\
\hline 24 & 80 & 198 & $1.063619 \mathrm{E}+02$ \\
\hline 24 & 83 & 198 & $1.076021 \mathrm{E}+02$ \\
\hline 24 & 85 & 198 & $1.088002 \mathrm{E}+02$ \\
\hline 24 & 86 & 198 & $1.095970 \mathrm{E}+02$ \\
\hline 24 & 87 & 198 & $1.109852 \mathrm{E}+02$ \\
\hline 24 & 88 & 198 & $1.132608 \mathrm{E}+02$ \\
\hline 24 & 90 & 198 & $1.134354 \mathrm{E}+02$ \\
\hline 24 & 92 & 198 & $1.204183 \mathrm{E}+02$ \\
\hline 24 & 105 & 198 & $1.385578 \mathrm{E}+02$ \\
\hline 24 & 106 & 198 & $1.396933 \mathrm{E}+02$ \\
\hline 24 & 112 & 198 & $1.492380 \mathrm{E}+02$ \\
\hline 24 & 127 & 198 & $1.974917 \mathrm{E}+02$ \\
\hline 24 & 133 & 198 & $2.060326 \mathrm{E}+02$ \\
\hline 24 & 136 & 198 & $2.088416 \mathrm{E}+02$ \\
\hline 24 & 138 & 198 & $2.126930 \mathrm{E}+02$ \\
\hline 24 & 140 & 198 & $2.133760 \mathrm{E}+02$ \\
\hline 24 & 154 & 198 & $2.598844 \mathrm{E}+02$ \\
\hline 24 & 155 & 198 & $2.729164 \mathrm{E}+02$ \\
\hline 24 & 156 & 198 & $2.789284 \mathrm{E}+02$ \\
\hline 24 & 157 & 198 & $2.858070 \mathrm{E}+02$ \\
\hline 24 & 158 & 198 & $2.993868 \mathrm{E}+02$ \\
\hline 24 & 159 & 198 & $3.010723 \mathrm{E}+02$ \\
\hline 24 & 160 & 198 & $3.089313 \mathrm{E}+02$ \\
\hline 24 & 162 & 198 & $3.411800 \mathrm{E}+02$ \\
\hline 24 & 167 & 198 & $3.787521 \mathrm{E}+02$ \\
\hline 24 & 168 & 198 & $3.863641 \mathrm{E}+02$ \\
\hline 24 & 183 & 198 & $5.077875 \mathrm{E}+02$ \\
\hline 24 & 3 & 199 & $1.415927 \mathrm{E}+01$ \\
\hline 24 & 4 & 199 & $1.431081 \mathrm{E}+01$ \\
\hline 24 & 6 & 199 & $1.584737 \mathrm{E}+01$ \\
\hline 24 & 7 & 199 & $1.595987 \mathrm{E}+01$ \\
\hline 24 & 8 & 199 & $1.603637 \mathrm{E}+01$ \\
\hline 24 & 9 & 199 & $1.664725 \mathrm{E}+01$ \\
\hline 24 & 53 & 199 & $8.537165 \mathrm{E}+01$ \\
\hline 24 & 56 & 199 & $9.034239 \mathrm{E}+01$ \\
\hline 24 & 58 & 199 & $9.136026 \mathrm{E}+01$ \\
\hline 24 & 61 & 199 & $9.251478 \mathrm{E}+01$ \\
\hline 24 & 67 & 199 & $9.559330 \mathrm{E}+01$ \\
\hline 24 & 69 & 199 & $9.667132 \mathrm{E}+01$ \\
\hline 24 & 71 & 199 & $9.702214 \mathrm{E}+01$ \\
\hline 24 & 73 & 199 & $9.754155 \mathrm{E}+01$ \\
\hline 24 & 77 & 199 & $1.019023 \mathrm{E}+02$ \\
\hline 24 & 80 & 199 & $1.037610 \mathrm{E}+02$ \\
\hline 24 & 82 & 199 & $1.048848 \mathrm{E}+02$ \\
\hline 24 & 83 & 199 & $1.049410 \mathrm{E}+02$ \\
\hline 24 & 85 & 199 & $1.060803 \mathrm{E}+02$ \\
\hline 24 & 86 & 199 & $1.068375 \mathrm{E}+02$ \\
\hline 24 & 90 & 199 & $1.104819 \mathrm{E}+02$ \\
\hline 24 & 92 & 199 & $1.170953 \mathrm{E}+02$ \\
\hline 24 & 136 & 199 & $1.990453 \mathrm{E}+02$ \\
\hline 24 & 140 & 199 & $2.031600 \mathrm{E}+02$ \\
\hline 24 & 156 & 199 & $2.617243 \mathrm{E}+02$ \\
\hline 24 & $\begin{array}{l}157 \\
157\end{array}$ & 199 & $2.677713 \mathrm{E}+02$ \\
\hline 24 & 158 & 199 & $2.796556 \mathrm{E}+02$ \\
\hline 24 & 161 & 199 & $2.997311 \mathrm{E}+02$ \\
\hline 24 & 166 & 199 & $3.447190 \mathrm{E}+02$ \\
\hline 24 & 167 & 199 & $3.477154 \mathrm{E}+02$ \\
\hline 24 & 183 & 199 & $4.535162 \mathrm{E}+02$ \\
\hline 24 & 3 & 200 & $1.413955 \mathrm{E}+01$ \\
\hline 24 & 5 & 200 & $1.455702 \mathrm{E}+01$ \\
\hline 24 & 6 & 200 & $1.582267 \mathrm{E}+01$ \\
\hline 24 & 7 & 200 & $1.593481 \mathrm{E}+01$ \\
\hline 24 & 9 & 200 & $1.661999 \mathrm{E}+01$ \\
\hline 24 & 56 & 200 & $8.954545 \mathrm{E}+01$ \\
\hline 24 & 57 & 200 & $9.030744 \mathrm{E}+01$ \\
\hline 24 & 60 & 200 & $9.114355 \mathrm{E}+01$ \\
\hline 24 & 61 & 200 & $9.167923 \mathrm{E}+01$ \\
\hline 24 & 66 & 200 & $9.406585 \mathrm{E}+01$ \\
\hline 24 & 67 & 200 & $9.470148 \mathrm{E}+01$ \\
\hline 24 & 68 & 200 & $9.515400 \mathrm{E}+01$ \\
\hline 24 & 69 & 200 & $9.575937 \mathrm{E}+01$ \\
\hline 24 & 73 & 200 & $9.661319 \mathrm{E}+01$ \\
\hline 24 & 75 & 200 & $9.956693 \mathrm{E}+01$ \\
\hline 24 & 78 & 200 & $1.012501 \mathrm{E}+02$ \\
\hline 24 & 80 & 200 & $1.027111 \mathrm{E}+02$ \\
\hline 24 & 86 & 200 & $1.057248 \mathrm{E}+02$ \\
\hline 24 & 87 & 200 & $1.070161 \mathrm{E}+02$ \\
\hline 24 & 88 & 200 & $1.091303 \mathrm{E}+02$ \\
\hline 24 & 90 & 200 & $1.092924 \mathrm{E}+02$ \\
\hline 24 & 92 & 200 & $1.157600 \mathrm{E}+02$ \\
\hline 24 & 136 & 200 & $1.952174 \mathrm{E}+02$ \\
\hline 24 & 140 & 200 & $1.991738 \mathrm{E}+02$ \\
\hline 24 & $\begin{array}{l}162 \\
162 \quad y=1\end{array}$ & 200 & $3.062616 \mathrm{E}+02$ \\
\hline 24 & 167 & 200 & $3.361992 \mathrm{E}+02$ \\
\hline 24 & 183 & 200 & $4.341209 \mathrm{E}+02$ \\
\hline
\end{tabular}

